\title{
MAGMATISM AND DYNAMICS OF CONTINENTAL BREAKUP IN THE Presence of a Mantle Plume
}

\author{
by \\ JUN KORENAGA \\ B.Sc., University of Tokyo, 1992 \\ M.Sc., University of Tokyo, 1994 \\ Submitted in partial fulfillment of the requirements for the degree of Doctor of Philosophy \\ at the \\ MASSACHUSETTS INSTITUTE OF TECHNOLOGY \\ and the \\ WOODS HOLE OCEANOGRAPHIC INSTITUTION
}

February, 2000

(C) 2000 Jun Korenaga. All rights reserved.

The author hereby grants to MIT and WHOI permission to reproduce and to distribute copies of this thesis document in whole or in part.

Signature of Author:

Joint Progrām in Oceanography

Massachusets Institute of Technology/Wood Hole Oceanographic Institution

December, 1999

Certified by:

Thomas H. Jordan

Professor, MIT

Thesis Co-Supervisor

Certified by:

Peter B. Kelemen

Associate Scientist, WHOI

Thesis Co-Supervisor

Accepted by:

Timothy L. Grove

Chairman, Joint Committee in Marine Geology and Geophysics

Massachusetts Institute of Technology/Woods Hole Oceanographic Institution 


\title{
Magmatism and Dynamics of Continental breakup in the Presence of a Mantle Plume
}

by

\author{
Jun Korenaga
}

\begin{abstract}
Submitted in partial fulfillment of the requirements for the degree of Doctor of Philosophy at the Massachusetts Institute of Technology and the Woods Hole Oceanographic Institution
\end{abstract}

February, 2000

\begin{abstract}
This thesis studies the dynamics of mantle melting during continental breakups by geophysical, geochemical, and numerical analyses. The first part focuses on the mantle melting and crustal accretion processes during the formation of the Southeast Greenland margin, on the basis of deep-crustal seismic data. A new seismic tomographic method is developed to jointly invert refraction and reflection travel times for a compressional velocity structure, and a long-wavelength structure with strong lateral heterogeneity is successfully recovered, including 30 - to $15-\mathrm{km}$-thick igneous crust within a $150-\mathrm{km}$-wide continent-ocean transition zone. A nonlinear Monte Carlo analysis is also conducted to establish the absolute uncertainty of model parameters. The derived crustal structure is first used to resolve the origin of a margin gravity high, with new inversion schemes using both seismic and gravity constraints. Density anomalies producing the gravity high seem to be confined within the upper crust, not in the lower crust as suggested for other volcanic margins. A new robust framework is then developed for the petrological interpretation of the velocity structure of igneous crust, and the thick igneous crust formed at the continentocean transition zone is suggested to have resulted from vigorous active upwelling of mantle with only somewhat elevated potential temperature. In the second part, the nature of mantle melting during the formation of the North Atlantic igneous province is studied on the basis of the major element chemistry of erupted lavas. A new fractionation correction scheme based on the $\mathrm{Ni}$ concentrations of mantle olivine is used to estimate primary melt compositions, which suggest that this province is characterized by a large degree of major element source heterogeneity. In the third part, the nature of preexisting sublithospheric convection is investigated by a series of finite element analyses, because the strength of such convection is important to define the "normal" state of mantle, the understanding of which is essential to identify any anomalous behavior of mantle such as a mantle plume. The results suggest that small-scale convection is likely in normal asthenosphere, and that the upwelling velocity in such convection is on the order of 1$10 \mathrm{~cm} / \mathrm{yr}$.
\end{abstract}




\author{
Thesis Committee: \\ Dr. Thomas H. Jordan, Professor of Geophysics (Thesis Co-Supervisor) \\ Dr. Peter B. Kelemen, Associate Scientist, WHOI (Thesis Co-Supervisor) \\ Dr. W. Steven Holbrook, Professor of Geophysics, University of Wyoming \\ Dr. Robert S. Detrick, Senior Scientist, WHOI \\ Dr. Bradford $H$. Hager, Professor of Geophysics \\ Dr. John A. Whitehead, Senior Scientist, WHOI \\ Dr. Maria T. Zuber, Professor of Geophysics (Chair)
}




\section{Table of Contents}

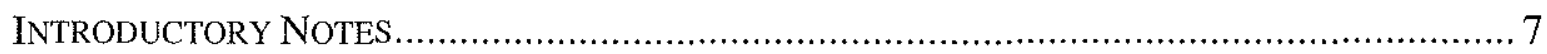

CHAPTER 1. CRUSTAL STRUCTURE OF THE SOUTHEAST GREENLAND MARGIN FROM JOINT REFRACTION AND REFLECTION SEISMIC TOMOGRAPHY .......................................... 9

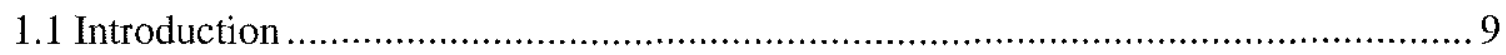

1.2 Data Acquisition and Processing............................................................. 11

1.3 Joint Refraction and Reflection Tomography ............................................ 15

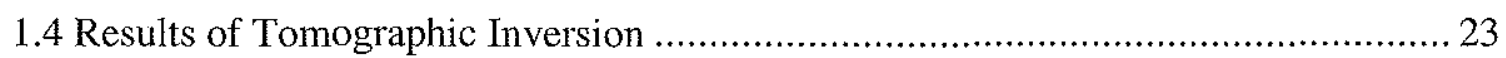

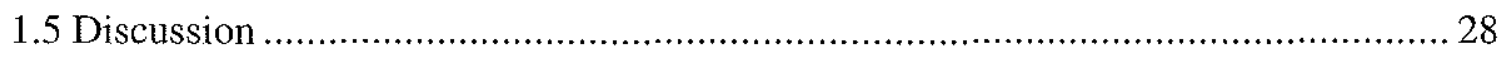

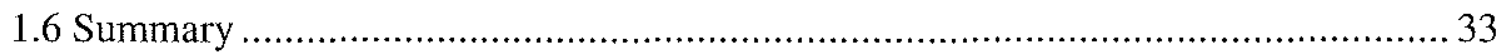

CHAPTER 2. GRAVITY ANOMALIES AND CRUSTAL STRUCTURE AT THE SOUTHEAST

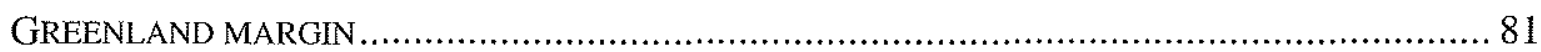

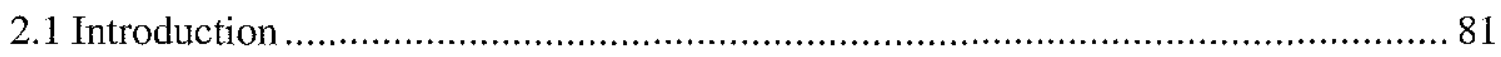

2.2 The Paradox of the Margin Gravity High ................................................. 83

2.3 Possible Origins of the Margin Gravity High ................................................. 88

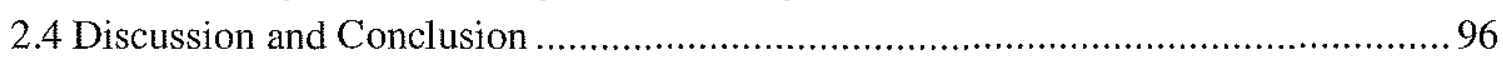

Appendix 2.A Inversion of Gravity Anomalies in the Presence of Upper and Bottom

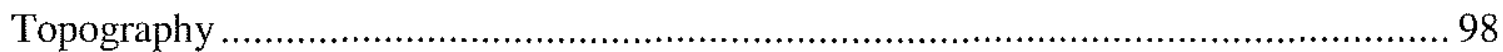

Appendix 2.B Joint Inversion of Seismic Travel Times and Gravity Anomalies.......... 99

CHAPTER 3. MANTLE MELTING AND CRUSTAL ACCRETION PROCESSES DURING THE FORMATION OF THE SOUTHEAST GREENLAND MARGIN …....................................... 119

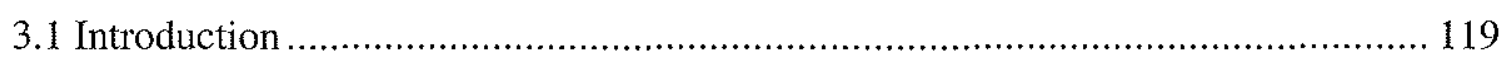

3.2 Mineralogies, Seismic Velocities and Densities of Igneous Lower Crust............. 122

3.3 Bulk Crustal Velocity of Normal Oceanic Crust............................................... 129

3.4 Mantle Melting Model with Active Upwelling Component ............................... 131

3.5 Nature of Mantle Melting During the Formation of the Southeast Greenland Margin

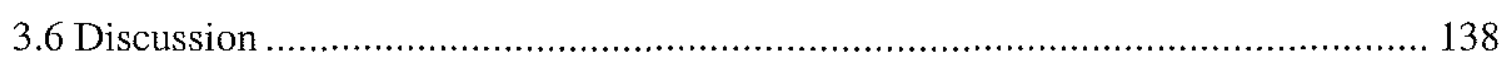

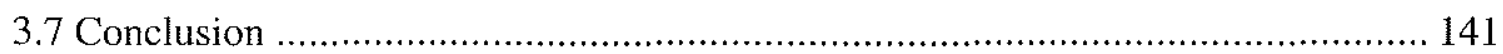

ChAPTER 4. MAJOR-ELEMENT SOURCE HETEROGENEITY IN THE NORTH ATLANTIC IGNEOUS

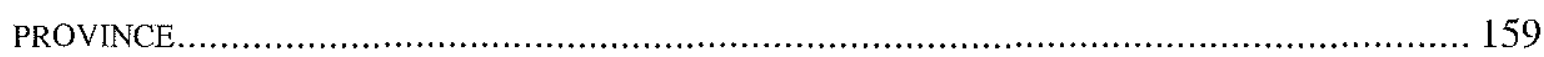

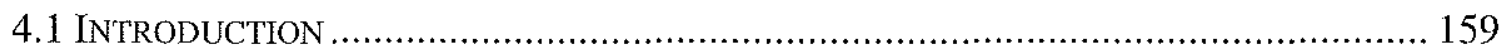

4.2 Behavior of Ni in Crustal Modification Processes .......................................... 162

4.3 Composition of Primary Mantle Melts for the North Atlantic Igneous Province ... 164

4.4 Discussion and Conclusion ...................................................................... 170

CHAPTER 5. EFFECTS OF VERTICAL BOUNDARIES ON INFINITE PRANDTL NUMBER THERMAL

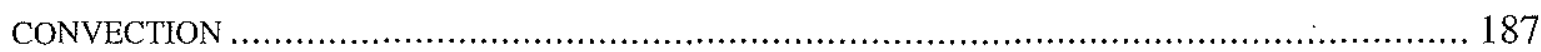




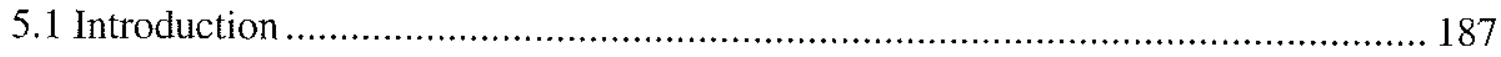

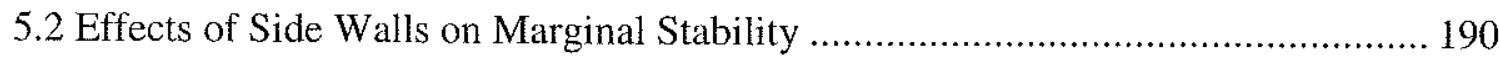

5.3 Stability of 2-D Steady-State Thermal Convection in a Bounded Fluid ................ 194

5.4 Characteristics of 3-D Convection in a Bounded Fluid ........................................... 202

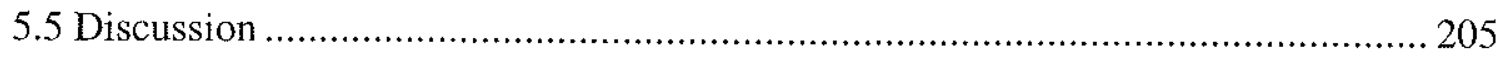

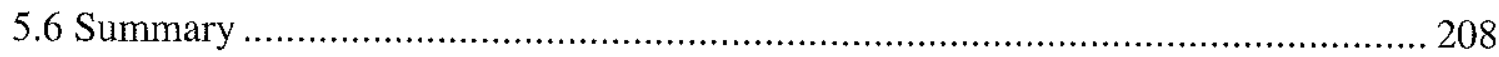

Appendix 5.A: Finite Element Formulation for 3-D Single-Mode Stokes Flow .......... 209

Appendix 5.B: Finite Element Formulation for 3-D Mean-Field Energy Equation ..... 210 CHAPTER 6. THE STRENGTH OF SUBLITHOSPHERIC MANTLE CONVECTION AND ITS BEARING

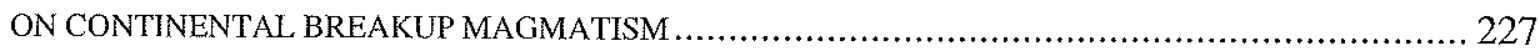

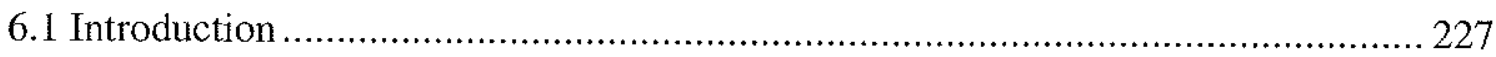

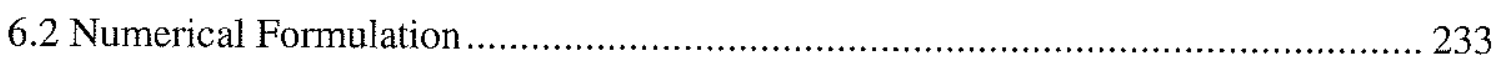

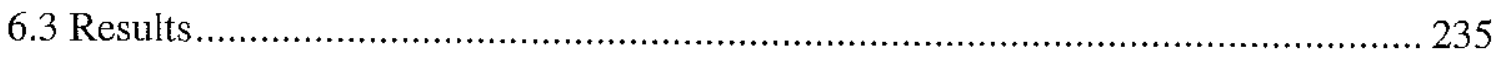

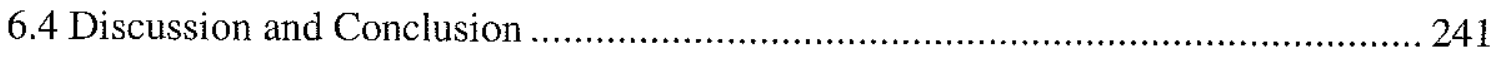

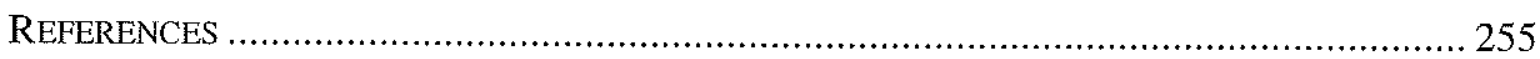

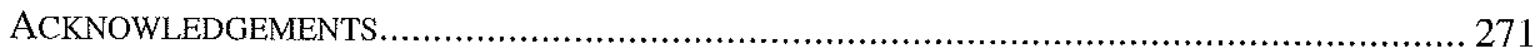




\section{Introductory Notes}

This thesis is composed of three parts, all of which are related to the dynamics of mantle melting during continental breakups. In the first part, rifting magmatism associated with the opening of the North Atlantic (at $\sim 60 \mathrm{Ma}$ ) is investigated on the basis of deep-crustal seismic data collected across the Southeast Greenland continental margin. A new seismic tomographic method is developed to jointly invert refraction and reflection travel times for a two-dimensional velocity structure (Chapter 1). Several key issues in travel time tomography such as velocity-depth ambiguity and absolute parameter uncertainty are identified, and a practical strategy is presented to resolve them. The derived crustal velocity structure is then used to investigate the origin of a conspicuous gravity high observed along the margin (Chapter 2). Two different inversion methods are implemented to utilize both seismic and gravity constraints. The igneous component of the margin crustal structure is a product of mantle melting during the opening of the North Atlantic, and a new interpretational framework is constructed to estimate the thermal and dynamical state of mantle based on the velocity structure of igneous crust (Chapter 3).

In the second part, the North Atlantic igneous province, which includes the Greenland continental margin and the currently active Iceland hotspot, is studied to derive geochemical constraints on the process of mantle melting from the major element chemistry of erupted lavas (Chapter 4). A new fractionation correction scheme is developed based on the Ni concentrations of mantle olivine, and it is used to estimate the primary melt compositions for this igneous province using a published geochemical database.

In the third part, the convective stability of asthenosphere is studied, with special attention to the influence of the heterogeneity in lithospheric structure. Because anomalous magmatism is often attributed to the anomalous state of mantle such as a mantle plume, it is important to first understand what the normal state of mantle is. The Earth's lithosphere is known to have variable thickness on a global scale, and there is a strong correlation between the distribution of cratons and the thickness of lithosphere. The effect of thick, cold cratonic lithosphere on the upper-mantle convection is first analyzed through a series

of stability analyses (Chapter 5). The convective instability of asthenosphere beneath a supercontinent is then investigated as a transient cooling problem (Chapter 6). 


\title{
Chapter 1
}

\section{Crustal structure of the Southeast Greenland margin from joint refraction and reflection seismic tomography}

\begin{abstract}
We present results from a combined multichannel seismic reflection (MCS) and wideangle on-shore/off-shore seismic experiment conducted in 1996 across the Southeast Greenland continental margin. A new seismic tomographic method is developed to jointly invert refraction and reflection travel times for a two-dimensional velocity structure. We employ a hybrid ray tracing scheme based on the graph method and the local ray-bending refinement to efficiently obtain an accurate forward solution, and smoothing and optional damping constraints to regularize an iterative inversion. We invert $2318 \mathrm{Pg}$ and $2078 \mathrm{PmP}$ travel times to construct a compressional velocity model for the $350-\mathrm{km}$-long transect, and a long-wavelength structure with strong lateral heterogeneity is successfully recovered, including (1) $-30 \mathrm{~km}$ thick, undeformed continental crust with a velocity of 6.0 to $7.0 \mathrm{~km} / \mathrm{s}$ near the landward end, (2) 30 - to $15-\mathrm{km}$-thick igneous crust within a $150-\mathrm{km}$-wide continent-ocean transition zone, and (3) 15- to 9-km-thick oceanic crust toward the seaward end. The thickness of the igneous upper crust characterized by a high velocity gradient also varies from $6 \mathrm{~km}$ within the transition zone to $-3 \mathrm{~km}$ seaward. The bottom half of the lower crust generally has a velocity higher than $7.0 \mathrm{~km} / \mathrm{s}$, reaching a maximum of 7.2 to $7.5 \mathrm{~km} / \mathrm{s}$ at the Moho. A nonlinear Monte Carlo uncertainty analysis is performed to estimate the a posteriori model variance, showing that most velocity and depth nodes are well determined with absolute errors of $0.04-0.08 \mathrm{~km} / \mathrm{s}$ and $0.25-1.0 \mathrm{~km}$, respectively. Despite significant variation in crustal thickness, the mean velocity of the igneous crust, which serves as a proxy for the bulk crustal composition, is surprisingly constant $(-7.0 \mathrm{~km} / \mathrm{s})$ along the transect. Based on a mantle melting model incorporating the effect of active mantle upwelling, this velocity-thickness relationship is used to constrain the mantle melting process during the breakup of Greenland from Europe. Our result is consistent with a nearly constant mantle potential temperature of $1270-1350^{\circ} \mathrm{C}$ throughout the rifting, but with a rapid transition in the style of mantle upwelling, from vigorous active upwelling during the initial rifting phase to passive upwelling in the later phase.
\end{abstract}

\subsection{Introduction}

Igneous crust emplaced at a rifted continental margin is the integrated product of the melting of an upwelling mantle during continental breakup. Its thickness is often taken as a good proxy for the total melt volume produced upon rifting, whereas its seismic velocity reflects the original melt composition [e.g., White and McKenzie, 1989; Kelemen and Holbrook, 1995]. Because the mantle melting process is highly sensitive to the temperature and composition of the mantle as well as its upwelling rate [e.g., McKenzie and Bickle, 
1988; Kinzler and Grove, 1992; Langmuir et al., 1992], the crustal seismic structure of a rifted margin is one of the primary sources of information regarding mantle dynamics in the rifting period.

Rifted continental margins are often associated with voluminous igneous activity as inferred from seaward dipping reflectors (SDRs), which were proposed to originate in subaerial eruptions [Hinz, 1981; Mutter et al., 1982], as later confirmed by deep-sea drilling [Roberts et al., 1984; Eldholm et al., 1989; Larsen et al., 1994]. Deep seismic studies have revealed thick sequences of mafic igneous crust below SDRs on several margins [e.g., White et al., 1987; Mutter et al., 1988; Holbrook and Kelemen, 1993]; the thickness of such igneous crust can be as much as several times that of normal oceanic crust, indicating some unusual condition in mantle melting, such as higher-than-normal potential temperature and/or rapid mantle upwelling. The Southeast Greenland margin, which was formed during the opening of the North Atlantic around $60 \mathrm{Ma}$ [Srivastava and Tapscott, 1986], is one of these volcanic rifted margins; SDRs have been mapped over $2000 \mathrm{~km}$ along the entire margin [Larsen and Jakobsdóttir, 1988], and the crustal structure of the conjugate margin [White et al., 1987; Mutter and Zehnder, 1988; Barton and White, 1997a] suggests the existence of similarly thick igneous crust beneath the SDRs. Because of its proximity to the Iceland hotspot, it has been proposed that a mantle plume played a major role in the formation of this volcanic margin [e.g., Richards et al., 1989; White and McKenzie, 1989; Hill et al., 1992; Coffin and Eldholm, 1994; Barton and White, 1997a], but the paucity of high-quality seismic data in this region has prevented a full understanding of the influence of a mantle plume on continental rifting magmatism.

To investigate the influence of the Iceland hotspot on the opening of the North Atlantic, an extensive deep seismic experiment, the 1996 Seismic Investigation of the Greenland Margin (SIGMA), was conducted along the Southeast Greenland margin jointly by Woods Hole Oceanographic Institution (WHOI) and the Danish Lithosphere Center. Four transects were located at latitudes of approximately $68^{\circ} \mathrm{N}, 66^{\circ} \mathrm{N}, 63^{\circ} \mathrm{N}$ and $59^{\circ} \mathrm{N}$, respectively, to systematically sample the structure of the margin at increasing distance from the Greenland-Iceland Ridge, the presumed Iceland hotspot track (Figure 1-1). This 
paper focuses on results from transect 2, whose data comprise about $300 \mathrm{~km}$ of multichannel seismic reflection data and coincident wide-angle refraction and reflection data recorded on seventeen ocean-bottom and nine onshore seismometers, covering from the Greenland coast through the continental shelf to the deep-water ocean basin at magnetic anomaly chron 19 . Unlike the two transects to the south, linear marine magnetic anomalies are not observed on the northwestern part of transect 2 (Figure 1-1b), suggesting a significant deviation from normal seafloor spreading. Owing to its proximity to the hotspot track, the extent of rifting-to-spreading magmatism on this transect is critical to assess the possible effects of a mantle plume.

In this paper, we develop a joint refraction and reflection tomographic method to invert wide-angle travel time data for a compressional velocity field. The forward travel time calculation takes a hybrid approach; a graph-theoretical method is used to ensure a global optimization, followed by ray bending refinement to achieve the desired accuracy. The inversion with a fine model parameterization is regularized by smoothness constraints on both velocity and reflector nodes. Velocity-depth ambiguity is always a serious concern for reflection tomography, and we show a simple and practical way to investigate the ambiguity using depth kernel weighting. The absolute uncertainty of a velocity model is also estimated by a nonlinear Monte Carlo approach. The crustal structure of the volcanic continental margin, which exhibits strong lateral heterogeneity both in velocity and crustal thickness, is shown to be fully resolved with high model fidelity, and we discuss its geological and geophysical implications.

\subsection{Data Acquisition and Processing}

The seismic data on transect 2 of the SIGMA experiment were acquired in September 1996 aboard R/V Maurice Ewing. Multi-channel seismic (MCS) profiling was conducted using a 20 -element, $8460 \mathrm{cu}$. in. airgun array fired every $-50 \mathrm{~m} \mathrm{(20} \mathrm{s)} \mathrm{and} \mathrm{a} \mathrm{4.0-km} \mathrm{hydrophone}$ streamer with 160 channels. Wide-angle data were recorded on ten WHOI ocean bottom hydrophones (OBH), eight U.S. Geological Survey (USGS) ocean bottom seismometers (OBS), and nine IRIS/PASSCAL on-land seismic recorders deployed along the transect 
(Figure 1-2). We will hereafter refer to model distance along the transect, which is measured from a point located about $5 \mathrm{~km}$ northwest of the most landward seismometer, ST 9. The total model distance of transect 2 is $350 \mathrm{~km}$.

\subsubsection{Multichannel Seismic Data}

Shot gathers were recorded at a sampling interval of $4 \mathrm{~ms}$. Streamer group spacing was $25 \mathrm{~m}$, which gives a common mid point (CMP) spacing of $12.5 \mathrm{~m}$. With 160 channels and a shot interval of $50 \mathrm{~m}$, CMP gathers sorted from raw shot gathers are thus 40 fold at maximum. After band-pass filtering from 5 to $40 \mathrm{~Hz}$ and a spherical divergence correction, stacking velocity analysis was conducted with 4-CMP supergathers. In addition to strong seafloor and basement reflections, several sedimentary reflections were available to estimate stacking velocities. There are a few sub-basement reflectors such as SDRs for some locations, but the majority of data do not exhibit coherent reflections below the basement. We thus used a velocity model based on wide-angle seismic data to supply stacking velocities for the crustal section. Because of severe sea conditions during the shooting of transect 2, the MCS data are only of modest quality, suffering from inconsistent gun signature, missing traces, and high ambient noise. Even with constant velocity stack analysis, no clear Moho reflection was identified throughout the transect. The following analysis, therefore, focuses only on the imaging of shallow crustal structure.

Our major task in MCS data processing was multiple suppression. Though it is not imperative for the deep-water environment where the first water multiple occurs far below the basement, contamination by strong water multiples becomes more problematic as the seafloor shallows from $\mathrm{km} 250$ landward on the transect. Unfortunately, the shallower bathymetry coincides with the occurrence of the SDR sequence, which is almost completely overlapped by high-energy water multiples. We first tried predictive deconvolution both in the $t-x$ and $\tau-p$ domains with little success, probably because of low data quality. We found that the $f$ - $k$ demultiple method [e.g., Yilmaz, 1987] was more robust with negligible transform artifacts. To avoid aliasing, we applied the $f-k$ multiple suppression to 4-CMP supergathers with optional zero traces filling in missing offsets. We further applied a $2-\mathrm{km}$ inside mute below the basement reflection, because water multiples 
tend to be more coherent at smaller offsets in CMP gathers. Finally, a stacked time section was produced with median stacking, which turned out to be very effective in reducing any residual multiple energy; this is mainly because of a differential moveout between primary reflections and water multiples.

To correctly relocate reflectors in the depth section and collapse diffraction hyperbolae due to rough reflector topography, post-stack depth migration was performed using the split-step migration algorithm [Stoffa et al., 1990]. The velocity model based on the wide-angle data was used again for the crustal section in the migration. Except for several migration artifacts originating in missing data spots on the landward side, the overall quality of the migrated image is good, clearly depicting the sedimentary layers, the basement topography, and the intra-basement SDR sequence (Figure 1-3).

Sedimentary layers start to appear at $\mathrm{km} 113$ and gradually thicken seaward, reaching a maximum thickness of about $1.5 \mathrm{~km}$ around the shelf break. The deep-water ocean basin is covered with less than 1-km-thick sediments. A distinct wedge of SDRs can be seen from $\mathrm{km} 190$ to $\mathrm{km} \mathrm{235;} \mathrm{the} \mathrm{reflectors} \mathrm{are} \mathrm{divergent-arcuate} \mathrm{with} \mathrm{dips} \mathrm{steepening}$ from subhorizontal at shallow crustal levels to $-4^{\circ}$ toward their base, and they can be traced to at least $4 \mathrm{~km}$ below the basement. The seaward end of the SDR sequence is abrupt, and it is marked by a basement high centered at $\mathrm{km} 240$. The basement topography becomes more irregular seaward of the basement high, suggesting a transition from subaerial volcanism with extensive lava flows to more restricted submarine volcanism at this location. The continental side of the main SDR sequence may continue subhorizontally to $\mathrm{km} 120$, though its reflectivity is more subdued. Apart from this, there is a strong dipping reflector running from $\mathrm{km} 80-150$ (Reflector A in Figure 1-3). Its reflectivity is unusually intense compared with other SDRs on this transect, generating its own strong water multiples. It is clear from its two-way travel times that the reflection is neither a seafloor multiple nor a basement multiple, so it must represent some sort of unconformity. The strong reflectivity, together with the lack of concurrent reflectors, suggests that it may represent a tectonic breakup unconformity as suggested by Larsen and Duncan [1996] for a similar reflector in the nearby EG66 seismic transect. However, as we will demonstrate 
with a tomographic image later, the reflector lies in the middle of the thick volcanic layer characterized by a high velocity gradient, so that its origin remains unresolved.

\subsubsection{Wide-Angle Seismic Data}

Most of the wide-angle data on transect 2 are of high quality (Figure 1-4). The sampling interval was $10 \mathrm{~ms}$, and $5-14 \mathrm{~Hz}$ band-pass filtering was applied to the data followed by predictive deconvolution and coherency weighting. Air gun firing at a 20 s interval at 4.7 knots yields densely spaced seismic traces at $50 \mathrm{~m}$, and resultant high phase correlation among traces is essential for accurate phase identification, especially for later arrivals such as $P m P$. One drawback of the short shooting interval is, however, that the reverberation of seismic energy in the water column can appear as strong "previous shot noise" [Nakamura et al., 1987]. Though the air gun firing adapted a shot time randomization of $\pm 1 \mathrm{~s}$ to reduce inter-trace coherency of the waterborne energy, the sheer intensity of previous shot noise can be a major annoyance in a deep-water environment where water multiples can propagate to large offsets with little attenuation. To mitigate this problem, additional shooting with a $70 \mathrm{~s}$ interval was conducted for the wide-angle data acquisition on this transect. Without substantially sacrificing phase correlation, the effect of previous shot noise is significantly reduced in the $70 \mathrm{~s}$ shot data, which were especially useful for phase interpretations with deep-water instruments.

The refraction phase through the crystalline crust, $P g$, is observed on all the instruments. The pattern of the $P g$ arrival varies significantly from deep-water instruments to shallow-water and on-land instruments, indicating a strong lateral variation in the crustal structure along the transect. The maximum range of the $P g$ arrivals increases from $-70 \mathrm{~km}$ in deep water to $\sim 150 \mathrm{~km}$ along the inner continental shelf, indicating landward thickening of the high-velocity-gradient upper crust. The near-offset asymmetry observed in $P g$ arrivals on $\mathrm{OBH} 20$ suggests a sharp transition in the shallow upper crustal velocity at $\mathrm{km} \sim 80$, because of the nearly flat basement and absence of sediment cover around the instrument. The reflection phase from the crust-mantle boundary, $P m P$, is observed on most of the instruments with variable quality. The smallest offset where $P m P$ arrivals can be recognized ranges from $-20 \mathrm{~km}$ in the deep ocean basin to $60-80 \mathrm{~km}$ on the continental 
shelf, and most of the $P m P$ arrivals can be traced to merge with the $P g$ arrivals. Several minor mid-crustal reflections are also observed on some instruments, but we do not attempt to model them. Being a later arrival, the $P m P$ phase tends to be obscured by source-generated noise, and it is also sometimes difficult to distinguish it from mid-crustal reflections and their multiples. The self-consistent identification of the $P m P$ phase was made possible by confirming reciprocity among a number of different source-receiver pairs. For the on-land stations, however, reciprocity cannot be confirmed owing to the lack of land sources, and we relied on the phase correlation among the closely located stations. Refraction and reflection travel times were picked manually, and we assigned a rather conservative picking error, varied from 50 to $200 \mathrm{~ms}$, to each picked travel time.

\subsection{Joint Refraction and Reflection Tomography}

\subsubsection{Model Parameterization}

A two-dimensional velocity model is parameterized as a sheared mesh hanging beneath seafloor and land surface [Toomey et al., 1994; Van Avendonk et al., 1998]. Bilinear interpolation is used in each parallelogram-shaped grid cell, so the velocity field is continuous everywhere. Nodal spacing can be variable both in the horizontal and the vertical directions, and it should be much finer than expected velocity variations, to avoid any bias introduced by a coarse parameterization. A fine mesh is also important to obtain accurate graph theoretical solutions. The sheared mesh representation allows accurate travel time calculation in the presence of large topographic variations, with much smaller computational resources than a rectangular grid [e.g., White and Clowes, 1990; Zhang and Toksöz, 1998].

A reflector is represented as an array of linear segments, whose nodal spacing is independent of that used in the velocity grid. The horizontal coordinate of reflector nodes is fixed so that each node has only one degree of freedom in the vertical direction. Currently only one reflector is supported in our method, which we used to model the $P m P$ phase. Although the velocity discontinuity at the Moho is fundamental for the generation of the $P m P$ phase, we do not explicitly treat this discontinuity in our modeling. With no $P n$ 
phase observed in our data, there is no information regarding the velocity beneath the Moho, so it is arbitrary how to assign a velocity discontinuity in the velocity grid. We therefore employ a floating reflector formulation as the simplest choice; a reflector can be updated freely without changing adjacent velocity nodes.

\subsubsection{Forward Problem}

The accurate and efficient calculation of travel times and ray paths is essential in seismic tomography. With a sampling interval of $10 \mathrm{~ms}$ and an average spacing of a few hundred meters in a velocity grid, our target accuracy is $1 \mathrm{~ms}$ in travel times and $100 \mathrm{~m}$ in ray path positions. We employ a hybrid method based on the graph method and the ray-bending method, similar to the one developed by Papazachos and Nolet [1997] and Van Avendonk et al. [1998], because it is probably most efficient in terms of both memory and computation time. The graph method, also known as the shortest path method, was developed in network theory [e.g., Dijkstra, 1959; Gallo and Pallottino, 1986], and it can calculate the shortest connections from an origin node to all other nodes in a network. If a seismic travel time between nodes is used as a nodal distance, this global optimization scheme can be used to generate a set of first-arrival travel times and corresponding ray paths [Nakanishi and Yamaguchi, 1986; Moser, 1991], and it has recently become popular in refraction tomography [Toomey et al., 1994; Zhang and Toksöz, 1998]. The calculation of later arrivals such as reflection phases can be formulated as a two-step application of the graph method [Moser, 1991], and it has been used in refraction and reflection tomography [Zhang et al., 1998].

One drawback of the graph method is that the representation of a ray path is restricted by a forward star, which defines the structure of possible node connections [Matarese, 1993; Van Avendonk, 1998; Zhang and Toksöz, 1998]. In a graph solution, therefore, seismic rays tend to zigzag, and travel times are often overestimated. Instead of using a higher-order forward star and a finer mesh to improve the accuracy, we use the raybending method [Um and Thurber, 1987; Prothero et al., 1988; Moser et al., 1992] to

refine a graph solution because we need this extra refinement only for a small portion of node connections in an entire network, i.e., actual source-receiver pairs used in a seismic 
experiment. A graph solution serves as a good initial guess, which is required for the raybending refinement to converge to the global minimum.

The ray-bending method can converge more rapidly if a better initial guess is given, so that there is a trade-off between computation times used in the graph method and the bending method. The graph solution with a higher-order forward star provides a better solution with extra computation time, and the order of the forward star that is optimal for this hybrid calculation depends on the node spacing of a velocity model and its complexity. We also note that, for a typical crustal velocity model in which vertical velocity gradient dominates horizontal one, a forward star that preferentially searches the downward direction is superior to an isotropic forward star with the same number of nodes [Van Avendonk, 1998; Van Avendonk et al., 1999]. In our case, we found that a mixed 5-th/10-th order forward star is sufficient for a rapid convergence in the bending refinement. Because the water column is outside the sheared mesh representation of a velocity model, a graph solution would be incomplete without supplying connections between marine sources and seafloor nodes. By Fermat's principle, an entry point for a ray starting from a particular marine source can be found by searching all seafloor nodes for a connection with the minimum travel time [e.g., Toomey et al., 1994].

We then apply the ray-bending procedure developed by Moser et al. [1992], which employs the conjugate gradient method to directly minimize the travel time along a ray path. Rays are parameterized as beta-splines, which can express a variety of curves with a small number of control points, thereby enhancing the convergence in a conjugate gradient search. The nodes used in a graph solution are used as initial control points for betasplines, with an optional dissection for too a long ray segment resulting from the use of a high-order forward star. A constrained conjugate gradient search is used to handle interfaces such as seafloor and a reflector [Moser et al., 1992].

Figure 1-5 shows an example from benchmark tests of our ray tracing code. A 1-D velocity profile representing a typical oceanic crust is used in this example. Analytical ray paths and travel times are calculated based on ray theory [e.g., Aki and Richards, 1980]. Three types of numerical ray tracing are compared: (1) the graph method with a velocity 
grid of $1 \times 0.25 \mathrm{~km}$ cells, (2) the graph method followed by the ray-bending refinement, with the same velocity grid, and (3) the graph method with a velocity grid of $0.25 \times 0.125 \mathrm{~km}$ cells. Errors in travel time and ray path are summarized in Figure $1-5 \mathrm{~g}-\mathrm{j}$. In the purely graph method applications, the ray path error is bounded by the characteristic spacing of the velocity model, but the travel time error exceeds the target accuracy of $1 \mathrm{~ms}$, which is achieved in the succeeding application of the bending method. Though the pure graph method with a finer grid can be as accurate as the hybrid method, it takes more than ten times more computation time in this example. Because the computation time used in the bending method is proportional to the number of rays, the computational efficiency will change with a different experiment geometry. It seems, however, that the hybrid approach is generally more compact both in memory and computation time, for typical experimental configurations employed in crustal seismology.

\subsubsection{Inverse Problem}

Given an initial reference velocity model, refraction travel time residuals $\delta T_{j}$ can be mapped into slowness perturbations $\delta u$ along the ray paths in a reference model, using the following path integral,

$$
\delta T_{j}=\int_{\Gamma_{j}} \delta u d \Gamma .
$$

Similarly, reflection travel time residuals can be written in the 2-D Cartesian coordinates as,

$$
\delta T_{j}=\int_{\Gamma_{j}} \delta u d \Gamma+\left.\frac{\partial T}{\partial z}\right|_{x=x_{j}} \delta z\left(x_{j}\right),
$$

where $x_{j}$ is the reflecting point of the $j$-th ray. By the finite dimensional approximation of velocity and reflector models, equations 1.1 and 1.2 can be written collectively as

$$
\mathbf{d}=\mathbf{G} \delta \mathbf{m},
$$

where $\mathbf{d}$ is the travel time residual vector, $\mathbf{G}$ is the Fréchet derivative matrix, and $\delta \mathbf{m}$ is the unknown model perturbation vector. This matrix equation forms the foundation of our travel time tomography. The velocity sensitivity part of the Fréchet matrix is simply a path length distributed to relevant velocity nodes according to the bilinear interpolation used for 
slowness interpolation. The depth sensitivity part can be expressed using an incident angle upon reflection, the slope of a reference reflector, and the velocity at the reflecting point as derived in Bishop et al. [1985]. When a starting model is far from the true model, the above linearized inversion must be applied iteratively until the model converges.

The uncertainty of observed travel times is usually nonuniform, and it can be expressed in the data covariance matrix $\mathbf{C}_{d}$. Though errors in hand-picked travel times can be correlated to some extent, no correlation in data error is assumed in $\mathbf{C}_{d}$ for simplicity. This simplified representation of data error does not pose a major problem, because we use our iterative inversion merely as an efficient solution finder and will quantify the model covariance by the nonlinear Monte Carlo method. For a controlled-source experiment in crustal seismology, there is often no significant a priori information available for the velocity structure of a study area, so the use of the a priori model covariance matrix is highly limited. Nonetheless, the normalization of model parameters is beneficial to minimize a possible solution bias due to the magnitude of reference model parameters. Equation 1.3 is thus normalized through the following relations,

$$
\begin{aligned}
\mathbf{G}^{\prime} & =\mathbf{C}_{d}^{-1 / 2} \mathbf{G C}_{m}^{-1 / 2} \\
\delta \mathbf{m}^{\prime} & =\mathbf{C}_{m}^{-1 / 2} \delta \mathbf{m} \\
\mathbf{d}^{\prime} & =\mathbf{C}_{d}^{-1 / 2} \mathbf{d}
\end{aligned}
$$

where the model scaling matrix $\mathbf{C}_{m}$ is simply a diagonal matrix whose elements are each a square of a model parameter. Primes will be dropped hereafter, and this normalization is always assumed.

With the typical resolving power of observed travel time data, a fine model parameterization prohibits inversion of equation 1.3 in its simplest form because of severe singularity, and we need to somehow regularize the linear system to stabilize the inversion. In the absence of a priori information on the scale of velocity variations, the only reasonable way appears to be to restrict the model space with some arbitrary additional constraints [cf. Scales and Snieder, 1997]. We choose to apply smoothness constraints on both velocity and depth perturbations using pre-defined correlation lengths. Because a fully 2-D smoothing constraint on velocity perturbations results in a rather dense matrix 
that requires large memory storage, we use two 1-D smoothing constraints, one each for horizontal and vertical directions [Toomey et al., 1994]. A regularized linear system can then be written as,

$$
\left[\begin{array}{c}
\mathbf{d} \\
\mathbf{0} \\
\mathbf{0} \\
\mathbf{0}
\end{array}\right]=\left[\begin{array}{cc}
\mathbf{G}_{v} & w \mathbf{G}_{d} \\
\lambda_{v} \mathbf{L}_{1 \mathrm{k}} & \mathbf{0} \\
\lambda_{v} \mathbf{L}_{\mathrm{v}_{v}} & \mathbf{0} \\
\mathbf{0} & w \lambda_{d} \mathbf{L}_{d}
\end{array}\right]\left[\begin{array}{c}
\delta \mathbf{m}_{v} \\
\frac{1}{w} \delta \mathbf{m}_{d}
\end{array}\right],
$$

where subscripts $v$ and $d$ for the Fréchet matrix and the model vector denote their velocity and depth components, respectively, and where $\lambda_{v}$ and $\lambda_{d}$ control the relative importance of the smoothing constraints with respect to the data resolution. $\mathbf{L}_{\mathrm{H} v}$ and $\mathbf{L}_{\mathrm{V}_{v}}$ are the horizontal and vertical smoothing matrices for slowness perturbations, respectively, and $\mathbf{L}_{d}$ is the smoothing matrix for depth perturbations. The depth-kernel weighting parameter, $w$, adjusts the relative weighting of depth sensitivity in the Fréchet matrix. Gaussian smoothing within one decay length is used in all smoothing matrices [e.g., Toomey et al., 1994], which are also normalized by the model scaling matrix to be consistent with the normalization of the Fréchet matrix. To reduce bias toward a starting model, the above formulation contains no information about a reference model except through the model normalization. When a reliable reference model is independently available, however, it is straightforward to modify equation 1.5 to incorporate a pure jumping strategy [Shaw and Orcutt, 1985; Toomey et al., 1994].

Equation 1.5 is a sparse system so it can be efficiently solved by the sparse matrix solver LSQR [Paige and Saunders, 1982]. The computation time used in solving one matrix equation is typically an order of magnitude less than that used for the forward ray tracing. When a starting model is far from a true solution, model updates can be so large that the succeeding iterations become unstable. To avoid this instability, when there are data with too large residuals, we repeat the inversion without the outliers. In addition, damping constraints for velocity and depth nodes are added to equation 1.5. Therefore, there are four weighting parameters in total in our inverse equation: two for smoothing and two for damping. Even though solving a single sparse system is computationally inexpensive, trying to find the optimal combination of the four parameters to satisfy some 
user-defined constraints such as desired model roughness requires a number of trial solutions at each iteration, which we regard as impractical. Our strategy is to first determine the smoothing weights by systematically testing velocity and depth smoothing with a preliminary single-step inversion, and then to fix these weights throughout all subsequent iterations. When model perturbations are found to be too large, optional sweeps on the damping weights are done by the secant and bisection method to satisfy userdefined upper limits on average velocity and depth perturbations. In the later phase of iterations, model perturbations tend to become very small, and weak but undesired model roughness can emerge with this fixed smoothing strategy. To amend this, post-inversion smoothing is applied at each iteration as,

$$
\mathbf{m}_{i+1}=\mathbf{m}_{i}\left(1+\mathbf{L}_{v}\left(\delta \mathbf{m}_{i}\right)-\delta \mathbf{m}_{c}\right)
$$

where $\mathbf{L}_{v}$ denotes the 2-D smoothing operator for velocity parameters, $\delta \mathbf{m}_{i}$ is the $i$-th model update, and $\mathbf{m}_{i}$ and $\mathbf{m}_{i+1}$ are the $i$-th and $(i+1)$-th model solutions, respectively. $\delta \mathbf{m}_{c}$ is a correction term to conserve data resolution during the a posteriori filtering [Deal and Nolet, 1996], and it is calculated by solving

$$
\mathbf{G} \delta \mathbf{m}_{c}=\mathbf{G}\left(\mathbf{L}_{v}\left(\delta \mathbf{m}_{i}\right)-\delta \mathbf{m}_{i}\right) .
$$

As well as compensating our compromise of using 1-D smoothing matrices in equation 1.5, this 2-D smoothing ensures model convergence with given correlation lengths.

A major concern in reflection tomography is the existence of nonuniqueness regarding the depth of a reflector and the velocity above it. Even in our joint refraction and reflection tomography, this nonuniqueness can still be present because the lower-crustal velocity is mainly resolved only by reflection travel times. There are a number of theoretical studies on the nature of this velocity-depth ambiguity [e.g., Bickel, 1990; Stork, 1992b; Stork, 1992a; Ross, 1994; Bube et al., 1995], but their treatments are limited to simple velocity structures and some particular source-receiver configurations. It is probably unrealistic to expect a general theory that can be versatile enough to handle a number of source-receiver pairs with different ray coverage, strongly heterogeneous velocity structure, and variable interface topography. Therefore we propose a practical 
strategy to investigate the degree of the velocity-depth ambiguity using the depth-kernel weighting parameter $w$ in equation 1.5. Even with smoothness constraints on velocity and depth, the linear system for joint refraction and reflection tomography can still be singular because of this velocity-depth ambiguity. For a singular system, iterative matrix solvers based on conjugate gradients such as LSQR provide the minimum-norm solution if starting with an initial null vector [Hestenes, 1975]. The weighting parameter of unity corresponds to the equal weighting of velocity and depth nodes [Stork and Clayton, 1991], and increasing the weighting parameter should lead to larger depth perturbations with smaller velocity perturbations if the system is singular. We can thus test the velocity-depth ambiguity by systematically exploring the solution space with the single controlling parameter.

A numerical example to demonstrate this approach is shown in Figure 1-6. In this example, a starting model already has the true velocity field, but the reflector is located $1 \mathrm{~km}$ shallower than the true one. With dense ray coverage as in Figure 1-6b, the inversion successfully recovers the true reflector location with negligible velocity perturbations, regardless of the value of $w$ (Figure 1-6c and $\mathrm{d}$ ). When ray coverage is sparse (Figure 1-6e), the inversion with equal weighting results in an insufficient recovery of the reflector depth with compensating velocity perturbations (Figure 1-6f), and a desirable recovery can be obtained with greater depth sensitivity (Figure 1-6g). The travel time fits for the two different recoveries are nearly the same, thus they are indistinguishable from data resolution. The weaker weighting of depth nodes would provide the other end of the valid model space. When there is no other information to discriminate among these different recoveries, one should present all of them as equally legitimate inverse solutions to characterize the velocity-depth ambiguity. The ambiguity is mainly controlled by available ray coverage and the scale of perturbations we want to resolve, and in general it can be reduced by using greater correlation lengths. Correlation lengths for depth nodes, therefore, must be comparable with those for velocity nodes to properly evaluate the extent of the ambiguity. 
The above depth-kernel weighting should not be confused with weighting reflection data against refraction data. A linear system corresponding to the latter case may be expressed as,

$$
\left[\begin{array}{c}
\mathbf{d}_{1} \\
\alpha \mathbf{d}_{2} \\
\mathbf{0} \\
\mathbf{0} \\
\mathbf{0}
\end{array}\right]=\left[\begin{array}{cc}
\mathbf{G}_{v}^{1} & \mathbf{0} \\
\alpha \mathbf{G}_{v}^{2} & \alpha \mathbf{G}_{d}^{2} \\
\lambda_{v} \mathbf{L}_{\mathrm{H} v} & \mathbf{0} \\
\lambda_{v} \mathbf{L}_{\mathrm{v}_{v}} & \mathbf{0} \\
\mathbf{0} & \lambda_{d} \mathbf{L}_{d}
\end{array}\right]\left[\begin{array}{l}
\delta \mathbf{m}_{v} \\
\delta \mathbf{m}_{d}
\end{array}\right],
$$

where $\mathbf{d}_{1}$ and $\mathbf{d}_{2}$ denotes refraction and reflection travel times, respectively, and $\alpha$ controls the importance of reflection data relative to refraction data. Changing the value of $\alpha$ results in a different linear system, whereas changing the depth-kernel parameter $w$ in equation 1.5 does not. This difference is vital. In addition, the use of equation 1.8 is not generally recommended because the relative importance between refraction and reflection data should be already incorporated as corresponding data variance.

\subsection{Results of Tomographic Inversion}

\subsubsection{Long-Wavelength Structure}

From the twenty five wide-angle data sets, $2318 \mathrm{Pg}$ and $2078 \mathrm{PmP}$ travel times were picked to invert for the crustal structure along transect 2 . The model domain is $350 \mathrm{~km}$ wide and $40 \mathrm{~km}$ deep from seafloor. The horizontal grid spacing is approximately $1 \mathrm{~km}$ on average, and the vertical grid spacing gradually increases from $50 \mathrm{~m}$ at the seafloor to $1 \mathrm{~km}$ at the model bottom, amounting to over 27000 velocity nodes (Figure 1-7). The number of reflector nodes is 351 with a uniform $1 \mathrm{~km}$ spacing. The horizontal correlation length for velocity nodes ranges from $10 \mathrm{~km}$ at seafloor, linearly increasing to $20 \mathrm{~km}$ at the bottom, and the vertical correlation length also varies linearly from $50 \mathrm{~m}$ at seafloor to $4 \mathrm{~km}$ at the bottom. The correlation lengths for the reflector nodes are sampled from the 2-D horizontal correlation length function for velocity nodes, using the reflector depths at each iteration. We choose to impose no lateral variation in the correlation length functions, to minimize the influence of the prescribed correlation lengths on the structural interpretation of the continent-ocean transition zone. 
The sedimentary section was already modeled with MCS vertical-incident travel times and wide-angle basement reflection travel times as reported in Holbrook et al. [1999], and it is incorporated in a starting model as the a priori information. We prepared a starting model for the crustal part by smoothing the preliminary crust model of Holbrook et al. [1999], which was constructed using the semi-forward modeling approach of Zelt and Smith [1992], with a 100-km wide and 10-km high Gaussian window (Figure 1-8a). The initial root-mean-square (RMS) travel time misfit is $137 \mathrm{~ms}$, with $\chi^{2}$ of 1.34 . Average model perturbations were less than $2 \%$ for both velocity and depth throughout the inversion, so no damping was applied. After ten iterations, model perturbations became $-0.05 \%$ for velocity and $\sim 0.1 \%$ for depth, and the RMS travel time misfit was reduced to $81 \mathrm{~ms}$ with $\chi^{2}$ of 0.42 (Figure $1-8 \mathrm{~b}$ ).

Three distinct types of crust can be identified in the final model: (1) $\sim 30-\mathrm{km}$-thick continental crust near the landward end of the transect, (2) 30- to 15-km-thick transitional crust from $\mathrm{km} 100$ to 250 , and (3) 15 - to $9-\mathrm{km}$-thick oceanic crust from $\mathrm{km} 250$ to the seaward end. The velocity of continental crust ranges almost linearly from $6.0 \mathrm{~km} / \mathrm{s}$ at the surface to $7.2 \mathrm{~km}$ at $30 \mathrm{~km}$ depth, which closely resembles the global average velocity profile for continental crust [Christensen and Mooney, 1995]. A high-velocity-gradient upper crust appears from $\mathrm{km} 60$, with this transition from low-velocity-gradient to highvelocity-gradient upper crust tentatively interpreted as the continent-ocean boundary. This continent-ocean boundary at shallow crustal levels roughly coincides with the mapped coast-parallel dike swarm [e.g., Larsen and Duncan, 1996]. The transitional crust has 6$\mathrm{km}$-thick upper crust, and the bottom half of its lower crust has a velocity exceeding $7.0 \mathrm{~km} / \mathrm{s}$. From $\mathrm{km} 100$ to 150 , the high-velocity lower crust is remarkably thickened, producing a good contrast to the lower-velocity continental crust. Although the lower crustal velocity of the transitional crust becomes higher toward the Moho than that of normal oceanic crust $(\sim 6.9 \mathrm{~km} / \mathrm{s})$ [White et al., 1992], we note that it is not quite as high as those found in other seismic transects on North Atlantic margins [White et al., 1987; Mutter and Zehnder, 1988], which are higher than $7.2 \mathrm{~km} / \mathrm{s}$ for most of the lower crust. The thickness of the upper crust reduces to $-3 \mathrm{~km}$ for the oceanic crust. Though this part of 
the crust is thinnest along the transect, it is still thicker than normal oceanic crust with 6 to $7 \mathrm{~km}$ thickness [White et al., 1992]. Its velocity is still higher than $7.0 \mathrm{~km} / \mathrm{s}$ in the bottom half of the lower crust, and a prominent high velocity anomaly is observed near the Moho from $\mathrm{km} 260$ to 300 .

To examine the sensitivity to starting models, we conducted another set of inversions with a different starting model. The second starting model was constructed by hanging a $1-D$ average crustal velocity profile calculated from Figure 1-8a beneath the basement, and the initial Moho was set flat at $25 \mathrm{~km}$ deep (Figure 1-9a). The initial RMS travel time misfit exceeded $800 \mathrm{~ms}$ with $\chi^{2}$ of 64.5 , but outliers with normalized residuals greater than 5 after the first iteration are only $0.25 \%$ of all data, indicating a minor role of outlier removal. Damping was applied to retain average slowness and depth perturbations below $3 \%$ and $9 \%$, respectively. After the first two iterations, damping was no more required, and all the travel time residuals were within the cutoff threshold. After ten iterations, the RMS travel time misfit reduced to $77 \mathrm{~ms}$ with $\chi^{2}$ of 0.36 (Figure $1-9 \mathrm{~b}$ ). Though the details differ, the main key structural features observed in Figure 1-8b remain the same. These features include the overall variation in crustal thickness, and the thickening of the high-velocity-gradient upper crust from the seaward end to the continental shelf, and its sudden thinning near the coast line. The crustal velocity structure from $\mathrm{km} 170$ to 330 , including the small-scale high velocity anomaly in the lower crust around $\mathrm{km} \mathrm{280,} \mathrm{is} \mathrm{almost} \mathrm{identical} \mathrm{between} \mathrm{the} \mathrm{two} \mathrm{models,} \mathrm{indicating} \mathrm{this} \mathrm{part} \mathrm{of} \mathrm{the}$ model is well resolved and virtually independent of starting models. An increase in the lower-crustal velocity at $\mathrm{km} 100$ to 150 is also found in the second model, though its overall velocity is lower. A majority of the continental crust seems to require an average velocity of $-6.5 \mathrm{~km} / \mathrm{s}$ with an almost linear velocity gradient, as found in both models. These close similarities between the two models satisfactorily demonstrate the robustness of the resolved velocity structure, and we will use the first model (Figure 1-8b) as our preferred choice in the following analysis.

To investigate the degree of velocity-depth ambiguity in our inversion, we repeated the inversion with the starting model of Figure 1-8a, using two different values, 0.01 and 
100 , for depth-kernel weighting. The inversion solutions after ten iterations are shown in Figure 1-10 along with the solution for equal weighting. The travel time misfits for the three models are very similar, though the model with $w$ of 100 has a slightly inferior fit. Since there is no major difference found among these models, we conclude that our inversion solution does not suffer from serious velocity-depth ambiguity, given the relatively wide spatial correlation we employed in the smoothing constraints. We will thus employ the equal weighting of the velocity and depth kernels in all the following inversions.

\subsubsection{Resolution and Accuracy}

The ray coverage in the model can be concisely represented by the derivative weight sum (DWS) [Toomey and Foulger, 1989], which is simply the column-sum vector of the Fréchet velocity kernel. The DWS for our velocity model is shown in Figure 1-11, as a crude measure of the linear sensitivity in our inversion. The lower crust at $\mathrm{km} 30$ to 130 is sparsely covered by reflections alone, implying lower resolution than the other parts of the model.

Because the DWS does not provide any quantitative information regarding the resolution and accuracy of the model, and because nonlinear sensitivity is important for iterative inversions [e.g., Zhang and Toksöz, 1998], we conduct two types of analyses: (1) resolution tests using synthetic data, and (2) a nonlinear Monte Carlo uncertainty analysis. In the resolution tests, we calculate synthetic data for a perturbed velocity model with the same sources and receivers as in the actual observation, add random noise of $50 \mathrm{~ms}$ to the synthetic data, and invert them with an initial unperturbed model to see how well given perturbations are recovered. Though not comprehensive, this is a succinct and computationally inexpensive way to demonstrate the resolving power of the available ray coverage. In the first resolution test, we only perturbed velocity nodes with $5 \%$ positive and negative anomalies of a 140 $\mathrm{km}$ wavelength aligned in the mid-crust (Figure 1-12a). Good recovery was obtained for the seaward half of the model (Figure 1-12b). Despite a significant deterioration toward the landward end, the result suggests that the ray coverage of our data is sufficient to resolve the continent-ocean boundary around $\mathrm{km} 100$. The 
recovered anomalies tend to be laterally stretched, resulting from our conservative choice of the horizontal correlation length. In the second resolution test, only the reflector nodes were perturbed with a $1.5-\mathrm{km}$ amplitude and a $100-\mathrm{km}$ wavelength (Figure $1-12 \mathrm{c}$ ). The perturbed Moho was well recovered for $\mathrm{km} 50$ to 100 and $\mathrm{km} 150$ to 330 . The parts that were not recovered correctly, such as $\mathrm{km} 100$ to 150 and near the model ends, are indeed not covered by $P m P$ (Figure 1-11). The unrecovered reflector perturbations leak into velocity perturbations above the reflector, but their amplitude is only $\sim 0.5 \%$, being consistent with the negligible velocity-depth ambiguity indicated in Figure 1-10.

The absolute uncertainty of the seismic velocity is fundamental for the reliable interpretation of crustal composition. The only practical way to estimate the model uncertainty for a large-scale, nonlinear inversion such as our tomography is the Monte Carlo method [e.g., Tarantola and Valette, 1982; Tarantola, 1987]. By inverting data with random errors with random initial models, we can construct a number of Monte Carlo realizations, with which the a posteriori model covariance matrix is estimated as

$$
\mathbf{C}=\int(\mathbf{p}-E(\mathbf{p})) \cdot(\mathbf{p}-E(\mathbf{p}))^{\mathrm{T}} \sigma_{\mathbf{p}}(\mathbf{p}) d \mathbf{p}
$$

where $\mathbf{p}$ is the solution vector of the realizations, $E(\mathbf{p})$ is the a posteriori expectation of $\mathbf{p}$, and $\sigma_{\mathrm{p}}(\mathbf{p})$ is the a posteriori marginal density function [Tarantola and Valette, 1982]. By assuming that all $N$ realizations have the same probability, we may approximate the above definition as [Matarese, 1993],

$$
\mathbf{C} \approx \frac{1}{N} \sum_{i=1}^{N}\left(\mathbf{p}_{i}-E(\mathbf{p})\right) \cdot\left(\mathbf{p}_{i}-E(\mathbf{p})\right)^{\mathrm{T}}
$$

where $\mathbf{p}_{i}$ is the solution of the $i$-th realization. Zhang and Toksöz. [1998] noted that modeling data errors with Gaussian errors for absolute travel times is unrealistic in light of the nature of travel time picking, and they suggested using common-shot errors (or common-receiver errors in the case of marine experiments) and travel time gradient errors. We thus randomized data by adding the following two error components to the original data: random common-receiver errors with a maximum amplitude of $50 \mathrm{~ms}$, and random travel time errors with the same maximum amplitude. The small-amplitude travel time errors have virtually the same function as the travel time gradient errors used by Zhang and 
Toksöz [1998]. The highly correlated nature of travel time pick errors can be taken into account by this data randomization. Random initial velocity models were prepared by adding random perturbations to the solution shown in Figure 1-8b. The 2-D smoothing $\mathbf{L}_{v}$ of equation 1.6 was applied to the velocity perturbations to ensure that the initial models are within the model space of our interest, which is restricted by the imposed correlation lengths. A similar Gaussian smoothing was applied for randomized reflector nodes. The amplitude of raw random perturbations was chosen so that the smoothed perturbations for velocity and reflector depth have a maximum amplitude of approximately $5 \%$ (Figure 1-13). With this degree of model perturbations, normalized $\chi^{2}$ was generally higher than 1.3 , with RMS travel time misfit of $\sim 140 \mathrm{~ms}$. On average, ten iterations were required to reduce normalized $\chi^{2}$ below 0.5 , which we set as a threshold for acceptable models. Using 100 realizations, the a posteriori model variance was calculated as shown in Figure 1-14. The standard deviation of the velocity nodes is lowest (less than $0.04 \mathrm{~km} / \mathrm{s}$ ) at the midcrust, where most of refraction rays turn (Figure 1-11). From $\mathrm{km} 50$ to 330, most of the velocity nodes have a standard deviation of less than $0.08 \mathrm{~km} / \mathrm{s}$ (Figure 1-14). The standard deviation of the Moho depths is less than $0.25 \mathrm{~km}$ for the thinner crust and about $1.0 \mathrm{~km}$ for the thicker crust.

Using the Monte Carlo ensembles, one can estimate the uncertainty of an averaged quantity by applying the averaging operation to each ensemble and then calculating the variance from the whole averaged ensembles. Even when model parameters have large errors, the variance of their average can be much smaller if the errors are negatively correlated. The above approach using the Monte Carlo realizations correctly handles this parameter correlation. The Monte Carlo ensembles obtained in this section will be used when we discuss the relationship between crustal thickness and average crustal velocity.

\subsection{Discussion}

\subsubsection{Short-Wavelength Structure}

The travel times predicted by the long-wavelength velocity structure consistently fail to fit the small undulations of $\sim 200 \mathrm{~ms}$ in the observed travel times around $\mathrm{km} 230$ to 245 
(Figure 1-4). This negative travel time misfit is found for all the refraction and reflection phases shot from that model range, which coincides with the basement high and the occurrence of the seaward termination of the SDR wedge (Figure 1-3), so that they must originate in a positive velocity anomaly in a shallow depth below the basement high. To resolve this positive velocity anomaly, we inverted a subset of the travel time data with shorter correlation lengths; the horizontal and vertical correlation lengths were set as $5 \mathrm{~km}$ and $0.5 \mathrm{~km}$, respectively, for a $150 \mathrm{~km}$ wide and $10 \mathrm{~km}$ deep sub-region including the basement high. After only one iteration, we were able to achieve excellent travel time fits (Figure 1-15a). The high-resolution velocity model shows that a $15-\mathrm{km}$ wide and $4-\mathrm{km}$ thick region beneath the basement high has velocities higher by $0.5-1.0 \mathrm{~km} / \mathrm{s}$ than the adjacent upper crust (Figure 1-15b).

The shallow positive velocity anomaly provides an important constraint on the origin of the basement high. A similar velocity anomaly identified beneath the Krafla central volcano in Iceland [Brandsdóttir et al., 1997; Staples et al., 1997] has been interpreted as a shallowing of the igneous intrusion depth. The termination of the SDR sequence adjacent to the basement high is consistent with this explanation on the basis of models of SDR evolution [Hinz, 1981; Mutter et al., 1982]. In addition, there must have been a small-scale, seaward ridge jump from the eruption center, to leave the high velocity anomaly region within the continental margin crust. The other possibility is that the positive velocity anomaly represents a later intrusion cutting through the preexisting SDRs, as suggested for the "outer high" on the Hatton Bank [White et al., 1987; Barton and White, 1997b]. Unlike the case of the Hatton Bank, however, almost no coherent linear structure is observed on the seaward side of the basement high to support this hypothesis (Figure 1-15c). Moreover, the thickness of the upper crust drastically changes across the basement high (e.g., Figure 1-8b), further reducing the likelihood that the data can be explained as the result of a later intrusion. An abandoned central volcano thus appears to be the most likely explanation for the basement high. 


\subsubsection{Some Remarks on Travel Time Modeling}

The compressional velocity model of transect 2 presented in Holbrook et al. [1999] was constructed using the iterative damped least square inversion of Zelt and Smith [1992]. The model consists of a few tens of trapezoidal velocity cells as shown in Figure 1-16a, and its RMS travel time misfit is about $100 \mathrm{~ms}$ with $\chi^{2}$ of $\sim 1.0$. Although the histogram of normalized travel time residuals shows how loosely the travel times are fitted (Figure 1$16 \mathrm{~b}$ ), the acquired misfit is considered to be reasonable, given the simplicity of the model parameterization. The inversion code RAYINVR developed by Zelt and Smith [1992] has been popular among crustal seismologists as an efficient tool to estimate long wavelength structures, but we would like to point out its two practical disadvantages that makes our tomography method preferable for seismic studies of continental margins.

First, the program RAYINVR requires a "good" model parameterization, which a user must find by trial and error. Because their inversion is only a damped least squares, one has to construct a model with a small number of nodes, whose spatial distribution should be also optimized for sensitivity to data. In addition, since ray tracing is done by the ray shooting method [e.g., Cerveny et al., 1977], it is not guaranteed that rays are traced to all observation points. A starting model must be prepared so that rays are traced to all data; otherwise, unmatched data points cannot contribute to the inversion. This is not a major obstacle when a true velocity structure can be well approximated as a stack of layers. However, for regions where considerable lateral heterogeneity is expected, such as continent-ocean transition zones, finding a good parameterization can be challenging. Increasing the number of nodes for expected heterogeneity may lead to weaker parameter sensitivities and result in an oscillatory solution. Model complexity can be thus limited, and we are often forced to deal with a trade-off between data resolution and model error, instead of a trade-off between model resolution and model error. Though one could construct a complex model with strong parameter damping, using RAYINVR in this manner becomes almost identical to forward modeling by trial-and-error. The ray shooting method also tends to fail to correctly calculate ray paths and travel times for complex velocity structures. 
More important, the coarse model parameterization used in RAYINVR often hinders realistic determination of model uncertainty. To estimate a parameter's absolute uncertainty, Zelt and Smith [1992] suggested that one should perturb it, hold it fixed, and invert for the rest of the parameters, to determine the maximum acceptable perturbation based on (1) the ability to trace rays to all observations and (2) the travel time misfit. A failure to trace rays to all observations, however, cannot be used to constrain model uncertainty because it may only mean that a chosen parameterization is not sufficiently flexible. This inflexibility in model adjustments may also lead to greater travel time misfits, by which model uncertainty is very likely to be underestimated.

Although tomographic methods have been mainly used to resolve small-scale velocity structures, we should also use them to construct large-scale crustal velocity models. It is important to use a tomographic method with a robust uncertainty analysis, such as the Monte Carlo method used in this study, especially when the geological interpretation of a velocity model critically depends on the absolute values of estimated parameters. Without knowing their realistic uncertainties, it would be difficult to judge the fidelity of such an interpretation.

\subsubsection{Interpretation of Long-Wavelength Velocity Structure}

Our interpretation of the continent-ocean transition zone is shown in Figure 1-17a. The stretched continent crust extends over $50 \mathrm{~km}$ below the extrusive layer, showing a similar geometry to other transects across the North Atlantic continental margins [White et al, 1987; Barton and White, 1997a; Holbrook et al., 1999]. Given the smoothed nature of the tomographic image, the actual continent-ocean boundary could be sharper, as observed for the US Atlantic margin [Holbrook et al., 1994a; Holbrook et al., 1994b; Lizarralde and Holbrook, 1997], but we use the diffuse boundary shown in Figure 1-17a to obtain a conservative estimate of the volume of igneous products. The thickness and the mean velocity of the igneous crust are then calculated as a function of the model distance (Figure 1-17b). Prior to averaging velocities, we applied a pressure correction of $0.22 \times 10^{-3} \mathrm{~km} / \mathrm{s} / \mathrm{MPa}$ [Christensen and Mooney, 1995] and a temperature correction of $-0.5 \times 10^{-3} \mathrm{~km} / \mathrm{s} /{ }^{\circ} \mathrm{C}$, with respect to a reference pressure of $600 \mathrm{MPa}$ and a reference 
temperature of $400^{\circ} \mathrm{C}$. For the temperature correction, we assume a linear conductive geotherm with $0^{\circ} \mathrm{C}$ at the seafloor and $750^{\circ} \mathrm{C}$ at $40 \mathrm{~km}$ depth. In addition, all crustal velocities less than $6.8 \mathrm{~km} / \mathrm{s}$ are assumed to be affected by alteration or porosity, so we replaced them with $6.8 \mathrm{~km} / \mathrm{s}$ to properly evaluate the bulk composition of the igneous crust [Kelemen and Holbrook, 1995; Holbrook et al., 1999]. Velocities and depths within a horizontal window of $20 \mathrm{~km}$ were averaged at each model distance, and we repeated this averaging procedure with the 100 Monte Carlo ensembles to estimate uncertainty.

Using the thickness and the mean velocity of the igneous crust as seismological constraints on the volume and the bulk composition of mantle-derived melt, we can place a first-order constraint on the style of mantle melting during continental breakup. Here we use an adiabatic mantle melting model extended from McKenzie and Bickle [1988] to incorporate active mantle upwelling, as described in Holbrook et al. [1999]. This gives mean pressure and fraction of melting, and melt volume for various combinations of potential temperature and upwelling velocity. These are connected to melt compositions and crustal velocity via the empirical methods developed by Kelemen and Holbrook [1995]. A predicted relationship between the mean crustal velocity and crustal thickness with the corresponding mantle potential temperatures is plotted for a passive upwelling case and three active upwelling cases, with active upwelling ratio of 2, 4, and 8 (Figure 118). The active upwelling ratio is defined as the mantle upwelling rate normalized by the surface divergence half-rate. One prominent feature of the observed mean velocity is that it is confined in a narrow range from $6.92 \mathrm{~km} / \mathrm{s}$ to $7.02 \mathrm{~km} / \mathrm{s}$, regardless of the corresponding crustal thickness (Figure 1-18). This translates into a relatively constant mantle potential temperature $\left(1270-1350^{\circ} \mathrm{C}\right)$, with a drastic temporal variation in the active upwelling ratio from $\sim 8$ during the initial phase of breakup to $\sim 1$ (passive) after $\sim 43 \mathrm{Ma}$. Interestingly, this is the same range of upwelling ratios obtained by Kelemen and Holbrook [1995] for the U.S. East Coast. In contrast, the velocity-thickness data from transect 1 suggest active upwelling of high-temperature $\left(1400-1450^{\circ} \mathrm{C}\right)$ mantle throughout the opening of the North Atlantic [Holbrook et al., 1999] (Figure 1-18). The uncertainty in average velocity based on the Monte Carlo method is smaller than that obtained by the preliminary sensitivity test 
of Holbrook et al. [1999], and it is sufficiently small to clearly define this marked velocity contrast between the two adjacent transects. Though the transect 1 data may be consistent with its coincidence with the presumed hotspot track, it is puzzling, from a viewpoint of a mantle plume hypothesis, that the more vigorous active upwelling of a cooler mantle during the initial rifting period is inferred for a location only $250 \mathrm{~km}$ from the presumed center of the hotspot track. The influence of the Iceland plume during the breakup of Greenland from Europe, therefore, may have been spatially restricted, and some contribution from non-plume components, such as in the convection models proposed by Mutter et al. [1988] and Boutilier and Keen [1999], may be required to account for the inferred complexity in the thermal and dynamic state of the mantle during the continental breakup. We note that, however, a few caveats need to be addressed for the interpretational method used in Kelemen and Holbrook [1995] and Holbrook et al. [1999]. First of all, replacing upper-crustal velocity uniformly with $6.8 \mathrm{~km} / \mathrm{s}$ may be too crude and can potentially introduce significant errors in the estimate of bulk crustal composition. Second, all mantle-derived melts are assumed to be emplaced as observed crust, and the possibility of subcrustal fractionation, which may have been important for melt migration through preexisting lithosphere, is completely ignored. Finally, the empirical relationship between melt compositions and crustal velocity derived by Kelemen and Holbrook [1995] has the uncertainty of as large as $0.2 \mathrm{~km} / \mathrm{s}$. Even though the transient contribution of vigorous active upwelling of the mantle still seems to be a robust conclusion, the interpretation of the details of the observed velocity-thickness curve requires a more accurate method that can resolve the above weaknesses. The construction of such a new framework and its application to this transect will be presented in Chapter 3.

\subsection{Summary}

Using the multichannel and wide-angle seismic data obtained in the 1996 SIGMA experiment across the Southeast Greenland margin, we were able to fully resolve crustal structure along the $350 \mathrm{~km}$ long transect, including nearly undeformed continental crust, thick igneous crust at the continent-ocean transition zone, and moderately thick oceanic 
crust near the seaward end. The depth-migrated MCS section clearly images the basement topography and the shallow crustal reflectivity, including seaward dipping reflectors. We developed a new tomographic method to invert wide-angle refraction and reflection travel time data for the long-wavelength structure along the entire transect. Strong lateral heterogeneity both in velocity and reflector depth is successfully recovered, and the Monte Carlo uncertainty analysis shows the most of model parameters are well constrained, with 0.04-0.08 km/s error for velocity nodes and 0.25-1.0 km error for depth nodes. Negligible trade-off between velocity and depth is also demonstrated.

The lateral variation in the mean velocity and thickness of the igneous crust is interpreted as a temporal variation in the composition and the volume of mantle melt produced during the opening of the North Atlantic. Based on a simple mantle melting model incorporating the effect of active upwelling, the inferred melt composition and volume seem to require a gradual transition from vigorous active upwelling during the initial stage of opening (56-50 Ma) to passive upwelling after $43 \mathrm{Ma}$, with moderately high potential temperatures of $1270-1350^{\circ} \mathrm{C}$. This result makes a sharp contrast to the almost unvarying active upwelling of high temperature mantle inferred for transect 1 , which is on the presumed Iceland hotspot track located $\sim 250 \mathrm{~km}$ north of transect 2 . This contrast may suggest a limited influence of the Iceland plume on the rifting magmatism and a considerable non-plume component in the mantle upwelling, though this needs to be revisited by a more accurate interpretational method for the observed crustal velocity structure. 

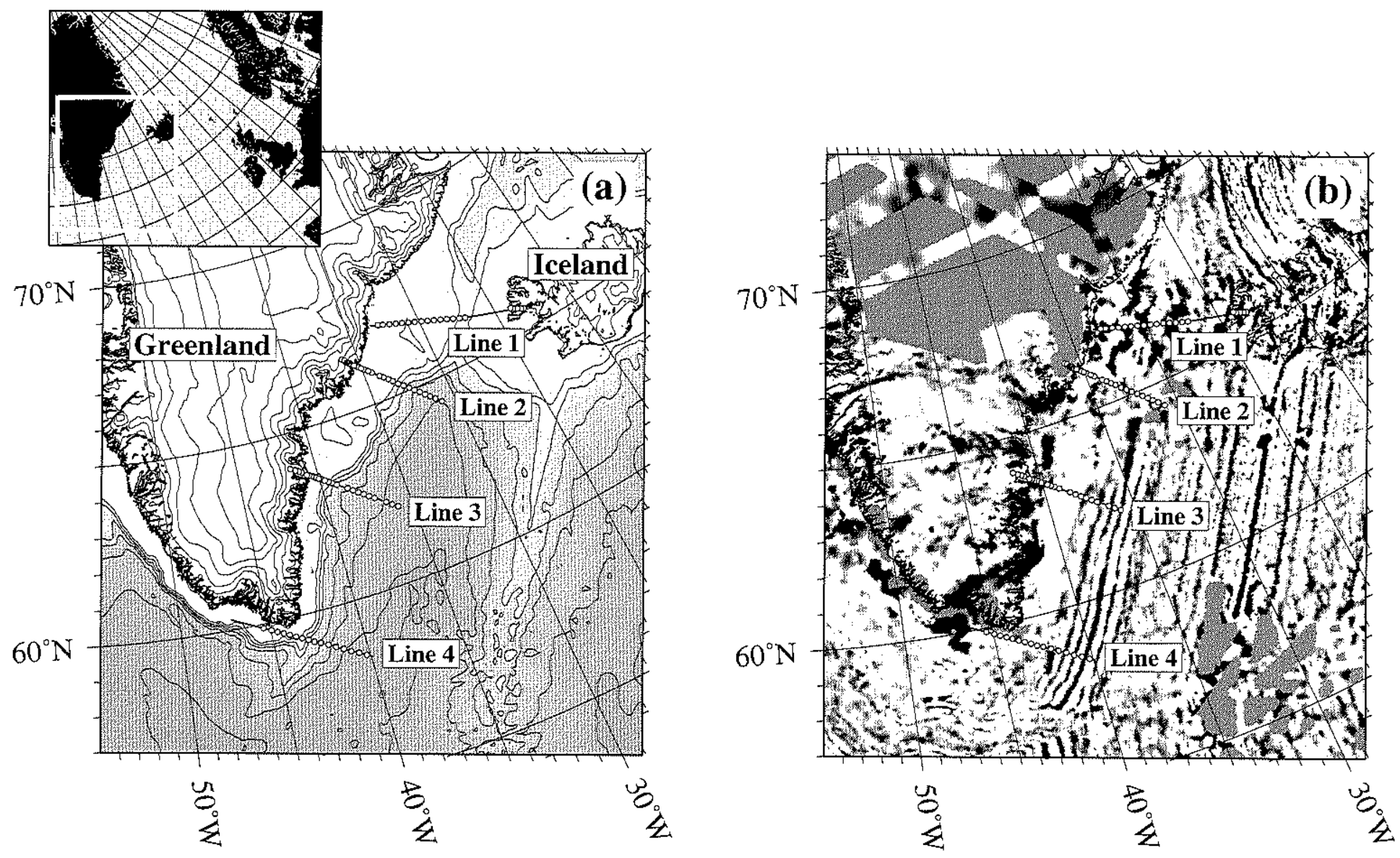

Figure 1-1. Location of the 1996 SIGMA seismic experiment. Circles denote location of onshore and offshore seismic instruments. (a) Bathymetry with $500 \mathrm{~m}$ contour interval. (b) Magnetic anomaly with positive anomaly shaded. 


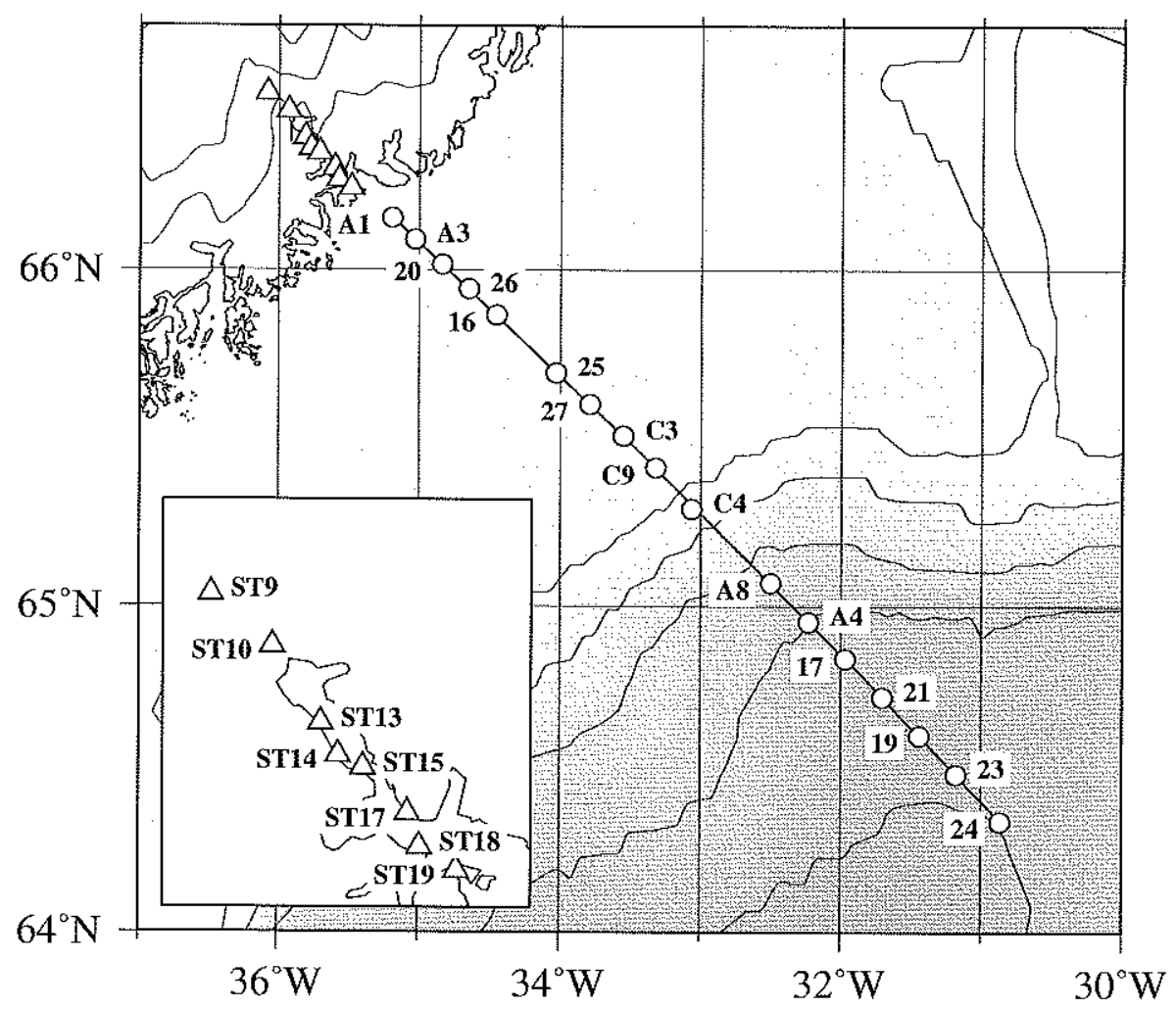

Figure 1-2. Configuration of transect 2 seismic experiment. Circles and triangles denote ocean-bottom instruments and onshore seismic recorders, respectively. Bathymetry contours are drawn at $500 \mathrm{~m}$ interval. 

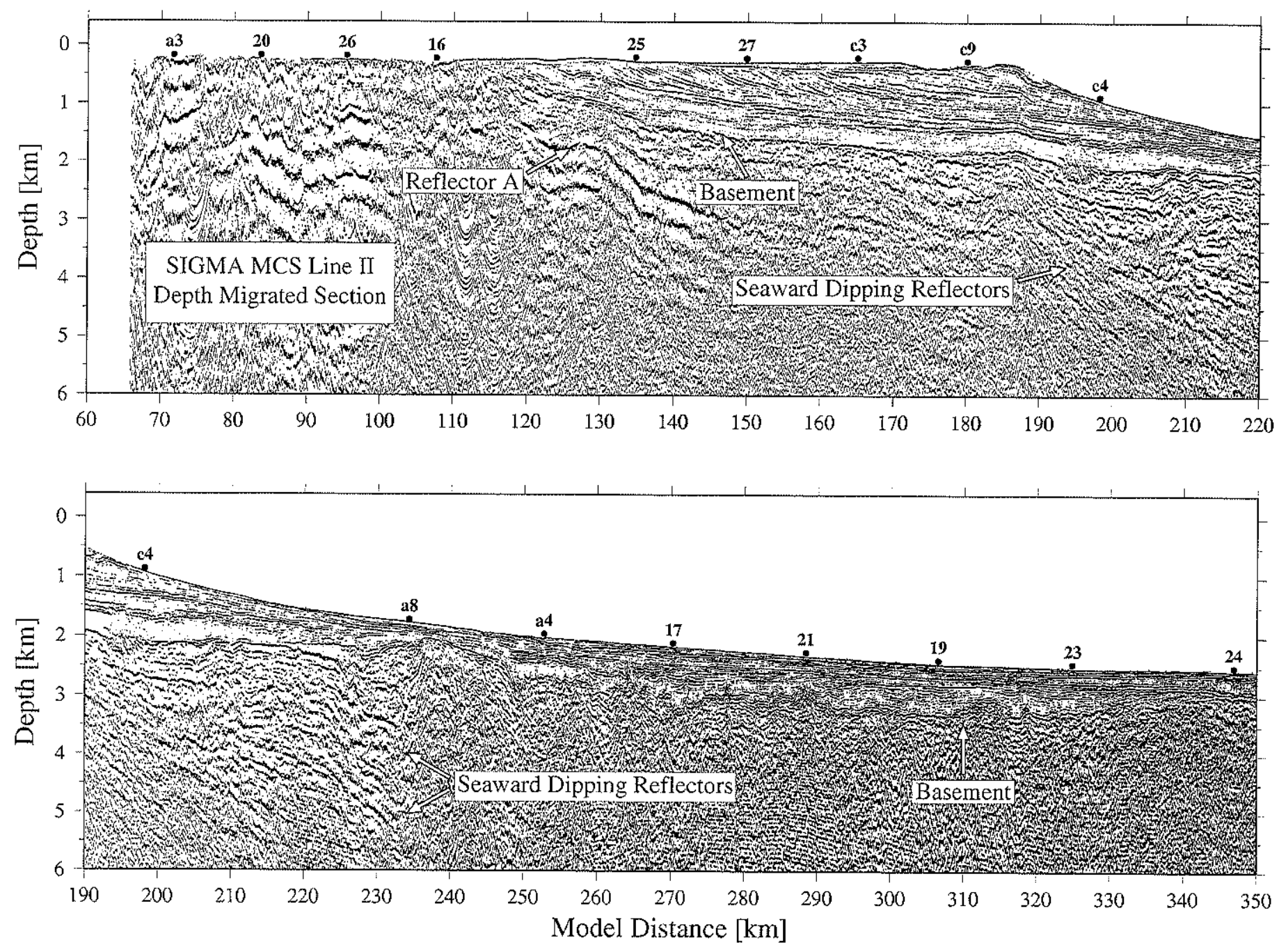

Figure 1-3. Depth migrated section of multichannel seismic reflection profile. Coherence filtering, automatic gain correction, and trace normalization were applied. Location of ocean bottom instruments is also shown by solid circles. 


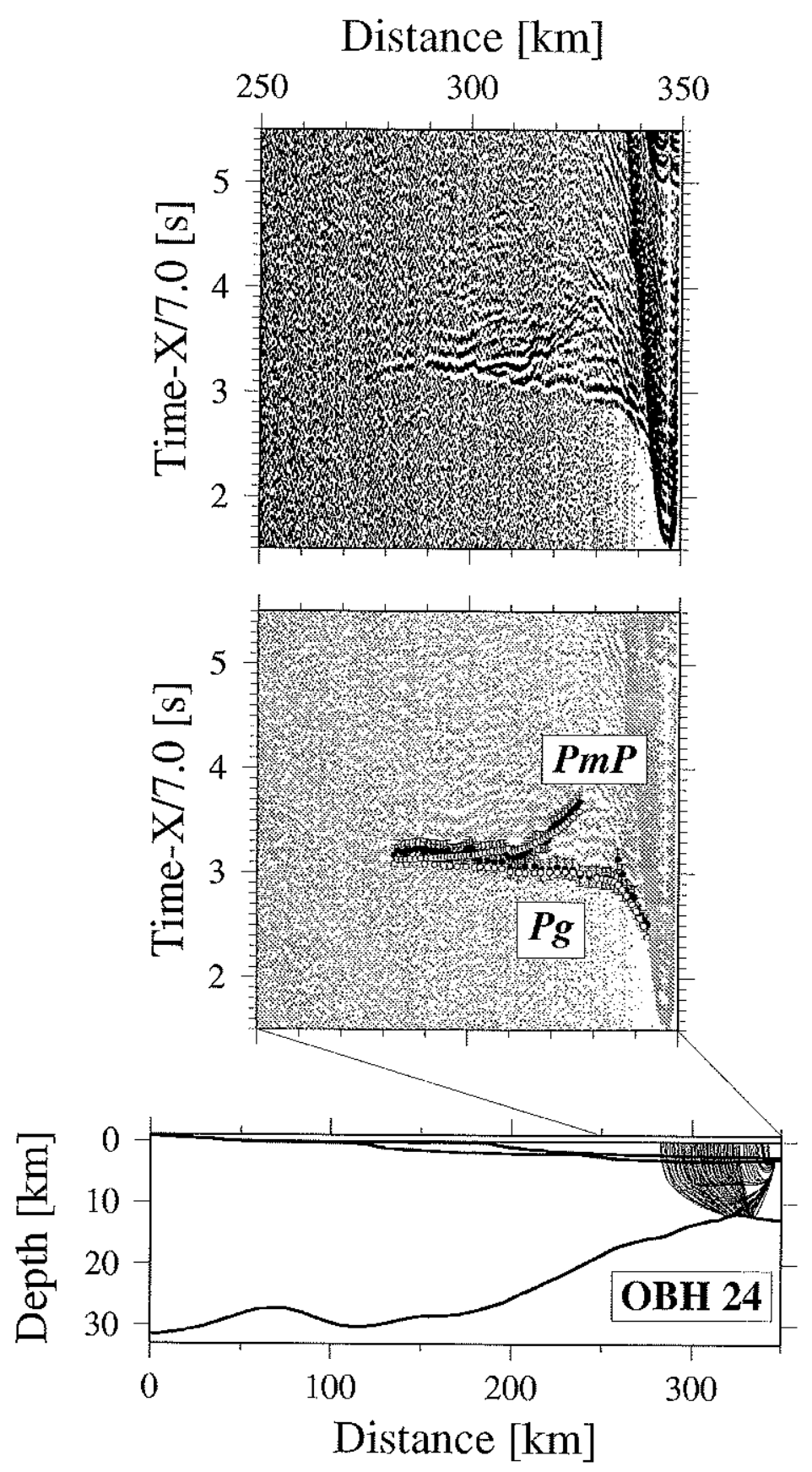

Figure 1-4. Wide-angle seismic data are plotted after range gain correction, with picked (solid circle with pick error) and predicted (open circle) travel times for $P g$ and $P m P$ phases. Synthetic travel times are based on the velocity model presented in Figure 1-8b, and corresponding ray paths are also plotted at the bottom. (a) $\mathrm{OBH} 24$, (b) $\mathrm{OBH} 23$, (c) $\mathrm{OBH} 19$, (d) $\mathrm{OBH} 21$, (e) OBH 17, (f) OBS A4, (g) OBS A8, (h) OBS C4, (i) OBS C9, (j) OBS C3, (k) OBH 27, (l) OBH 25, (m) OBH 16, (n) OBH 26, (o) OBH 20, (p) OBS A3, (q) OBS A1, (r) ST 19, (s) ST 18, (t) ST 17, (u) ST 15, (v) ST 14, (w) ST 13, (x) ST 10, and (y) ST 9. 70 s shot data are shown for most of deep-water and on-land instruments. For USGS OBS data, we show the hydrophone component, which has better quality than the geophone components. 


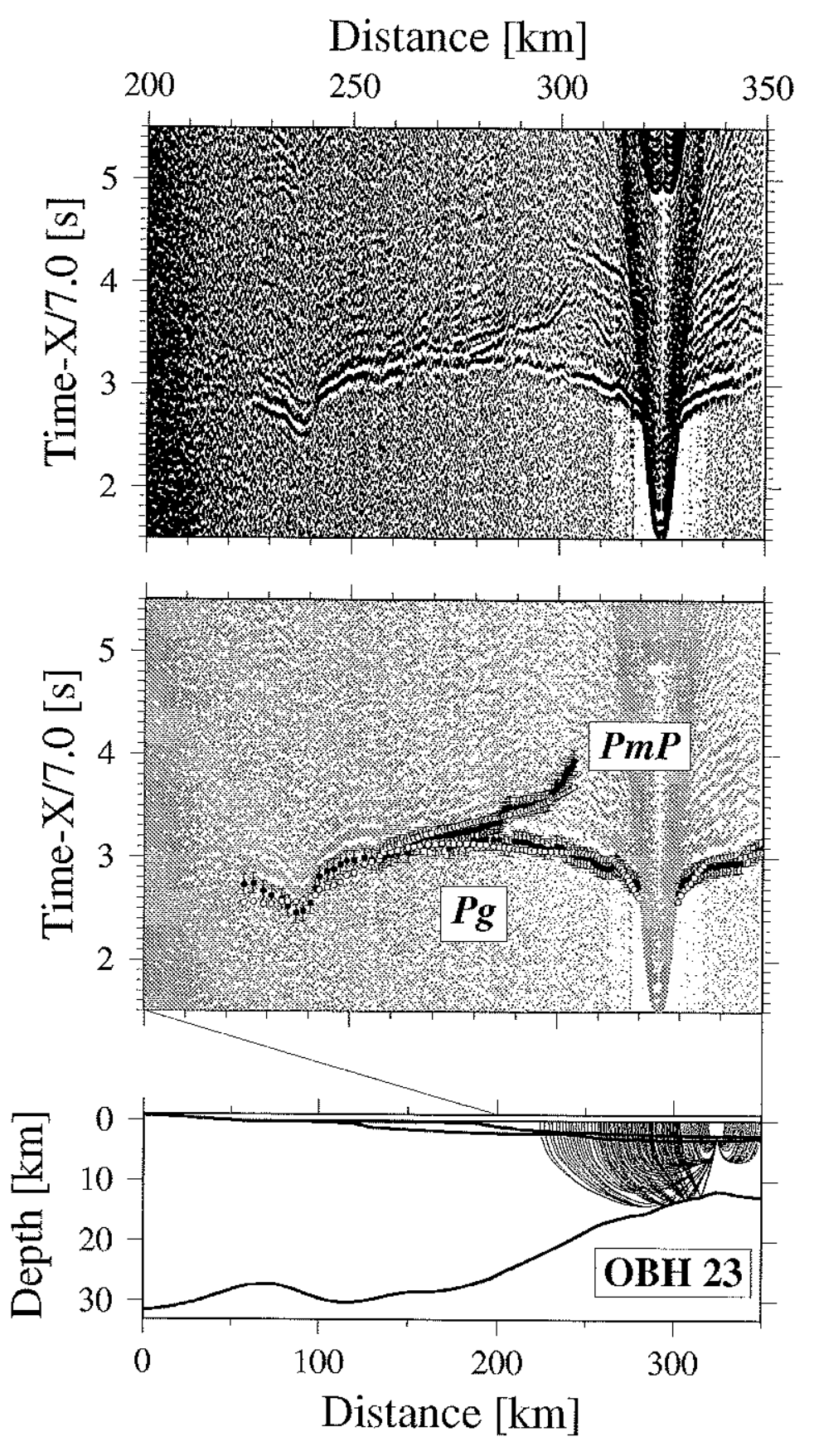



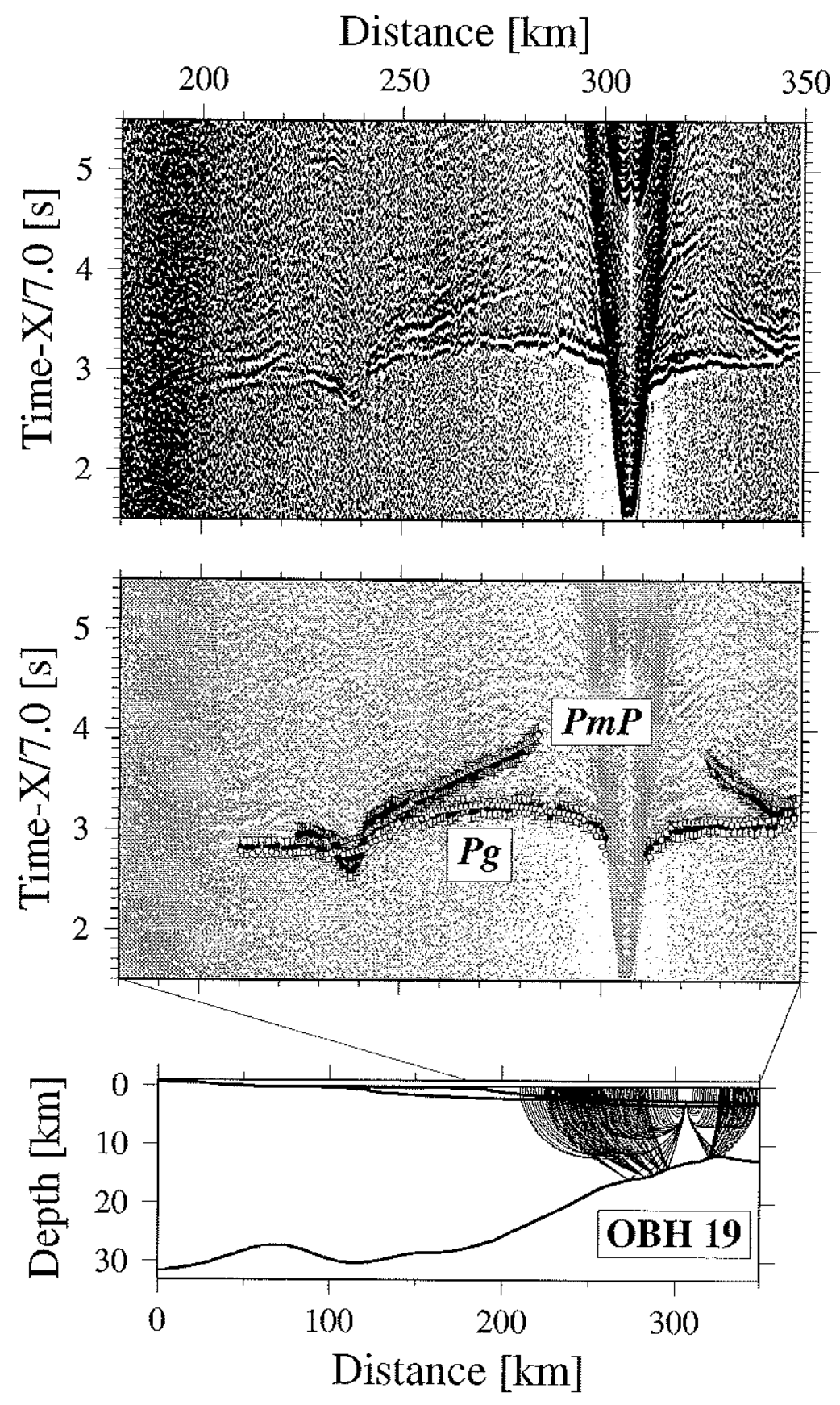

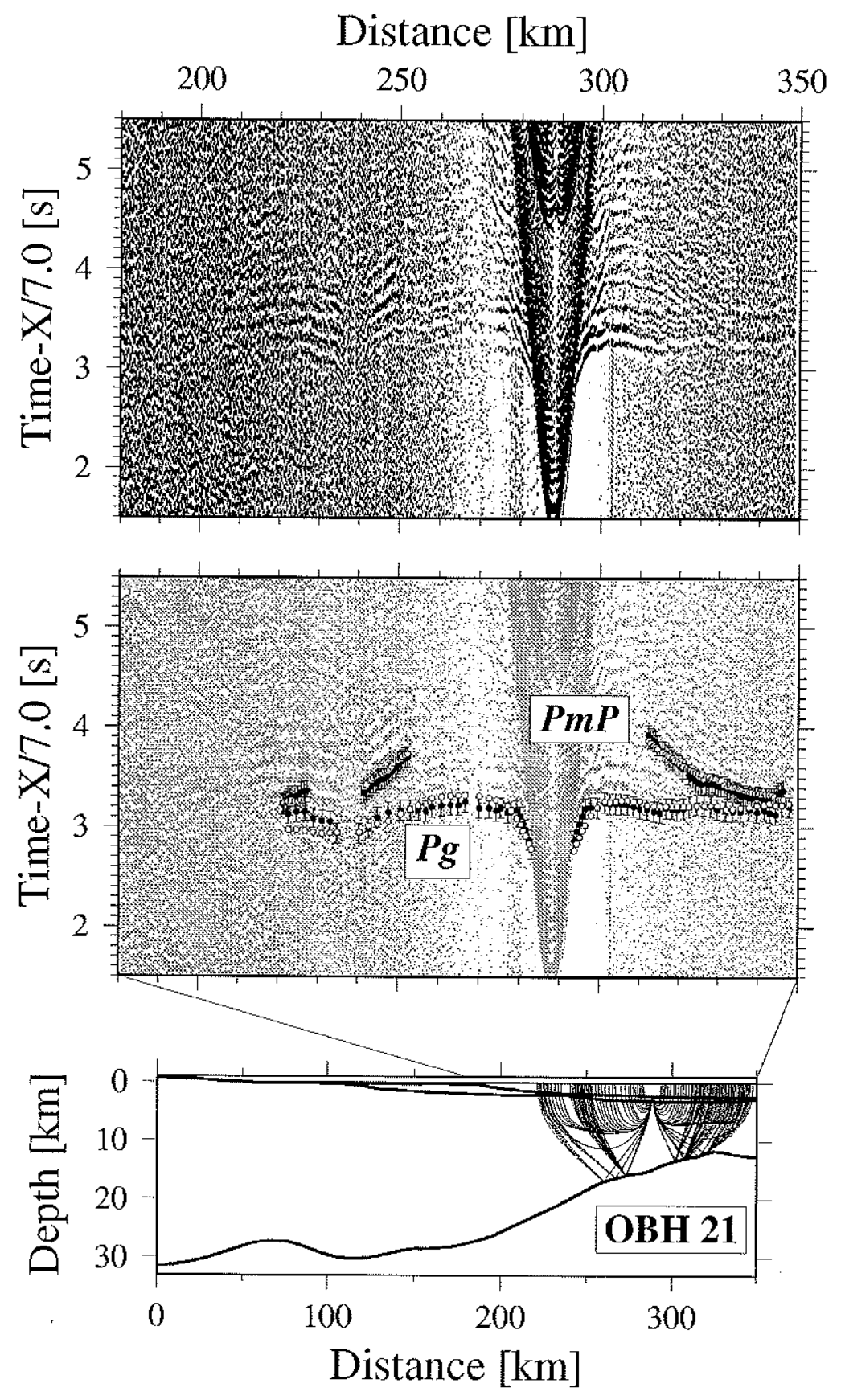

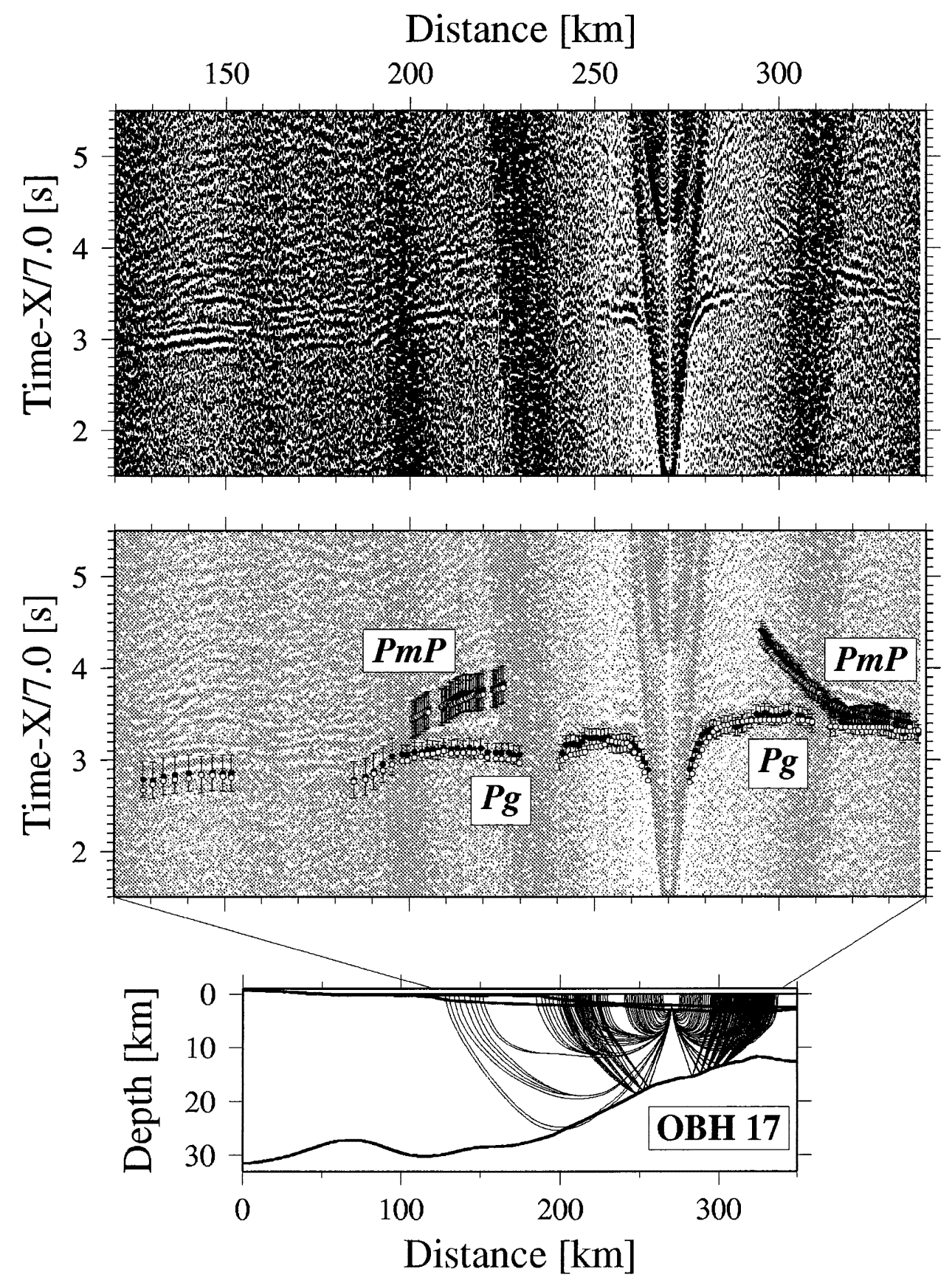

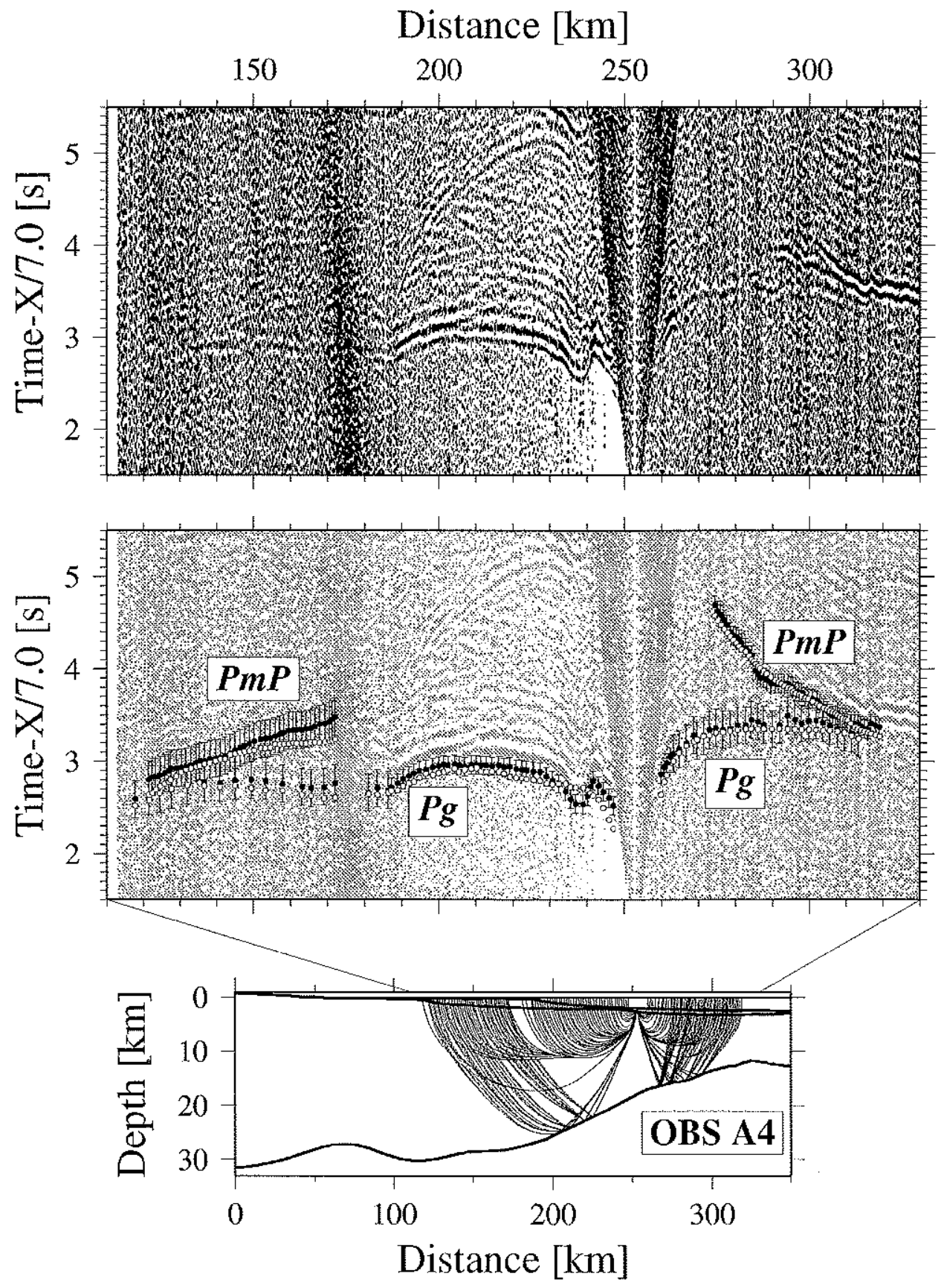

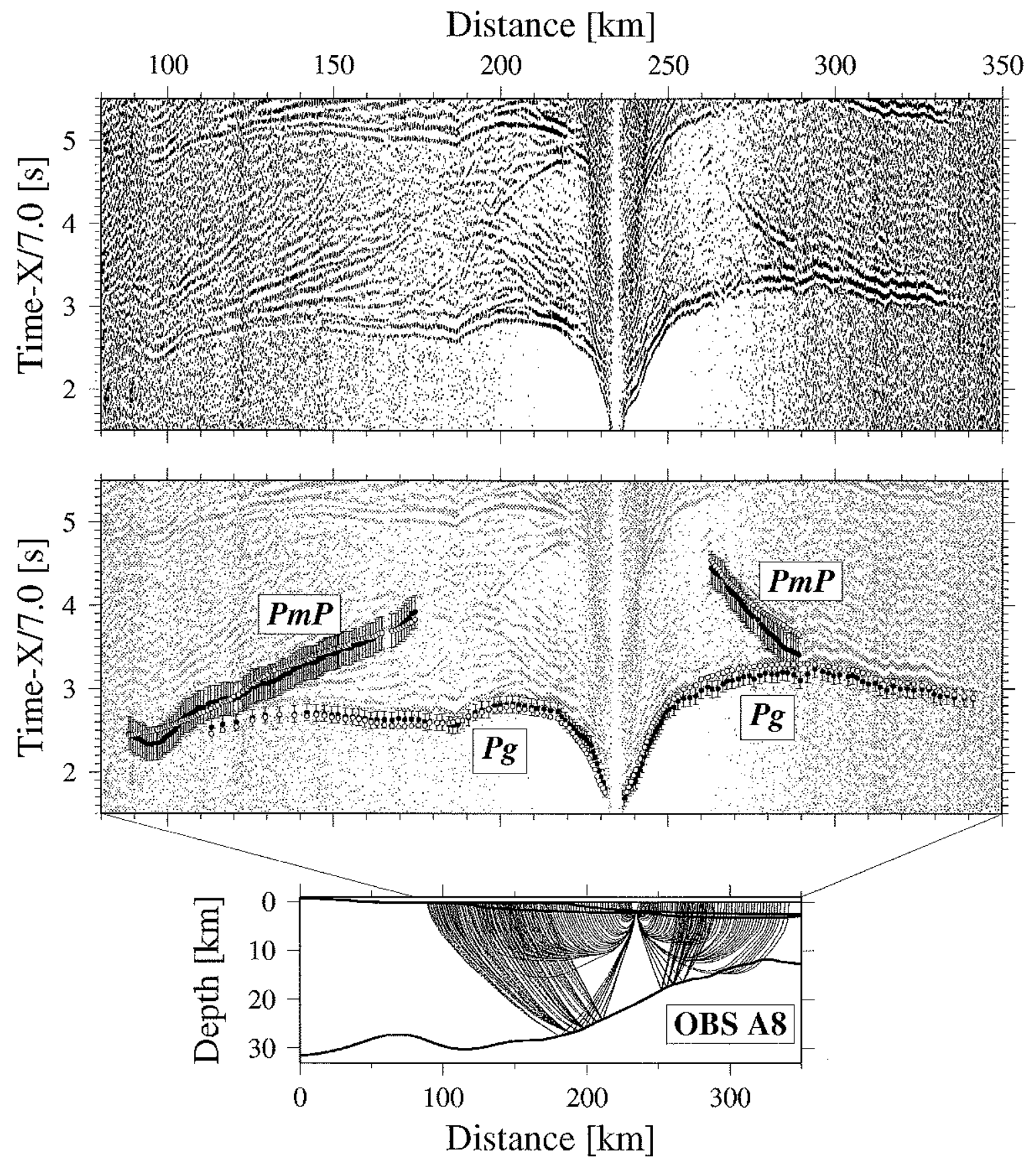

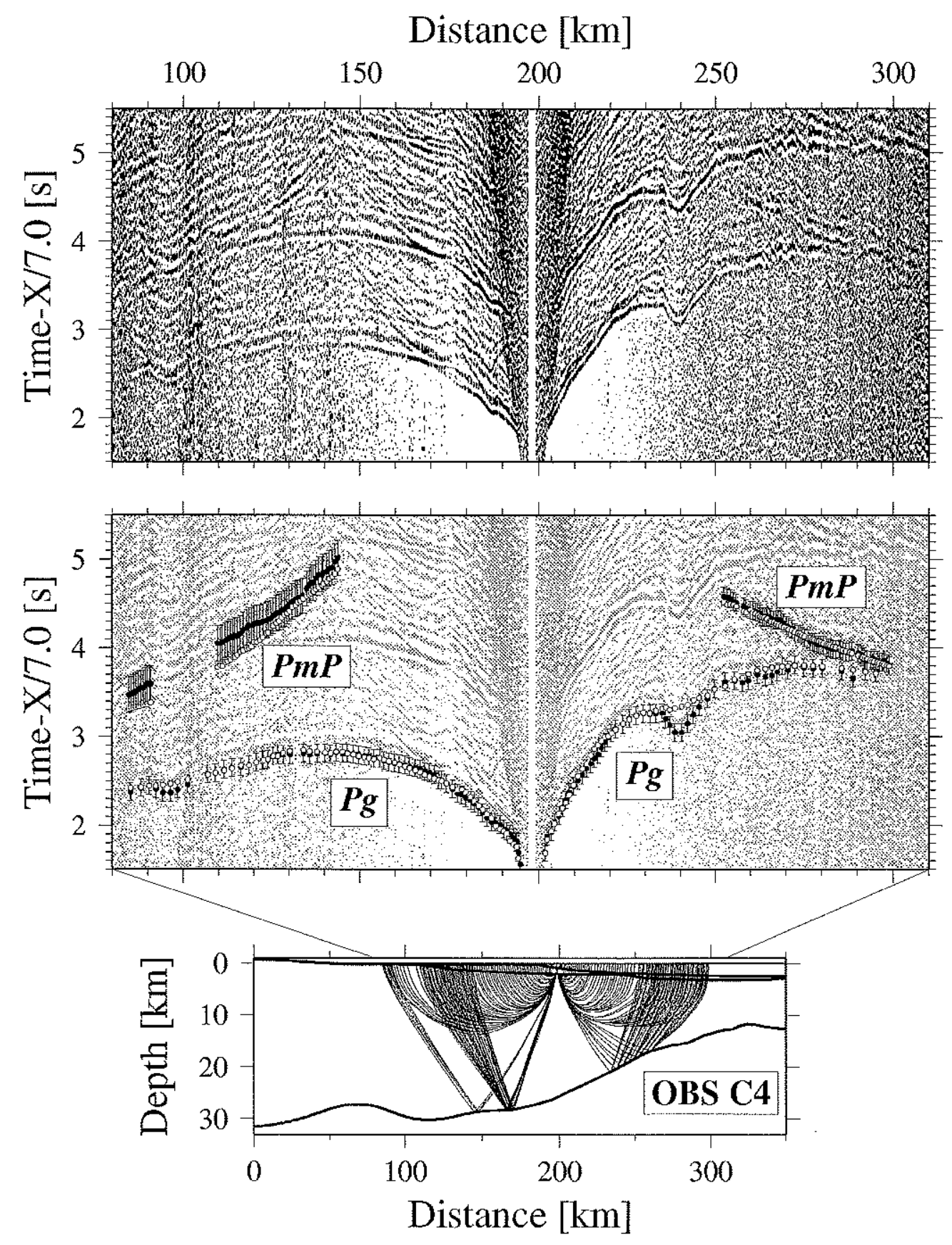

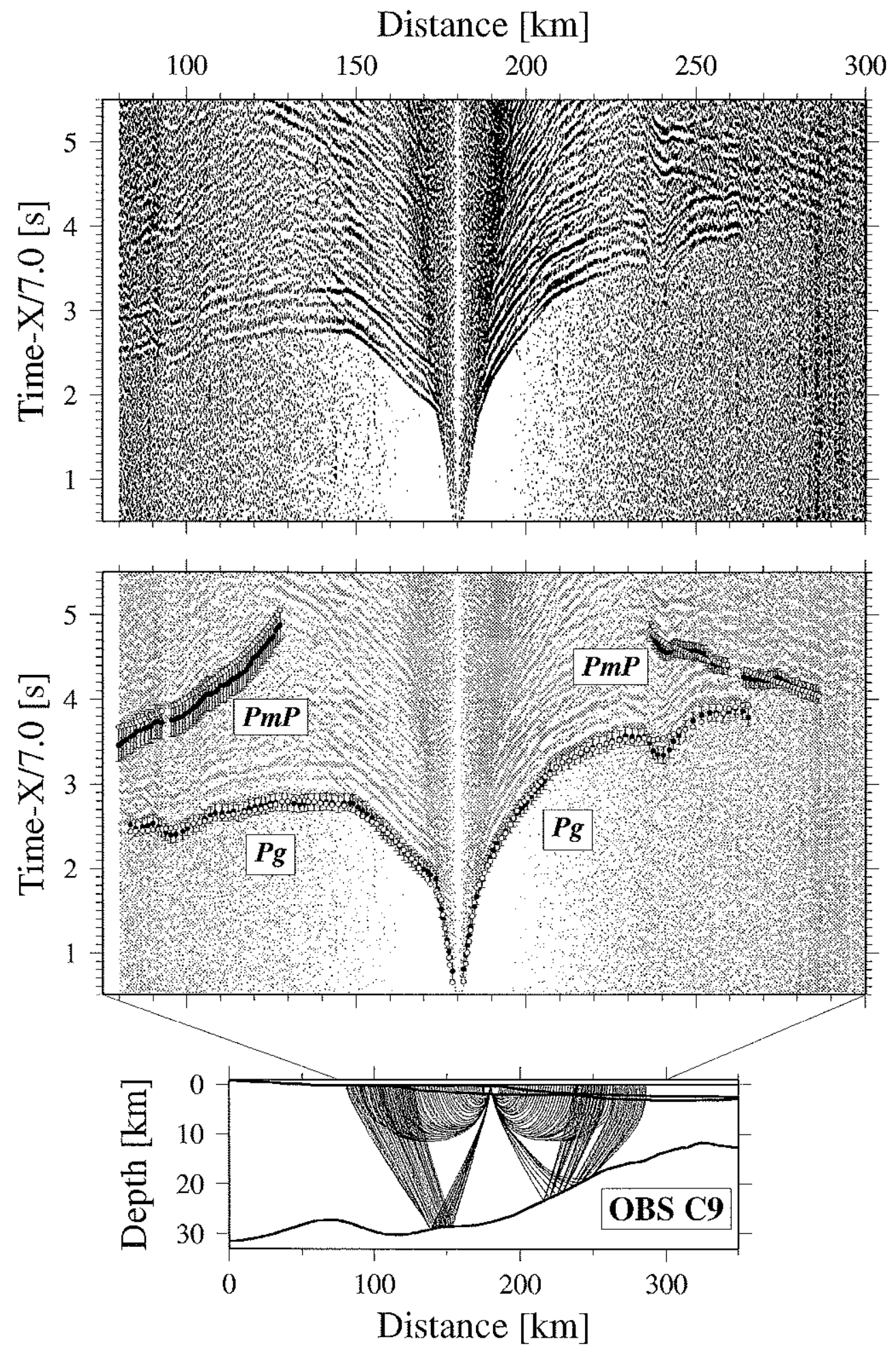

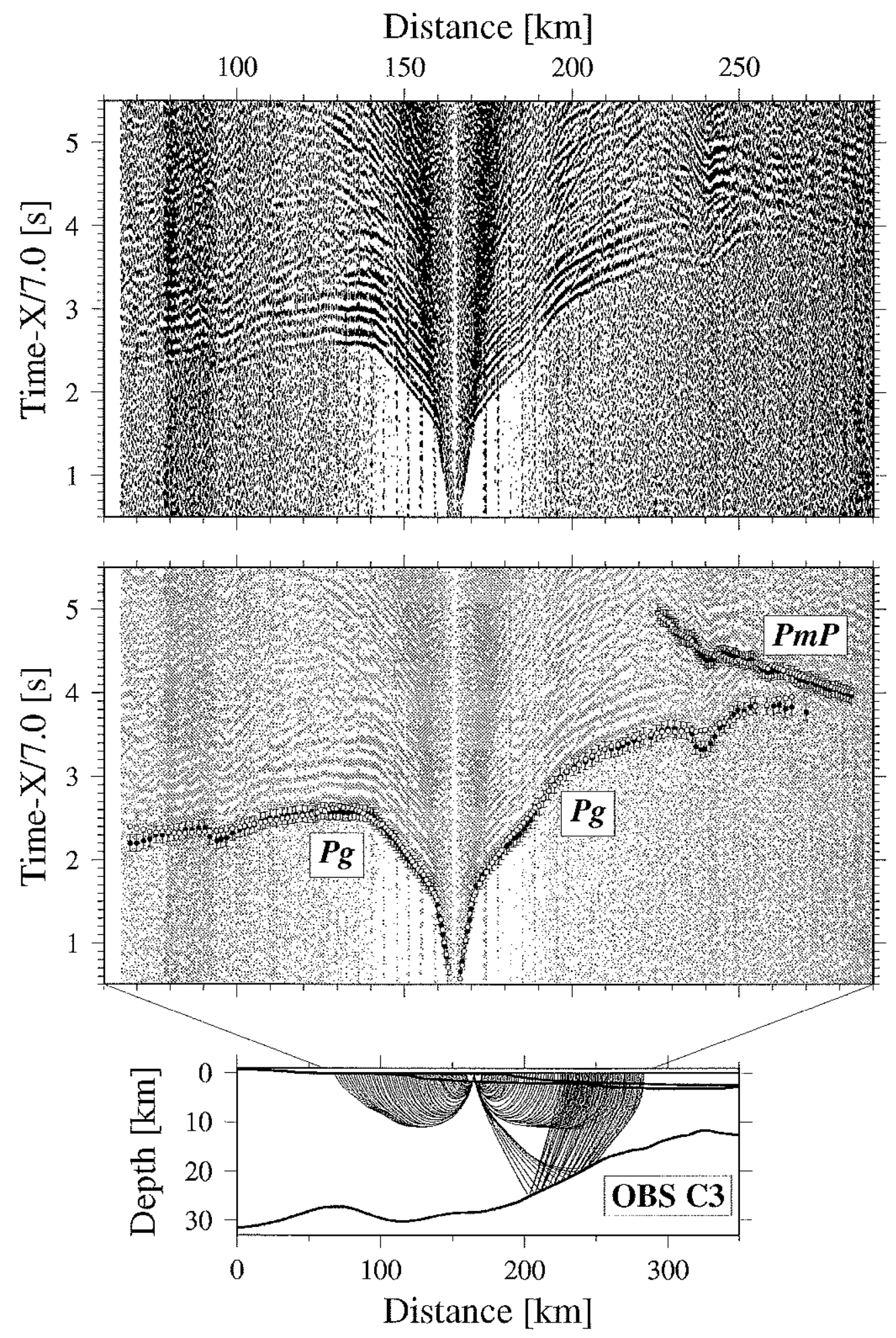

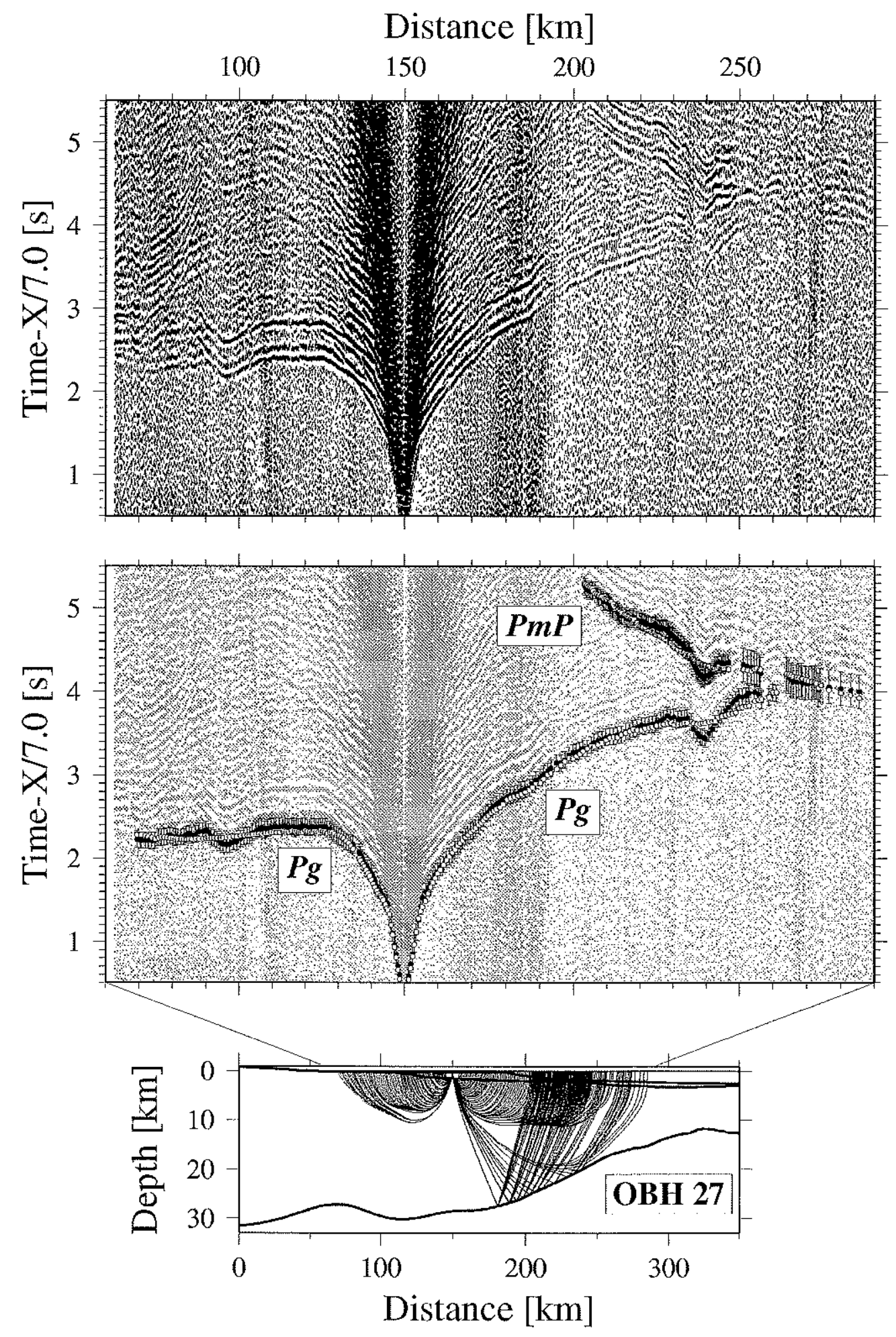

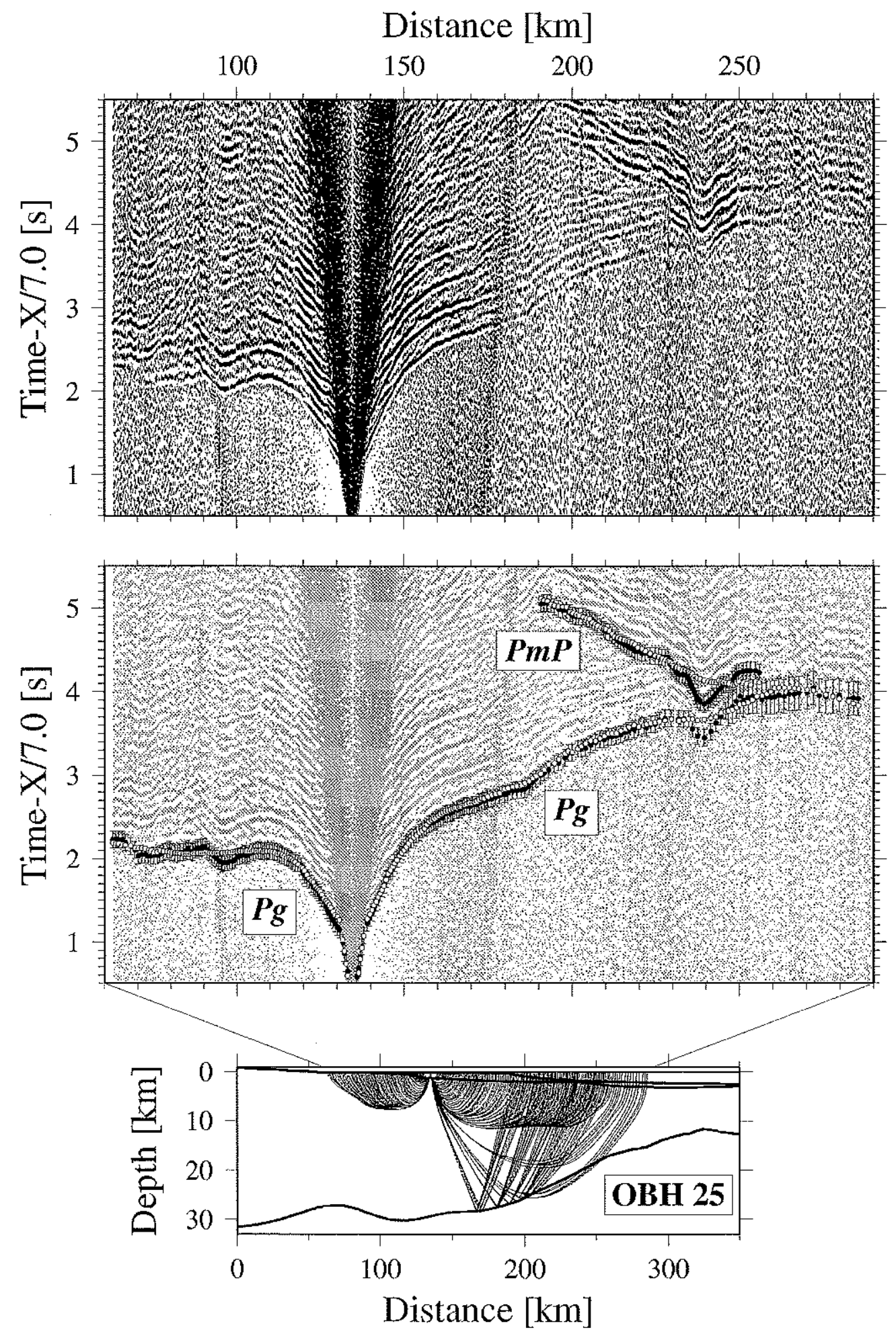

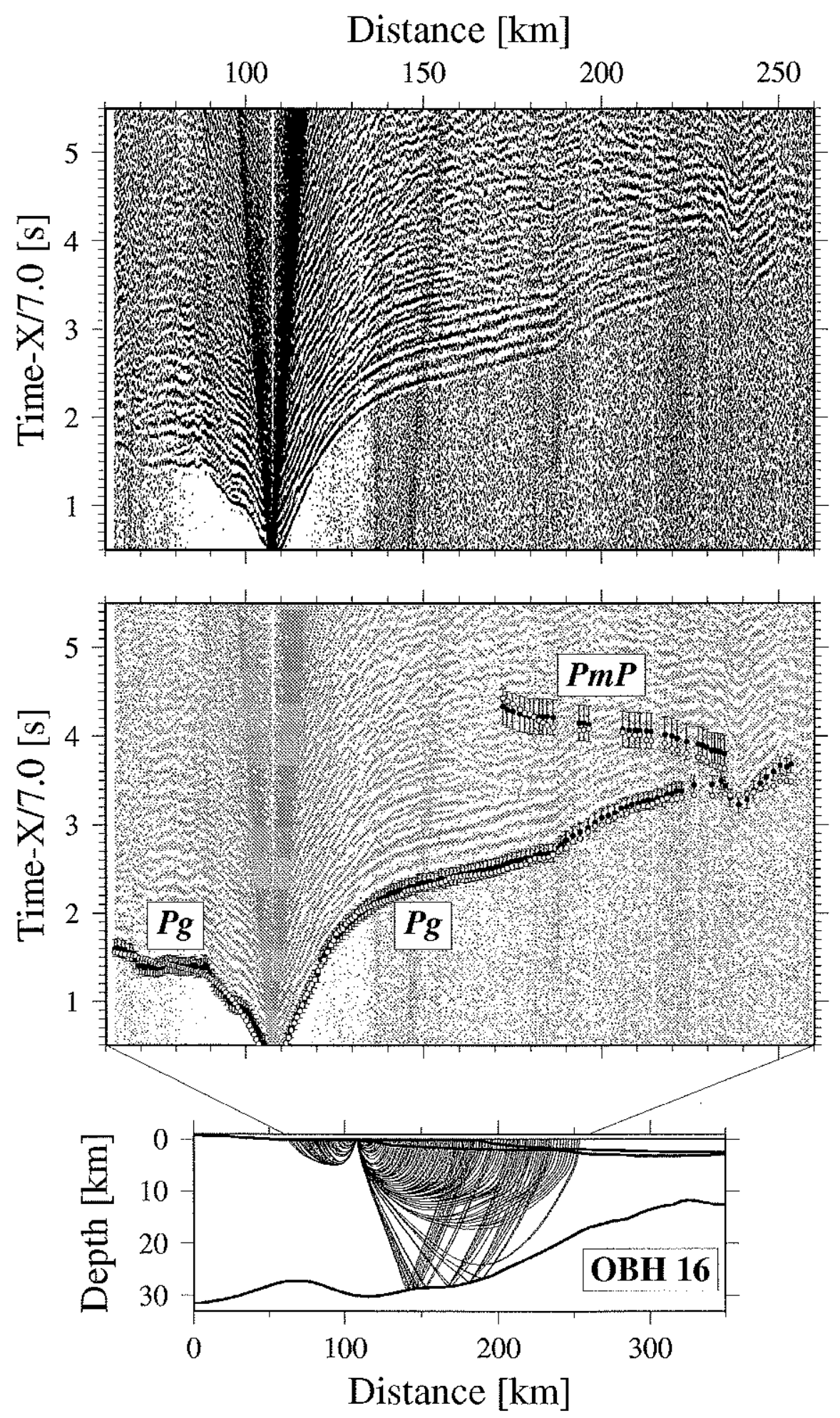


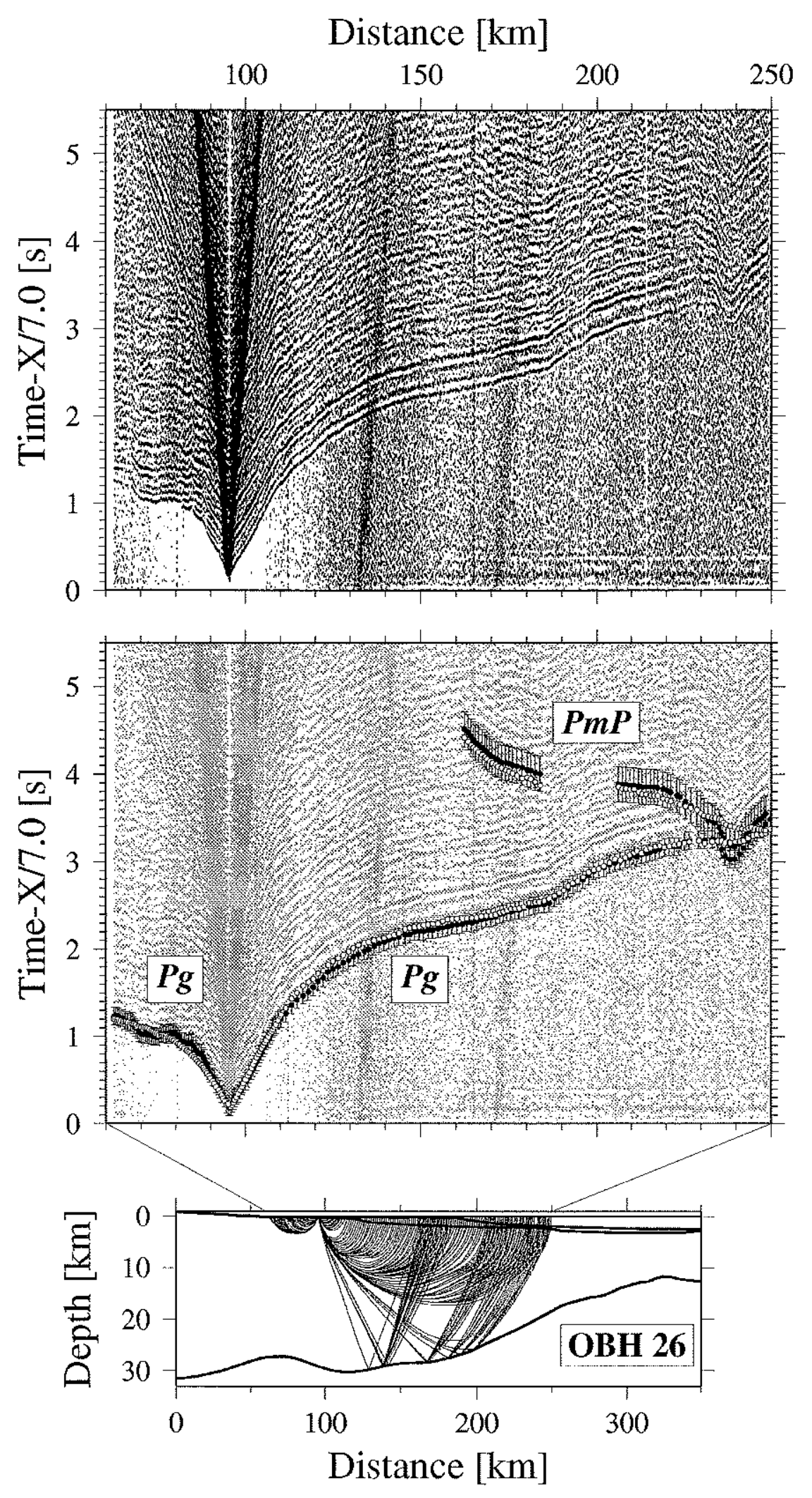




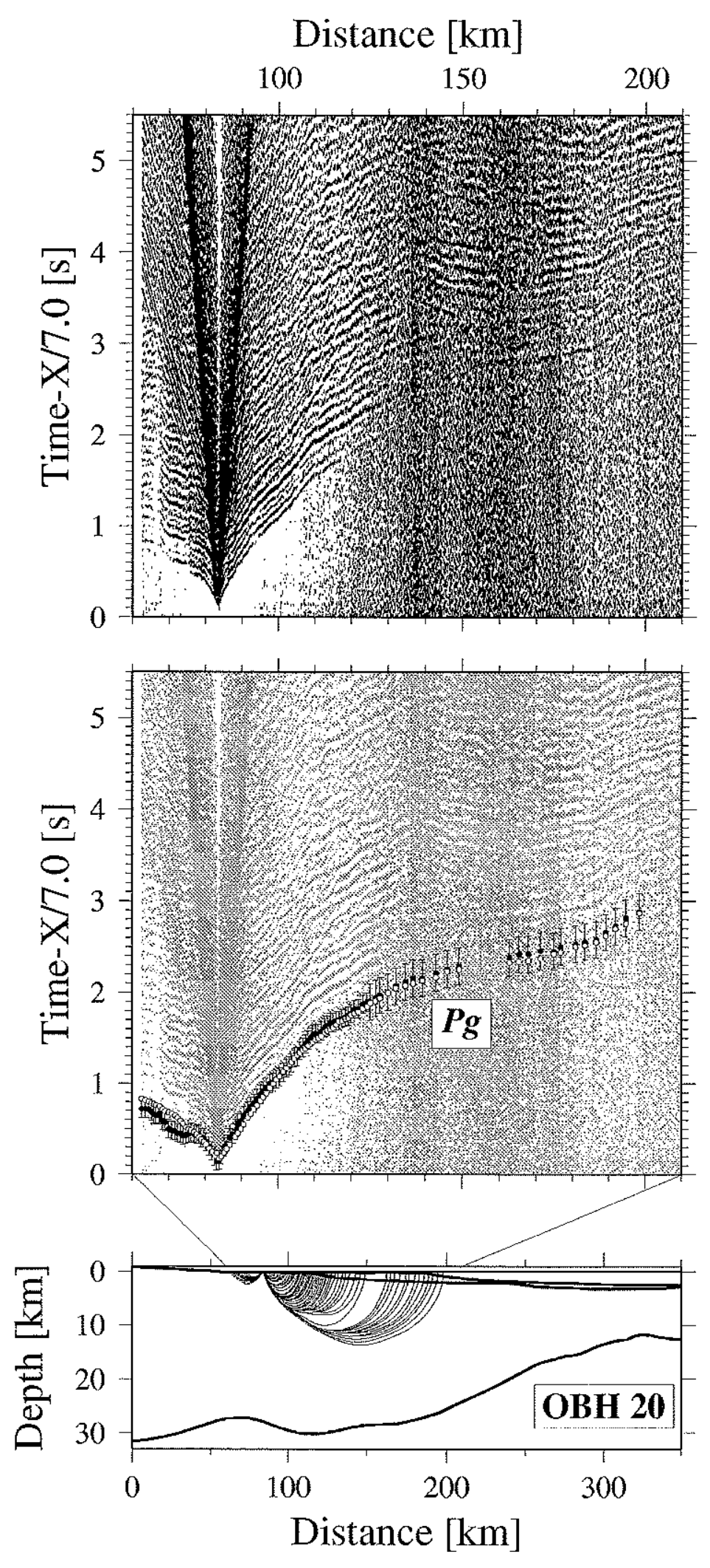



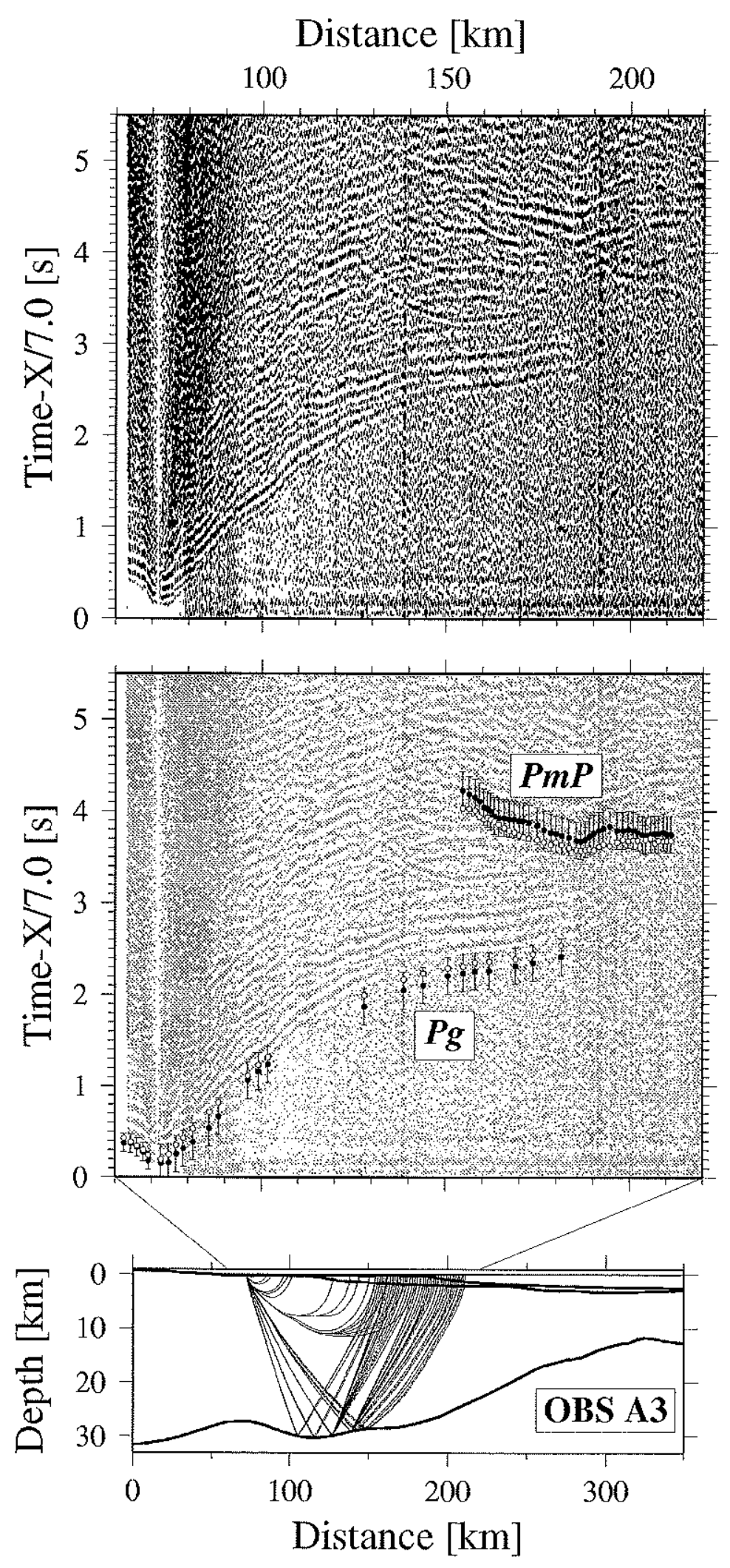

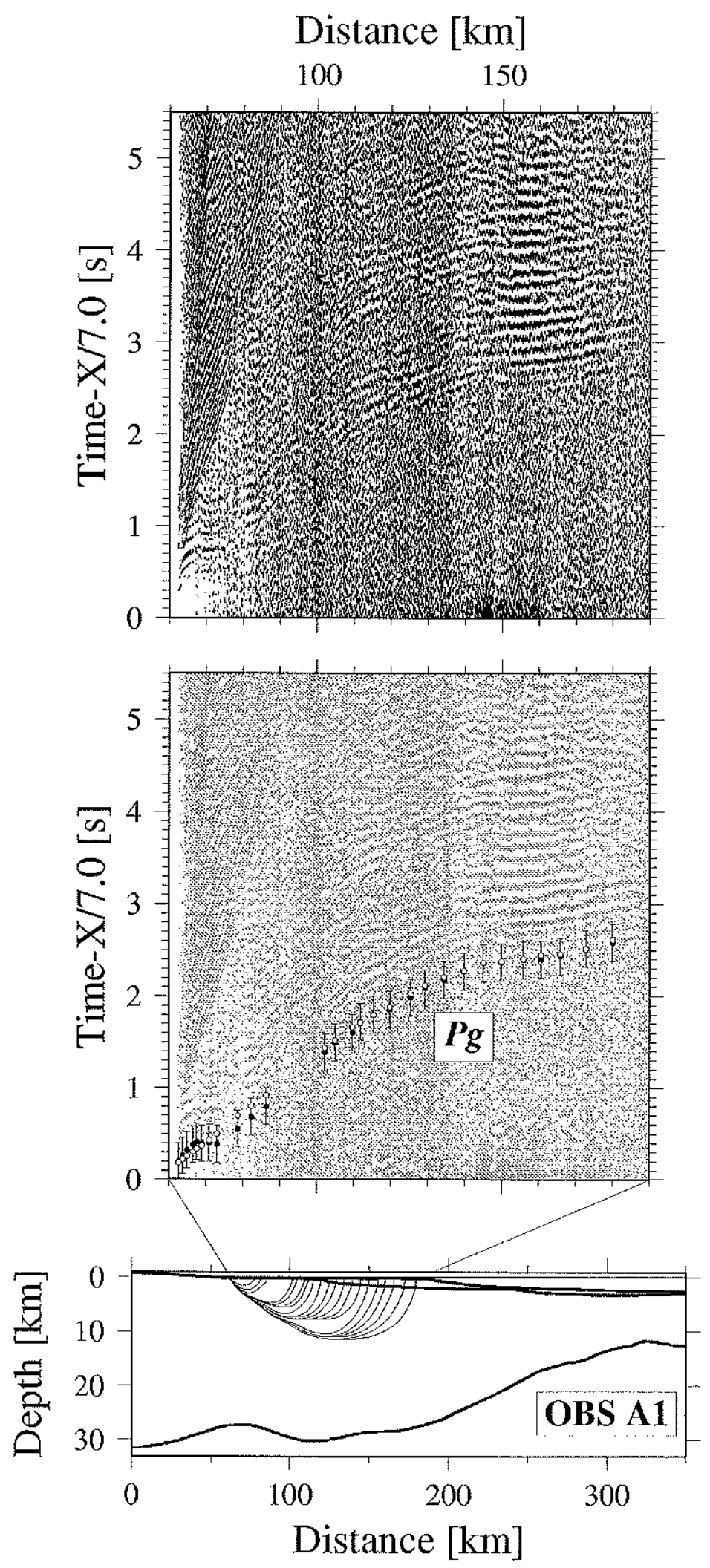


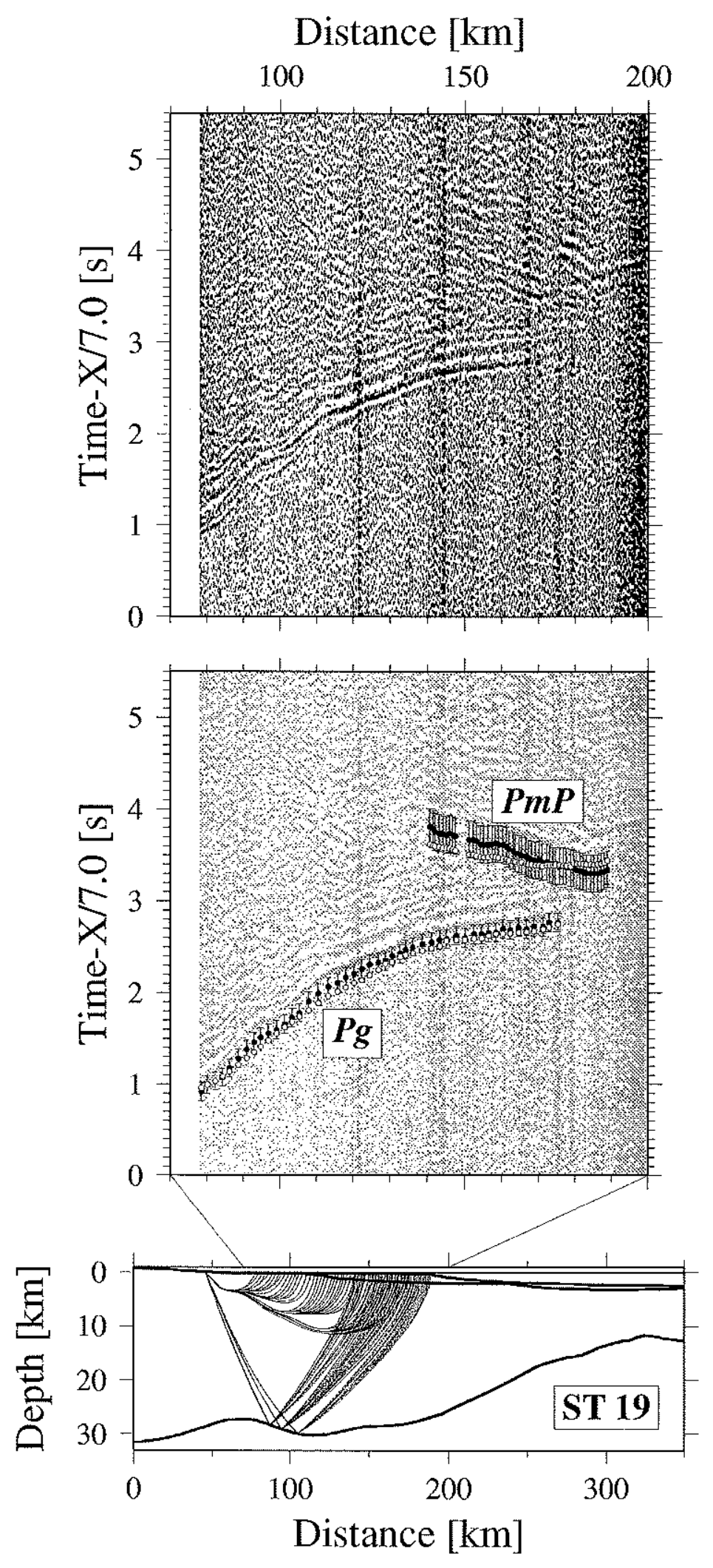




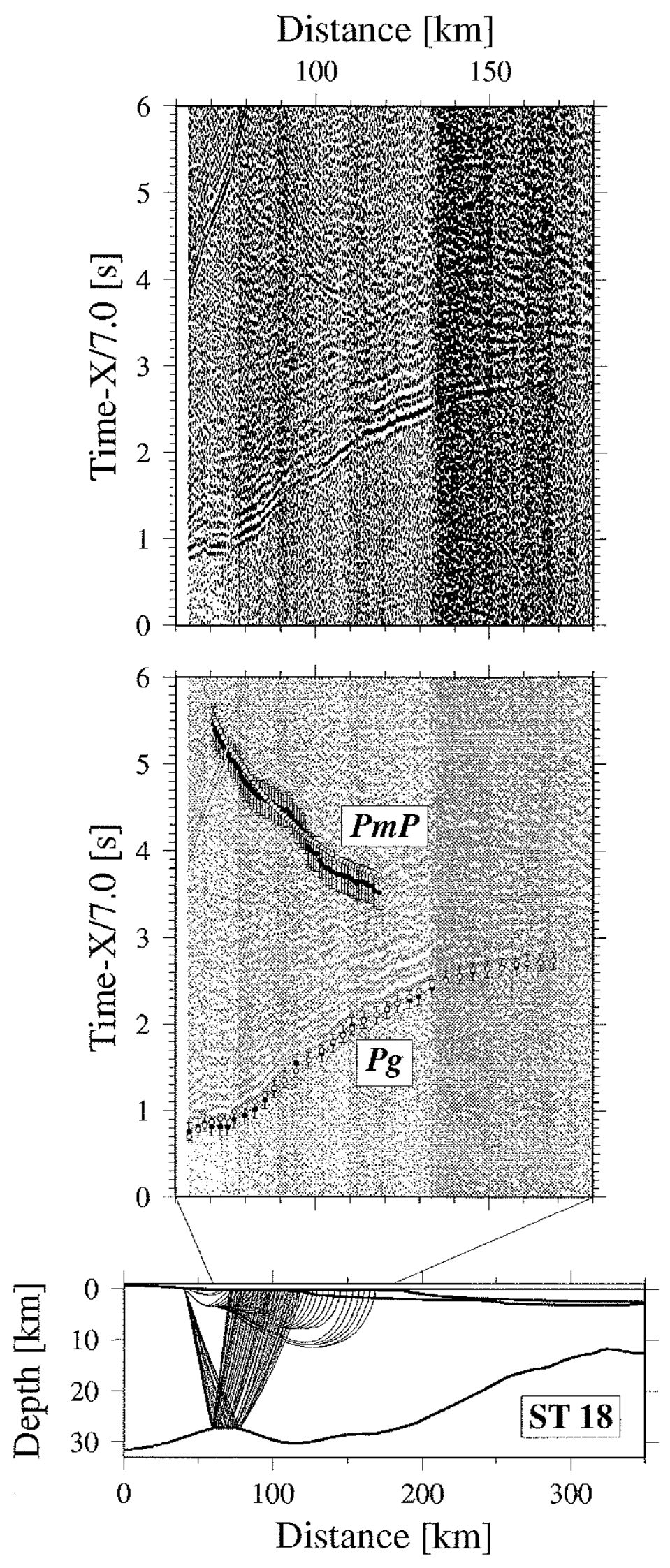




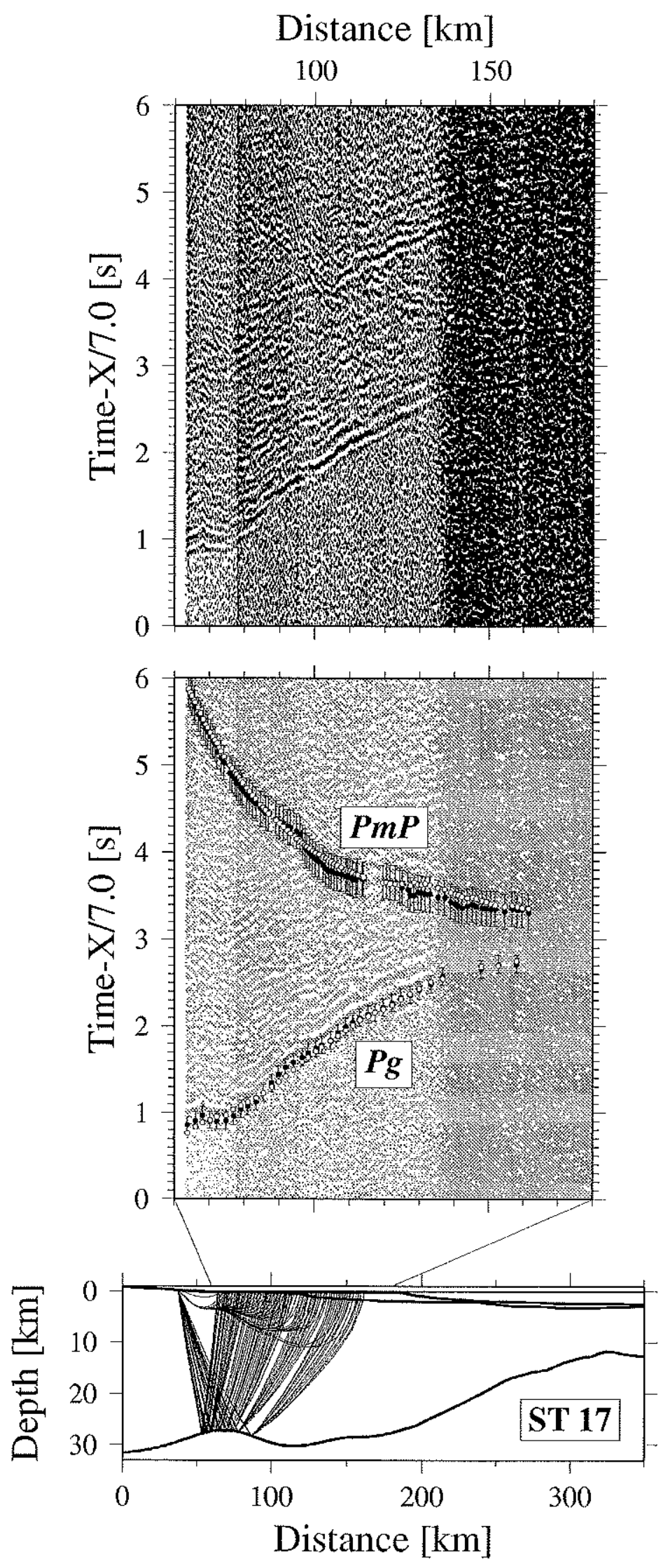




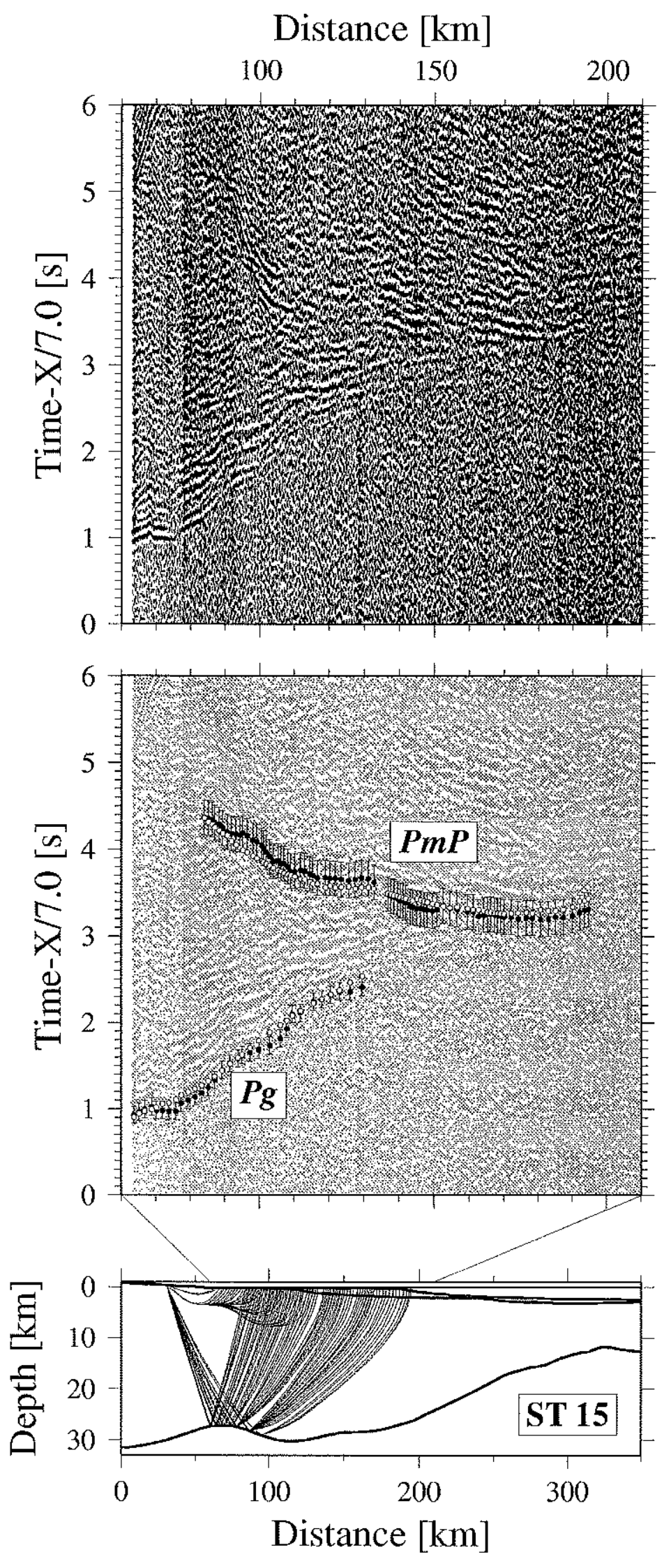



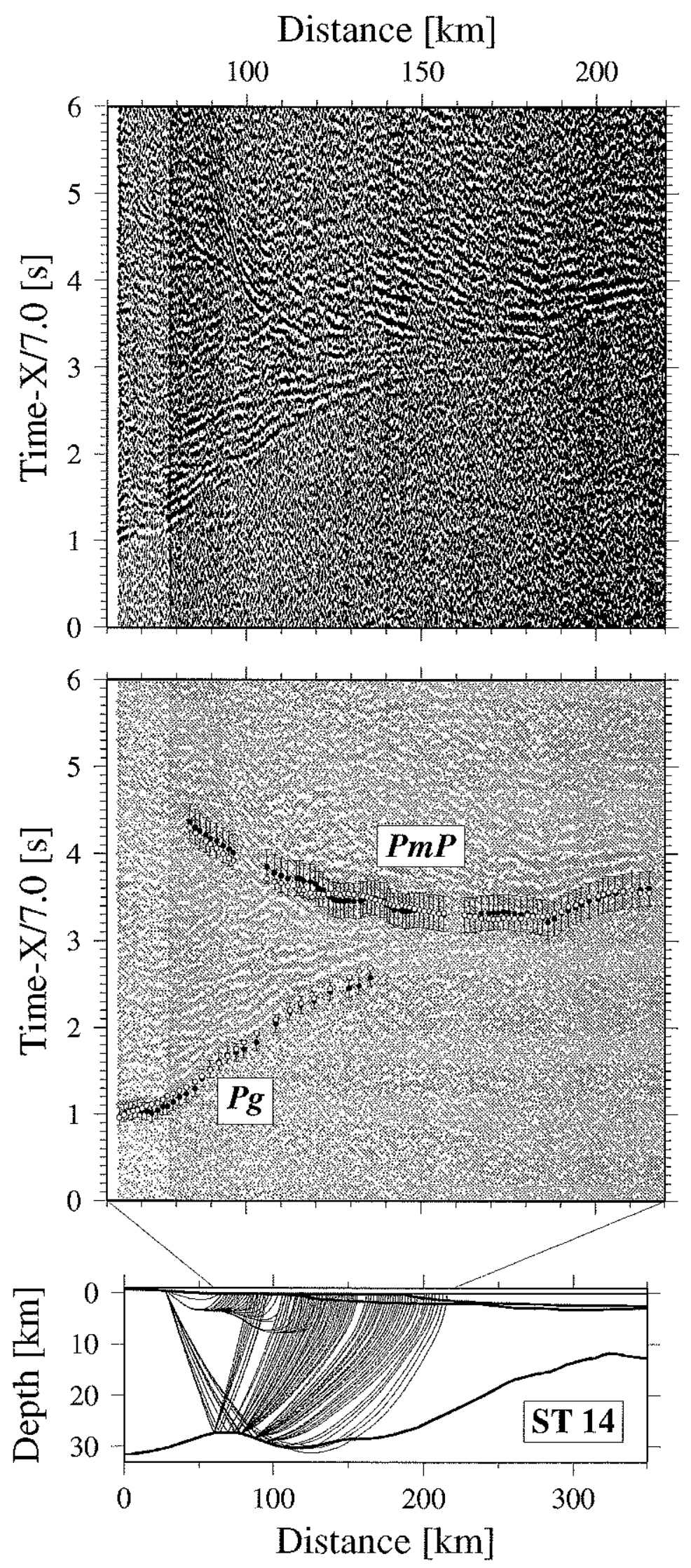


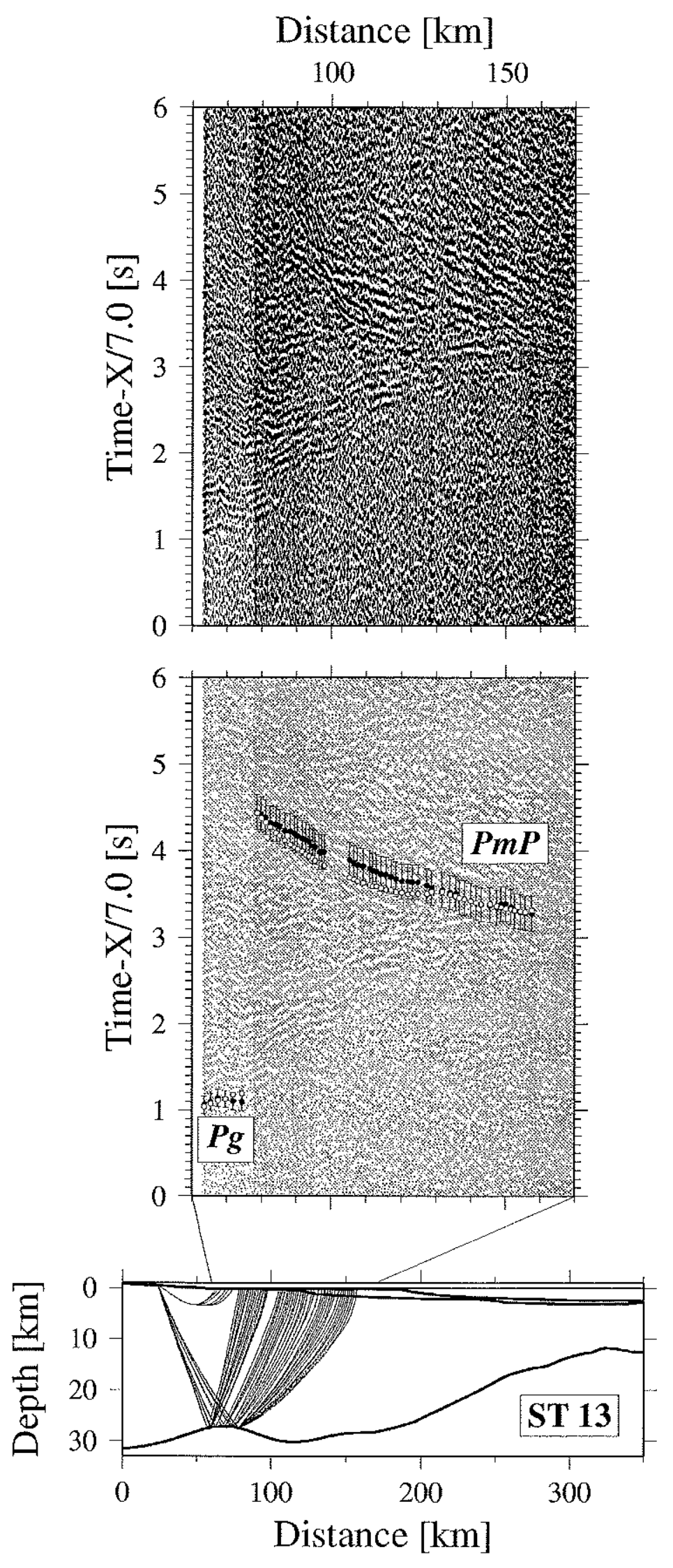




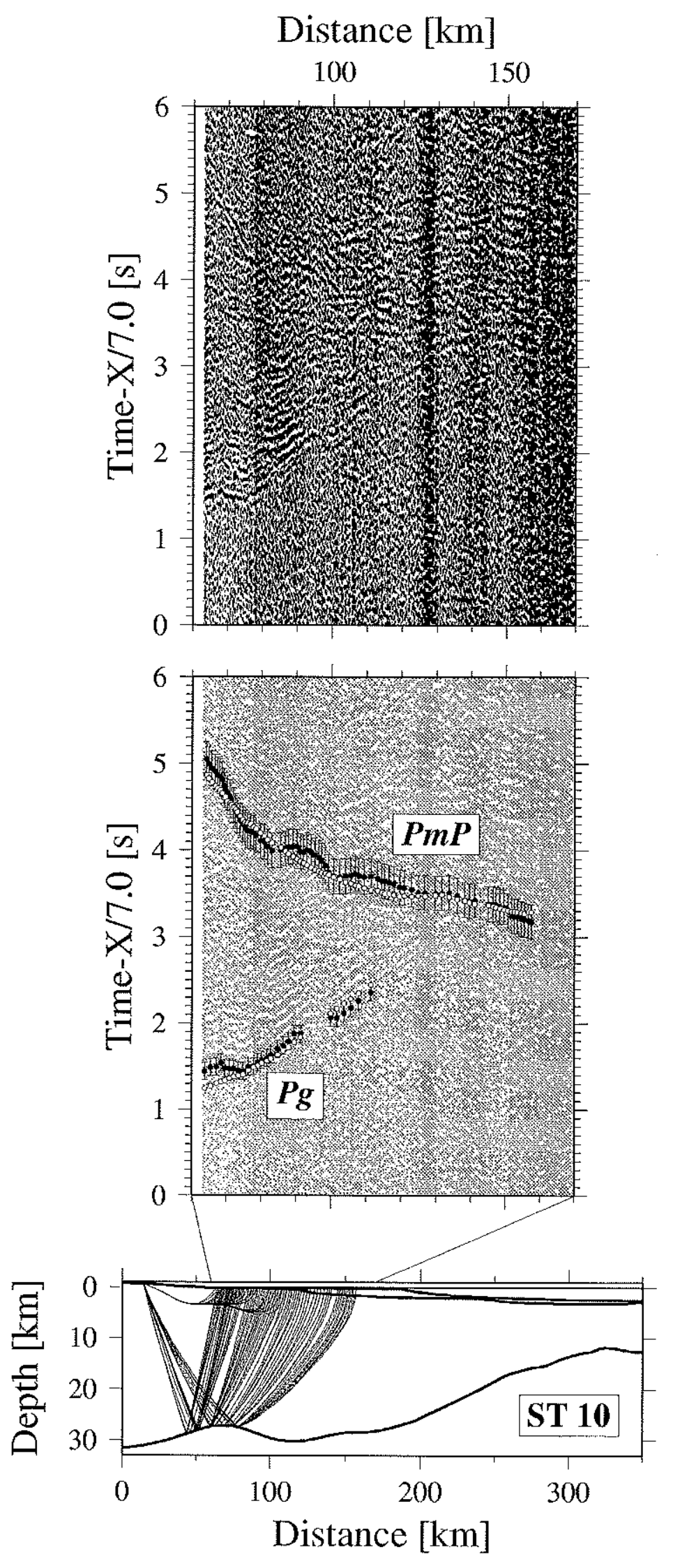




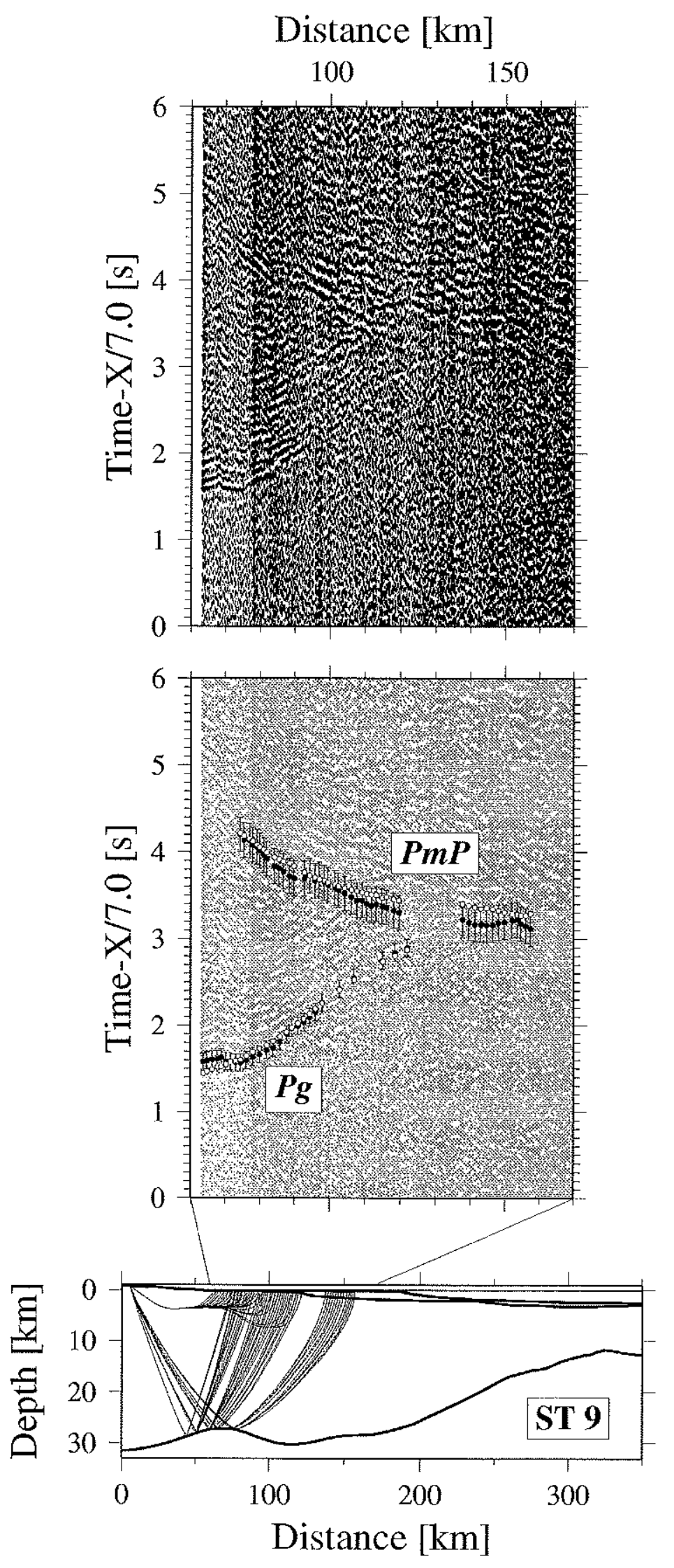


Figure 1-5. Benchmark test of the ray tracing algorithm. (a) 1-D velocity model of typical oceanic crust. (b) Ray paths and (c) travel times are calculated for the model using analytical integration for both refraction and reflection phases. Receiver is located on seafloor, and sources are located on sea surface at approximately $10-\mathrm{km}$ interval. (d) Ray paths by the graph method with a velocity grid of $1-\mathrm{km}$ horizontal spacing and $0.25-\mathrm{km}$ vertical spacing. (e) Ray paths refined by the ray-bending method. (f) Another purely graph-theoretical paths with a velocity grid of $0.25-\mathrm{km}$ horizontal spacing and $0.125-\mathrm{km}$ vertical spacing. Error in travel times is plotted as a function of source-receiver offset in (g) for refraction and in (h) for reflection. Open circle, solid star, and solid circle correspond to the pure graph solution (d), the hybrid solution (e), and the pure graph solution with the finer grid, respectively. Ray dissimilarity is also plotted in the same way in (i) for refraction and in (j) for reflection. Ray dissimilarity is a measure of ray path misfit [e.g., Fischer and Lees, 1993], and our definition here is $\Delta x=\int_{0}^{1}\left|\mathbf{x}(s)-\mathbf{x}_{\text {true }}(s)\right| d s$, where $\mathbf{x}$ and $\mathbf{x}_{\text {true }}$ are numerical and analytical ray paths, respectively, as a function of the normalized path coordinate, $s$, defined along each ray. 
(a) 1-D test model

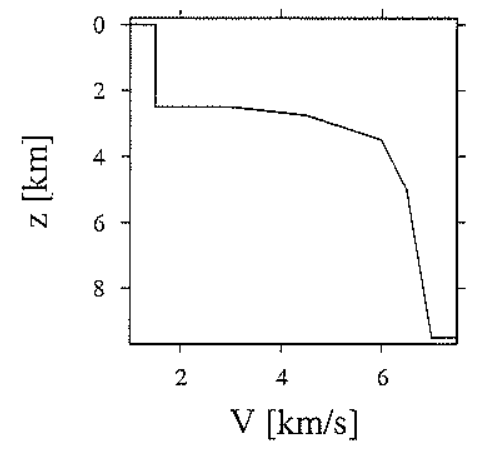

(d) graph only (1x0.25)

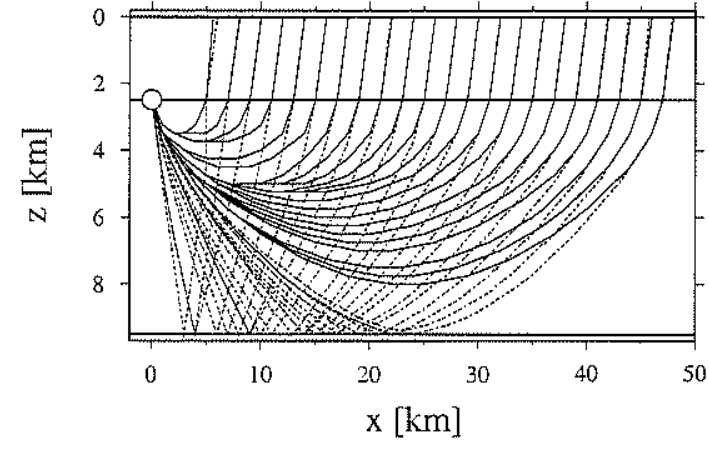

(g)

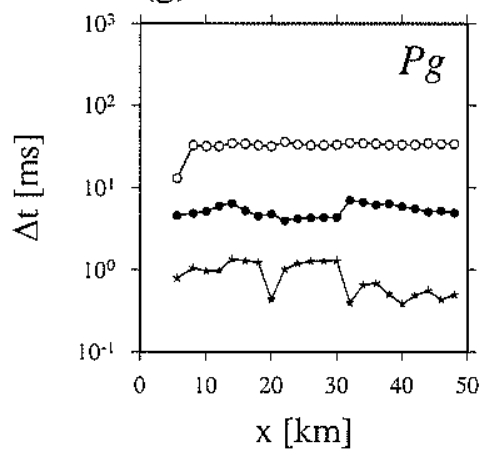

(h)

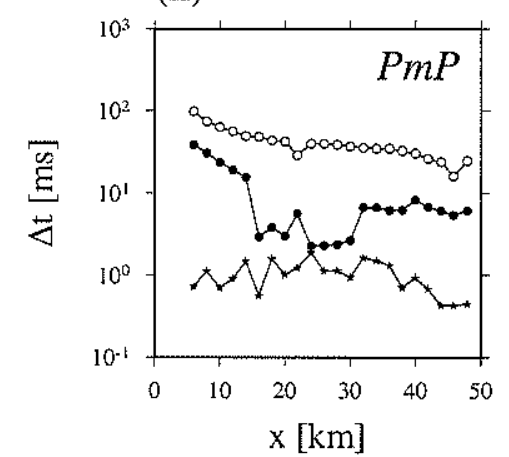

(c) analytical travel times

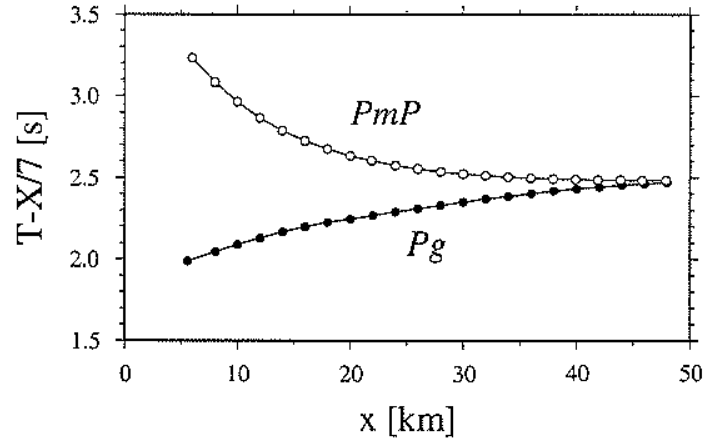

(f) graph only $(0.25 \times 0.125)$

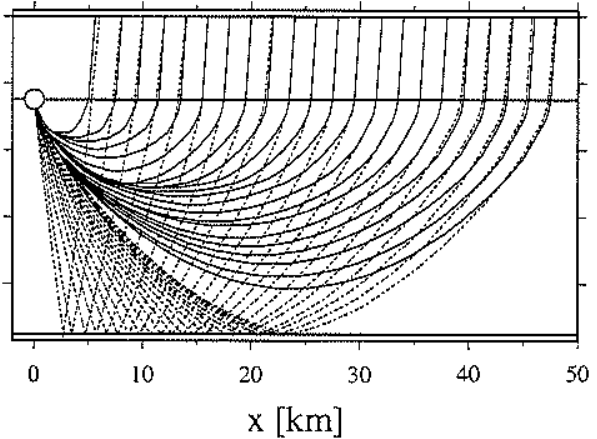

(e) graph+bending $(1 \times 0.25)$

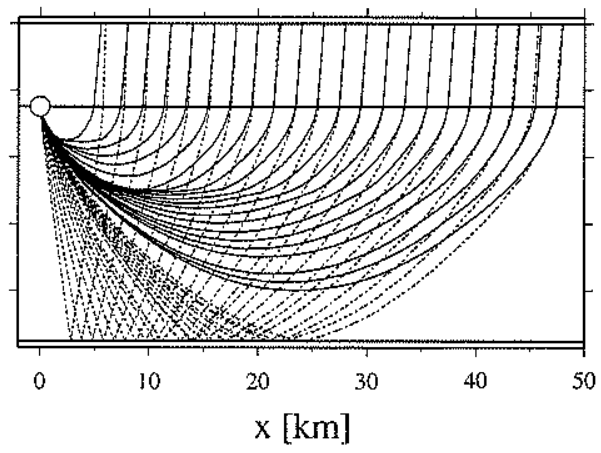

(i)

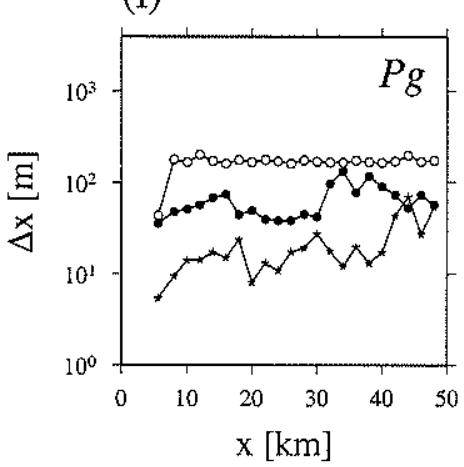

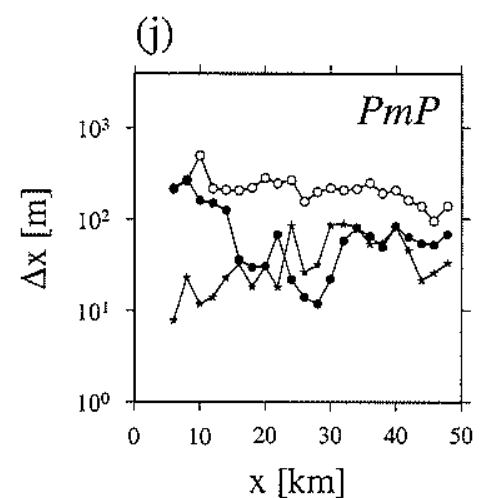


Figure 1-6. Numerical examples to demonstrate velocity-depth ambiguity in reflection tomography. In a true model, 1-D velocity profile similar to Figure 1-5a was hung beneath the seafloor, and a reflector is located at depth of $9 \mathrm{~km}$. (a) Starting model has true velocity structure (i.e., no velocity perturbation), but starting reflector (solid) is located 1-km shallower than true reflector (dotted). Two types of geometry were tested with this starting model. (b) Geometry A has five ocean bottom receivers and twenty sources. Excellent recovery is obtained both with (c) the case of $w=1$, and (d) the case of $w=100$. (e) Geometry B has the same sources but only three receivers. (f) Recovery with $w=1$ shows unwanted velocity perturbation, and the reflector is not completely restored. (g) Higher weighting of depth sensitivity $(\mathrm{w}=100)$ results in better recovery. The corresponding travel time fits in (f) and (g) are virtually the same. In all inversions, horizontal correlation length is set as $5 \mathrm{~km}$ at the seafloor and $10 \mathrm{~km}$ at the model bottom, and vertical correlation length is set as $0.5 \mathrm{~km}$ at the seafloor and $1 \mathrm{~km}$ at the bottom. 
(a) Starting Model
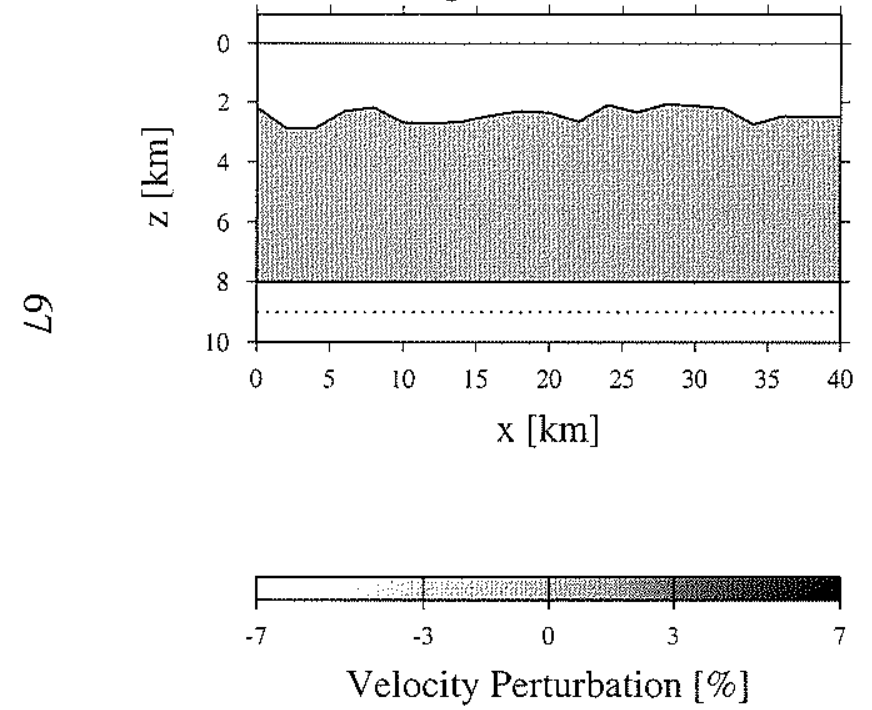

(b) Geometry A

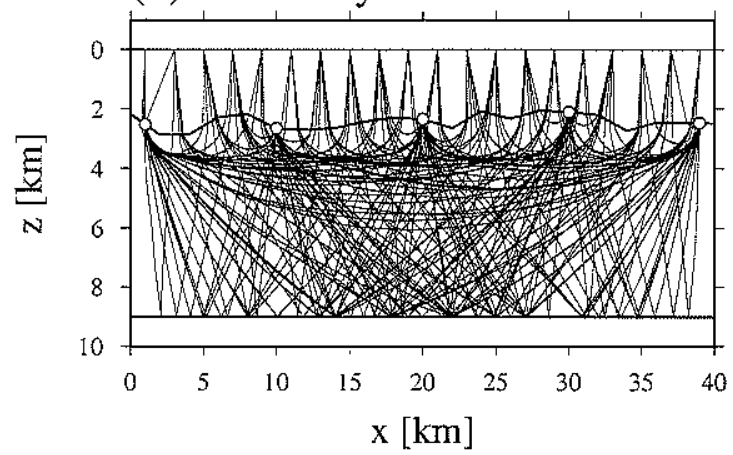

(c) Recovery $(w=1)$
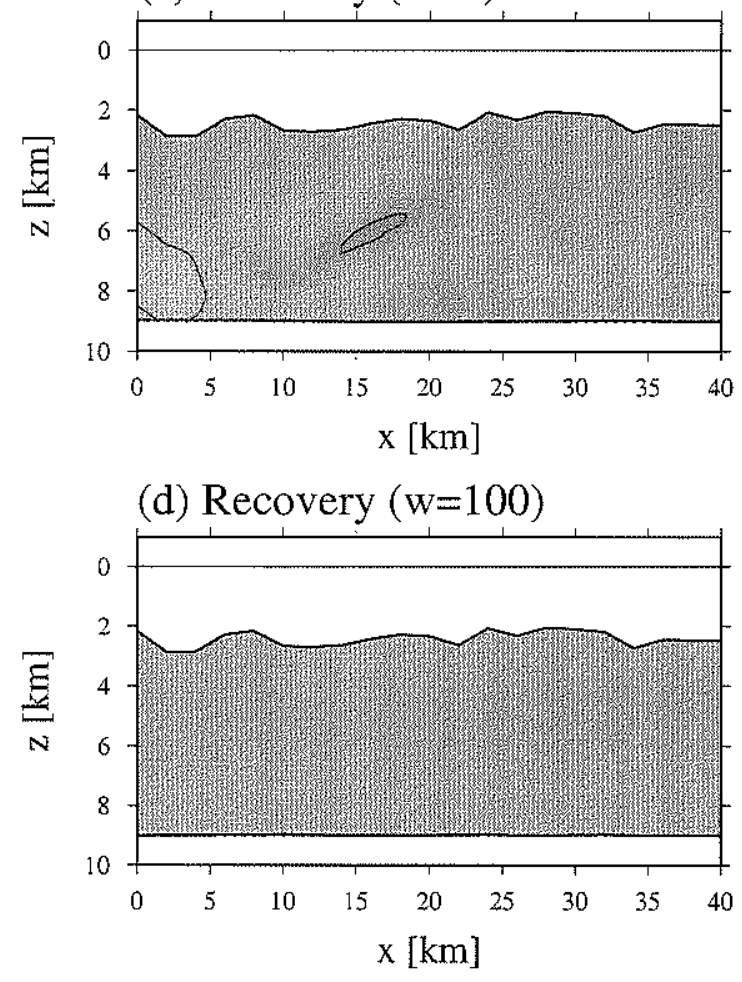

(e) Geometry B

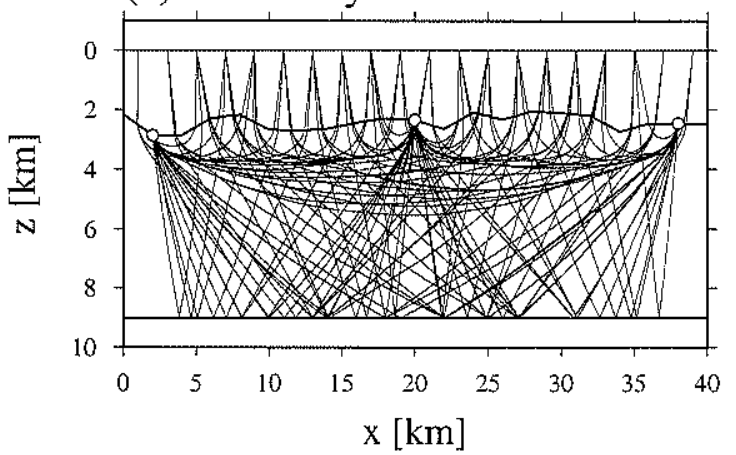

(f) Recovery $(\mathrm{w}=1)$
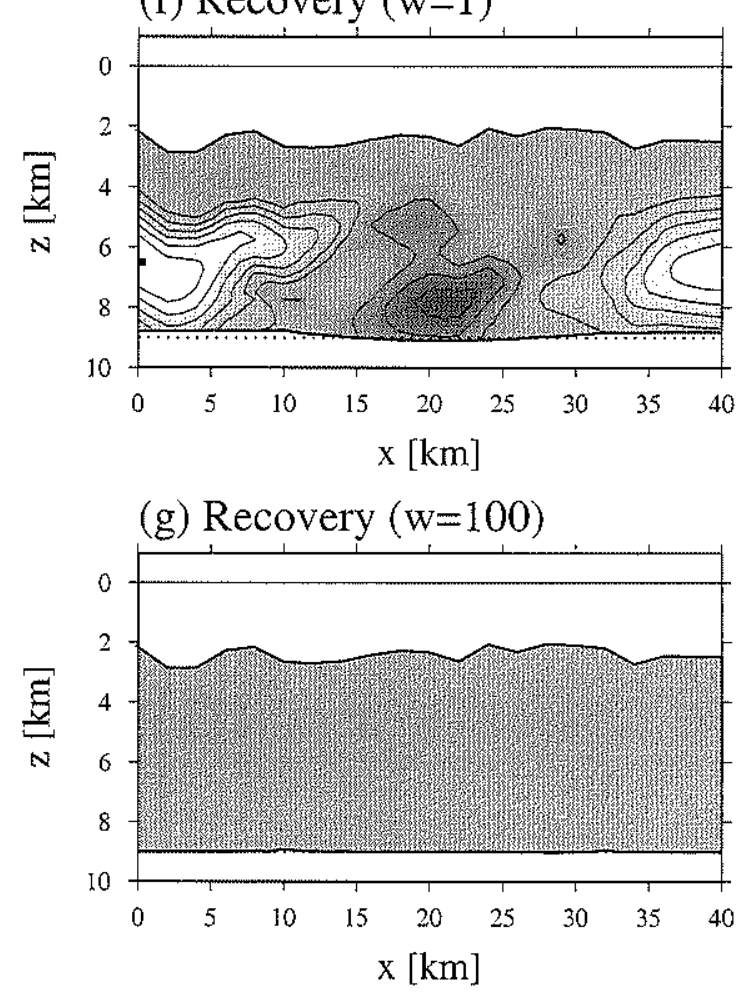


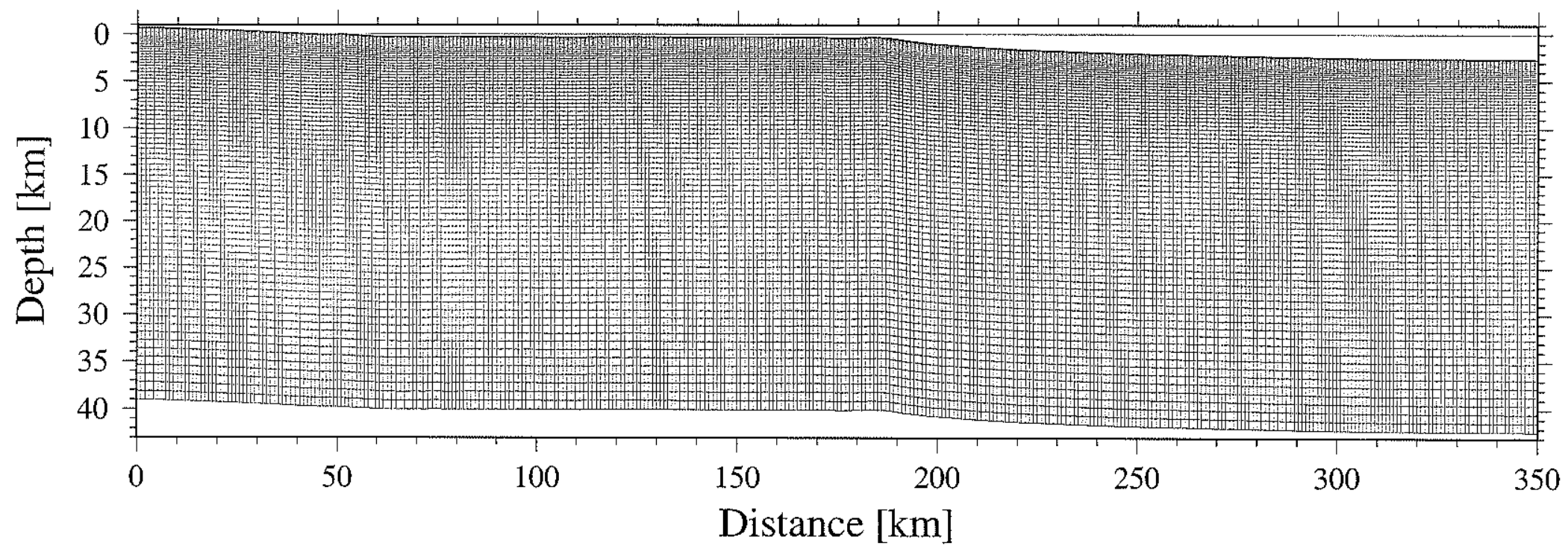

Figure 1-7. A velocity mesh is composed of $351 \times 77$ variable size cells hanging from seafloor. 

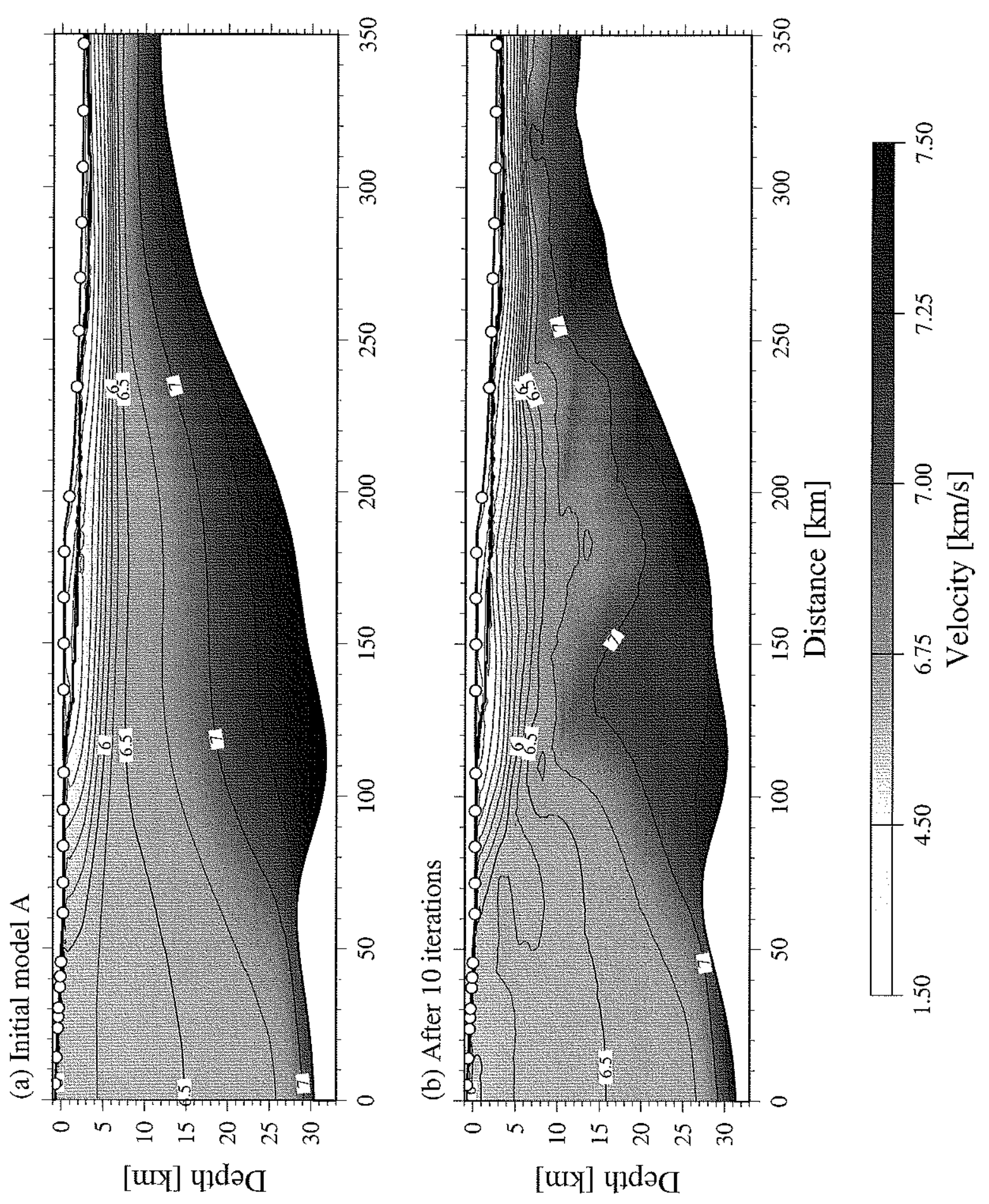

Figure 1-8. Result of tomographic inversion. (a) Initial model was constructed by filtering the preliminary model reported in Holbrook et al. [1999]. Gaussian filtering with horizontal window of $100 \mathrm{~km}$ and vertical window of $10 \mathrm{~km}$ was applied. (b) After ten iterations, RMS travel time misfit was reduced to $81 \mathrm{~ms}$ with $\chi^{2}$ of 0.42 . 

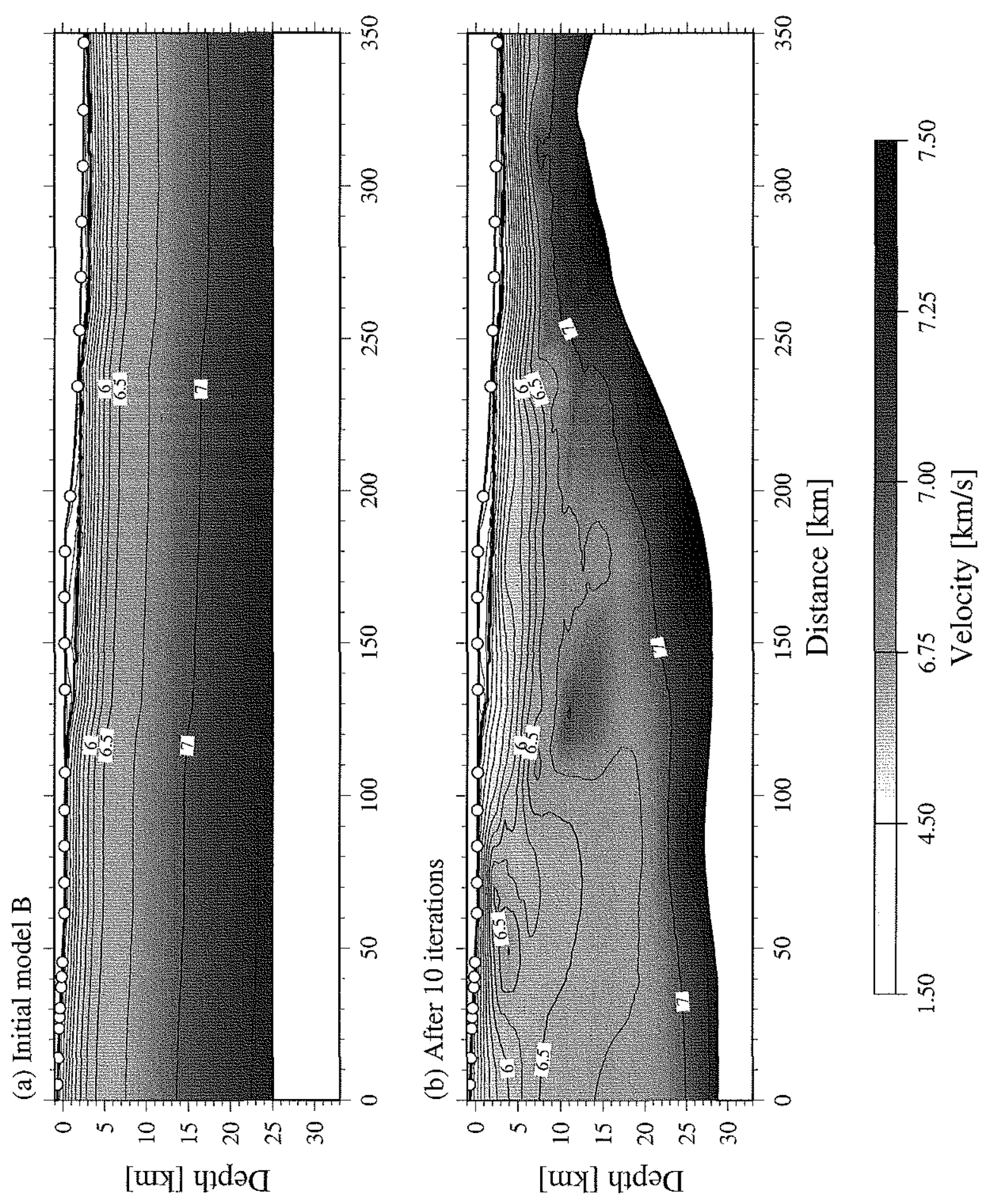

Figure 1-9. Result of tomographic inversion with a different starting model. (a) Initial model was constructed by hanging the 1-D average crustal velocity profile of the model shown in Figure 1-8a beneath the basement. Initial Moho is flat at depth of $25 \mathrm{~km}$. (b) After 20 iterations, RMS travel time misfit was reduced to $77 \mathrm{~ms}$ with $\chi^{2}$ of 0.36 . 
(a) $\mathrm{w}=0.01$

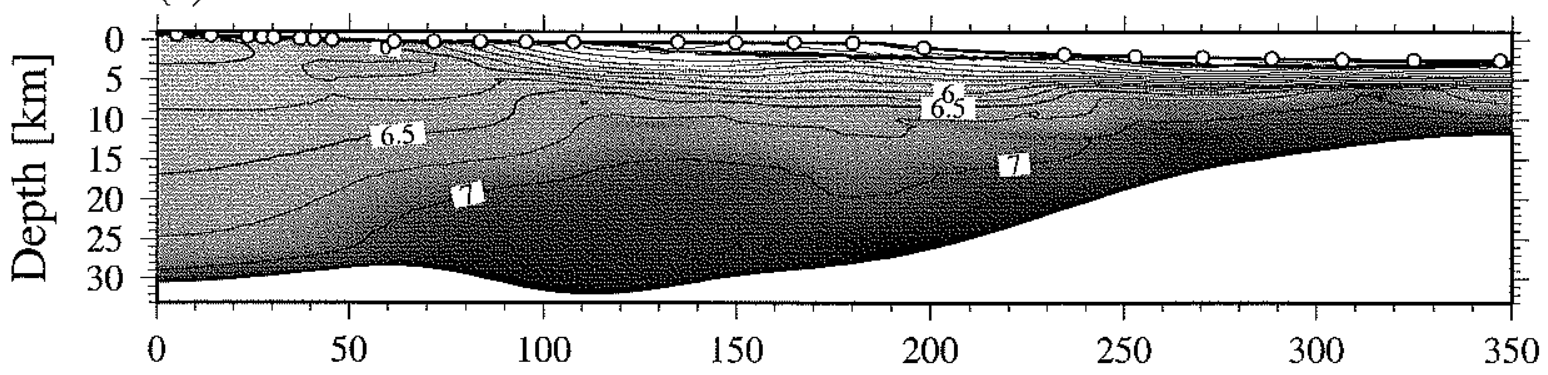

(b) $w=1$

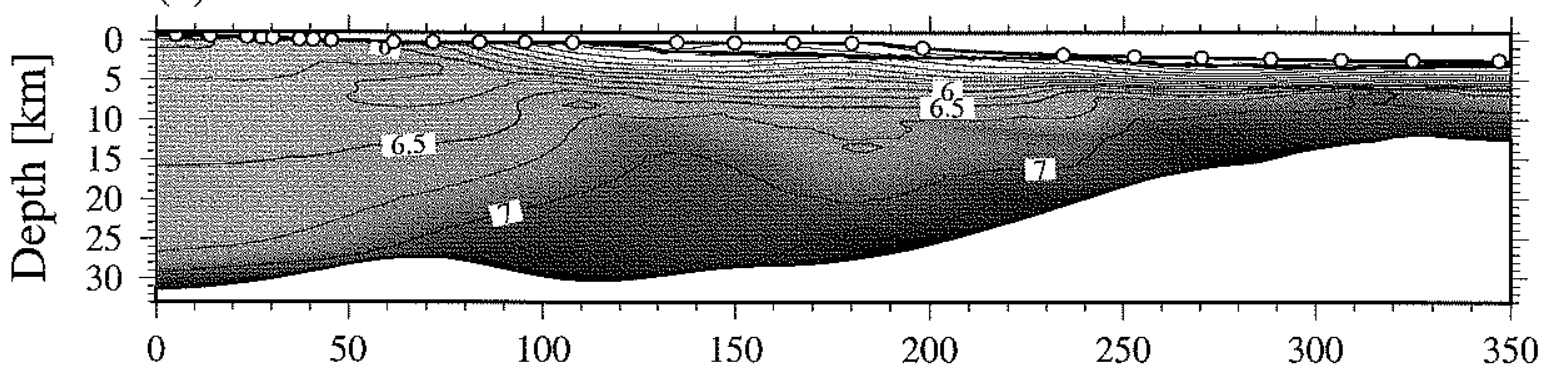

(c) $\mathrm{w}=100$
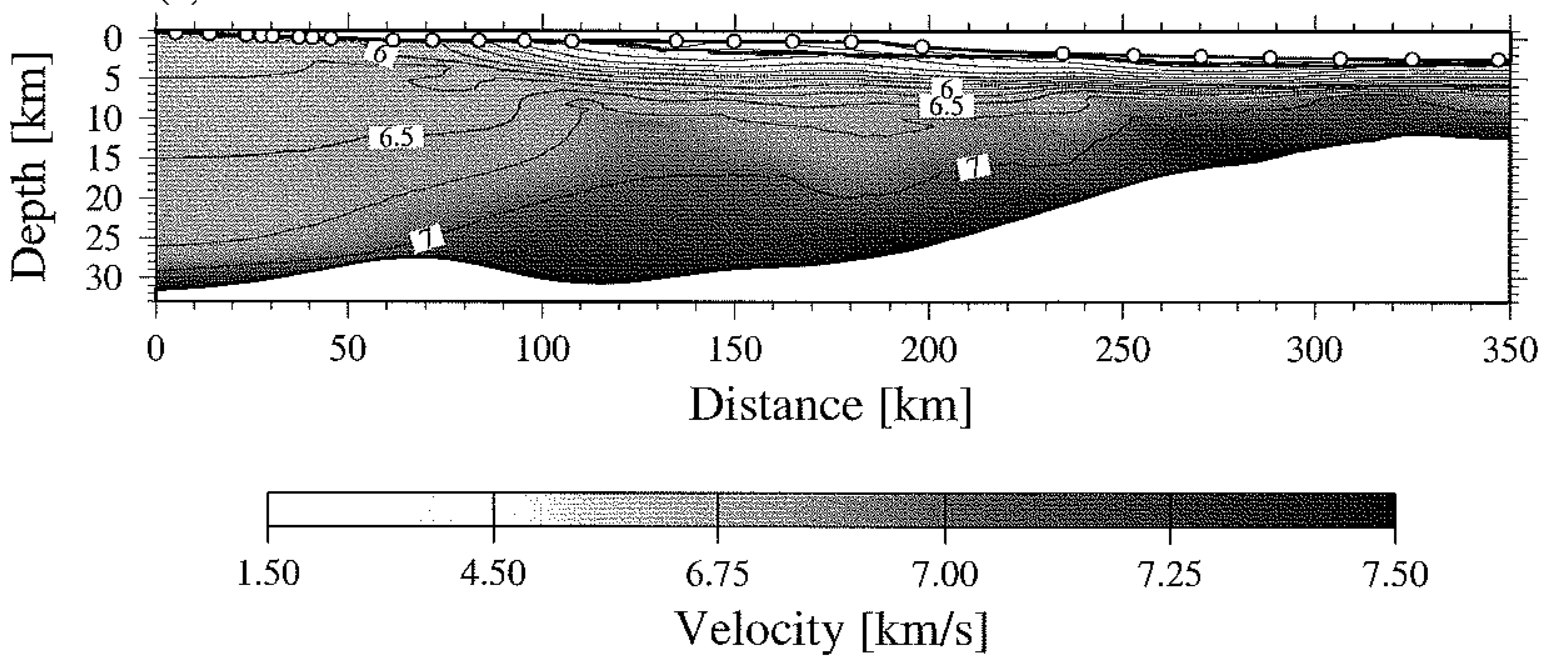

Figure 1-10. Test of velocity-depth ambiguity using different weighting of the depth kernel. Initial model is shown in Figure 1-8a and ten iterations were applied. (a) $w=0.01$, (b) $w=1$ (same as Figure 1-8b), and (c) $w=100$. RMS travel time misfits are $82 \mathrm{~ms}, 81 \mathrm{~ms}$, and $84 \mathrm{~ms}$, respectively. 

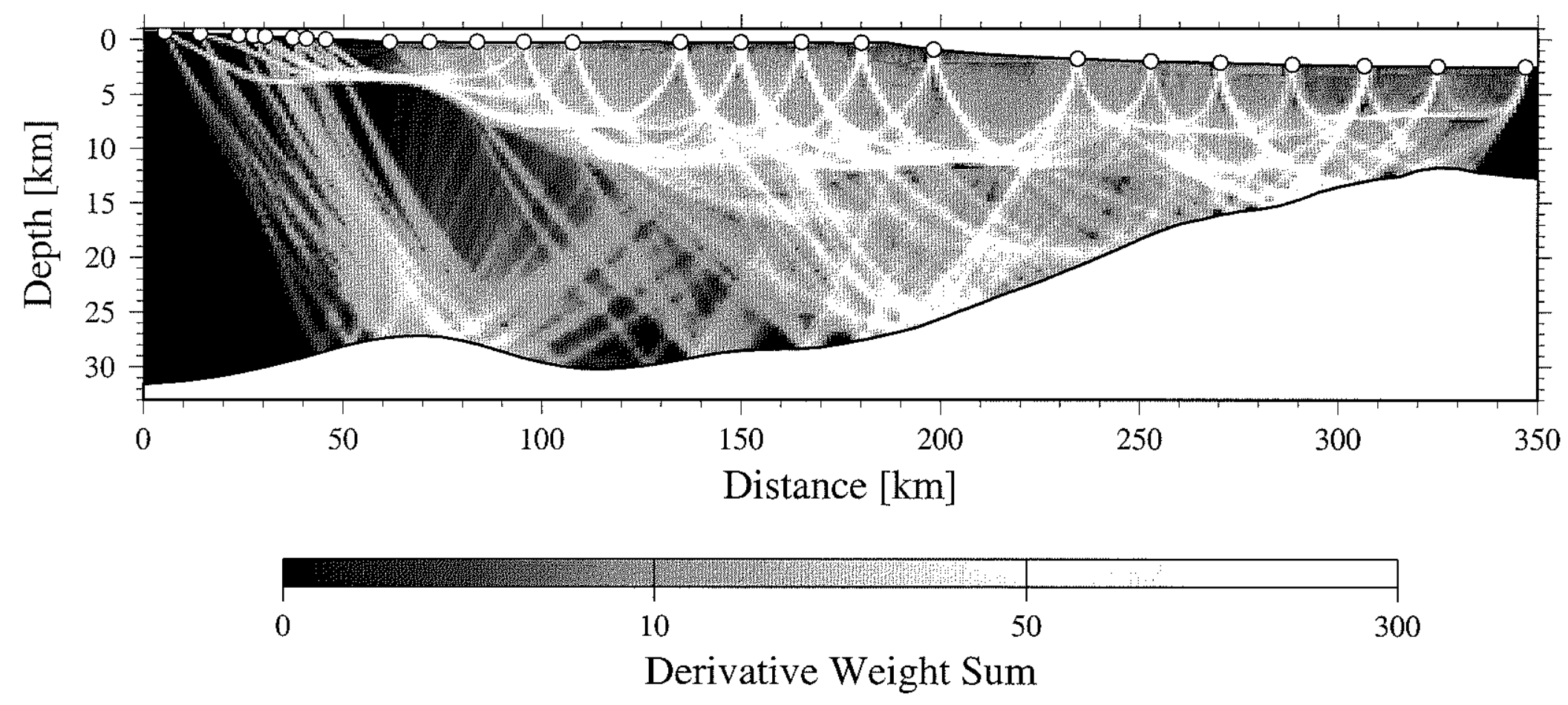

Figure 1-11. Derivative weight sum in the case of the solution shown in Figure 1-8b. 
(a) Model A: Input

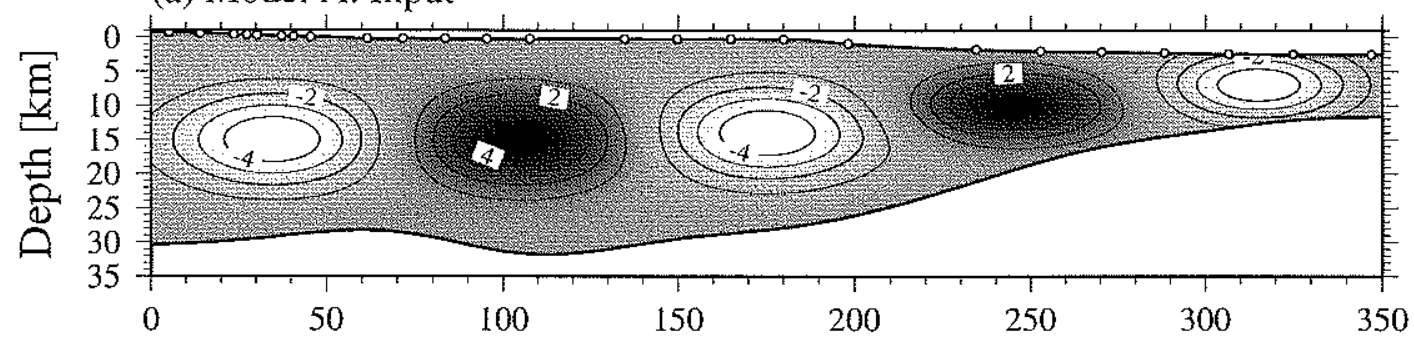

(b) Model A: Recovery (after 4 iterations)

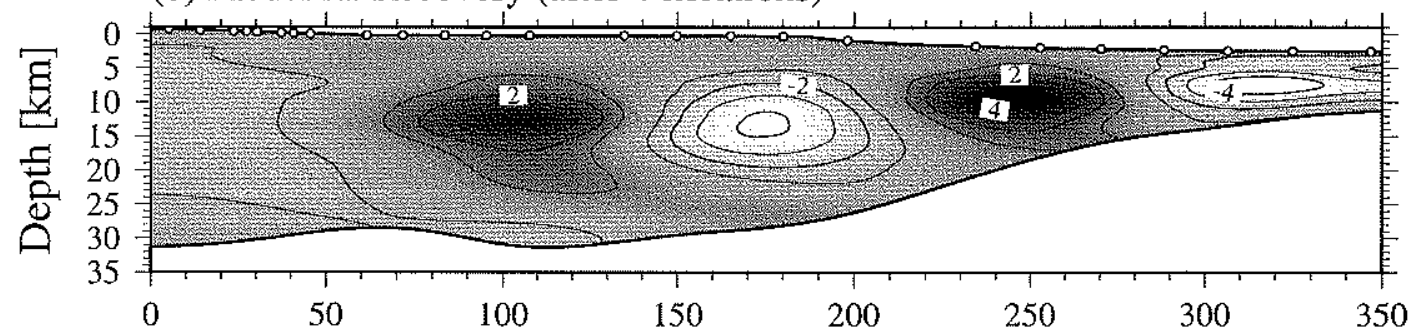

(c) Model B: Input

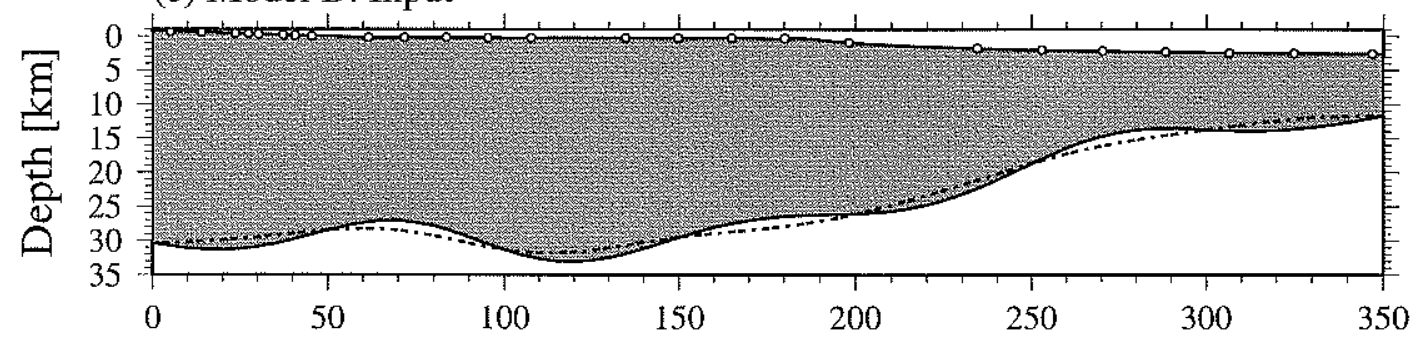

(d) Model B: Recovery (after 4 iterations)
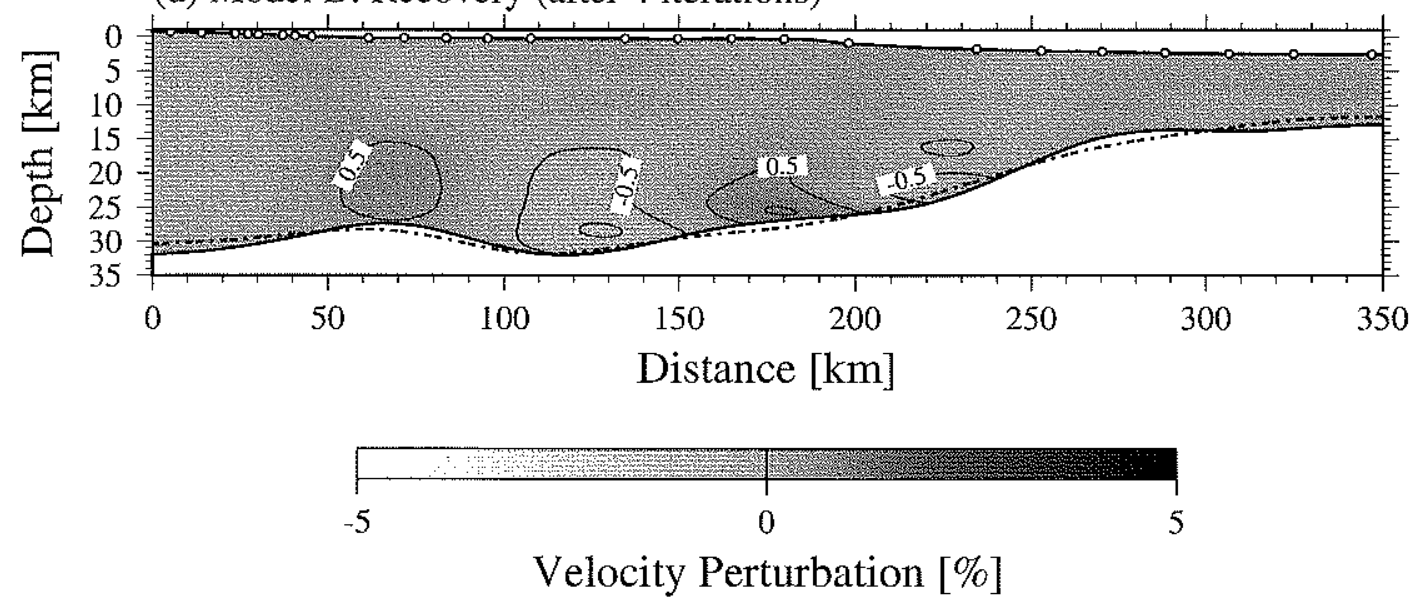

Figure 1-12. Resolution tests. Reference velocity model is the same as in Figure 1-8a. We use the same source and receiver locations for synthetic travel time calculation, and random noise of $100 \mathrm{~ms}$ was added to synthetic data prior to inversion. (a) True model perturbation consists of five alternating $5 \%$ velocity anomalies. (b) Recovery obtained after four iterations. Almost no change is observed for reflector depths. (c) Model reflector (solid) was constructed by adding $1.5-\mathrm{km}$ perturbation with $100-$ $\mathrm{km}$ wavelength to the reference reflector (dashed). (d) Starting from the reference reflector, good recovery is obtained for most parts with small leaking into velocity perturbations. 

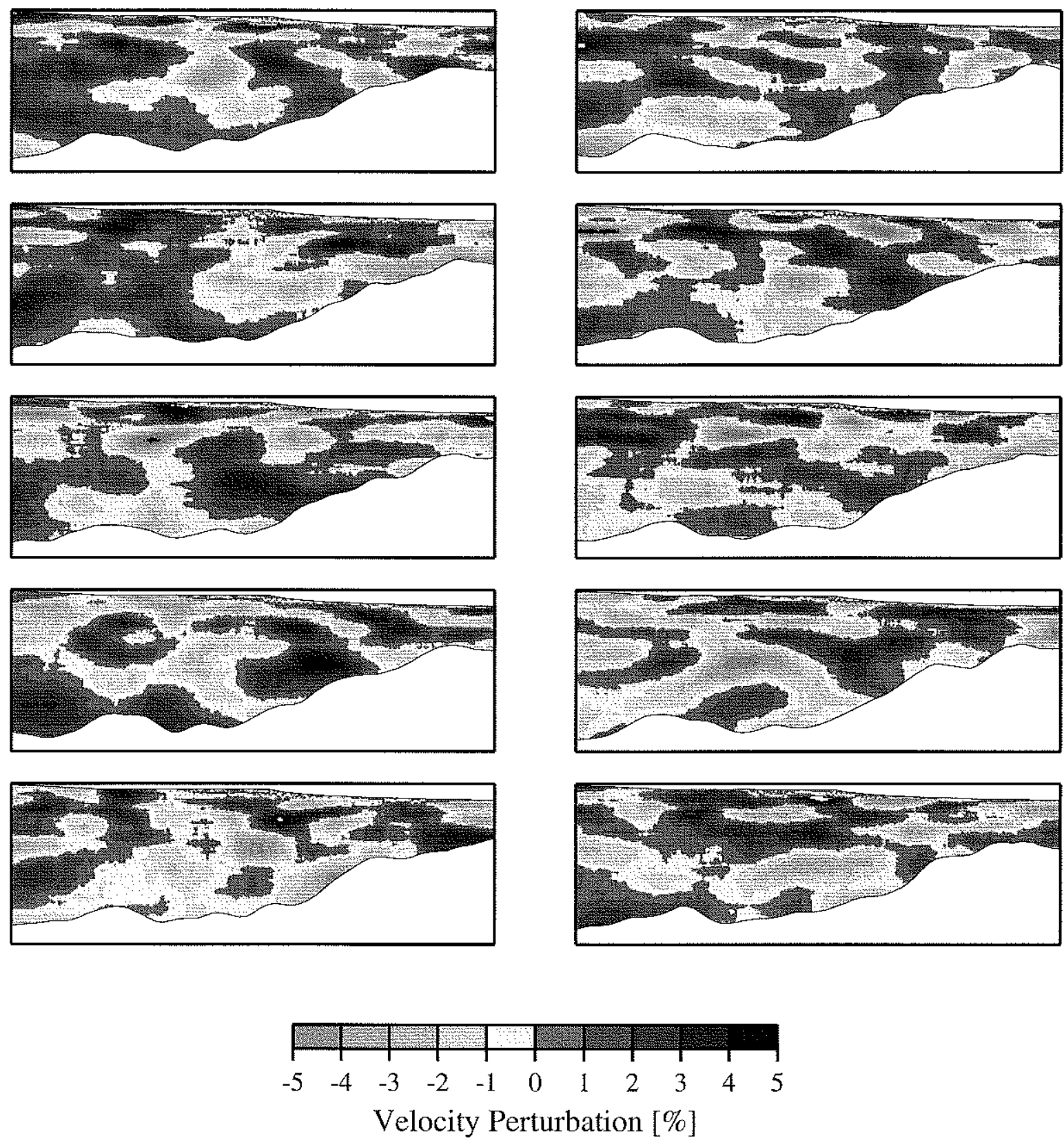

Figure 1-13. Example of randomized starting models in Monte Carlo uncertainty analysis. 
(a)
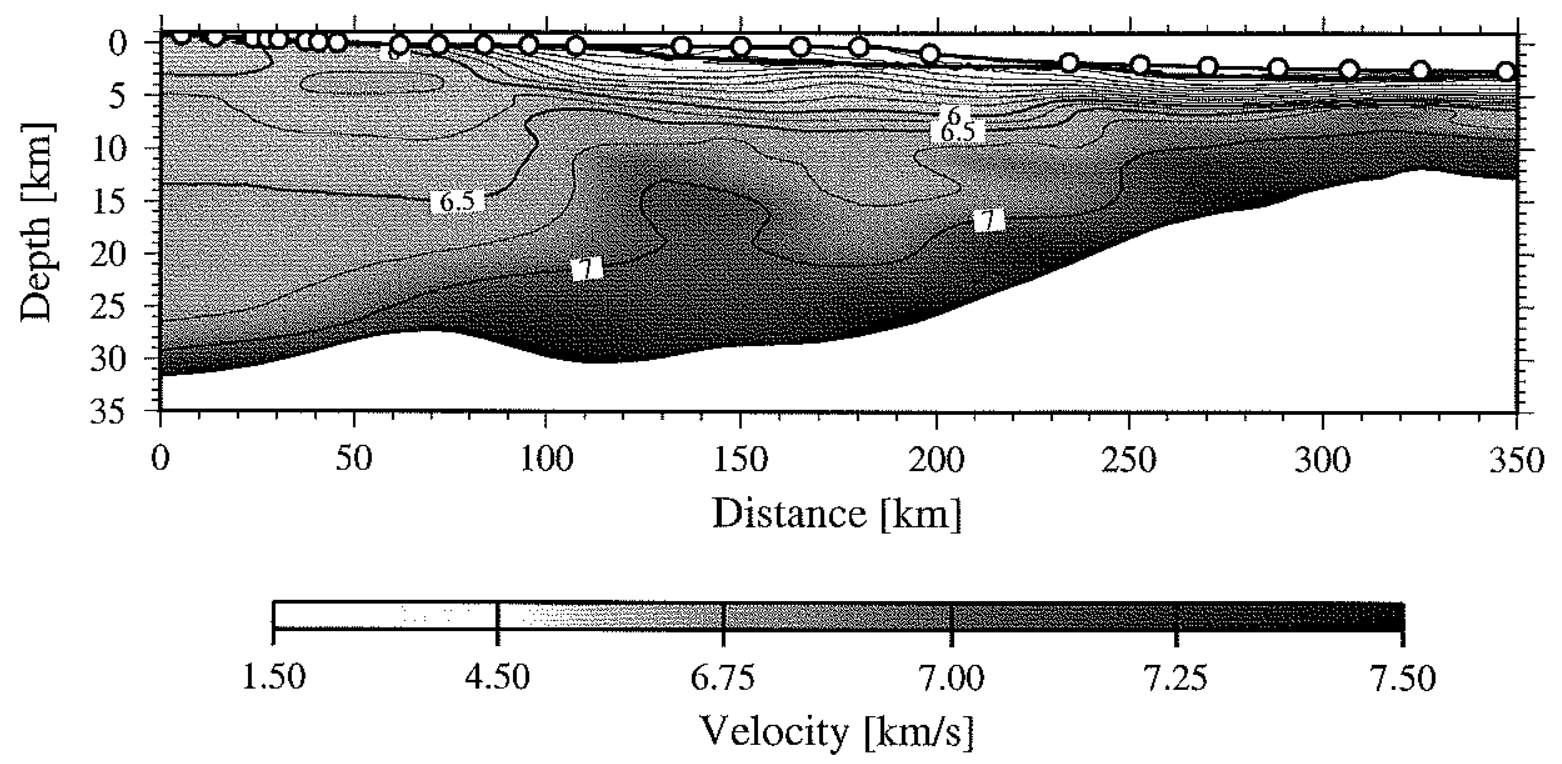

(b)
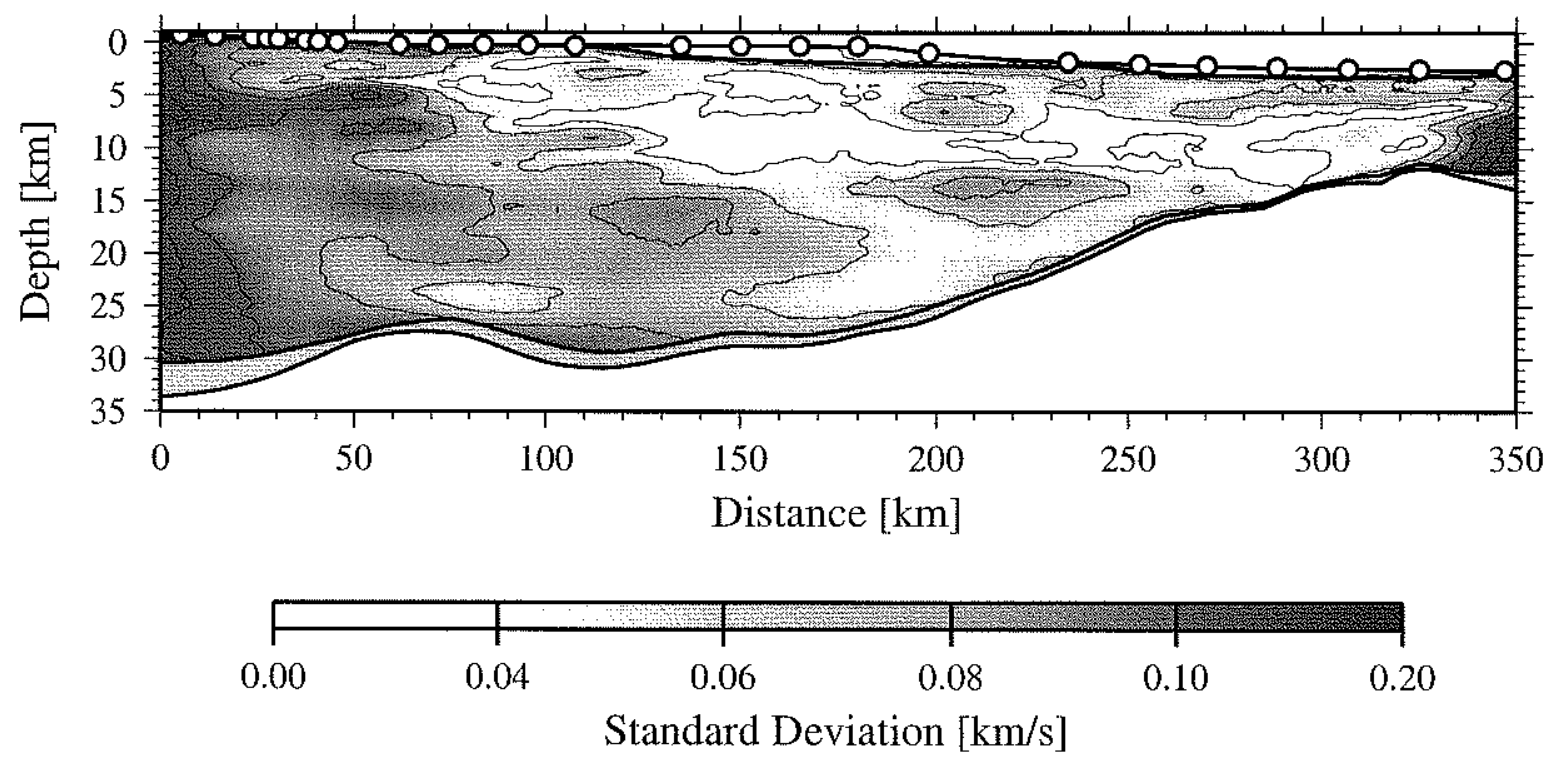

Figure 1-14. (a) Final velocity model derived by averaging all Monte Carlo ensembles. (b) Corresponding standard deviation for velocity and depth nodes. Contours are drawn at $0.02 \mathrm{~km} / \mathrm{s}$ interval. 
(a)

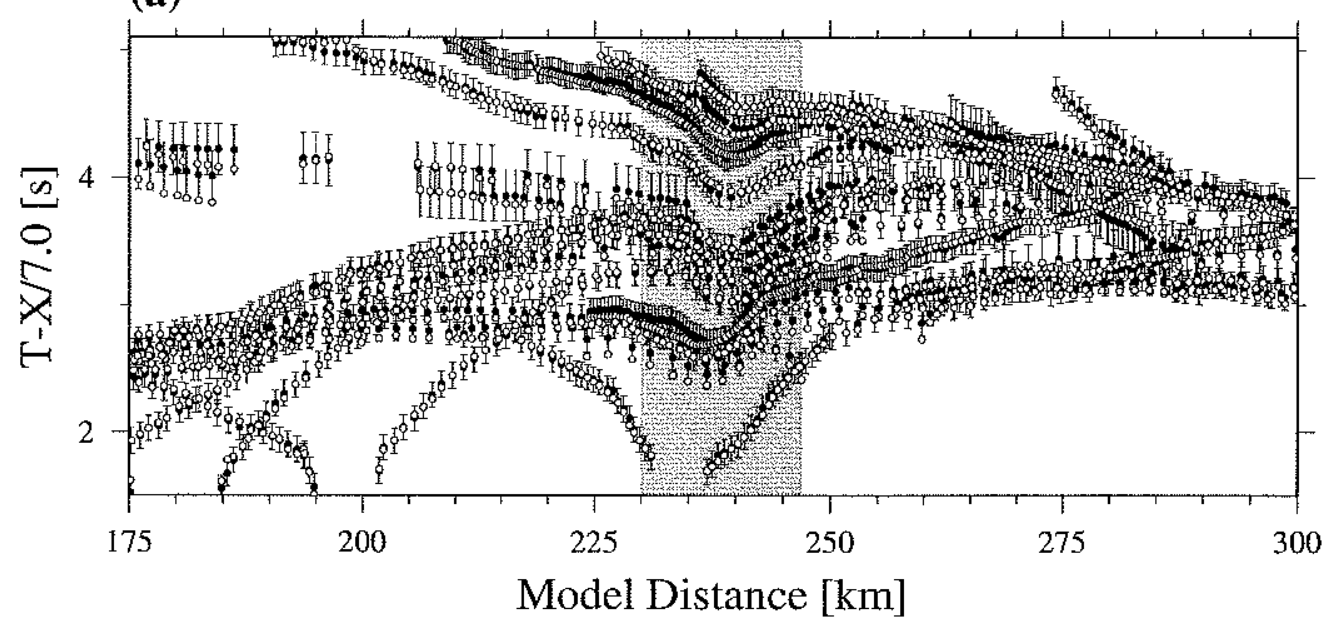

(b)

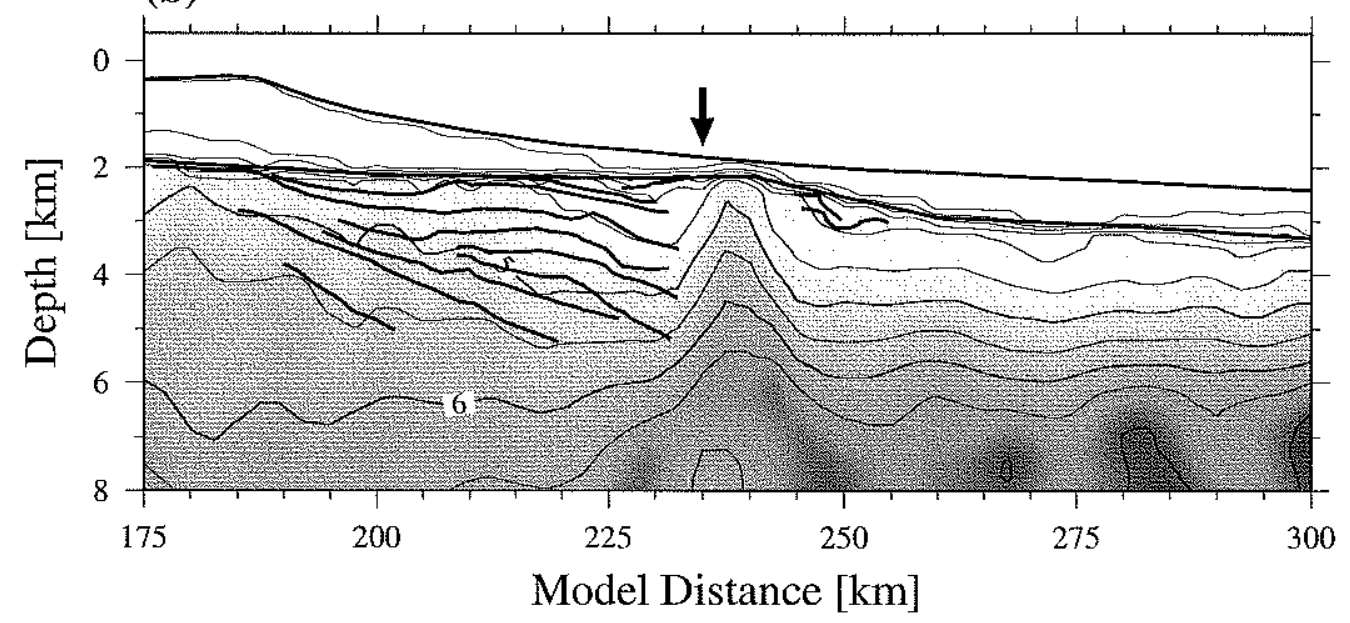

(c)

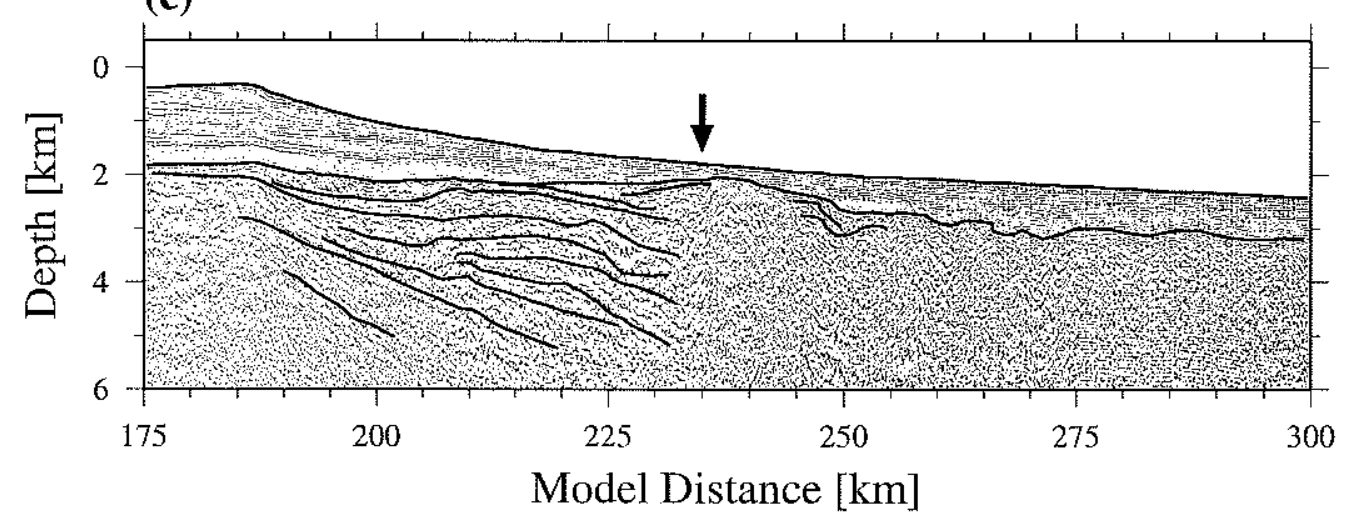

Figure 1-15. Short-wavelength features derived by inverting with shorter correlation lengths. (a) Observed travel times (solid circle with error bar) are plotted with predicted travel times (open circle) based on the high-resolution model shown in (b). Better fits are obtained for bumps in travel times around km 230-245. (c) Depth migrated section of seismic reflection data overlaid with line drawing. The seaward end of the wedge of seaward dipping reflectors (shown by arrow in $b$ and c), correlates well with the high velocity anomaly. 
(a)
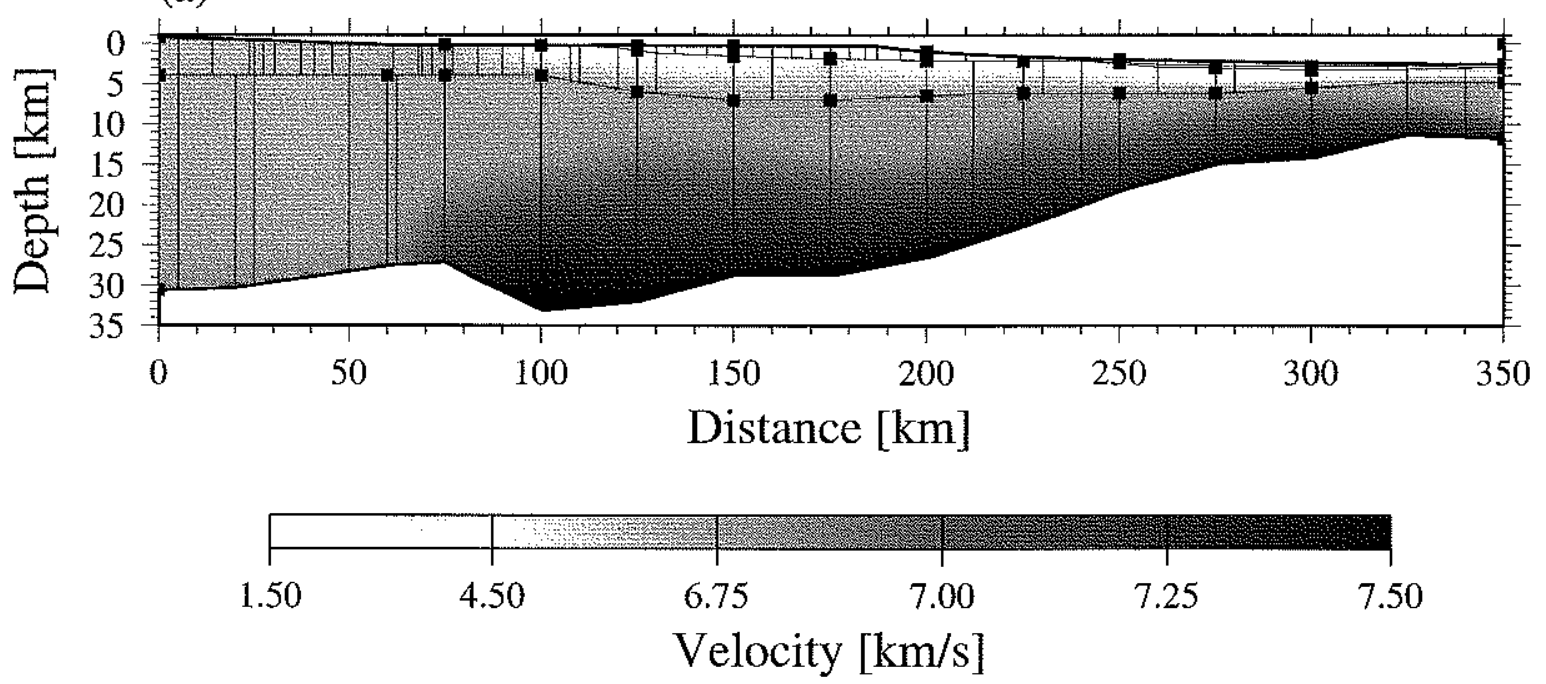

(b)

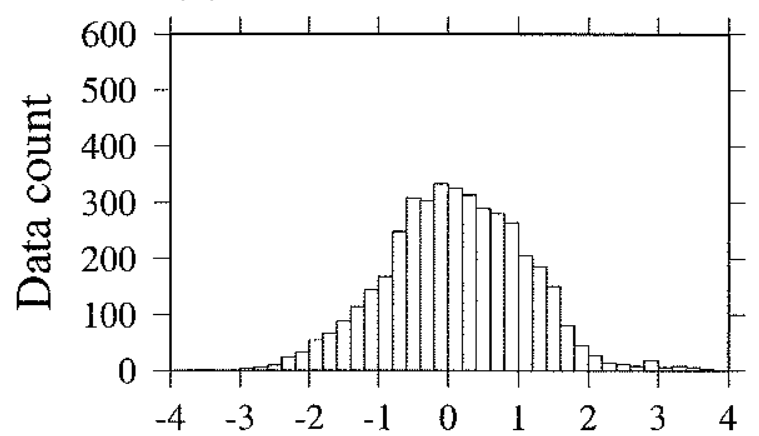

Normalized traveltime residual (c)

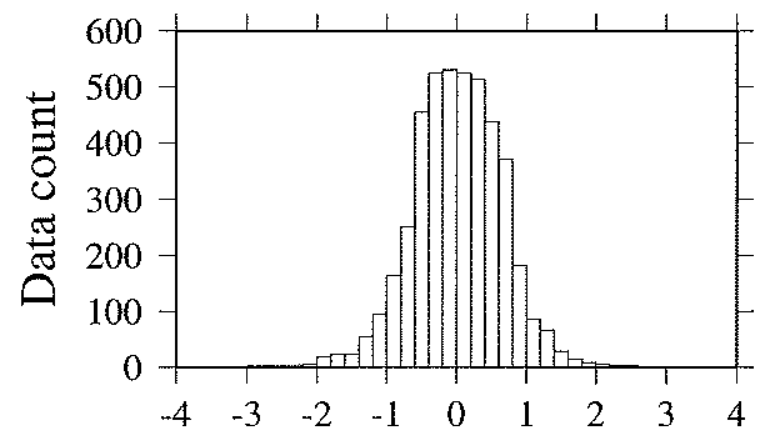

Normalized traveltime residual

Figure 1-16. (a) A preliminary compressional velocity model based on the travel time inversion scheme of Zelt and Smith [1992] (after Holbrook et al. [1999].) Solid squares denote velocity nodes; lower crustal velocity is controlled by the velocity at the upper boundary and user-defined velocity gradients. Beneath seafloor, the model consists of two sedimentary layers, one upper crustal layer, and one lower crustal layer. Also shown are trapezoidal cells, which are defined by either depth nodes or velocity nodes. Distribution of normalized travel time residuals is shown for (b) the preliminary model and (c) the model of Figure 1-8b. 

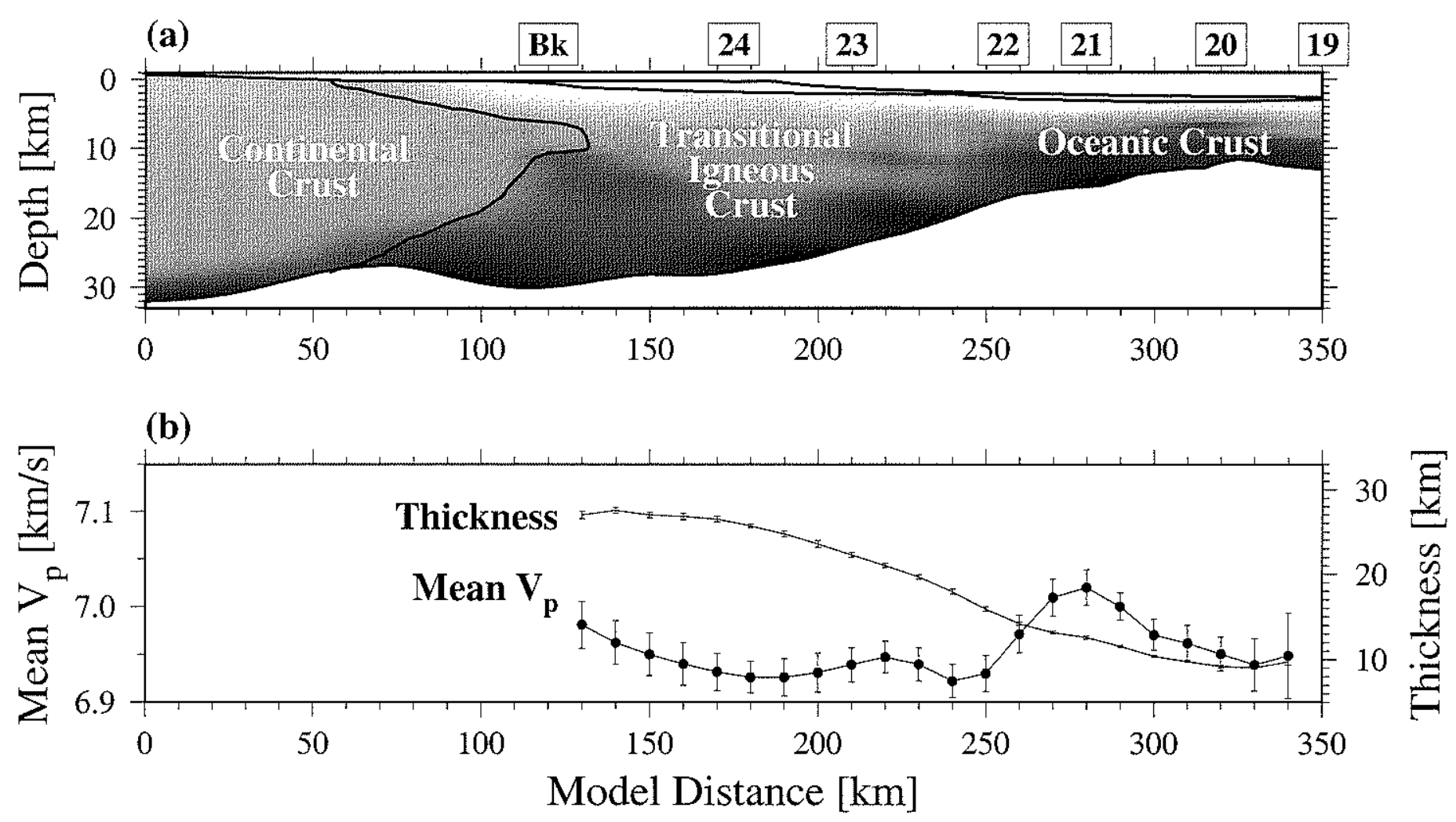

Figure 1-17. (a) Geological interpretation of the long-wavelength velocity model of Figure 1-14a. Solid line denotes the seaward extent of the stretched continental crust. Anomalies 19 to 24 identified on other southern transects are projected on this transect to provide age control. Bk denotes the breakup point interpreted by Holbrook et al. [1999]. (b) Mean velocity and thickness of the igneous crustal part are calculated at $10 \mathrm{~km}$ interval with $20 \mathrm{~km}$ wide averaging window, using the 100 Monte Carlo ensembles. 


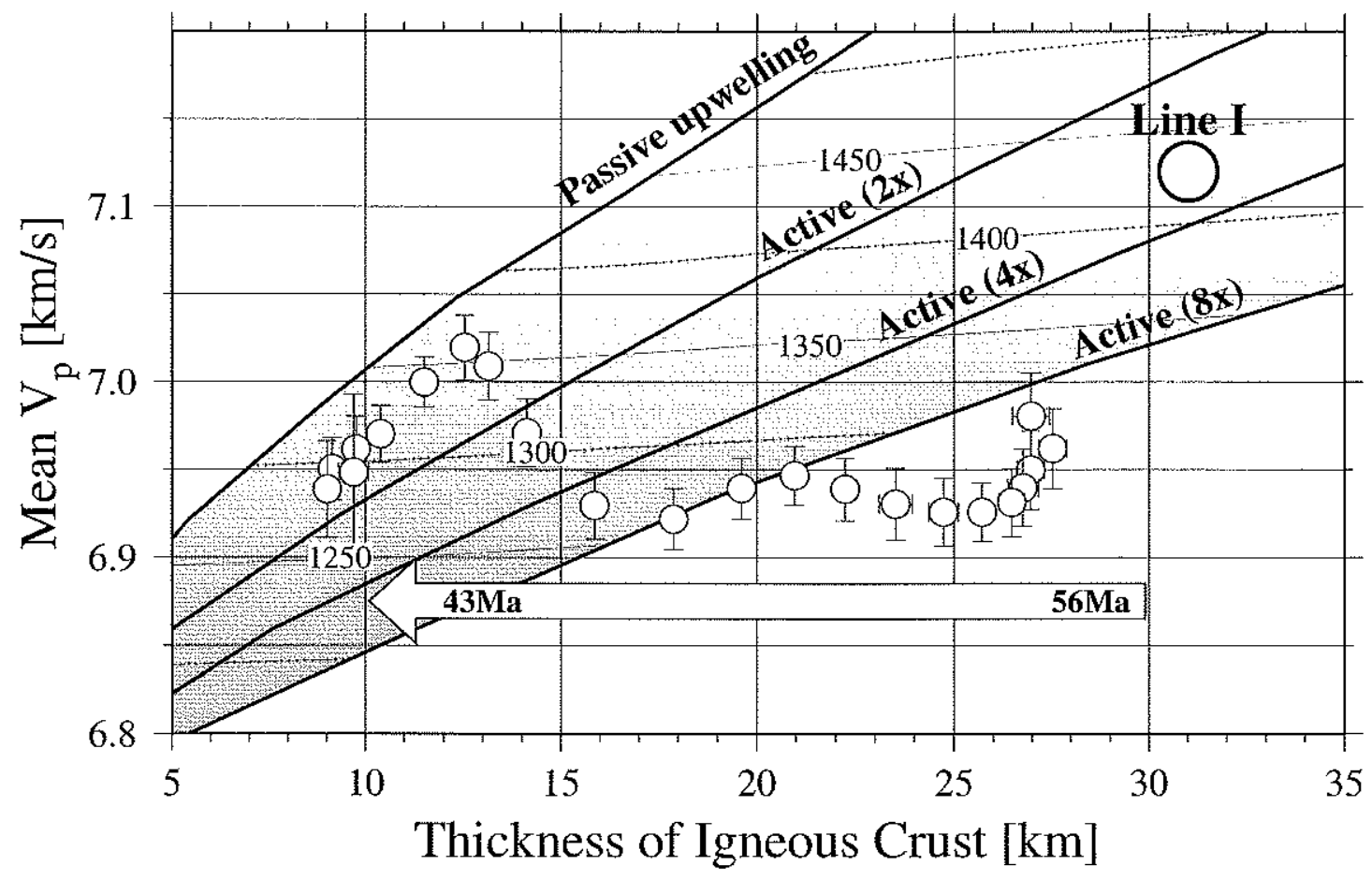

Figure 1-18. Mean velocity of the igneous crust is plotted as a function of corresponding thickness (open circles with error bars). The large open circle indicates the range of the velocity-thickness data (which are nearly constant) for transect 1 . Also shown is the predicted velocity-thickness curves based on the mantle melting model, for passive and three active upwelling cases. The contours of corresponding mantle potential temperature are drawn at $50^{\circ} \mathrm{C}$ interval. 


\title{
Chapter 2 \\ Gravity anomalies and crustal structure at the Southeast Greenland margin
}

\begin{abstract}
Free-air gravity anomalies across the Southeast Greenland margin are investigated in conjunction with a well-constrained seismic velocity model to provide a constraint on subsurface density structure. A new systematic approach is adopted for gravity modeling, which consists of (1) full error propagation from the velocity model to predicted gravity anomalies through a posteriori model covariance represented by Monte Carlo ensembles, (2) the inversion of residual gravity anomalies for density variations within geological subdomains, and (3) the joint inversion of seismic travel times and gravity anomalies. A density model derived from the velocity model, using conventional conversion laws for the continental and oceanic crust, substantially underpredicts the observed gravity by $\sim 70 \mathrm{mGal}$ over the continental shelf. A possible range of mantle contribution is first investigated by modeling various thermal evolution and depletion scenarios, which suggests that the maximum contribution is only -20 mGal. Most of the residual gravity anomaly therefore must have a crustal origin, but neither errors in the velocity model nor the uncertainty of the chosen conversion laws is sufficient for such a large gravity misfit. Applying a conversion law with a denser upper crust in the continent-ocean transition zone seems to be the only plausible option to resolve this difficulty. Contrasting eruption environments for the transition zone crust (subaerial) and the oceanic crust (submarine) probably result in different porosity structures, to which a velocity-density relationship is highly sensitive. The wire-log and laboratory measurements of plateau basalts recovered from recent drilling legs on North Atlantic margins are shown to support this explanation.
\end{abstract}

\subsection{Introduction}

In the past decade or so, there have been an increasing number of deep-crustal seismic investigations on rifted continental margins, which have often revealed thick igneous crust at continent-ocean transition zones [LASE Study Group, 1986; White et al., 1987; Mutter and Zehnder, 1988; Tréhu et al., 1989; Zehnder et al., 1990; Hopper et al., 1992; Holbrook and Kelemen, 1993]. The thick igneous crust at these volcanic passive margins is typically $20-30 \mathrm{~km}$ thick, and its lower-crustal velocity is usually higher than $7.2 \mathrm{~km} / \mathrm{s}$. The correlation between the velocity and density of crustal rocks [e.g., Birch, 1961] implies the existence of a corresponding high-density lower crust, which has been used to explain positive gravity anomalies observed at the continent-ocean transition zones [Morgan and 
Barton, 1990; Holbrook et al., 1994a; Holbrook et al., 1994b; Lizarralde and Holbrook, 1997]. The high-velocity and high-density crust indicates an unusually high $\mathrm{MgO}$ content compared to the normal oceanic crust, implying the melting of hotter mantle during continental breakup [e.g., White and McKenzie, 1989; Kelemen and Holbrook, 1995].

In 1996, deep seismic experiments were conducted across the Southeast Greenland margin to systematically investigate the margin crustal structure with respect to the presumed Iceland hotspot track [Holbrook et al., 1999], and one of the seismic transects (transect 2) has been analyzed with joint refraction and reflection tomography to construct a high-fidelity seismic velocity model (Chapter 1). Transect 2 was located about $250 \mathrm{~km}$ south of the Iceland hotspot track (Figure 2-1a) and extended from the deep-water ocean basin, across the continental slope and shelf, to the coastal region. The free-air gravity anomaly along the transect is marked by a positive gravity high with an amplitude of $\sim 60 \mathrm{mGal}$ over the continental shelf (Figure 2-1b), which is also characteristic of gravity anomalies at other North Atlantic margins [e.g., Morgan and Barton, 1990]. There is significant lateral variation in crustal thickness along the transect, from $\sim 9 \mathrm{~km}$ at the seaward end to $-30 \mathrm{~km}$ beneath the continental shelf (Figure 2-1c). Whereas a water-crust density contrast at the shallowing bathymetry produces a positive gravity anomaly, a crustmantle density contrast at this deepening Moho results in a larger anomaly with an opposite sign. The observed gravity high thus requires a variation in crustal density that can offset the effect of crustal thickening. The $P$-wave velocity of the thick igneous crust is, however, not significantly higher than the thinner oceanic crust, so that there is an apparent discrepancy between the velocity model and the observed free-air gravity anomaly on transect 2 .

The crustal velocity structure of transect 2 is well constrained by the unprecedented quality of seismic data with a dense receiver array and deep-penetrating sources, and its reliability has been comprehensively demonstrated by a nonlinear Monte Carlo uncertainty analysis (Chapter 1). Therefore we have an opportunity to resolve the possible origin of the conspicuous gravity high, which may also be applicable to other volcanic margins. The purpose of this paper is to construct a density model that is consistent with both the 
observed free-air gravity anomaly and the crustal velocity structure, and to discuss the geological and geophysical implications of the inferred density structure. Though inference on density structure solely from gravity anomalies is notoriously nonunique, it is possible to considerably reduce the nonuniqueness and to place reasonable bounds on our estimate of density structure by utilizing the seismic velocity information and by considering possible geological processes involved in the formation of a volcanic passive margin. Our approach is based on (1) the full characterization of the absolute uncertainty of the velocity model (Chapter 1), which enables us to isolate other sources of uncertainty in regional gravity modeling, and (2) the inversion of residual gravity anomalies for density variation within a variable-shaped domain. We begin with a critical evaluation of existing velocitydensity relationships for igneous rocks, which is fundamental to the use of a seismic velocity model as a constraint on density structure.

\subsection{The Paradox of the Margin Gravity High}

The gravity profile along transect 2 was constructed by combining shipboard free-air gravity data and Bouguer-corrected land gravity data; high-resolution marine gravity data based on satellite altimetry are also available [Smith and Sandwell, 1995], which are virtually identical with the shipboard data except at very short wavelengths (less than 1$2 \mathrm{~km}$ ). Because the land data included a Bouguer correction, we will consider only a subhorizontal region to model the observed gravity anomaly. Density models are $350 \mathrm{~km}$ wide and $35 \mathrm{~km}$ deep and are gridded with uniform rectangular cells, each of which is $200 \mathrm{~m}$ wide and $50 \mathrm{~m}$ deep, to accurately represent sharp density discontinuities such as the seafloor and the Moho. We assume that observed gravity anomalies can be modeled in 2-D with reasonable accuracy, since gravity anomalies and bathymetry both have nearly linear patterns aligned perpendicular to transect 2 . Gravity anomalies are obtained as the vertical integral of contributions from horizontal density layers, each of which is calculated in the 1-D Fourier domain [e.g., Blakely, 1995]. The horizontal ends of the model are continuously padded for an additional $200 \mathrm{~km}$ to avoid truncation artifacts, and the model was then mirrored to minimize the leaking of the transcendent component into shorter 
wavelengths within the Fourier transform. We benchmarked our gravity calculations against various analytical solutions for simple bodies [e.g., Telford et al., 1990], and the numerical errors were found to be less than $1 \%$. In all gravity calculations in this paper, a reference density structure is taken from $\mathrm{km} \sim 300$ to match the predicted anomaly with the observed anomaly toward the seaward end of the transect.

We first considered a density model comprised of four constant-density layers: water $\left(1.0 \mathrm{Mg} / \mathrm{m}^{3}\right)$, sediment $\left(2.0 \mathrm{Mg} / \mathrm{m}^{3}\right)$, crust $\left(2.86 \mathrm{Mg} / \mathrm{m}^{3}\right)$, and mantle $\left(3.3 \mathrm{Mg} / \mathrm{m}^{3}\right)$. The basement and Moho boundaries are adopted from the seismic velocity model. A gravity anomaly calculated from this simple density model largely underpredicts the observed gravity anomaly, especially for the positive gravity high observed over the continental shelf (Figure 2-2). This clearly illustrates that the effect of the deepening Moho along the transect is significantly larger than the effect of the shallowing bathymetry, despite the fact that the seafloor has a larger density contrast and is located closer to the surface. Thus, strong density variations somewhere in the crust or mantle are necessary to explain the observed gravity anomaly. We will consider crustal density variations first, based on the seismic velocity model, and to do so, a conversion rule from $P$-wave velocity to density needs to be determined. For the sedimentary layer, we use Hamilton's empirical relation for shale [Hamilton, 1978], $\rho=0.917+0.747 V_{p}-0.08 V_{p}^{2}$, which is the best fit to data on the ODP Leg 152 sediment core [Larsen et al., 1994]. For the continental crust, we use a nonlinear velocity-density regression curve estimated for average continental crust at $20 \mathrm{~km}$ depth [Christensen and Mooney, 1995], $\rho=5.055-14.094 / V_{p}$, throughout the whole continental crust; this crude application of the conversion law is not very critical because of the relatively uniform crustal thickness and the weak velocity heterogeneity within the continental crust. The high velocity gradients observed in the upper crust in the transitional and oceanic regions are most likely due to the effects of porosity and alteration, and we use the following empirical relation, $\rho=3.61-6.0 / V_{p}$, which is based on DSDP and ODP core data [Carlson and Herrick, 1990].

The lower crust in the transitional and oceanic regions occupies the largest volume in the model, so a conversion law for this subdomain probably has the most significant 
influence on the predicted gravity anomaly. The velocity-density systematics for igneous lower crust, however, have not been firmly established. This is because laboratory measurements of velocity and density are mostly limited to gabbroic rocks sampled from the oceanic crust and ophiolites [e.g., Christensen and Shaw, 1970; Christensen and Salisbury, 1975; Christensen and Smewing, 1981], whose velocity is usually strongly affected by metamorphism and porosity. Whereas empirical relations based on these data are probably appropriate for the normal oceanic crust, the majority of which seems to be influenced by hydrothermal alteration [e.g., Christensen and Salisbury, 1975; Gregory and Taylor, 1981; McCollom and Shock, 1998], it is not certain whether they are also applicable to the lower section of thick $(>15 \mathrm{~km})$ igneous crust, where crack-free, pristine gabbroic rocks unaffected by hydrothermal circulation probably predominate. In addition, though rock types expected for igneous lower crust are much more limited than those for continental crust [Christensen and Mooney, 1995], even mineral assemblages composed only of olivine, plagioclase, and clinopyroxene can have considerable compositional diversity, for which a single velocity to density conversion may not be appropriate.

To investigate the relationship between velocity and density expected for primary gabbroic rocks, we have conducted fractional crystallization modeling starting from several primary mantle melt compositions, corresponding to a wide range of mean pressures and mean fractions of melting. Because the chemical composition of igneous lower crust must be bounded by the composition of primary mantle melts and that of fractionated, "cumulate" mineral assemblages, by conducting the crystallization modeling at a range of crustal pressures, we should be able to delineate the permissible extent of velocity and density variation for igneous lower crust. Readers are referred to Chapter 3 for the details of the modeling procedures. The results are presented in Figure 2-3, with other published empirical relations for mafic and ultramafic rocks. In this modeling, we assume that seismic anisotropy is insignificant for igneous lower crust, the degree of anisotropy in which is unknown. For a given density, fractionated assemblages usually have higher velocity than unfractionated ones because FeO is preferentially partitioned into residual liquids. The observed scatter in the velocity-density diagram results from nonlinearity in 
mixing the elastic properties of different minerals. Note that all crack-free, synthetic assemblages lack the most commonly observed velocity-density values for normal oceanic crust $\left(6.9 \mathrm{~km} / \mathrm{s}\right.$ and $\left.2.9 \mathrm{Mg} / \mathrm{m}^{3}\right)$. Birch's law for rocks with mean atomic weight of $\sim 21$ [Birch, 1961] is broadly consistent with the modeling results. Since we are interested in explaining the gravity variation as a result of crustal density variation, we adopt Birch's law for diabase, gabbro, and eclogite [Birch, 1961], $\rho=0.375+0.375 V_{p}$, which can serve as an upper bound for the density of primary gabbroic rocks (Figure 2-3). For velocities of $6.8-7.0 \mathrm{~km} / \mathrm{s}$, this conversion law is very close to traditional empirical relationships for the oceanic crust [e.g., Christensen and Shaw, 1970; Carlson and Herrick, 1990], so this upper bound only becomes significant for higher velocities. For the lowermost section of thick igneous crust, there is a possibility of garnet growth [Ringwood and Green, 1964; Ringwood and Green, 1966]. For anhydrous igneous rocks with a typical cooling history, however, this garnet growth is unlikely because of kinetic barriers to reaction [Ahrens and Schubert, 1975]. Even if garnet forms, the velocity and density of resultant garnet granulites and eclogitic rocks both increase following Birch's law [e.g., Sobolev and Babeyko, 1994]. Therefore, our approach focusing on gabbroic assemblages is probably sufficient for igneous lower crust in general. This fractionation modeling also provides theoretical estimates for the temperature and pressure derivatives of velocity and density (Chapter 3). Using these derivatives, a conversion law defined at specific temperature and pressure can be used to calculate in situ density, $\rho$, from in situ velocity, $V$, as,

$$
\begin{gathered}
V_{r}\left(p_{r}, T_{r}\right)=V(p, T)+\frac{\partial V}{\partial p}\left(p_{r}-p\right)+\frac{\partial V}{\partial T}\left(T_{r}-T\right) \\
\rho_{r}\left(p_{r}, T_{r}\right)=f\left(V_{r}\left(p_{r}, T_{r}\right)\right) \\
\rho(p, T)=\rho_{r}\left(p_{r}, T_{r}\right)+\frac{\partial \rho}{\partial p}\left(p-p_{r}\right)+\frac{\partial \rho}{\partial T}\left(T-T_{r}\right)
\end{gathered}
$$

where $p_{r}$ and $T_{r}$ are reference pressure and temperature $\left(1000 \mathrm{MPa}\right.$ and $\left.25^{\circ} \mathrm{C}\right)$, respectively, at which velocity to density conversion, $\rho=f(V)$, is defined, and $p$ and $T$ are in situ pressure and temperature, respectively.

Using the above conversion laws for the four subdomains, i.e., the sedimentary layer, the continental crust, the upper crust, and the lower crust, a gravity anomaly was 
calculated based on the $P$-wave velocity model for transect 2 . Although pressure and temperature derivatives are not constant and have a roughly linear dependence on velocity and density (Chapter 3), the calculation of gravity anomalies using average derivatives for expected ranges of velocity and density is sufficiently accurate, with an error of less than $0.5 \mathrm{mGal}$. The pressure and temperature derivatives we used for velocity are $0.2 \times 10^{-3} \mathrm{~km} / \mathrm{s} / \mathrm{MPa}$ and $-0.4 \times 10^{-3} \mathrm{~km} / \mathrm{s} / \mathrm{C}^{\circ}$, respectively, and those for density are $0.01 \times 10^{-3} \mathrm{Mg} / \mathrm{m}^{3} / \mathrm{MPa}$ and $-0.03 \times 10^{-3} \mathrm{Mg} / \mathrm{m}^{3} / \mathrm{C}^{\circ}$, respectively. Temperature at seafloor is set as $0^{\circ} \mathrm{C}$, and the geotherm is calculated with a vertical thermal gradient of $20^{\circ} \mathrm{C} / \mathrm{km}$, which is appropriate for 40- to 60-m.y.-old lithosphere [e.g., Parsons and Sclater, 1977].

To propagate the uncertainty of the velocity model to that of a predicted gravity anomaly, we utilize 100 Monte Carlo ensembles of the velocity model obtained in Chapter 1. Because any model parameter in a tomographic model is correlated with other parameters to some extent, the model covariance is fundamental to evaluate error propagation for averaged quantities such as the gravity anomaly. In particular, the uncertainty of the lower-crustal velocity is expected to negatively correlate with the uncertainty of Moho depth, because reflection travel times are the major constraint on the lower-crustal structure. Therefore, the uncertainty of the corresponding gravity anomaly correctly estimated using the model covariance can be smaller than that estimated with the model variance only. Though the explicit expression of the full model covariance matrix and its use for error propagation are computationally intractable, by repeating the same procedure of gravity calculation for each Monte Carlo ensemble, and by taking the statistics of the resultant gravity anomalies, we can correctly incorporate the covariance of the velocity model into the uncertainty of the predicted gravity. Predicted gravity anomalies and densities obtained by this procedure are shown in Figure 2-4. To separate the effects of pressure and temperature corrections, two more anomalies are also shown in Figure 2-4a; the corrections for velocity, which has a much larger effect than those for density, result in a $-30 \mathrm{mGal}$ increase at most compared with the case of no correction. The calculated gravity anomaly, however, still significantly underpredicts the observed anomaly by $\sim 70 \mathrm{mGal}$ over the continental shelf. 


\subsection{Possible Origins of the Margin Gravity High}

The preceding section demonstrates that the seismic velocity model cannot explain the margin gravity high with the adopted velocity-density conversion. A constant mantle density assumed in our gravity calculation may be a source of this discrepancy, but a possible density variation in our mantle section has only a small contribution, as we will show below. Remaining possibilities are then limited to crustal contributions; (1) the true velocity structure may be somehow very different from our velocity model, and/or (2) the standard velocity-density conversion may not apply to our transect. We will test these possibilities in turn by quantifying a source density anomaly using the inversion of gravity anomalies and by revisiting travel time tomography with the additional constraint of observed gravity anomalies.

\subsubsection{Mantle Contributions}

A mantle Bouguer anomaly (MBA) is calculated by subtracting the predicted gravity anomaly based on the density model shown in Figure $2-4 b$ from the observed free-air anomaly (Figure 2-5a). The non-zero MBA indicates a density variation in the mantle section or an error in the crustal density model or both. Though the separation of the MBA into mantle and crustal components is nonunique, we can place a bound on the mantle contribution by modeling a plausible thermal and depletion history for the mantle underlying the igneous crust. We first estimated the order of required density variations in the mantle to explain the MBA, using an inversion technique described in Appendix A. The resultant 1-D density variations for the mantle section, with different maximum depth extents for the anomalous region, are shown in Figure 2-5b. The amplitude of the mantle density anomaly varies from 0.04 to $0.06 \mathrm{Mg} / \mathrm{m}^{3}$ for the range of the maximum depth, corresponding to a temperature variation of $400-600^{\circ} \mathrm{C}$ or a compositional variation of 2 to $3 \%$ in the $\mathrm{Mg}$ number [Jordan, 1988], neither of which seems reasonable for our mantle section. The crustal contribution to the MBA is thus indisputable, but at the same time it is important to limit how much of the MBA can be explained by a possible density variation in the mantle. 
The formation age of the igneous crust varies from $61 \mathrm{Ma}$ to $43 \mathrm{Ma}$ along the transect, and older lithospheric mantle beneath the thicker igneous crust would be colder and thus denser. The mantle thermal structure is calculated using the plate cooling model of McKenzie [1978]; a geotherm is obtained as a function of time by,

$$
\frac{T(t, z)}{T_{a}}=\frac{z}{a}+\frac{2}{\pi^{2}} \sum_{n=1}^{\infty} \frac{(-1)^{n+1} a}{n^{2} b} \sin \left(\frac{n \pi b}{a}\right) \sin n \pi\left(1-\frac{z}{a}\right) \exp \left(-\frac{n^{2} \pi^{2} \kappa t}{a^{2}}\right),
$$

where $T_{a}, a, b$, and $\kappa$ are asthenospheric temperature, plate thickness, the initial thickness of a lithospheric lid, and thermal diffusivity, respectively. We use the observed crustal thickness for the initial lid thickness and set the thermal diffusivity of mantle peridotite as $10^{-6} \mathrm{~m}^{2} / \mathrm{s}$. The $2-\mathrm{D}$ thermal structure is converted to a mantle density model using the thermal expansion coefficient of $3 \times 10^{-5} \mathrm{~K}^{-1}$ and the adiabatic compressibility of $8 \times 10^{-3} \mathrm{GPa}^{-1}$ [Turcotte and Schubert, 1982]. A density difference due to an age difference is mostly confined to depths less than $100 \mathrm{~km}$. Although the density contrast can be slightly increased by increasing the asthenospheric temperature and the plate thickness, those effects are trivial for the already cold lithosphere with the age difference of less than 20 m.y. (Figure 2-6a). For simplicity, we first model the mantle beneath the continental crust as 60-m.y.-old oceanic lithosphere in this and succeeding examples. However, this simplified treatment results in an positive gravity anomaly increasing toward the landward end of the transect (Figure 2-6a, profiles A-C), which is incompatible with the overall trend of the MBA. The assumption that the subcontinental mantle is the same as that beneath the new, oceanic crust offshore is probably inappropriate because the Greenland continental crust is of the Archean age and the Greenland mantle lithosphere is probably highly depleted compared to the oceanic lithosphere [Bernstein et al., 1998]. Considering its depleted nature, the continental lithosphere at model distances less than $50 \mathrm{~km}$ can be less dense than oceanic mantle despite its greater age and consequently lower temperature [e.g., Jordan, 1988]. To account for this effect, the required buoyancy is modeled to reduce predicted gravity at the landward end to zero (Figure 2-6a, profile $A+$ ). The amplitude of the resultant gravity anomaly due to mantle density anomaly is $\sim 20 \mathrm{mGal}$. 
Differentiation processes associated with continental rifting can significantly modify the mantle density structure, so that we need to include the effect of mantle depletion in addition to the temperature and pressure effects discussed above. We first used the mantle depletion model of Klein and Langmuir [1987]; the fertile plagioclase lherzolite and garnet-spinel therzolite have densities of $3.27 \mathrm{Mg} / \mathrm{m}^{3}$ and $3.34 \mathrm{Mg} / \mathrm{m}^{3}$, respectively, at atmospheric pressure and $25^{\circ} \mathrm{C}$, and the boundary between plagioclase and spinel lherzolite is set at $0.8 \mathrm{GPa}$. Igneous crust is generated by the passive upwelling of mantle, so thicker crust requires a higher-degree of depletion of hotter asthenosphere, which results in more buoyant present-day mantle. A mantle density model predicted by this passive upwelling scenario has a gravity anomaly with a trend opposite to the trend of the observed MBA (Figure 2-6b). As suggested by the petrologic interpretation of the seismic velocity model of transect 2, active mantle upwelling seems to have been significant during the formation of this continental margin, and the mantle potential temperature was probably almost constant despite the strong variation in crustal thickness (Chapter 1). Therefore, we next considered a mantle depletion model with an average degree of melting of $12 \%$ throughout the transect (Figure 2-6c). A positive amplitude is then recovered, and, after correcting for the density of the continental lithosphere, the amplitude of the gravity anomaly is $\sim 15 \mathrm{mGal}$ (Figure 2-6c); the effect of mantle depletion only reduces the mantle contribution in the MBA.

The subcrustal fractionation of mantle melt to form ultramafic "cumulates" could also affect the bulk density of shallow mantle. Geochemical studies of the North Atlantic igneous province indicate an important role for high-pressure fractionation (8-15 kbar) during the ascent of magma through lithosphere [Morrison et al., 1985; Thompson et al., 1986; Bernstein, 1994; Fram and Lesher, 1997]. The effect of preexisting lithosphere might persist during continental rifting, so crystallization from primary melt might have started within the mantle lithosphere. At high pressures, crystallizing phases from basaltic liquids are generally olivine, then clinopyroxene, and then plagioclase [e.g., Bender et al., 1978; Presnall et al., 1978; Grove et al., 1992; Langmuir et al., 1992]. During olivine-only fractionation and olivine+clinopyroxene fractionation, the forsterite content of crystallizing 
olivine gradually decreases because of Fe-Mg partitioning between olivine and liquid [Roeder and Emslie, 1970], if fractionating liquid does not react with surrounding mantle. Relatively Fe-rich olivine cumulates at subcrustal levels could increase bulk mantle density. The density of crystal assemblages calculated in the fractionation modeling (Figure 2-3) is used to quantify this effect (Figure 2-7). The magnitude of the positive density anomaly depends on the initial liquid Mg-number; a lower Mg-number results in a shaper decrease in the forsterite content (Figure 2-7a). The overall impact on mantle density is limited by the appearance of the second crystallizing phase, and the resulting bulk density anomaly rarely exceeds $0.02 \mathrm{Mg} / \mathrm{m}^{3}$ (Figure $2-7 \mathrm{~b}$ ). For 30-km-thick crust, for example, as much as $20 \%$ subcrustal fractionation would merely result in a $7.5-\mathrm{km}$-thick layer with a density anomaly of $0.02 \mathrm{Mg} / \mathrm{m}^{3}$. This is an order of magnitude smaller than the mantle density anomaly required to explain the MBA (Figure 2-5b), indicating that this effect is only of minor significance in this study.

\subsubsection{Crustal Contributions}

\section{Crustal Density Anomalies}

Having explored a reasonable range of thermal and depletion history for our mantle section, we can conclude that the mantle contribution to the observed MBA is about $20 \mathrm{mGal}$ at largest, and that the rest of the MBA must originate in the crust. Given the uncertainty in our modeling of mantle-origin gravity anomalies, we will hereafter consider three different residual gravity anomalies including the original MBA to investigate the crustal contribution (Figure 2-8); the residual anomalies are inverted for density anomalies in the whole crust, the lower crust, and the upper crust (Figure 2-9). An inversion fit similar to the one shown in Figure 2-5a was obtained for all inversions. Note that these inversion results depend on the choice of reference density structure in the gravity calculation. If we choose to define the reference model to match a predicted anomaly over the continental shelf with the observed anomaly, for example, the resultant MBA would be all negative except over the shelf, and the required variation in crustal density would nearly double, because the largest residual anomaly would now correspond to the thinnest crust. Since the seaward section of the transect is best resolved in the seismic velocity model and 
is similar to the normal oceanic crust, which has tightly constrained velocity-density systematics, it can be confidently treated as the reference for our gravity modeling.

The whole-crustal density anomaly has an amplitude of $0.06-0.08 \mathrm{Mg} / \mathrm{m}^{3}$ (Figure 2-9a). A systematic error of this degree in our upper-crustal density model could be acceptable, because the velocity to density conversion law of Carlson and Herrick [1990] has a similar uncertainty. Since the conversion law we adopt for the lower crust provides the greatest possible density for a given velocity, however, this density anomaly must be attributed to a systematic error of greater than $0.16-0.21 \mathrm{~km} / \mathrm{s}$ in the lower-crustal velocity, which substantially exceeds the absolute error of $0.04-0.08 \mathrm{~km} / \mathrm{s}$ estimated by the nonlinear Monte Carlo analysis (Chapter 1). In addition, the average crustal velocity has a smaller error of $\sim 0.03 \mathrm{~km} / \mathrm{s}$ because of the negative correlation of parameter uncertainty, further reducing the possibility of such a large systematic error in the lower-crustal velocity.

Restricting the density anomaly to the lower crust causes an even larger density anomaly (Figure 2-9b), so we are unable to explain this density anomaly for the same reason. The upper-crustal density anomaly naturally has a much greater amplitude (Figure $2-9 \mathrm{c}$ ), and the anomaly of about $0.2 \mathrm{Mg} / \mathrm{m}^{3}$ cannot be explained by either the uncertainty of the conversion law or an error in the upper-crustal velocity. The upper crust is the best constrained part of the tomographic model owing to the dense coverage of refraction rays (Chapter 1). Although there is a possibility of having a completely different conversion law for the upper crust, as we will discuss later, we would like to first make a conservative review of our previous estimate of the uncertainty of the seismic velocity model and reexamine its reliability. One may argue that, for example, the number of ensembles used in our Monte Carlo analysis is not sufficiently large to provide the definitive estimate of the a posteriori covariance. This type of criticism is always valid for the Monte Carlo method unless the number of ensembles is extremely large, which we regard as an unrealistic ideal for our large-scale tomography, so we must seek an alternative, independent check on our uncertainty analysis. Since the majority of the lower crust is constrained only by reflection travel times, we think it beneficial to revisit the tomography 
problem by jointly inverting seismic travel times and gravity anomalies. The virtue of this joint inversion is that the exploration of the model space is biased to some subspace that can explain an input gravity anomaly, thereby supplementing the possibly limited search of the model space by Monte Carlo randomization.

\section{Traveltime Tomography Revisited}

The formulation of the joint inversion of seismic travel times and gravity anomalies is given in Appendix B. A starting model is the velocity model shown in Figure 2-1c, and correlation length functions for velocity and depth nodes are the same as in Chapter 1. Depth-kernel weighting is set as unity for all inversions. The slowness derivatives of density are based on the velocity-density conversion laws as chosen above except for the sedimentary layer, for which we assign zero sensitivity. The travel time data consist of $2318 \mathrm{Pg}$ and $2078 \mathrm{PmP}$ picks from 25 instruments, and the gravity anomaly data consist of 76 data points sampled from the observed free-air gravity anomaly with a $5 \mathrm{~km}$ interval. With the smoothing weights fixed, the gravity-kernel weighting parameter, $\lambda_{g}$, is varied from 0.01 to 1 . For each choice of $\lambda_{g}$, the inversion converged within five iterations. The results are summarized in Figure 2-10a. There is almost no reduction in a root-mean-square (RMS) gravity residual with small $\lambda_{g}$, and the corresponding final velocity model (Figure $2-10 b)$ is virtually identical to the starting model. The RMS gravity residual starts to decrease drastically around $\lambda_{g}$ of 0.1 , and reaches $-3 \mathrm{mGal}$ for the largest $\lambda_{g}$. The long wavelength variations in the observed gravity anomaly are completely fitted using a density model corresponding to the velocity model shown in Figure 2-10c, and the small residual is mostly due to the short wavelength feature observed at model distances of $\mathrm{km}$ 60-90 (Figure $2-1 \mathrm{~b}$ ). This final velocity model has a high-velocity root $(\sim 7.5 \mathrm{~km} / \mathrm{s})$ in the lowermost crust at $\mathrm{km} 80-230$. There is, however, a strong trade-off between the travel time constraint and the gravity constraint; while the RMS gravity residual reduces by increasing the gravity-kernel weighting, the travel time fits significantly deteriorate, especially for the PmP phase (Figure 2-10a).

This exercise demonstrates that there is no hidden model space that we might have missed in our previous Monte Carlo uncertainty analysis; if there were such model space, 
we should observe no change in the travel time fits, while gravity fits are improved. Although the joint inversion does place the needed high-velocity material at the least resolved part of the velocity model, the travel time constraint is largely violated, so that the final velocity model such as shown in Figure 2-10c can be rejected. One may note that a part of the gravity misfit can be explained without strongly violating the travel time data, such as the case of setting $\lambda_{g}$ to 0.1 (Figure 2-10a). Considering that the densest possible conversion was used for the lower crust, however, it is very unlikely that we can accept a denser lower-crustal model than derived by the original velocity model. Therefore, the residual gravity anomaly cannot be attributed to the lower crust despite the fact that it has the largest volume contribution and the largest model uncertainty.

\section{Is Upper Crust Denser?}

The upper crust of transect 2 constitutes about $30 \%$ of the whole-crustal volume, and its velocity structure has an uncertainty of $\sim 0.04 \mathrm{~km} / \mathrm{s}$ (Chapter 1 ). The density conversion law of Carlson and Herrick [1990] is well constrained with one standard deviation of $0.07 \mathrm{Mg} / \mathrm{m}^{3}$. The upper-crustal density anomaly of $\sim 0.2 \mathrm{Mg} / \mathrm{m}^{3}$ required to explain the residual gravity anomaly (Figure 2-9c), therefore, seems difficult to reconcile with the seismic data. The velocity-density systematics for the upper oceanic crust is largely controlled by porosity, and Carlson and Herrick's empirical law is based on a number of laboratory measurements of samples collected from the oceanic crust and ophiolites (see references cited in Carlson and Raskin [1984]). We note, however, that the velocity and density of lavas recovered from the ODP Site 642 (Vøring margin) and Site 917 (Southeast Greenland margin) are systematically offset, despite large scatters, from Carlson and Herrick's law [Planke, 1994; Planke and Cambray, 1998] (Figure 2-11a). Though there are only two drilling sites that show this trend and the penetration depths into basaltic rocks are less than $1 \mathrm{~km}$, this systematic offset of about $0.2 \mathrm{Mg} / \mathrm{m}^{3}$ in density or $1.0 \mathrm{~km} / \mathrm{s}$ in velocity might be a fundamental difference between normal oceanic upper crust and the upper crust of North Atlantic continent-ocean transition zones. Volcanic passive margins are characterized by the occurrence of seaward-dipping reflectors, which resulted from vigorous subaerial eruptions [e.g., Hinz, 1981]. Multichannel seismic reflection data on our 
transect show several seaward-dipping reflectors within the transitional upper crust, and the basement high located around $\mathrm{km} 240$ likely marks a transition from subaerial eruption to submarine eruption (Chapter 1). Most of the upper-crustal density anomaly is confined to the thick transitional upper crust (Figure 2-9c), suggesting that Planke's conversion law should be preferred for that part of the upper crust.

Several possibilities have been proposed for the difference between the two conversion laws for the igneous upper crust, including compositional effects, porosity structure, and the degree of alteration [Planke, 1994]. Plateau basalts tend to have lower Mg-numbers than mid-ocean ridge basalts (MORBs), and the corresponding increase in the iron content may be a major reason to modify the velocity-density systematics (e.g., Figure 2-3). Whereas Icelandic plateau basalts do indeed have higher grain densities [Christensen and Wilkens, 1982], however, reported grain densities for samples from the Greenland margin are virtually the same as that for average MORB [Larsen et al., 1994; Duncan et al., 1996], so it is unlikely that the systematic offset results from a difference in matrix density. Alternatively, subaerial lava emplacement could result in a different porosity structure, and we note that the velocity of porous rocks is highly sensitive to the spectrum of pore aspect ratios [e.g., Wilkens et al., 1991]. Porosity structure is also affected by alteration, largely controlled by hydrothermal circulation and cooling history, which can substantially differ between plateau basalts and MORBs. Simple forward modeling was conducted to illustrate the effect of porosity structure (Figure 2-11); the spectra of pore aspect ratios are modeled to fit the overall trend of the two empirical conversion laws. The chosen spectra are of course arbitrary, in the sense that there are many other solutions to generate similar fits, but the potential of porosity structure in modifying velocity-density systematics is clear from this example.

The residual gravity anomaly of $40-60 \mathrm{mGal}$ over the transitional crust might, therefore, be due to a denser upper crust, compared to the oceanic upper crust. Our preferred density model, incorporating this upper-crustal density anomaly, is presented in Figure 2-12c. The corresponding gravity anomaly shows an excellent fit with observations, providing an independent check on the accuracy of our inversion method (Figure 2-12a). A 
mass anomaly based on the final density model is shown in Figure $2-12$ b, which is broadly comparable with a mass anomaly based on an observed sedimentary load, indicating that most of the current sedimentary load is not compensated by the underlying lithosphere. Since the thickest sediment column is only about $1.5 \mathrm{~km}$, however, the transect is close to isostatic equilibrium; required surface elevations to attain local isostasy are less than $0.6 \mathrm{~km}$ along the transect (Figure 2-12b). The mode of isostacy is of a mixed nature; the basement topography is balanced by both the crustal thickness variation (Airy-type isostacy) and the lateral density variation within the crust (Pratt-type isostacy).

\subsection{Discussion and Conclusion}

Gravity modeling has been a common exercise in studies of deep-crustal structure [e.g., Holbrook et al., 1994b; Horsefield et al., 1994; Chian et al., 1995; Barton and White, 1997a; Lizarralde and Holbrook, 1997; Reid and Jackson, 1997; Mjelde et al., 1998]; through the correlation between velocity and density, it can provide an independent check on a velocity model. There are, however, several problems with this type of gravity modeling, which have often been overlooked in previous studies. Because the velocitydensity systematics can have considerable uncertainty even for a single rock type, due to compositional and porosity effects, and because a velocity model itself always has some estimation errors, constructing a density model that can satisfy a given gravity constraint could become arbitrary, considering the nonuniqueness of the derived density distribution. Being able to present a 'consistent' pair of velocity and density models may only mean that an original velocity model is poorly constrained. We therefore need to (1) demonstrate how well a velocity model is constrained and (2) translate its uncertainty into a density model. Velocity-depth ambiguity is inherent in the seismic constraint of reflection travel times, and the corresponding correlation between velocity and depth parameters should be used to provide a proper error estimate in gravity modeling. The Monte Carlo approach used in this study fulfills these requirements.

Uncertainty in velocity to density conversion is more problematic. For the continental crust, because of the variety of rock types we can expect [e.g., Christensen and 
Mooney, 1995], rigorous error propagation does not seem to provide any useful outcome [Barton, 1986]. For the thick igneous and oceanic crust, however, we can reasonably assume a one-step crustal production from mantle melting, so that less ambiguous conversion is possible. This is especially true for the lower-crustal section of thick igneous crust, which is hardly affected by seawater alteration. Even though there is still considerable scatter in the velocity-density relation of gabbroic rocks (Figure 2-3), a bounding approach such as our densest possible conversion is effective to systematically isolate density anomalies, together with the inversion method developed in this paper. Whereas the inversion of gravity anomalies is usually formulated to estimate the shape of a causative body [e.g., Oldenburg, 1974; Granser, 1987; Guspi, 1990], we showed that an alternative formulation to estimate density is particularly useful when reliable seismic information is available. Our success in this systematic approach is, however, somewhat fortuitous because the bounding approach largely depends on having well-constrained oceanic crust within the transect. Based on the presence of oceanic crust, we were able to test various possibilities for the thicker transitional crust. If we did not have such a reliable benchmark, the gravity modeling could have been almost meaningless, as lucidly demonstrated by Barton [1986].

Owing to limited experimental capability in the past, it is for only the last several years that we have been able to collect dense, high-quality seismic data and to confidently derive deep-crustal seismic structure. Deep-crustal seismic studies in previous generations relied heavily on expanding-spread profiling, suffering from serious velocity-depth ambiguity for lower-crustal structure. Because of this, gravity studies based on an assumption of local or regional isostacy were common, and their validity is open to question. As demonstrated by Lizarralde and Holbrook [1997] and by this study, isostatic compensation at passive continental margins can be quite complex, suggesting that previous geophysical studies on passive margins based on simple isostacy assumptions [e.g., Karner and Watts, 1982; Watts, 1988; Watts and Marr, 1995] need to be revisited.

Many passive continental margins show a gravity high similar to that in our transect. Whereas Vogt et al. [1998] proposed densified crust resulting from a gabbro- 
eclogite phase change as the most likely, general explanation for these gravity highs, such an explanation does not apply to our transect, because the crustal velocity is too low to be interpreted as eclogite. Though we do not believe that there should necessarily be a single explanation for all passive margin gravity highs, the dense upper crust made of plateau basalts might be an important factor for gravity highs at volcanic margins worldwide. Because the number of joint seismic and gravity studies at volcanic margins is fairly limited, and because direct comparison with our result is hindered by the difference in the quality and quantity of seismic data and in gravity modeling methods, it is difficult to discuss this hypothesis further.

\section{Appendix 2.A Inversion of Gravity Anomalies in the Presence of Upper and Bottom Topography}

A 2-D gravity anomaly, $g_{z}$, caused by a 1-D density variation, $\rho(x)$, bounded by $z=z_{l}(x)$ and $z=z_{2}(x)$ (Figure 2.A1) may be expressed in the Fourier domain as [Parker, 1972],

$$
\mathrm{F}\left[g_{z}\right]=-2 \pi G \exp \left(|k| z_{0}\right) \sum_{n=1}^{\infty} \frac{(-|k|)^{n-1}}{n !} \mathrm{F}\left[\rho\left(z_{1}^{n}-z_{2}^{n}\right)\right],
$$

where F[] denotes the 1-D Fourier transform with respect to the horizontal coordinate, and $G, z_{0}$, and $k$ are the universal gravity constant, the vertical coordinate of an observation plane $\left(z_{0}<\min \left(z_{l}\right)\right)$, and the horizontal wavenumber, respectively. A restriction to constant thickness is usually applied to separate the $\mathrm{F}[\rho]$ term from other higher-order terms with topography variations and construct an iterative inversion formula for the density variation [e.g., Parker and Huestis, 1974]. This limitation to constant layer thickness is only superficial, and an extension to variable thickness is straightforward. Noting that the 1-D density variation between the two boundaries can be also expressed as a layer of constant thickness and two topographic corrections, equation $2 \mathrm{~A} .1$ may be rewritten as,

$$
\begin{aligned}
\frac{\mathrm{F}\left[g_{z}\right]}{2 \pi G \mathrm{e}^{|k| z_{0}}}= & \frac{1}{|k|}\left(\mathrm{e}^{-|k| z_{a}}-\mathrm{e}^{-|k| z_{b}}\right) \mathrm{F}[\rho]+\sum_{n=1}^{\infty} \frac{(-|k|)^{n-1}}{n !} \times \\
& \left\{\mathrm{e}^{-|k| z_{a}} \mathrm{~F}\left[\rho\left(z_{1}-z_{a}\right)^{n}\right]-\mathrm{e}^{-|k| z_{b}} \mathrm{~F}\left[\rho\left(z_{2}-z_{b}\right)^{n}\right]\right\},
\end{aligned}
$$


where $z_{a}=\min \left(z_{1}\right)$ and $z_{b}=\max \left(z_{2}\right)$. Since the convergence of the infinite series summation is fastest when a reference plane is located between the top and bottom boundaries [Parker, 1972], the more numerically efficient form of equation 2A.2 may be expressed as,

$$
\begin{aligned}
\frac{\mathrm{F}\left[g_{z}\right]}{2 \pi G \mathrm{e}^{|k| z_{0}}}= & \frac{1}{|k|}\left(\mathrm{e}^{-|k| z_{n}}-\mathrm{e}^{-|k| z_{b}}\right) \mathrm{F}[\rho]+\sum_{n=1}^{\infty} \frac{(-|k|)^{n-1}}{n !} \times \\
& \left\{\mathrm{e}^{-|k| z_{r 1}} \mathrm{~F}\left[\rho\left\{\left(z_{a}-z_{r 1}\right)^{n}-\left(z_{1}-z_{r 1}\right)^{n}\right\}\right]\right. \\
& \left.+\mathrm{e}^{-|k| z_{r 2}} \mathrm{~F}\left[\rho\left\{\left(z_{2}-z_{r 2}\right)^{n}-\left(z_{b}-z_{r 2}\right)^{n}\right\}\right]\right\},
\end{aligned}
$$

where $z_{r 1}=\left(z_{a}+\max \left(z_{1}\right)\right) / 2$ and $z_{r 2}=\left(z_{b}+\min \left(z_{2}\right)\right) / 2$. An iterative inversion formula for the density variation is thus obtained as

$$
\begin{aligned}
\mathrm{F}[\rho]= & \frac{|k|}{\mathrm{e}^{-|k| z_{r}}-\mathrm{e}^{-|k| z_{b}}}\left(\frac{\mathrm{F}\left[g_{z}\right]}{2 \pi G \mathrm{e}^{|k| z_{0}}}-\sum_{n=1}^{\infty} \frac{(-|k|)^{n-1}}{n !} \times\right. \\
& \left\{\mathrm{e}^{-|k| z_{r 1}} \mathrm{~F}\left[\rho\left\{\left(z_{a}-z_{r 1}\right)^{n}-\left(z_{1}-z_{r 1}\right)^{n}\right\}\right]\right. \\
& \left.\left.+\mathrm{e}^{-|k| z_{r 2}} \mathrm{~F}\left[\rho\left\{\left(z_{2}-z_{r 2}\right)^{n}-\left(z_{b}-z_{r 2}\right)^{n}\right\}\right]\right\}\right),
\end{aligned}
$$

Note that, for $k=0$, the term $|k| /\left(\mathrm{e}^{-|k| z_{a}}-\mathrm{e}^{-\mid k z_{z_{b}}}\right)$ reduces to the reciprocal of the constant layer thickness, $1 /\left(z_{b}-z_{a}\right)$. An annihilator can be constructed by setting $\mathrm{F}\left[g_{z}\right]=0$ and using $\delta(k)$ as an initial guess for $\mathrm{F}[\rho]$. This inversion is essentially a downward continuation so that proper low pass filtering must be applied at each iteration to avoid the divergence of high-wavenumber components.

\section{Appendix 2.B Joint Inversion of Seismic Travel Times and Gravity Anomalies}

Following the notation adopted in Chapter 1, a linearized inverse equation for slowness and depth perturbations that are constrained by seismic travel times and gravity anomalies may be expressed as,

$$
\left[\begin{array}{c}
\mathbf{d}_{t} \\
\lambda_{g} \mathbf{d}_{g} \\
\mathbf{0} \\
\mathbf{0} \\
\mathbf{0}
\end{array}\right]=\left[\begin{array}{cc}
\mathbf{G}_{v} & w \mathbf{G}_{d} \\
\lambda_{g} \mathbf{F}_{v} & \lambda_{g} w \mathbf{F}_{d} \\
\lambda_{v} \mathbf{L}_{\mathrm{Hv}} & \mathbf{0} \\
\lambda_{v} \mathbf{L}_{\mathrm{V} v} & \mathbf{0} \\
\mathbf{0} & \lambda_{d} w \mathbf{L}_{d}
\end{array}\right]\left[\begin{array}{c}
\delta \mathbf{m}_{v} \\
\frac{1}{w} \delta \mathbf{m}_{d}
\end{array}\right],
$$


where $\mathbf{d}_{t}$ and $\mathbf{d}_{g}$ are residual travel times and residual gravity anomalies, respectively, $\mathbf{G}$ and $\mathbf{F}$ are Fréchet derivative matrices for travel times and gravity anomalies, respectively, the $\mathbf{L}$ matrices are for smoothing constraints, and the $\delta \mathbf{m}$ vectors are for model perturbations. The subscripts $v$ and $d$ denote slowness and depth components, respectively. The weighting parameters, $\lambda_{g}, \lambda_{v}$, and $\lambda_{d}$, control the significance of the gravity anomaly constraint and the smoothness constraints on the velocity model, respectively, relative to the travel time constraint. The depth-kernel weighting parameter, $w$, determines the relative weighting of depth sensitivity in the Fréchet matrices. The details of inversion strategy are identical to those described in Chapter 1.

To be consistent with the node-oriented parameterization employed in Chapter 1 , the cell volumes of the slowness mesh are redistributed to nodes, and we assign a centroid, $\left(x_{i}, z_{i}\right)$, for each nodal volume of $d V_{i}$. The 2-D gravity Fréchet matrix for slowness nodes may be then written as,

$$
\begin{aligned}
F_{y}^{i, j} & =\frac{d \rho}{d s} K\left(x_{i}, z_{i} ; x_{o}^{j}, z_{v}^{j}\right) d V_{i} \\
& =2 G \frac{d \rho}{d s} \frac{z_{i}-z_{o}^{j}}{\left(x_{i}-x_{o}^{j}\right)^{2}+\left(z_{i}-z_{o}^{j}\right)^{2}} d V_{i},
\end{aligned}
$$

where $d \rho / d s$ is the slowness derivative of density, $K(\cdots)$ is a 2-D gravity kernel for a point mass source, and $\left(x_{o}^{j}, z_{o}^{j}\right)$ is an observation point for the $j$-th residual gravity anomaly. Additional sensitivity is required for horizontal edge nodes to be consistent with the padding applied in the forward gravity calculation, so the following sensitivity as,

$$
F_{v}^{i, j}=2 G \frac{d \rho}{d s} d z\left[\frac{\left(z_{i}-z_{o}^{j}\right) d x}{\left(x_{i}-x_{o}^{j}\right)^{2}+\left(z_{i}-z_{o}^{j}\right)^{2}}+\frac{\pi}{2}+\tan ^{-1}\left(\frac{x_{\min }-x_{o}^{j}}{z_{r}-z_{o}^{j}}\right)\right],
$$

is applied for the edge nodes at the horizontal minimum of the model domain, $x_{\min }$, based on the semi-infinite slab formula [e.g., Telford et al., 1990]. A nodal volume $d V_{i}$ is decomposed as $d x \times d z$ in the above expression, and for the nodes at the horizontal maximum, $x_{\max }$, the term $\left(x_{\min }-x_{o}^{j}\right)$ should be replaced with $\left(x_{o}^{j}-x_{\max }\right)$. Similarly, the Fréchet matrix for depth nodes, $\left(x_{i}, z_{i}\right)$, can be derived as,

$$
F_{d}^{i, j}=2 G\left[\rho\left(x_{i}, z_{i}\right)-\rho_{\mathrm{n}}\right] \frac{\left(z_{i}-z_{o}^{j}\right) \overline{d x}}{\left(x_{i}-x_{o}^{j}\right)^{2}+\left(z_{i}-z_{o}^{j}\right)^{2}},
$$


where $\rho_{m}$ is mantle density, and $\overline{d x}$ is average distance to neighboring nodes.

An example of the gravity Fréchet matrix for slowness nodes is shown in Figure 2.B1. The radial part of equation 2 B.2 has strong sensitivity below the observational point with a lateral spread of $\sim 15 \mathrm{~km}$, and it rapidly attenuates away from the core (Figure 2.B1a). Because the nodal volume distribution is not regular and gradually increases downward due to the variable grids (see Figure 7 of Chapter 1), and because different geological subdomains such as the upper and lower igneous crust have different derivatives of slowness with respect to density, the total sensitivity has a few discontinuities and a diffuse character (Figure 2.B1b). Even after applying a cutoff criterion, therefore, the gravity sensitivity matrix is no more sparse. The number of residual gravity anomaly data, however, can be limited because we are interested only in long-wavelength gravity variations, so that it is possible to maintain the memory compactness of the inverse equation. 

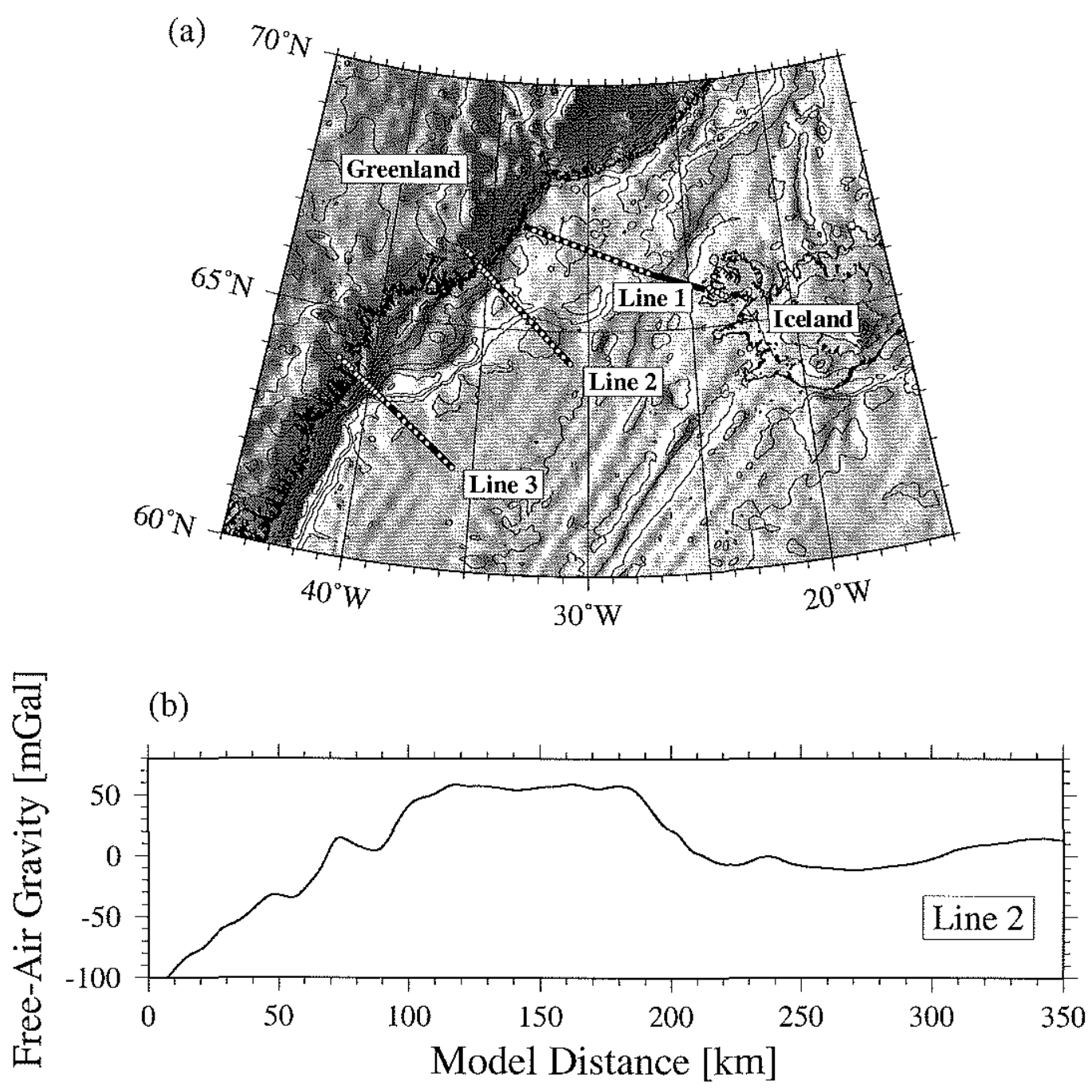

(c)

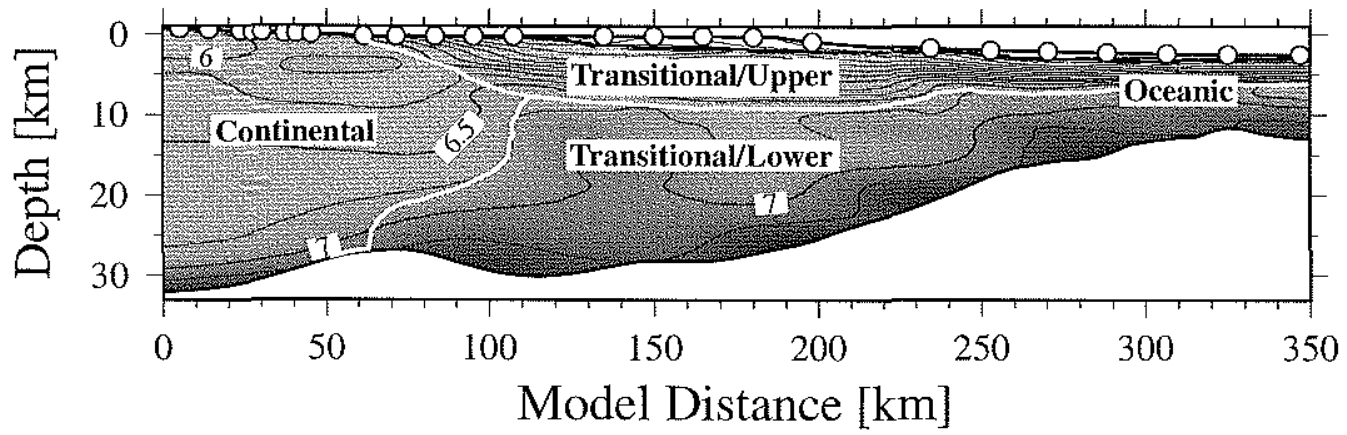

Figure 2-1. (a) Shaded gravity anomaly map with location of 1996 SIGMA seismic transects. Contour interval is $25 \mathrm{mGal}$. Solid lines denote airgun shot lines whereas open circles denote onshore/offshore seismic instruments. (b) Free-air gravity anomaly on transect 2. Land data $(<\mathrm{km} 50)$ were applied with Bouguer correction. (c) $P$-wave crustal velocity model for transect 2 , with geological interpretation of continent-ocean transition zone (Chapter 1). 


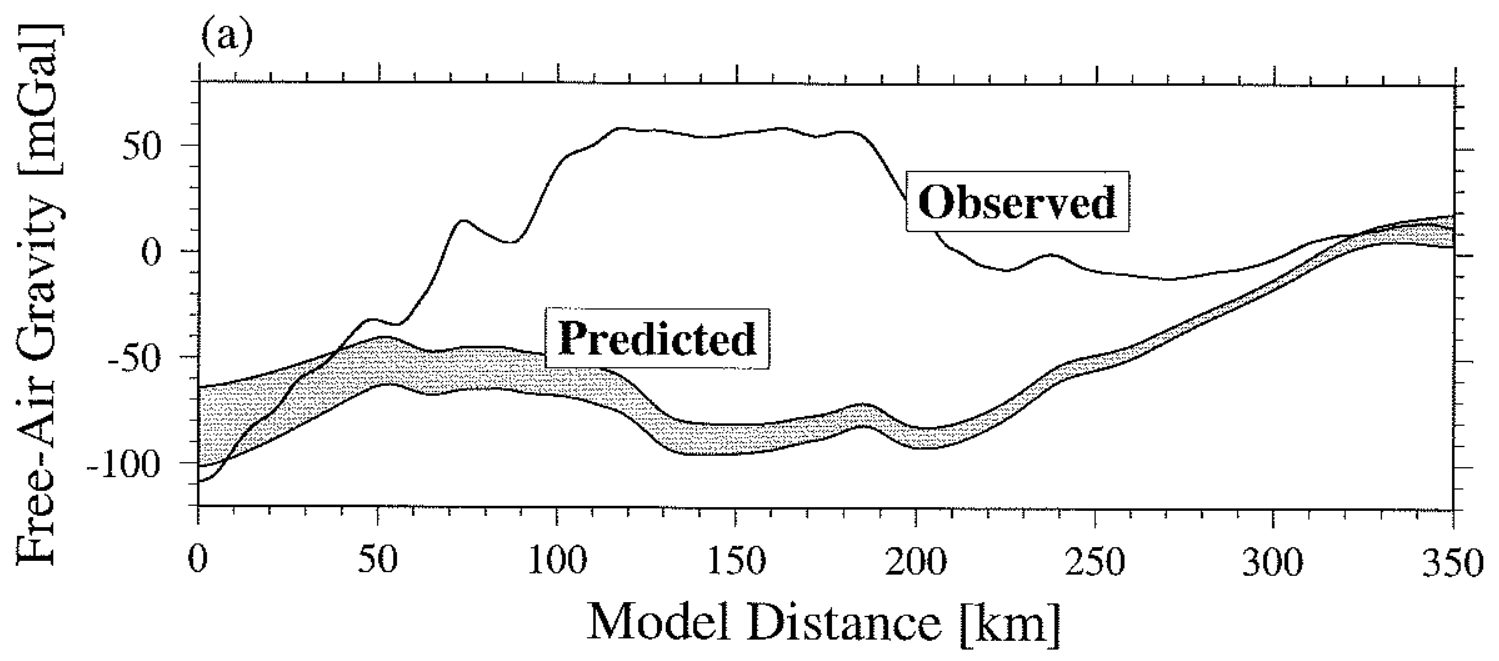

(b)

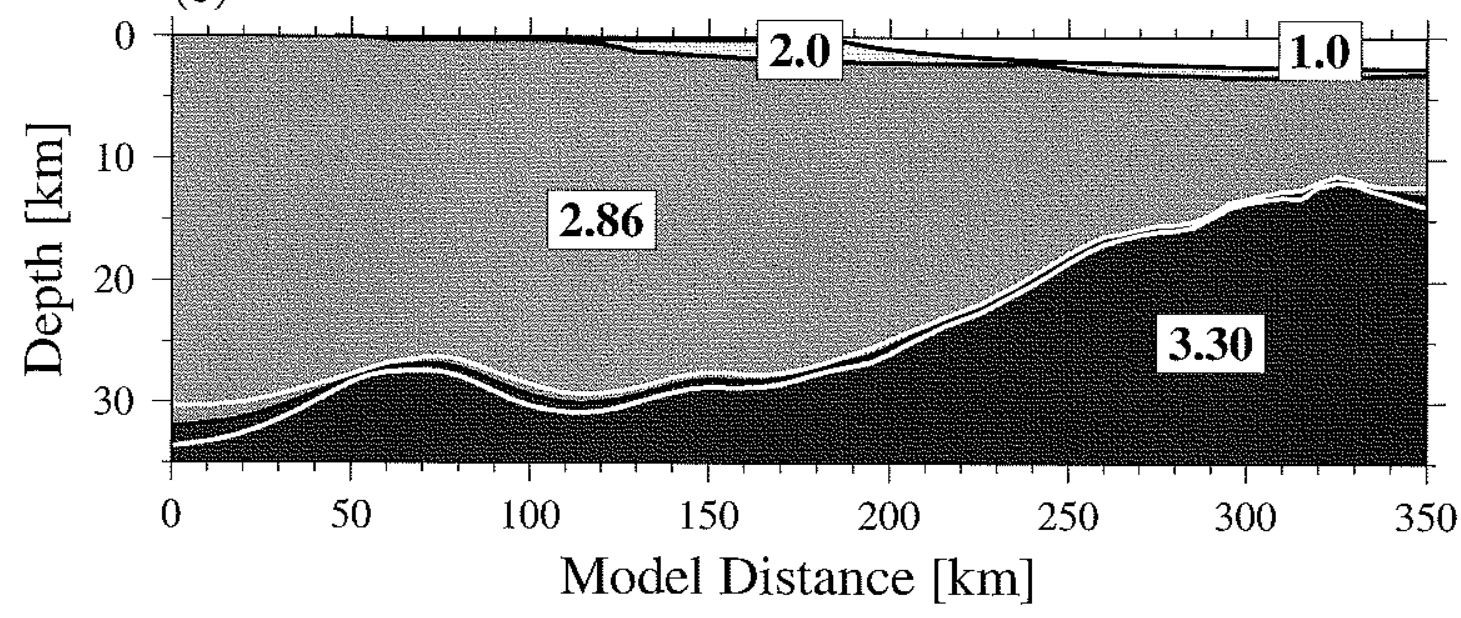

Figure 2-2. (a) Observed free-air gravity anomaly is plotted with predicted free-air gravity anomaly $( \pm 1 \sigma)$ based on 4-layer constant density model shown in (b). Standard deviation of predicted gravity anomaly is based on standard deviation of the Moho depths as shown as white lines in (b). 


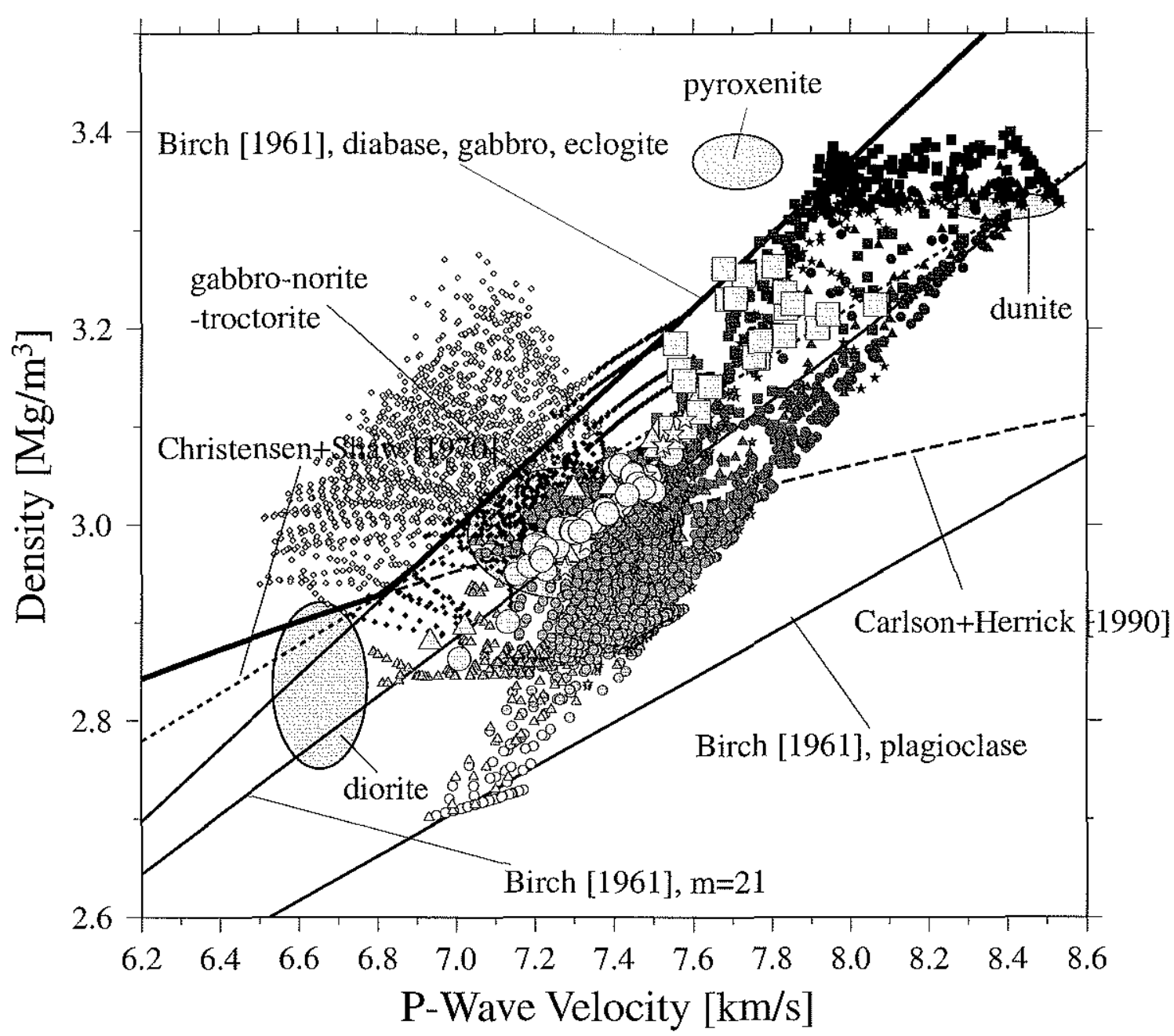

Figure 2-3. Covariation of $P$-wave velocity and density of mafic and ultramafic rocks at $1000 \mathrm{MPa}$ and $25^{\circ} \mathrm{C}$. Large symbols denote values based on the CIPW norm calculation of mantle primary melts (circles [Kinzler and Grove, 1992; Kinzler and Grove, 1993; Kinzler, 1997], triangles [Hirose and Kushiro, 1993], stars [Baker and Stolper, 1994], and squares [Walter, 1998]), and small symbols denote values of fractionated crystal assemblages based on fractional crystallization modeling at 100 , 200,400 , and $800 \mathrm{MPa}$ (see Chapter 3 for details). Velocity-density relationship adopted in this study is shown as thick solid line (Birch's law for diabase, gabbro, and eclogite). Also plotted are velocity and density of residual liquid compositions (solid diamonds for solid fractions less than 0.5 , and open diamonds for solid fractions greater than 0.5 ). Though density for residual liquids at late fractionation stages exceeds the adopted conversion law because of high $\mathrm{FeO}$ content, its contribution to lower crust is minimal, considering its small volume proportion. Ellipses denote laboratory data for diorite, gabbro-norite-troctolite, pyroxenite, and dunite, reported by Christensen and Mooney [1995]. Three solid lines are taken from Birch [1961] for plagioclase, rocks with mean atomic weight of $\sim 21$, and diabase-gabbro-eclogite. Dashed and dotted lines are for normal oceanic crust based on ODP/DSDP core data [Carlson and Herrick, 1990] and on samples dredged from the Mid-Atlantic Ridge [Christensen and Shaw, 1970]. 


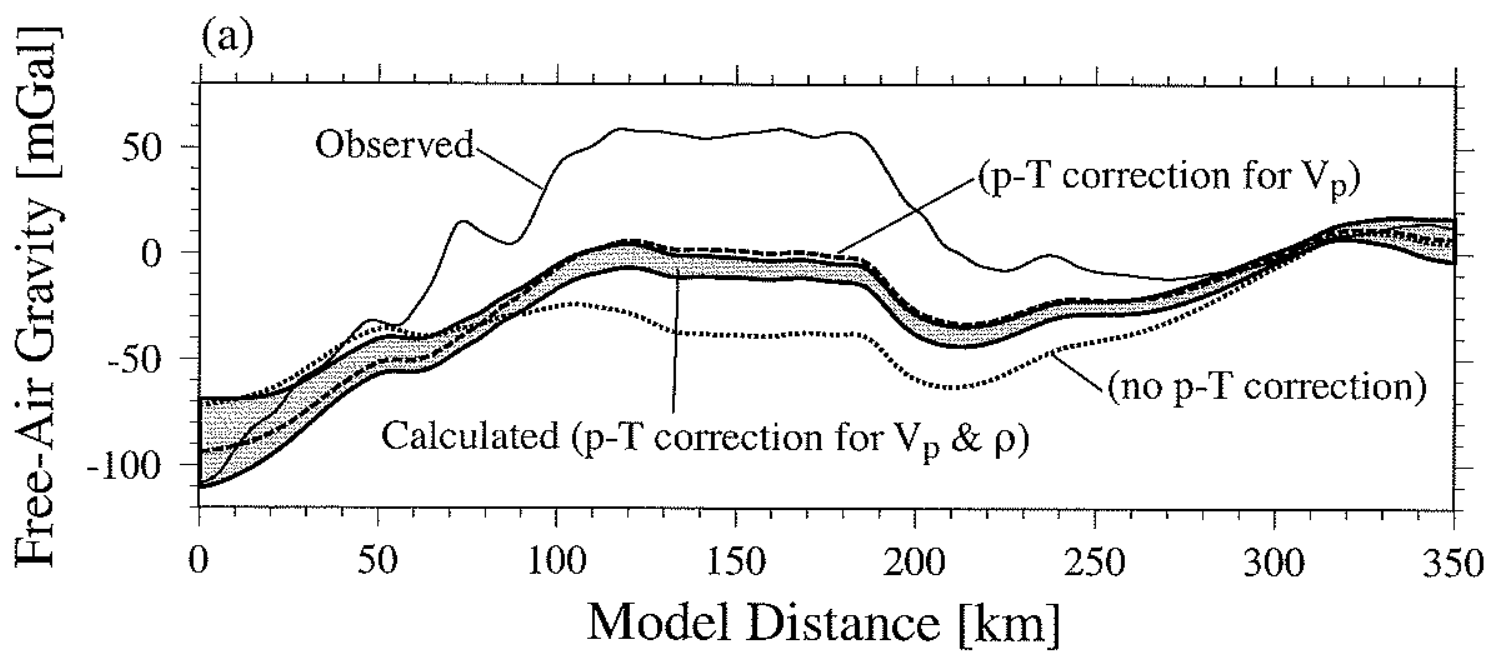

(b)

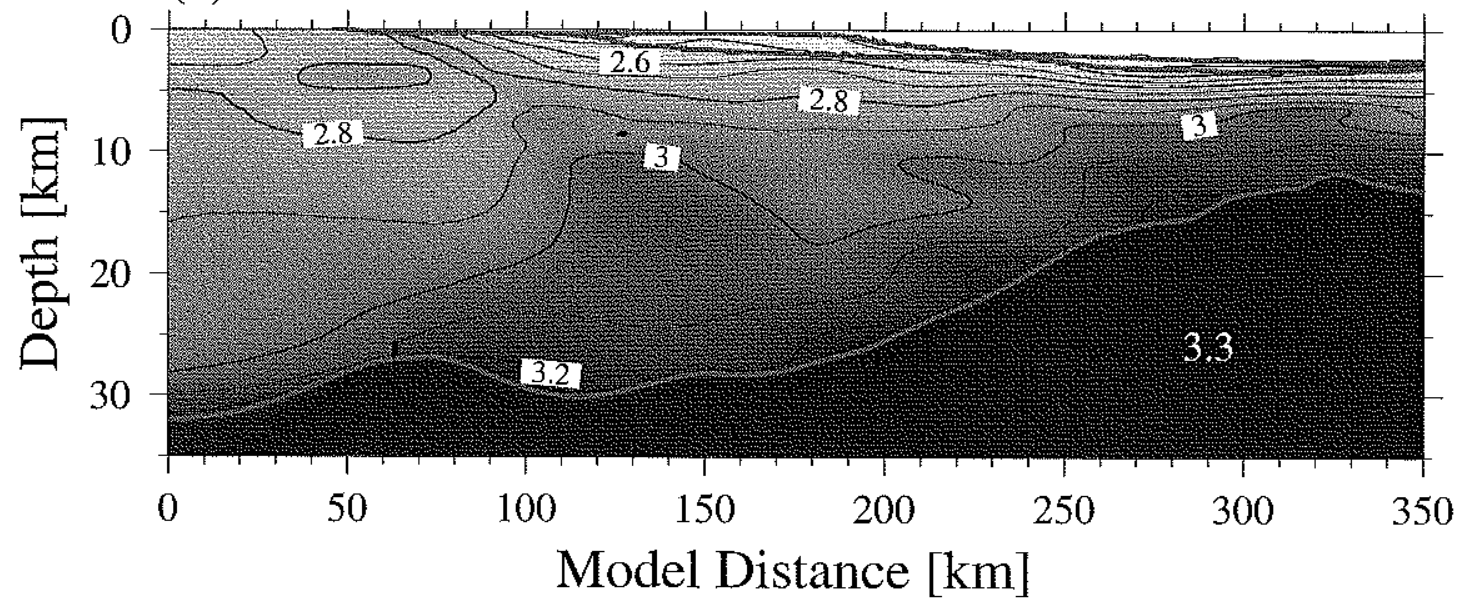

Figure 2-4. (a) Predicted free-air gravity anomaly $( \pm 1 \sigma)$ based on the density model shown in (b) is plotted with observed free-air gravity anomaly. Pressure and temperature corrections are applied to both velocity and density conversions. Also shown are gravity anomalies with no pressure and temperature corrections (dotted) and with pressure and temperature corrections for velocity conversion only (dashed). 


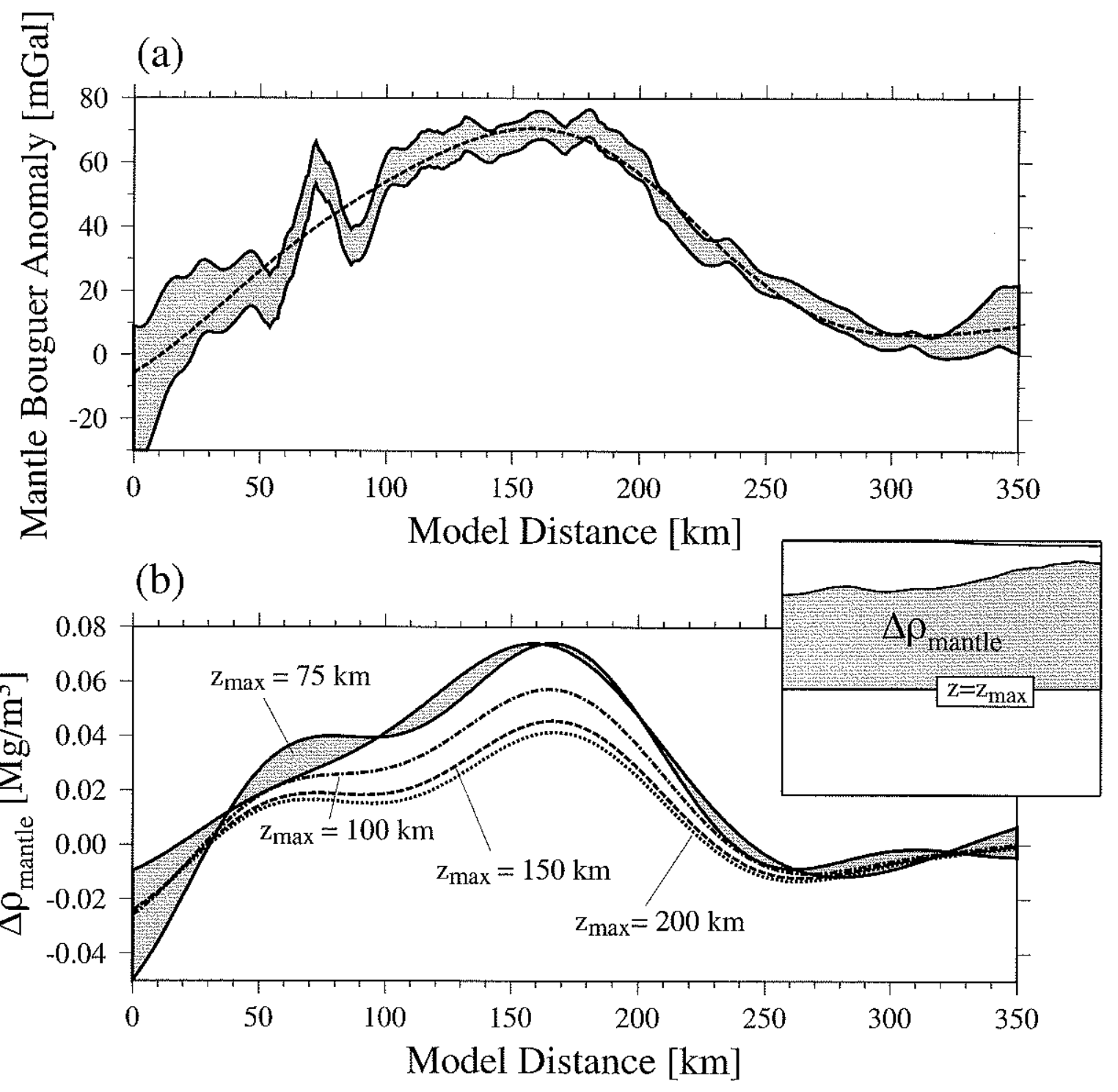

Figure 2-5. (a) Mantle Bouguer anomaly based on crustal density model shown in Figure 2-4(b). Dashed line denotes fitted gravity anomaly obtained in the inversion for mantle density anomaly. Virtually identical fits are obtained for different maximum compensation depths. (b) Mantle density anomalies for different maximum compensation depths (solid: $75 \mathrm{~km}$, dash-dotted: $100 \mathrm{~km}$, dashed: $150 \mathrm{~km}$, and dotted: $200 \mathrm{~km}$ ). One standard deviation is shown for the case of maximum depth of $75 \mathrm{~km}$. A low-pass filter used in this inversion is cosine-tapered with cut wavelength of $80 \mathrm{~km}$ and pass wavelength of $100 \mathrm{~km}$. 
Figure 2-6. Three different models for mantle density anomaly (lower panel) and corresponding gravity anomalies (upper panel). Mantle density anomaly is relative to $3.3 \mathrm{Mg} / \mathrm{m}^{3}$, the constant value assumed in the other density models. (a) Mantle density anomaly from pressure and temperature effects only. Mantle thermal field is based on 1-D thermal evolution model. A (solid): $T_{a}=1350^{\circ} \mathrm{C}$ and $a=200 \mathrm{~km}, \mathrm{~B}$ (dotted): $T_{a}=1450^{\circ} \mathrm{C}$ and $a=200 \mathrm{~km}$, and $\mathrm{C}$ (gray): $T_{a}=1350^{\circ} \mathrm{C}$ and $a=125 \mathrm{~km}$. A+ (dashed) is obtained by adding negative density anomaly of $0.015 \mathrm{Mg} / \mathrm{m}^{3}$ for the region enclosed by solid box (continental lithosphere). (b) In addition to temperature and pressure corrections, the effect of mantle depletion required to produce the observed crustal thickness based on passive mantle upwelling model is incorporated (solid). Asthenospheric temperature is varied accordingly and plate thickness is held constant at $200 \mathrm{~km}$. A positive density anomaly of $0.032 \mathrm{Mg} / \mathrm{m}^{3}$ is added to continental lithosphere to increase the landward end of gravity anomaly to zero (dashed). (c) Average degree of melting is held constant at $12 \%$ with plate thickness of $200 \mathrm{~km}$ (solid). A negative density anomaly of $0.009 \mathrm{Mg} / \mathrm{m}^{3}$ is added to continental lithosphere to reduce the landward end of gravity anomaly to zero (dashed). 
(a) $\mathrm{p}-\mathrm{T}$ correction only

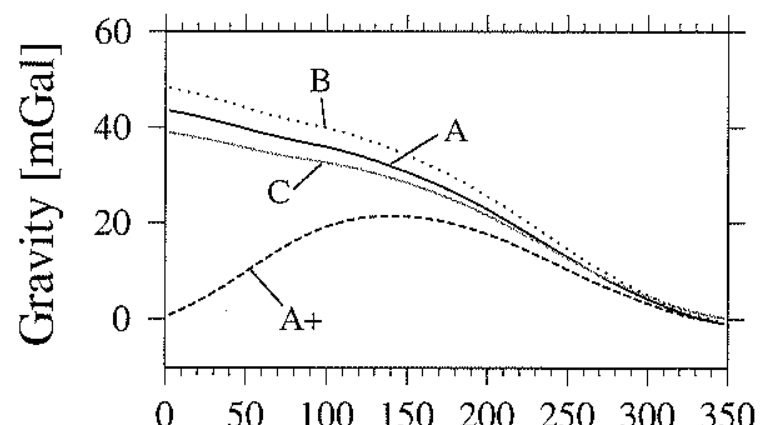

5

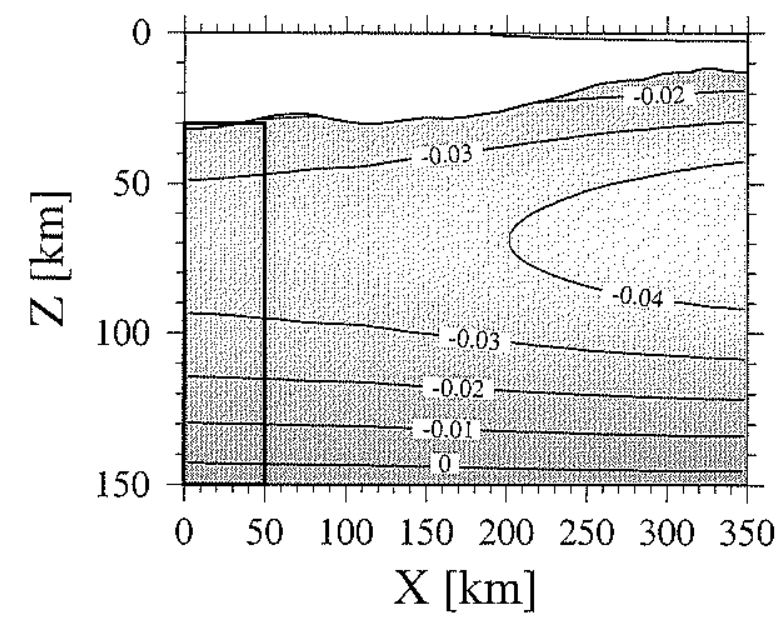

(b) variable depletion model
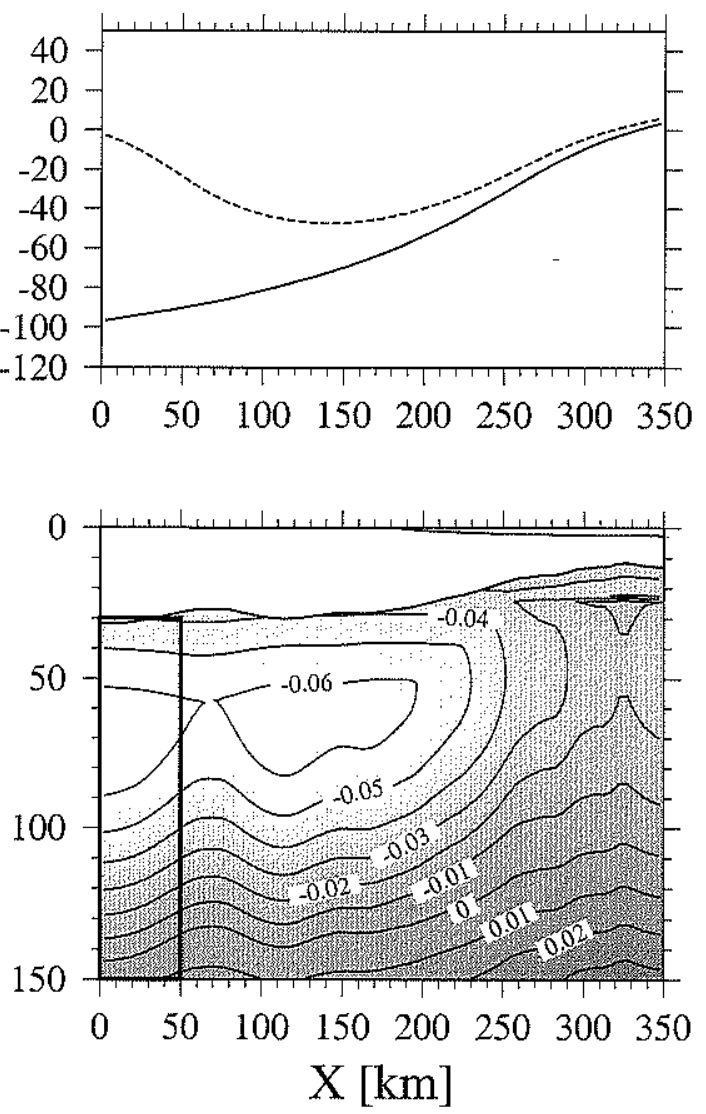

(c) constant depletion model
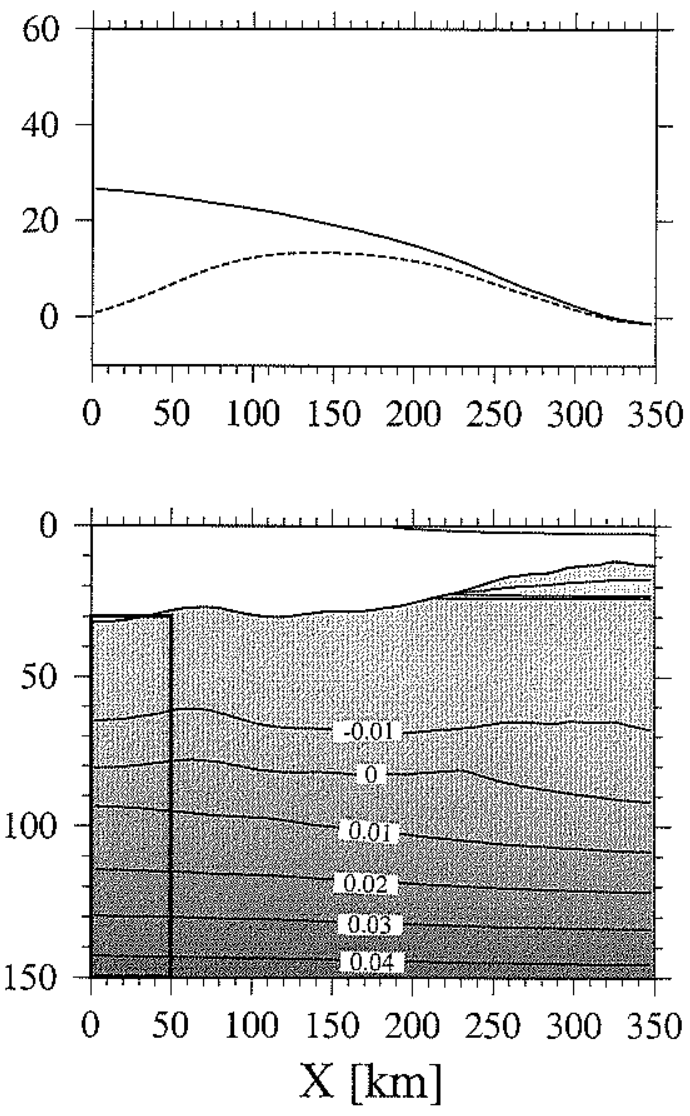
(a)

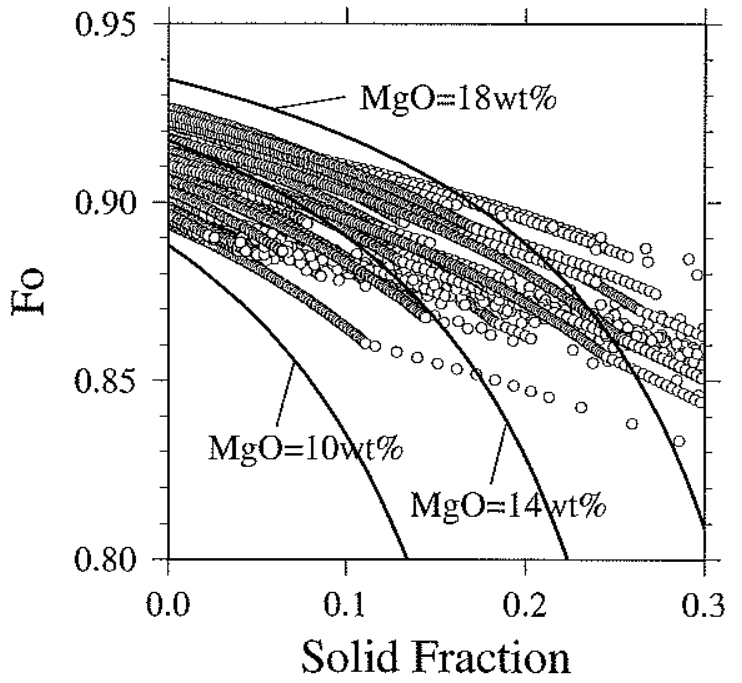

(b)

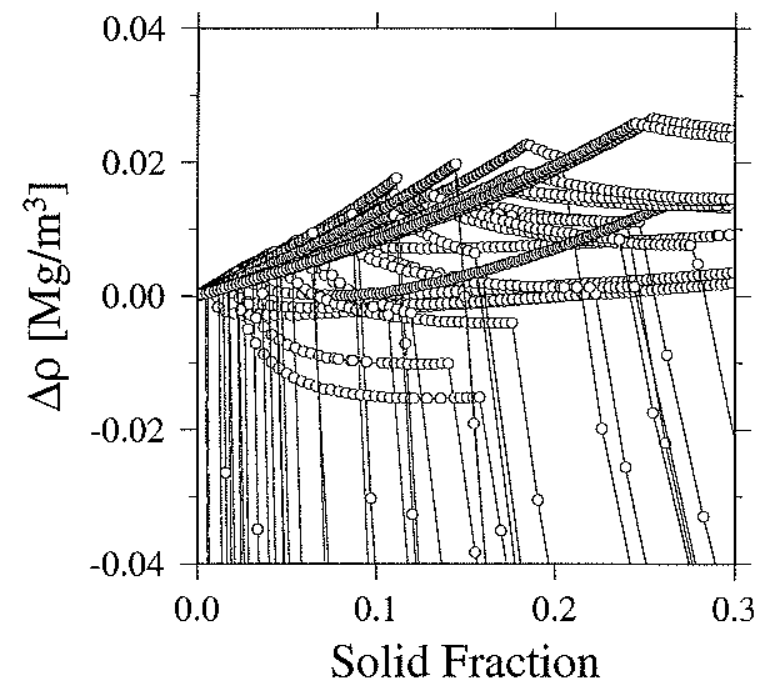

Figure 2-7. Summary of high-pressure fractionation in terms of olivine composition and density anomaly based on fractional crystallization modeling at $800 \mathrm{MPa}$. Densities are calculated at $800 \mathrm{MPa}$ and $480^{\circ} \mathrm{C}$ (deep Moho condition). (a) The olivine composition of the incremental crystal assemblage is plotted as a function of total solid fraction. Also shown are hypothetical olivine fractionation paths starting from initial liquids with $7.5 \mathrm{FeO}$ wt $\%$ and 10,14 , and $18 \mathrm{MgO}$ wt $\%$, using olivine-liquid Fe-Mg exchange coefficient of 0.30 . Because of addition of clinopyroxene and/or plagioclase in fractionating assemblage at a solid fraction $>0.1$, decrease in the forsterite content is more reduced in more realistic fractionation paths. (b) Increase in the density of cumulative fractionated assemblage with respect to the initial olivine density is plotted as a function of solid fraction. Because of appearance of clinopyroxene and plagioclase, the effect of the low forsterite content on the bulk density is limited up to $\sim 0.025 \mathrm{Mg} / \mathrm{m}^{3}$. 


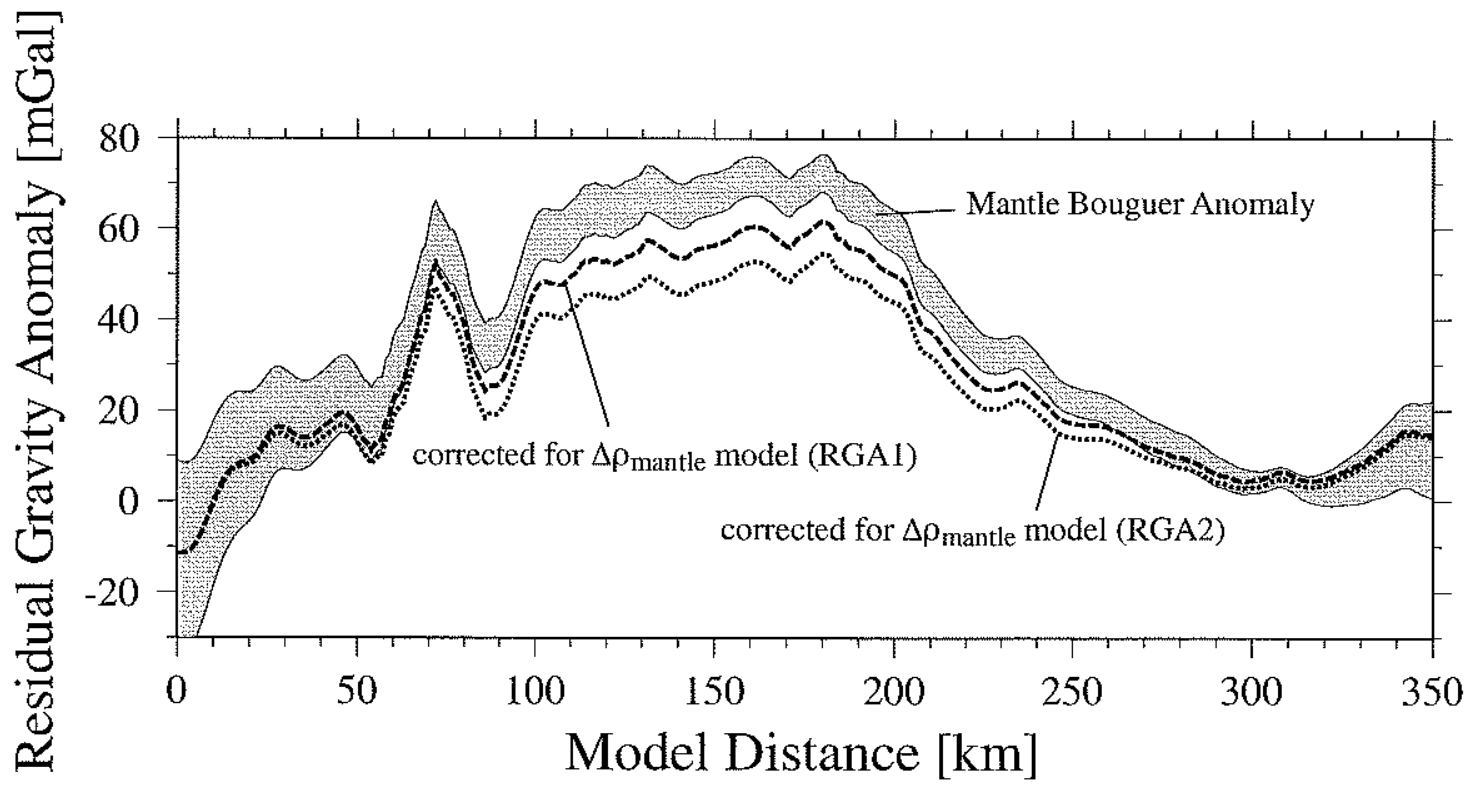

Figure 2-8. Three candidates for residual gravity anomalies. MBA (solid with one standard deviation): original mantle Bouguer anomaly as shown in Figure 2-5(a), RGA1 (dashed): corrected for the mantle density model shown in Figure 2-6(c), and RGA2 (dotted): corrected for the mantle density model shown in Figure 2-6(a). RGA1 is our preferred choice, since it incorporates the most likely thermal and depletion history of the mantle beneath the transect. Standard deviation for RGA1 and RGA2 are the same as for $\mathrm{MBA}$, and are omitted for clarity. 
(a)

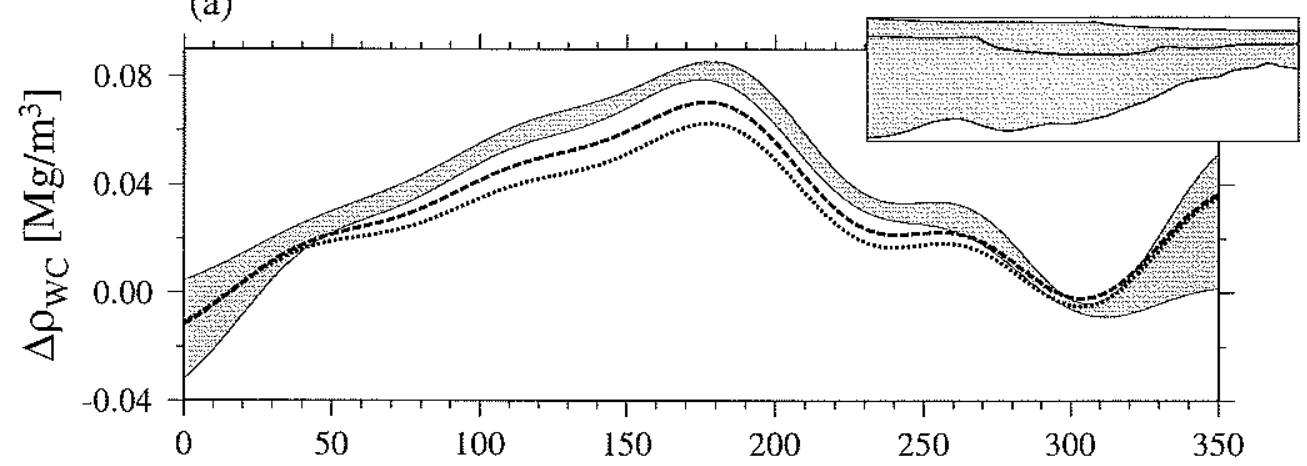

(b)
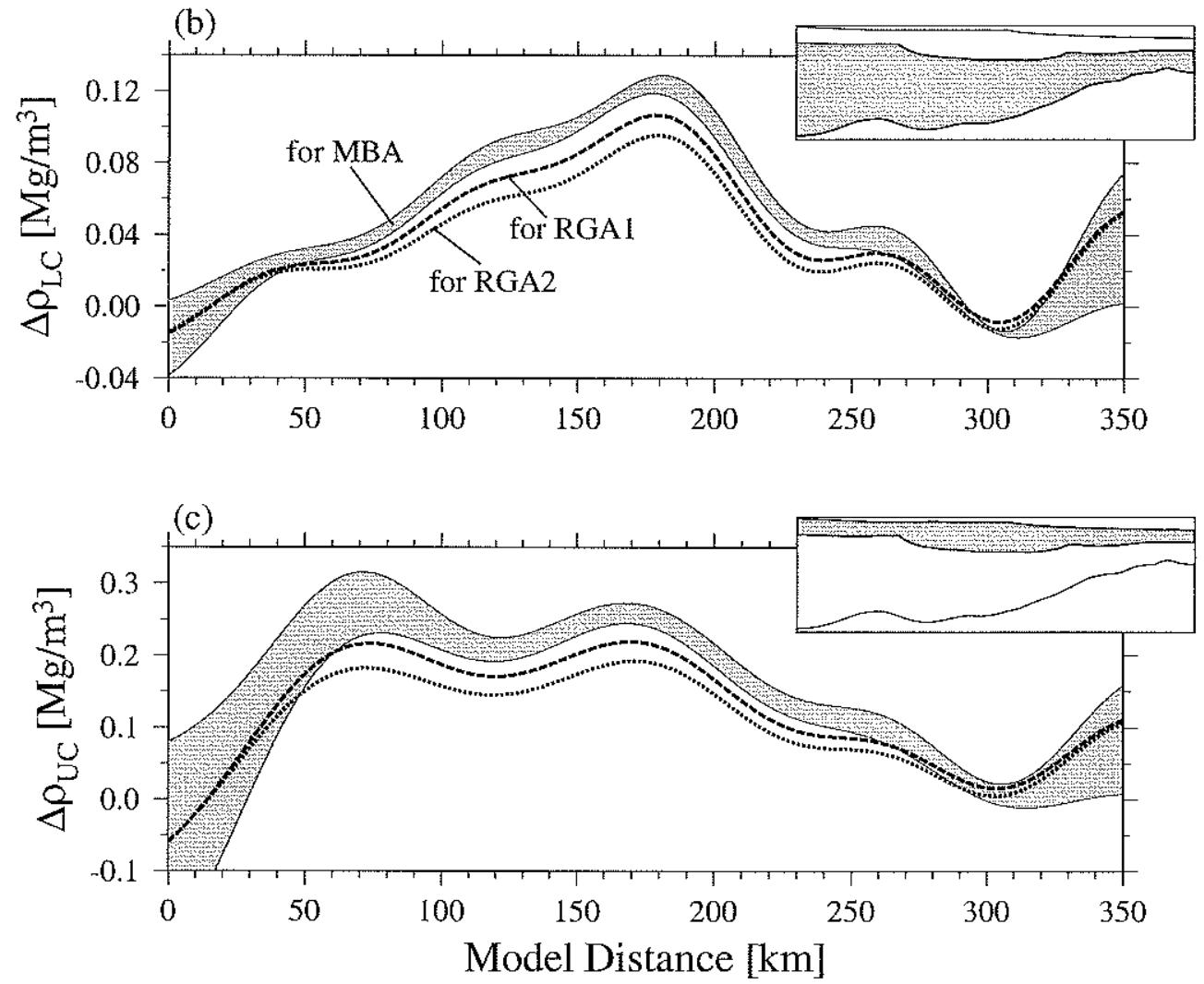

Figure 2-9. Results of inversion for crustal density anomalies. Solid lines show the density anomalies for the gravity anomaly input of MBA, dashed for RGA1, and dotted for RGA2. For clarity, one standard deviation is shown only for MBA results. (a) Whole-crustal density anomalies, (b) lower-crustal density anomalies, and (c) uppercrustal density anomalies. Note that we slightly modified the upper/lower crust boundary from that shown in Figure 2-1c to avoid zero thickness in the upper crust for the onshore region. A low-pass filter used in this inversion is cosine-tapered with cut wavelength of $50 \mathrm{~km}$ and pass wavelength of $70 \mathrm{~km}$. All crustal density anomalies

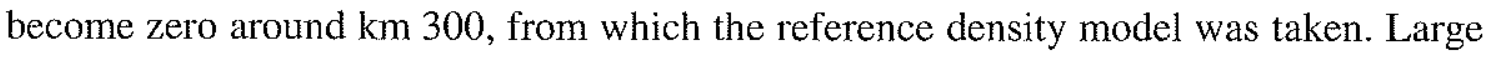
uncertainty toward the seaward end originates in the uncertainty of the original velocity model, which is amplified by inverting gravity anomalies for a thin crustal layer. 
(a)

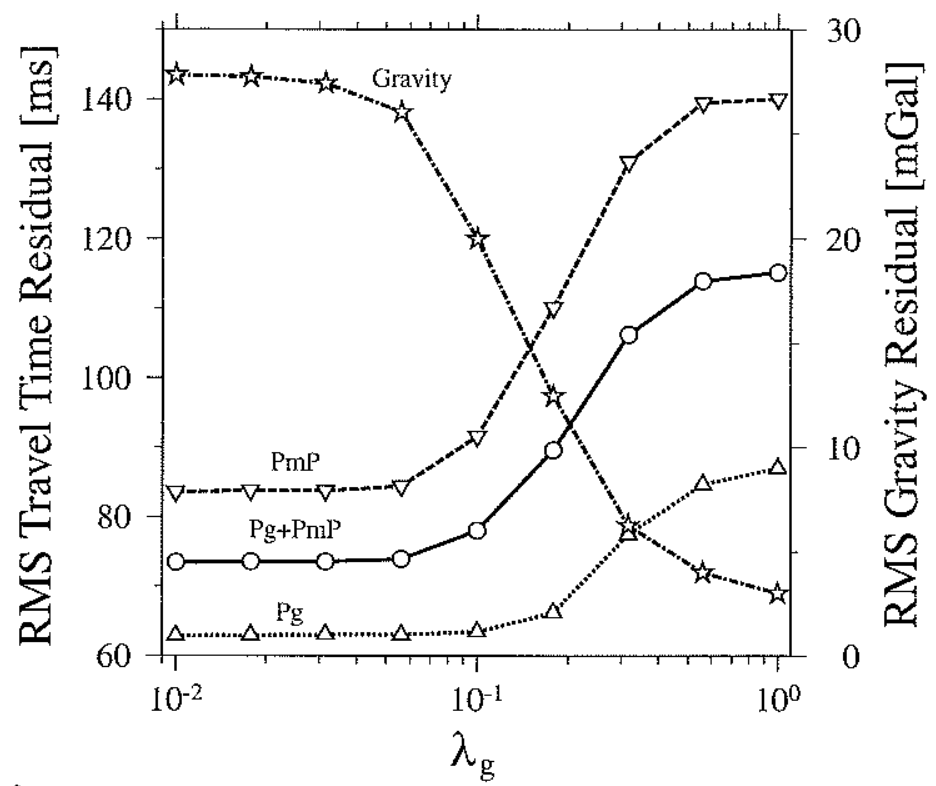

(b) $\lambda_{\mathrm{g}}=0.01$

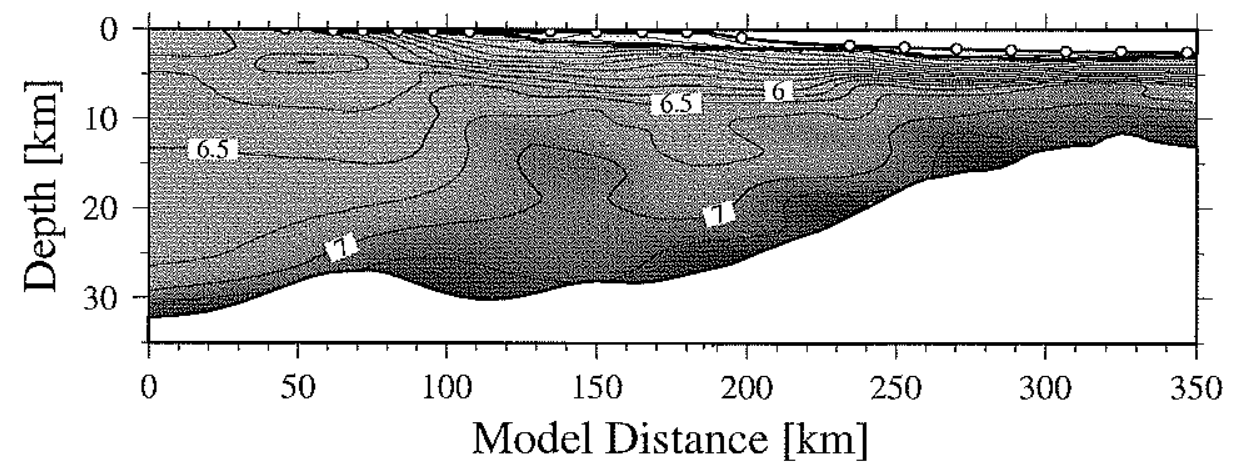

(c) $\lambda_{\mathrm{g}}=1.0$

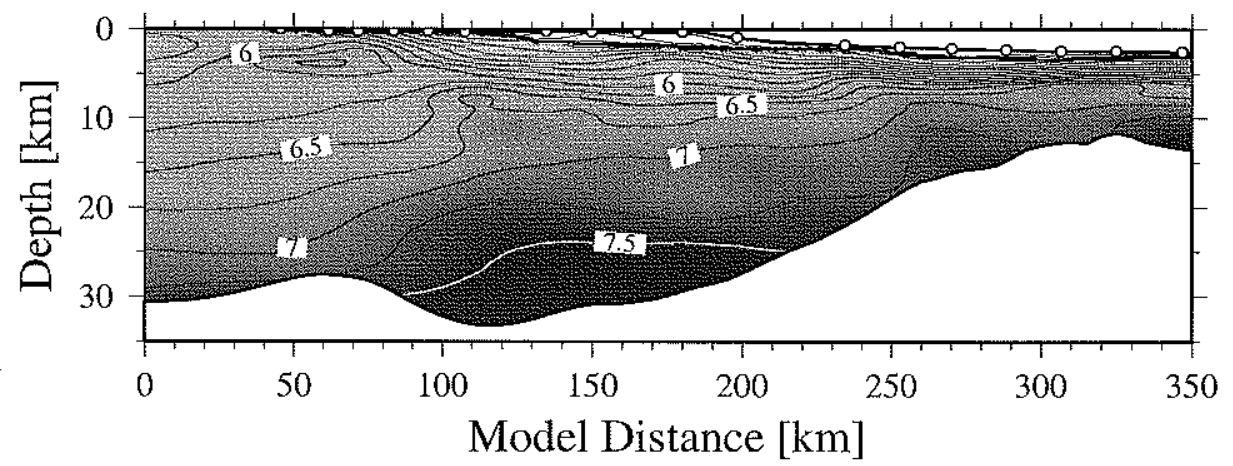

Figure 2-10. Result of joint inversion of seismic travel times and gravity anomalies. (a) RMS travel time residuals for $P g$ (triangle), $P m P$ (inverse triangle), and both (circle), and RMS gravity residual (star), are plotted as a function of the gravity weighting parameter, $\lambda_{g}$. (b) Final velocity model obtained with $\lambda_{g}$ of 0.01 . (c) Final velocity model obtained with $\lambda_{g}$ of 1.0 . Open circles denote the location of onshore/offshore seismometers deployed during the SIGMA experiment. 
(a)

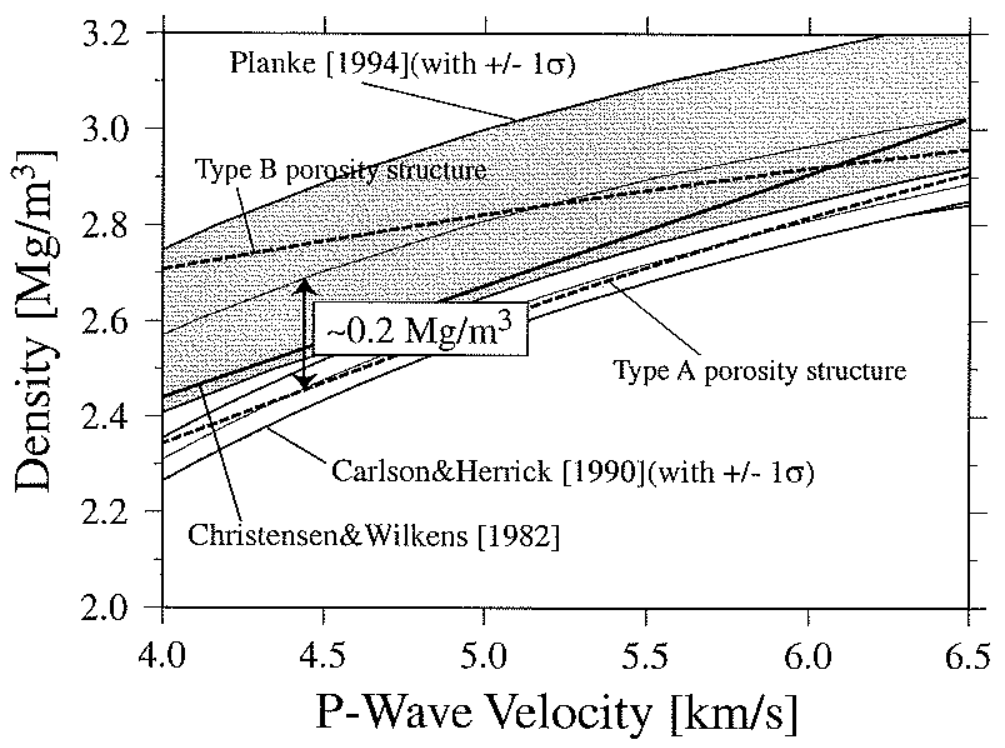

(b)

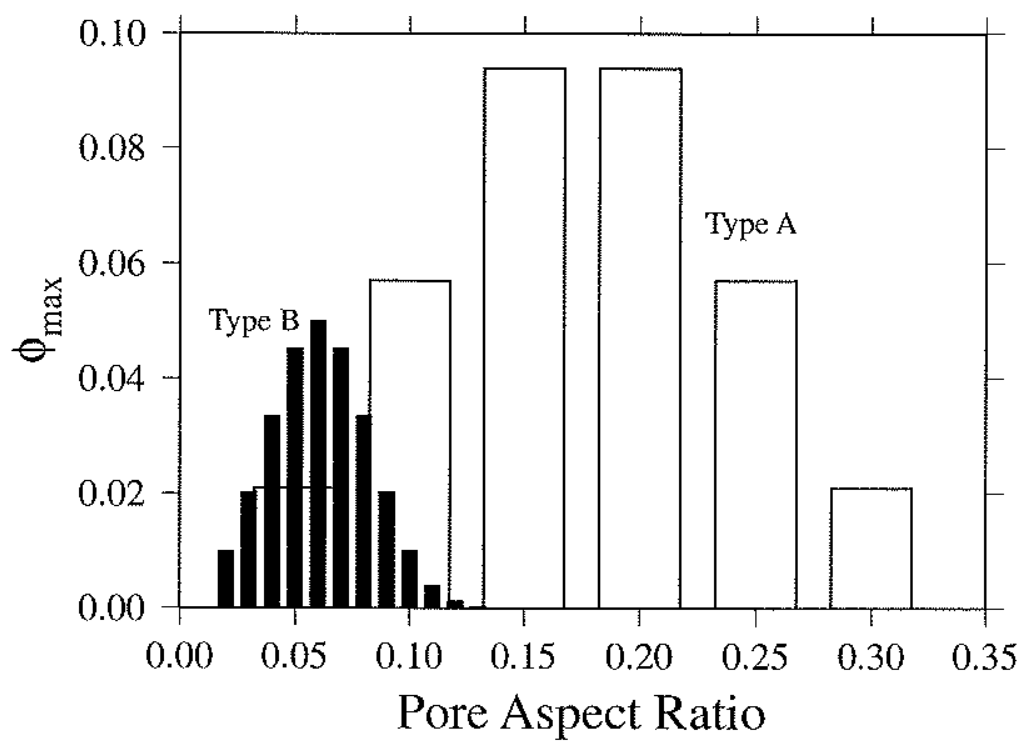

Figure 2-11. (a) Relationship of $P$-wave velocity with density of mafic rocks established for upper oceanic crust [Carlson and Herrick, 1990], Iceland plateau basalt sequences [Christensen and Wilkens, 1982], and seaward dipping reflector sequences at the Vøring margin [Planke, 1994]. Also shown as dashed are results of forward modeling of velocity-density systematics using the pore aspect ratio spectrum shown in (b). We first calculated effective elastic moduli of dry porous rock with the formula of Kuster and Toksöz [1974], and then applied the Gassmann low-frequency relations to obtain effective moduli for fluid saturated porous rock [e.g., Mavko et al., 1998]. Elastic moduli for basalt and seawater are taken from Wilkens et al. [1991]. 

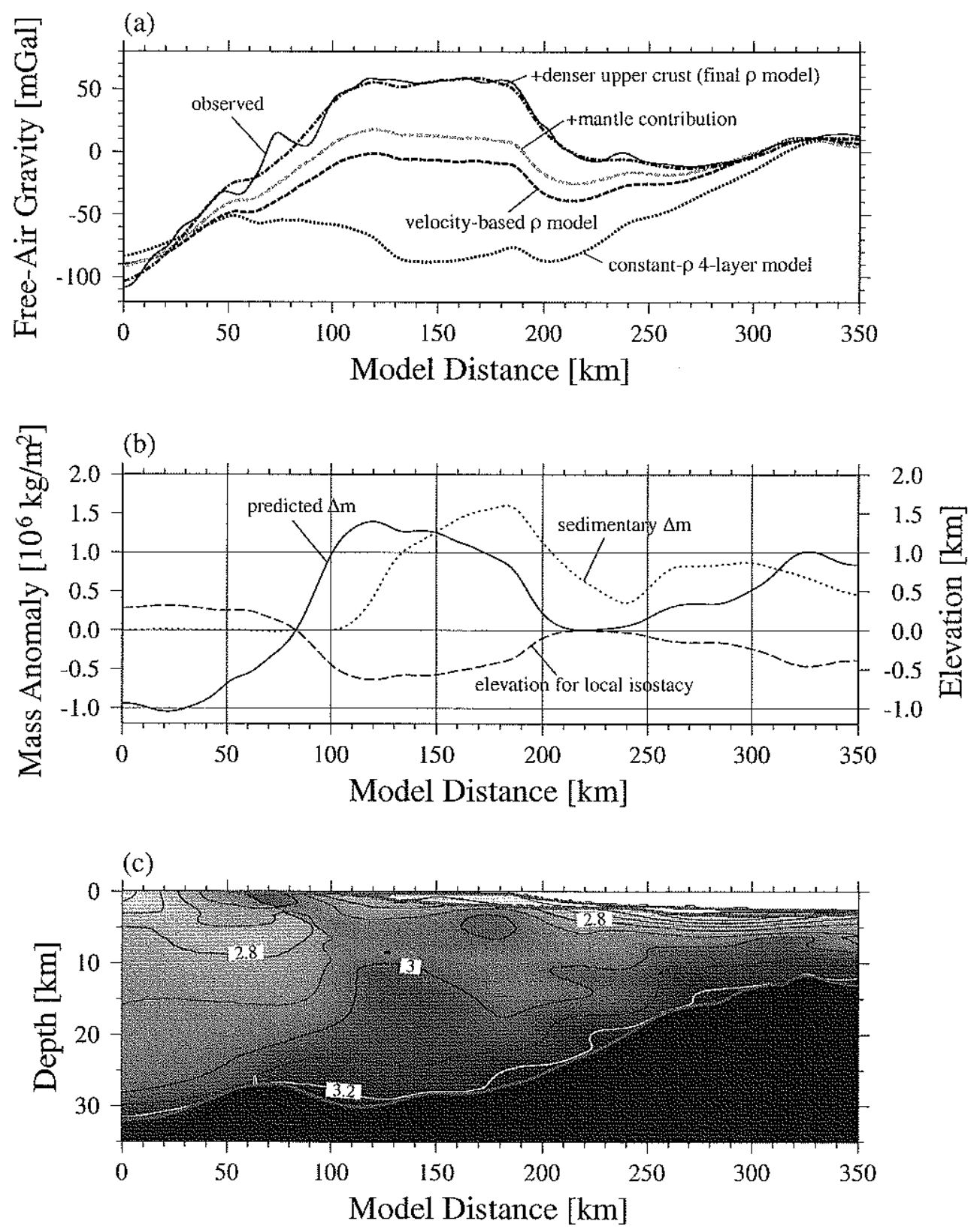

Figure 2-12. (a) Free-air gravity anomaly corresponding to the final density model shown in (c) (dot-dashed) is plotted with observed anomaly (solid) and other preliminary predicted anomalies. (b) Mass anomaly for a 36-km-thick vertical column (from $1 \mathrm{~km}$ above the sea surface to $35 \mathrm{~km}$ deep) along the transect (solid) is plotted based on the final density model shown in (c). Hypothetical mass anomaly based on observed sedimentary load, $\left(\rho_{\text {sediment }}-\rho_{\text {water }}\right) H_{\text {sediment }}$, is shown as dotted. Also shown is elevation required to attain local isostatic equilibrium (dashed). (c) Final density model is constructed by adding upper-crustal density anomaly corresponding to RGAl to the density model shown in Figure 2-4(b). Cosine window is used to taper down the density anomaly toward the bottom boundary of the upper crust, and the density anomaly is then scaled up to conserve the total density anomaly. 


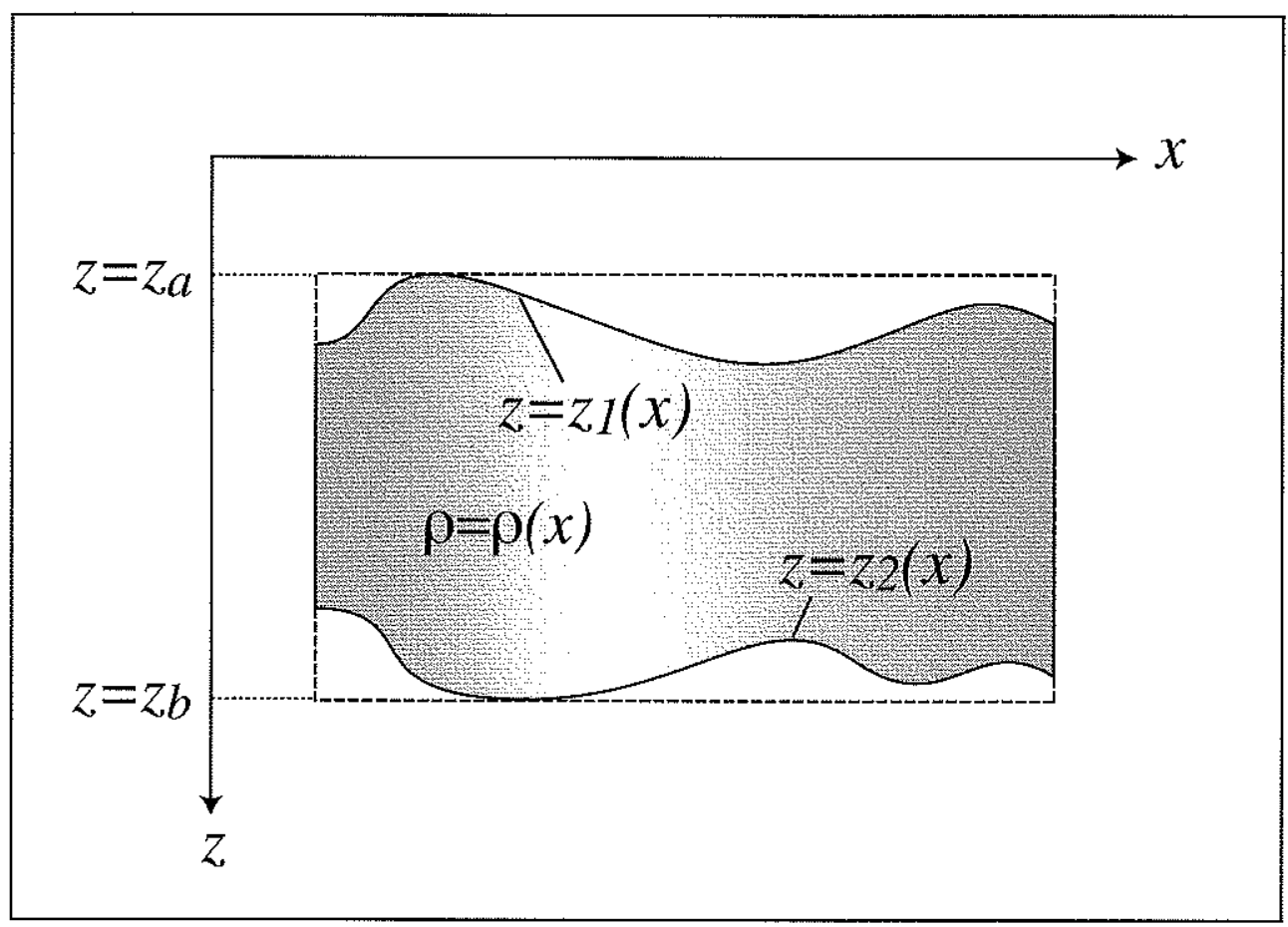

Figure 2.A1. Schematic diagram showing the 2-D model geometry for the inversion of gravity anomalies in the presence of upper and bottom topography. 
(a)
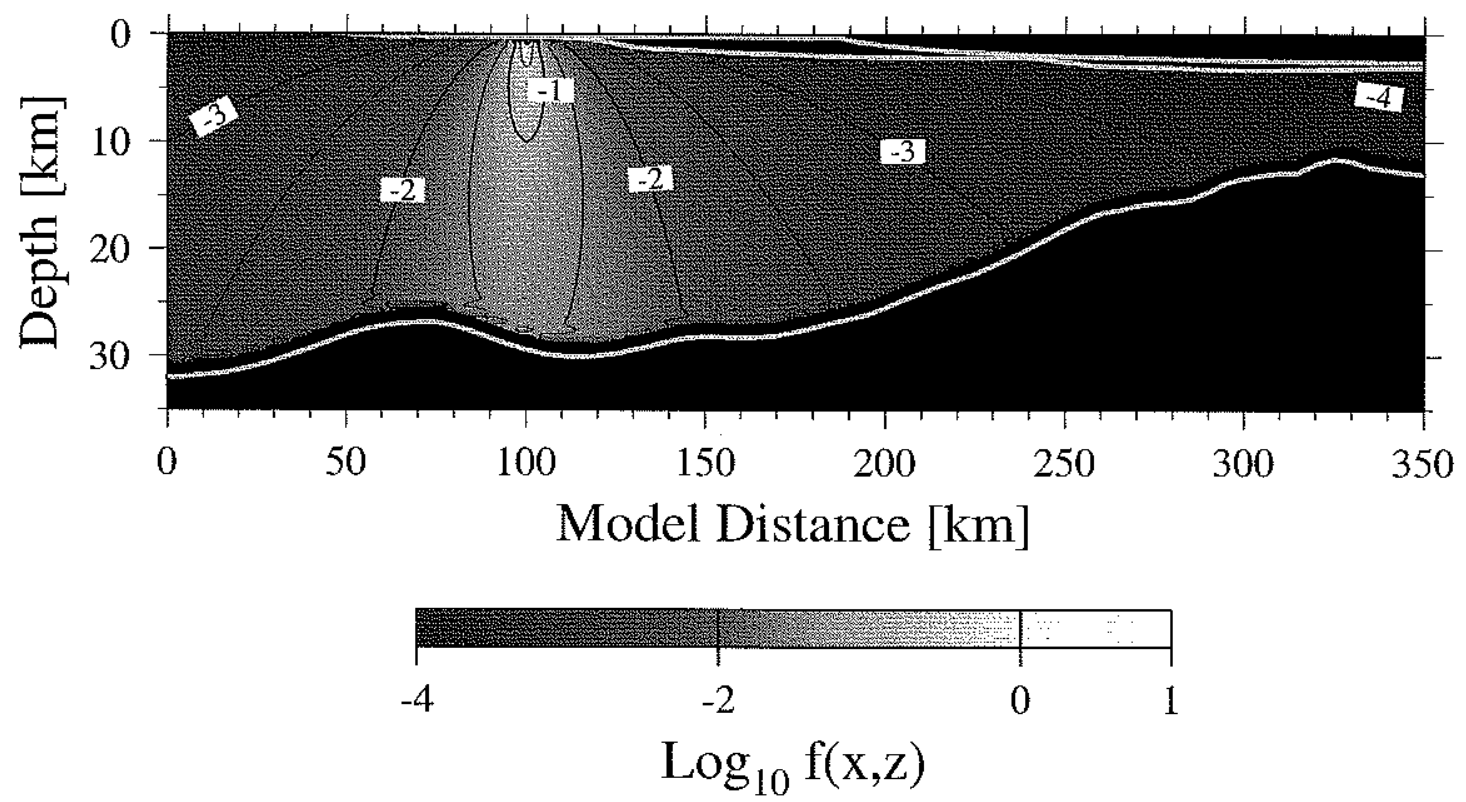

(b)
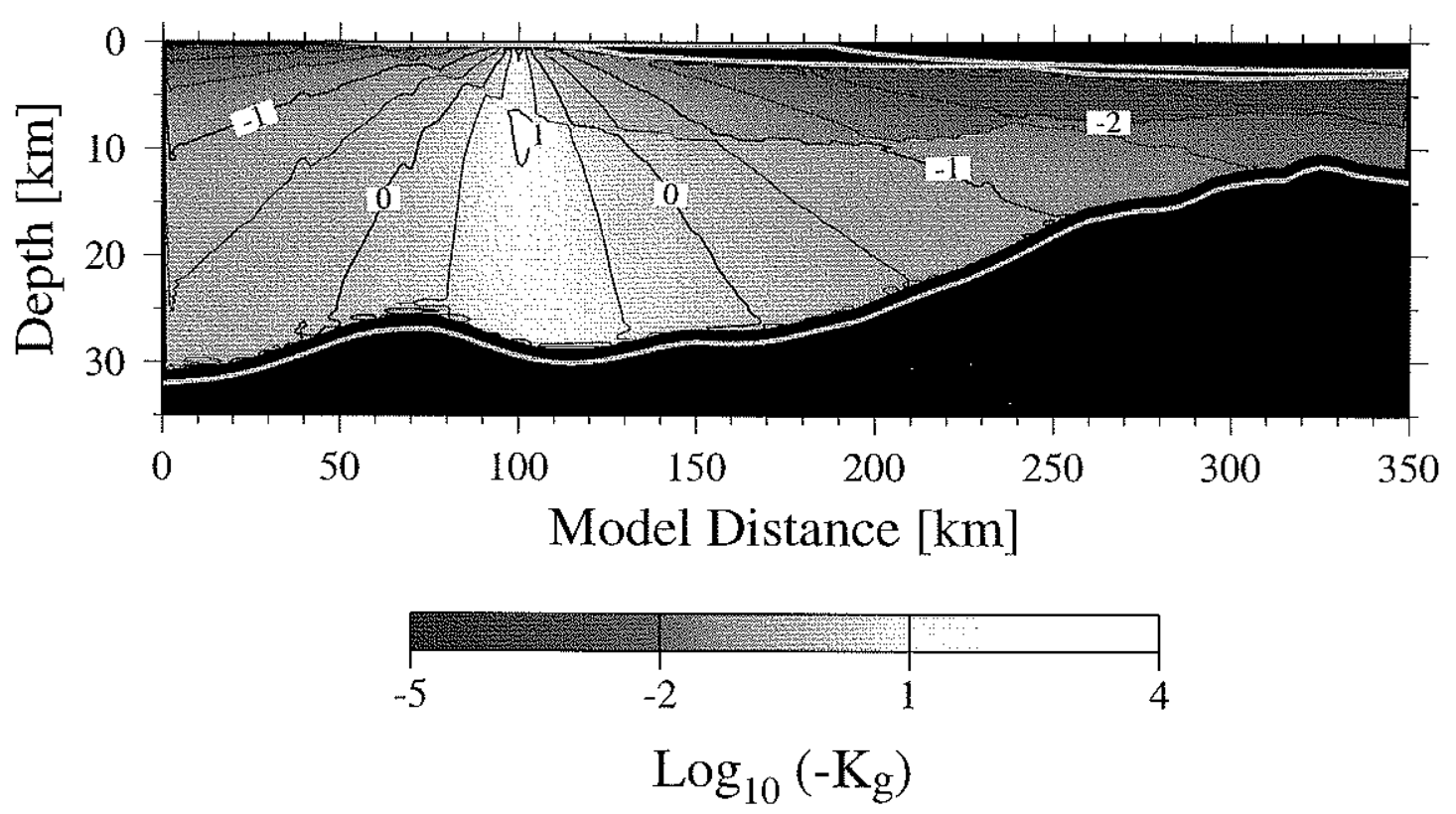

Figure 2.B1. An example of gravity kernel for an input datum located at $x=100 \mathrm{~km}$ and $z=0 \mathrm{~km}$. (a) Radial part of the gravity kernel, i.e., $f(x, z)=z /\left(x^{2}+z^{2}\right)$. (b) Total gravity kernel including nodal volume distribution and the slowness derivatives of density. The sensitivity in the sedimentary layer is set to zero for both cases. 


\title{
Chapter 3
}

\section{Mantle melting and crustal accretion processes during the formation of the Southeast Greenland margin}

\begin{abstract}
We present a new robust framework to understand the process of mantle melting based on the velocity structure of resultant igneous crust. Our approach focuses on a lower-crustal section, which is expected to be least affected by porosity and seawater alteration, especially for thick igneous crust of large igneous provinces. Though bulk crustal velocity cannot be uniquely estimated from observed lower-crustal velocity because of ambiguity in crustal emplacement processes, it is possible to place a bound on the expected range of bulk crustal velocity for a given lower-crustal velocity, by modeling fractional crystallization processes at a range of crustal pressures. A quantitative relation between bulk crustal velocity and mantle melting parameters is established based on an extensive database of mantle melting experiments, and a simple melting model is constructed to illustrate the effects of mantle potential temperature, active mantle upwelling, and a preexisting lithospheric lid on predicted crustal thickness and velocity. This new interpretation is applied to a seismic transect across the Southeast Greenland margin to resolve the mantle dynamics during the opening of the North Atlantic. We assume constant mantle composition throughout continental rifting and subsequent sea-floor spreading. Our result indicates that thick $(\sim 30 \mathrm{~km})$ igneous crust formed at the continent-ocean transition zone resulted from vigorous active upwelling of mantle with only somewhat elevated potential temperature $\left(\sim 1300^{\circ} \mathrm{C}\right)$, arguing against plume head hypotheses proposed for the North Atlantic igneous province.
\end{abstract}

\subsection{Introduction}

Large igneous provinces (LIPs) are characterized as products of unusually extensive magmatism, with respect to the 'normal' state of terrestrial magmatism such as observed at the majority of mid-ocean ridges [e.g., Coffin and Eldholm, 1994]. Though LIPs are the largest expression of magmatism on this planet, their origins are still poorly understood; mantle plumes have been the most commonly accepted explanation for focused supply of mantle-derived melt [e.g., Richards et al., 1989; White and McKenzie, 1989; Hill et al., 1992], whereas small-scale convection as a result of lithospheric response to surface tectonics has also been advocated as a possible formation mechanism of LIPs [e.g., Mutter et al., 1988; Anderson, 1994; Boutilier and Keen, 1999]. Given our limited understanding of realistic mantle dynamics, especially when melting is involved [e.g., Cordery and 
Phipps Morgan, 1993; Tackley and Stevenson, 1993; Spiegelman, 1996], it is difficult at present to distinguish different hypotheses solely by commonly-employed arguments based on geological coincidence (e.g., plume head impacts for LIPs and plume tails for succeeding hotspot chains). Obtaining quantitative constraints on basic parameters, such as the potential temperature of mantle and its upwelling rate, is an essential step in investigating the formation of LIPs as well as improving our understanding of multi-scale mantle dynamics.

The temperature and upwelling rate of the mantle are reflected in the composition and volume of melt generated by adiabatic decompression. Since seismic velocity is sensitive to rock composition, the velocity structure of resultant igneous crust can provide, in principle, robust constraints on the composition of mantle-derived melt, from which we can estimate past mantle dynamics. In particular, for LIPs formed near divergent plate boundaries where lithospheric controls on melt migration are supposed to be minimal, the whole-crustal velocity structure is a promising proxy for parental melt composition [Kelemen and Holbrook, 1995]. There have been, however, two critical difficulties in this geophysical inference. The first one is regarding the reliability of crustal velocity models. Because upper crust is usually highly porous and seismic velocity is more sensitive to porosity structure than to composition, only the lower crust remains useful for petrological interpretation. The seismic structure of lower crust is, however, more difficult to confidently determine; Moho reflection travel times are often the sole seismic constraint so that accurate determination of lower-crustal velocity tends to be hindered by a tradeoff with Moho depth. In addition, uncertainty analysis for velocity models has often been incomplete, with respect to the nonlinear nature of tomographic inversions, so it is hard to assess the validity of resulting petrological interpretations.

The second difficulty lies in the estimation of parental mantle melt from crustal seismic velocity. Kelemen and Holbrook [1995] conducted an early, quantitative attempt to estimate the volume and composition of mantle melt from crustal velocity structure. They replaced upper crustal velocity uniformly with $6.8 \mathrm{~km} / \mathrm{s}$ to minimize the effects of porosity and alteration, and calculated the whole-crustal average velocity to estimate bulk crustal 
composition. It is difficult, however, to test the assumption of the uniform upper-crustal velocity, and errors in the averaged velocity introduced by this assumption are thus uncertain. The relationship between upper- and lower-crustal compositions and parental melt composition can be complicated because of possible fractionation and assimilation processes during crustal accretion. Figure 3-1 shows some proposed crustal accretion models for normal oceanic crust. Modification processes acting on mantle-derived melt ascending through a crustal section are controlled by the thermal structure of crust, which is balanced with heating by magma injection and its solidification and cooling by hydrothermal circulation and conductive heat loss [e.g., Sleep, 1991; Phipps Morgan and Chen, 1993]. Whereas slow spreading ridges have low-temperature axial crust, which may often lead to the in situ crystallization of incoming melt batches (Figure 3-1c), fast spreading ridges tend to have steady-state axial magma chambers, in which crystal fractionation can take place (Figure 3-1a and b). Although the importance of crustal thermal structure in modifying melt composition is generally accepted [e.g., Sinton and Detrick, 1992], how oceanic crust is actually constructed is still highly controversial [Boudier et al., 1996; Kelemen et al., 1997b; Chen, 1998; Kelemen and Aharonov, 1998; Korenaga and Kelemen, 1998]. Our understanding is even more limited for the formation of igneous crust during continental breakups, for which the preexisting geotherm is probably influential and the transient evolution of crustal thermal structure may be significant. With this uncertainty in, for example, the degree of lower-crustal crystal fractionation, therefore, it is impossible to uniquely determine bulk composition from lower-crustal velocity structure. Instead, we must seek to place reasonable bounds on the bulk composition.

To resolve the first difficulty, joint refraction and reflection tomography with a comprehensive uncertainty analysis has been formulated in Chapter 1, and the purpose of this paper is to construct a robust interpretational framework for the seismic structure of igneous crust. We will focus on the relationship between averaged lower-crustal velocity and parental melt composition. Because of the fundamental ambiguity in this relationship, our strategy is to derive plausible bounds on the petrological interpretation. Fractionation 
processes depend mostly on melt composition, crystallization pressure, and the mode of fractionation such as fractional and equilibrium crystallization, and any specific assumption regarding these factors must be avoided. Our approach is based on crystallization modeling at a range of crustal pressures using extensive experimental data sets on mantle melt composition at various temperatures and pressures. After establishing velocity-composition systematics, we then consider a mantle melting process incorporating the effects of active mantle upwelling and preexisting lithospheric lid, to explore possible variations in predicted crustal thickness and velocity. Finally, we will apply this new framework to interpret a crustal velocity model of the Southeast Greenland margin (Figure $3-2$ ), in terms of mantle melting and crustal accretion processes associated with the opening of the North Atlantic.

\subsection{Mineralogies, Seismic Velocities and Densities of Igneous Lower Crust}

\subsubsection{Fractional Crystallization Processes and Bulk Crustal Velocity}

The degree of fractionation expected in igneous lower crust ranges from no fractionation (i.e., in situ crystallization of parental melt) to perfect fractionation (i.e., cumulate formation by fractional crystallization). Because igneous cumulates are always more mafic than their parental liquids, and because more mafic rocks have higher seismic velocity, cumulate lower crust formed by perfect fractional crystallization should have the highest velocity for a given parental melt composition, thus providing an upper limit on the deviation of lower-crustal velocity from bulk crustal velocity (throughout this paper, we use the term 'bulk crustal velocity' to denote a hypothetical crustal velocity corresponding to the case of $100 \%$ equilibrium crystallization of a primary, mantle-derived melt.). The uncertainty in the estimation of parental melt composition from lower-crustal velocity thus depends on the magnitude of this deviation, and we will try to constrain it by modeling fractional crystallization processes. We use the method of Weaver and Langmuir [1990] as extended by Langmuir et al. [1992] for high-pressure crystallization. Though actual crustal fractionation processes are probably more complex, including periodically replenished 
magma chambers [O'Hara, 1977], combined wall-rock assimilation and fractional crystallization [DePaolo, 1981], and in situ fractionation [Langmuir, 1989], the case of pure fractional crystallization provides an upper limit on lower-crustal seismic velocity.

Major crystallizing minerals from low $-\mathrm{H}_{2} \mathrm{O}$, basaltic liquids at $\leq 1 \mathrm{GPa}$ are olivine, plagioclase, and clinopyroxene, and crystallizing assemblages range from dunite to gabbro. The elastic properties of minerals depend on temperature, pressure, and composition, and we use the compilation of Sobolev and Babeyko [1994] to calculate effective isotropic moduli and density at a given temperature and pressure for each mineral with a certain composition, and then calculate the Hashin-Shtrikman bounds [e.g., Hashin and Shtrikman, 1963; Watt et al., 1976; Berryman, 1995] for the compressional-wave velocity of a crystallizing assemblage. For mafic mineral assemblages, the bounds are typically tighter than $0.03 \mathrm{~km} / \mathrm{s}$, so that the average of the upper and lower bounds is sufficient for our purposes. To calculate hypothetical solid velocities for primary and residual liquids, the weight proportions of minerals and their compositions are calculated based on the CIPW norm, the weight proportions are converted to the volume proportions using mineral densities, and effective compressional velocities of their assemblages are calculated in the same manner for crystallizing assemblages. Hereafter we will refer to this velocity based on the CIPW norm as the norm-based velocity. Although in principle the use of the CIPW norm may not be as accurate as thermodynamic calculations for estimating equilibrium mineral assemblages [e.g., Sobolev and Babeyko, 1994], norm-based velocity for bulk crustal composition can serve as a useful, easily reproduced reference to evaluate the influence of fractionation on lower-crustal velocity.

An example of crystallization modeling is shown in Figure 3-3. A starting liquid composition is an estimate of primary melt for normal mid-ocean ridges as calculated by Kinzler [1997] (polybaric near-fractional melt aggregated from a triangular shaped melting regime, with mean melt fraction of $9 \%$ and mean pressure of melting of $1.5 \mathrm{GPa}$ ). Three different crystallization paths are shown in this example: (1) fractional crystallization at $100 \mathrm{MPa}$, (2) polybaric fractional crystallization from $800 \mathrm{MPa}$ to $100 \mathrm{MPa}$, and (3) equilibrium crystallization at $100 \mathrm{MPa}$. Compressional-wave velocities of crystallizing 
phases as well as of residual phases are calculated as a function of solid fraction (Figure 3-3a). The velocities are calculated at $100 \mathrm{MPa}$ and $100^{\circ} \mathrm{C}$, i.e., a typical mid-crustal state of normal oceanic crust. For fractionally-crystallizing phases, velocities are shown for both incremental and cumulative assemblages, the latter being of primary interest because of its relevance to average lower-crustal velocity. Corresponding densities are also shown in Figure 3-3b, with liquid densities of residual phases calculated by the method of Bottinga and Weill [1970]. Though the residual liquids become progressively denser with crystal fractionation, they are always more buoyant than the solid phases, so that the lower crust does not act as a density filter [e.g., Sparks et al., 1980; Stolper and Walker, 1980] in this example. Higher crystallization pressure increases the stability of clinopyroxene [e.g., Bender et al., 1978; Presnall et al., 1978; Grove et al., 1992] (Figure 3-3d), leading to a slight reduction in the velocity of the resultant assemblage (Figure 3-3a). Olivine is the sole crystallizing phase at early fractionation stages (Figure 3-3c-e), and mostly because of this, the deviation of the maximum lower-crustal velocity from the bulk crustal velocity $(-7.17 \mathrm{~km} / \mathrm{s}$ in this case) is too large to be useful when lower crust occupies only a small fraction of the whole crust. The deviation sharply decreases, however, at $\sim 20 \%$ solidification. In fact, the fraction of the crust composed of plutonic rocks (i.e., lower crust) is usually greater than 50\% in igneous crust [e.g., Mutter and Mutter, 1993].

\subsubsection{Primary Mantle Melts and Melting Systematics}

To establish a general relation between normative bulk crustal velocity and lower-crustal velocity of igneous crust using the bounding approach outlined above, a reasonably wide range of mantle melt compositions needs to be considered. We therefore compiled recent, high-quality melting experiments of mantle peridotites [Kinzler and Grove, 1992; Hirose and Kushiro, 1993; Kinzler and Grove, 1993; Baker and Stolper, 1994; Kinzler, 1997; Walter, 1998], and a norm-based velocity is calculated for each melt composition. The norm-based velocities of the compiled melt compositions at a pressure of $600 \mathrm{MPa}$ and a temperature of $400^{\circ} \mathrm{C}$ (we will refer to this as the reference state) vary from $6.8 \mathrm{~km} / \mathrm{s}$ to $7.8 \mathrm{~km} / \mathrm{s}$ (Figure 3-4), the range of which is sufficiently wide to cover expected variations in bulk crustal velocity. Since we are interested in a connection between mantle melting 
process and resultant igneous crustal structure, following the approach of Kelemen and Holbrook [1995] (hereafter referred to as KH95), the compressional-wave velocities for mantle melts are related to their pressures and degrees of melting using multiple linear regression as (Figure 3-4),

$$
V_{p}=6.87+0.09 P+1.38 F-0.16 P F,
$$

where $P$ is the pressure of melting in GPa, and $F$ is melt fraction. All mantle melts are included in this regression except those of Hirose and Kushiro [1993]. The calculation of melt fraction by Hirose and Kushiro [1993] is based on the $\mathrm{Na}_{2} \mathrm{O}$ concentration in melt, which could lead to overestimation [e.g., Kogiso et al., 1998]. In addition, half of their melting data is from the melting of the pyroxenite HK66, which has a substantially different melting function. All other data are for melting of mantle peridotites, with minor differences in fertility. One standard deviation of this regression is about $0.06 \mathrm{~km} / \mathrm{s}$. This uncertainty is probably due to a nonlinear, second-order relation between norm-based velocity and the mantle melting parameters, and to variation in source composition (the spread of whole rock $\mathrm{Mg}$ numbers of mantle peridotites in the cited studies is $1.3 \%$ ) and the difference of melting styles (i.e., batch and fractional).

The majority of melt data used here are from batch melting experiments, and others are calculated, aggregated melts from polybaric fractional melting, modeled by Kinzler and Grove [1992; 1993] and Kinzler [1997]. For the latter, we used the mean pressure and degree of melting for the aggregate melts in the linear regression, and Figure 3-4 suggests that, at least in terms of normative bulk crustal velocity, the difference in melting styles is only of minor importance. Because the importance of fractional melting in the generation of mid-ocean ridge basalts (MORB) is clear from geochemical evidence [e.g., Klein and Langmuir, 1987; Johnson et al., 1990; Sobolev and Shimizu, 1993] as well as physical arguments [e.g., McKenzie, 1984; Daines and Richter, 1988], this weak sensitivity of bulk velocity to melting styles is particularly encouraging for our approach, which relies heavily on batch melting experiments. The actual melting style is probably somewhere between pure batch and pure fractional modes, owing to likely melt-rock reaction associated with melt migration [e.g., Kelemen et al., 1995; Lundstrom et al., 1995; Kelemen et al., 1997a], 
further reducing the possible importance of fractional melting in our interpretation of crustal velocity.

This empirical relation between normative bulk crustal velocity and the mantle melting parameters such as $P$ and $F$ will be used later to calculate the crustal manifestation of various mantle melting processes. A similar form of empirical relation was derived by KH95 as,

$$
V_{p}(\mathrm{KH} 95)=6.712+0.16 P+0.661 F,
$$

at the reference state of $600 \mathrm{MPa}$ and $400^{\circ} \mathrm{C}$. These two equations indicate different relations between normative bulk crustal velocity and the pressure and degree of melting (Figure 3-5a), and the possible causes of this discrepancy need to be clarified. First of all, our regression is based on an expanded data set of mantle melt compositions, including higher-pressure data of Kinzler [1997] and Walter [1998]. A more important difference, however, lies in the way of assigning velocity to melt composition. Compiling the laboratory measurements of the velocities and compositions of igneous and metamorphic rocks, $K H 95$ derived the following empirical relation,

$$
V_{p}(\mathrm{KH} 95)=7.854-0.024 \mathrm{wt} \% \mathrm{SiO}_{2}+0.029 \mathrm{wt} \% \mathrm{MgO},
$$

for the reference state. One standard deviation of this regression is $0.19 \mathrm{~km} / \mathrm{s}$, and adding additional oxides did not significantly improve the fit. Though the regression for equation 3.2 itself has a standard deviation of only $0.02 \mathrm{~km} / \mathrm{s}$, the large error of equation 3.3 essentially determines the prediction error of equation 3.2. There are several possibilities to explain the large standard deviation of equation 3.3; (1) laboratory measurements often suffer from residual porosity, (2) alteration introduces secondary mineral phases that are not relevant to pristine igneous crust, and (3) chemical analyses and velocity measurements are often conducted on different samples from the same rock, so that local heterogeneity in mineralogy and texture may obscure the relation between velocity and composition [e.g., Jackson et al., 1990]. Figure 3-5b compares the velocities predicted by the equation for mantle melts with their norm-based velocities. Compared to our method, equation 3.3 systematically underpredicts velocity, suggesting that the 
influence of residual porosity and alteration may be significant in the approach of $K H 95$ based on laboratory data.

Compared to the KH95 approach, our new equation 3.1 is more sensitive to melt fraction and less sensitive to the pressure of melting (Figure 3-5a). In particular, for a melting condition that is believed to be appropriate for the generation of normal MORB (i.e., mean pressure of melting of $1 \mathrm{GPa}$ and mean melt fraction of 0.1 ), our equation predicts bulk crustal velocity of $7.1 \mathrm{~km} / \mathrm{s}$, whereas the equation of $K H 95$ predicts $6.95 \mathrm{~km} / \mathrm{s}$. Though the latter value is more close to the global average of oceanic lowercrustal velocity [e.g., White et al., 1992], this coincidence is merely fortuitous, because the majority of oceanic lower crust is supposed to be affected by hydrothermal circulation so that this sort of direct comparison cannot be applied. The bulk crustal velocity of igneous crust formed by normal mantle melting does not have to be equal to the seismic velocity of normal oceanic crust. This point has not been always appreciated in previous studies, but it can make a substantial difference in the interpretation of thick igneous crust. We will discuss this issue further in a later section.

\subsubsection{Theoretical Upper- and Lower-Crustal Velocities}

We modeled perfect fractional crystallization for all mantle melt data, at pressures of 100 , 400 , and $800 \mathrm{MPa}$. We denote the velocity of the cumulative fractionated assemblage as lower-crustal velocity, and its deviation from normative bulk crustal velocity is summarized in Figure 3-6. The deviation provides a lower bound on the possible range of bulk crustal velocity, for a given average lower-crustal velocity with the corresponding proportion of lower crust. For a lower-crustal fraction of 0.5 , for example, normative bulk crustal velocity can be lower than the observed lower-crustal velocity by about $0.20 \mathrm{~km} / \mathrm{s}$ at most. Though this bound is highly variable for low solid fractions, suggesting the strong dependence of a crystallization path on starting melt composition, the bound and its scatter both decrease gradually as a solid fraction increases (Figure 3-6), because the difference will eventually converge to null at complete solidification. At solid fractions of $0.7-0.8$, the deviation is about $0.15 \mathrm{~km} / \mathrm{s}$ with a standard error of $0.02 \mathrm{~km} / \mathrm{s}$ for a typical range of crustal velocities, nearly independent of crystallization pressures (Figure 3-6a and b). 
Similarly, the velocity for a residual liquid composition is denoted as upper-crustal velocity, and its difference from bulk crustal velocity is also shown in Figure 3-6. Though this velocity could never be observed because of high porosity and variable degrees of alteration in actual upper crust, we note that there are a wide range of possible uppercrustal velocities $(6.3-7.3 \mathrm{~km} / \mathrm{s})$. The deviation shows greater scatter as a solid fraction increases, implying that it is impractical to try to estimate hypothetical velocity for a residual liquid phase with reasonable accuracy. This further supports our strategy based on lower-crustal velocity as the most practical approach to extract compositional information from crustal velocity structure.

\subsubsection{Velocity-Density Systematics}

One of the benefits of the crystallization modeling described above is that we can also construct theoretical velocity-density systematics for pristine igneous rocks, with temperature and pressure derivatives. The compressional-wave velocities and densities of fractionated assemblages, at a pressure of $1 \mathrm{GPa}$ and a temperature of $25^{\circ} \mathrm{C}$, are plotted in Figure 3-7, together with those for parental melt compositions. The reference state used here is chosen so as to facilitate comparisons with published empirical relations based on laboratory data.

Theoretical temperature and pressure derivatives for density and velocity are shown in Figure 3-8. The nonlinear effect of mixing is clearly seen, but the overall behavior can be well approximated with linear relationships, which are useful in correcting observed velocities at various temperatures and pressures to the reference state. The calculated derivatives for velocity are qualitatively similar to those based on laboratory measurements of mafic and ultramafic rocks [e.g., Christensen, 1979; Kern and Tubia, 1993], but this theoretical confirmation, based on single crystal data and mixture theories of rock properties, is important because the laboratory determination of pressure and temperature derivatives is prone to be affected by residual porosity and hysteresis, both of which are insignificant at geologic time scales [Christensen, 1974; Christensen, 1979]. In addition, the virtue of our theoretical velocity-density data is that we can comprehensively explore possible regions in the $V$ - $\rho$ space for pristine igneous rocks originating from mantle 
melting. The mapping of this permissible space is particularly useful when velocity structure is used to infer possible density structure to study gravity anomalies (e.g., Chapter 2).

\subsection{Bulk Crustal Velocity of Normal Oceanic Crust}

To interpret the velocity structure of 'abnormal' igneous crust constituting LIPs, a proper understanding of the seismic velocity of normal oceanic crust is essential. The lowercrustal velocity of normal oceanic crust with a thickness of 6-7 $\mathrm{km}$ has been known to be consistently around $6.9 \mathrm{~km} / \mathrm{s}$ worldwide [e.g., Raitt, 1963; Shor et al., 1970; White et al., 1992] (Figure 3-9), and crustal velocities exceeding this value have been commonly interpreted as an indication of anomalous crustal composition. White and McKenzie [1989] are the first to attempt to quantitatively model the velocity of igneous crust in connection to mantle melting, and, based on the melting model of McKenzie and Bickle [1988], they predicted bulk crustal velocities of 6.9 to $7.2 \mathrm{~km} / \mathrm{s}$ as the possible outcome of the melting of mantle with potential temperatures of $1280-1580^{\circ} \mathrm{C}$. In the framework of McKenzie and Bickle [1988], the passive upwelling of mantle with a potential temperature of $1280^{\circ} \mathrm{C}$ results in the formation of normal oceanic crust, and velocities higher than $6.9 \mathrm{~km} / \mathrm{s}$ may thus appear to indicate unusually high potential temperatures. The assumption that the melting of normal mantle should result in bulk crustal velocity of around $6.9 \mathrm{~km} / \mathrm{s}$ was adopted by $K H 95$, whose interpretation of a high bulk crustal velocity of $7.3 \mathrm{~km} / \mathrm{s}$ observed in thick igneous crust of the U.S. East Coast was primarily based on its deviation from the 'normal' crustal velocity of $6.9 \mathrm{~km} / \mathrm{s}$. On the other hand, Zehnder et al. [1990] interpreted a lower-crustal velocity of as high as $7.5 \mathrm{~km} / \mathrm{s}$ observed at the Vøring margin as the result of the melting of normal mantle (but with active upwelling to generate thick crust), based on its similarity to the measured velocities of gabbroic rocks sampled from ophiolites. Interestingly, this interpretation appears to be one of the motivations to advocate the active upwelling of normal mantle during the opening of the North Atlantic [Mutter et al., 1988], as opposed to the plume head hypothesis presented by White and 
McKenzie [1989]. There seems, therefore, no consensus among marine seismologists on the 'normal' velocity of igneous crust.

It has been well known from laboratory studies that the compressional-wave velocity of gabbroic rocks at a pressure and temperature condition appropriate for normal oceanic crust is generally higher than $7.0 \mathrm{~km} / \mathrm{s}$, and that the typical lower-crustal velocity of $6.9 \mathrm{~km} / \mathrm{s}$ does not indicate the existence of gabbroic cumulates [e.g., Christensen and Salisbury, 1975; Spudich and Orcutt, 1980]. The example of crystallization modeling presented in Figure 3-3, which used a potential primary melt composition for normal MORB as a starting liquid, also clearly demonstrates that the normative bulk crustal velocity for normal MORB is higher than $7.1 \mathrm{~km} / \mathrm{s}$. The fractional cumulative assemblages have velocities higher than $7.3 \mathrm{~km} / \mathrm{s}$, comparable with the laboratory data for relatively unaltered mafic cumulates sampled from ophiolites [e.g., Christensen and Smewing, 1981]. Thus, hydrothermal circulation may be influential in reducing lower-crustal velocity. A number of petrological and geochemical studies of lower crust from ophiolites [e.g., Gregory and Taylor, 1981; Pallister and Hopson, 1981] and drilled samples of oceanic crust [e.g., Dick et al., 1991; Manning and MacLeod, 1996] indicate some hydrothermal activity, and the lower-crustal velocity of $6.9 \mathrm{~km} / \mathrm{s}$ was once thought to reflect the dominant presence of hornblende metagabbro [Christensen and Salisbury, 1975].

Field evidence from the Oman ophiolite, however, suggests that hydrothermal alteration is localized within cracks and veins, and that the majority of the cumulate lower crust is relatively unaltered with the model proportion of hornblende less than a few percent [e.g., Gregory and Taylor, 1981]. This low degree of alteration is not sufficient to reduce the velocity of gabbroic rocks to as low as $6.9 \mathrm{~km} / \mathrm{s}$ (Figure 3-10a). A more likely explanation seems to be the effect of residual crack porosity. Figure 3-10b shows that, if pore aspect ratio is low (i.e., more crack-like), porosity as low as $0.5 \%$ can significantly lower velocity. Oceanic gabbroic rocks recovered by drilling exhibit a broad correlation between velocity and porosity, which is consistent with the trend calculated for low aspect ratio porosity. We note that, while reported porosities for these gabbroic rocks were measured at atmospheric pressure, the corresponding velocities shown in Figure 3-10b 
were measured at a pressure of $200 \mathrm{MPa}$. The observed correlation between porosity and velocity, therefore, suggests that residual porosity may remain open even at a typical Moho pressure. Thus, once the lower crust is invaded by hydrothermal circulation, its originally high velocity corresponding to cumulate gabbros may be considerably lowered by residual crack porosity. Though the depth of hydrothermal circulation into oceanic crust is difficult to predict [e.g., Lister, 1974; Lister, 1983], it is reasonable to expect that the effect of hydrothermal circulation should decrease as total crustal thickness increases, because of greater lithostatic pressure. Indeed, the studies of microearthquakes at mid-ocean ridges show that the depths of hypocenters do not exceed $8 \mathrm{~km}$ beneath seafloor [e.g., Toomey et al., 1985; Huang and Solomon, 1988; Wolfe et al., 1995]. Given this possible porosity effect for thinner crust, therefore, the global average of normal crustal velocity may not be a useful reference value in interpreting the seismic structure of thick igneous crust. Thus, in this paper we rely instead on calculation of normative bulk crustal velocity directly from melt composition.

\subsection{Mantle Melting Model with Active Upwelling}

Based on the relation between bulk crustal velocity and the pressure and degree of mantle melting (equation 3.1), a possible connection between mantle melting processes and resultant crustal structure can be made. We shall consider a simple 1-D steady-state melting model, which includes the effects of preexisting lithosphere and active mantle upwelling (Figure 3-11). The following treatment may be too simple to describe realistic melting dynamics, but our primary interest is to illustrate the first-order influence of three main parameters on mantle melting during continental rifting: mantle potential temperature, lithospheric lid, and active mantle upwelling. The importance of mantle potential temperature has been widely recognized as the fundamental control on magmatism at mid-ocean ridges [e.g., Klein and Langmuir, 1987; McKenzie and Bickle, 1988; Kinzler and Grove, 1992; Langmuir et al., 1992] as well as LIPs [e.g., White and McKenzie, 1989; Coffin and Eldholm, 1994; White and McKenzie, 1995]. A lithospheric lid such as preexisting continental lithosphere and cold oceanic lithosphere imposes the 
upper limit of a mantle melting zone, and its importance is probably greatest during continental breakup [e.g., Fram and Lesher, 1993; Kelemen and Holbrook, 1995]. In addition, the mantle upwelling rate is not necessarily equal to the surface divergence rate, and it can be much higher than passive upwelling in the presence of either a mantle plume [e.g., Ribe et al., 1995; Ito et al., 1996], or a sharp lateral temperature gradient in the lithospheric lid [Mutter et al., 1988; Keen and Boutilier, 1995; Boutilier and Keen, 1999].

Adiabatically upwelling mantle, if not too cold, eventually intersects its solidus and begins to melt. We adopt the following solidus of dry peridotite [Takahashi and Kushiro, $1983]$

$$
T_{o}=1150^{\circ} \mathrm{C}+120^{\circ} \mathrm{C} / \mathrm{GPa} P_{o},
$$

where $P_{o}$ is the initial pressure of melting, and $T_{o}$ is the mantle temperature at the pressure. Following McKenzie and Bickle [1988], we define the potential temperature, $T_{p}$, as the hypothetical temperature of mantle adiabatically brought to the surface without melting,

$$
T_{p}=T_{o}-20^{\circ} \mathrm{C} / \mathrm{GPa} P_{o} \text {. }
$$

Given the above solidus and mantle adiabat, the amount of melting can be determined by the heats of fusion and heat capacities of mantle materials and a relation between temperature above the solidus and the extent of melting. To first-order, melt fraction can be approximated as a linear function of pressure decrease from the initial pressure of melting as,

$$
F(P)=\left(\frac{\partial F}{\partial P}\right)_{S}\left(P_{o}-P\right),
$$

where $(\partial F / \partial P)_{S}$ is a change in melt fraction with a change in pressure above the solidus during adiabatic decompression. This parameter is probably most important in relating the melting process to observable crustal thickness (melt volume), but its estimate varies from 10\%/GPa to 20\%GPa [e.g., Ahern and Turcotte, 1979; McKenzie, 1984; Langmuir et al., 1992; Asimow et al., 1997]. Therefore, we will test several choices for this parameter later in this section. The final pressure of melting is limited by a lithospheric lid, which includes newly formed igneous crust $(H)$ and preexisting lithosphere or cold oceanic mantle $(b)$,

$$
P_{f}=(H+b) / 30
$$


in which we assumed $30 \mathrm{~km} / \mathrm{GPa}$ for depth to pressure conversion. The mean fraction of melting is defined as,

$$
\bar{F}=\frac{\int_{P_{f}}^{P_{o}} F(P) d P}{\int_{P_{f}}^{P_{o}} d P} .
$$

This is the bulk mean fraction of melting [Plank et al., 1995]. For the linear melting function of equation 3.6, it reduces to $\bar{F}=0.5 F\left(P_{f}\right)$. Similarly, the mean pressure of melting is defined as,

$$
\bar{P}=\frac{\int_{P_{f}}^{P_{o}} P F(P) d P}{\int_{P_{f}}^{P_{o}} F(P) d P} .
$$

For a triangular melting regime associated with mantle corner flow, the mean pressure of melting, assuming a linear melting function, is $\left(P_{0}+2 P_{f}\right) / 3$ for batch melting, and $\left(2 P_{0}+P_{f}\right) / 3$ for fractional melting [Langmuir et al., 1992], and more complex melting regimes would lead to different mean pressures [e.g., Plank and Langmuir, 1992]. A realistic mixing formula for mean melting pressure is probably somewhere between purely batch and purely fractional cases, especially for major element compositions [e.g., Kelemen et al., 1997a], and we adopt $\bar{P}=\left(P_{o}+P_{f}\right) / 2$ as a neutral choice. If all melt is emplaced as igneous crust, the crustal thickness is then calculated as,

$$
H=30 \chi\left(P_{o}-P_{f}\right) \bar{F},
$$

where $\chi$ is the ratio of the mantle upwelling rate to the surface divergence rate. $\chi$ is equal to or greater than unity, and $\chi$ of 1.0 corresponds to passive upwelling. Another seismic observable, bulk crustal velocity, can be calculated from $\bar{P}$ and $\bar{F}$, using equation 3.1. For given $T_{p}, b$, and $\chi$, equations 3.6-3.10 can be solved in a closed form; the final pressure of melting can be expressed as,

$$
P_{f}=P_{o}+\frac{1}{\chi}\left(\frac{\partial F}{\partial P}\right)_{S}^{-1}\left\{1-\left[1+2 \chi\left(\frac{\partial F}{\partial P}\right)_{S}\left(P_{o}-b / 30\right)\right]^{1 / 2}\right\},
$$


and the crustal thickness is then calculated using equation 3.10. For a more complex formulation of the melting function, however, the above equations must be solved numerically.

Our definition of "active upwelling" is different from that which has been commonly used in the literature on mid-ocean ridge dynamics, so some clarification is necessary. Several authors have demonstrated that a decoupling of solid mantle flow from the surface divergence is possible when extra buoyancy due to high melt retention and lower Fe/Mg in residual solids is considered [e.g., Rabinowicz et al., 1987; Scott, 1992; Turcotte and Phipps Morgan, 1992; Su and Buck, 1993; Barnouin-Jha et al., 1997]. The resultant rapid solid upwelling is often referred as active mantle upwelling, and it has been suggested as a possible mechanism of melt focusing beneath mid-ocean ridges. In an extreme case in which all melts are retained, mantle melt would be at equilibrium at the shallowest pressure of melting; mean pressure of melting and mean melt fraction are simply the final pressure of melting and the maximum melt fraction, respectively, attained in the melting zone [Langmuir et al., 1992]. This type of mantle dynamics associated with melting would add important complexity to the interpretation of igneous crustal structure. Its likelihood, however, has been largely discounted by a recent large-scale seismic investigation of the East Pacific Rise [The MELT Seismic Team, 1998], and we do not include it in our model parameterization. For continental rifting, however, the decoupling of mantle flow from the surface motion is still viable. Since rifting is a transient process in which surface divergence evolves from null to some finite value, mantle upwelling velocity due to preexisting sublithospheric convection, such as in a mantle plume, or to rifting-induced mantle convection can be significantly greater than that of plate-driven flow. The active upwelling ratio, $\chi$, in our model is designated to quantify this decoupling.

Figure 3-12 shows some sample calculations based on our melting model, using a linear melting function with $(\partial F / \partial P)_{S}$ of $12 \% / \mathrm{GPa}$ (standard model). The mean pressure of melting, the mean melt fraction, crustal thickness, and bulk crustal velocity are calculated as a function of mantle potential temperature, with a range of active upwelling ratio and preexisting lid thickness. A higher active upwelling ratios results in thicker crust, 
which in turn limits the melting zone, leading to slightly higher mean melting pressure and lower melt fraction (Figure 3-12a-c). High pressure of melting and low melt fraction have competing effects on bulk crustal velocity, and the net effect is a small decrease in crustal velocity (Figure 3-12d). A preexisting lid has similar but larger effects on melting pressure and melt fraction, and a reverse effect on crustal thickness (Figure 3-12e-g). A thicker lid also lowers the resultant crustal velocity (Figure 3-12h). There are of course an infinite number of other combinations of active upwelling ratio and lid thickness, but for the sake of discussion, we limit ourselves to two end-member cases: (1) active upwelling with zero lid thickness, and (2) non-zero lid thickness and passive upwelling. As shown in Figure 3-13a, these two cases can cover a considerable range of crustal thickness and velocity, and we think that more complicated cases should be considered only when some independent geological information requires them. This crustal thickness and velocity $\left(H-V_{p}\right)$ diagram concisely illustrates a possible relationship between seismic observables and mantle melting parameters. For comparison, we also construct a $H$-V $V_{p}$ diagram using the velocity equation of KH95 (Figure 3-13b). For given melting parameters, this diagram consistently underpredicts crustal velocity.

To illustrate the sensitivity of the $H$ - $V_{p}$ diagram to the choice of a melting function, two more diagrams were made with (1) a linear function with a higher melting rate of $16 \% / \mathrm{GPa}$ (Figure 3-14a), and (2) a three-stage melting function incorporating the effect of initial, small degrees of melting due to, e.g., $\mathrm{H}_{2} \mathrm{O}$ and $\mathrm{K}_{2} \mathrm{O}$, and the effect of clinopyroxene exhaustion, similar to that calculated by Asimow et al. [1997](Figure 3-14b). The overall features of all of these $H-V_{p}$ diagrams such as the location of passive upwelling curve and the effects of active upwelling and lithospheric lid are very similar. The only critical difference is mantle potential temperature; for a given pair of crustal thickness and velocity, the estimate of mantle potential temperature would be systematically higher for a melting function with smaller $(\partial F / \partial P)_{S}$. Considering that our understanding of a mantle melting function is still limited, therefore, the estimate of potential temperature based on our $H-V_{p}$ diagram is most useful for comparative purposes. The estimate of active 
upwelling ratio or lid thickness seems to be more robust if bulk crustal velocity is reasonably well determined.

\subsection{Nature of Mantle Melting During the Formation of the Southeast Greenland Margin}

The 1996 SIGMA seismic experiment across the Southeast Greenland margin employed dense receiver arrays and deep-penetrating air-gun sources to provide deep-crustal seismic data of unprecedented quality on four transects (Figure 3-2a). The transect 2 data were analyzed with joint refraction and reflection tomography to construct a $P$-wave velocity model (Chapter 1). A nonlinear Monte Carlo uncertainty analysis showed that the model was tightly constrained; the absolute uncertainty for vertically-averaged velocity is less than $0.03 \mathrm{~km} / \mathrm{s}$. The interpretation of the velocity model based on the framework of KH95 suggested that the thick igneous crust observed in the continent-ocean transition zone was the result of active upwelling of mantle with almost normal potential temperature $(\chi>8)$. This interpretation, however, remained inconclusive because the framework of KH95 involved other sources of uncertainty, such as the poorly constrained velocity-composition relationship and the assumption of uniform upper-crustal velocity. Given the high quality of the seismic data and interpretation, the comparatively large uncertainties in the KH95 approach to inferring crustal composition and mantle dynamics, were the main motivations for this study. In this section, we revisit the transect 2 velocity model with our new interpretational strategy.

The thickness of the igneous crust on transect 2 varies from $-30 \mathrm{~km}$ in the continent-ocean transition zone to $\sim 9 \mathrm{~km}$ in the deep-ocean basin near the seaward end (Figure 3-2b). The thickness of the upper crust, which is marked by high velocity gradients, also changes along the transect, and the proportion of the upper crust is nearly constant at around 30\% (Figure 3-15b). Despite its great thickness, the transition zone lower crust does not exhibit a notable increase in its velocity (Figure 3-2b and c), being conspicuously different from thick, high velocity $(>7.2 \mathrm{~km} / \mathrm{s})$ transition zone crust observed at other volcanic margins [e.g., White et al., 1987; Mutter and Zehnder, 1988; 
Holbrook and Kelemen, 1993]. After applying a pressure correction of $0.2 \times 10^{-3} \mathrm{~km} / \mathrm{s} / \mathrm{MPa}$ and a temperature correction of $-0.4 \times 10^{-3} \mathrm{~km} / \mathrm{s} /{ }^{\circ} \mathrm{C}$ (Figure 3-8), with respect to a reference pressure of $600 \mathrm{MPa}$ and a reference temperature of $400^{\circ} \mathrm{C}$, the harmonic mean velocity of the igneous lower crust is calculated as a function of the model distance (Figure 3-15a). For the temperature correction, we assume a linear conductive geotherm with a thermal gradient of $20^{\circ} \mathrm{C} / \mathrm{km}$ and a surface temperature of $0^{\circ} \mathrm{C}$. Velocities within a horizontal window of $20 \mathrm{~km}$ were averaged at each model distance, and we repeated this averaging procedure with 100 Monte Carlo ensembles to estimate uncertainty as in Chapter 1.

The average lower-crustal velocity is plotted with the corresponding whole-crustal thickness on the standard $H-V_{p}$ diagram (Figure 3-15c). Note that the lower-crustal velocity is the upper bound of the possible range of bulk crustal velocity. For the lower-crustal proportion of $\sim 70 \%$ and the lower-crustal velocity of $\sim 7.0 \mathrm{~km} / \mathrm{s}$, the bulk crustal velocity can be as low as $6.85 \mathrm{~km} / \mathrm{s}$, considering the maximum effect of fractionation (Figure 3-6a and b). For the thinner crust observed toward the seaward end, this upper bound is most likely invalid because an effect of hydrothermal alteration or cracks, lowering the lowercrustal velocities, is expected. As described in a previous section, we thus focus on the thick $(>15 \mathrm{~km}$ ) transitional crust, for which the correction for hydrothermal alteration may not be necessary. The $H-V_{p}$ diagram suggests that an anomalously strong active upwelling of mantle (more than eight times faster than the surface divergence) is responsible for the formation of the thick igneous crust on transect 2 . As already noted, the estimate of mantle potential temperature ranges from $1250^{\circ} \mathrm{C}-1350^{\circ} \mathrm{C}$, depending on the choice of a melting function (Figure 3-13a and Figure 3-14). However, in all cases the estimated potential temperature is not significantly higher than a normal potential temperature of $-1300^{\circ} \mathrm{C}$ [e.g.,McKenzie and Bickle, 1988; Langmuir et al., 1992]. Though the regression for equation 3.1, which is a key link between crustal velocity and mantle melting parameters, has a standard deviation of as large as $0.08 \mathrm{~km} / \mathrm{s}$, the vigorous mantle upwelling with almost normal temperature, as inferred by Holbrook et al. [1999] and in Chapter 1, still seems to be a robust conclusion. Based on the new interpretational method, therefore, the preliminary conclusion made in Chapter 1 seems to be further substantiated, and this result 
strongly argues against the plume head hypotheses proposed for the North Atlantic igneous province [e.g., Richards et al., 1989; White and McKenzie, 1989; Barton and White, 1997a; Holbrook et al., 1999]. Since the use of the $\mathrm{H}$ - $V_{p}$ diagram would be more robust in comparison with adjacent seismic transects, the accurate determination of whole-crustal velocity structure on all four SIGMA transects would be essential to resolve outstanding questions on margin-wide mantle dynamics during the opening of the North Atlantic.

\subsection{Discussion}

\subsubsection{Effects of Subcrustal Fractionation and Mantle Source Heterogeneity}

So far we have assumed that all mantle melt is emplaced as seismically observable crust, and that the mantle source composition is similar to pyrolite [e.g., Ringwood, 1975; Hart and Zindler, 1986; McDonough and Sun, 1995]. In this section, we will examine the validity of these two fundamental assumptions. First of all, a significant portion of primary melt may crystallize within a mantle column or as ultramafic cumulates at the base of the crust. As discussed in the Introduction, however, it is difficult to quantify the likelihood of this subcrustal fractionation, because the transient thermal structure of a newly forming rifting axis is not well understood. Though there are several geochemical studies of the North Atlantic igneous province that indicate relatively high-pressure crystallization [Morrison et al., 1985; Thompson et al., 1986; Bernstein, 1994; Fram and Lesher, 1997] (0.8-1.5 GPa), such high-pressure fractionation can also result from deep-crustal fractionation. The possibility of subcrustal fractionation is critical for our bounding approach with the $H-V_{p}$ diagram, because it is probably the only mechanism, except for hydrothermal alteration, that can violate the upper limit on bulk crustal velocity provided by observed lower-crustal velocity. Early fractionating phases at high pressures are most likely olivine and clinopyroxene [e.g., Bender et al., 1978; Presnall et al., 1978; Grove et al., 1992; Langmuir et al., 1992], both of which have high seismic velocities, so normative bulk crustal velocity can become higher than observed lower-crustal velocity if there is a missing subcrustal component composed of the high-velocity minerals. To quantify this effect, we repeated fractional crystallization modeling at $1 \mathrm{GPa}$, and compared the bulk 
crustal velocities of residual liquids with those of primary melts, at several proportions of subcrustal fractionation (Figure 3-16). The effect of subcrustal fractionation is more significant for higher bulk crustal velocities, and it can be as much as $0.2 \mathrm{~km} / \mathrm{s}$. For bulk crustal velocities lower than $7.0 \mathrm{~km} / \mathrm{s}$, however, its effect is about $0.05 \mathrm{~km} / \mathrm{s}$ even for $30 \%$ of subcrustal fractionation. This is because plagioclase appears early on fractionation paths for primary liquids corresponding to normative bulk crustal velocities less than $7.0 \mathrm{~km} / \mathrm{s}$. Based on this modeling, therefore, we conclude that the effect of subcrustal fractionation on the observed low velocity $(-7.0 \mathrm{~km} / \mathrm{s})$ of the thick transitional crust is minimal. The possibility of a missing subcrustal component would increase total melt volume and mantle potential temperature, but with a negligible influence on the estimate of active upwelling ratio.

Second, our regression of bulk crustal velocity to mantle melting parameters (equation 3.1) is only valid for mantle compositions similar to pyrolite model compositions. The majority of abyssal peridotites are best explained as solid residues from the melting of pyrolitic mantle [e.g., Dick et al., 1984; Kelemen et al., 1992; Asimow, 1999], and the assumption of pyrolitic source compositions is probably justified for the melting of an average oceanic upper mantle. The local heterogeneity of the mantle source composition is, however, another issue, and the degree of major element heterogeneity and its spatial scale are not well known. Whereas isotopic and trace elemental heterogeneity in the convecting upper mantle is firmly established, especially in relation to hotspots, major elemental heterogeneity is difficult to quantify because the major element compositions of erupted lavas are strongly sensitive to melting and fractionation processes as well as source compositions. The importance of major element source heterogeneity has been suggested for several hotspots including Iceland, Azores, Hawaii, and Yellowstone [e.g., Langmuir and Hanson, 1980; Schilling et al., 1983; Hauri, 1996; Takahashi et al., 1998], but its effect on melting processes has not been well quantified. Different mantle source compositions have different solidi and melting functions as well as different melt compositions, and there are too few melting experiments [e.g., Hirose and Kushiro, 1993; Kogiso et al., 1998] to develop a quantitative melting model incorporating source 
heterogeneity. The interpretational method developed in this study, therefore, should be regarded as a baseline, on which we must elaborate for source heterogeneity, as more data on mantle melting accumulate. Since seismic data provide only two observables (velocity and thickness) and we need to resolve at least three unknowns (potential temperature, active upwelling ratio, and source composition), it will be important to include geochemical information obtained from sampled lavas to correctly interpret seismic crustal structure.

\subsubsection{Previous Deep-Crustal Studies of the North Atlantic Margins}

As emphasized by KH95 and Holbrook et al. [1999], active mantle upwelling is important when crustal thickness is used to infer mantle potential temperature (e.g., Figure 3-13). The estimate of potential temperature simply assuming passive upwelling [e.g., Barton and White, 1997a] may considerably deviate from the actual thermal state of mantle. Although accurate information regarding lower-crustal velocity is essential to assess the active upwelling ratio, lower-crustal velocity is, unfortunately, generally the least constrained part of crustal structure. Though the North Atlantic margins are so far the volcanic margins best studied by deep-crustal seismic surveys [White et al., 1987; Mutter and Zehnder, 1988; Fowler et al., 1989; Morgan et al., 1989; Zehnder et al., 1990; Barton and White, 1997a; Holbrook et al., 1999], some caution is required in compiling published crustal velocity models to infer the whole picture of the mantle melting process during the opening of the North Atlantic.

The lower crust is usually characterized by a small vertical velocity gradient, so that its velocity structure is constrained mostly with $P m P$ travel times. During the 1980 s, expanding spread profiling (ESP) using two ships was popular to acquire wide-angle refraction and reflection data for nearly 1-D geological settings. Because $P m P$ phases are usually identifiable only at large offsets, the $P m P$ travel time data in an ESP common-midpoint gather, which have a very limited range of ray aperture, does not provide a good constraint on lower-crustal velocity structure. The waveform information such as the minimum range of post-critical $P m P$ phases was, therefore, commonly used to determine a velocity contrast at the Moho. Since shallow mantle velocity can be inferred from $P n$ 
arrivals or its lower limit can be reasonably assumed to be $-7.9 \mathrm{~km} / \mathrm{s}$, this method seems to be able to derive a lower limit on lower-crustal velocity from the waveform information. This approach implicitly assumes, however, that lower crust can be accurately described as a single block with a uniform velocity or a constant velocity gradient. This is a dangerous assumption because the Moho can be a diffuse discontinuity with complex structure, as geological studies of ophiolites indicate [Nicolas and Prinzhofer, 1983; Benn et al., 1988; Boudier and Nicolas, 1995; Boudier et al., 1996]. The waveform data are only sensitive to short-wavelength structure [e.g., Jannane et al., 1989], so that extending a velocity contrast at the Moho to all of lower crust is an unstable extrapolation.

It is thus preferable to use only travel time data of $P m P$ phases. Because $P m P$ travel times are sensitive to both the depth of the Moho and velocity above it, dense ray coverage with a number of source and receiver pairs is required to minimize velocity-depth ambiguity (Chapter 1). We emphasize that velocity-depth ambiguity has a serious influence on the estimate of crustal composition; an error of $3 \%$ in velocity, for example, means an error of $0.2 \mathrm{~km} / \mathrm{s}$ for $7.0 \mathrm{~km} / \mathrm{s}$, whereas the same degree of uncertainty in the Moho depth has only a trivial effect on the estimate of melt volume. Except for our tomography in Chapter 1 , however, the issue of velocity-depth ambiguity may not have been properly treated in earlier crustal seismic studies. In addition, a robust uncertainty analysis has rarely been done for published velocity models (see Discussion in Chapter 1), whereas placing an error bound on estimated velocity is extremely important for petrological interpretation. The significant difference in lower-crustal structure between our transect 2 and other North Atlantic transects (Figure 3-9) may simply reflect the difference in the quality of original seismic data and the thoroughness of uncertainty analysis.

\subsection{Conclusion}

We developed a new methodology to relate the seismic crustal structure of large igneous provinces with the process of causative mantle melting. Following the approach taken by Kelemen and Holbrook [1995], we first established a relation between bulk crustal velocity 
and mantle melting parameters including the pressure and degree of melting, based on an extended database of mantle melting experiments. A more accurate calculation of velocity from a given composition through the CIPW norm results in a much tighter relation than that of $K H 95$, with one standard deviation of only $0.08 \mathrm{~km} / \mathrm{s}$. Noting that upper-crustal velocity does not contain useful petrological information, and that it is impossible to uniquely determine a bulk crustal composition from lower-crustal velocity alone, we estimated the possible range of compositional variations in the lower crust during crustal accretion by modeling fractional crystallization at a range of pressures. Based on the results of this crystallization modeling, a bound on the possible range of bulk crustal velocity for a given lower-crustal velocity was obtained as a function of lower-crustal fraction. Finally, a simple mantle melting model was developed to illustrate the effects of active mantle upwelling and a preexisting lithospheric lid, which are prominent complicating factors in the formation of large igneous provinces, and a relationship between mantle melting parameters and corresponding seismic observables was succinctly summarized in a single $H-V_{p}$ diagram.

The crustal structure of the Southeast Greenland margin was revisited, and the preliminary conclusion made in Chapter 1 was reinforced with this new interpretational method; the thick $(-30 \mathrm{~km})$ transitional crust with an average lower-crustal velocity of $-7.0 \mathrm{~km} / \mathrm{s}$ is the result of vigorous active upwelling of mantle with almost normal potential temperature. Even though our melting model contains several sources of uncertainties such as a melting function and the style of melting, this conclusion seems to be robust. The effect of major element heterogeneity in a mantle source composition is probably the only significant remaining uncertainty in this conclusion. Future work to incorporate source heterogeneity is warranted. 


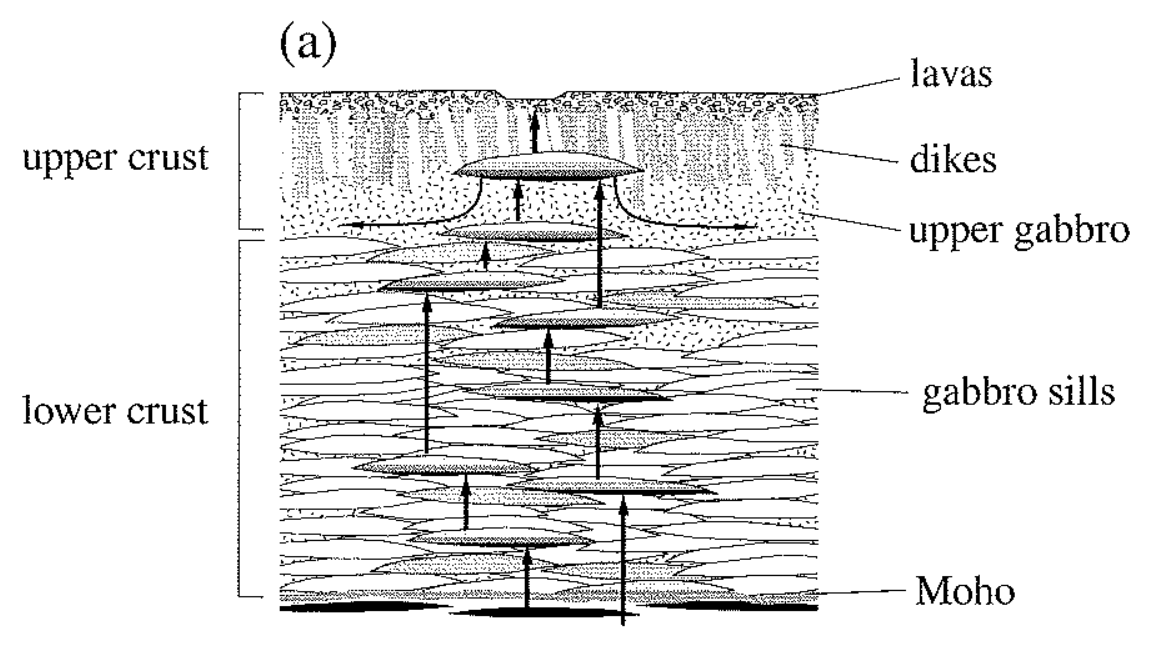

(b)

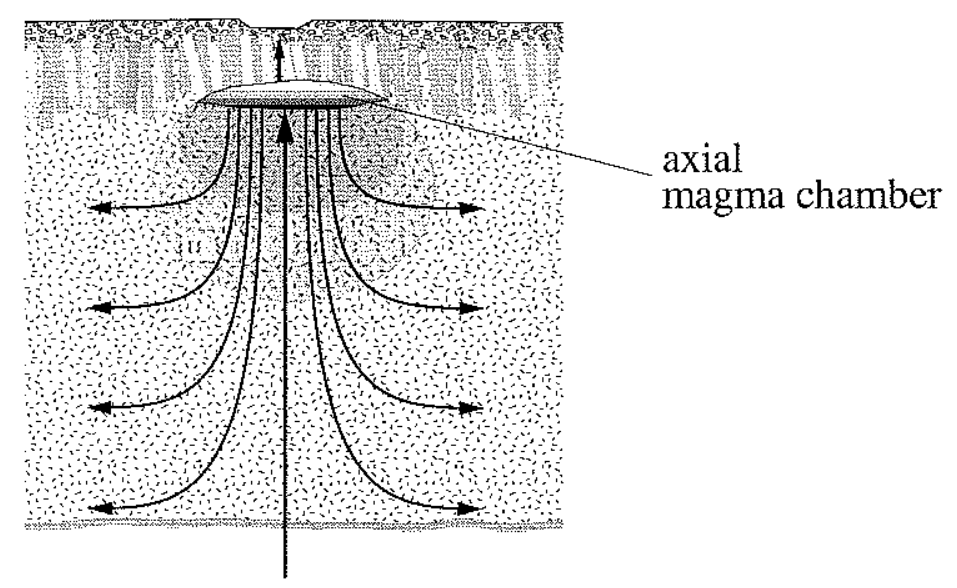

(c)

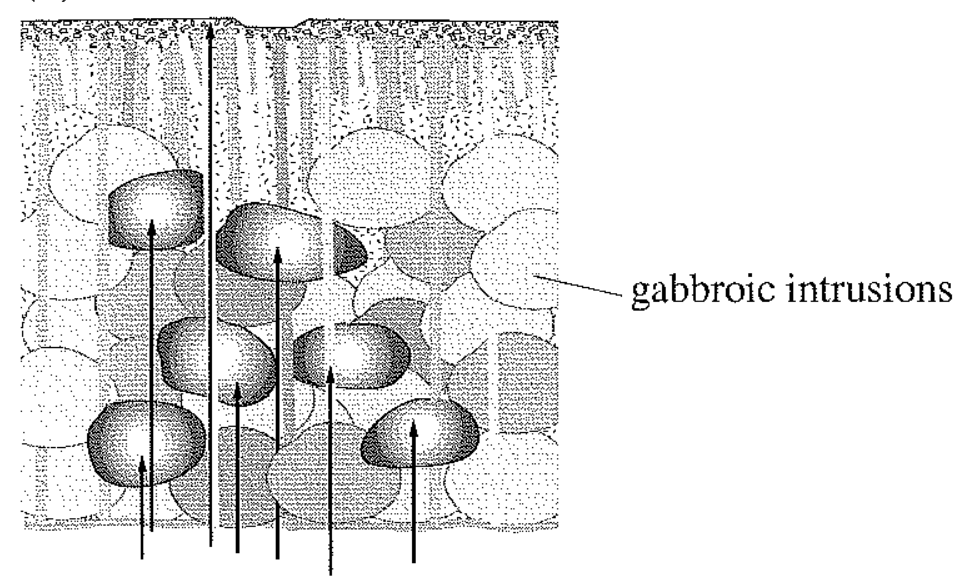

Figure 3-1. Schematic drawings for recently proposed accretion models for igneous crust. (a) Sheeted sill model [Kelemen et al., 1997b; Kelemen and Aharonov, 1998], (b) gabbro glacier model [Nicolas et al., 1988; Henstock et al., 1993; Phipps Morgan and Chen, 1993; Quick and Denlinger, 1993; Menke and Sparks, 1995], and (c) intrusion model [Dick et al., 1992; Cannat, 1996]. 


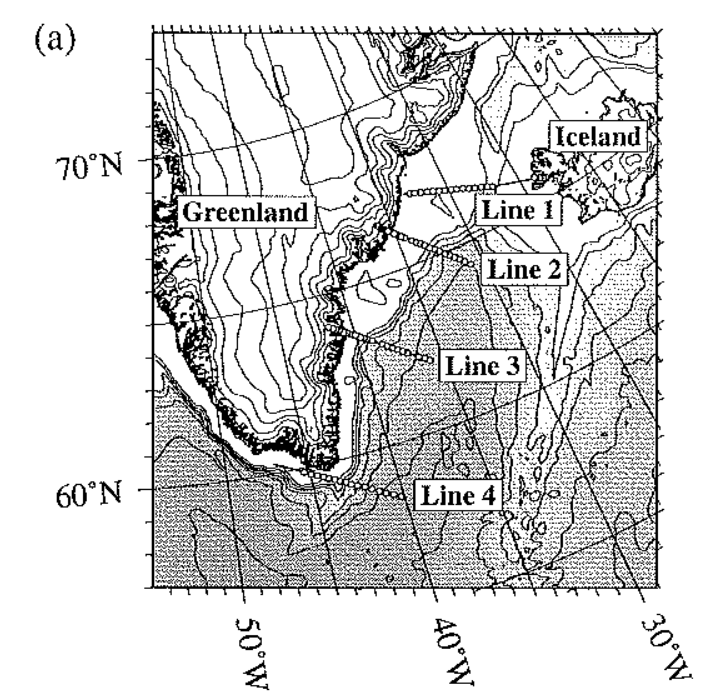

(b)

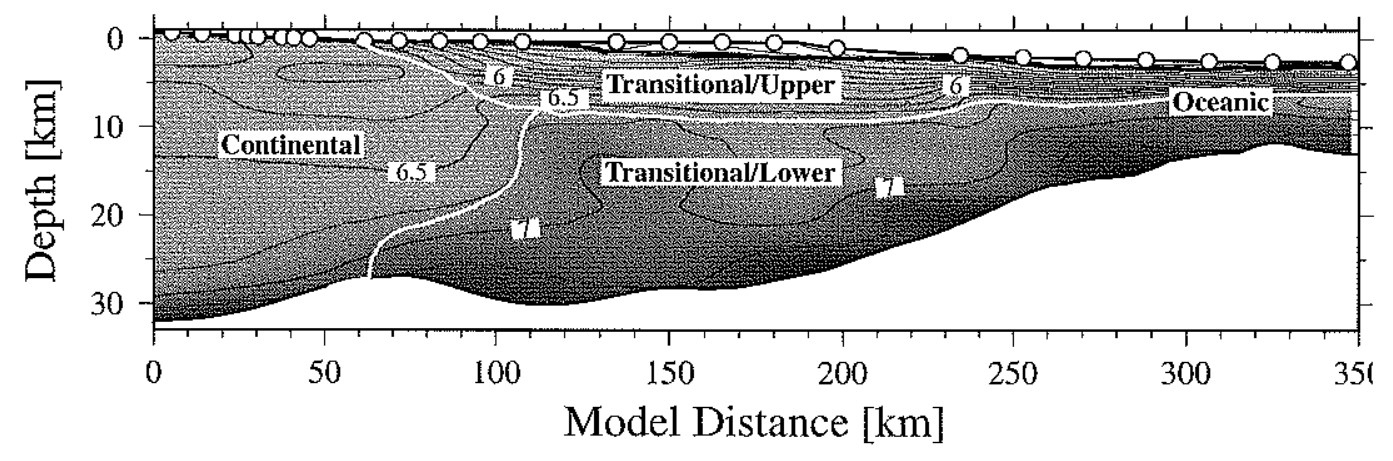

(c)

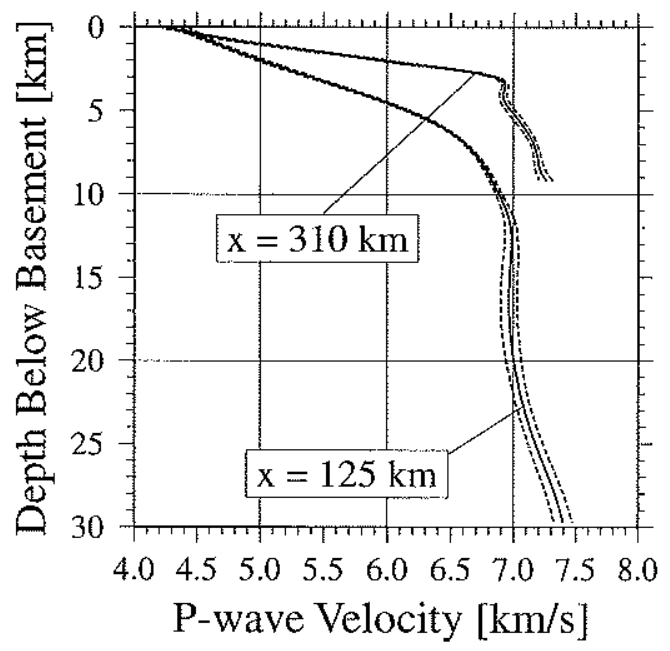

Figure 3-2. (a) Location of 1996 SIGMA transects. Open circles denote the location of seismic receivers, and solid lines denote shot lines. (b) $P$-wave velocity model for transect 2 (from Chapter 1), with geological interpretation of continental, transitional and oceanic crust. (c) 1-D velocity profiles sampled from (b) at model distances of $125 \mathrm{~km}$ and $310 \mathrm{~km}$. One standard deviation is shown as dashed, based on 100 Monte Carlo ensembles. 

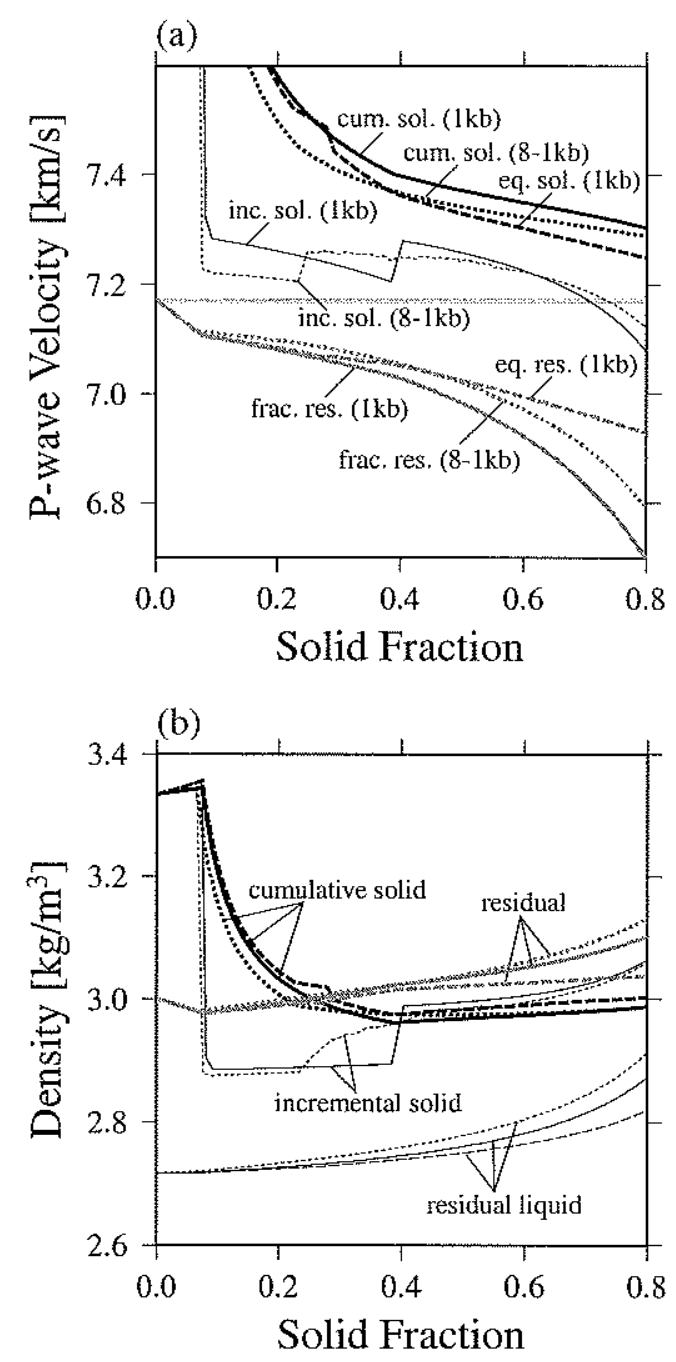
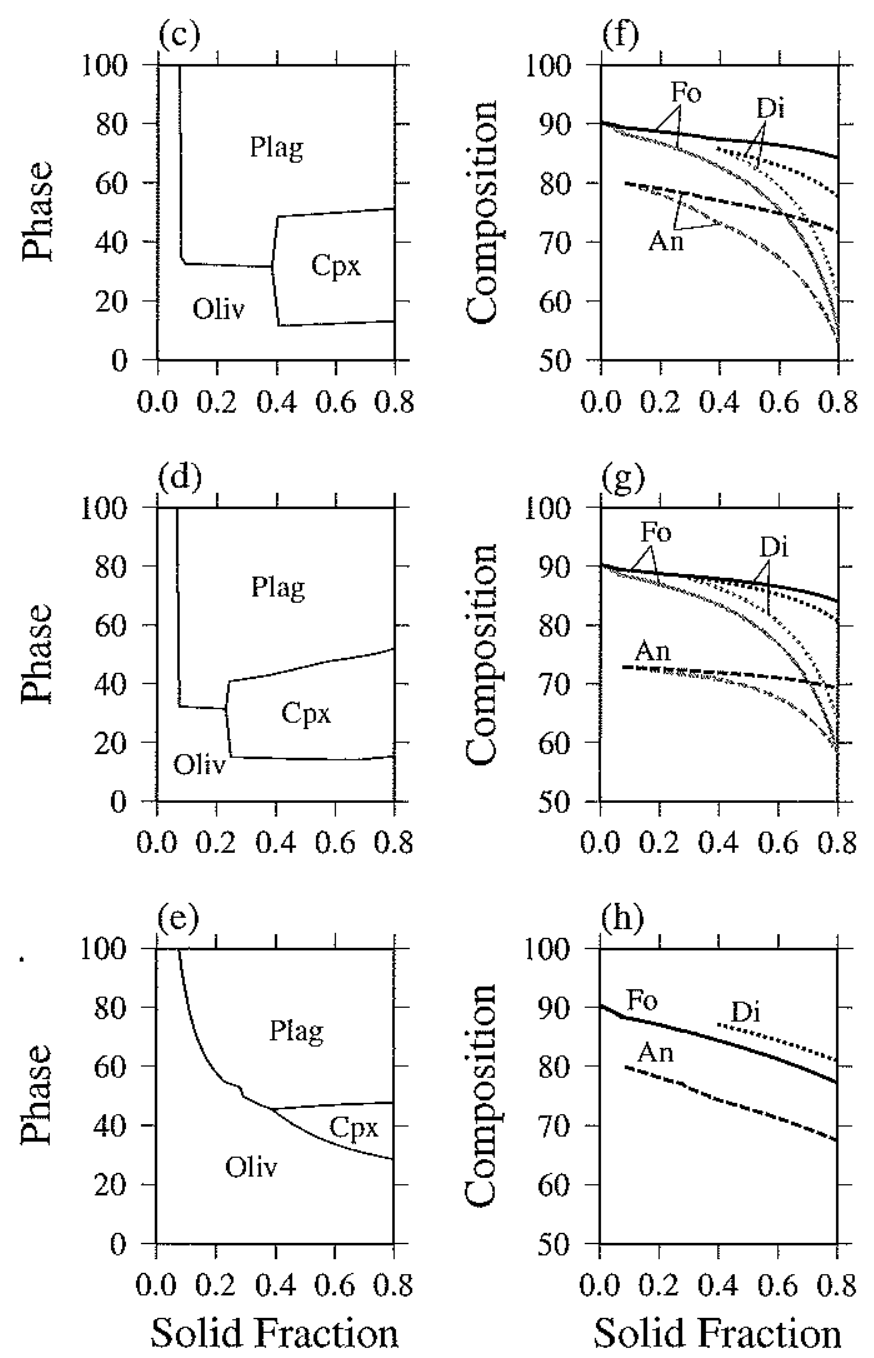

Figure 3-3. Example of crystal fractionation modeling. Starting liquid is polybaric nearfractional melt aggregated from a triangular shaped melting regime, with mean melt fraction of $9 \%$ and mean pressure of melting of $1.5 \mathrm{GPa}$, as calculated by Kinzler [1997] $\left(\mathrm{SiO}_{2}: 48.2 \%\right.$, $\mathrm{TiO}_{2}: 0.94 \%, \mathrm{Al}_{2} \mathrm{O}_{3}: 16.4 \%, \mathrm{Cr}_{2} \mathrm{O}_{3}: 0.12 \%$, FeO: $7.96 \%, \mathrm{MgO}: 12.5 \%, \mathrm{CaO}: 11.4 \%, \mathrm{~K}_{2} \mathrm{O}:$ $0.07 \%, \mathrm{Na}_{2} \mathrm{O}: 2.27 \%$ ). Three fractionation paths are considered: (1) fractional crystallization at $100 \mathrm{MPa}$ (solid), (2) equilibrium crystallization at $100 \mathrm{MPa}$ (dashed), and (3) polybaric fractional crystallization at 800 to $100 \mathrm{MPa}$ (dotted). (a) $P$-wave velocities of cumulative fractionating assemblage and normative assemblage for residual liquid phase are calculated at a pressure of $100 \mathrm{MPa}$ and a temperature of $100^{\circ} \mathrm{C}$ and shown as black and gray lines, respectively. Velocity of incremental fractionating assemblage is also shown as a thin gray line for fractional crystallization cases. (b) Solid densities are calculated at the same reference state. Residual liquid densities of residual phases are also shown. Phase proportions and compositions of fractionating assemblage are shown in (c)-(h): (c,f) fractional crystallization at $100 \mathrm{MPa},(\mathrm{d}, \mathrm{g})$ polybaric fractional crystallization at $800-100 \mathrm{MPa}$, and (e,h) equilibrium crystallization at $100 \mathrm{MPa}$. Abbreviations are Oliv (olivine), Plag (plagioclase), Cpx (clinopyroxene), Fo (forsterite content), Di (diopside content), and An (anorthite content). 


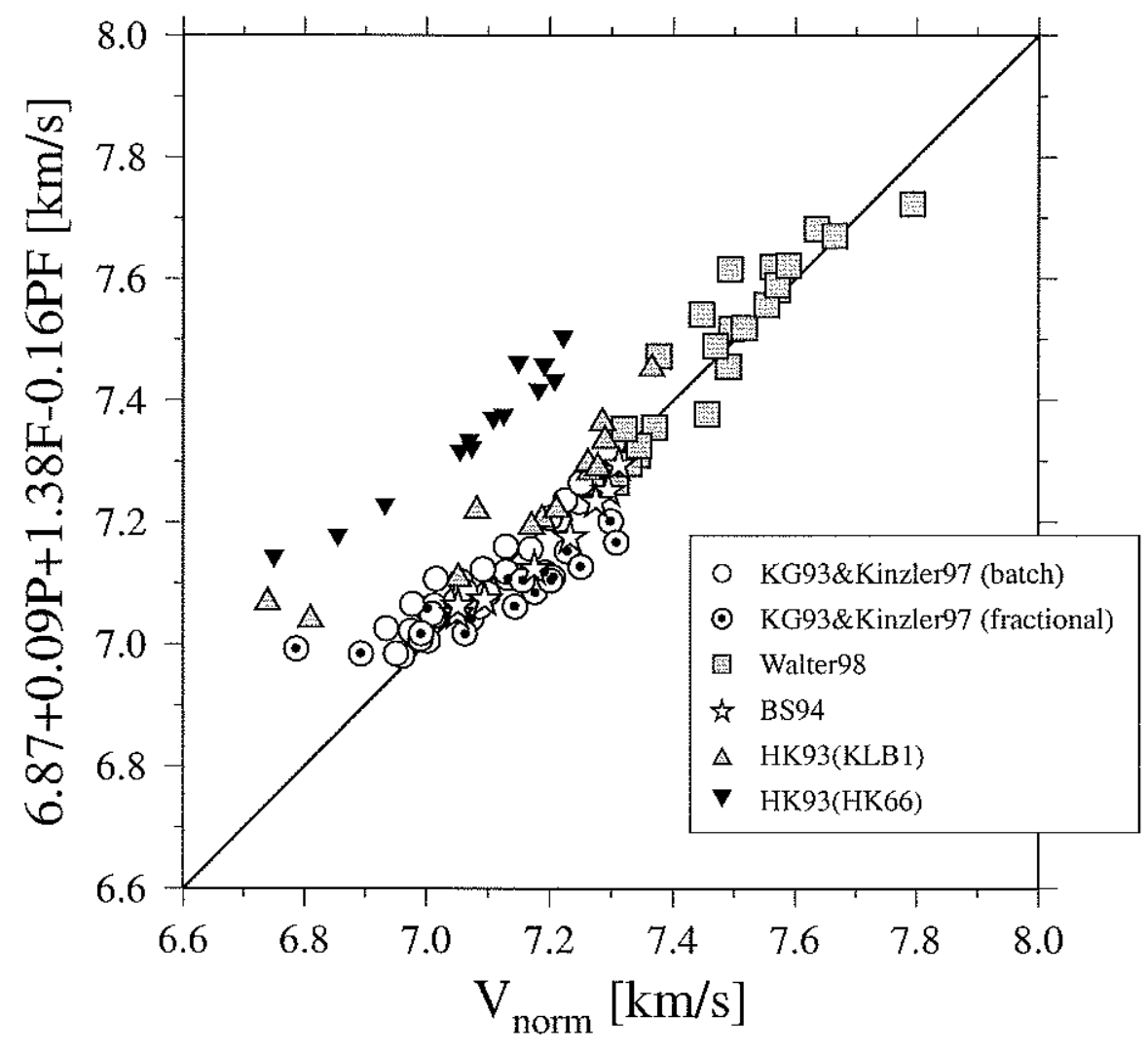

Figure 3-4. Relationship of norm-based $P$-wave velocity to the pressure and fraction of melting, for experimental and calculated liquids for the melting of mantle therzolite [Kinzler and Grove, 1992; Hirose and Kushiro, 1993; Kinzler and Grove, 1993; Baker and Stolper, 1994; Kinzler, 1997; Walter, 1998]. One standard deviation is $0.08 \mathrm{~km} / \mathrm{s}$. Open circles with dots denote polybaric fractional melts calculated by Kinzer and Grove [1993] and Kinzler [1997]. Predicted velocities for HK66 melts using equation 3.1 are systematically higher than their norm-based velocities, mainly because the melting of HK66 is associated with much higher degrees of melting compared to the melting of peridotites. 


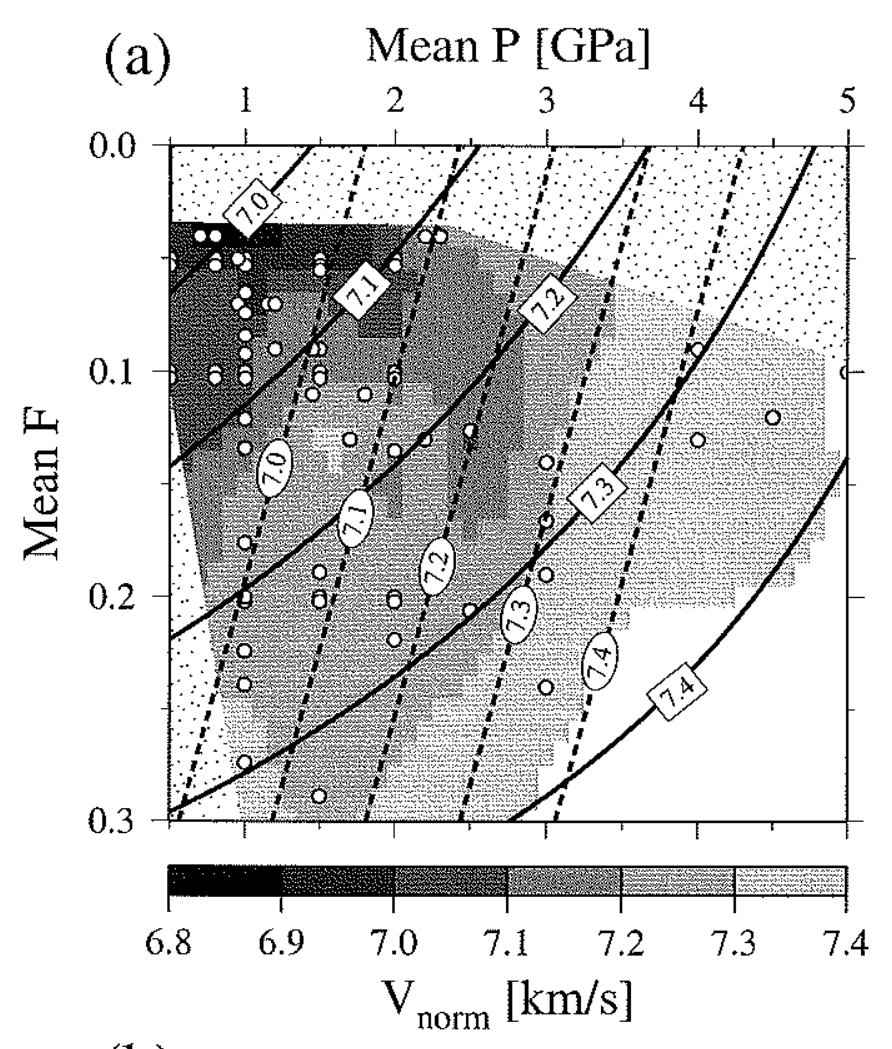

(b)

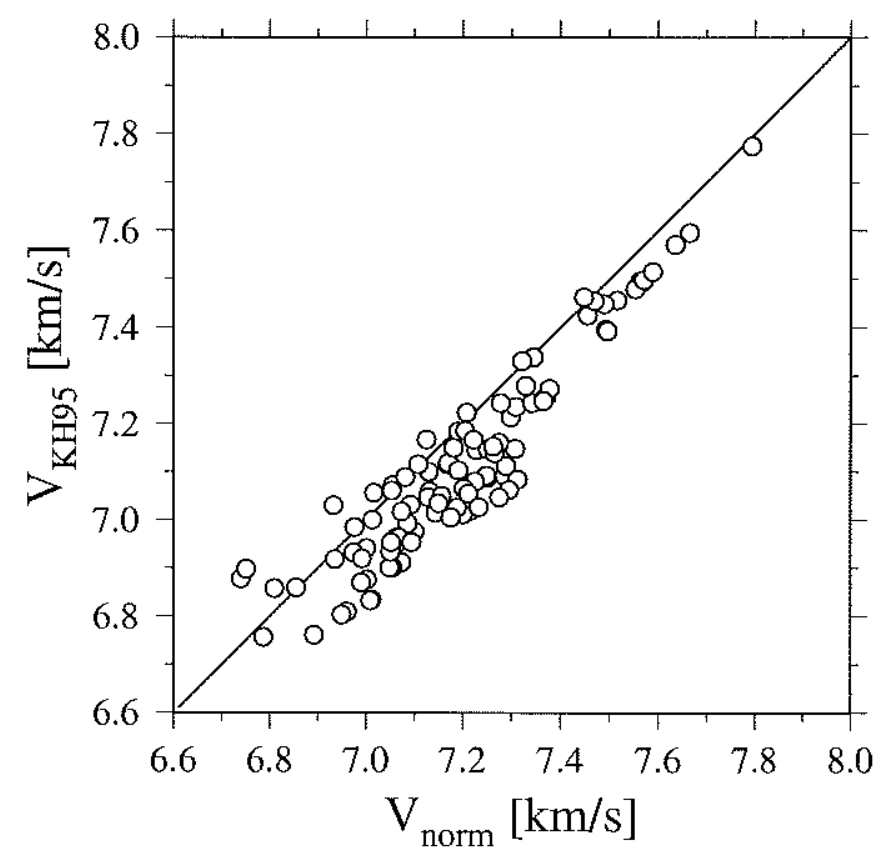

Figure 3-5. (a) mantle melt data used for regression (shown as open circles) are gridded as a function of the pressure and fraction of melting, and contours of predicted velocity based on our new regression are overlaid as solid lines. Prediction based on the empirical relation derived by KH95 (equation 3.2) is also shown as dashed lines. (b) Comparison of norm-based velocity with velocity calculated using equation 3.3. 
(a) $\mathrm{P}_{\mathrm{xl}}=100 \mathrm{MPa}$

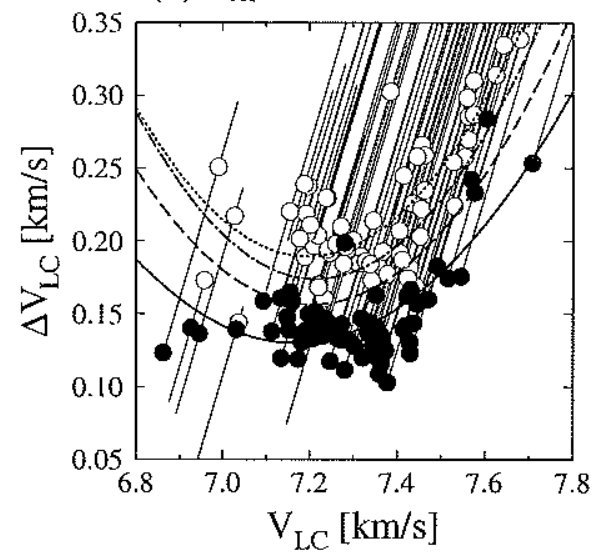

(c) $\mathrm{P}_{\mathrm{xl}}=100 \mathrm{MPa}$

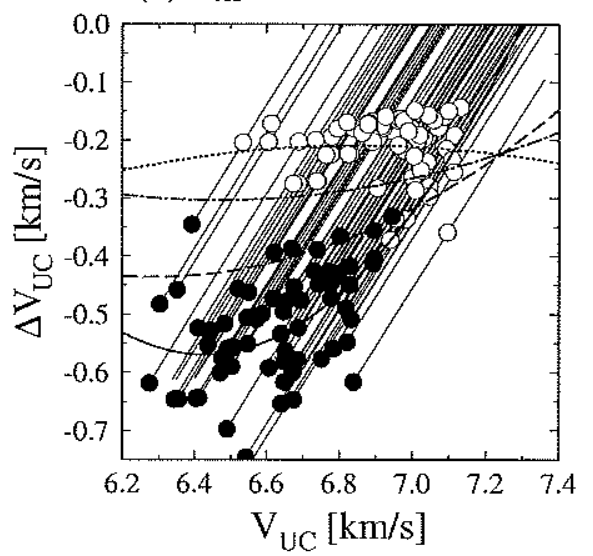

(b) $\mathrm{P}_{\mathrm{xl}}=400 \mathrm{MPa}$

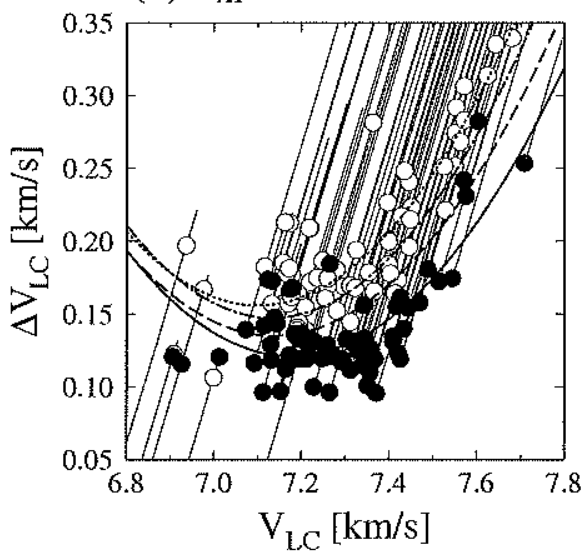

(d) $\mathrm{P}_{\mathrm{xl}}=400 \mathrm{MPa}$

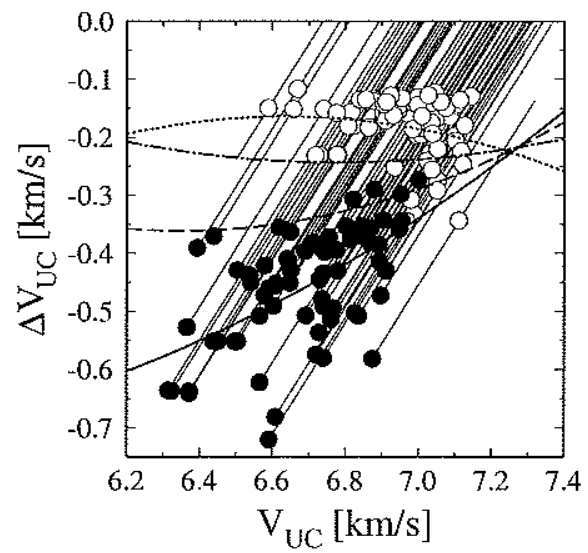

$$
\begin{array}{r}
0-F_{x l}=0.5 \\
-\cdots-F_{x l}=0.6 \\
---F_{x l}=0.7 \\
-F_{x l}=0.8
\end{array}
$$

Figure 3-6. Effects of fractional crystallization on $P$-wave velocities of fractionating solid phase and residual liquid phase. (a) $P$-wave velocity of cumulative fractionating phase is denoted as $V_{\mathrm{LC}}$, and its deviation from bulk crustal velocity as $\Delta V_{\mathrm{LC}}$ is plotted as a function of $V_{\mathrm{LC}}$, for the fractionation paths of all mantle melts at $100 \mathrm{MPa}$ (thin line). Velocities are values at $600 \mathrm{MPa}$ and $400^{\circ} \mathrm{C}$. Open and solid circles denote the loci of fractionation paths at solid fractions of 0.5 and 0.8 , respectively. To quantify the scatter of $\Delta V_{\mathrm{LC}}$ for a given solid fraction, quadratic regressions are made at solid fractions of 0.5 to 0.8 , and one standard deviation gradually decreases from $0.03\left(F_{x l}=0.5\right)$ to $0.02\left(F_{x l}=0.8\right)$. (b) Same as (a) but at a crystallization pressure of $400 \mathrm{MPa}$. (c) Same as (a) but with $P$-wave velocity of residual liquid phase $\left(V_{\mathrm{UC}}\right)$ and its deviation from bulk crustal velocity $\left(\Delta V_{\mathrm{Uc}}\right)$. One standard deviation of quadratic regression increases from $0.06\left(F_{x l}=0.5\right)$ to $0.09\left(F_{x l}=0.8\right)$. (d) Same as (c) but at a crystallization pressure of $400 \mathrm{MPa}$. Similar results are obtained for a crvstallization pressure of $800 \mathrm{MPa}$. 


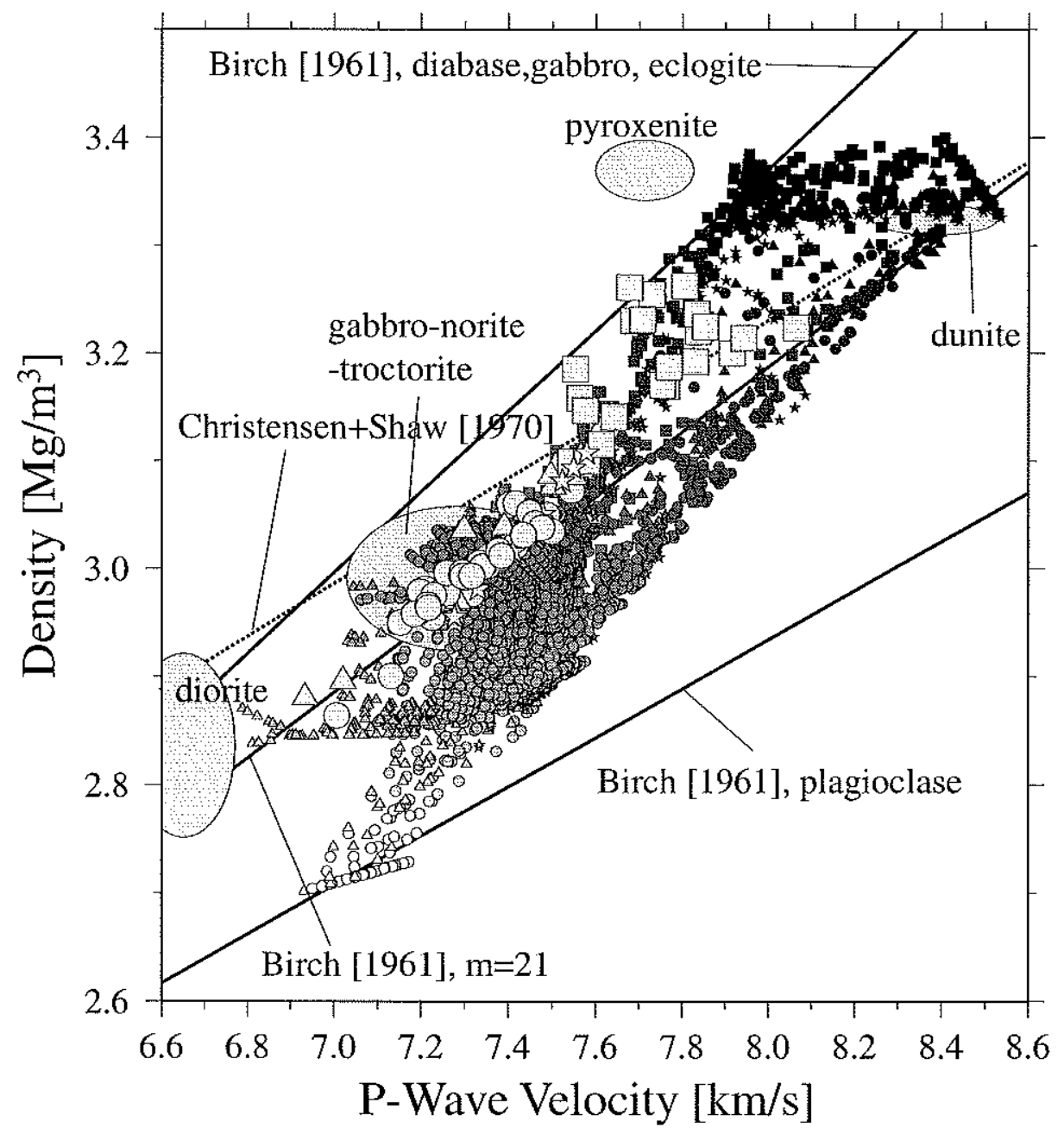

Figure 3-7. Covariation of $P$-wave velocity and density of mafic and ultramafic rocks at $1000 \mathrm{MPa}$ and $25^{\circ} \mathrm{C}$. Large symbols denote norm-based calculations for mantle melts, and small symbols denote fractionated crystal assemblages formed at a range of crustal pressures (100-800 MPa). Ellipses denote laboratory data for diorite, gabbro-noritetroctorite, pyroxenite, and dunite, reported by Christensen and Mooney [1995]. Three solid lines are taken from Birch [1961] for plagioclase, rocks with mean atomic weight of $\sim 21$, and diabase-gabbro-eclogite. Dotted line is for normal oceanic crust based on samples dredged from the Mid-Atlantic Ridge [Christensen and Shaw, 1970]. 
(a)

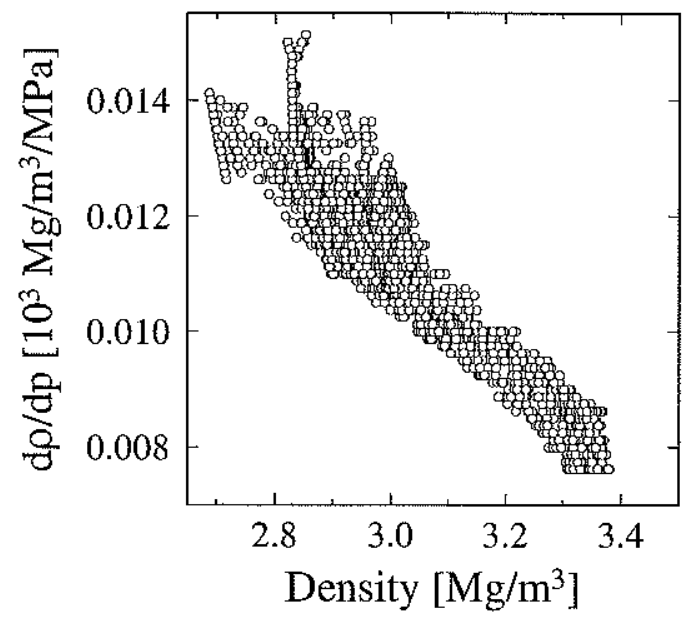

(b)

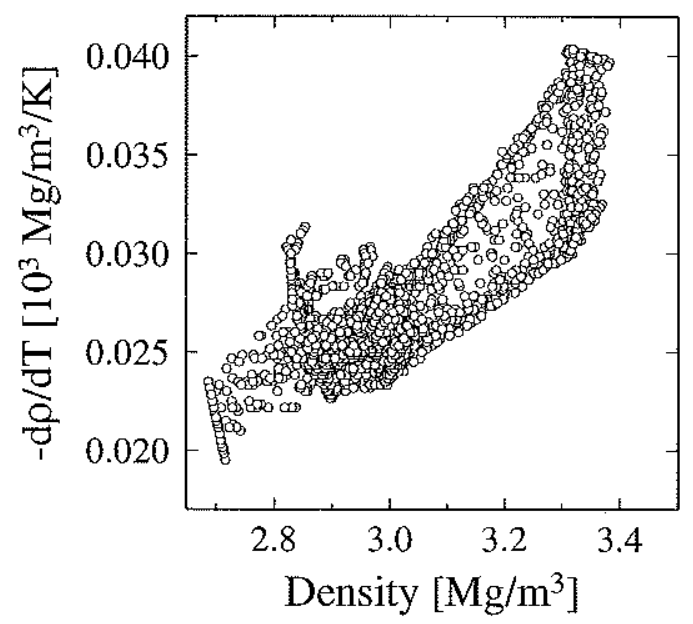

(c)

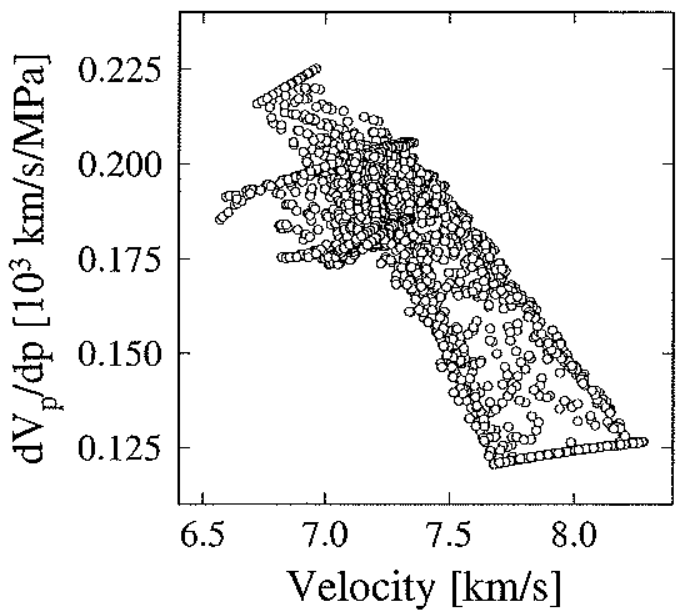

(d)

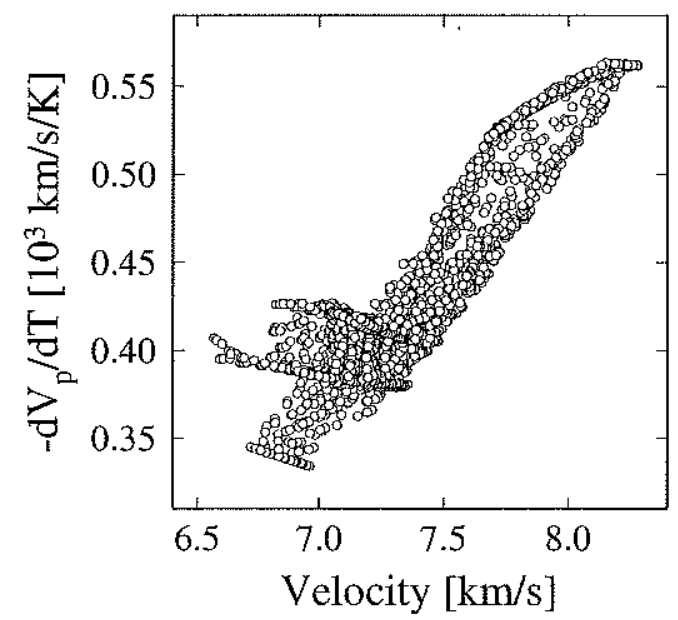

Figure 3-8. Theoretical pressure and temperature derivatives for density and velocity, calculated at $600 \mathrm{MPa}$ and $400^{\circ} \mathrm{C}$, using fractionated crystal assemblages formed at a range of crustal pressures (100-800 MPa). 


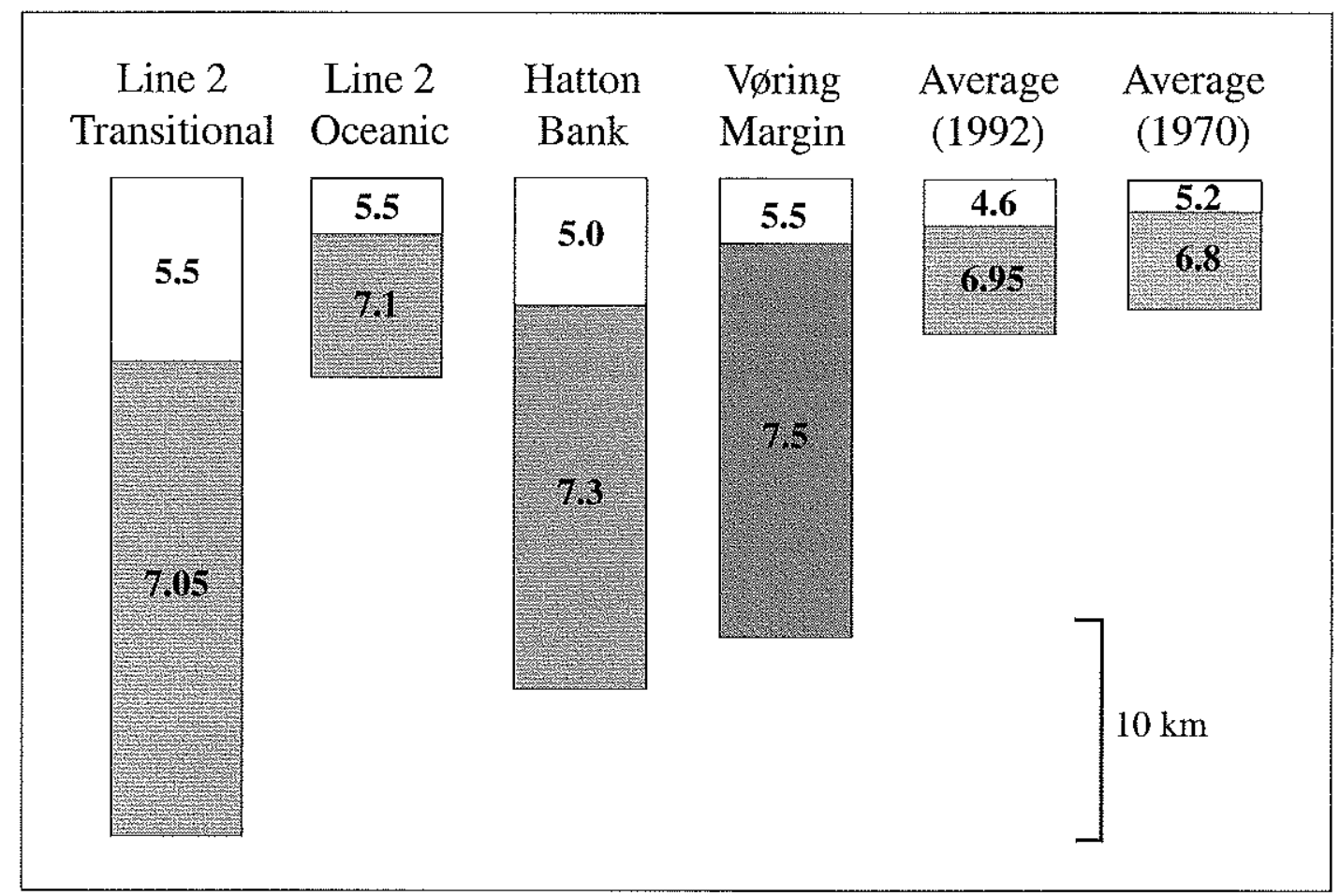

Figure 3-9. Two-layer $P$-wave velocity models (upper and lower crust) for North Atlantic margins and normal oceanic crust. From left to right: transitional and oceanic crust from SIGMA transect 2 (Southeast Greenland margin; Figure 3-2c), Hatton Bank transitional crust [Fowler et al., 1989], Vøring Margin transitional crust [Zehnder et al., 1990], global average of normal oceanic crust compiled by White et al. [1992] and Shor et al. [1970]. 
(a)

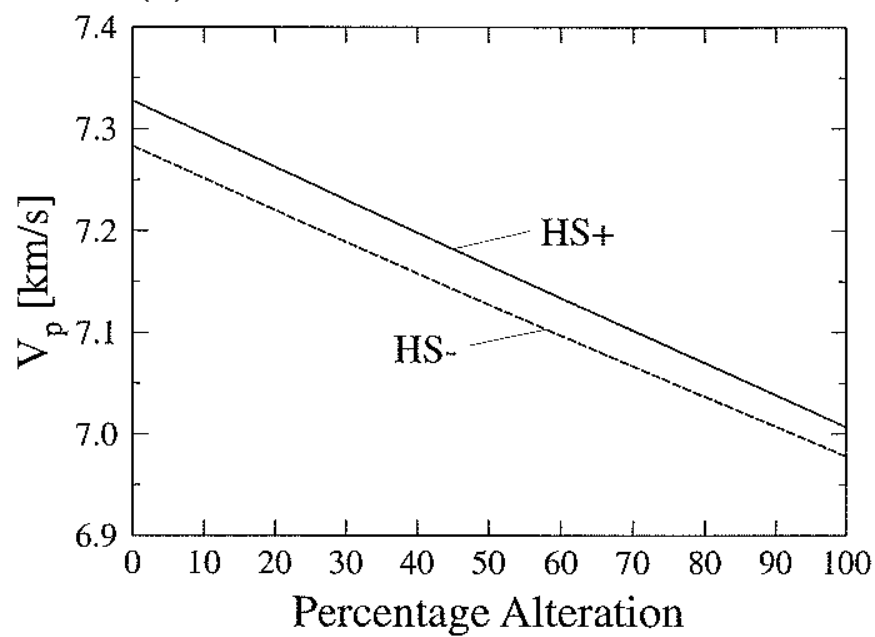

(b)

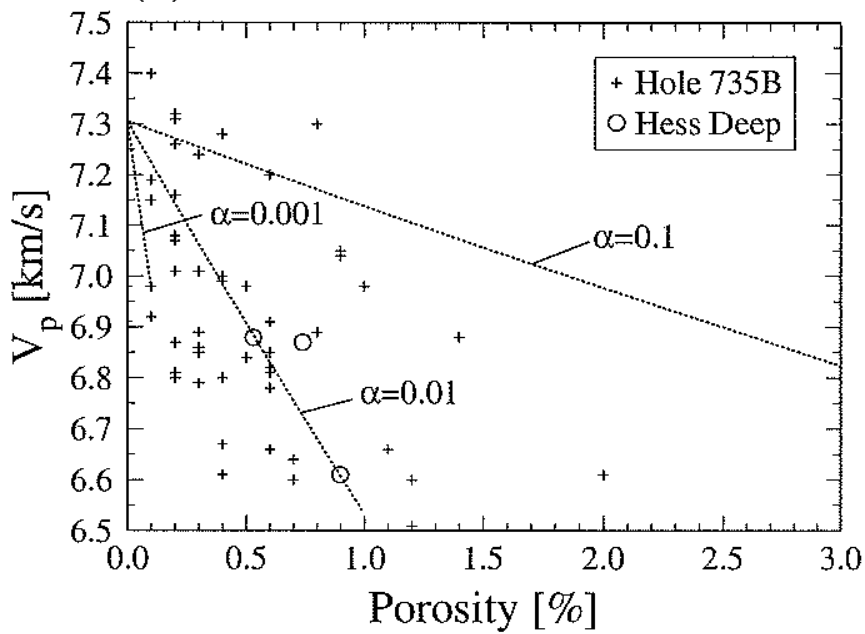

Figure 3-10. (a) Effect of hydrothermal alteration on gabbro velocity. Unaltered gabbro composition used here has $47 \%$ plagioclase $\left(\mathrm{An}_{60}\right), 35 \%$ clinopyroxene $\left(\mathrm{Di}_{80}\right)$, and $18 \%$ olvine $\left(\mathrm{FO}_{75}\right)$, which is the average of oceanic gabbroic rocks recovered from ODP Hole 735B, as reported by Dick et al. [1991]. We assume hydrothermal alteration simply results in the replacement of clinopyroxene with hornblende. Elastic moduli and density are first calculated for each mineral at $100 \mathrm{MPa}$ and $100^{\circ} \mathrm{C}$, and Hashin-Shtrikman bounds for mineral assemblage are then calculated. (b) Effect of residual crack porosity on gabbro velocity. We first calculated effective elastic moduli of dry porous rock with diffrent pore aspect ratio (0.1, 0.01, and 0.001) using the formula of Kuster and Toksöz [1974], and then applied the Gassmann low-frequency relations to obtain effective moduli for fluid saturated porous rock [e.g., Mavko et al., 1998]. Elastic moduli for seawater are taken from Wilkens et al. [1991], and elastic moduli for pore-free gabbro are taken from the average of HashinShtrikman bounds as calculated in (a). The results are shown as dotted lines. Also shown are velocity-porosity data of gabbroic rocks recovered from ODP Hole 735B (cross) [Iturrino et al., 1991] and Hess Deep (open circle) [Iturrino et al., 1996]. While porosity data are measured at atmospheric pressure, velocity data are measured at $200 \mathrm{MPa}$. 


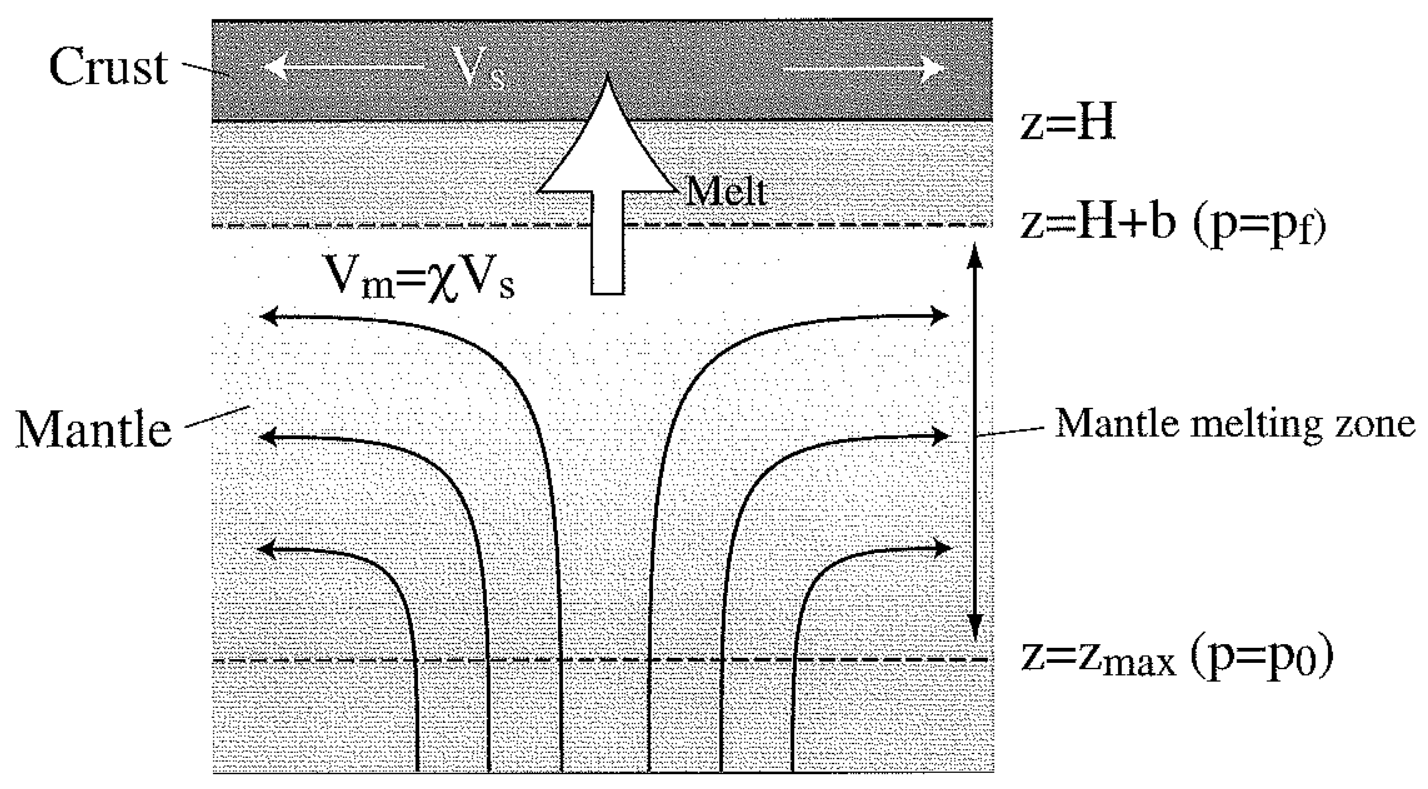

Figure 3-11. Schematic diagram of mantle melting model used in this study. Mantle upwelling rate $\left(V_{m}\right)$ can be faster than surface divergence rate $\left(V_{s}\right)$ by a factor of $\chi$ (active upwelling ratio). The mantle melting zone starts when the mantle adiabat intersects the solidus, and its upper limit is imposed by a lithospheric lid, which is composed of newly formed igneous crust $(H)$, and preexisting lithosphere or cold oceanic mantle $(b)$. 
Effect of active upwelling $(b=0)$

Effect of lithospheric lid $(\chi=1)$
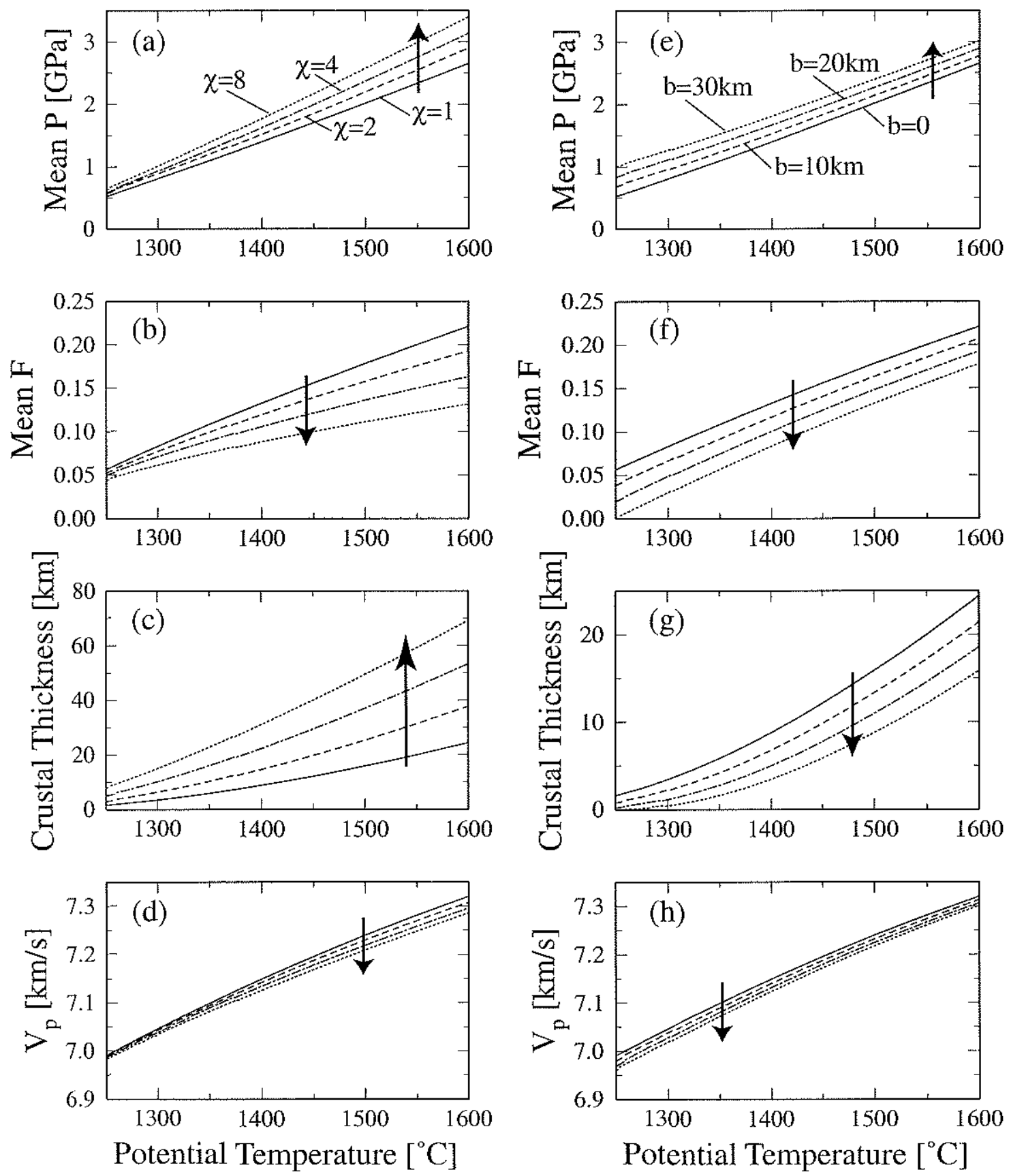

Figure 3-12. (a) Mean pressure of melting, (b) mean fraction of melting, (c) predicted crustal thickness, and (d) predicted bulk crustal velocity, are calculated as a function of mantle potential temperature, for several different active upwelling ratios and zero thickness of preexisting lid. A linear melting function with $(\partial F / \partial P)_{S}$ of $12 \% / \mathrm{GPa}$ is used. Similar calculations with different thicknesses of preexisting lid and passive upwelling are presented in (e)-(h). 
(a) Standard $\mathrm{H}-\mathrm{V}_{\mathrm{p}}$ diagram

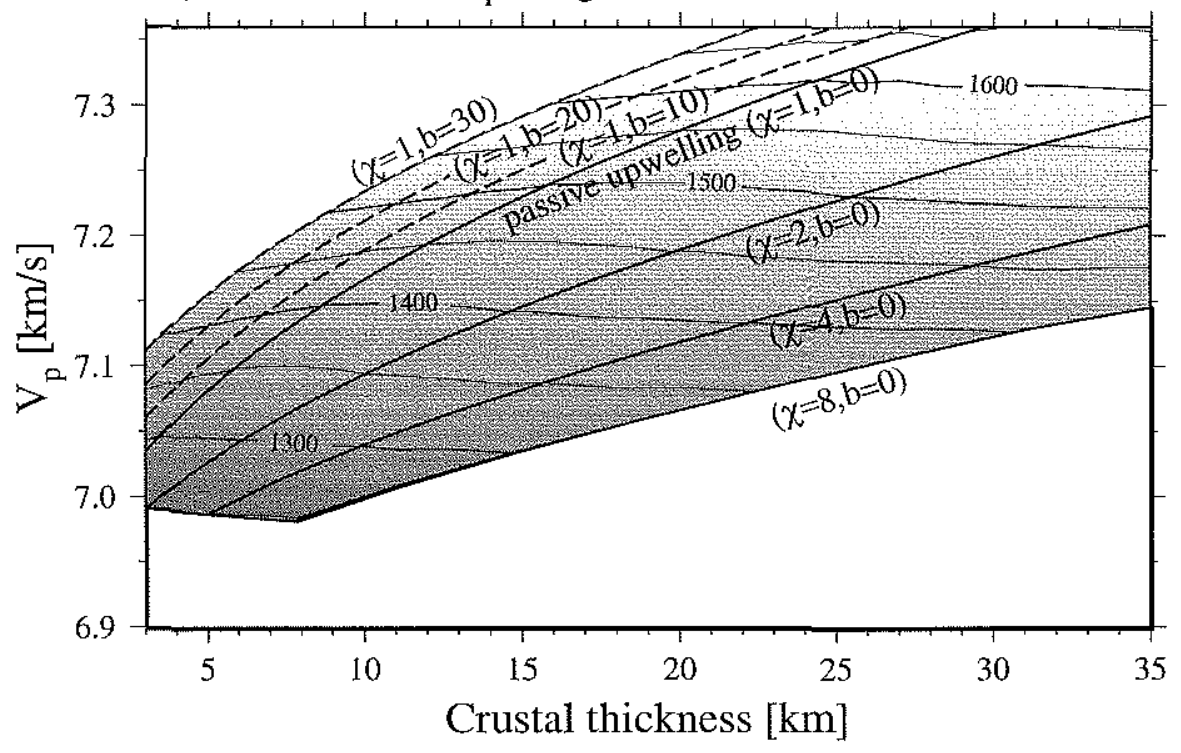

(b) $\mathrm{KH} 95 \mathrm{H}-\mathrm{V}_{\mathrm{p}}$ diagram

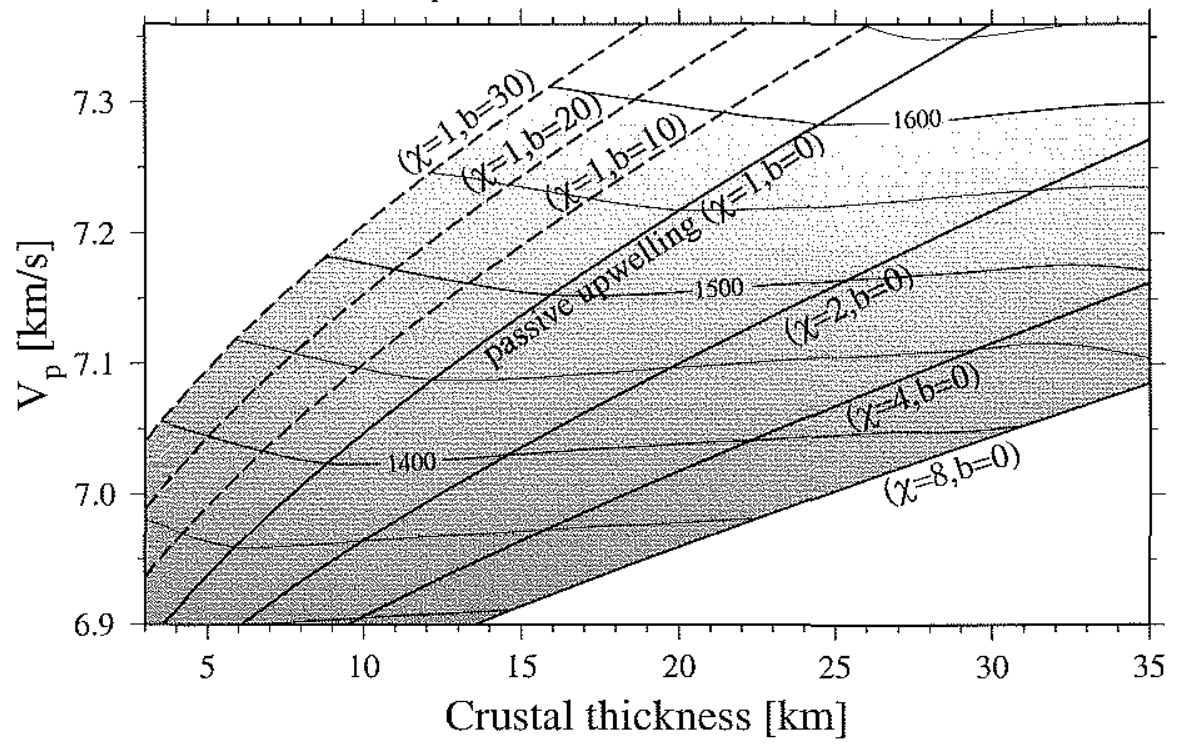

Figure 3-13. (a) Standard $H-V_{p}$ diagram based on calculations presented in Figure 3-12. The contours of mantle potential temperature are drawn at $50^{\circ} \mathrm{C}$ interval. (b) Same as (a), but with the empirical relation of KH95 (equation 3.2). 
(a) $(\mathrm{dF} / \mathrm{dP})_{\mathrm{S}}=16 \% / \mathrm{GPa}$

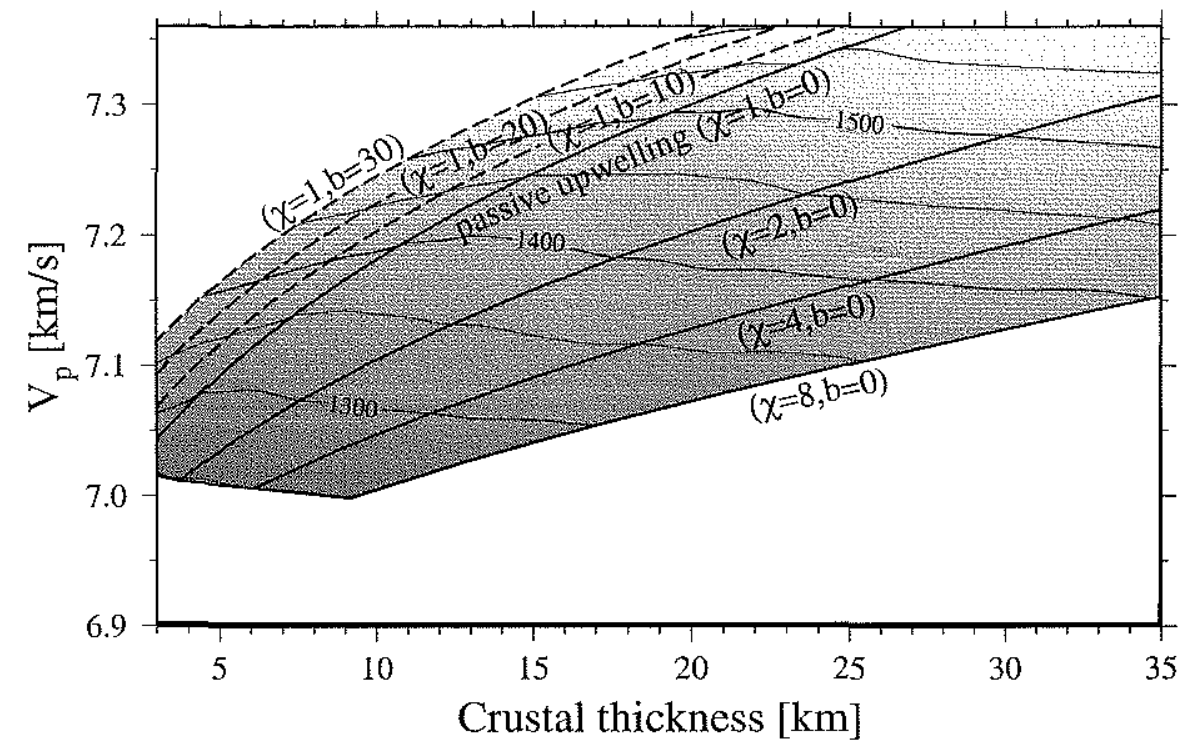

(b) variable $(\mathrm{dF} / \mathrm{dP})_{\mathrm{S}}$ model

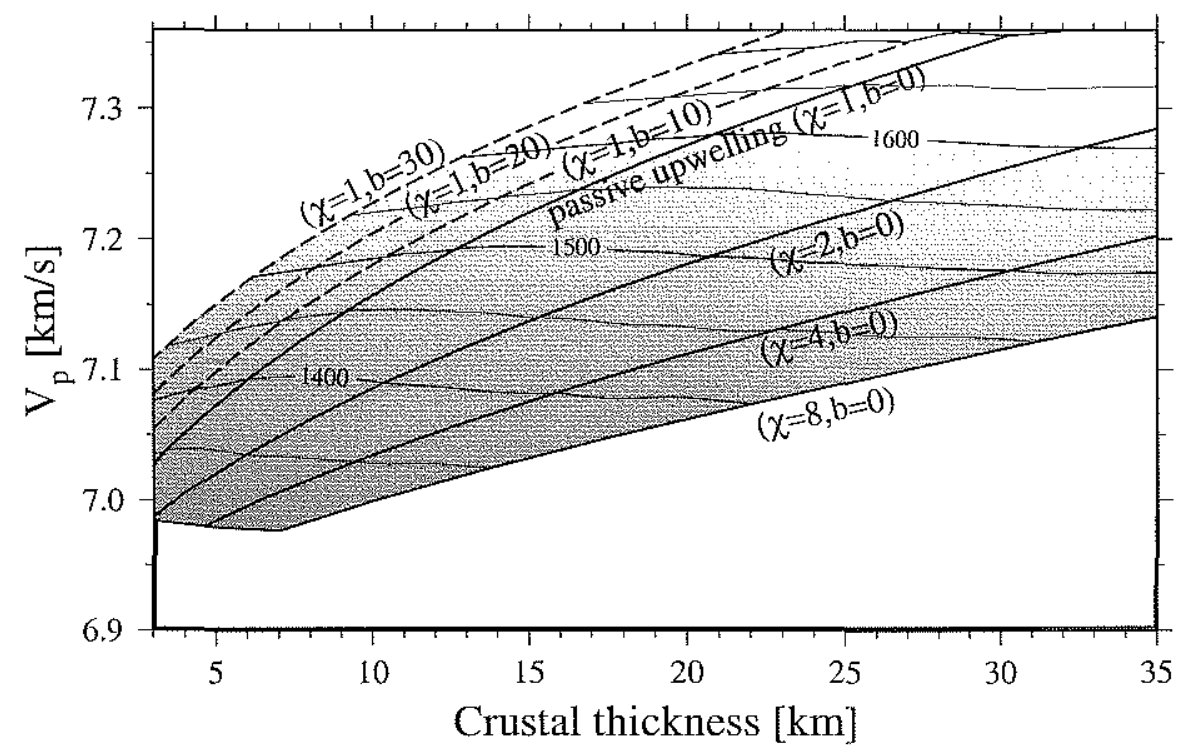

Figure 3-14. (a) Same as Figure 3-13a, but with $(\partial F / \partial P)_{S}$ of $16 \% / \mathrm{GPa}$. (b) Same as Figure 3-13a, but with a three-stage melting function to simulate the effect of clinopyroxene exhaustion; $F_{1}=0.035 d P(d \quad P<1.15), \quad F_{2}=0.23(d P-1.15)+0.04$ $(0.15 \leq d P<0.175)$, and $F_{3}=0.113(d P-1.75)+0.18(d P \geq 1.75)$, where $d P$ denotes $P_{o}-P$. This is a crude approximation of the results of thermodynamic calculations by Asimow et al. [1997] 
(a)

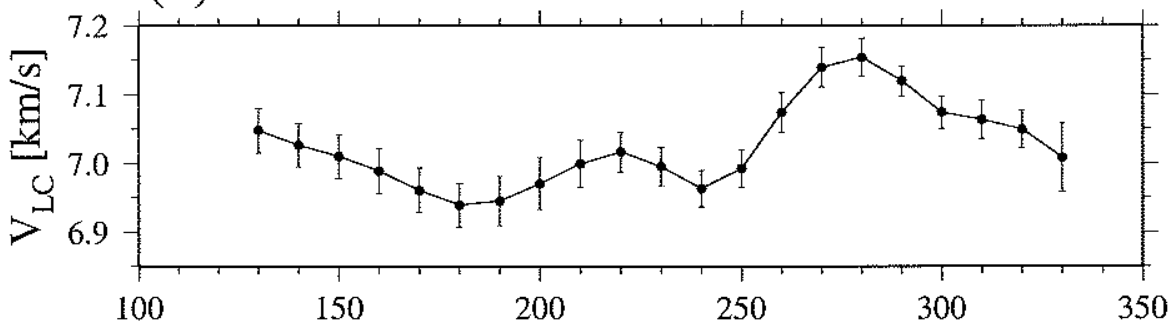

(b)

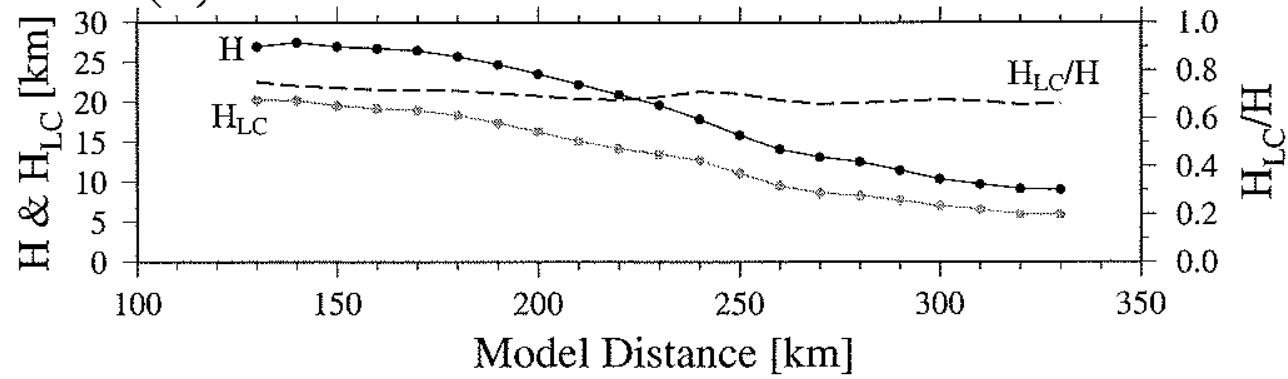

(c)

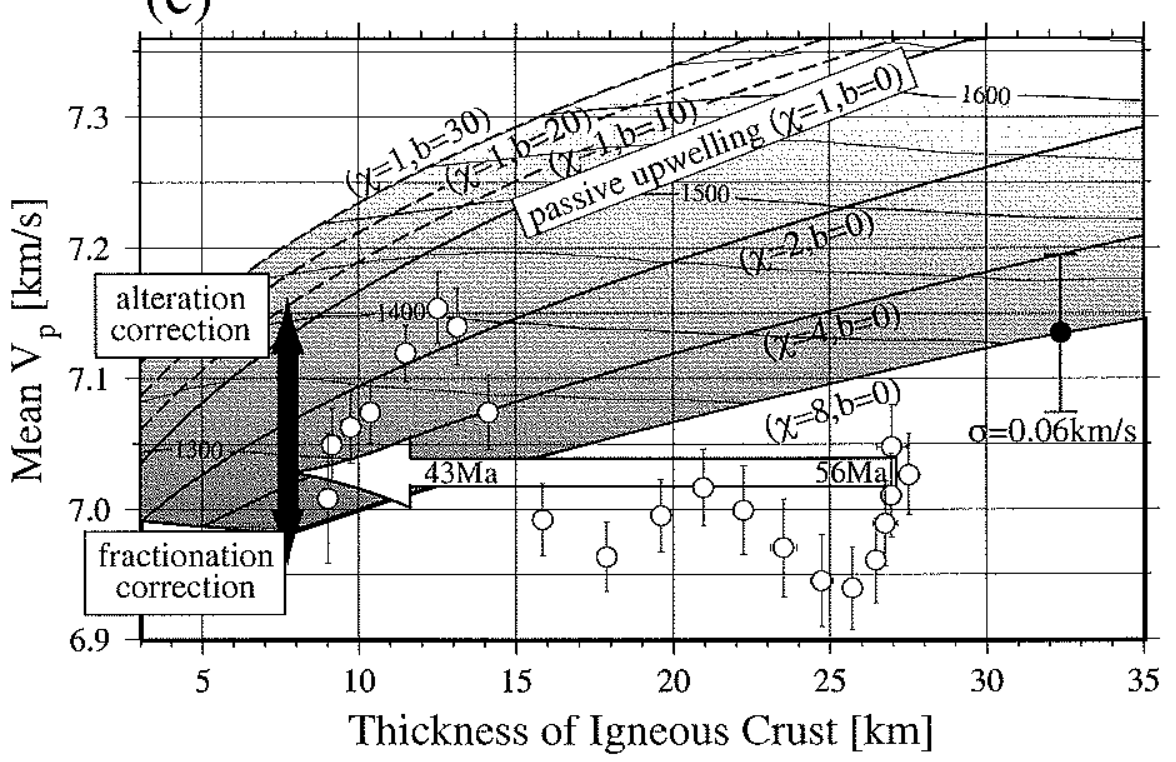

Figure 3-15. (a) Harmonic-mean velocity of the lower-crustal part of SIGMA transect 2 is calculated at $10 \mathrm{~km}$ interval with a $20 \mathrm{~km}$ wide averaging window, using 100 Monte Carlo ensembles (Chapter 1). (b) Whole-crustal (solid) and lower-crustal (gray) thicknesses are calculated in a similar manner. Lower-crustal fraction is also shown as dashed. (c) Mean velocity of the igneous lower crust is plotted as a function of corresponding whole-crustal thickness (open circles with error bars), on the standard $H$ $V_{p}$ diagram (Figure 3-13a). 


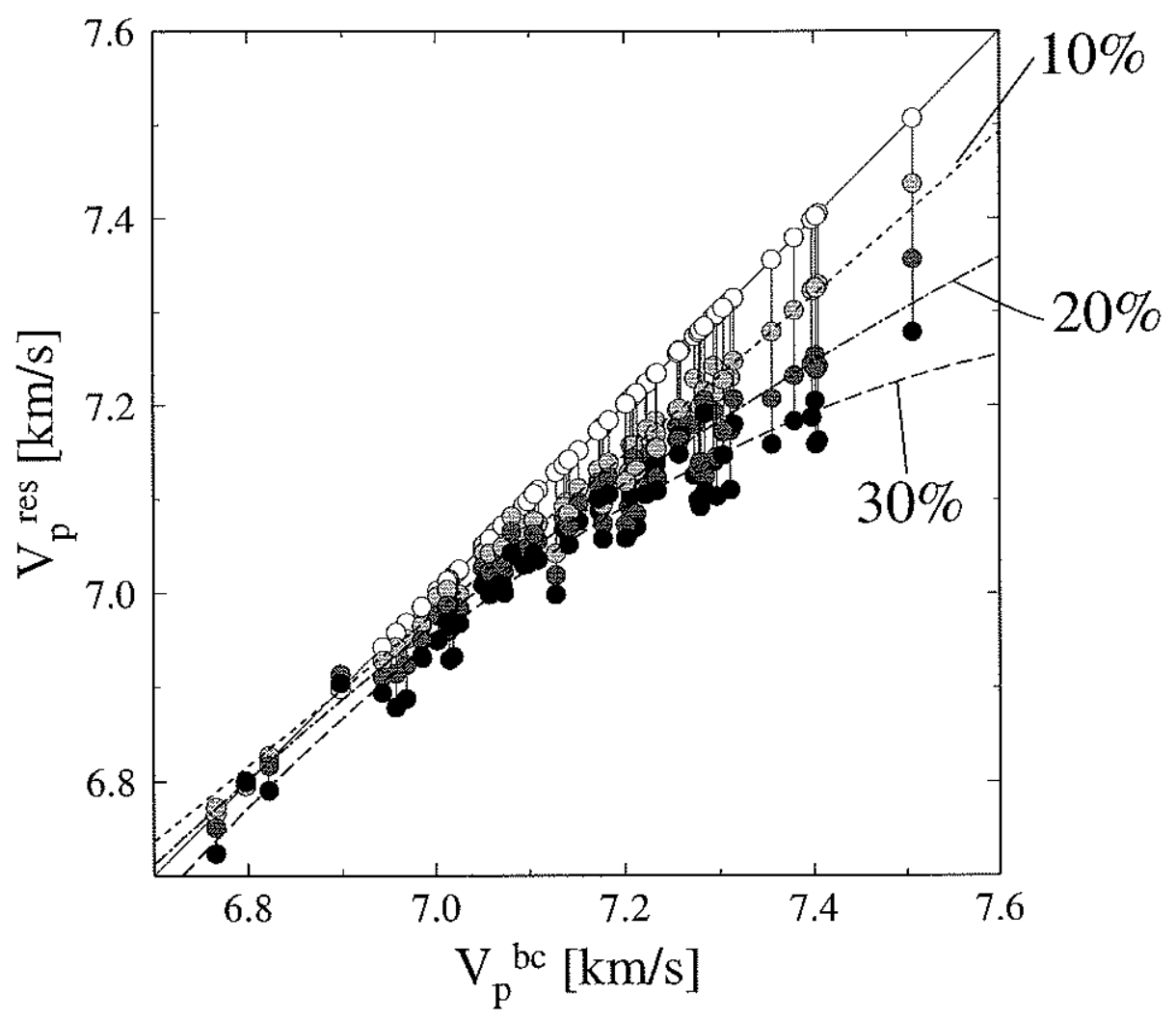

Figure 3-16. Effect of subcrustal fractionation. $P$-wave velocities of residual phases $\left(V^{r e s}\right)$ formed by fractional crystallization at $1 \mathrm{GPa}$ are plotted as a function of original bulk crustal velocity $\left(V^{b c}\right)$, for subcrustal fractionation of 10,20 , and $30 \%$. Results of quadratic regression are: $V^{\text {res }}=3.52+0.16 \mathrm{~V}+$ $0.046 V^{2}(1 \sigma=0.014)$ for $F=10 \%, V^{r e s}=-9.56+3.97 V-0.225 V^{2}(1 \sigma=0.020)$ for $F=20 \%$, and $V^{r e s}=-22.63+7.65 V-0.489 V^{2}(1 \sigma=0.025)$ for $F=30 \%$. 


\title{
Chapter 4
}

\section{Major-element source heterogeneity in the North Atlantic igneous province}

\begin{abstract}
High-MgO ( $>8.5 \mathrm{wt} \%)$, aphyric lavas erupted at various locations in the North Atlantic igneous province are analyzed to characterize the nature of mantle melting during the formation of this province. Based on the observation that the Ni concentration in residual mantle olivine mostly falls in the range of $2000-3500 \mathrm{ppm}$, these high-MgO samples are corrected for olivine fractionation until the $\mathrm{Ni}$ concentration of equilibrium olivine reaches $3500 \mathrm{ppm}$, to estimate the composition of primary mantle-derived melt. Estimated primary melt compositions suggest that this province is characterized by a large degree of major element source heterogeneity possibly resulting from basalt addition prior to melting. Primary melts for North Iceland and Southwest Iceland are shown to require different source mantle compositions. Whereas the North Iceland primary melt may be explained by the melting of normally assumed pyrolitic mantle, the source mantle for Southwest Iceland must be enriched in iron, having molar $\mathrm{Mg} /(\mathrm{Mg}+\mathrm{Fe})<0.87$. This compositional dichotomy in Iceland seems to continue to adjacent Mid-Atlantic Ridge segments, i.e., the Kolbeinsey and Reykjanes Ridges. The primary melts for East and Southeast Greenland also indicate a fertile mantle source, and the estimate of molar $\mathrm{Mg} /(\mathrm{Mg}+\mathrm{Fe})$ is the lowest for the East Greenland source mantle $(<0.86)$ among all regions we investigated. The inferred spatial extent of source heterogeneity suggests an important and complex role for a long-lived compositional anomaly in this igneous province since the opening of the North Atlantic.
\end{abstract}

\subsection{Introduction}

When Greenland rifted from Europe at about $60 \mathrm{Ma}$, vigorous magmatism took place along the $\sim 2000$-km-long rifting axis, as indicated by the distribution of seaward-dipping reflectors on the North Atlantic continental margins [Mutter et al., 1984; Larsen and Jakobsdóttir, 1988; Mutter and Zehnder, 1988; White and McKenzie, 1989]. Within $10 \mathrm{~m} . \mathrm{y}$. or so, this spatially extensive voluminous magmatism seems to have rapidly reduced in area, but it continued to exist as the current Iceland hotspot (Figure 4-1). The impact of an ancestral Iceland plume has been proposed to explain the formation of this North Atlantic igneous province in a unified fashion [e.g., Richards et al., 1989; White and McKenzie, 1989; Hill, 1991; White and McKenzie, 1995]. One way to test this plume impact hypothesis is to use the geochemistry of erupted lavas in this province to deduce the process of mantle melting and its causal mantle dynamics. The major element composition 
of mantle-derived melt is sensitive to the pressure and degree of melting as well as the style of melting (i.e., batch vs. fractional) [e.g., Klein and Langmuir, 1987; McKenzie and Bickle, 1988; Kinzler and Grove, 1992; Langmuir et al., 1992; Kinzler, 1997]. If one can estimate primary melt composition from observed lavas, therefore, useful constraints on mantle melting may be obtained based on the major element systematics. Mantle potential temperature can be estimated, and if the thickness of igneous crust is known, the rate of active mantle upwelling can also be calculated. Both types of information are necessary to constrain the thermal state of mantle and its dynamics.

Since erupted lavas are expected to have been modified from their primary composition during ascent to the surface, we need to correct for possible modification processes. Fractional crystallization is probably the most important of these processes. At low pressures, fractionating phases from basaltic liquids are generally olivine, then plagioclase, and then clinopyroxene. At high pressures ( $>0.5 \mathrm{GPa})$, clinopyroxene tends to appear earlier than plagioclase [e.g., Bender et al., 1978; Presnall et al., 1978; Grove et al., 1992; Langmuir et al., 1992]. In either case, it is difficult to accurately correct for multiphase fractionation, and it is common to apply up-temperature correction only to $\mathrm{MgO}$-rich samples by incrementally adding equilibrium olivine, assuming that olivine was the only fractionating phase. In this correction, some criterion has to be adopted to determine how much olivine must be added. For example, Fram and Lesher [1997], applied an olivine fractionation correction until the liquid $\mathrm{Mg}$ number (defined as molar $\mathrm{Mg} /(\mathrm{Mg}+\mathrm{Fe})$ ) reaches 0.70 or 0.76 , using an olivine/liquid $\mathrm{Fe} / \mathrm{Mg} \mathrm{K}_{\mathrm{D}}$ of 0.31 , assuming that all iron as $\mathrm{FeO}$, and assuming that the forsterite content of residual mantle olivine may vary from 0.88 to 0.91 .

We found problems in trying to use this approach to study melting dynamics in the North Atlantic igneous province. Figure 4-2 shows the compilation of recent mantle melting experiments in terms of liquid $\mathrm{Mg}$ number and melt fraction. The $\mathrm{Mg}$ number of primary melt depends on source olivine composition and melting processes. The $\mathrm{Mg}$ number criterion, therefore, cannot serve our purpose of using primary melt composition to infer melting processes. Another problem with the $\mathrm{Mg}$ number criterion is that it is 
impossible to detect source heterogeneity from estimated primary melts. Though major element heterogeneity in source mantle is still poorly understood compared to isotope and trace element heterogeneity, its importance has been suggested for several hotspots including Iceland [e.g., Langmuir and Hanson, 1980; Schilling et al., 1983; Hauri, 1996; Takahashi et al., 1998]. The main process responsible for major element heterogeneity is probably the removal or addition of a basaltic component [e.g., Langmuir and Hanson, 1980; Frey et al., 1985; Allègre and Turcotte, 1986]. If this is the case, source olivine composition can serve as a proxy for the degree of heterogeneity. Correcting olivine fractionation based on some pre-determined composition of residual olivine, therefore, can mask the signature of major element source heterogeneity that may exist in lava samples.

An alternative criterion that can be used in olivine fractionation correction is the $\mathrm{Ni}$ content of residual olivine [Allègre et al., 1977; Minster et al., 1977; Sato, 1977]. Because $\mathrm{Ni}$ is compatible in olivine, its concentration in mantle olivine is relatively insensitive to the past history of depletion, and the addition of a basaltic component only lowers the concentration. A recent compilation of $\mathrm{Ni}$ content in mantle olivine in continental peridotites [Kelemen et al., 1998] is shown as histograms in Figure 4-3. Despite variability in the forsterite content, which is most likely the result of variable depletion history, $\mathrm{Ni}$ concentration clusters around $3000 \mathrm{ppm}$, and most of the data are between $2500 \mathrm{ppm}$ and $3500 \mathrm{ppm}$. The Ni content of olivine in oceanic peridotites (H.J.B. Dick, personal communication) is also shown in Figure 4-3, and it shows a similarly tight distribution, but with a lower mean value $(-2500 \mathrm{ppm})$. Thus, a value of $3500 \mathrm{ppm}$ can be confidently used as the upper limit on the Ni content of residual mantle olivine, regardless of the degree of major element heterogeneity. Our procedure for olivine fractionation correction is, therefore, (1) calculate olivine composition in equilibrium with liquid using an $\mathrm{Fe}-\mathrm{Mg}$ exchange coefficient, $K_{\mathrm{D}}^{\mathrm{Fc}-\mathrm{Mg} g}$ and the Ni partition coefficient, $D_{\mathrm{Ni}}{ }^{\mathrm{o} / / \mathrm{iq}}$, (2) add $0.1 \mathrm{wt} \%$ of the equilibrium olivine to liquid, and (3) repeat 1 and 2 until the Ni content of equilibrium olivine reaches $3500 \mathrm{ppm} . K_{\mathrm{D}}^{\mathrm{Fe}-\mathrm{Mg}}$ is nearly constant around 0.30 , for a wide range of liquid composition, temperature, and pressure [e.g., Roeder and Emslie, 1970; Ulmer, 1989; Johnes, 1995], but $D_{\mathrm{Ni}}^{\text {ol/iiq }}$ is strongly compositionally-dependent [e.g., Hart and Davis, 
1978; Takahashi, 1978; Nabelek, 1980; Kinzler et al., 1990](Figure 4-4). We use the equation of Kinzler et al. [1990], which incorporates all previous experimental studies, to calculate $D_{\mathrm{Ni}}^{\mathrm{o} / \mathrm{l} \mathrm{ig}}$ at each correction step,

The purpose of this paper is to estimate primary mantle-derived melts, using this olivine fractionation correction, for the North Atlantic igneous province on the basis of published geochemical data. The estimated melt compositions will then be discussed in light of recent experimental studies of mantle melting. Our results indicate an important role of major element source heterogeneity in the formation of this igneous province. To begin with, we will review the validity of olivine-only fractionation correction for high$\mathrm{MgO}$ samples through a series of crystallization modeling.

\subsection{Behavior of Ni in Crustal Modification Processes}

Fractional crystallization is not the only process to affect the composition of mantlederived melt. To facilitate our discussion on possible crustal modification processes, the modeling of crystal fractionation is conducted at a range of crustal pressures, starting from several sample melt compositions. This exercise is also instructive to identify the type of volcanic suites to which our olivine fractionation correction can be safely applied. To calculate the liquid line of descent, we use the method of Weaver and Langmuir [1990] with extension for high-pressure crystallization by Langmuir et al. [1992]. Initial liquids are chosen to cover a reasonably wide range of $\mathrm{MgO}$ concentration (10-18\%; Figure 4-5), and are assumed to be in equilibrium with mantle olivine that has a Ni concentration of $3000 \mathrm{ppm}$ (Figure 4-4b). The Ni concentration of liquid is tracked using the equation of Kinzler et al. [1990] for olivine-liquid partitioning, and assuming that the partitioning between clinopyroxene and liquid is one sixth of that between olivine and liquid and that $\mathrm{Ni}$ is perfectly incompatible with plagioclase [e.g., Irving, 1978; Johnes, 1995]. The modeling results are shown in terms of the $\mathrm{Ni}$ and $\mathrm{MgO}$ contents of liquid as well as the phase proportions of fractionated minerals (Figure 4-5). Both fractional and equilibrium crystallization paths are calculated, and as Langmuir [1989] showed, the liquid line of descent resulting from a periodically replenished magma chamber [O'Hara, 1977; O'Hara 
and Matthews, 1981] is bounded by these two fractionation paths. Except for the initial liquid with the lowest $\mathrm{MgO}$ content, olivine is the first phase to crystallize, and at low pressure, it is the only fractionating phase until the liquid $\mathrm{MgO}$ content drops below $10 \%$ (Figure 4-5a).

The appearance of plagioclase or clinopyroxene as the second fractionating phase introduces some complication in the $\mathrm{Ni}-\mathrm{MgO}$ covariation, because the bulk partition coefficients of $\mathrm{Ni}$ and $\mathrm{MgO}$ are both modified by the second phase. Whereas olivine+plagioclase fractionation leads to a continuing decrease in both $\mathrm{Ni}$ and $\mathrm{MgO}$ concentration (e.g., curve B in Figure 4-5a), olivine+clinopyroxene fractionation reduces the variation in $\mathrm{Ni}$ content as $\mathrm{MgO}$ continues to decrease (e.g., curve $\mathrm{C}$ in Figure 4-5b). This difference is vital for the use of an olivine-only fractionation correction. If the correction is applied to a sample on the olivine+plagioclase fractionation path, the MgO content of primary melt tends to be overestimated, and if applied to a sample on the olivine+clinopyroxene fractionation path, it would be underestimated. Since the $\mathrm{MgO}$ content of mantle-derived melt strongly depends on the degree of melting [e.g., McKenzie and Bickle, 1988; Kinzler and Grove, 1992; Langmuir et al., 1992; Baker and Stolper, 1994; Walter, 1998], the nature of mantle melting inferred from estimated primary melt composition is highly sensitive to the validity of the assumption of olivine-only fractionation. Though the possibility of plagioclase fractionation may be avoided by using samples only with more than $10 \mathrm{wt} \% \mathrm{MgO}$, clinopyroxene can appear above this threshold in high-pressure crystallization (Figure 4-5b). However, that clinopyroxene fractionation without plagioclase would result in a large change in $\mathrm{CaO} / \mathrm{Al}_{2} \mathrm{O}_{3}$, and such a signal at $\mathrm{MgO}$ higher than 10wt\% has not been found in our study area [e.g., Meyer et al., 1985; Nicholson et al., 1991; Fram and Lesher, 1997; Thy et al., 1998]. Therefore, we regard the high-pressure liquid line of descent with early clinopyroxene fractionation (e.g., Figure $4-5 b$, liquid C) as a rather rare case, and will not consider its possibility hereafter.

Assimilation and fractional crystallization (AFC) [DePaolo, 1981] and in situ crystallization [Langmuir, 1989] are also plausible modification processes, but their importance is limited in our study. The AFC process in the oceanic environment is, for 
example, equivalent to a process in a periodically replenished magma chamber, because an assimilant may typically have a primary melt composition, so its effect is already represented by our crystallization modeling. Though this simplification does not apply to continental flood basalts, in which an assimilant can be continental crust, potential complexity introduced by the AFC process can be circumvented by restricting our analysis to samples likely to be uncontaminated by continental crust. In situ crystallization leads to the mixing of primary liquid with more differentiated liquids. Because the Ni-MgO covariation is almost linear for high $\mathrm{MgO}$ liquids except for high-pressure clinopyroxene fractionation, this process does not significantly affect the modeled liquid lines of descent.

It thus seems that olivine-only fractionation correction is a robust procedure to estimate primary melt composition, if it is applied only to uncontaminated samples with $>10 \mathrm{wt} \% \mathrm{MgO}$. We found, however, that this criterion for sample screening is so strict that the number of available data is severely reduced. By relaxing the $\mathrm{MgO}$ threshold down to $8.5 \mathrm{wt} \%$, we will attempt to obtain the upper bound on the amount of olivine used in correction. Possible plagioclase fractionation could only lower the amount of olivine addition required for a given $\mathrm{Ni}$ criterion. This upper bound approach also conforms to our use of a "maximum" value of $3500 \mathrm{ppm} \mathrm{Ni}$ in mantle olivine as the endpoint of the fractionation correction. The estimated $\mathrm{MgO}$ content, therefore, can be regarded as the upper limit on its primary content. On the other hand, plagioclase fractionation can substantially increase liquid $\mathrm{SiO}_{2}$ and $\mathrm{FeO}$, and if ignored in a fractionation correction, could lead to an overestimate of $\mathrm{SiO}_{2}$ and $\mathrm{FeO}$ contents in primary melt. Care must be taken, therefore, to verify that the $\mathrm{SiO}_{2}$ and $\mathrm{FeO}$ contents of estimated primary melt are not biased by results of fractionation correction on low-MgO samples.

\subsection{Composition of Primary Mantle Melts for the North Atlantic Igneous Province}

To apply our olivine fractionation correction with the olivine Ni criterion, samples must be aphyric, have $\mathrm{MgO}$ higher than $8.5 \mathrm{wt} \%$, and have reported Ni concentration. Though there are numerous petrological and geochemical studies on the North Atlantic igneous province 
(see, for example, references cited in Fram and Lesher [1997]), the number of samples that can satisfy the above three conditions is limited. The majority of reported lava samples are polyphyric. When a modal analysis is given, we classified samples with less than 5\% phenocrysts as "sub-aphyric", and both aphyric and "sub-aphyric" samples are used for the fractionation correction. Six regions are identified to have sufficient data for the estimation of primary melt composition: Southeast Greenland, East Greenland, North Iceland, Southwest Iceland, the Reykjanes Ridge, and the Kolbeinsey Ridge [Wood et al., 1979; Schilling et al., 1983; Hemond et al., 1993; Slater, 1996; Fram and Lesher, 1997; Larsen et al., 1998] (Figure 4-1).

All data are normalized on an anhydrous basis. All iron is treated as ferrous (i.e., $\left.\mathrm{Fe}^{2+} /\left(\mathrm{Fe}^{2+}+\mathrm{Fe}^{3+}\right)=1.0\right)$, but the following results would be only slightly modified if we used $\mathrm{Fe}^{2+} /\left(\mathrm{Fe}^{2+}+\mathrm{Fe}^{3+}\right)=0.9$. A constant $K_{\mathrm{D}}^{\mathrm{Fe}-\mathrm{Mg}}$ of 0.31 is used to calculate equilibrium olivine composition throughout the correction; changing this value by \pm 0.01 has only minor influence on our results. In addition to the correction for fractional crystallization as already described, we also calculate correction for equilibrium crystallization of olivine, to illustrate the extent of ambiguity introduced by a periodically replenished magma chamber. The equilibrium crystallization correction does not always converge to the target $\mathrm{Ni}$ concentration of equilibrium olivine, because it leads to large increases in liquid $\mathrm{MgO}$ combined with very small increases in Ni content. Since Ni becomes less compatible with increasing liquid $\mathrm{MgO}$ (Figure 4-4), equilibrium olivine for such high $\mathrm{MgO}$ and low $\mathrm{Ni}$ liquid often fails to reach the $\mathrm{Ni}$ concentration of $3500 \mathrm{ppm}$. The total amount of olivine that can be fractionated from mantle-derived melt is, however, usually less than $30 \%$ of initial liquid (e.g., Figure 4-5), so that we use a maximum olivine fraction of 0.3 as a second criterion when correcting for equilibrium crystallization.

Data for Southeast Greenland are from submarine flood basalt sequences drilled in Leg 152 of the Ocean Drilling Program [Larsen et al., 1994; Larsen et al., 1998]. Following Larsen et al. [1998], we use $\mathrm{Ba} / \mathrm{Zr}$ as the index of crustal contamination, and screen out samples with $>1.5 \mathrm{Ba} / \mathrm{Zr}$. Uncontaminated lavas are then almost entirely confined to the Upper Series of Hole 917A. The results of fractionation correction are 
shown in Figure 4-6. The correction paths are plotted with the corresponding Ni concentrations in equilibrium olivine. The $\mathrm{SiO}_{2}, \mathrm{MgO}, \mathrm{FeO}, \mathrm{TiO}_{2}$, and $\mathrm{Na}_{2} \mathrm{O}$ contents of liquid are displayed as well as the Fo content of equilibrium olivine. The correction paths for equilibrium crystallization generally deviate from overall trends shown by original lavas, and we infer that fractional crystallization is the dominant modification process. Fractionation correction tends to increase $\mathrm{MgO}$ variability, compared to the lavas, because of the compositional dependence of Ni partitioning. The estimated primary $\mathrm{MgO}$ contents at $3500 \mathrm{ppm} \mathrm{Ni}$ in olivine vary from $12.7 \mathrm{wt} \%$ to $18.0 \mathrm{wt} \%$, but most are below $16.0 \mathrm{wt} \%$. Olivine addition does not significantly modify $\mathrm{FeO}$, and the mean of the primary FeO contents is $11.8 \mathrm{wt} \%$. $\mathrm{SiO}_{2}, \mathrm{TiO}_{2}$, and $\mathrm{Na}_{2} \mathrm{O}$ all decrease as a result of fractionation correction. Their variability in the estimated primary liquids reflects the original sample variability, without noticeable amplification. Two high-MgO, aphyric lavas have equilibrium olivine with $>3500$ ppm Ni (Figure 4-6b), so they are not considered in our correction. These aphyric lavas have higher $\mathrm{MgO}$ and lower $\mathrm{FeO}$ than the average of estimated primary melt compositions, suggesting that they may have resulted from the dissolution of cumulative olivine by superheated melt. Interestingly, Thy et al. [1998] interpreted them (referred as Unit 14 and Unit 16 in their Table 7) as a candidate of primary melt, mainly because they are aphyric with very high $\mathrm{MgO}$ contents.

The data from East Greenland early Tertiary flood basalts are from Fram and Lesher [1997], and the results of fractionation correction are shown in Figure 4-7. The identification of uncontaminated lavas follows their approach based on ${ }^{143} \mathrm{Nd} /{ }^{144} \mathrm{Nd}, \mathrm{La} / \mathrm{Zr}$, and $\mathrm{Zr} / \mathrm{Ti}$. Only two aphyric lavas can be used in our correction, so the statistical reliability of our estimate is uncertain. It should be noted, however, that samples in this region, including phyric lavas, are systematically higher in $\mathrm{SiO}_{2}$ and $\mathrm{TiO}_{2}$ and lower in $\mathrm{Na}_{2} \mathrm{O}$ than those in Southeast Greenland, implying some fundamental difference in the nature of mantle melting between these two regions. Olivine in equilibrium with our estimated primary melt compositions has a forsterite content of 0.86 , significantly lower than conventionally assumed residual olivine compositions $\left(\mathrm{FO}_{89-91}\right)$. Fram and Lesher [1997] also conducted an olivine fractionation correction using the same data by assuming 
residual olivine of $\mathrm{Fo}_{88-91}$, resulting in much higher $\mathrm{MgO}(15.2-19.7 \mathrm{wt} \%)$ and lower $\mathrm{SiO}_{2}$ $(48.2-47.2 \mathrm{wt} \%)$. As emphasized in the Introduction, their estimate of primary melt composition depends on the assumption of a primary liquid $\mathrm{Mg}$ number, which now seems questionable, especially for lavas sampled from hotspots. Correction for fractional crystallization to a liquid in equilibrium with $\mathrm{Fo}_{90}$ olivine yields a Ni-rich liquid, with corresponding $\mathrm{Ni}$ in olivine $>6000 \mathrm{ppm}$.

Iceland has three major neovolcanic zones: the western rift zone (Southwest Iceland), the mid-Iceland belt, and the eastern rift zone (North Iceland) [e.g., Hemond et al., 1993]. As already noted, liquid FeO varies little during olivine-only fractionation, so that the $\mathrm{FeO}$ content of high-MgO lava is a good proxy for its primary $\mathrm{FeO}$ content [Klein and Langmuir, 1987]. Since there is a distinct difference in $\mathrm{FeO}$ between samples from Southwest Iceland and those from North Iceland, these two segments are considered separately in the fractionation correction (Figure 4-8 and Figure 4-9). In general, primary melt from Southwest Iceland is lower in $\mathrm{SiO}_{2}$ and higher in $\mathrm{FeO}$ and $\mathrm{TiO}_{2}$, compared to that of North Iceland. The differences in $\mathrm{MgO}$ and $\mathrm{Na}_{2} \mathrm{O}$ are small. The Fo content of equilibrium olivine for Southwest Iceland $(\sim 0.87)$ is thus lower than that for North Iceland $(\sim 0.90)$, and the primary melt of Southwest Iceland is more similar to that of Southeast Greenland. This contrast in the Fo content observed in Icelandic lavas seems to continue to the adjacent segments of the Mid-Atlantic Ridge. The Reykjanes Ridge data seem to be more akin to Southwest Iceland (Figure 4-8), whereas the Kolbeinsey Ridge data are almost indistinguishable from the North Iceland data (Figure 4-9). Again, due to the limited number of available samples, this inference regarding spatial continuity remains preliminary.

Finally, several samples from 'normal' mid-ocean ridges (i.e., located far from known hotspots) are also considered to provide a reference frame in the following discussion. The results of fractionation correction to glass samples from the East Pacific Rise (EPR) at $23^{\circ} \mathrm{N}$ [Bender et al., 1984] and from the Southeast Indian Ridge (SEIR) near the Australian-Antarctic Discordance [Klein et al., 1991] are shown in Figure 4-10. These samples, together with the Kolbeinsey data, constitute the major part of the "global trend" 
of mid-ocean ridge basalt chemistry, identified by Klein and Langmuir [1987]. The primary melt for the EPR segment has higher $\mathrm{FeO}$ and lower $\mathrm{Na}_{2} \mathrm{O}$ than that for the SWIR segment, which is consistent with the global trend of $\mathrm{Na}_{8.0}$ and $\mathrm{Fe}_{8.0}$ [Klein and Langmuir, 1987; Langmuir et al., 1992]. The Fo content of equilibrium olivine is $\sim 0.90$ for both regions.

The regional average of estimated primary melt composition is summarized in Table 4.1. An estimation based on fractionation correction to an equilibrium olivine with a maximum Fo content of 0.90 is also shown as an example of a more conventional correction. This Fo-based correction results in a very high Ni concentration (>5000 ppm) in equilibrium olivine for East Greenland, Southeast Greenland, Southwest Greenland, and the Reykjanes Ridge. The amount of olivine addition required by this $\mathrm{Fo}_{90}$ criterion is thus too high for these regions. However, the differences between results of the two methods for $\mathrm{FeO}, \mathrm{TiO}_{2}$, and $\mathrm{Na}_{2} \mathrm{O}$ are relatively minor.

The $\mathrm{MgO}$ and FeO contents of estimated primary melts based on the Ni criterion are plotted in Figure 4-11, together with those of mantle melts from recent experimental studies at a range of mantle pressures [Kinzler and Grove, 1992; Hirose and Kushiro, 1993; Kinzler and Grove, 1993; Baker and Stolper, 1994; Kinzler, 1997; Kogiso et al., 1998; Walter, 1998]. Most of these studies used mantle peridotites with whole rock $\mathrm{Mg}$ numbers of $0.89-0.90$ as a source material. Exceptions are the pyroxenite HK66 used by Hirose and Kushiro [1993] (whole rock Mg number of 0.854), and two homogeneous mixtures of peridotite and basalt KG1 and KG2 used by Kogiso et al. [1998] (whole rock $\mathrm{Mg}$ numbers of 0.811 and 0.848 , respectively). The composition of incipient melt is in equilibrium with the forsterite content of source olivine, or approximately with the whole rock $\mathrm{Mg}$ number. As melting proceeds, the liquid $\mathrm{Mg}$ number always increases due to source depletion regardless of the style of melting (e.g., batch and fractional), so that the $\mathrm{Mg}$ number of primary melt can be used to determine the $\mathrm{Mg}$ number of residual olivine and to place an upper bound on the Fo content of source olivine. Therefore, Figure 4m11 indicates that, whereas source mantle for North Iceland and the Kolbeinsey Ridge as well as EPR and SEIR probably has compositions similar to pyrolite (i.e., whole rock $\mathrm{Mg}$ 
number of $\sim 0.895$ ) [e.g., Ringwood, 1975; Hart and Zindler, 1986; McDonough and Sun, 1995], other regions in the North Atlantic igneous province must have significantly different source mantle with $\mathrm{Mg}$ numbers lower than $0.86-0.87$, which could have resulted from $20-30 \%$ addition of a basaltic component to typical mantle "pyrolite".

Being an essential structural constituent [Hanson and Langmuir, 1978], liquid $\mathrm{SiO}_{2}$ concentration is not very sensitive to source composition, and a number of experimental studies show that it is a good proxy for the pressure of melting [Jaques and Green, 1980; Fujii and Scarfe, 1985; Falloon et al., 1988; Hirose and Kushiro, 1993; Kinzler, 1997; Walter, 1998]. Using experimental mantle melts, liquid $\mathrm{SiO}_{2}$ is fitted as a function of the pressure and degree of melting (Figure 4-12a). In Figure 4-12b, the $\mathrm{SiO}_{2}$ contents of estimated primary melts are plotted to constrain the mean pressure of melting for each region. For the regions with 'normal' source mantle similar to pyrolite, the inferred pressure of melting increases, in the order SEIR, EPR, Kolbeinsey, and North Iceland. This seems to be consistent with common hypotheses regarding correlations between seafloor depth, crustal thickness, and mantle potential temperature [Klein and Langmuir, 1987; Langmuir et al., 1992]. Similar systematics, but with systematically higher pressures, can also be found for the regions with anomalous source mantle, if East Greenland is excluded. The East Greenland data indicate the lowest pressure of melting among all regions considered in this study, contrary to our expectation for this region which lies along the presumed Iceland hotspot track [White and McKenzie, 1989; Lawver and Müller, 1994]. The low melting pressure is also puzzling considering the possible effect of a preexisting lithospheric lid on mantle melting [Fram and Lesher, 1993]. We note, however, that there may be a compositional dependence of Si partitioning between melt and residue, as inferred from the systematic underprediction of $\mathrm{SiO}_{2}$ by our regression for melts derived from low-Mg-number peridotite (Figure 4-12a). Though only tentative due to the paucity of appropriate melting experiments, a separate regression based solely on melts of low$\mathrm{Mg}$-number compositions indicates that enrichment in a basaltic component may increase liquid $\mathrm{SiO}_{2}$ by as much as $2 \%$ (Figure 4-12b). Because the East Greenland primary melt has the lowest estimated $\mathrm{Mg}$ number of residual mantle (Figure 4-11), its apparent low 
pressure of melting may simply be an artifact due to source heterogeneity, not reflecting the true pressure of the melting process.

\subsection{Discussion and Conclusion}

Our case study of the North Atlantic igneous province has demonstrated that olivine fractionation correction with the $\mathrm{Ni}$ criterion is a promising approach to estimate the nature of primary melt. Though the number of samples qualified for our scheme is small, this method is especially useful to constrain the extent of major element source heterogeneity. Because the major element composition of mantle-derived melt is very sensitive to both source composition and melting process, the interpretation of lava chemistry solely on the basis of compositional systematics is often equivocal. The high FeO content of the Southwest Iceland lavas, for example, has been known for decades [Jakobsson et al., 1978; Zindler et al., 1979; Langmuir and Hanson, 1980], but it has been attributed only to the melting process or previous depletion history, based on a correlation with $\mathrm{La} / \mathrm{Sm}$ ratio. Fram and Lesher [1997] estimated primary melts for various regions of the North Atlantic igneous province using olivine fractionation with the liquid $\mathrm{Mg}$ number criterion, and based on a correlation with $\mathrm{Dy} / \mathrm{Yb}$ ratio, they concluded that the East Greenland Tertiary flood basalts resulted from the melting of Fe-poor mantle, which is at odds with our result. On the other hand, Scarrow and Cox [1995] proposed Fe-rich source mantle like HK66 for the Skye Main Lava Series in Northwest Scotland, but their work is based on the whole rock composition of polyphyric lavas, which contain up to $20 \%$ olivine phenocrysts of $\mathrm{Fo}_{80-86}$.

The Ni criterion is also useful to discriminate high- $\mathrm{MgO}$ samples with an olivinecumulative origin. The most primitive lavas, i.e., aphyric lavas with the highest $\mathrm{MgO}$ content, can place a lower bound on the primary $\mathrm{MgO}$ content. This inference, however, must also be supported by liquid Ni concentration, because petrographic criteria may often be misleading [Clarke, 1970; Hart and Davis, 1978; Clarke and O'Hara, 1979; Kinzler et $a l ., 1990$ ]. Figure 4-6b and Figure 4-7b clearly show that the misinterpretation of picritic samples can be avoided in some cases by calculating the Ni content of equilibrium olivine. 
The most forsteritic olivine phenocrysts are also commonly used to estimate primary liquid $\mathrm{MgO}$ content [Clarke, 1970; Pedersen, 1985]. The Southeast Greenland samples, for example, include large euhedral olivine phenocrysts with $\mathrm{Fo}_{90.2-92.4}[$ Demant, 1998]. Thy et al. [1998] used this observation to support their interpretation of picritic lavas $(\sim 18 \mathrm{wt} \%$ $\mathrm{MgO}$ ) as a possible primary melt. Such high-Fo olivine, however, could be xenocrysts derived from depleted continental mantle. Indeed, mantle xenoliths from the Greenland cratonic lithosphere have highly forsteritic olivines $\left(\mathrm{FO}_{87-94}\right.$ with a mean of $\left.\mathrm{Fo}_{92.7}\right)$ [Bernstein et al., 1998] (Figure 4-3e). The possibility of xenocrysts can, therefore, introduce significant ambiguity in the interpretation of phenocryst composition, especially for continental flood basalts.

Although trace element source heterogeneity in the North Atlantic igneous province is beyond dispute [e.g., Schilling et al., 1982; Schilling et al., 1983; Schilling, 1985; Langmuir et al., 1992], trace element systematics during melting based on an assumed source composition have commonly been used to infer the process of mantle melting in this region [Fram and Lesher, 1993; White and McKenzie, 1995; Fram et al., 1998; Tegner et al., 1998]. By combining trace element information with inference based on major element chemistry, one may be able to place additional constraints on the degree of source heterogeneity as well as the dynamics of the melting process. However, for this purpose, major element systematics for melting of a range of different source compositions have to be firmly established by further melting experiments.

In Table 4.1 , theoretical compressional-wave velocity at $600 \mathrm{MPa}$ and $400^{\circ} \mathrm{C}$ is also reported for each primary melt composition, to compare with the seismic velocity structure of igneous crust in this province. The bulk crustal velocity of 30 -km-thick igneous crust at the Southeast Greenland margin is, for example, observed to be lower than $\sim 7.0 \mathrm{~km} / \mathrm{s}$ (Chapter 3). The Southeast Greenland primary melt based on the $3500 \mathrm{ppm} \mathrm{Ni}$ criterion has a velocity of $-7.15 \mathrm{~km} / \mathrm{s}$, substantially exceeding the seismic estimate. Because seismic velocity is generally most sensitive to $\mathrm{MgO}$ content, this discrepancy may simply result from our upper bound approach in terms of $\mathrm{MgO}$ content, so that the true primary melt may have even lower $\mathrm{MgO}$ content. Its equilibrium olivine may thus be less 
forsteritic, further enhancing the inferred degree of major element source heterogeneity. Though our understanding of the spatial extent of source heterogeneity is still at a preliminary stage due to the limited number of suitable data, this study suggests that a long-standing compositional anomaly may have had an important role in the formation of the North Atlantic igneous province. 
Table 4.1. Estimated primary melt compositions for the North Atlantic igneous province and other normal mid-ocean ridges.

A. Correction with maximum Ni content of $3500 \mathrm{ppm}$ in equilibrium olivine

\begin{tabular}{|c|c|c|c|c|c|c|c|c|c|c|}
\hline Location & $\mathrm{N}^{\mathrm{a}}$ & $\mathrm{SiO}_{2}$ & $\mathrm{TiO}_{2}$ & $\mathrm{Al}_{2} \mathrm{O}_{3}$ & $\mathrm{FeO}^{*}$ & $\mathrm{MgO}$ & $\mathrm{CaO}$ & $\mathrm{Na}_{2} \mathrm{O}$ & Fo & $V_{D}^{b}$ \\
\hline E Greenland & 2 & 49.87 & 2.07 & 11.69 & 11.64 & 12.61 & 10.41 & 1.70 & 0.862 & 7.14 \\
\hline SE Greenland & 11 & 47.44 & 1.27 & 13.58 & 11.75 & 14.71 & 9.18 & 2.08 & 0.877 & 7.16 \\
\hline SW Iceland & 5 & 46.52 & 1.45 & 12.89 & 12.64 & 15.09 & 9.65 & 1.75 & 0.873 & 7.21 \\
\hline $\mathrm{N}$ Iceland & 69 & 47.67 & 0.85 & 13.26 & 10.36 & 15.45 & 10.84 & 1.57 & 0.895 & 7.25 \\
\hline Reykjanes & 2 & 49.04 & 0.91 & 12.73 & 11.23 & 13.78 & 10.39 & 1.93 & 0.875 & 7.16 \\
\hline Kobeinsey & 2 & 47.96 & 0.71 & 13.32 & 9.58 & 15.25 & 11.70 & 1.48 & 0.903 & 7.27 \\
\hline EPR $23^{\circ} \mathrm{N}$ & 9 & 48.30 & 1.14 & 13.72 & 9.79 & 14.63 & 10.45 & 1.95 & 0.895 & 7.19 \\
\hline SE Indian Ridge & 10 & 49.34 & 1.25 & 14.42 & 8.80 & 13.67 & 9.84 & 2.68 & 0.899 & 7.10 \\
\hline
\end{tabular}

B. Correction with maximum forsterite content of 0.90

\begin{tabular}{|c|c|c|c|c|c|c|c|c|c|c|}
\hline Location & $\mathrm{N}$ & $\mathrm{SiO}_{2}$ & $\mathrm{TiO}_{2}$ & $\mathrm{Al}_{2} \mathrm{O}_{3}$ & $\mathrm{FeO}^{*}$ & $\mathrm{MgO}$ & $\mathrm{CaO}$ & $\mathrm{Na}_{2} \mathrm{O}$ & Ni(oliv) & $V_{p}^{b}$ \\
\hline E Greenland & 2 & 48.40 & 1.75 & 9.85 & 11.60 & 18.20 & 8.77 & 1.43 & 6240 & 7.29 \\
\hline SE Greenland & 11 & 46.70 & 1.12 & 12.15 & 11.66 & 18.29 & 8.21 & 1.86 & 5030 & 7.26 \\
\hline SW Iceland & 5 & 45.75 & 1.26 & 11.20 & 12.41 & 19.46 & 8.39 & 1.53 & 5220 & 7.32 \\
\hline $\mathrm{N}$ Iceland & 69 & 47.53 & 0.81 & 12.96 & 10.34 & 16.23 & 10.61 & 1.53 & 3870 & 7.27 \\
\hline Reykjanes & 2 & 48.14 & 0.81 & 11.34 & 11.19 & 17.55 & 9.26 & 1.72 & 5140 & 7.26 \\
\hline Kobeinsey & 2 & 47.99 & 0.71 & 13.54 & 9.49 & 14.87 & 11.91 & 1.49 & 3370 & 7.27 \\
\hline $\mathrm{EPR} 23^{\circ} \mathrm{N}$ & 9 & 48.14 & 1.12 & 13.42 & 9.80 & 15.38 & 10.23 & 1.91 & 3820 & 7.21 \\
\hline SE Indian Ridge & 10 & 49.35 & 1.22 & 14.39 & 8.77 & 13.76 & 9.83 & 2.66 & 3670 & 7.10 \\
\hline
\end{tabular}

C. Difference between A and B

\begin{tabular}{lrrrrrrrrr}
\hline Location & \multicolumn{2}{c}{$\mathrm{SiO}_{2}$} & \multicolumn{2}{c}{$\mathrm{TiO}_{2}$} & \multicolumn{2}{c}{$\mathrm{Al}_{2} \mathrm{O}_{3}$} & \multicolumn{2}{c}{$\mathrm{FeO} *$} & \multicolumn{2}{c}{$\mathrm{MgO}$} & \multicolumn{2}{c}{$\mathrm{CaO}$} & $\mathrm{Na}_{2} \mathrm{O}$ \\
\hline E Greenland & 2 & -1.47 & -0.32 & -1.84 & -0.04 & 5.59 & -1.64 & -0.27 \\
SE Greenland & 11 & -0.74 & -0.15 & -1.43 & -0.09 & 3.58 & -0.97 & -0.22 \\
SW Iceland & 5 & -0.77 & -0.19 & -1.69 & -0.23 & 4.37 & -1.26 & -0.22 \\
N Iceland & 69 & -0.14 & -0.04 & -0.30 & -0.02 & 0.78 & -0.23 & -0.04 \\
Reykjanes & 2 & -0.90 & -0.10 & -1.39 & -0.04 & 3.77 & -1.13 & -0.21 \\
Kobeinsey & 2 & 0.03 & 0.00 & 0.22 & -0.09 & -0.38 & 0.21 & 0.01 \\
EPR 23 $3^{\circ} \mathrm{N}$ & 9 & -0.16 & -0.02 & -0.30 & 0.01 & 0.75 & -0.22 & -0.04 \\
SE Indian Ridge & 10 & 0.01 & -0.03 & -0.03 & -0.03 & 0.09 & -0.01 & -0.02 \\
\hline
\end{tabular}

Values for oxide concentration are in weight percent.The oxides have been normalized to a total of 100 , and all iron $(\mathrm{FeO} *$ ) is calculated as $\mathrm{FeO}$.

"Number of analyses.

${ }^{b}$ Theoretical compressional-wave velocity of solidified phase at $600 \mathrm{MPa}$ and $400^{\circ} \mathrm{C}$. Values are in $\mathrm{km} / \mathrm{s}$. (see Chapter 3 for the details of calculation). 


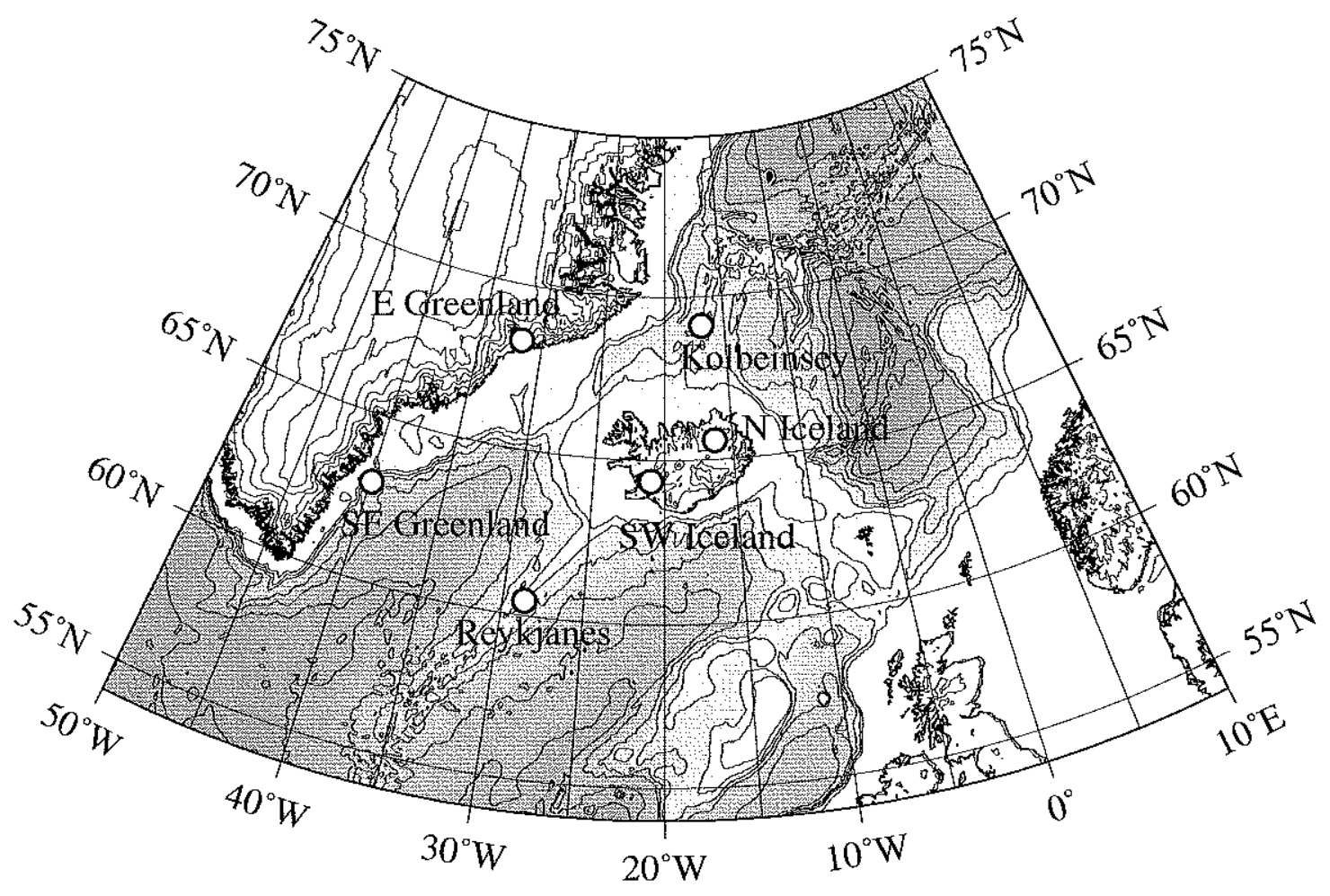

Figure 4-1. Map of North Atlantic igneous province. Bathymetric contours are at $500 \mathrm{~m}$ interval. 


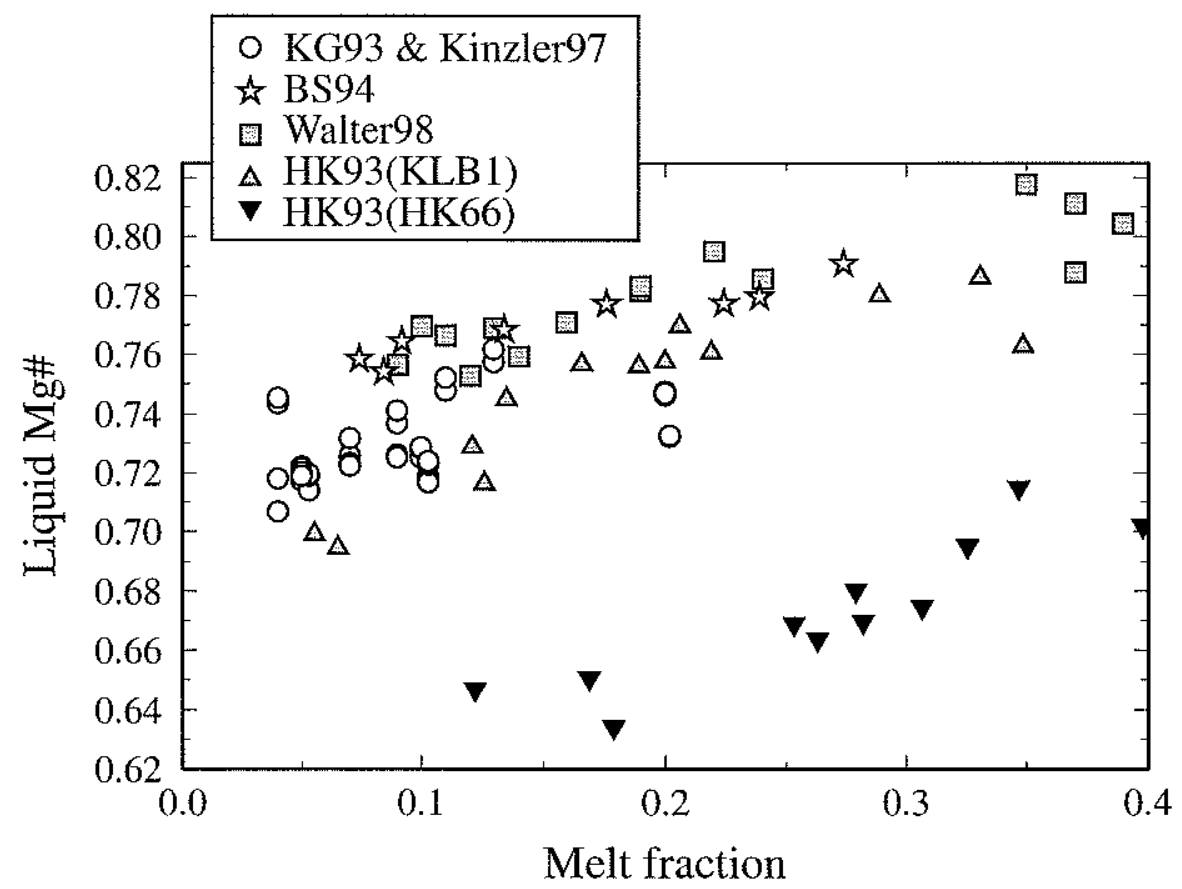

Figure 4-2. Liquid $\mathrm{Mg}$ numbers of experimental mantle melts are plotted as a function of melt fraction. Data are from Kinzler and Grove [1993], Hirose and Kushiro [1993], Baker and Stolper [1994], Kinzler [1997] and Walter [1998]. 

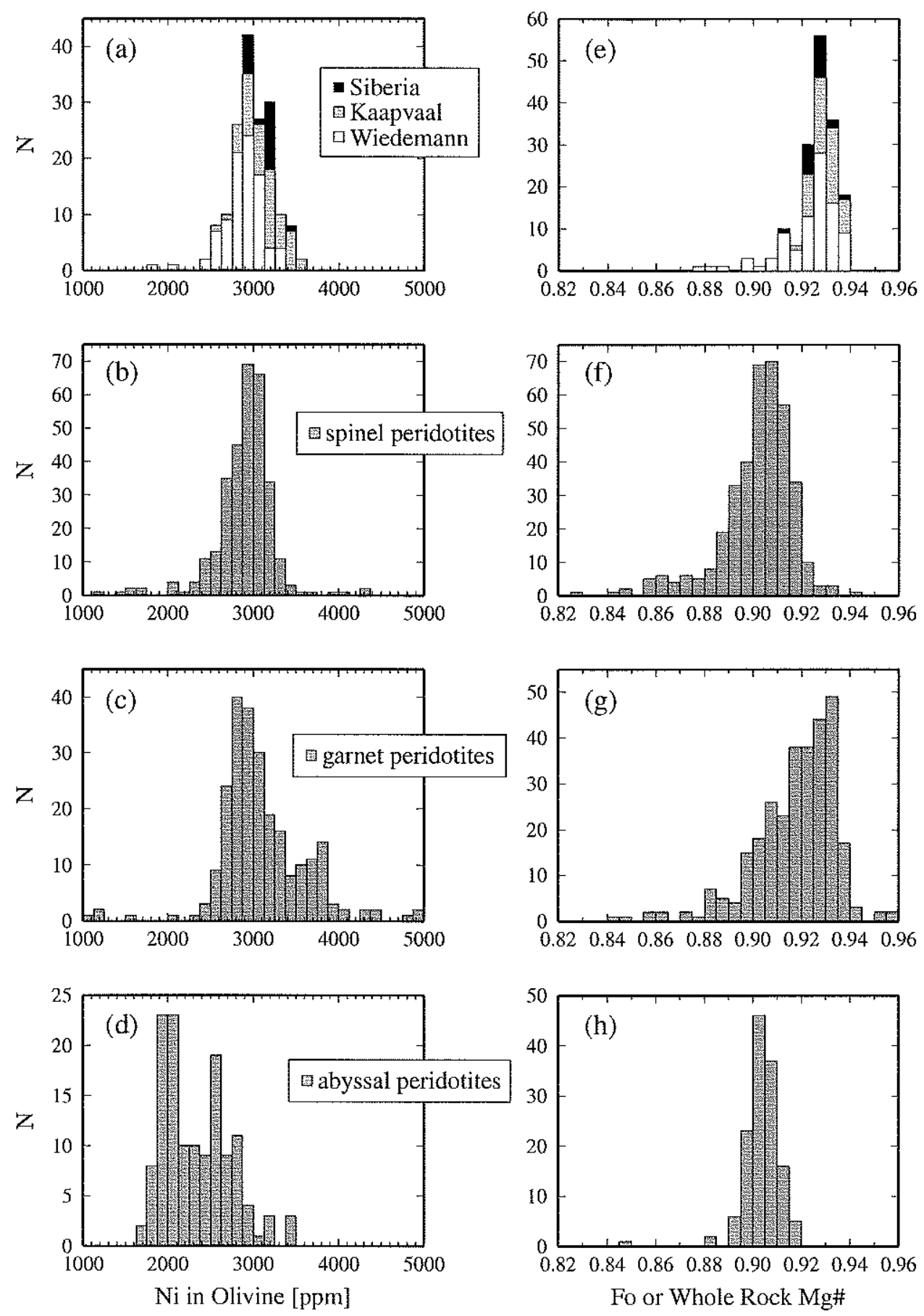

Figure 4-3. Histograms of the $\mathrm{Ni}$ and Fo contents of olivine in continental and oceanic mantle peridotites. Data plotted outside of the shown ranges constitute less than $1 \%$ of total data. (a) and (e): mantle xenoliths from East Greenland [Bernstein et al., 1998], Siberia [Boyd et al., 1997], and Kaapvaal (Boyd, pers. comm.). (b) and (f): Spinel peridotites from McDonough and Frey [1989], with normative mineral proportions and $\mathrm{Ni}$ contents of minerals calculated using the spinelTherzolite norm [Kelemen et al., 1992]. (c) and (g): Gamet peridotites from McDonough and Frey [1989], with normative mineral proportions and $\mathrm{Ni}$ contents of minerals calculated using the garnet-lherzolite norm [Kelemen et al., 1992]. (d) and (h): Abyssal peridotites (Dick, pers. comm.). 

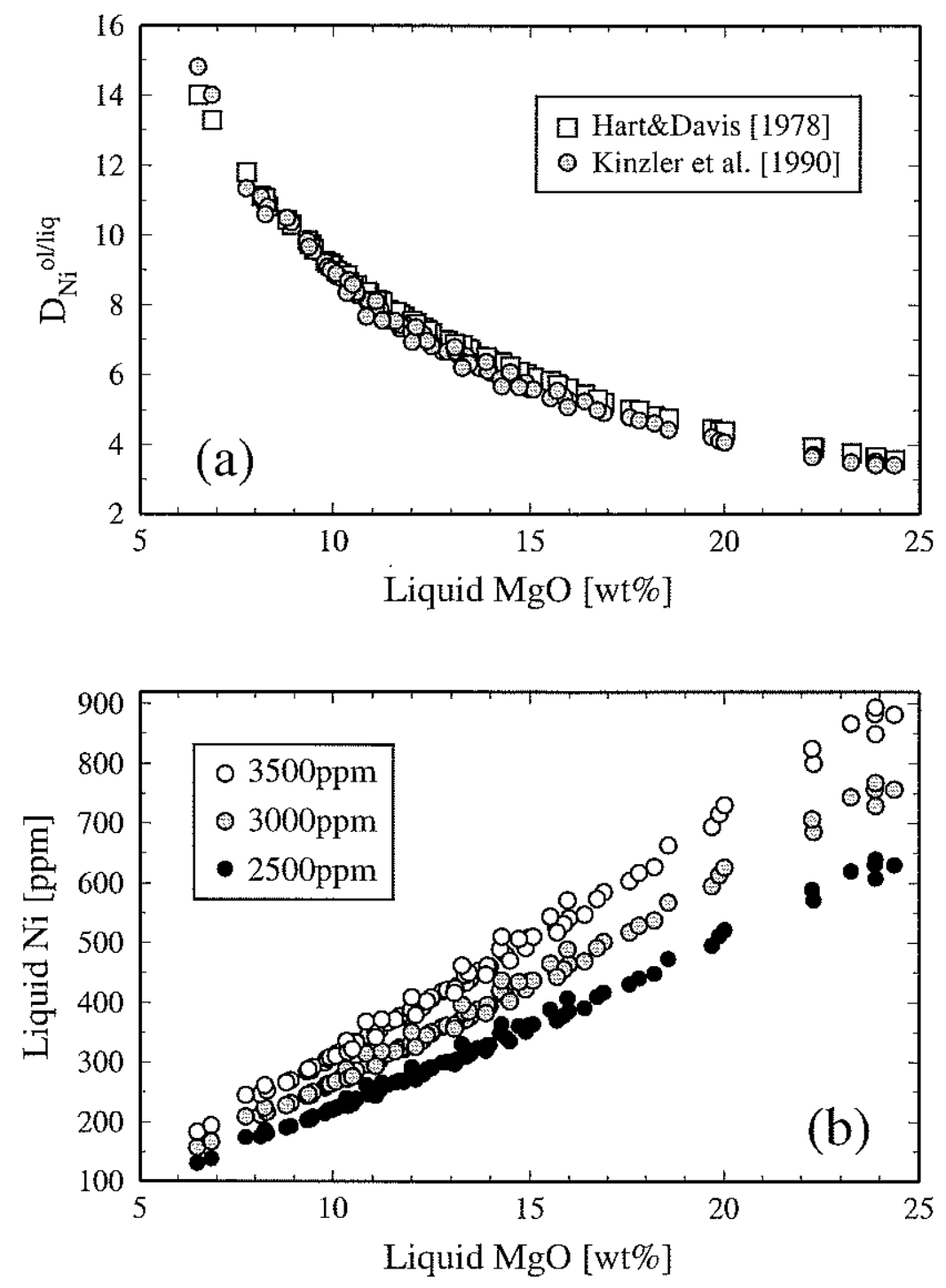

Figure 4-4. (a) A range of molar $D_{\mathrm{Ni}}^{\text {ol/iiq }}$ (defined as $X_{\mathrm{NiO}}{ }^{\text {ol }} / X_{\mathrm{NiO}}$ liq, which is 1.5 times smaller than the definition of Kinzler et al. [1990], $X_{\mathrm{Ni2SiO4} 4}{ }^{\text {ol }} / X_{\mathrm{NiO}}$ lig $)$ are calculated using the equation of Kinzler et al. [1990] and the compositions of experimental mantle melts used in Figure 4-2. The result based on the equation of Hart and Davis [1978] is also shown. (b) The Ni concentration of liquid in equilibrium with olivine with $2500-3500 \mathrm{ppm} \mathrm{Ni}$ is shown as a function of liquid $\mathrm{MgO}$, using the equation of Kinzler et al. [1990] and the same experimental mantle melts used in (a). 
(a)
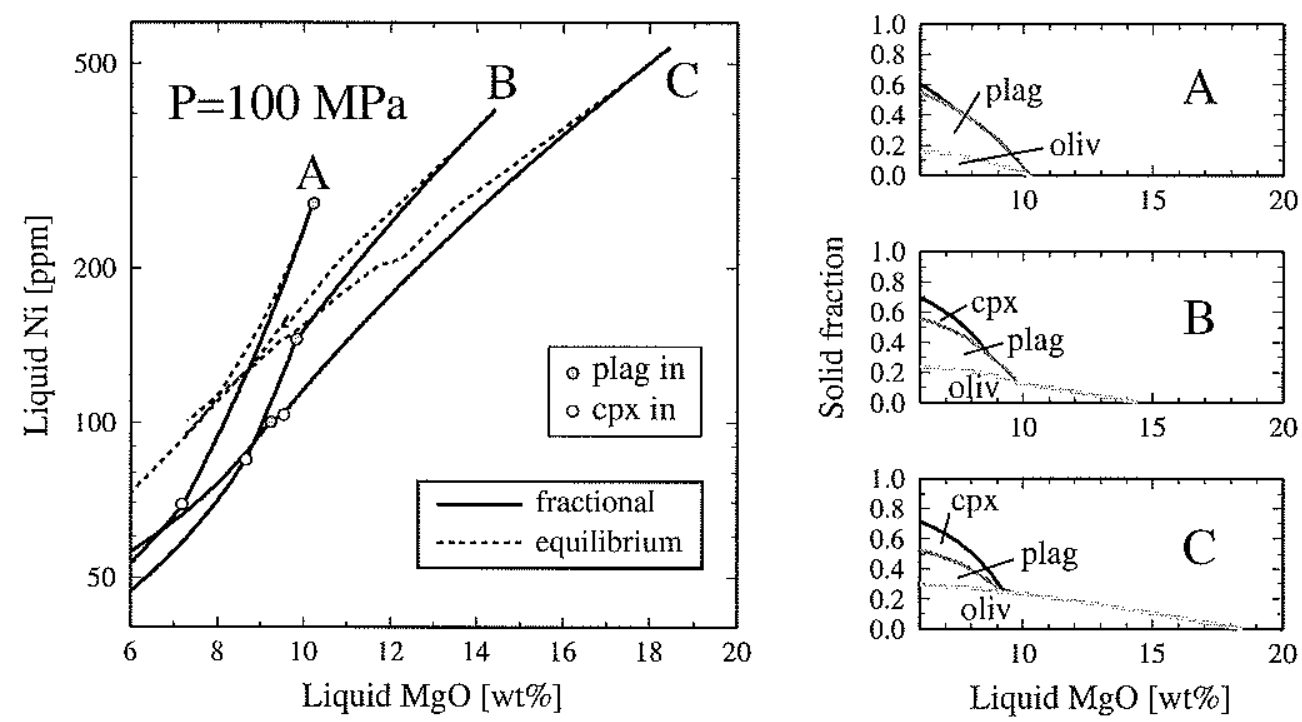

(b)
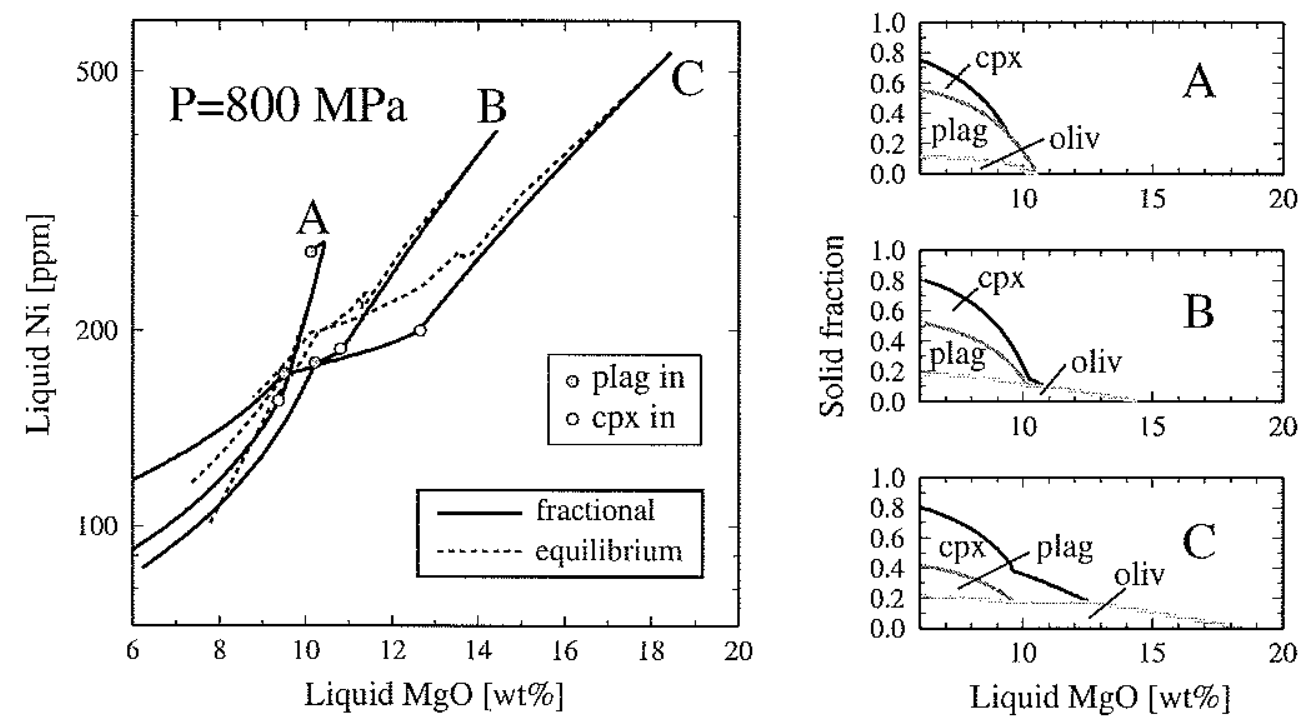

Figure 4-5. Results of fractional and equilibrium crystallization modeling are shown in terms of liquid $\mathrm{MgO}$ and $\mathrm{Ni}$ contents. Temperature increment is $1^{\circ} \mathrm{C}$ for fractional crystallization. Cumulative proportion of fractionating phases are also shown for fractional crystallization cases. Starting liquids are A (12\% melting of KLBI at $1 \mathrm{GPa}$ [Hirose and Kushiro, 1993]), B (aggregated, nearly fractional polybaric melts with melt fraction of $11 \%$ and mean melting pressure of $1.8 \mathrm{GPa}$ [Kinzler, 1997]), and C (24\% melting of garnet peridotite at $3 \mathrm{GPa}$ [Walter, 1998]). Crystallization pressures are (a) $100 \mathrm{MPa}$ and (b) $800 \mathrm{MPa}$. 

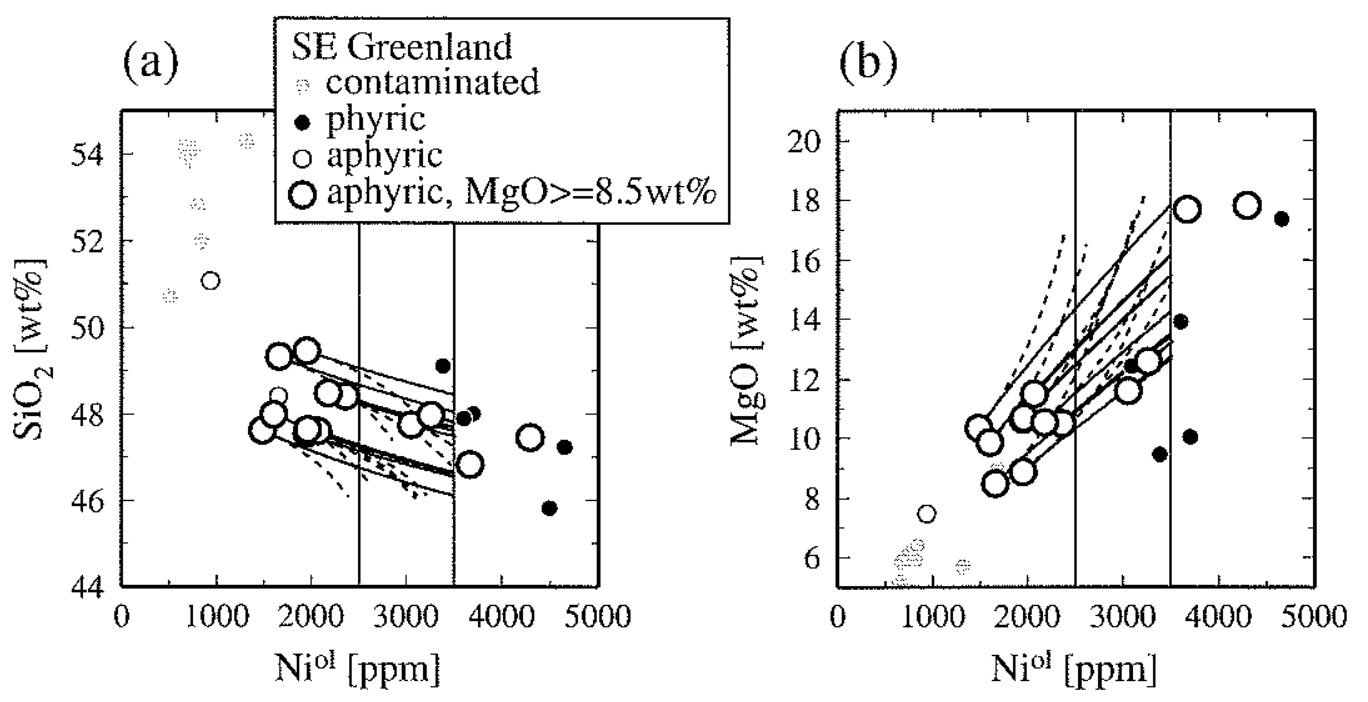

(c)

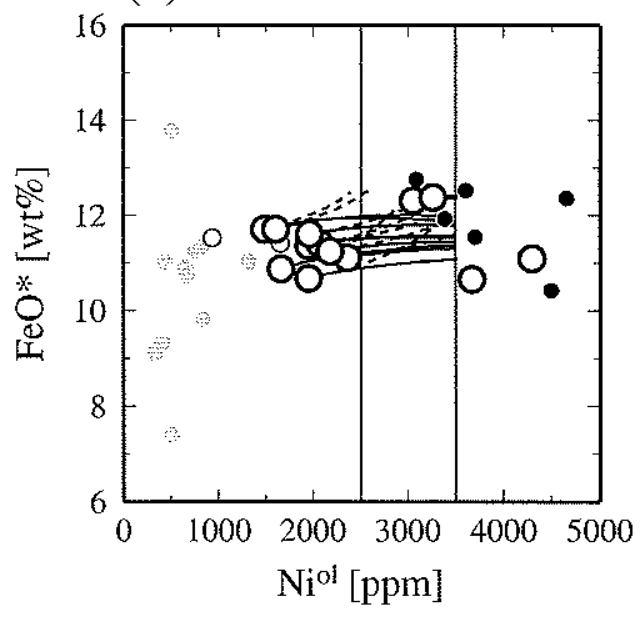

(d)

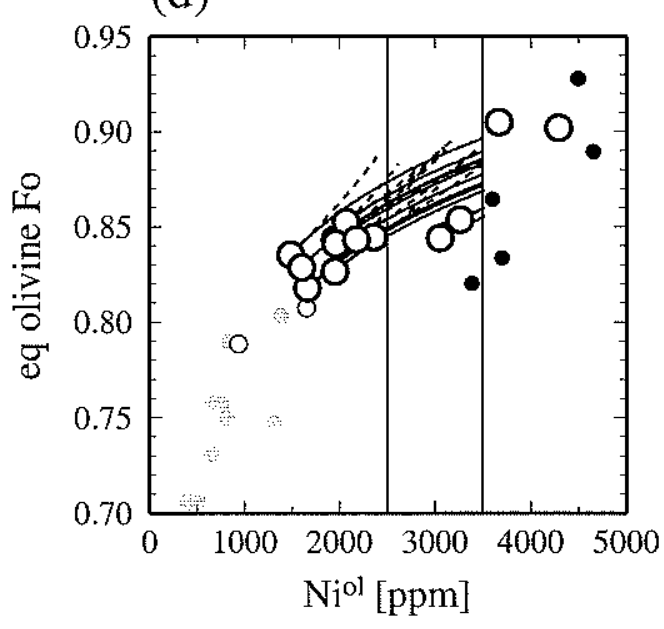

(e)

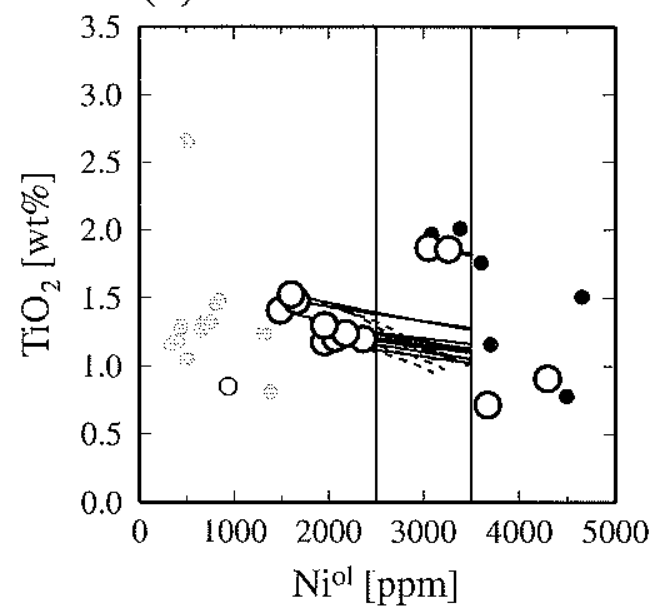

(f)

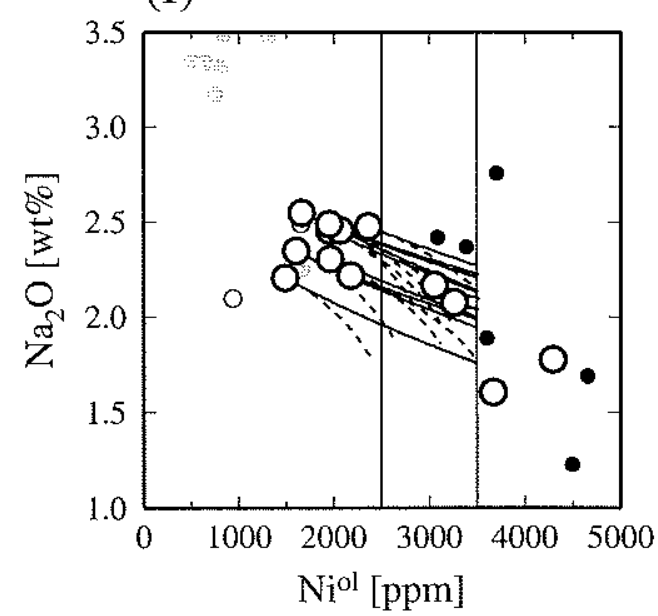

Figure 4-6. Southeast Greenland lava samples [Larsen et al., 1998] with fractionation correction. Solid curves denote correction for fractional crystallization, and dashed curves for equilibrium crystallization. 


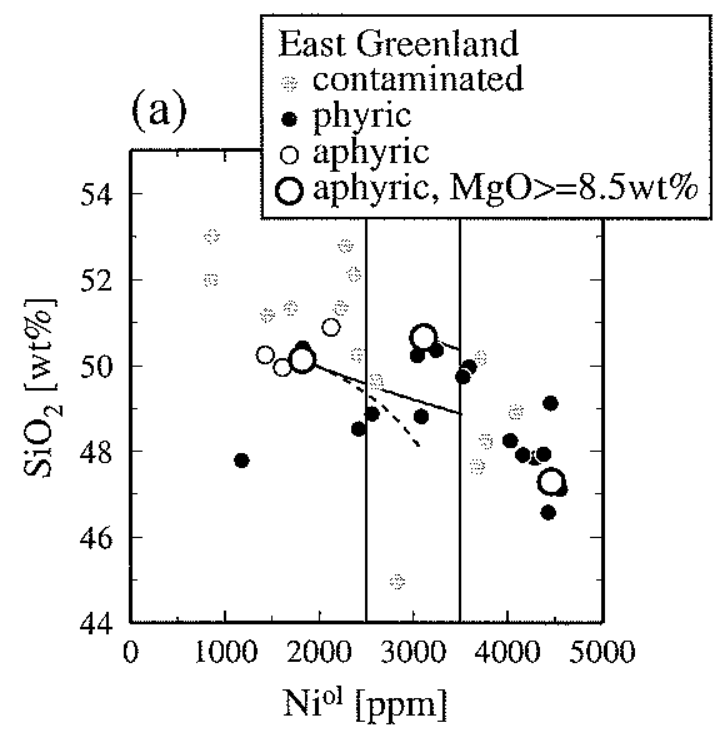

(b)

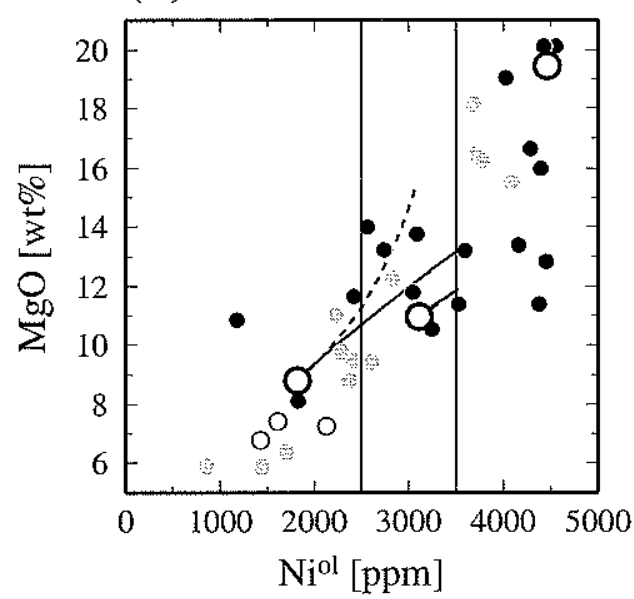

(c)

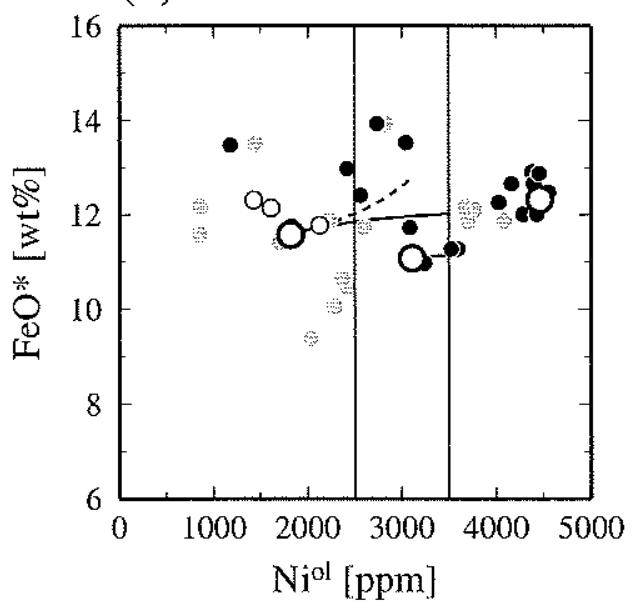

(d)

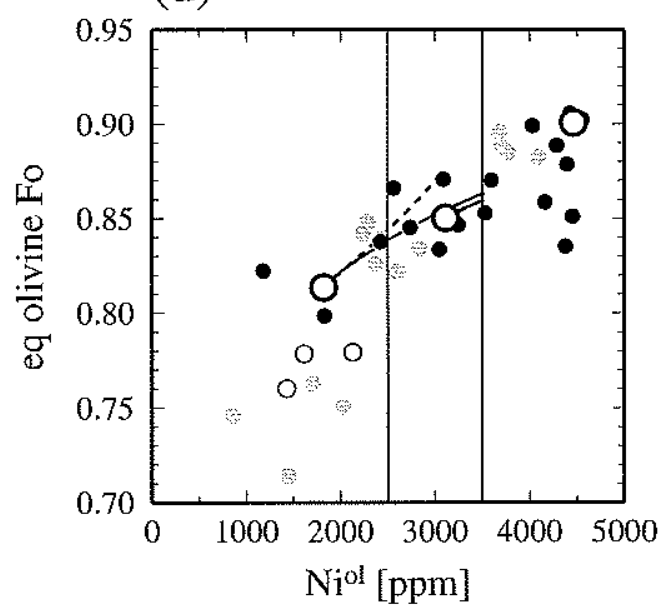

(e)

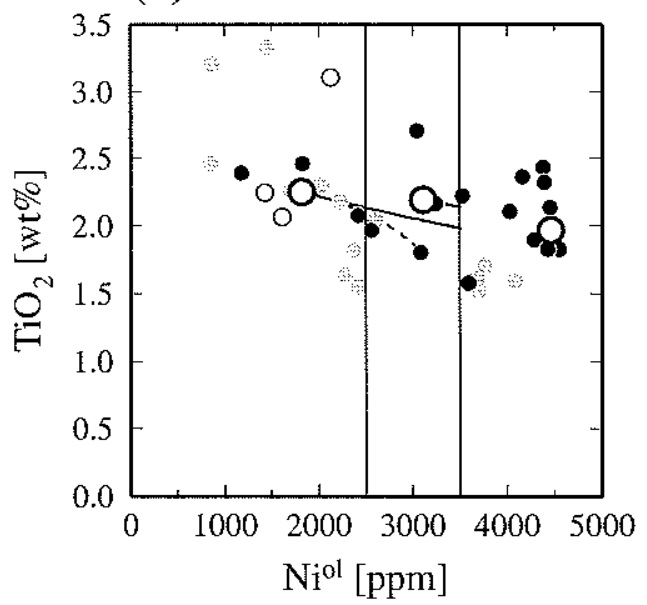

(f)

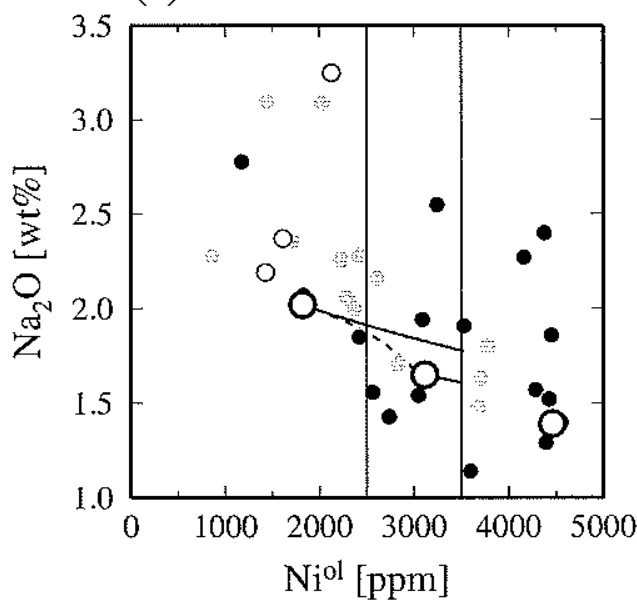

Figure 4-7. Same as Figure 4-6, but for the East Greenland samples [Fram and Lesher, 1997]. 


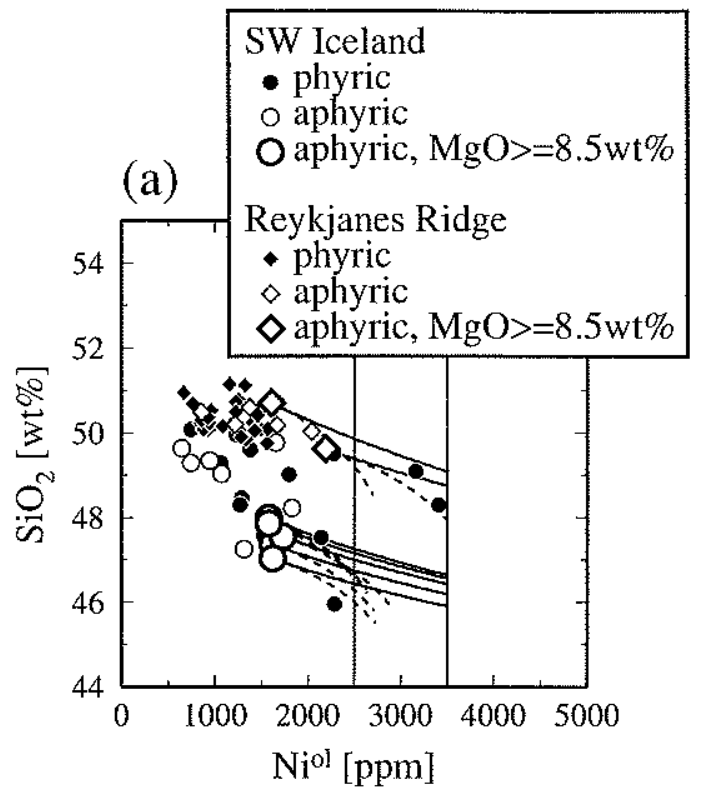

(b)

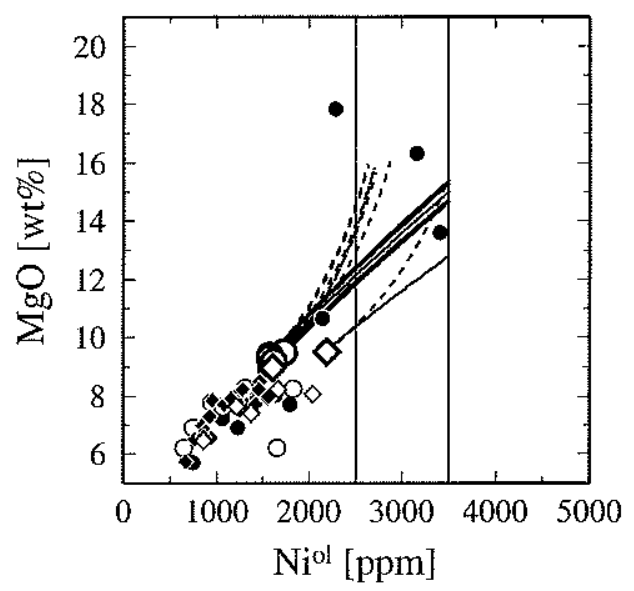

(c)

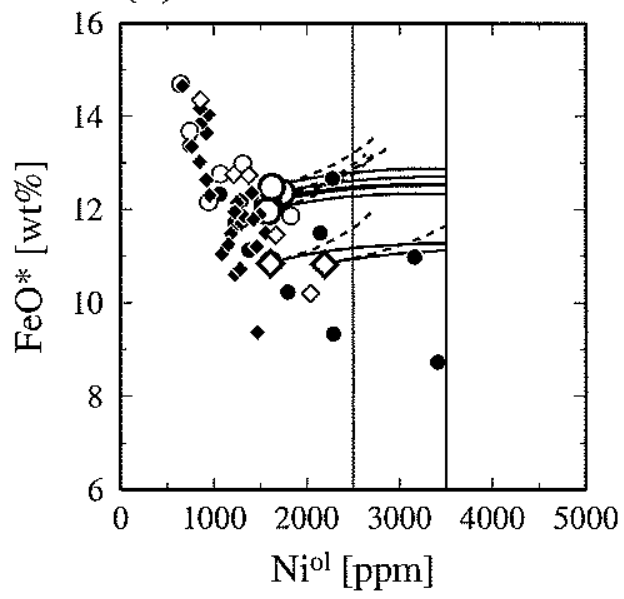

(d)

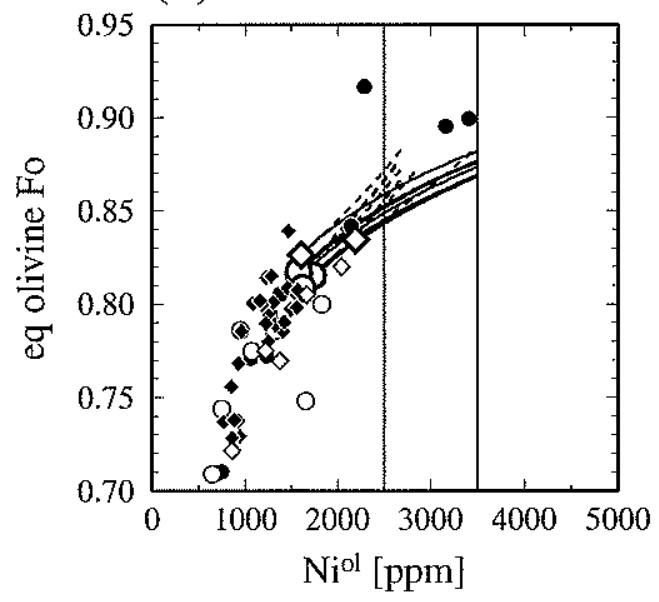

(e)

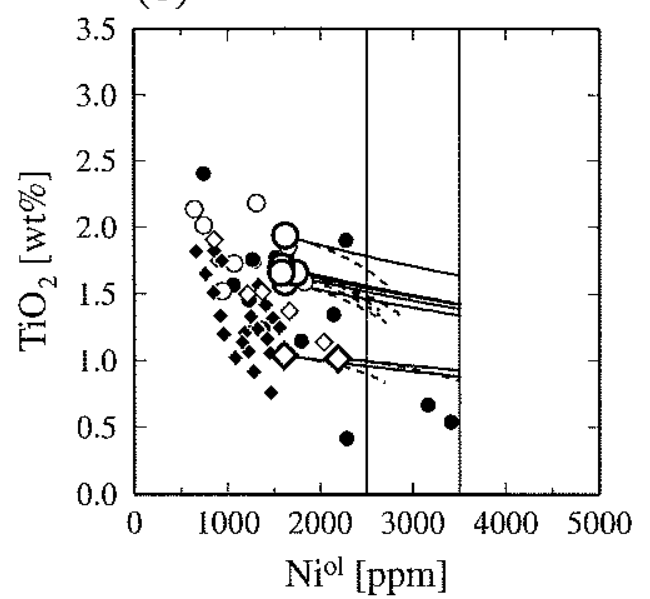

(f)

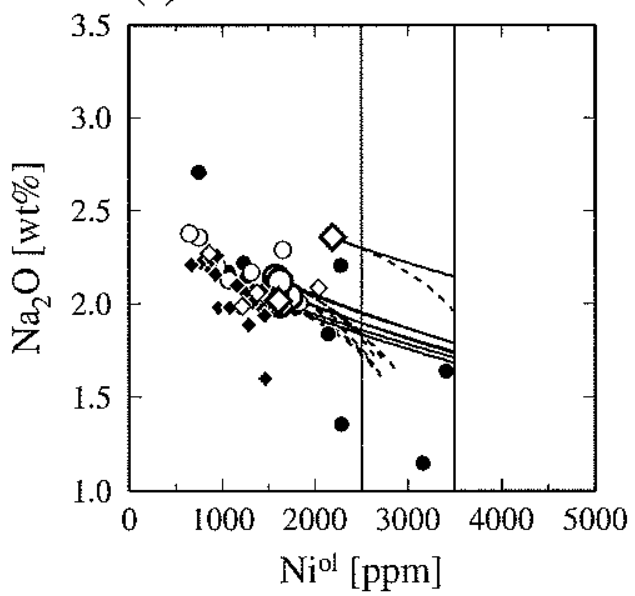

Figure 4-8. Same as Figure 4-6, but for samples from Southwest Iceland and the Reykjanes Ridge [Wood et al., 1979; Schilling et al., 1983; Hemond et al., 1993]. 


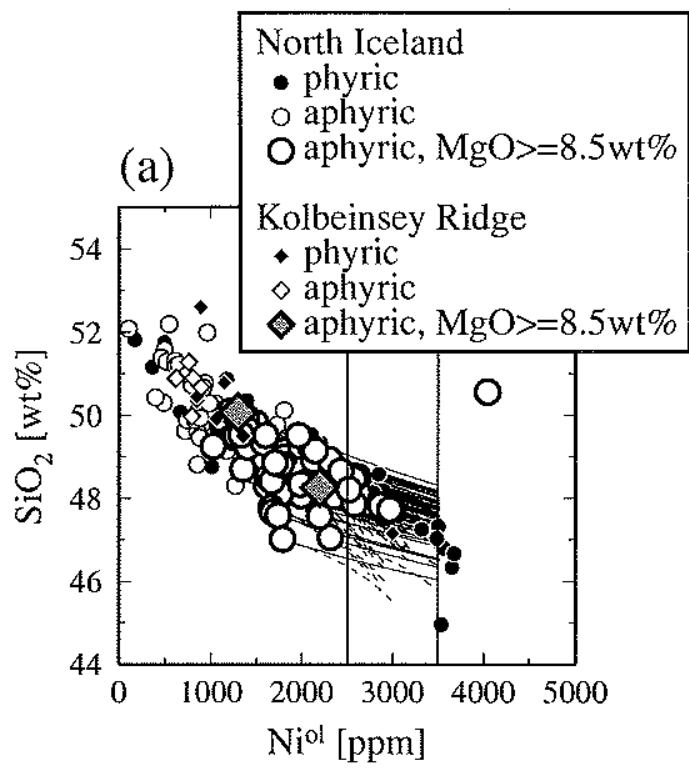

(b)

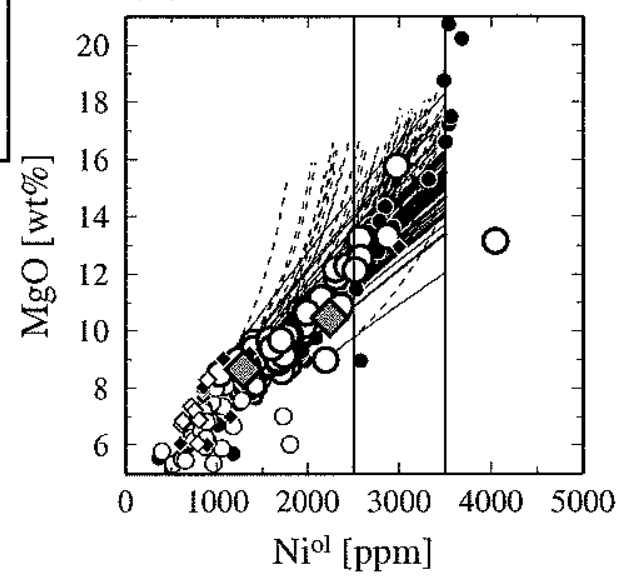

(c)

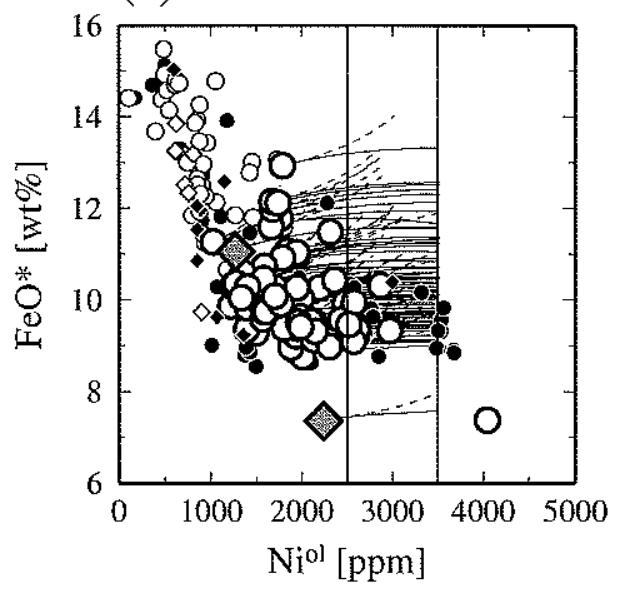

(d)

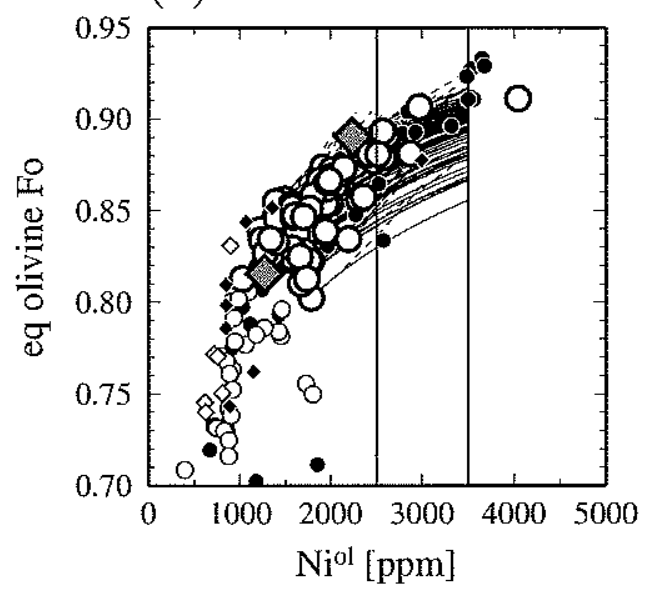

(e)

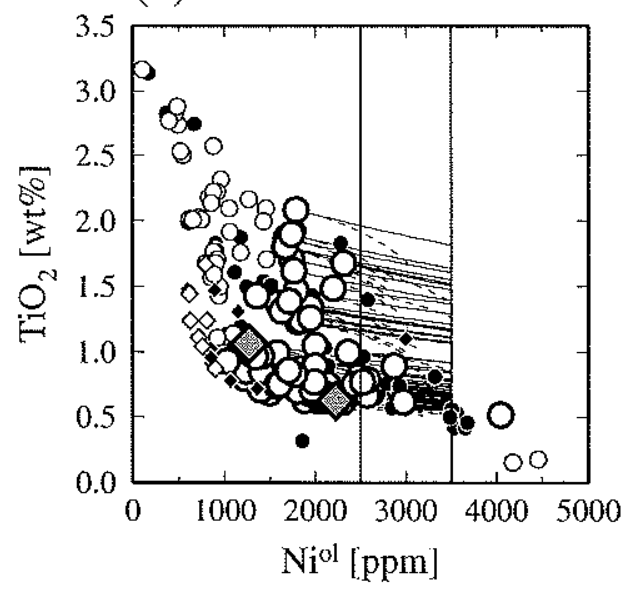

(f)

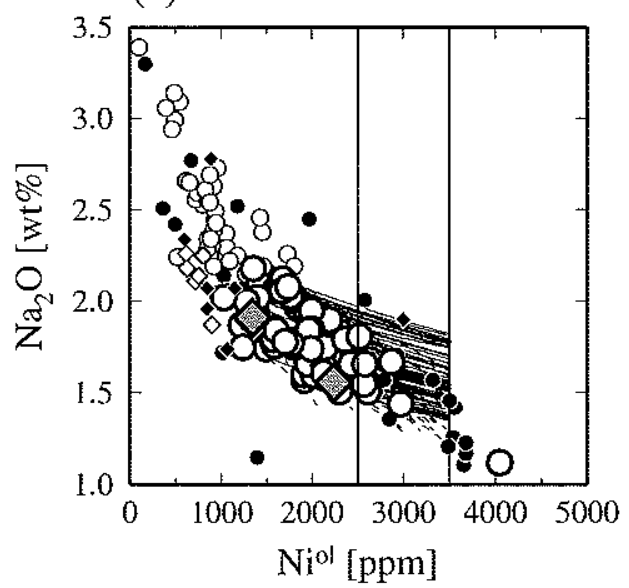

Figure 4-9. Same as Figure 4-6, but for samples from North Iceland and the Kolbeinsey Ridge [Wood et al., 1979; Schilling et al., 1983; Hemond et al., 1993; Slater, 1996]. 

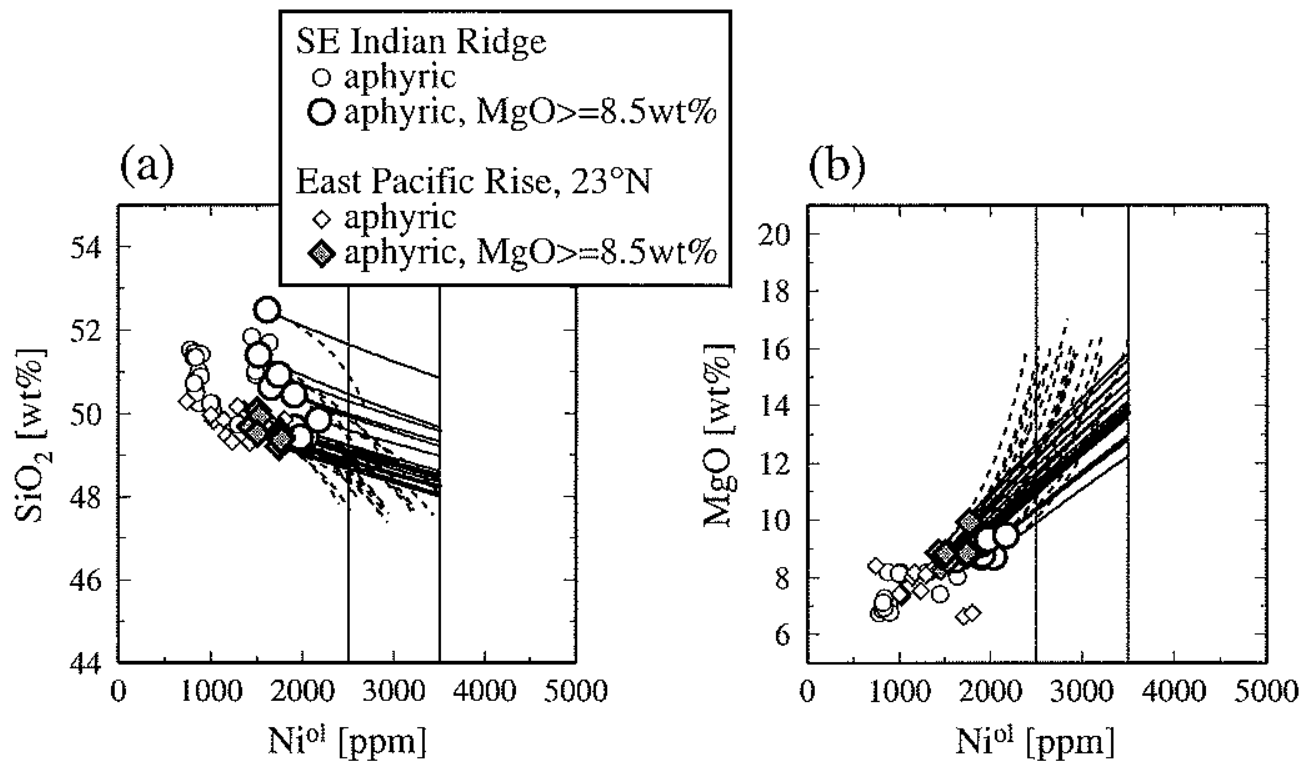

(c)

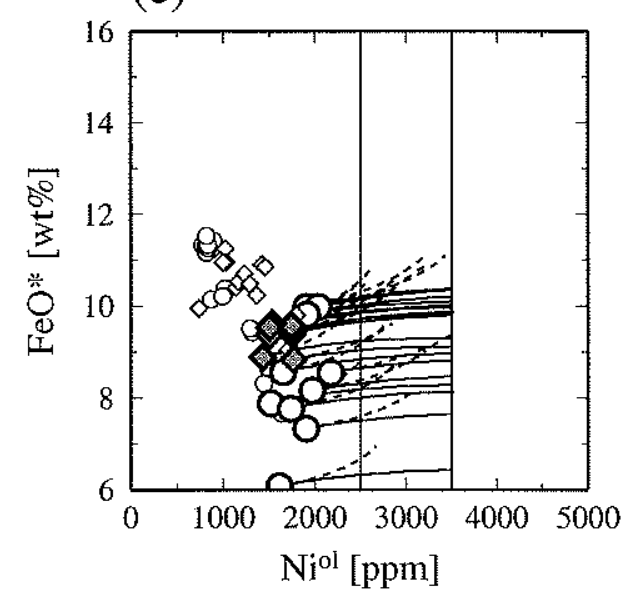

(d)

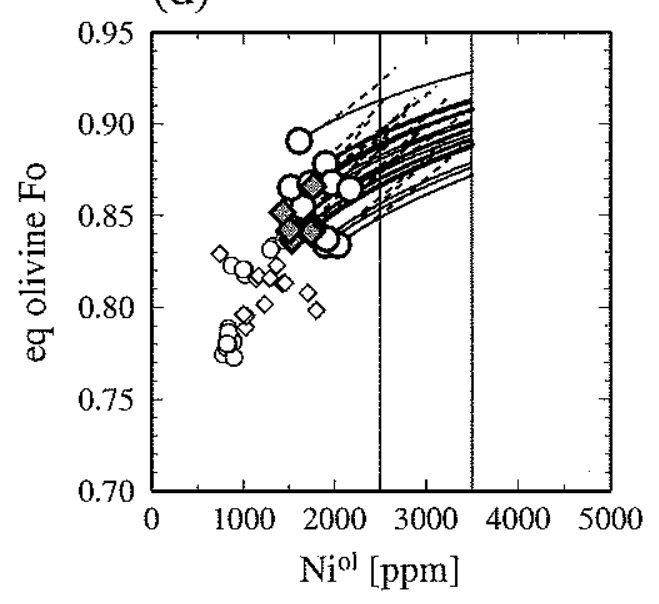

(e)

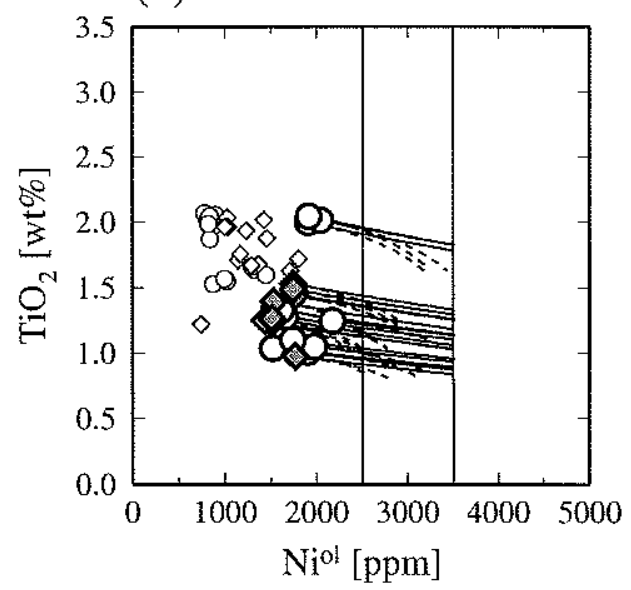

(f)

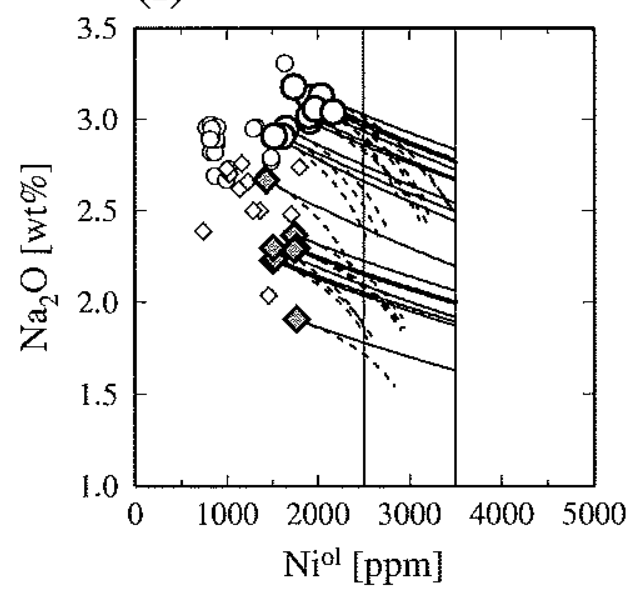

Figure 4-10. Same as Figure 4-6, but for samples from the Southeast Indian Ridge [Klein et al., 1991] and the East Pacific Rise $23^{\circ} \mathrm{N}$ [Bender et al., 1984]. 
O KG93 \& Kinzler97

t BS94

$\square$ Walter98

$\triangle$ HK93(KLB1)

$\nabla$ HK93(HK66)

$\diamond$ KHT98

$\bigcirc \begin{aligned} & \text { Estimated primary melts } \\ & \text { (with } 3500 \mathrm{ppm} \mathrm{Ni})\end{aligned}$

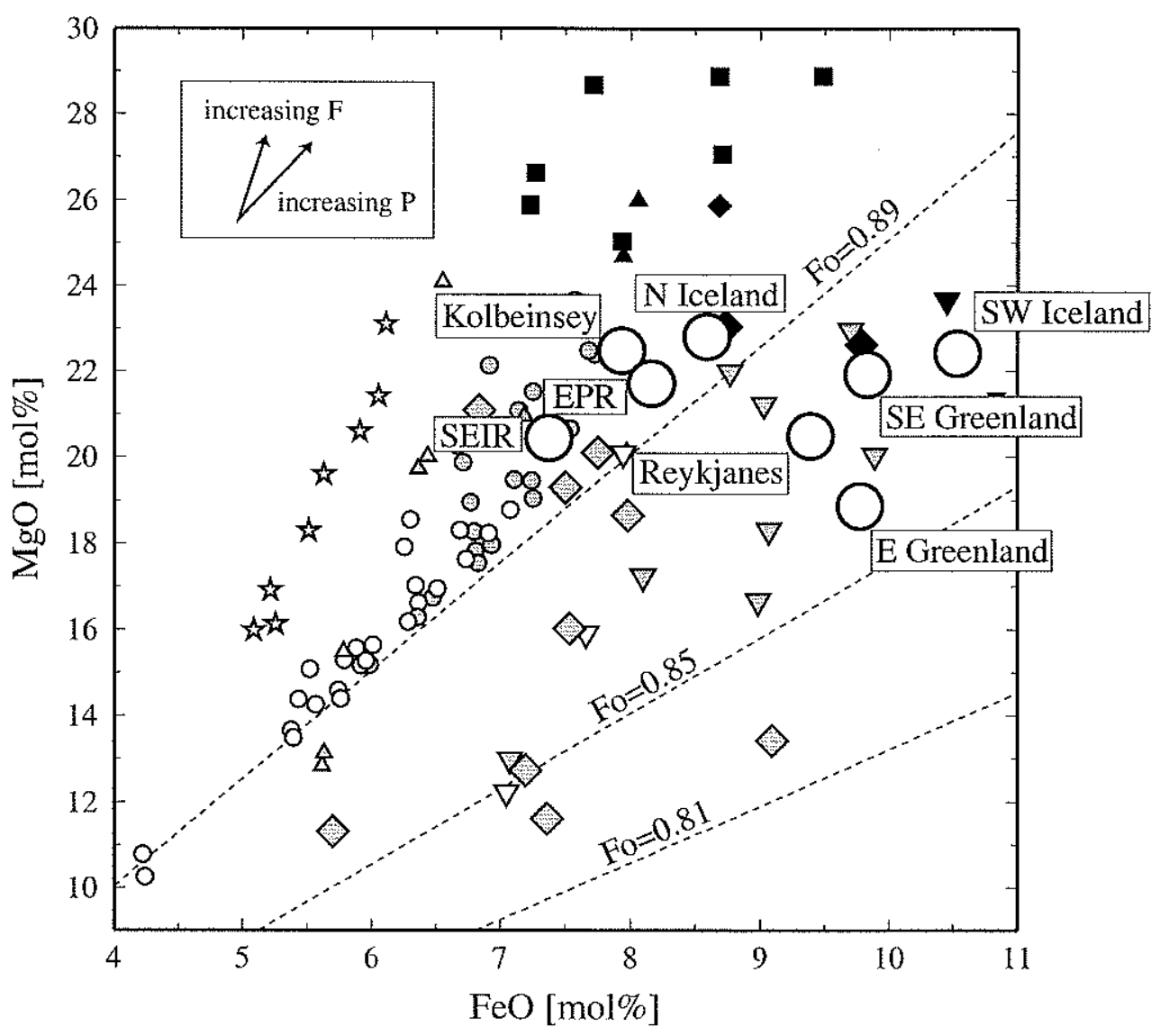

Figure 4-11. The molar $\mathrm{FeO}$ and $\mathrm{MgO}$ contents of estimated primary melts based on the $3500 \mathrm{ppm}$ olivine Ni criterion are plotted with those of experimental mantle melts. In addition to mantle melts used in Figure 4-2, data from Kogiso et al. [1998] are also plotted. The Fo content of equilibrium olivine is shown as dashed

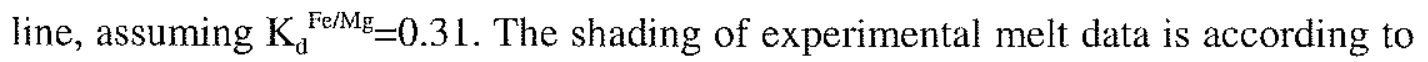
the pressure of melting: open $(P<1.5 \mathrm{GPa})$, gray $(P=1.5-3.0 \mathrm{GPa})$, and solid $(P>3.0 \mathrm{GPa})$. 

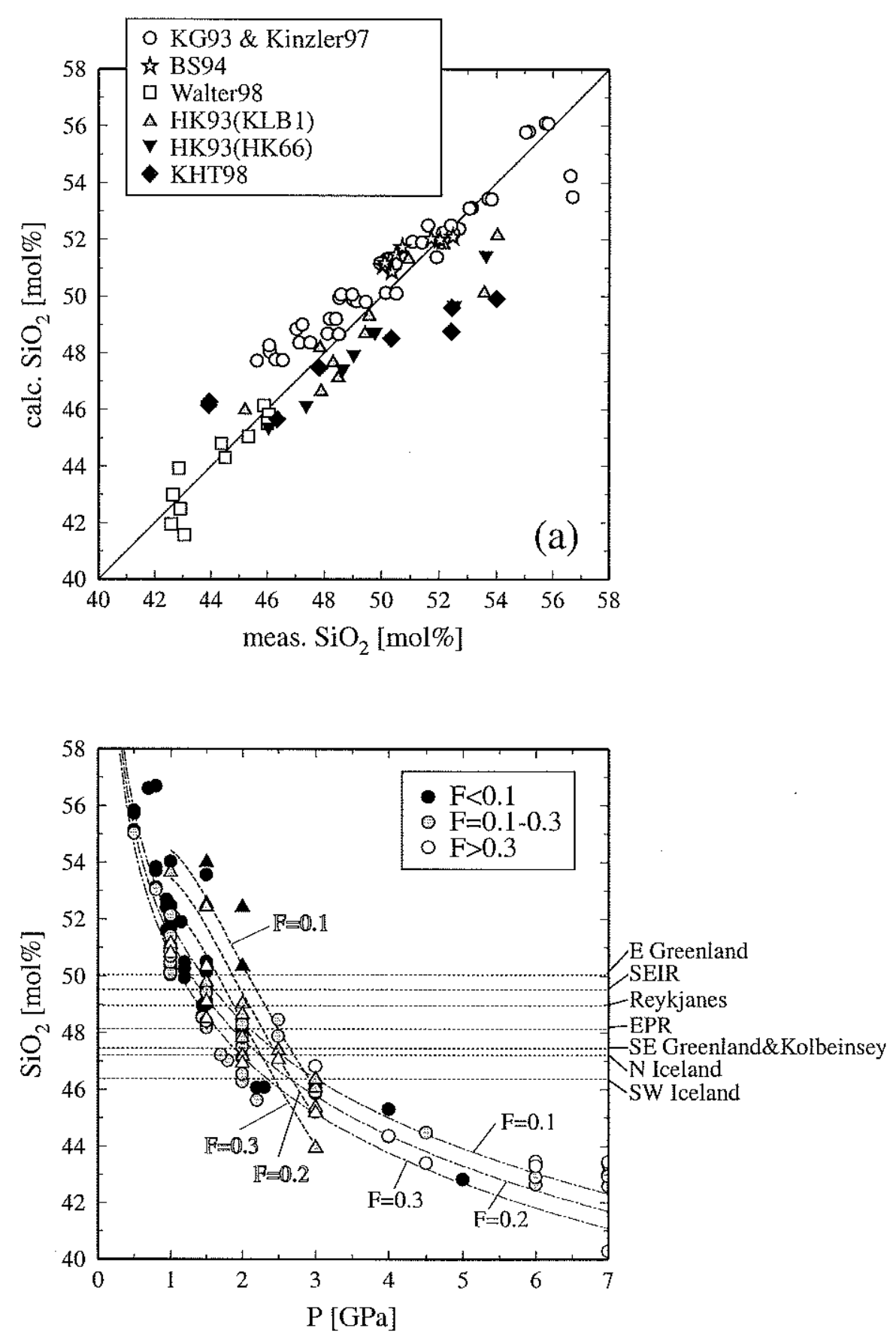

Figure 4-12. (a) The molar $\mathrm{SiO}_{2}$ contents of experimental melts are fitted as a function of the pressure and fraction of melting: $\mathrm{SiO}_{2}(\mathrm{~mol} \%)=51.92+0.636 / P-4.668 \log (P)-6.145 F$. One standard deviation is 1.348. Only data with $F<=0.3$ are used for this regression, because $\mathrm{SiO}_{2}$ systematics become complicated after clinopyroxene exhaustion. (b) The $\mathrm{SiO}_{2}$ contents of experimental melts are plotted as a function of melting pressure, together with the estimated primary $\mathrm{SiO}_{2}$ contents for the North Atlantic igneous province and representative normal midocean ridges. Dashed-dotted lines are based on the regression in (a). Dashed lines illustrate results of another regression based solely on experimental melts of relatively Fe-rich peridotite compositions from Hirose and Kushiro (HK66) [1993] and Kogiso et al. [1998]. 


\title{
Chapter 5
}

\section{Effects of vertical boundaries on infinite Prandtl number thermal convection}

\begin{abstract}
The influence of the basal topography of lithosphere on mantle convection is studied using a simple hydrodynamic model in which a fluid is heated from below and is bounded by rigid side walls. Rayleigh number up to $10^{6}$ is considered. A series of finite element stability analyses are employed to systematically characterize the nature of convective instability in such a system. The marginal stability of a bounded fluid is increased not only by the rigid boundary condition on side walls, but also by heat conduction across side walls. The planform of convection at the marginal state is $3-\mathrm{D}$, having convective rolls aligned perpendicular to side walls. The characteristics of nonlinear convection at supercritical Rayleigh numbers are investigated by first calculating 2-D steady-state solutions and then applying 2-D and 3-D stability analyses. The 2-D stability analysis shows that the presence of side walls significantly reduces the transition Rayleigh number for time-dependent convection, and the 3-D stability analysis shows that heat conduction through side walls strongly prefers $3-\mathrm{D}$ convective motion, as opposed to the case of insulating side walls. The characteristics of 3-D convection are then analyzed through a 3D mean-field approximation. Both 2-D and 3-D mean-field solutions demonstrate that conducting side walls reduce the strength of convection, with a negligible influence at higher Rayleigh numbers. The principal role of thick lithosphere is thus to modulate the planform of small-scale convection in the upper mantle.
\end{abstract}

\subsection{Introduction}

The thickness of the Earth's lithosphere (used as a cold thermal boundary layer throughout this paper) varies significantly on a global scale, especially in association with continental lithosphere, as inferred from global seismic tomography [e.g., Montagner and Tanimoto, 1991; Zhang and Tanimoto, 1993; Su et al., 1994; Ritzwoller and Lavely, 1995] as well as regional seismic studies [e.g., Jordan, 1988; Grand, 1994; Gaherty and Jordan, 1995], and a change in lithospheric thickness of as large as $200 \mathrm{~km}$ is fairly common at continentocean boundaries. Characterizing sublithospheric convection with such a variable thickness of lithosphere is important to understand how near surface structure can interact with mantle convection [e.g., Anderson, 1998a]. A strong spatial association between continental margins and flood basalts, for example, led Anderson [1994] to suggest that lithosphere has a primary control on the dynamics of exceptionally voluminous volcanism, 
as opposed to a more common explanation based on deep-seated mantle plumes [e.g., Richards et al., 1989; White and McKenzie, 1989; Hill et al., 1992]. Compared to mantle plumes, however, the importance of a heterogeneous top boundary on mantle convection has attracted less attention, and there have been only a limited number of geodynamical studies for this problem.

A few authors have considered the influence of a variable lithospheric thickness through a perturbation approach [Busse, 1978; Zhang and Busse, 1997], but this type of treatment is limited to a small amplitude of thickness variation with respect to the vertical scale of a convective system. Although the Earth's mantle has a depth extent of about $3000 \mathrm{~km}$, a proper vertical scale for the convective interaction with near surface structure may be that of the upper mantle, because the mantle viscosity structure is very likely to be layered, the lower mantle being more viscous than the upper mantle [Hager et al., 1985; Forte and Mitrovica, 1996; Simons and Hager, 1997], and because an endothermic phase transition at the base of the upper mantle may retard material flux [Honda et al., 1993; Tackley et al., 1993; Solheim and Peltier, 1994; Tackley, 1995].

In this case, the thickness variation of lithosphere, such as expected at continentocean transitions would exceed the valid range of linear approximations in the perturbation analyses. The influence of a sharp spatial variation in boundaries on a convective system is a highly nonlinear problem. It has been considered with some specific model geometries, such as for convection due to slab cooling [Rabinowicz et al., 1980; Nataf et al., 1981; Christensen, 1983], and for convection due to cratonic lithosphere or rifting-generated lithospheric necking [e.g., Richter, 1973; Elder, 1976; Mutter et al., 1988; Keen and Boutilier, 1995; King and Anderson, 1998]. Because these studies focus on specific geological problems, it is difficult to extract the general effects of a heterogeneous top boundary. Moreover, an initial-value approach is common in these convection studies [Mutter et al., 1988; Keen and Boutilier, 1995; Boutilier and Keen, 1999], so their results critically depend on a particular choice of initial conditions.

The main objective of this paper is to derive scaling laws that will be useful to assess how the characteristics of mantle convection can be affected by the structure of the 
top boundary, based on a boundary-value approach. The essence of the strongly varying basal topography of lithosphere on underlying mantle convection can be reduced to the influence of bounding vertical boundaries on a convecting fluid. Throughout this paper, therefore, a simple hydrodynamic model, in which a fluid is bounded with both horizontal and vertical rigid boundaries, will be systematically analyzed with a series of stability analyses and be characterized by several scaling laws such as the Rayleigh number-Nusselt number relationship. Although this model has no direct analog in the actual mantle convection system, we can deduce the influence of side walls on a convection system, by comparing these model results with the classical model of an infinite horizontal layer. The virtue of our approach therefore lies in its simplicity and comprehensiveness, not in an effort to simulate realistic complexity in mantle convection.

\subsubsection{Model Configuration}

The geometry of the model boundaries is two-dimensional. As shown in Figure 5-1, while a fluid is bounded laterally in one of the horizontal coordinates $(x$, hereafter called the inplane coordinate), its extent is infinite in the other horizontal coordinate ( $y$, the out-of plate coordinate). This model configuration is motivated by the fact that a stepwise change in lithospheric thickness associated with a continent-ocean transition can be reasonably approximated as a linear feature for the scale of upper-mantle convection. A fluid is heated from below, i.e., the surface and the bottom temperature are fixed at zero and some positive temperature, $\Delta T$, respectively. Convective motions in such systems are not necessarily $2-\mathrm{D}$, and we will investigate both $2-\mathrm{D}$ and $3-\mathrm{D}$ cases. The aspect ratio of the model domain, $r$, is defined as width over depth, and several different aspect ratios will be considered. The side wall has both kinematic and thermal effects on convection, through its rigid and conducting boundary conditions. Two different thermal boundary conditions, i.e., conducting and insulating, will be applied for the side walls to determine their contrasting effects. For conducting side walls, a linear thermal profile is used to retain the symmetry of the system. The mantle "fluid" is assumed to have an infinite Prandtl number and uniform viscosity. We also employ the Boussinesq approximation to the conservation equations of mass and momentum. Throughout this paper, we will employ the finite 
element method to solve viscous momentum equations and thermal advection-diffusion equations. Uniform 2-D quadrilateral elements with a non-dimensional size of 0.02 by 0.02 are used to discretize the model domain. With a temperature difference of $1000 \mathrm{~K}$ and viscosity of $10^{20}-10^{21} \mathrm{~Pa} \mathrm{~s}$, the Rayleigh number for upper mantle convection is on the order of $10^{5}-10^{6}$, so we will consider Rayleigh numbers up to $10^{6}$.

\subsection{Effects of Side Walls on Marginal Stability}

The equations for infinite Prandtl number thermal convection are governed by the Rayleigh number. The critical Rayleigh number corresponds to the onset of convection, and it plays a fundamental role in various scaling laws, such as the relationship between the Rayleigh number and the amplitude of convection [e.g., Chandrasekhar, 1981]. For example, the critical Rayleigh number varies with different boundary conditions. A fluid bounded by rigid horizontal boundaries, for example, has a higher critical Rayleigh number $(\sim 1708)$ than that by free-slip boundaries $(\sim 650)$; a fluid becomes less prone to convect when a free-slip condition is replaced with a rigid condition. A similar argument can be made for the addition of vertical side walls to a convective system, and some increase in the critical Rayleigh number is expected. Since the separation of variables is impossible when a fluid is bounded by both rigid horizontal and vertical boundaries, we need to employ the variational method to obtain approximate critical Rayleigh numbers [e.g., Davis, 1967;

Charlson and Sani, 1971; Chen, 1992].

\subsubsection{Variational Formulation}

The nondimensionalized governing equations for the marginally stable state are:

Conservation of mass

$$
\nabla \cdot \mathbf{u}=0
$$

Conservation of momentum

$$
-\nabla P+\nabla\left[\mu\left(\nabla \mathbf{u}+\nabla \mathbf{u}^{\mathrm{T}}\right)\right]-\operatorname{Ra} \theta \mathbf{e}_{z}=0
$$

Conservation of energy

$$
\mathbf{u} \cdot \nabla \theta-\nabla^{2} \theta=0
$$


where $\mathbf{u}$ is normalized velocity, $P$ is normalized pressure, $\mu$ is normalized viscosity, and $\theta$ is temperature perturbation from a linear conductive thermal profile, normalized by $\Delta T$. The spatial scale is normalized with a system height of $d$, and the temporal scale is normalized with a diffusion time scale of $d^{2} / \kappa$, where $\kappa$ is thermal diffusivity. Velocity is thus normalized with $\kappa / d$. The Rayleigh number, $\mathrm{Ra}$, is defined as,

$$
\mathrm{Ra}=\frac{\alpha \rho_{r} g \Delta T d^{3}}{\kappa \mu_{r}},
$$

where $\alpha$ is thermal expansivity, $\rho_{r}$ is a reference density, $g$ is gravitational acceleration, and $\mu_{r}$ is a reference viscosity. For some specific values of $\mathrm{Ra}$, the governing equations with the boundary conditions given above allow a non-zero solution, and the critical Rayleigh number is the minimum of such a solution family as,

$$
\operatorname{Ra}_{\mathrm{c}}=\min _{\theta, \hat{w}} \frac{\int_{V} \theta \nabla^{2} \theta d V}{-\int_{V} \theta \hat{w} d V},
$$

where $\hat{w}$ is vertical velocity field corresponding to the given temperature field, $\theta$, with unit Rayleigh number. This is the temperature-based variational formulation for the marginally stable state [Chandrasekhar, 1981]. The use of a unit velocity field requires that all boundary conditions are homogeneous, which is satisfied by our model configuration.

Using a finite dimensional approximation as,

$$
\begin{aligned}
\theta & =\sum_{i=1}^{n} \beta_{i} \theta_{i} \\
\hat{w} & =\sum_{i=1}^{n} \beta_{i} \hat{w}_{i}
\end{aligned}
$$

the variational statement leads to the following eigenvalue problem,

$$
\mathbf{A} \vec{\beta}=\lambda \mathbf{D} \vec{\beta}
$$

where

$$
\begin{aligned}
A_{i j} & =\int_{V} \theta_{i} \hat{w}_{j} d V \\
D_{i i} & =\int_{V} \theta_{i} \nabla^{2} \theta_{i} d V \\
\lambda & =\frac{1}{\mathrm{Ra}}
\end{aligned}
$$


Both $\mathbf{A}$ and $\mathbf{D}$ are positive-definite, so all eigenvalues are real and positive. The critical Rayleigh number corresponds to the largest eigenvalue, $\lambda_{\max }$. We choose the temperature trial functions, $\theta_{i}$, as,

$$
\theta_{i}=\sin \left(p_{i} \pi x\right) \cos (\psi y) \sin \left(q_{i} \pi z\right)
$$

for conducting side walls, and

$$
\theta_{i}=\cos \left(p_{i} \pi x\right) \cos (\psi y) \sin \left(q_{i} \pi z\right)
$$

for insulating side walls. $p_{i}$ and $q_{i}$ are non-negative integers, and $\psi$ is a real number. A velocity field corresponding to a temperature trial function is calculated by a finite element solver for Stokes flow. Since the matrix $\mathbf{D}$ can be calculated analytically, only 2-D numerical integration is required to calculate the matrix $\mathbf{A}$.

\subsubsection{Results for 2-D Perturbations}

We use all possible trial functions with $\psi=0$ and $p_{i}+q_{i} \leq N$, where $N$ denotes the maximum mode of the finite dimensional approximation. The variational solution is merely an upper bound for the true critical Rayleigh number, so that $N$ must be increased until convergence is obtained. Convergence is defined here such that a relative change between solutions for $N$ and $N+2$ does not exceed $0.1 \%$. A monotonic convergence is guaranteed in the variational method, and we found that $N$ less than 10 is usually sufficient (e.g., Figure 5-2). A comparison with the critical Rayleigh numbers calculated by Velte [1964] for the conducting case shows only about $0.5 \%$ difference in general, which is consistent with the expected accuracy from our finite element discretization. Since we are mainly interested in the first-order characteristics of stability curves for relatively small aspect ratios, this level of accuracy is sufficient.

The stability curves for 2-D perturbations are presented in Figure 5-3b as a function of aspect ratio, for insulating and conducting side walls. For conducting side walls, critical Rayleigh number is 36440,5050 , and 2397 at $r=0.5,1.0$, and 2.0, respectively. The case with no side walls has a critical Rayleigh number of 1708 , so the influence of the side walls on marginal stability is almost insignificant for aspect ratios larger than 2 . The divergent character of the stability curves for small aspect ratios is also evident in the stability curve for a layer of fluid with an infinite horizontal extent, if the latter is plotted as 
a function of a given perturbation wavelength (Figure 5-3a). This indicates that the increase in the critical Rayleigh number for a narrower channel simply results from wavenumber selection imposed by a small aspect ratio. At a given aspect ratio, the critical Rayleigh number for conducting side walls is always larger than that for insulating side walls. Because the insulating boundary condition is a natural boundary condition, which is automatically satisfied in the variational formulation, the trial function space in the variation formulation associated with the insulating case is larger than that with the conducting case [Charlson and Sani, 1970]. Therefore, the critical Rayleigh number of the conducting case should be always an upper bound for that of the insulating case. The inflections in the stability curves, which are more clearly observed for the insulating case, correspond to changes in the number of convective rolls and indicate the composite nature of the stability curves [e.g., Davis, 1967].

\subsubsection{Results for 3-D Perturbations}

Because of the infinite horizontal extent in the out-of-plane coordinate, a general 3-D perturbation in the model can be decomposed into the sum of single-mode perturbations, each of which has a fixed out-of-plane wavenumber. 3-D Stokes flow generated by a single-mode perturbation in the temperature field can be calculated efficiently by 2-D finite element procedures (see Appendix A), and the variational method used for the above 2-D case can be easily extended to single-mode 3-D perturbations. Stability curves as a function of out-of-plane wavenumber were calculated for each value of aspect ratio (Figure 5-4a), and by taking the minimum of each stability curve, the final 3-D stability curve was constructed as a function of aspect ratio (Figure 5-4b).

For the reason stated above regarding the relative size of function space, critical Rayleigh numbers for 3-D perturbations are always smaller than those for 2-D perturbations. The out-of-plane wavelength at which a stability curve takes the minimum is always finite, varying from $\sim 1$ to $\sim 2$ for aspect ratios between 0.25 and 2.0 , showing that, in the most stable convective pattern, convective rolls are aligned perpendicular to side walls. Because of the increased degree of freedom allowed by out-of-plane convection, there should be negligible influence of in-plane wavenumber selection imposed by aspect 
ratio. The calculated stability curves therefore reflect the effect of wall friction along the sides of convective rolls. It is interesting, therefore, that the most stable out-of-plane wavelength is close to 1 for aspect ratios less than 0.5 ; a strong influence of wall friction seems to prevent otherwise stable cellular convection with a wavelength of $\sim 2$. Nonetheless, this wall friction has a much weaker influence on critical Rayleigh number compared to the mode selection in the in -plane coordinate, as seen from a drastic decrease in 3-D critical Rayleigh number at small aspect ratios.

\subsection{Stability of 2-D Steady-State Thermal Convection in a Bounded Fluid}

For supercritical Rayleigh numbers, thermal convection becomes highly nonlinear and generally time-dependent. One way to characterize such a convective system is to conduct fully time-dependent calculations and calculate some statistical measures from them. Alternatively, the characteristics of convection past marginal stability may be succinctly represented by those of steady-state solutions if they exist. We take the latter approach because steady-state solutions can be quickly obtained by the temperature underrelaxation method [Christensen, 1984]. A stability analysis is then applied to steady-state solutions, because they could be artificially stabilized by the relaxation method. This boundary-value approach supplemented with a stability analysis provides a concise summary for the nature of supercritical convection, and it can efficiently determine a transition to time-dependent convection. Most of our analyses in this section are restricted to a purely 2-D framework. A 3-D stability analysis will be conducted at the end to test the validity of this 2-D assumption.

\subsubsection{Perturbation Analysis}

With the Boussinesq approximation, the governing equations for the convective system with a supercritical Rayleigh number are comprised of (1) the equation of mass conservation (equation 5.1), (2) the equation of momentum conservation, which is the same as equation 5.2 except that perturbation temperature $\theta$ is replaced with total temperature $T$, and (3) the equation of energy conservation, 


$$
\frac{\partial T}{\partial t}+\mathbf{u} \cdot \nabla T=\nabla^{2} T
$$

A temporal derivative appears only in the energy equation, so a steady-state solution can be obtained by dropping the left-hand side of equation 5.11 ab initio and, with an initial guess for a steady-state temperature field, iterating between the Stokes flow equation and the modified energy equation until convergence is attained. A purely implicit method is used to solve the steady-state energy equation [e.g., Hughes, 1987]. Rapid convergence is possible by temperature underrelaxation during iterations [Christensen, 1984]. We start with an underrelaxation factor of 0.5 , which is gradually increased when convergence is not obtained in given iterations. As a criterion for convergence, we use a root-mean-square difference of $0.05 \%$ in the velocity fields of consecutive iterations.

Convergence obtained in the underrelaxation method does not necessarily mean that a resultant 'steady-state' solution is stable. If a high degree of underrelaxation is required for convergence, for example, it is generally the sign of an unstable solution. A solution is called stable if it can maintain its structure against any kind of perturbation. The stability of a steady-state solution can be tested by integrating the time-dependent energy equation (equation 5.11) with an explicit time-stepping method [e.g., Christensen, 1987; Hansen and Ebel, 1988], or by a perturbation analysis described below [e.g., Busse, 1967], which is more quantitative and complete.

Denoting steady-state temperature and velocity fields by $T_{0}$ and $\mathbf{u}_{0}$, respectively, the energy equation for a perturbed state may be expressed to first-order as

$$
\frac{\partial \theta}{\partial t}=-\mathbf{u}_{0} \cdot \nabla \theta-\mathbf{u} \cdot \nabla \theta_{0}+\nabla^{2} \theta
$$

where $\theta$ is a deviation from steady-state temperature, and $\mathbf{u}$ is a perturbation velocity field generated by $\theta$ (equation 5.2). For a real steady-state solution, any kind of initial perturbation temperature must decay to null. Assuming an exponential time-dependence for perturbation temperature as $\theta \sim \exp (\sigma t)$, and using the same finite-dimensional approximation as in equation 5.6, the above perturbation equation leads to the following eigenvalue problem:

$$
\mathbf{A} \vec{\beta}=\sigma \mathbf{B} \vec{\beta}
$$


where

$$
\begin{gathered}
A_{i j}=\int_{V}\left[\theta_{i} \nabla^{2} \theta_{j}-\theta_{i}\left(\mathbf{u}_{0} \cdot \nabla\right) \theta_{j}-\theta_{i}(\mathbf{u} \cdot \nabla) \theta_{0}\right] d V \\
B_{i i}=\int_{V} \theta_{i}^{2} d V
\end{gathered}
$$

Although $\mathbf{B}$ is diagonal, $\mathbf{A}$ is nonsymmetric, so eigenvalues are generally complex numbers. The maximum real part of the eigenvalues, which is called a growth exponent, determines the stability of the steady-state solution in consideration; a steady-state solution must have a negative growth exponent.

\subsubsection{Stability for 2-D Perturbations}

Without any formal analysis, a qualitative understanding of stability may be obtained from the structure of a steady-state temperature field (Figure 5-5). Whereas the rigid boundary condition on side walls does not strongly alter the temperature field, the influence of the conducting boundary condition is significant; the steady-state solution with a Rayleigh number of $10^{5}$ for conducting side walls, for example, seems very unlikely to be stable. As in the variational formulation for the critical Rayleigh number, the number of trial functions used in the perturbation analysis must be increased until convergence is attained for a growth exponent. Because the perturbation equation is not self-adjoint, monotonical convergence is not guaranteed. Nevertheless, we found that a finite dimensional approximation with $N=10-15$ is sufficient for convergence in all cases we considered.

Since the system of steady-state convection is nonlinear, a steady-state solution is generally nonunique. In fact, at least three different modes of a steady-state solution, i.e., one odd mode and two even modes (Figure 5-6a), are easily found by using an appropriate initial guess in the temperature underrelaxation method. The two even modes share the same stability curve, due to their symmetrical relation. Though the odd mode and the even modes have different growth exponents, the first-order behaviors of these growth exponents as a function of the Rayleigh number are quite similar (Figure 5-6b). In the following analysis, we chose to use the growth exponent of the odd mode solution. Our preference to the odd mode is solely because there is no overall heat loss or gain through side walls for the odd-mode solutions. This removes an ambiguity in defining the Nusselt number, which is used to characterize the convective system in the next section. 
Growth exponents as a function of the Rayleigh number are shown in Figure 5-7 for three different aspect ratios $(0.5,1.0$, and 2.0) and for conducting and insulating boundary conditions. Also shown is the stability curve for the case of no side wall (i.e., reflecting boundary condition at sides) with a unit aspect ratio. Regardless of the type of thermal boundary condition on the side wall, the transition to an unstable regime is abrupt. This sharp transition makes a good contrast with a much more gradual transition for the case of no side wall. The Rayleigh number that marks this transition to time-dependent solutions is sometimes called the critical Rayleigh number of the second kind [e.g., Busse, 1967]. The Rayleigh number of the second kind is larger for insulating side walls, and it is bounded by the limiting case of no side wall, which becomes unstable at a Rayleigh number of $4 \times 10^{5}$ [e.g., Mitrovica and Jarvis, 1987]. The influence of side walls on stability may be more systematically seen when Rayleigh number is scaled with the system's own critical value, especially in the conducting case (Figure 5-7). That conducting side walls are more destabilizing than insulating side walls can be understood in terms of thermal boundary layer instability. Following Howard [1966], we define the local Rayleigh number as,

$$
\mathrm{Ra}_{l}=\frac{\alpha \rho_{r} g \Delta T_{l} h^{3}}{\kappa \mu_{r}},
$$

where $\Delta T_{l}$ is a temperature difference across a thermal boundary layer with a thickness of $h$. Because the conducting boundary condition leads to a thicker thermal boundary layer (e.g., Figure 5-5), the local Rayleigh number is higher for conducting side walls than for insulating side walls at a given global Rayleigh number. Thus, the effects of a conducting side wall on thermal convection can be summarized by the two kinds of critical Rayleigh numbers, i.e., higher critical Rayleigh number and lower transition Rayleigh number, compared to an insulating side wall. Conducting side walls reduce the system's convective potential and, at the same time, increase the tendency to time-dependent convection.

\subsubsection{Characteristics of 2-D Convection in a Bounded Fluid}

Two kinds of measures are used to characterize the strength of convection. The first one is the Nusselt number defined as, 


$$
\mathrm{Nu}=\frac{q d}{k \Delta T},
$$

where $q$ and $k$ are heat flux averaged over a horizontal boundary and thermal conductivity, respectively. $\mathrm{Nu}$ is nondimensional heat flux scaled by conductive heat flux at a given temperature difference. A higher Nusselt number indicates more efficient heat transport realized by convection. The second measure is maximum vertical velocity. Our choice of a vertical velocity component over horizontal velocity components is its relevance to terrestrial magmatism. When a vertical movement of mantle results in decompressional melting at shallow depths, vertical velocity becomes a critical parameter to affect the melt production rate. For high Rayleigh numbers with which no stable steady-state solution exists, we conducted fully time-dependent calculation with an explicit time-stepping method. Starting from an artificial steady-state solution obtained by the temperature underrelaxation method, the system was integrated for a unit thermal diffusion time, which is equivalent to $\sim 10^{3}$ to $10^{4}$ time steps for a range of the Rayleigh number we considered. Typically, the system reached its stationary state within the first 0.1 diffusion time, and the time-averaged values of the Nusselt number and vertical velocity were then calculated for these stationary-state solutions. Results are presented in nondimensionalized values, as well as dimensionalized ones scaled by relevant parameters appropriate for convection in the upper mantle: $d=600 \mathrm{~km}, \kappa=10^{-6} \mathrm{~m}^{2} \mathrm{~s}^{-1}, k=3.3 \mathrm{Wm}^{-1} \mathrm{~K}^{-1}$, and $\Delta T=1300 \mathrm{~K}$.

For Rayleigh numbers from $10^{4}$ to $10^{6}$, the average Nusselt number and maximum vertical velocity are plotted in Figure 5-8, for both conducting and insulating side walls. The case of no side wall is also shown as a reference. As expected from the extra constraints on convective motion imposed by side walls, both the Nusselt number and vertical velocity are smaller in the bounded cases. The influence of the side walls is systematic; the system with a larger aspect ratio almost always showing higher values. Heat flux at a rigid boundary is essentially determined by the thickness of a thermal boundary layer, so that the very low Nusselt numbers for the conducting case with the Rayleigh number less than $10^{5}$ result from the imposed, fixed temperature condition on the vertical boundaries. For the Rayleigh number close to $10^{6}$, the Nusselt numbers of the bounded cases are almost equal to that of the unbounded convection. This weakening of 
side wall effect on heat transfer at higher Rayleigh numbers has also been detected by theoretical and experimental studies on the relationship between $\mathrm{Nu}$ and $\mathrm{Ra}$ for convection bounded by insulating side walls [e.g., Frick and Clever, 1982]. Even for conducting side walls, the tendency to thicken thermal boundary layers and thus to lower the Nusselt number becomes negligible at higher Rayleigh numbers; a thick thermal boundary layer imposed by conductive side walls becomes unstable and is constantly destroyed, so timeaveraged Nusselt number increases.

In contrast, vertical velocity is persistently affected by the presence of side walls, and there is no significant difference between the different thermal boundary conditions. For an aspect ratio of 1.0 , for example, about a factor of two reduction in maximum vertical velocity can be observed for both kinds of side walls, up to the highest Rayleigh number we considered. One might expect that a scaling law for vertical velocity should behave similarly as that for heat flux, which is indeed the case for convection in an infinite horizontal layer [e.g., McKenzie et al., 1974]. From this perspective, the observed discrepancy in the two scaling laws may be puzzling. This, however, can be understood by considerting the kinetic energy budget. For a stationary state, velocity is determined mainly by a balance between the potential energy release and the energy dissipation at bounding surfaces [e.g., Chandrasekhar, 1981]. Whereas the growth of a thermal boundary layer is limited by the local Rayleigh number so that the Nusselt number becomes little affected by side walls, the kinematic effect of side walls is persistent.

One of the features of a stationary-state solution that is not evident in these scaling laws is the nature of temporal variability. Figure 5-9 shows examples of stationary state solutions for the conducting side walls at two different Rayleigh numbers. For Rayleigh numbers slightly exceeding the transition value for time dependency, the temporal variation of observables such as the Nusselt number and total kinetic energy is periodic with small amplitudes (Figure 5-9a). As clearly seen from a series of snapshots of the temperature field, time dependency originates in boundary layer instabilities. Instabilities form near side walls, where the thermal boundary layer is thickest, and they grow inward into the model domain. As the Rayleigh number increases, this periodic character 
diminishes, and more random, higher frequency variation dominates with much larger amplitudes (Figure 5-9b). It still appears that time dependence is controlled by instabilities at thermal boundary layers, but their occurrence is no longer limited to the vicinity of side walls.

A simple relation between the scale of time dependency and the Rayleigh number may be derived based on boundary layer theory, following the approach taken by Howard [1966]. A thermal boundary layer becomes unstable when its local Rayleigh number reaches a threshold, $\mathrm{Ra}^{\delta}$,

$$
\mathrm{Ra}_{l}=\mathrm{Ra}^{\delta} .
$$

The growth of a thermal boundary layer can be approximated as half-space cooling, so its thickness $h$ may be expressed as $h \approx 2 \sqrt{\kappa t}$. Corresponding temperature difference across the boundary layer is half of the total temperature difference across the entire model depth, $\Delta T / 2$. Equations 5.4, 5.15, and 5.17 can then be solved for the period over which boundary layer instability repeats. The solution may be expressed as

$$
\frac{t}{d^{2} / \kappa} \approx\left(\frac{\mathrm{Ra}^{\delta}}{4 \mathrm{Ra}}\right)^{2 / 3} \approx \frac{25}{\mathrm{Ra}^{2 / 3}}
$$

where we assumed $\mathrm{Ra}^{\delta}$ of $\sim 500$ [Parsons and McKenzie, 1978]. Figure 5-10 shows the power spectra of Nusselt numbers in the case of conducting side walls, for a range of Rayleigh number. Regardless of the aspect ratio of the bounded region, there is a broad agreement between the above, order-of-magnitude prediction and the actual measurements, in terms of the highest frequency excited. The effect of side walls is simply to lower the transition Rayleigh number for time-dependent behavior, and the periodicity of boundary layer instability is simply related to the actual Rayleigh number itself, not to the one scaled by the system's own critical Rayleigh number.

\subsubsection{Stability for 3-D Perturbations}

The stability analysis of 2-D steady-state solutions can be easily extended to a full 3-D perturbation analysis, in a manner similar to the 3-D marginal stability analysis. For each 2-D steady-state solution, a growth exponent was first calculated as a function of the outof-plane wavenumber, and its maximum value was then taken to determine the 3-D 
stability of the given 2-D solution. For the conducting case, the growth exponent is always positive from the marginally stable state, and it rapidly increases as the Rayleigh number increases (Figure 5-11a). The stability curve for the insulating case shows very different dependence on the Rayleigh number (Figure 5-11b). The growth exponent is generally much smaller than that of the conducting case, and it becomes negative for a certain range of the Rayleigh number. For an aspect ratio of 1.0 , the growth exponent is negative for Ra of $10^{4.15}-10^{4.55}$, and for an aspect ratio of 0.5 , it is negative for $R a \geq 10^{4.75}$ (Figure $5-11 \mathrm{~b}$ ). The stability curve for an aspect ratio of 2.0 shows a minimum at $\mathrm{Ra}$ of $10^{4.1}$, which is almost zero, but it never becomes negative. Thus, for insulating side walls with small aspect ratios, a 2-D steady-state solution can represent a steady-state convective state in a realistic 3-D framework, for a limited range of Rayleigh numbers. This limited stability of a 2-D solution was also observed for convective systems with an infinite horizontal extent [Busse, 1967; Clever and Busse, 1974]. In most cases, the most unstable wavenumber lies between $\pi$ and $2 \pi$ for the Rayleigh numbers we investigated and, except where a growth exponent becomes negative, there is an overall increasing trend with increasing Rayleigh number.

Based on a comparison of stability curves for the two different thermal boundary conditions on side walls, we see that heat conduction through side walls has a large effect on the pattern of convection. In the marginal stability analysis, 3-D perturbations are always more destabilizing than 2-D perturbations, regardless of a thermal boundary condition, so that the negative growth exponents calculated for 3-D perturbations in systems with insulating side walls are somewhat surprising. We note that, even for conducting side walls, single-mode 3-D perturbations are not always destabilizing if the out-of-plane wavenumber is sufficiently large $(>2 \pi)$, as Figure 5-11a implies. Negative growth exponents for some 2-D steady-state solutions for insulating side walls result from their stability even for low out-of-plane wavenumbers. We infer that insulating side walls can generate a stable 2-D pattern of convection, because a tendency for 3-D stability increases for a narrower channel (Figure 5-11b). Conducting side walls are more relevant to geophysical applications, however, and our stability analysis demonstrates that 3-D 
convective motion is fundamentally important for thermal convection bounded by vertical side walls. In the next section, therefore, we will investigate the nature of 3-D convection.

\subsection{Characteristics of 3-D Convection in a Bounded Fluid}

Instead of solving a fully 3-D convection problem, we employ a mean-field approximation to the out-of-plane coordinate. Only one characteristic wavenumber is considered to represent convective cells aligned perpendicular to side walls. The mean-field approximation [Malkus, 1954; Herring, 1963] reduces the dimension of the system by one, so fewer computational resources are required compared to a fully $3-\mathrm{D}$ case. Though the details of a mean-field solution, of course, depend on the prescribed out-of-plane wavenumber and are expected to be different from those of a fully 3-D solution, the 3-D mean-field approximation is an efficient way to evaluate the effect of the increased degree of freedom on domain-wide observables such as the Nusselt number. Care must be taken, however, not to overinterpret our results, because it is known that the Nusselt number based on a 2-D mean-field solution is systematically higher than that of a fully 2-D solution [Quareni et al., 1985; Quareni and Yuen, 1988]. Olson [1987] ascribed this systematic bias to the fact that vertical velocity and temperature in the out-of-plane coordinate are perfectly correlated in 2-D mean-field solutions. The effect of this perfect correlation should be somewhat reduced in our 3-D mean-field solutions, in which meanfield temperature is 2-D and has its own velocity field. In any case, results presented below must be regarded as a preliminary attempt to understand 3-D effects, until their validity is confirmed by fully 3-D calculations. In all calculations, we use the out-of-plane wavenumber of $\pi$, which corresponds to cellular convection with a unit aspect ratio. Only a conducting boundary condition is considered for side walls.

\subsubsection{3-D Mean-Field Formulation}

We apply a single-mode approximation with a particular wavenumber, $\psi$, for the out-ofplane coordinate, $y$, as,

$$
T(x, y, z)=T(x, z)+\theta(x, z) \cos (\psi y)
$$

for a temperature field, and 


$$
\begin{array}{r}
\mathbf{u}(x, y, z)=(U(x, z)+u(x, z) \cos (\psi y), \\
v(x, z) \sin (\psi y), \\
W(x, z)+w(x, z) \cos (\psi y))
\end{array}
$$

for a velocity field. The system of thermal convection equations can then be written as:

Conservation of mass

$$
\begin{gathered}
\frac{\partial U}{\partial x}+\frac{\partial W}{\partial z}=0 \\
\frac{\partial u}{\partial x}-\psi v+\frac{\partial w}{\partial z}=0
\end{gathered}
$$

Conservation of momentum

$$
\begin{aligned}
& -\nabla P+\nabla\left[\mu\left(\nabla \mathbf{U}+\nabla \mathbf{U}^{\mathrm{T}}\right)\right]-\operatorname{Ra} T \mathbf{e}_{z}=0 \\
& -\nabla P+\nabla\left[\mu\left(\nabla \mathbf{u}+\nabla \mathbf{u}^{\mathrm{T}}\right)\right]-\operatorname{Ra} \theta \mathbf{e}_{z}=0
\end{aligned}
$$

and

Conservation of energy

$$
\begin{aligned}
& \frac{\partial T}{\partial t}+U \frac{\partial T}{\partial x}+W \frac{\partial T}{\partial z}+\frac{1}{2}\left(u \frac{\partial \theta}{\partial x}-\psi v \theta+w \frac{\partial \theta}{\partial z}\right)=\frac{\partial^{2} T}{\partial^{2} x}+\frac{\partial^{2} T}{\partial^{2} z} \\
& \frac{\partial \theta}{\partial t}+U \frac{\partial \theta}{\partial x}+W \frac{\partial \theta}{\partial z}+u \frac{\partial T}{\partial x}+w \frac{\partial T}{\partial z}=\frac{\partial^{2} \theta}{\partial^{2} x}-\psi^{2} \theta+\frac{\partial^{2} \theta}{\partial^{2} z}
\end{aligned}
$$

Because mean-field velocity and perturbation velocity are completely decoupled in the equations of mass and momentum conservation, they are solved separately by a 2-D Stokes-flow solver and a 3-D single-mode Stokes-flow solver, respectively, for given temperature fields. A 2-D finite element procedure has been developed to solve the equations of energy conservation, and its implementation is presented in Appendix B. Using these finite element solvers, a steady-state solution can be obtained with the temperature relaxation method in the same manner as for 2-D convection. For all Rayleigh numbers from $10^{4}$ to $10^{6}$, steady-state solutions are easily obtained with a moderate relaxation factor. We do not attempt to investigate their stability, because they are likely to be artificially stabilized by the imposed symmetry in the out-of-plane coordinate.

\subsubsection{Results}

By inspecting the above equations, it can be shown that mean-field and out-of-plane

temperature fields must have the same type of reflecting symmetry with respect to a vertical plane. Because the most fundamental mode in out-of-plane temperature is even, 
even-mode solutions are generally preferred over odd-mode solutions, and odd-mode solutions indeed have much weaker velocity fields. Because of its $\cos (\psi y)$ dependency, the odd-mode out-of-plane temperature field corresponds to convection cells with interchanging direction of convection. Such planform of convection is expected to be unstable, and indeed we were able to obtain odd-mode solutions only at low Rayleigh numbers $\left(\sim 10^{4}\right)$ by the temperature underrelaxation method. There are two equivalent even-mode solutions, i.e., cooling and heating modes (e.g., Figure 5-6), and only coolingmode solutions are presented here, considering its relevance to the cooling effect of cratonic lithosphere on upper-mantle convection, which is our primary interest. In general, heat flux at the top and bottom boundaries can be different for even-mode solutions, and we take a logarithmic average of top and bottom Nusselt numbers. Similarly, a logarithmic average of the absolute values of maximum and minimum vertical velocities is calculated as a second measure of convective strength. This averaging eliminates the dependence of the following results on our choice of cooling-mode solutions.

Examples of temperature fields are shown in Figure 5-12, and their 3-D rendition is shown in Figure 5-13 with velocity fields. At Ra of $10^{4}$, reflecting symmetry with respect to a horizontal plane is nearly maintained, so the difference between cooling and heating modes is small. Convective motion is almost entirely driven by out-of-plane temperature at this low Rayleigh number. As Ra increases, the degeneration of the two even-mode solutions disappears, and complex interactions between mean-field and out-of-plane temperature are observed. Higher Ra leads to a thinner thermal boundary layer, and because of the conducting boundary condition, a thermal boundary layer is also developed along side walls. This confinement of buoyancy to the vicinity of the model boundaries generates center upwelling in mean-field velocity.

The average Nusselt number and average maximum vertical velocity of these steady-state solutions are summarized in Figure 5-14. For comparison, values for the case of no side wall, obtained by 2-D mean-field and fully 2-D calculations are also shown. As already noted, 2-D mean-field solutions consistently overestimate the Nusselt number (Figure 5-14a), and this may explain why some of the 3-D mean-field estimates for a 
bounded fluid exceed the values of the unbounded case. Thus, an accurate estimate of the side wall effect on heat flux is difficult to obtain. Based on a gross similarity among different aspect ratios, however, we can infer that the side wall effect on heat transfer is negligible for Ra larger than $10^{5}$; the Rayleigh number that marks this transition is about an order of magnitude lower than that for 2-D convection (Figure 5-8a). Maximum vertical velocities in 3-D mean-field solutions (Figure 5-14b) are approximately 50\% higher than those in 2-D solutions (Figure 5-8b), regardless of aspect ratio. Maximum velocity in the case of an aspect ratio of 2 slightly exceeds that of the limiting case for Ra larger than $2 \times 10^{4}$, and this may be again due to the mean-field approximation. We may argue, however, that the influence of the mean-field approximation does not significantly degrade the estimate of velocity. Because a fluid is not bounded in the out-of-plane coordinate, the observed $50 \%$ increase in velocity compared to the purely $2-\mathrm{D}$ case is what we may expect as a result of increasing the system's dimension from 2 to 3 , based on a balance between potential energy release and energy dissipation along rigid boundaries.

The proportion of mean-field kinetic energy to total kinetic energy (Figure 5-14c) shows a systematic influence of aspect ratio. This proportion is a good measure of the two dimensionality of a 3-D mean-field flow field. At Ra of $10^{6}$ and with a unit aspect ratio, for example, mean-field convection involves more than $50 \%$ of total kinetic energy, and the total temperature field shows that a sheet-like upwelling at the model center dominates the convective system (Figure 5-13). Because only one out-of-plane mode is treated in our 3-D mean-field calculation, this does not necessarily mean that a sheet-like upwelling is preferred to a cylindrical plume. A cylindrical plume involves a wide range of wavelengths, so it cannot be represented by our single-mode approximation. Nevertheless, a sheet-like feature might be a plausible upwelling style in a fully 3-D solution, because its driving force originates in sheet-like downwellings along 2-D conducting side walls.

\subsection{Discussion}

Our idealized model configuration is a reflection of the difficulty in understanding the role of variable lithospheric thickness on mantle convection. Given the nonlinear nature of this 
convection problem, it is unwarranted to generalize results based on some specific variation in lithospheric thickness. We therefore chose an extreme case that maximizes the influence of side walls. In the Earth, continental lithosphere does not extend to the base of the upper mantle, and the variation in thickness is more diffuse, so that our study can be taken as a limiting case. A more serious limitation is the use of constant viscosity. The rheology of the upper mantle is clearly temperature-dependent and non-Newtonian, and pressure-dependence also plays an important role [e.g., Karato and $W u, 1993$ ]. We believe, however, that this study is a necessary step toward a comprehensive understanding of small-scale mantle convection. A wide variety of lithospheric structure along continental margins is expected on the Earth, and site-specific modeling with realistic rheology is of course important. Considering our limited understanding of mantle rheology and of initial and boundary conditions, however, a systematic study with more simplified models must be also employed. The latter has been lacking from recent studies of small-scale convection.

For a conducting side wall, we used a linear thermal profile, because it is the simplest choice and retains reflecting symmetry, which facilitates the calculation of steadystate solutions and stability analyses. It is obvious from the variational formulation that heat conduction at side walls always results in a higher critical Rayleigh number compared to insulation, regardless of the shape of an imposed temperature profile. In a strongly convecting regime, however, the influence of conducting side walls may be significantly modified by specifying different temperature. Rabinowicz et al. [1980] and Nataf et al. [1981], for example, investigated the effect of a uniformly cold side wall, and found that lateral cooling led to the generation of convection cells with a wide aspect ratio. Their studies were designed to model cooling by a subducting slab, and efficient heat transfer at a side wall can result in additional negative buoyancy to overcome side wall resistance. In this regard, our linear temperature profile minimizes the cooling effect of a geological side wall. Our primary interest, however, is to study the effect of thick, cratonic lithosphere on mantle convection, not the effect of slab cooling. Because of low continental heat flow and high concentration of radiogenic elements in continental crust and possibly in the 
lithosphere, the estimated thermal structure of cratonic lithosphere suggests only a moderate cooling effect of conducting, cratonic sidewalls adjacent to asthenospheric mantle [Jordan, 1988; Rudnick et al., 1998]. The linear thermal profile used in this study, therefore, does not severely limit the application of our results to small-scale mantle convection associated with cratonic lithosphere.

The effect of cratonic lithosphere on mantle convection has been recently studied by King and Anderson [1995; 1998], with the focus on the relative importance of smallscale, edge-driven convection to large-scale background convection. Most of their calculations are limited to instantaneous Stokes flow generated by a few types of prescribed temperature fields. Their conclusions thus strongly depend on the nature of the background temperature field. For example, a strong upwelling from the base of cratonic lithosphere toward thin lithosphere, as observed in their model, is a variant of classical Rayleigh-Bénard convection deformed by the top rigid boundary. Their temperature field is designed to place a wide convection cell beneath a continent-ocean boundary, and the coincidence of upwelling and a continent-ocean boundary should not be taken as the effect of cratonic lithosphere. Several time-dependent calculations in King and Anderson [1998] show that calculated Stokes flow does not sustain the initial temperature field. The influence of large-scale background flow is unarguably important for the study of smallscale convection, and a more self-consistent approach to generate preexisting large-scale flow is necessary to draw some general conclusion. Though a conducting side wall does not enhance the strength of convection, it certainly acts to modulate the pattern of convection, preferring convective rolls aligned perpendicular to the side wall. This may be relevant to some previous speculations about the effect of cratonic keels on mantle convection, based on the spatial association of between cratons and continental flood basalt provinces [Anderson, 1994] and between continental margins and hotspots [Vogt, 1991; Anderson, 1998b].

The present study is limited to a boundary-value approach, which is appropriate to study the stationary-state of convection. In order to investigate transient mantle dynamics, an initial-value approach must be adopted as done in the studies of rifting-related 
convection [Mutter et al., 1988; Keen and Boutilier, 1995; Boutilier and Keen, 1999]. Juxtaposing a hot mantle to a cold side wall is gravitationally unstable, and it always results in convection because of finite viscosity. The strength of convection is highly sensitive to an initial lateral thermal gradient across the side wall, so it is essential to assess how sharp this gradient can be. This initial-value problem potentially contains a wealth of dynamic behaviors, so a systematic study regarding the formation of the initial thermal structure seems to have the first priority. Otherwise, modeling results starting with ad hoc initial conditions are probably of limited use. Compositional heterogeneity, both in vertical and horizontal dimensions, and corresponding rheological structure must have significant influence on the deformation of continental lithosphere during rifting and resultant basal topography, so further work on this issue is warranted.

\subsection{Summary}

To understand the effect of lithospheric thickness variation on mantle convection, we have investigated a simple convection system in which a fluid is bounded by rigid side walls. Both conducting and insulating boundary conditions on side walls are considered to isolate kinematic and thermal effects. 2-D and 3-D marginal stability analyses show that heat conduction at side walls always reduces convective instability and generally favors convective rolls aligned perpendicular to side walls. For supercritical Rayleigh numbers, the strength of thermal convection is characterized by surface heat flux and maximum vertical velocity. The presence of side walls becomes negligible for heat flux at higher Rayleigh number, due to the instability of thermal boundary layers, but its influence on velocity is shown to be persistent. The nature of convective stability is assessed by 2-D and 3-D stability analyses of 2-D steady-state solutions. In a purely 2-D framework, side walls enhance a tendency toward time-dependent convection. The periodicity in a stationary state is related to the development of boundary layer instability, and it follows a simple scaling with the Rayleigh number. The 3-D stability analysis reveals the contrasting effects of the side wall thermal boundary conditions on the planform of convection; conducting side walls strongly prefer $3-\mathrm{D}$ convective motion. The characteristics of $3-\mathrm{D}$ convection 
bounded by conducting side walls are thus studied through a 3-D mean-field approximation, and results indicate that side wall effects are reduced in 3-D convection, for both heat transfer and vertical velocity, compared to the 2-D results. As the Rayleigh number increases, a 3-D flow field gradually strengthens its 2-D component, and in the case of a side wall cooling mode, a sheet-like upwelling dominates the convective pattern. Thick, cratonic lithosphere may thus have a modulating effect on the planform of smallscale convection in the upper mantle.

\section{Appendix 5.A: Finite Element Formulation for 3-D Single-Mode Stokes Flow}

Following the notation adopted by Hughes [1987], the Galerkin formulation of the equations of Stokes flow (equations 5.1 and 5.2) may be expressed as,

$$
\mathbf{K d}=\mathbf{F},
$$

where $\mathbf{K}, \mathbf{d}$, and $\mathbf{F}$ are a stiffness matrix, a velocity vector, and a force vector, respectively. The stiffness matrix and the force vector are constructed by summing all elemental contributions as,

$$
\mathbf{K}=\mathbf{A}_{e=1}^{n_{e f}}\left(k^{e}\right), \quad \mathbf{F}=\mathbf{A}_{e=1}^{n_{e l}}\left(f^{e}\right),
$$

where $\mathbf{A}$ denotes the finite element assembly operator, and $k^{e}$ and $f$ are the stiffness matrix and force vector of the $e$-th element. Since the degree of freedom is three, $k^{e}$ has dimensions $3 n_{e n} \times 3 n_{e n}$, where $n_{e n}$ is the number of element nodes. Using the following subscripts,

$$
\begin{gathered}
p=3(a-1)+i, \quad q=3(b-1)+j \\
1 \leq a, b \leq n_{e n}, \quad 1 \leq i, j \leq 3
\end{gathered}
$$

element stiffness matrices may be further decomposed as,

$$
k_{p q}^{e}=\mathbf{e}_{i}^{\mathrm{T}} k_{a b}^{e} \mathbf{e}_{j}, \quad k_{a b}^{e}=\bar{k}_{a b}+\overline{\bar{k}}_{a b},
$$

where the penalty formulation is assumed in the second expression. Now introducing a 3-D flow field with a single wavenumber of $\psi$ in the out-of-plane coordinate as,

$$
\mathbf{u}(x, z, y)=\left(u_{1}(x, z) \cos (\psi y), u_{2}(x, z) \cos (\psi y), u_{3}(x, z) \sin (\psi y)\right),
$$

the explicit form of element stiffness matrices can be obtained as, 


$$
\begin{aligned}
& \bar{k}_{a b, 11}=\int_{\Omega^{e}} \mu\left(2 N_{a, 1} N_{b, 1}+\psi^{2} N_{a} N_{b}+N_{a, 2} N_{b, 2}\right) d \Omega, \\
& \bar{k}_{a b, 12}=\int_{\Omega^{e}} \mu N_{a, 2} N_{b, 1} d \Omega, \quad \bar{k}_{a b, 13}=-\int_{\Omega^{e}} \mu \psi N_{a} N_{b, 1} d \Omega, \\
& \bar{k}_{a b, 21}=\int_{\Omega^{e}} \mu\left(N_{a, 2} N_{b, 1}+N_{a, 1} N_{b, 2}\right) d \Omega, \\
& \bar{k}_{a b, 22}=\int_{\Omega^{e}} \mu\left(2 N_{a, 2} N_{b, 2}+\psi^{2} N_{a} N_{b}+N_{a, 1} N_{b, 1}\right) d \Omega, \\
& \bar{k}_{a b, 23}=-\int_{\Omega^{e}} \mu \psi N_{a} N_{b, 2} d \Omega, \\
& \bar{k}_{a b, 31}=-\int_{\Omega^{e}} \mu \psi N_{a, 1} N_{b} d \Omega, \quad \bar{k}_{a b, 32}=-\int_{\Omega^{e}} \mu \psi N_{a, 2} N_{b} d \Omega, \\
& \bar{k}_{a b, 33}=\int_{\Omega^{e}} \mu\left(2 \psi^{2} N_{a} N_{b}+N_{a, 2} N_{b, 2}+N_{a, 1} N_{b, 1}\right) d \Omega,
\end{aligned}
$$

and

$$
\begin{array}{ll}
\overline{\bar{k}}_{a b, 11}=\int_{\Omega^{e}} \lambda N_{a, 1} N_{b, 1} d \Omega, & \overline{\bar{k}}_{a b, 12}=\int_{\Omega^{e}} \lambda N_{a, 2} N_{b, 1} d \Omega, \\
\overline{\bar{k}}_{a b, 13}=\int_{\Omega^{e}} \lambda \psi N_{a, 1} N_{b} d \Omega, & \overline{\bar{k}}_{a b, 21}=\int_{\Omega^{e}} \lambda N_{a, 2} N_{b, 1} d \Omega, \\
\overline{\bar{k}}_{a b, 22}=\int_{\Omega^{e}} \lambda N_{a, 2} N_{b, 2} d \Omega, & \overline{\bar{k}}_{a b, 23}=\int_{\Omega^{e}} \lambda \psi N_{a, 2} N_{b} d \Omega, \\
\overline{\bar{k}}_{a b, 31}=\int_{\Omega^{e}} \lambda \psi N_{a} N_{b, 1} d \Omega, & \overline{\bar{k}}_{a b, 32}=\int_{\Omega^{e}} \lambda \psi N_{a} N_{b, 2} d \Omega, \\
\overline{\bar{k}}_{a b, 33}=\int_{\Omega^{e}} \lambda \psi \psi^{2} N_{a} N_{b} d \Omega, &
\end{array}
$$

where $N_{a}$ is an element shape function, and $\Omega^{e}$ is the domain of the $e$-th element. Element force vectors are calculated as,

$$
f_{p}^{e}=\int_{\Omega^{e}} N_{a} f_{i} d \Omega+\int_{\Gamma_{i}^{e}} N_{a} h_{i} d \Gamma-\sum_{q \in \eta_{g}} k_{p q}^{e} g_{q}^{e},
$$

where $f_{i}$ is an element body force vector, $(0, \operatorname{Ra} \theta, 0), h_{i}$ is a traction boundary condition specified at $\Gamma_{h}{ }^{e}$, and $g_{q}{ }^{e}$ is a velocity boundary condition.

\section{Appendix 5.B: Finite Element Formulation for 3-D Mean-Field Energy Equation}

Treating mean-field temperature, $T$, and perturbation temperature, $\theta$, collectively as a two-

dimensional vector field, d, the Galerkin formulation of 3-D mean-field energy conservation (equation 5.23) may be expressed as,

$$
\mathbf{M d}+\mathbf{K d}+\mathbf{F}=0
$$


where $\mathbf{M}, \mathbf{K}$, and $\mathbf{F}$ is the capacity matrix, the stiffness matrix, and the force vector. As in Appendix A, they are constructed by element matrices and vectors. Element capacity matrices, $m^{e}$, have dimensions $2 n_{e n} \times 2 n_{e n}$, and they may be expressed as,

$$
m_{p q}^{e}=\mathbf{e}_{i}^{\mathrm{T}} m_{a b}^{e} \mathbf{e}_{j}
$$

where

$$
m_{a b, 11}^{e}=\int_{\Omega^{e}}\left(N_{a}+p\right) N_{b} d \Omega, \quad m_{a b, 22}^{e}=\int_{\Omega^{e}} N_{a} N_{b} d \Omega,
$$

with zero off-diagonal components. $p$ is the stream-line Petrov-Galerkin shape function defined as [Brooks and Hughes, 1982],

$$
p=\kappa_{\mathrm{ad}}\left(U N_{a, 1}+W N_{a, 2}\right)
$$

where $\kappa_{\mathrm{ad}}$ is artificial diffusion determined by the mean-field velocity field. Similarly, element stiffness matrices may be expressed using nodal submatrices as,

$$
k_{p q}^{e}=\mathbf{e}_{i}^{\mathrm{T}} k_{a b}^{e} \mathbf{e}_{j},
$$

and

$$
\begin{aligned}
& k_{a b, 11}^{e}=\int_{\Omega^{e}}\left[\left(N_{a}+p\right)\left(U N_{b, 1}+W N_{b, 2}\right)+N_{a, 1} N_{b, 2}+N_{a, 2} N_{b, 2}\right] d \Omega, \\
& k_{a b, 12}^{e}=\frac{1}{2} \int_{\Omega^{e}} N_{a}\left(u N_{b, 1}+w N_{b, 2}-\psi v N_{b}\right) d \Omega, \\
& k_{a b, 21}^{e}=\int_{\Omega^{e}} N_{a}\left(u N_{b, 1}+w N_{b, 2}\right) d \Omega, \\
& k_{a b, 22}^{e}=\int_{\Omega^{e}}\left[\left(N_{a}+p\right)\left(U N_{b, 1}+W N_{b, 2}\right)+N_{a, 1} N_{b, 1}+N_{a, 2} N_{b, 2}+\psi^{2} N_{a} N_{b}\right] d \Omega .
\end{aligned}
$$

Note that we only have to apply the Petrov-Galerkin method to the mean-field temperature, because single-mode out-of-plane velocity does not cause artificial oscillations in thermal advection. Element force vectors are calculated from temperature boundary conditions, $g_{q}^{e}$, and heat flux boundary conditions, $h_{i}$, as,

$$
f_{p}^{e}=\sum_{q \in \eta_{g}} k_{p q}^{e} g_{q}-\int_{\Gamma_{h}^{e}} N_{a} h_{i} d \Gamma
$$



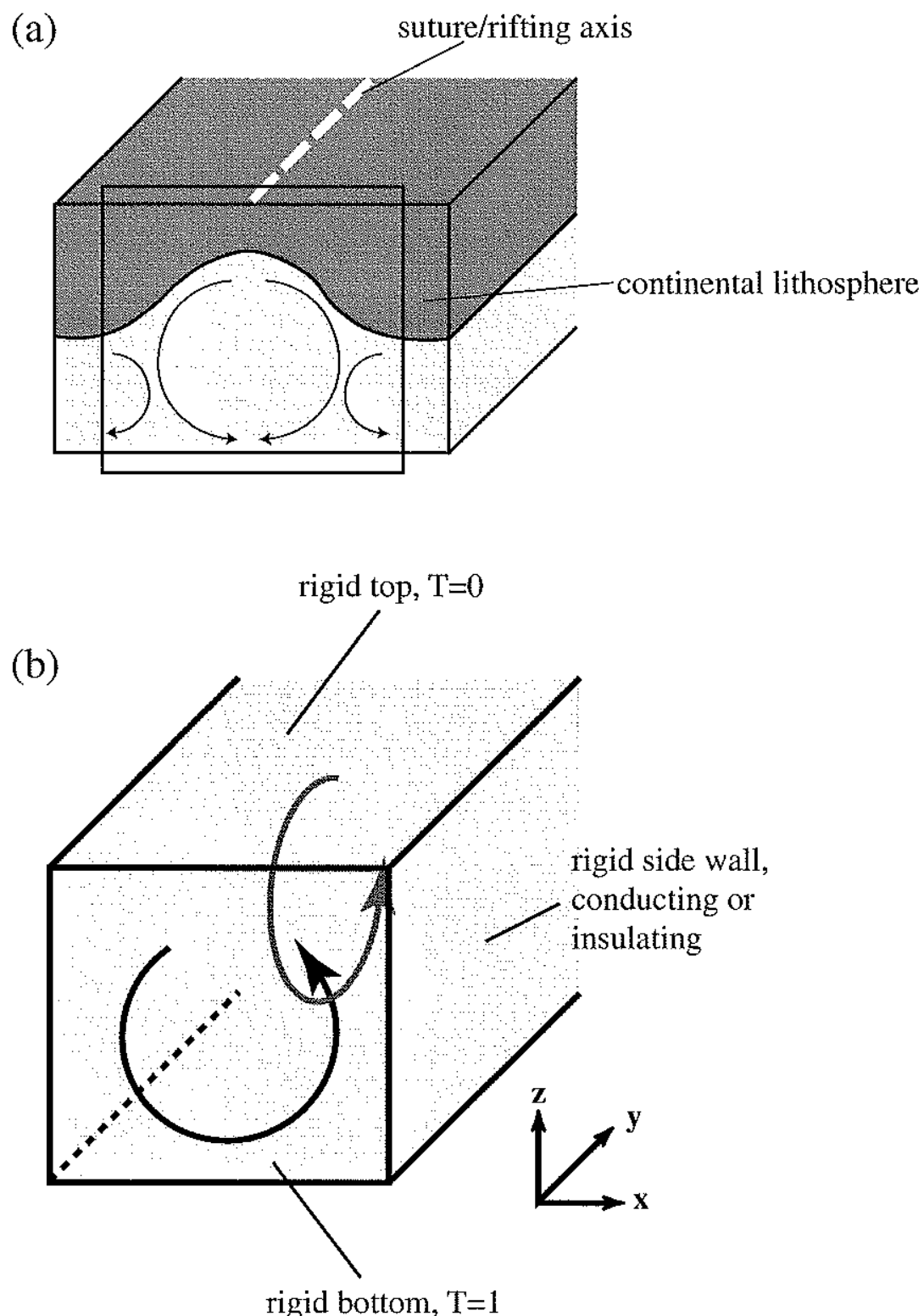

Figure 5-1. (a) Schematic drawing for sublithospheric convection beneath continental lithosphere with a variable thickness. A large variation in thickness with a relatively short wavelength may be expected beneath a suture zone, where asthenospheric mantle is bounded by adjacent thick cratonic lithosphere. (b) An idealized hydrodynamic model considered in this study, to investigate the nature of sublithospheric convection within a domain enclosed by a box in (a). 
(a)

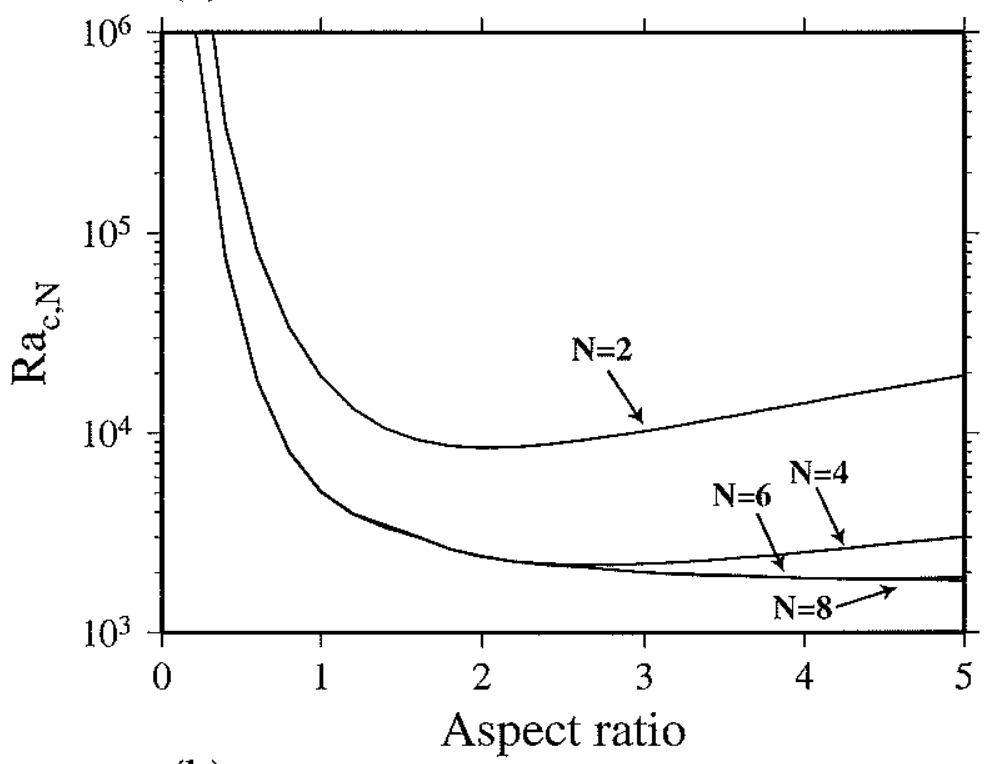

(b)

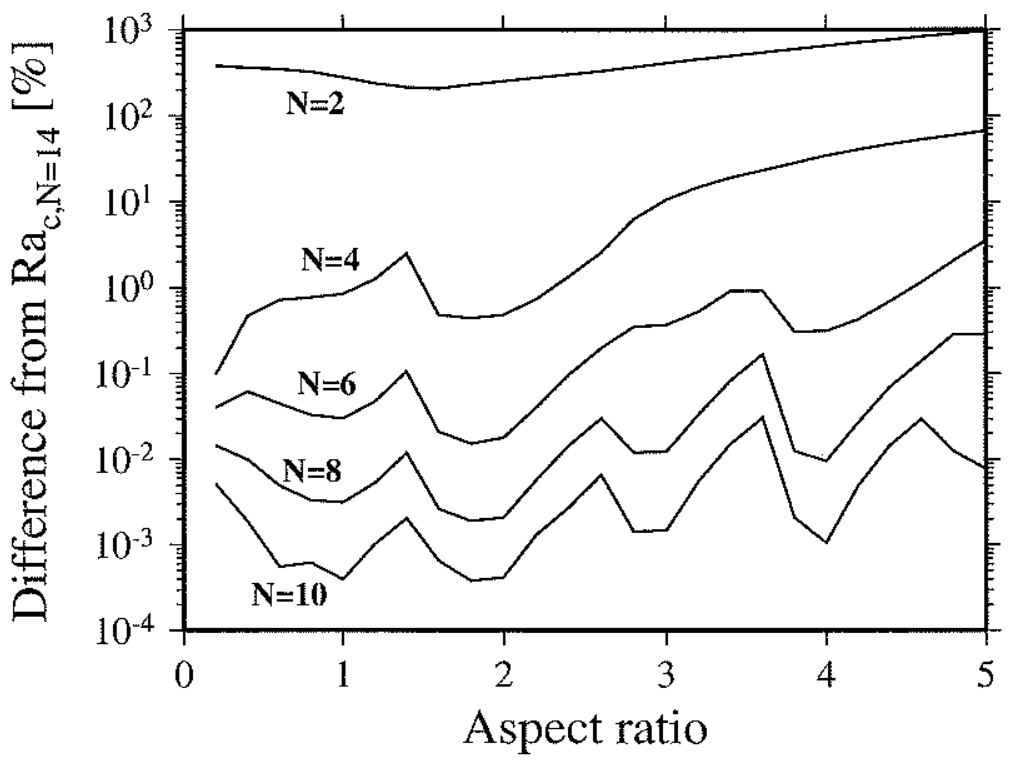

Figure 5-2. Example of convergence in the variational method. (a) Approximate critical Rayleigh numbers for 2-D perturbations with conducting side walls are shown for different numbers of modes. (b) Percentage differences from critical Rayleigh numbers calculated with $N=14$ are shown for $N=2-10$ as a function of aspect ratio. Note that the differences are reduced by about an order of magnitude as the number of modes is increased by 2 . Solutions obtained with $N=10$ have a less than $0.01 \%$ difference for aspect ratios smaller than 3 . 

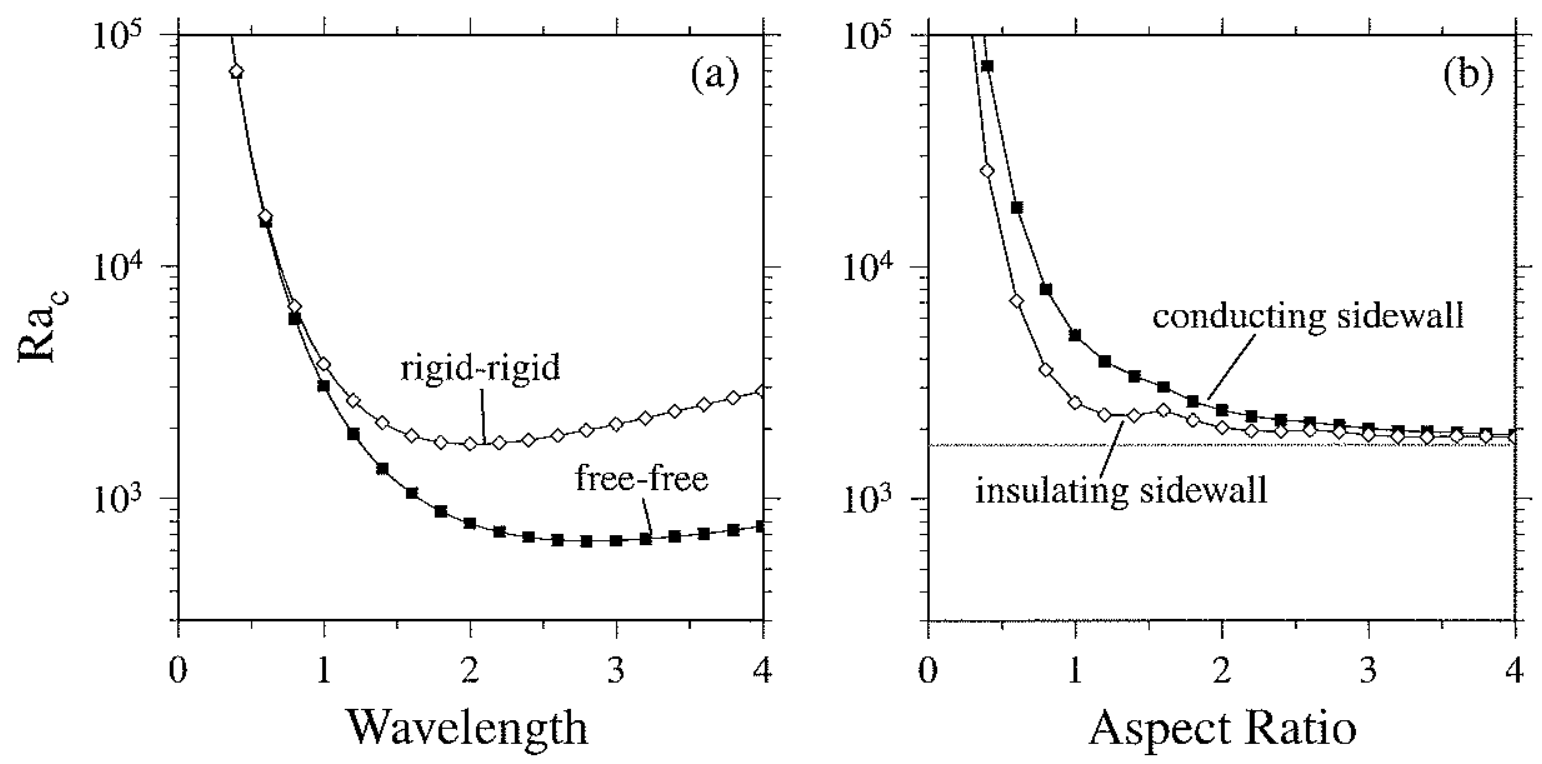

Figure 5-3. Critical Rayleigh numbers for 2-D perturbations. (a) $\mathrm{Ra}_{\mathrm{c}}$ for the classical case with an infinite horizontal extent is plotted as a function of wavelength (solid lines) [Chandrasekhar, 1981]. Overlaid symbols are solutions obtained by our variational method. (b) $\mathrm{Ra}_{\mathrm{c}}$ for a fluid heated from below and bounded by 2-D rigid side walls is plotted as a function of model aspect ratio. Open diamonds denote variational solutions for insulating side walls, and solid squares for conducting side walls. 

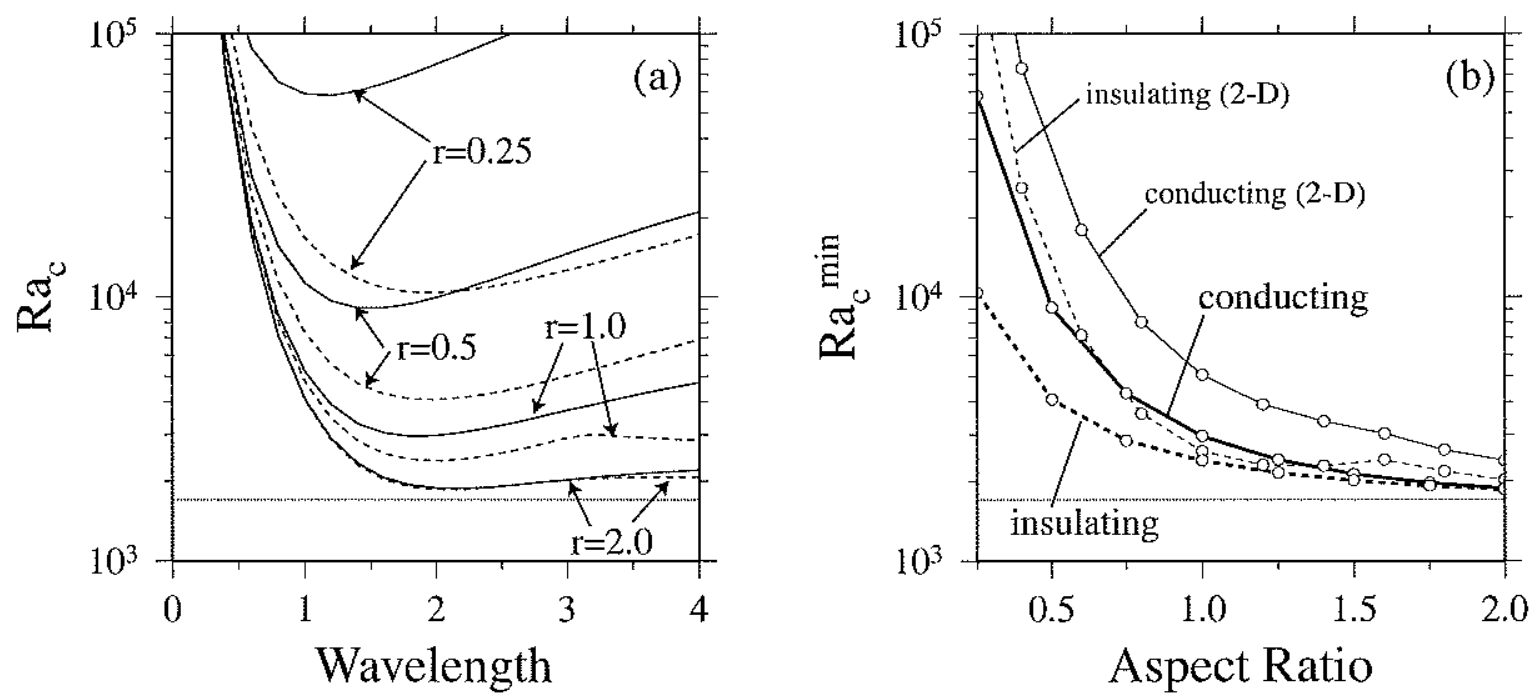

Figure 5-4. Critical Rayleigh number for 3-D perturbations. (a) $\mathrm{Ra}_{\mathrm{c}}$ as a function of out-of-plane wavelength, $2 \pi / \psi$, is plotted for insulating (dashed) and conducting (solid) cases, at aspect ratios of 0.25-2.0. (b) $\mathrm{Ra}_{\mathrm{c}}$ is plotted as a function of aspect ratio. Thick solid and dashed lines are $3-\mathrm{D} \mathrm{Ra}_{\mathrm{c}}$ for conducting and insulating cases, respectively. Also shown as thin curves are 2-D $\mathrm{Ra}_{\mathrm{c}}$. Gray horizontal line denotes $\mathrm{Ra}_{\mathrm{c}}$ for the system with an infinite horizontal extent (1708). 
(a) no sidewall
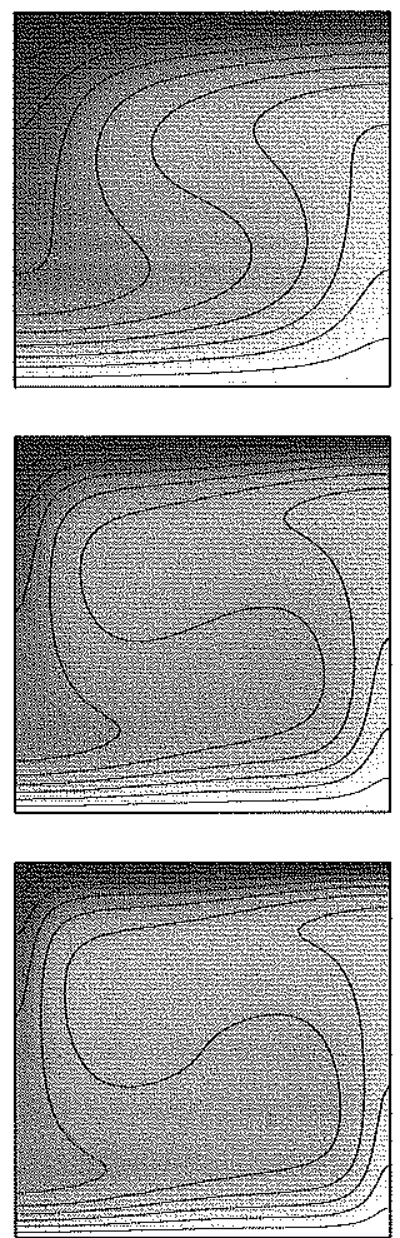

(b) insulating sidewall
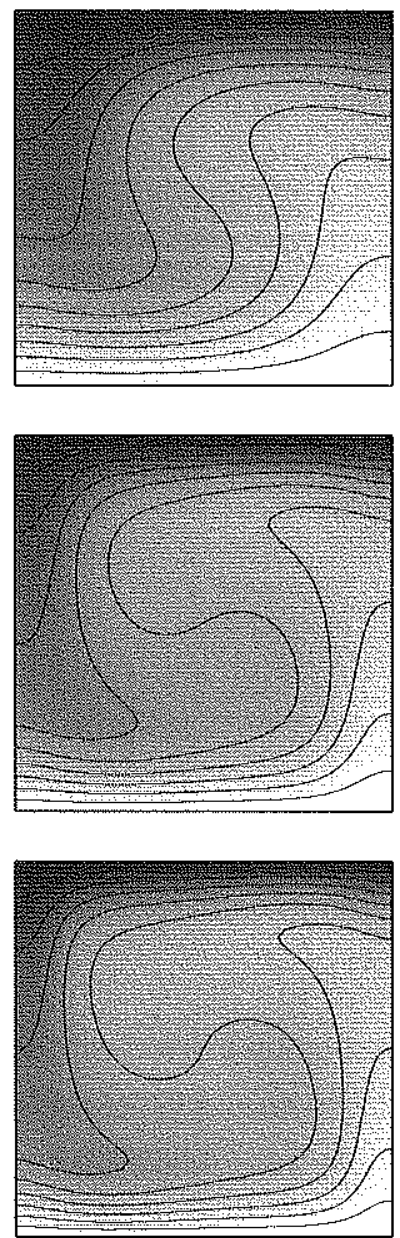

(c) conducting sidwall
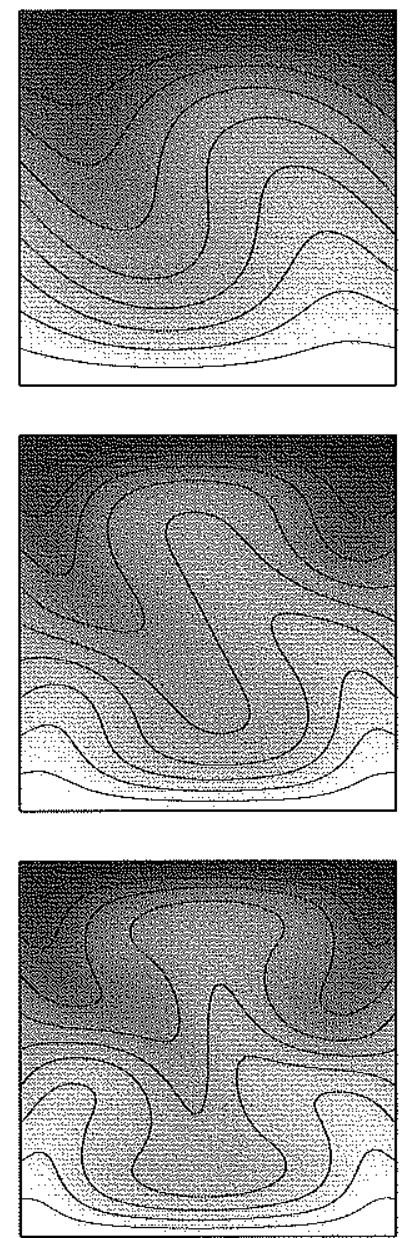

Figure 5-5. Temperature contour plots for examples of 2-D steady-state convection, for (a) no side wall case, (b) insulating side wall case, and (c) conducting side wall case, at $\mathrm{Ra}=10^{4}$ (top panel), $\mathrm{Ra}=4 \times 10^{4}$ (middle panel), and $\mathrm{Ra}=10^{5}$ (bottom). Contour interval is 0.1 . 
(a) Three possible modes of steady-state solution: $\mathrm{Ra}=5 \times 10^{4}$

(i) odd mode

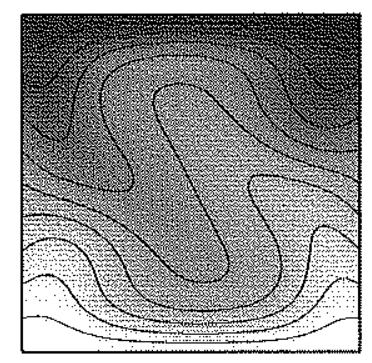

(ii) even mode (cooling)

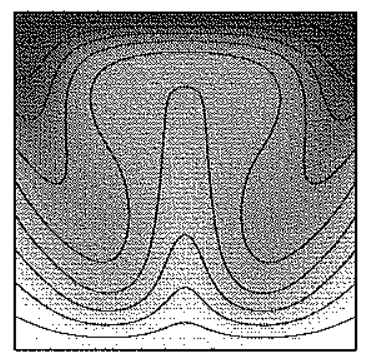

(iii) even mode (heating)

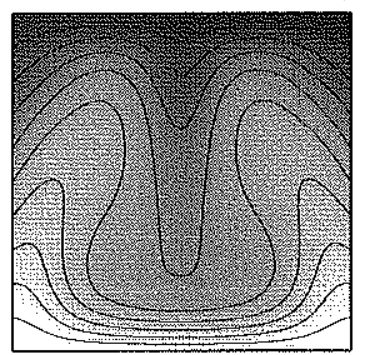

(b) 2-D Stability of steady-state solutions: conducting wall $(\mathrm{r}=1.0)$

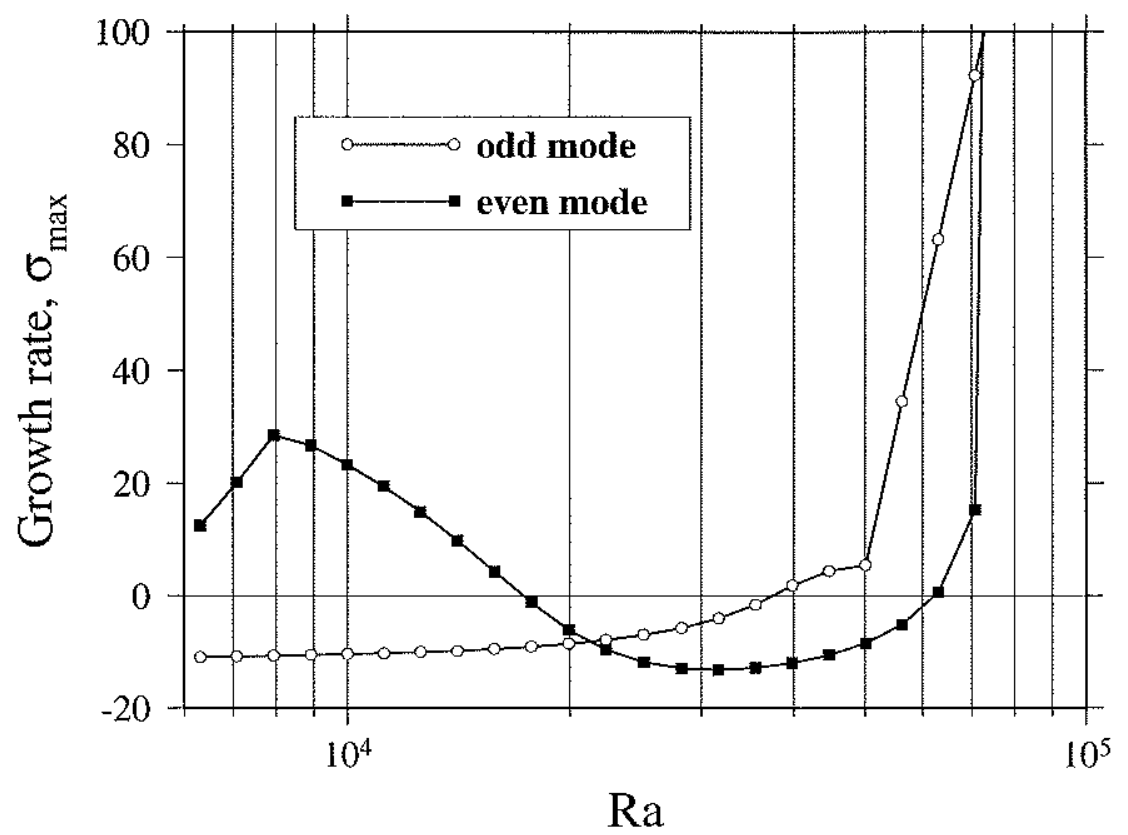

Figure 5-6. nonuniqueness of steady-state solutions. (a) Temperature contour plots for examples of steady-state solutions for conducting side walls with an aspect ratio of 1 , obtained at $R a=5 \times 10^{5}$. (b) Growth exponents for the above steady-state solutions are plotted as a function of Ra. Open circles and solid squares denote the stability curve of the odd-mode and even-mode solutions, respectively. 
(a) conducting sidewall

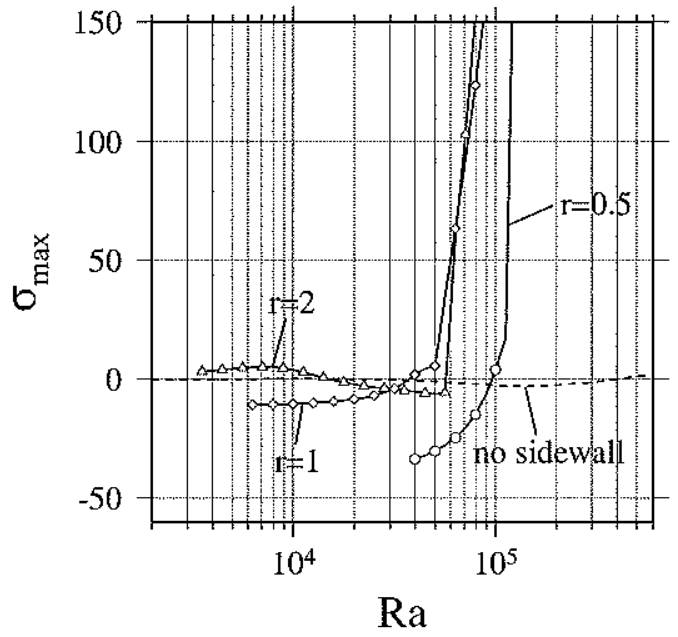

(c) conducting sidewall

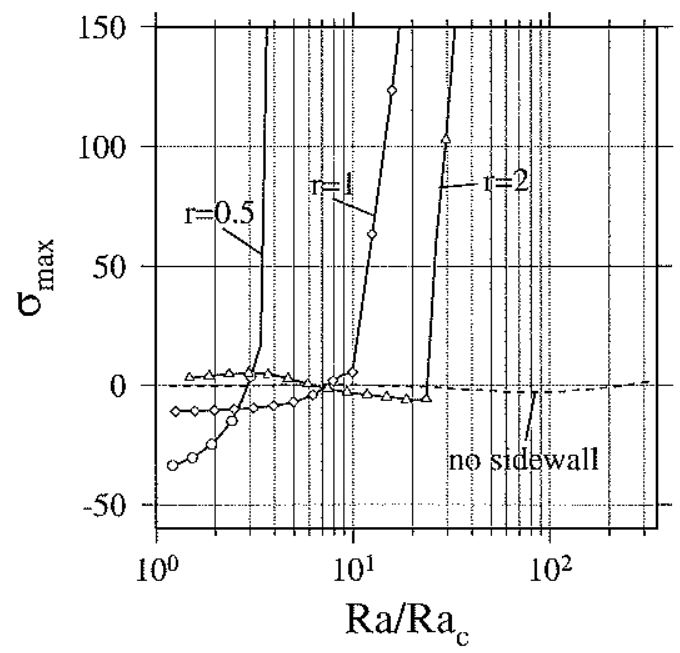

(b) insulating sidewall

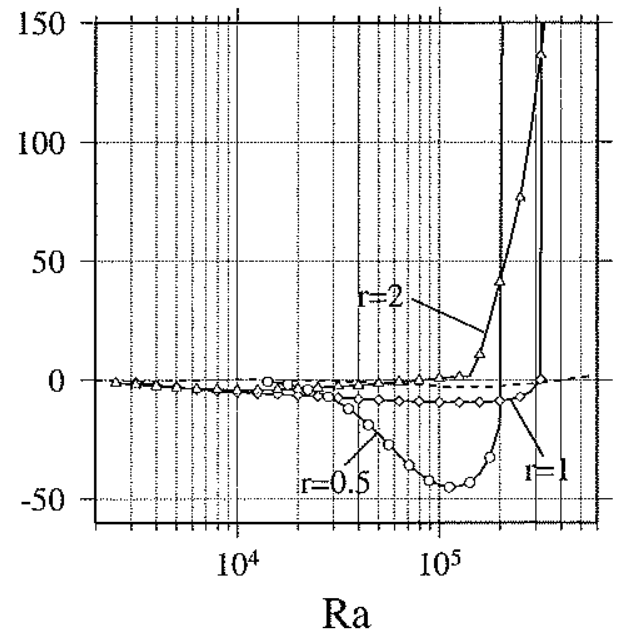

(d) insulating sidewall

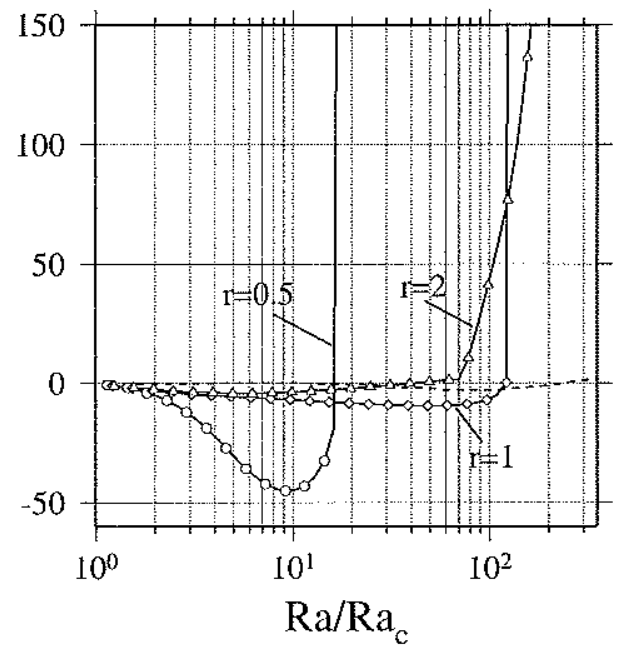

Figure 5-7. 2-D stability of 2-D steady-state convection. Growth exponents are shown for three aspect ratios (circle: $r=0.5$, diamond: $r=1$, and triangle: $r=2$ ) as a function of $\mathrm{Ra}$, for (a) conducting side walls and (b) insulating side walls. Dashed curve denotes the stability curve for the limiting case with no side wall. Stability curves scaled with the system's own critical Rayleigh numbers are shown in (c) and (d). 
(a) conducting sidewall
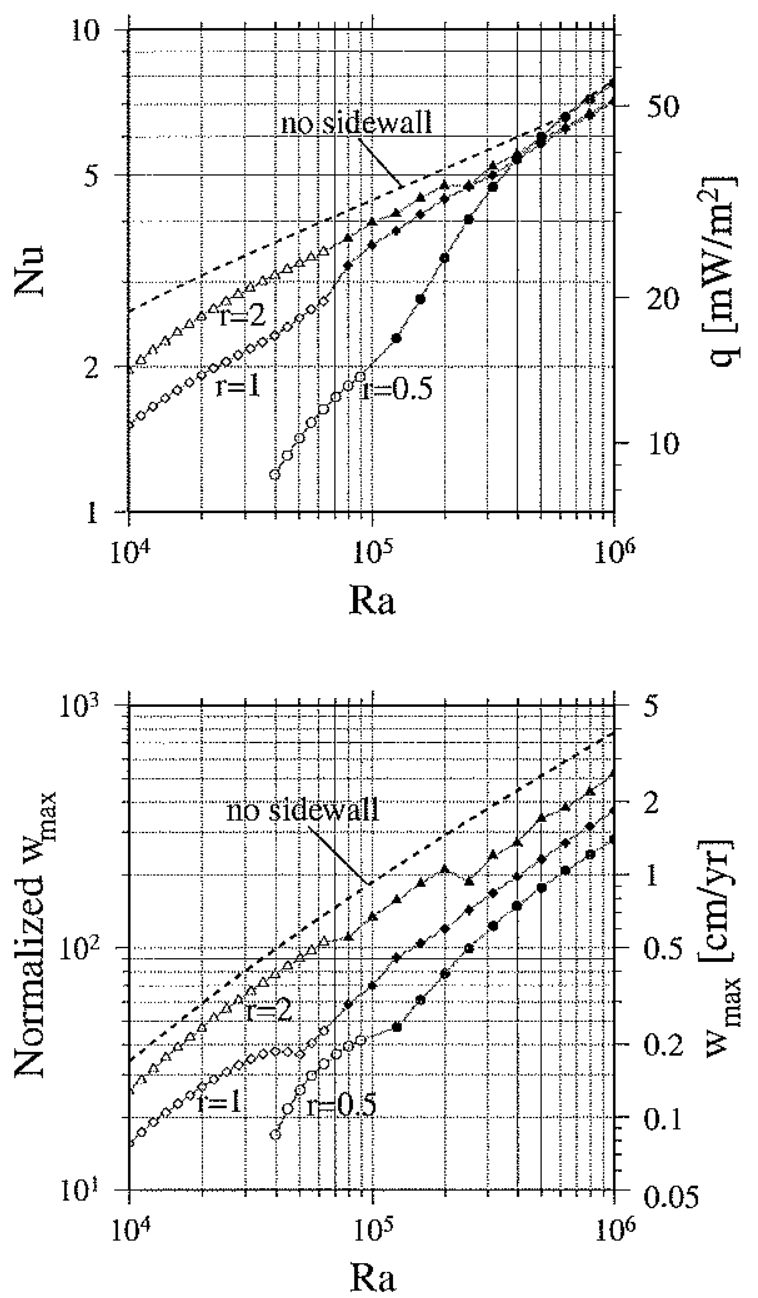

(b) insulating sidewall
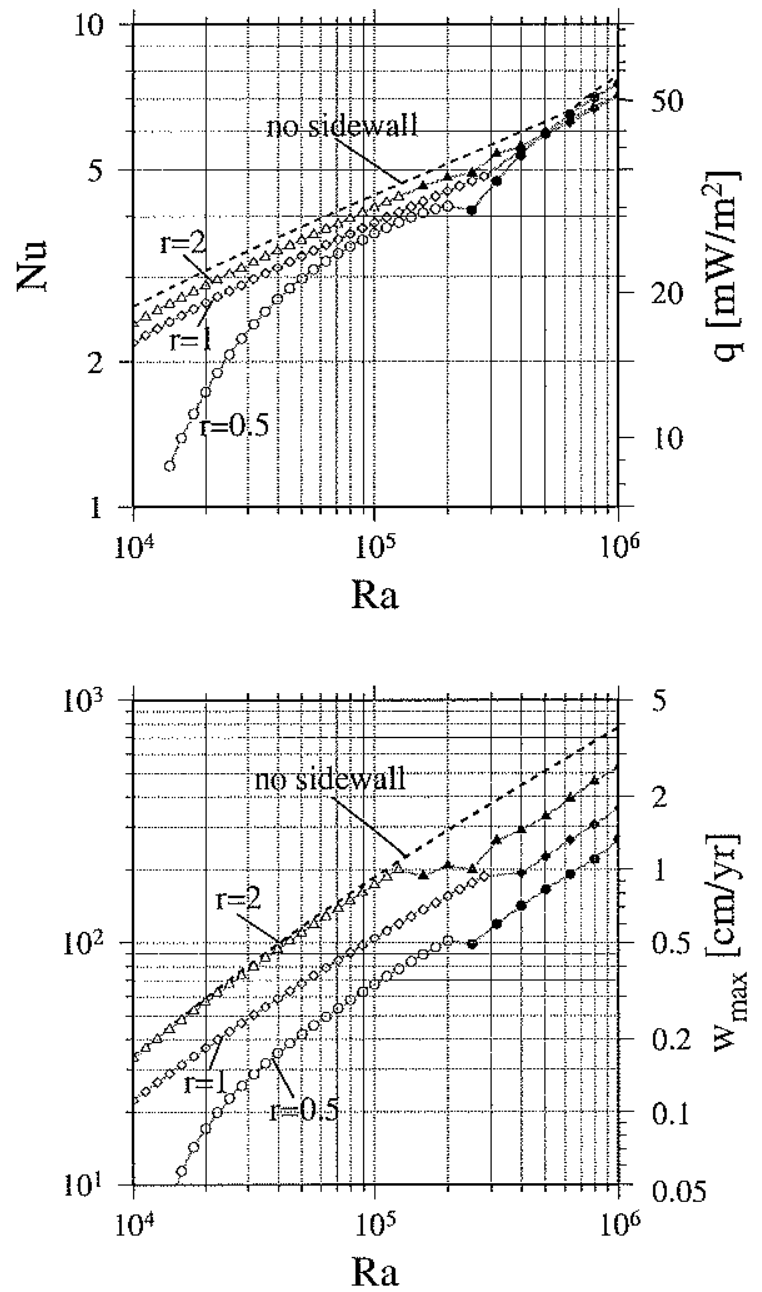

Figure 5-8. Characteristics of 2-D stationary-state convection, in terms of Nusselt number (top panel) and maximum vertical velocity (bottom panel) are plotted for (a) conducting and (b) insulating cases. Open symbols denote measurements from steady-state solutions, and solid symbols from timedependent solutions. Three aspect ratios ( $\mathrm{r}=0.5,1$, and 2) are considered. Dashed curve denotes the limiting case with no side wall. The statistics of

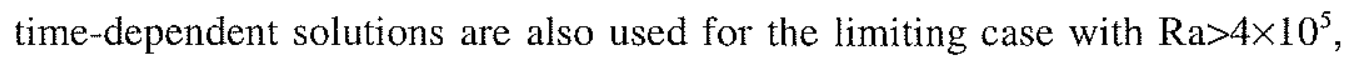
where no steady-state solution exists (Figure 5-7). 
(a) $\mathrm{Ra}=10^{5.1}, \mathrm{r}=1$, conducting wall
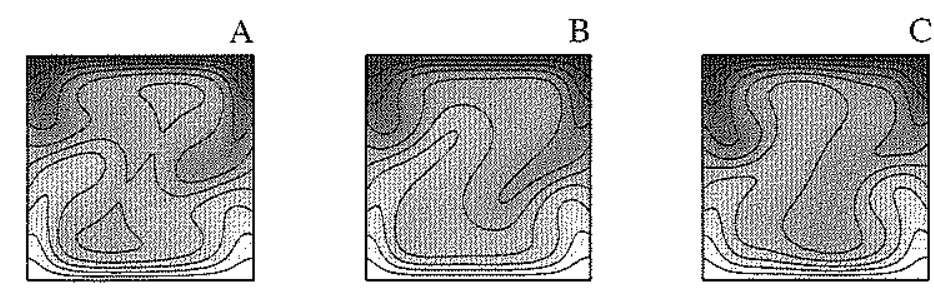

Time [m.y.]

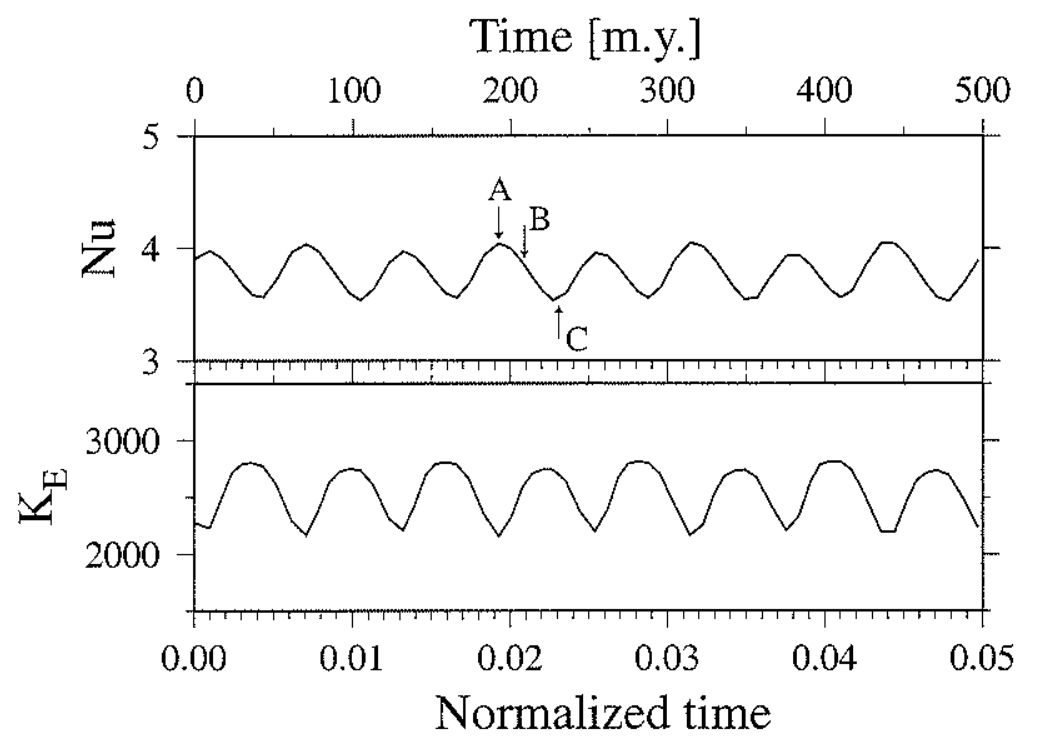

(b) $\mathrm{Ra}=10^{5.7}, \mathrm{r}=1$, conducting wall
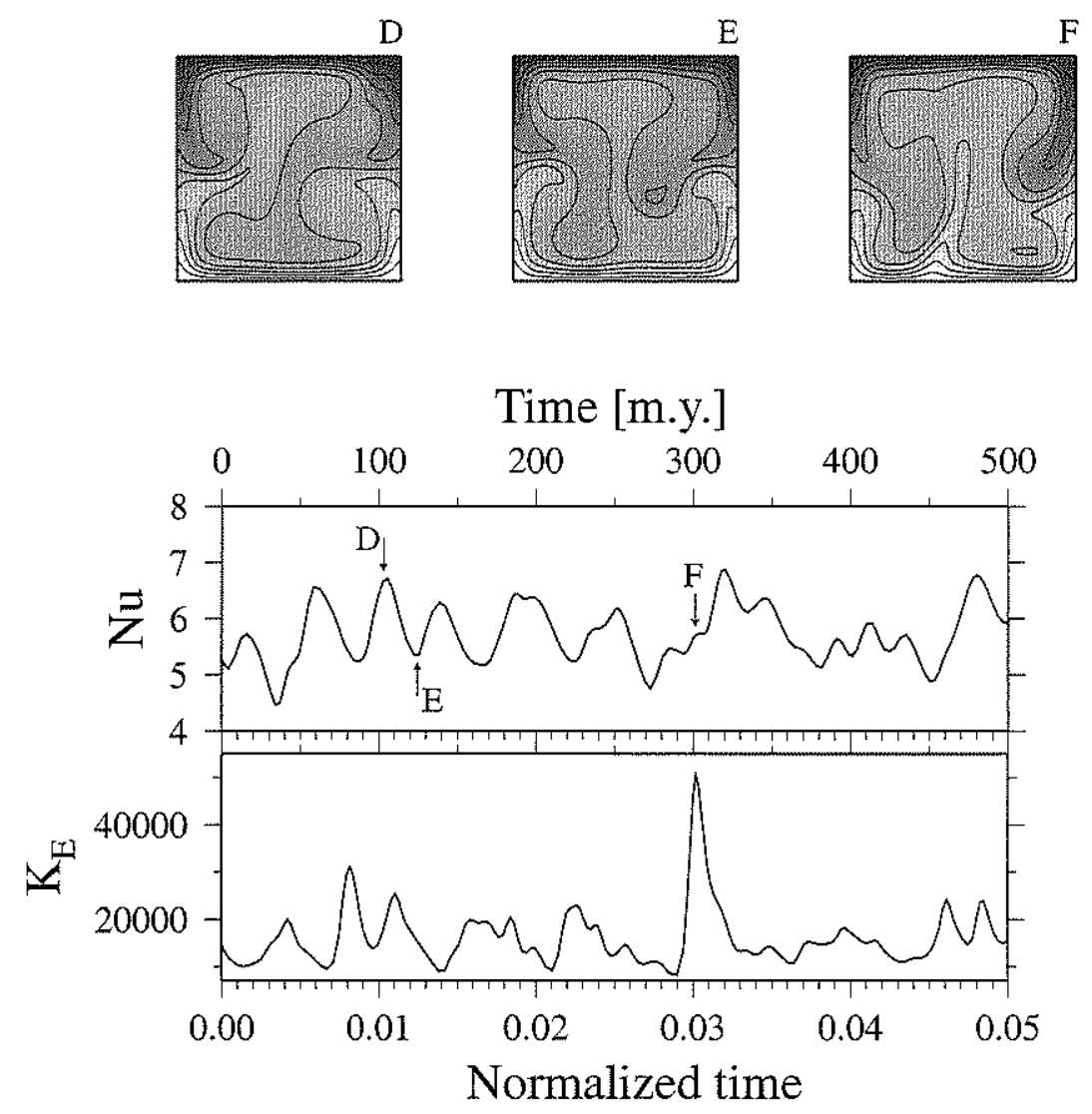

Figure 5-9. Example of 2-D time-dependent convection, with conducting side walls and a unit aspect ratio. Time series of surface Nusselt number and total kinetic energy are shown with snapshots of contoured temperature field. (a) $\mathrm{Ra}=10^{5.1}$, and (b) $\mathrm{Ra}=10^{5.7}$. 


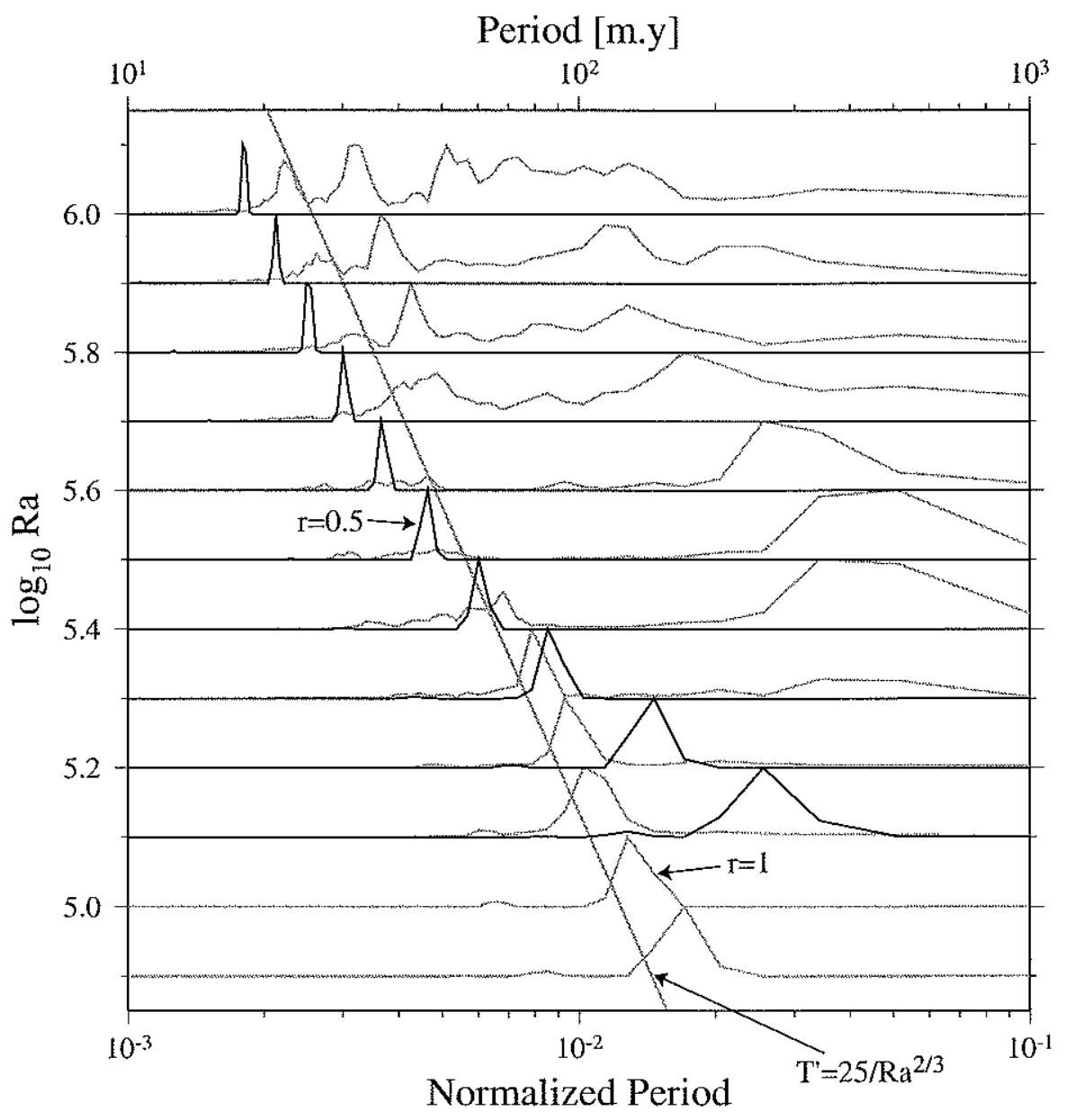

Figure 5-10. Spectral characterization of time-dependent convection, in terms of surface Nusselt number. The power spectrum of temporal variations in surface Nusselt numbers is plotted for a range of Rayleigh numbers. Solid lines denote the case of $r=0.5$, and gray lines denote the case of $r=1$. Theoretical estimate based on boundary layer theory is also shown. 
(a) conducting sidewall
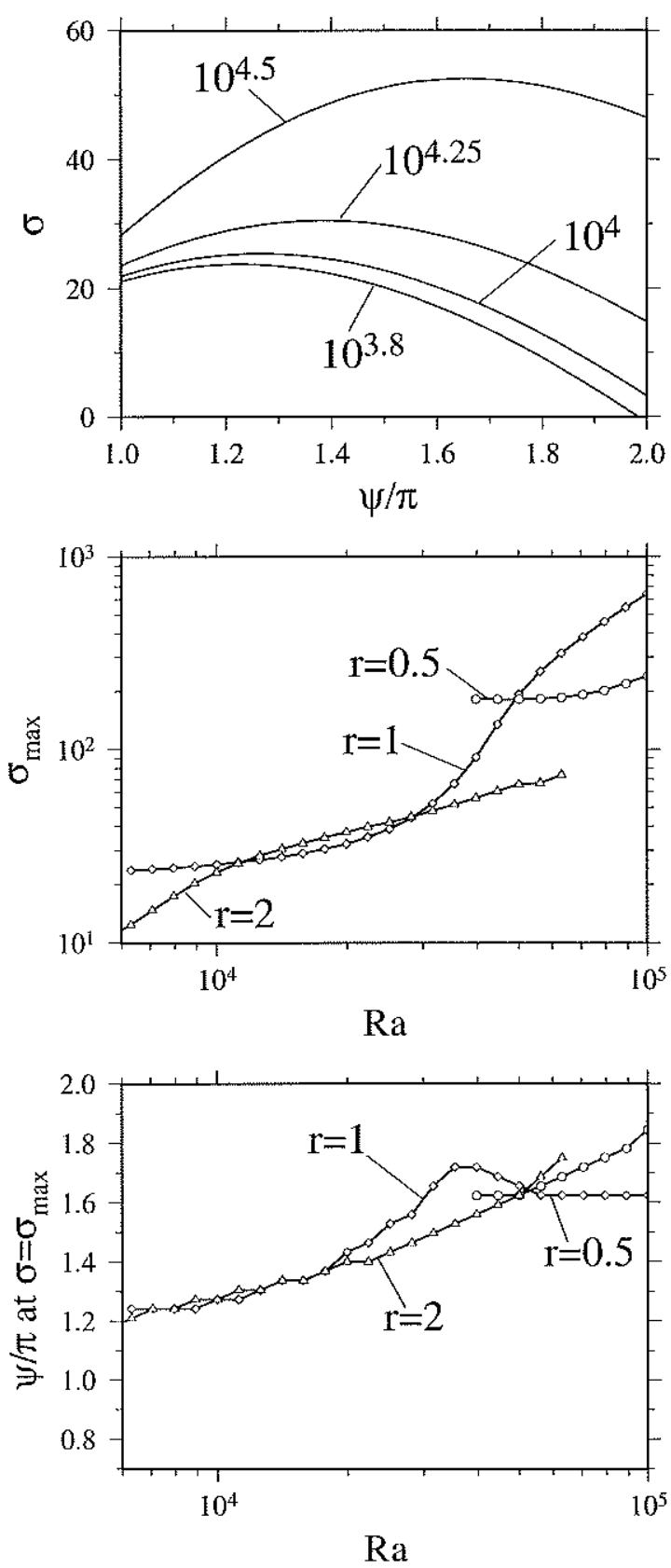

(b) insulating sidewall
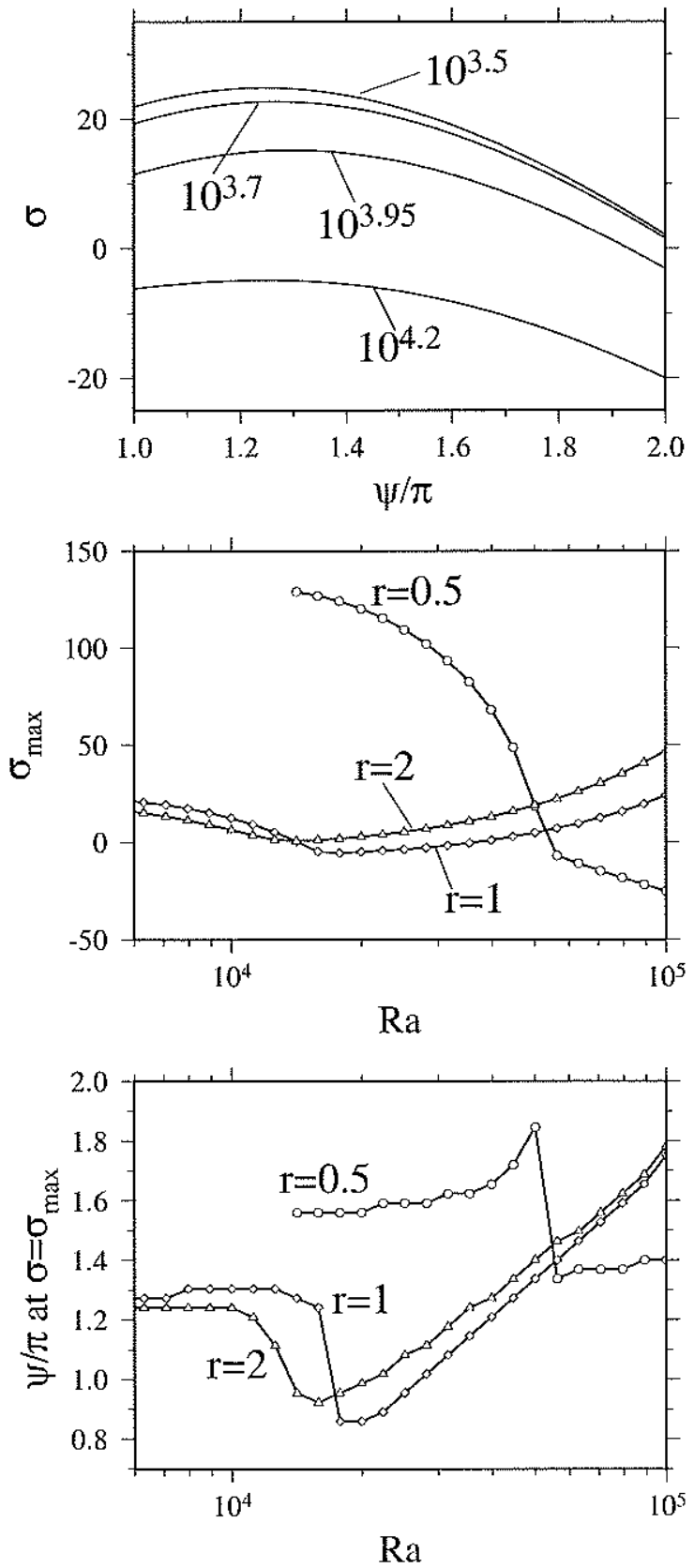

Figure 5-11. 3-D stability of 2-D steady-state convection, for (a) conducting side walls and (b) insulating side walls. In top panels, growth exponent in the case of a unit aspect ratio is plotted as a function of out-of-plane wavenumber, at several Rayleigh numbers. Maximum growth exponent and corresponding out-of-plane wavenumber are shown in middle and bottom panels, respectively, for three aspect ratios. 
$\mathrm{T}$
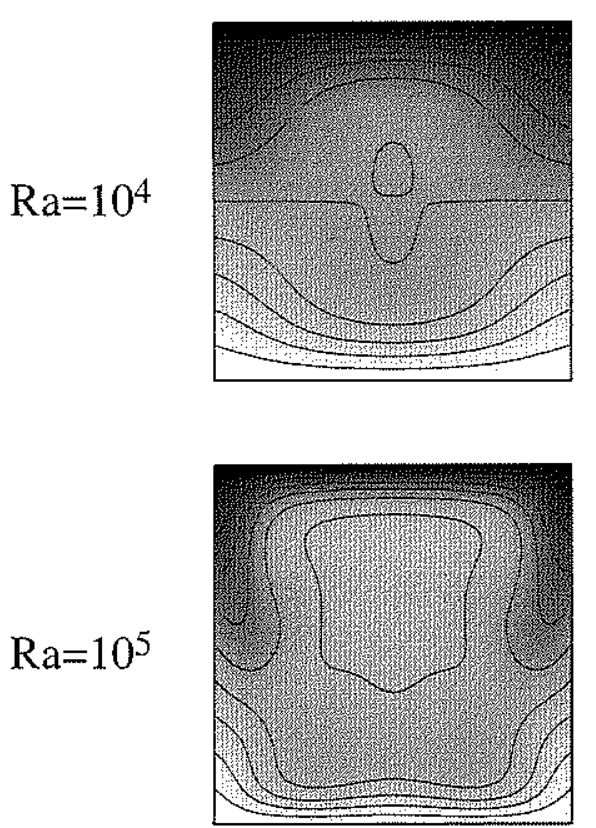

N $\theta$
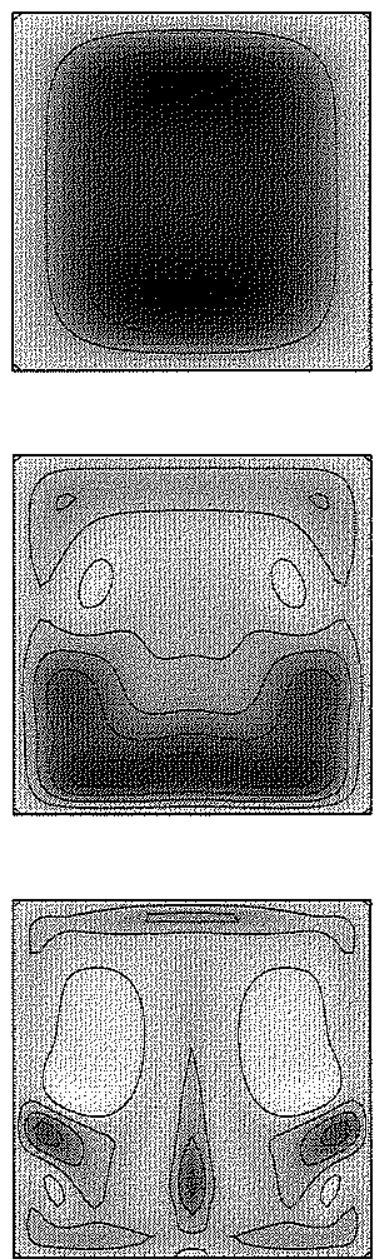

$\mathrm{T}+\theta$
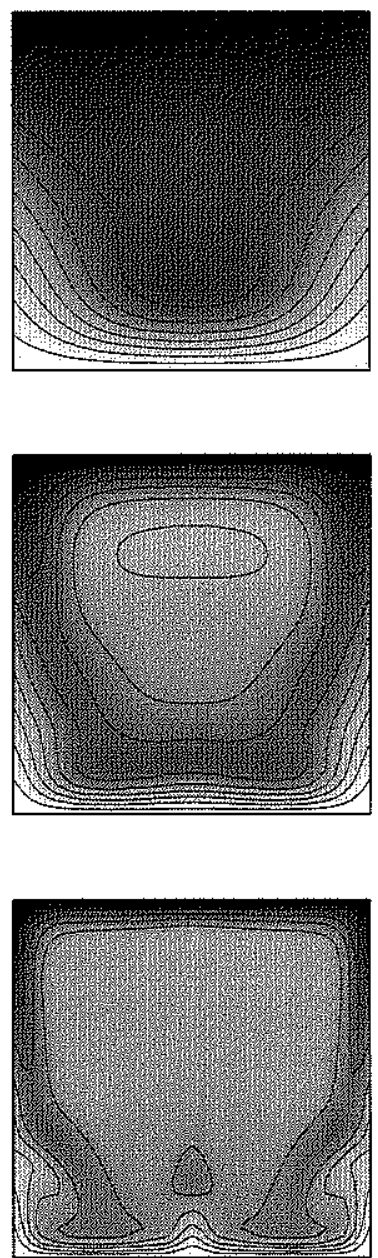

$\mathrm{T}-\theta$
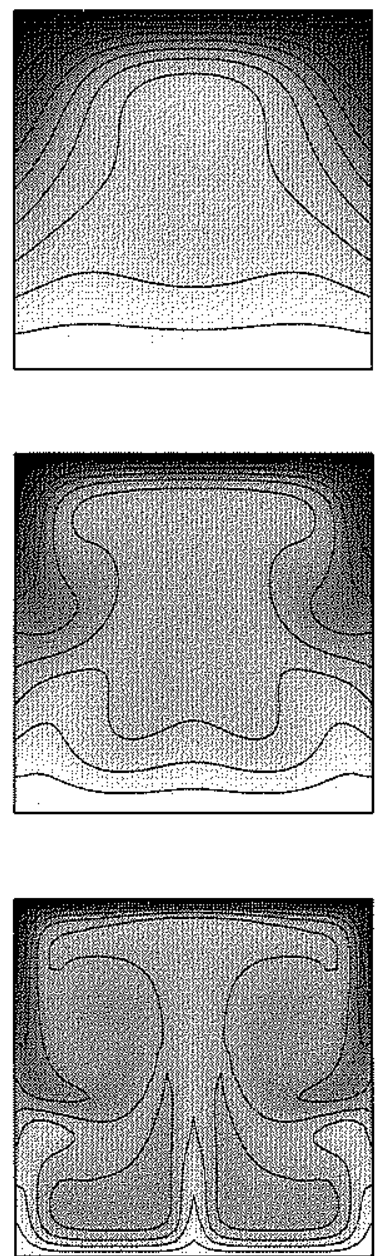

Figure 5-12. Example of 3-D mean-field convection, bounded by conducting side walls with a unit aspect ratio. The out-of-plane wavenumber is set as $\pi$. For $\mathrm{Ra}=10^{4}, 10^{5}$, and $10^{6}$, mean-field and out-of-plane temperature fields are shown along with slices at $y=1$ and -1 . Contour intervals are 0.1 , except for out-plane-temperature field, for which intervals are 0.05 . 

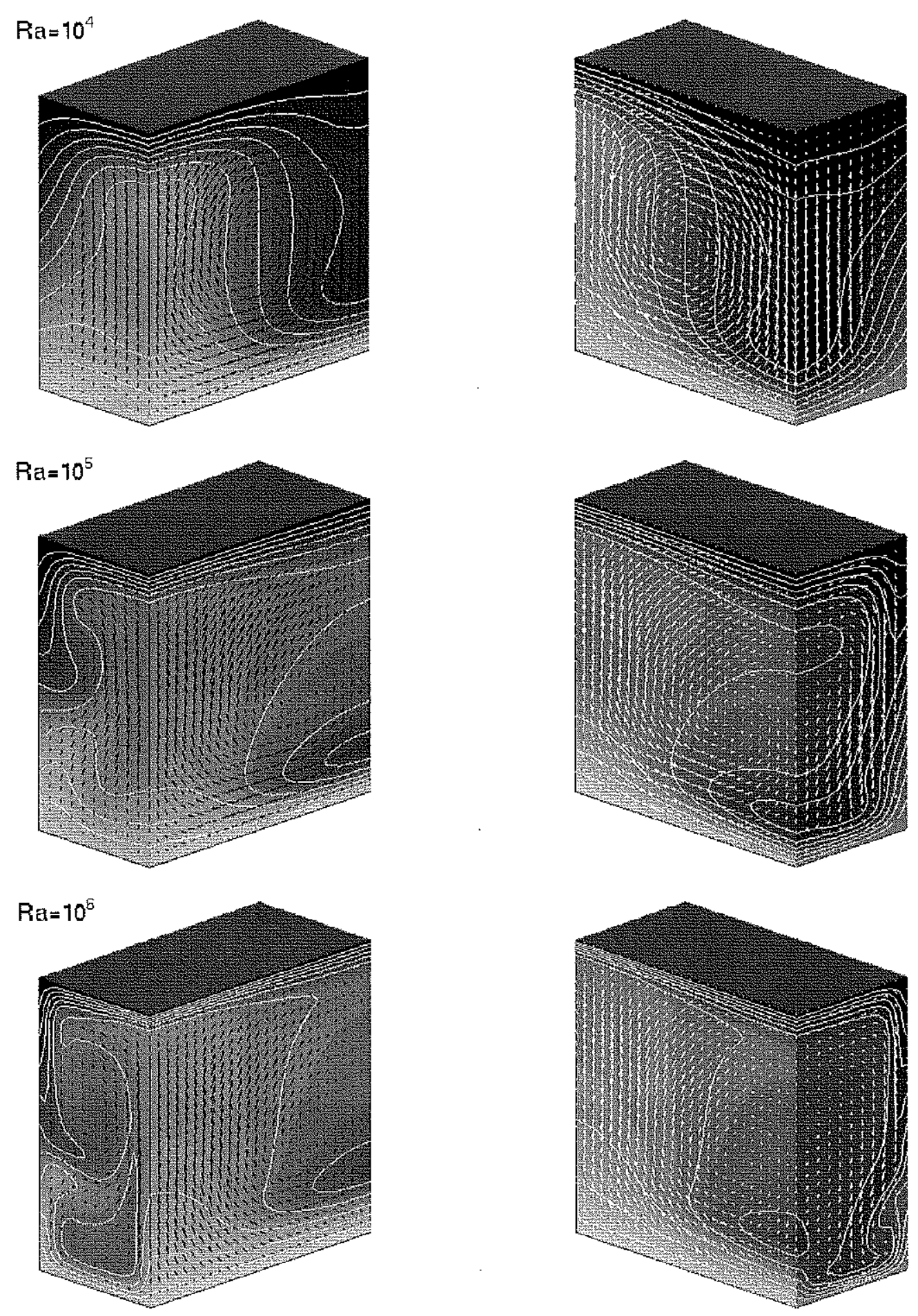

Figure 5-13. Perspective rendition of 3-D mean-field convection for $\mathrm{Ra}=10^{4}$, $10^{5}$, and $10^{6}$ The domain of $0 \leq x \leq 0.5,0 \leq y \leq 1.0$, and $0 \leq z \leq 1.0$ is shown, in its front view (left) and back view (right). Temperature contours are at a 0.1 interval. Velocity arrows are also shown, and their scale is normalized to the maximum value at each Ra. 
(a)

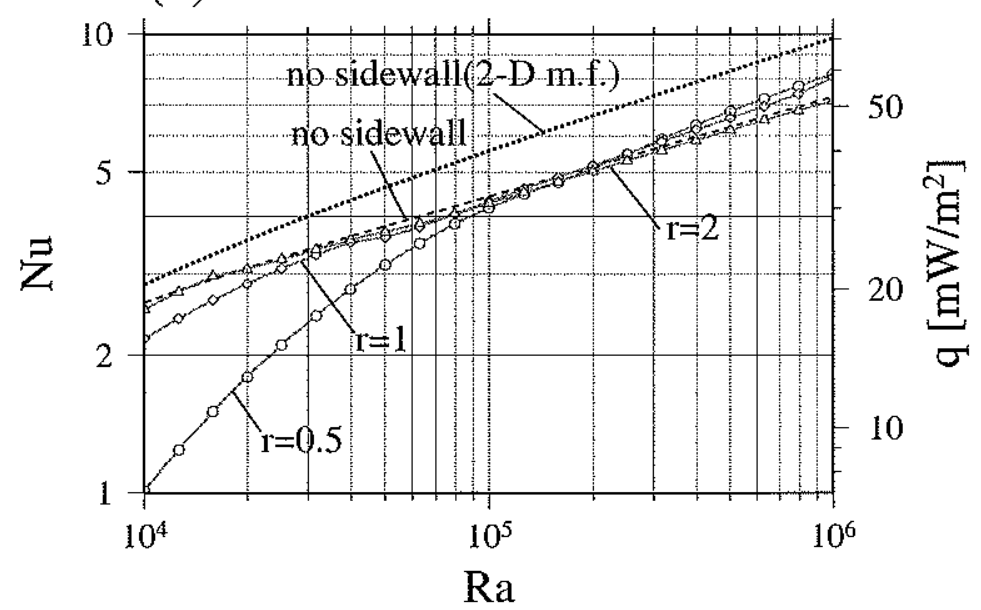

(b)

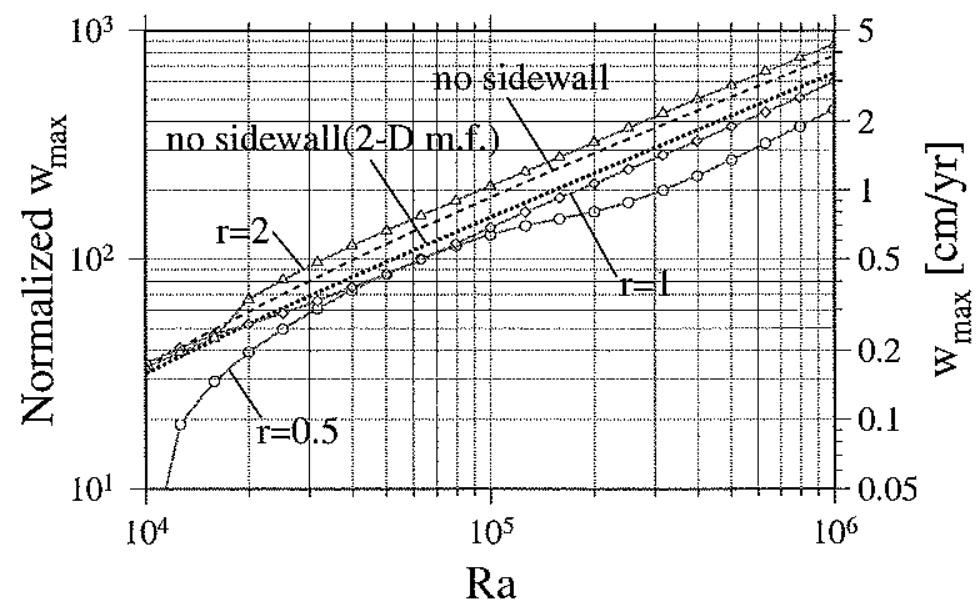

(c)

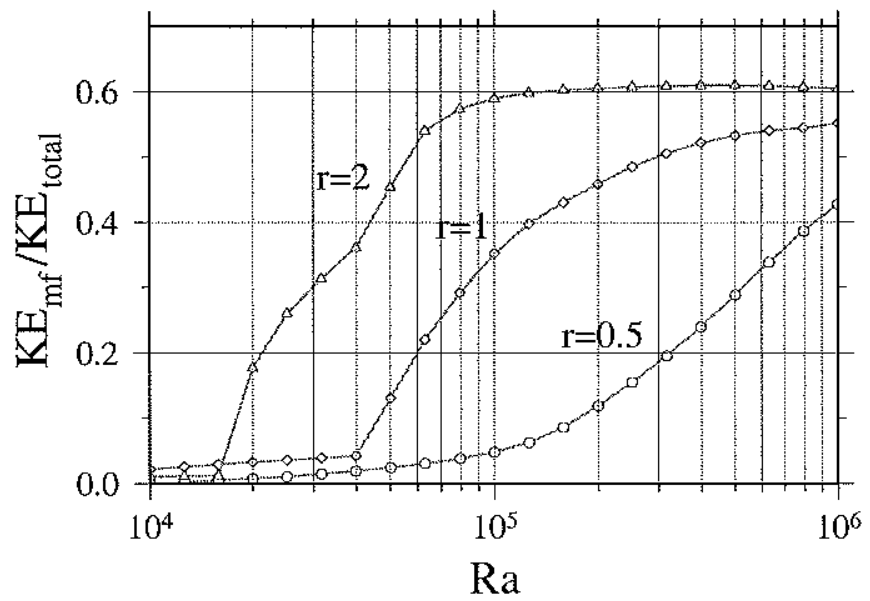

Figure 5-14. Characteristics of 3-D mean-field convection bounded by conducting side walls. The out-of-plane wavenumber is $\pi$. (a) Average Nusselt number and (b) average maximum velocity are plotted with open symbols for three aspect ratios (circle: $r=0.5$, diamond: $r=1$, and triangle: $r=2$ ). Dashed curve denotes the classical case with no side wall, and dotted curve denotes its 2-D mean-field approximation. (c) The proportion of mean-field kinetic energy is plotted as a function of Rayleigh number. 


\title{
Chapter 6
}

\section{The strength of sublithospheric mantle convection and its bearing on continental breakup magmatism}

\begin{abstract}
An assumption of isothermal and static asthenosphere as the normal state of sublithospheric mantle, as commonly employed in studies of terrestrial magmatism, may be physically implausible for a system cooled from above. The growth of an unstable thermal boundary layer at the base of the lithosphere can lead to small-scale convection, so asthenosphere is expected to be usually convecting. A first-order estimate based on the energetics of convection suggests that mantle upwelling rate associated with such smallscale convection may exceed $10 \mathrm{~cm} / \mathrm{yr}$ for asthenospheric viscosity of $10^{18}-10^{19} \mathrm{~Pa}$. To quantify the strength of small-scale convection with temperature-dependent viscosity, a simple transient cooling problem is considered through finite element modeling. Our primary interest is in the role of sublithospheric convection in anomalous magmatism associated with rifting of the supercontinent Pangea, and continental lithosphere with variable thickness is incorporated in our convection model to identify the effect of cratonic lithosphere on small-scale convection. Using asthenospheric viscosity of $10^{18}-10^{19} \mathrm{~Pa} \mathrm{~s}$, transition zone viscosity of $10^{20}-10^{21} \mathrm{~Pa} \mathrm{~s}$, and an activation energy of $200-400 \mathrm{~kJ} / \mathrm{mol}$, we find that the maximum vertical velocity is on the order of $1-10 \mathrm{~cm} / \mathrm{yr}$. A wide variety of temporal evolution and the planform of convection is also observed, including the formation of large-scale mantle flow due to the differential surface cooling imposed by the thickness variation of lithosphere. The potential importance of such sublithospheric convection in magmatism during continental rifting is discussed.
\end{abstract}

\subsection{Introduction}

Continental rifting during the dispersal of the supercontinent Pangea was often accompanied by massive magmatism as evidenced by the frequent occurrence of flood basalts and thick igneous crust emplaced along a number of rifted margins [White and McKenzie, 1989; Holbrook and Kelemen, 1993; Coffin and Eldholm, 1994]. The thickness of igneous crust formed at such volcanic rifted margins is typically $20-30 \mathrm{~km}$ [LASE Study Group, 1986; White et al., 1987; Mutter and Zehnder, 1988; Tréhu et al., 1989; Zehnder et al., 1990; Holbrook et al., 1994a; Holbrook et al., 1994b; Holbrook et al., 1999]. Because normal oceanic crust, which most likely results from decompression melting of passively upwelling mantle with normal potential temperature $\left(-1300^{\circ} \mathrm{C}\right)$ [Klein and Langmuir, 1987; McKenzie and Bickle, 1988; Kinzler and Grove, 1992], is only 6-7 km thick [e.g., 
White et al., 1992], the voluminous magmatism implied by several times thicker igneous crust formed during rifting has been commonly attributed to the upwelling of unusually hot mantle originating in a large plume head [Richards et al., 1989; White and McKenzie, 1989; Campbell and Griffiths, 1990; Hill et al., 1992; White and McKenzie, 1995]. On the other hand, continents seem to rift at preexisting weak zones such as cratonic edges [Wilson, 1966; Anderson, 1994]. Mutter et al. [1988] proposed that a sharp lateral thermal gradient produced by lithospheric necking can result in vigorous, small-scale mantle convection, which may account for the observed voluminous rifting magmatism. As Keen and Boutilier [1995] showed, however, such sharp necking is very unlikely in the deformation of ductile mantle. Intense lithospheric necking is fundamental for this type of mechanism, as is assumed a priori even in the most recent modeling effort presented by Boutilier and Keen [1999].

Both types of explanations, i.e., plume models and necking models, assume that the 'normal' state of asthenospheric mantle is isothermal and thus static. Anything that cannot be explained by the passive upwelling of mantle with normal potential temperature, therefore, seems to require some special mechanism. Even if asthenospheric mantle was indeed isothermal at some time, however, cooling from above results in the growth of a thermal boundary layer, which may eventually become unstable and lead to sublithospheric, small-scale convection [Foster, 1965; Howard, 1966; Parsons and McKenzie, 1978; Fleitout and Yuen, 1984; Yuen and Fleitout, 1984; Jaupart and Parsons, 1985; Buck and Parmentier, 1986; Sparks et al., 1993; Davaille and Jaupart, 1994], after which the resultant thermal structure is no longer isothermal. Static and isothermal asthenosphere, therefore, seems to be a rather extreme case, despite the fact that it is commonly assumed to be normal in studies of terrestrial magmatism. If asthenospheric mantle is usually convecting, how does this affect inferences regarding mantle dynamics based on the products of melting? The importance of small-scale convection may be significant, particularly for magmatism during continental rifting. Unlike mid-ocean ridge magmatism, which may be adequately modeled with steady-state, passive mantle upwelling driven by large-scale plate motion [e.g., McKenzie and Bickle, 1988], 
continental rifting is essentially a transient process, in which an initially null surface divergence evolves into finite-rate spreading. The dynamic aspect of preexisting sublithospheric convection may be important in calculating the amount of melt generated during rifting, because, when the convective upwelling rate exceeds the surface divergence rate, this can produce "excess" magmatism due to rapid mantle fluxing through the melting zone.

A first-order estimate of the strength of such small-scale convection may be obtained by considering its energetics. The conservation of momentum implies that the temporal variation in the kinetic energy of a thermally convective system confined in a volume $V$ with bounding surfaces $S$ is determined by the balance between the rate of potential energy release and the rate of viscous dissipation as [e.g., Golitsyn, 1979; Chandrasekhar, 1981],

$$
\frac{1}{2} \rho_{o} \frac{\partial}{\partial t} \int_{V} u_{i}^{2} d V=\rho_{o} \int_{V} \alpha g w \theta d V+\int_{S} u_{i} \tau_{i j} d S_{j}-\int_{V} \tau_{i j} \frac{\partial u_{i}}{\partial x_{j}} d V,
$$

where $\rho_{o}$ is a fluid density at some reference temperature, $u_{i}$ is a velocity vector, $\alpha$ is thermal expansivity, $g$ is gravitational acceleration, $w$ is the vertical component of velocity, $\theta$ is a deviation from the reference temperature, and $\tau_{i j}$ is a stress tensor. The Boussinesq approximation has already been applied. The last two terms of the right-hand side of the above equation represent the boundary dissipation and the volume dissipation, respectively. For small-scale convection, the contribution of the volume dissipation is usually negligible compared to other terms, so the energetics of steady-state convection are determined mostly by the balance between the first two terms on the right-hand side of equation 6.1 . The rate of potential energy release can be estimated by the boundary layer theory [e.g., Turcotte and Schubert, 1982]. In a system cooled from above, a thermal boundary layer forms only at the top boundary, so the rate of potential energy release may be approximated as,

$$
\Phi_{p} \approx \alpha \rho_{o} g U D \Delta T_{q} \delta
$$

where $U$ is the velocity scale of convection, $D$ is the depth extent of convection, and $\Delta T_{q}$ is the temperature difference across the thermal boundary layer with thickness of $\delta$. 
Assuming 2-D cellular convection with a unit aspect ratio, the growth of the thermal boundary layer may be expressed as,

$$
\delta \approx \sqrt{\frac{\kappa D}{U}}
$$

where $\kappa$ is the thermal diffusivity. The temperature difference is then related to the surface heat flux, $q$, as,

$$
\Delta T_{q} \approx \frac{q}{k} \sqrt{\frac{\kappa D}{U}},
$$

where $k$ is the thermal conductivity. For a fluid with constant viscosity of $\mu$, the boundary dissipation may be approximated as,

$$
\Phi_{v} \approx 8 \mu U^{2}
$$

Thus, using $\Phi_{p} \approx \Phi_{v}$, equations $6.2-6.5$ can be solved for the velocity scale as a function of surface heat flux,

$$
U \approx \sqrt{\frac{\alpha \rho_{o} g \kappa D^{2} q}{8 \mu k}} .
$$

Note that this analysis is valid only if the system evolves sufficiently slowly because a system cooled from above (and not heated from below) does not have a steady state without an internal heat source.

With strongly temperature-dependent viscosity, which characterizes the rheology of the Earth's mantle, stagnant lid convection prevails in a system cooled from above [Booker, 1976; Nataf and Richter, 1982; White, 1988; Ogawa et al., 1991; Davaille and Jaupart, 1993]. Because most of the temperature variation occurs in the stagnant lid, the energetics of small-scale convection beneath the lid can be approximated by that of isoviscous convection [e.g., Solomatov, 1995]. The thickness of the stagnant lid controls heat flux at the top of the convecting domain. Thicker lithosphere can transfer less heat, so the strength of sublithospheric convection is reduced with increasing lid thickness (equation 6.6). The generation of negative buoyancy for small-scale convection is also constrained by temperature-dependent viscosity. The thickness of the cold thermal boundary layer that can participate in convection is limited by a viscosity ratio 
corresponding to the temperature drop across the unstable boundary layer, and the viscosity ratio is about 3 for marginally stable convection and can be as high as 10 for fully developed convection [Davaille and Jaupart, 1993]. The temperature dependency of mantle rheology can be modeled by the following Arrhenius law [e.g., Weertman, 1970],

$$
\mu(T)=\mu_{o} \exp \left(\frac{E}{R T}-\frac{E}{R T_{o}}\right),
$$

where $E$ is activation energy, $R$ is the universal gas constant, and $\mu_{o}$ is reference viscosity at temperature $T_{o}$, for which we use the interior temperature of the convecting domain. Denoting $p=\log \left(\mu_{\max } / \mu_{o}\right)$, where $\mu_{\max }$ is the highest viscosity in the unstable boundary layer, the maximum temperature drop allowed in the boundary layer may be expressed as,

$$
\Delta T_{p} \approx-\frac{p R T_{o}^{2}}{E}
$$

The velocity scale derived for isoviscous convection (equation 6.6) can be applied to convection with temperature-dependent viscosity, if $\Delta T_{q}<\left|\Delta T_{p}\right|$.

Using values appropriate for the shallow upper mantle, i.e., $\alpha=3 \times 10^{-5}$, $\rho_{o}=3.3 \times 10^{3} \mathrm{kgm}^{-3}, \quad \kappa=10^{-6} \mathrm{~m}^{2} \mathrm{~s}^{-1}, k=3.3 \mathrm{Wm}^{-1} \mathrm{~K}^{-1}$, and $g=9.8 \mathrm{~ms}^{-2}$, the velocity scale expected for small-scale convection with a depth extent of $300 \mathrm{~km}$ is calculated as a function of asthenospheric viscosity, for several values of surface heat flux (Figure 6-1a). With the above thermal conductivity and a temperature difference of $1300 \mathrm{~K}$ across the rigid lithosphere, heat fluxes of $40-10 \mathrm{mWm}^{-2}$ approximately correspond to lithospheric thicknesses of $100-400 \mathrm{~km}$, respectively. The viscosity of the asthenosphere is probably in a range of $10^{18}-10^{19} \mathrm{~Pa} \mathrm{~s}$, based on laboratory studies [Karato and $\mathrm{Wu}, 1993$; Hirth and Kohlstedt, 1996] and geophysical observations [Pasay, 1981; Weins and Stein, 1985; Hager and Clayton, 1989]. Though the depth extent of this weak asthenosphere is not well known, the mantle transition zone $\left(400-670 \mathrm{~km}\right.$ ) seems to have a higher viscosity, of $10^{20}$ $10^{21} \mathrm{~Pa} \mathrm{~s}$, and a further increase in viscosity is also indicated for the lower mantle [Hager et al., 1985; Hager and Clayton, 1989; Forte and Mitrovica, 1996; Simons and Hager, 1997]. The depth extent of convection adopted here $(300 \mathrm{~km})$ was chosen in consideration of this likely viscosity layering. For the expected range of asthenospheric viscosity, 
therefore, small-scale convection beneath $100-\mathrm{km}$-thick lithosphere may have a velocity scale exceeding $10 \mathrm{~cm} / \mathrm{yr}$, which is an order-of-magnitude higher than a typical rifting rate $(\sim 1 \mathrm{~cm} / \mathrm{yr})$. Corresponding temperature variations in the unstable boundary layer are compared with the maximum temperature drop allowed in temperature-dependent mantle rheology (Figure 6-1b). Estimates of activation energy for ductile deformation of mantle peridotite vary from $240 \mathrm{~kJ} / \mathrm{mol}$ to $540 \mathrm{~kJ} / \mathrm{mol}$, depending on creep mechanism and the water content in the mantle [Karato and $W u, 1993]$. Though dislocation creep has a higher activation energy $(\sim 500 \mathrm{~kJ} / \mathrm{mol})$ than diffusion creep, its large stress exponent (3.0-3.5) can cause a $\sim 50 \%$ reduction in the effective activation energy [e.g., Christensen, 1984]. Indeed, a recent geophysical study of seamount loading suggests activation energy less than $200 \mathrm{~kJ} / \mathrm{mol}$ for oceanic upper mantle [Watts and Zhong, 1999]. For such low activation energy, the effect of temperature-dependent viscosity on small-scale asthenospheric convection seems to be small (Figure 6-1b).

The estimated velocity scale for small-scale convection in the asthenosphere is comparable to or larger than that for typical plate motion. Thus, small-scale convection cannot be regarded as a small perturbation to large-scale plate-driven flow, and fully 3-D convection must be considered to investigate the nature of small-scale convection beneath a rapidly moving plate such as the Pacific plate. Our current interest, however, lies in the strength of small-scale convection expected beneath the supercontinent Pangea. The rifting axes for the Pangea breakup were located far from subduction zones, so the influence of plate-driven flow is expected to have been minimal for subcontinental mantle. On the other hand, continental lithosphere has a variable thickness, and cratonic lithosphere may be as thick as $300 \mathrm{~km}$ [Jordan, 1988; Gaherty and Jordan, 1995]. Because the presence of cratonic lithosphere can reduce convective vigor (e.g., Chapter 5), lateral heterogeneity in lithospheric structure, expected for the amalgamated continental masses, has to be incorporated to properly evaluate the velocity field of small-scale convection. The purpose of this paper is, therefore, to explore the possibility of small-scale convection beneath the supercontinent in the pre-rifting period based on finite element modeling, with particular 
focus on the effect of continental lithosphere, and to quantify the strength of preexisting convection that may have contributed to rifting magmatism.

\subsection{Numerical Formulation}

The nondimensionalized governing equations for thermal convection of an incompressible fluid are:

Conservation of mass

$$
\nabla \cdot \mathbf{u}=0
$$

Conservation of momentum

$$
-\nabla P+\nabla\left[\mu\left(\nabla \mathbf{u}+\nabla \mathbf{u}^{\mathrm{T}}\right)\right]-\operatorname{Ra} T \mathbf{e}_{z}=0
$$

Conservation of energy

$$
\frac{\partial T}{\partial t}+\mathbf{u} \cdot \nabla T=\nabla^{2} T
$$

where $P$ is normalized pressure, and $\mathrm{Ra}$ is the Rayleigh number defined as,

$$
\mathrm{Ra}=\frac{\alpha \rho_{o} g \Delta T d^{3}}{\kappa \mu_{o}} .
$$

The spatial scale is normalized with a system height of $d$, and the temporal scale is normalized with a diffusion time scale of $d^{2} / \kappa$. Temperature is normalized with $\Delta T$, which is the difference between surface temperature $(273 \mathrm{~K})$ and initial asthenospheric temperature $(1573 \mathrm{~K})$. The temperature-dependent viscosity of equation 6.7 is employed, and the initial asthenospheric temperature is used as the reference temperature. The model domain is $650 \mathrm{~km}$ deep and $1300 \mathrm{~km}$ wide (Figure 6-2). The top and bottom boundaries are rigid. The surface temperature is fixed at $T=273 \mathrm{~K}$, and the bottom boundary is insulated. A reflecting boundary condition is applied to the side boundaries. No internal heat production is considered, so the system is simply cooled from above. Two sets of model geometry are considered (Figure 6-2). In model A, 100-km-thick lithosphere is juxtaposed with a 300km-thick lithosphere (Figure 6-2a). The initial temperature field is prepared by solving heat conduction subjected to an additional temperature boundary condition of $\mathrm{T}=1573 \mathrm{~K}$ at $\mathrm{z} \geq 300 \mathrm{~km}$ (for $0 \leq x<650 \mathrm{~km}$ ) and $z \geq 100 \mathrm{~km}$ (for $x \geq 650 \mathrm{~km}$ ). In model B, 200-km-wide and $100-\mathrm{km}$-thick lithosphere is surrounded by $300-\mathrm{km}$-thick lithosphere (Figure 6-2b), and its 
initial temperature field is prepared in a similar manner for model A. The velocity of lithospheric mantle with initial temperature of less than $1473 \mathrm{~K}$ is then fixed as zero. The survival of thick cratonic lithosphere for several billion years [e.g., Jordan, 1988] requires high activation energy ( $>500 \mathrm{~kJ} / \mathrm{mol})$ or high intrinsic viscosity [Shapiro, 1995; Doin et al., 1997], so the rheology of continental lithosphere is probably different from that of oceanic mantle. Lenardic and Moresi [1999] suggested that cratonic lithosphere should be intrinsically $10^{3}$ times more viscous than oceanic mantle to account for the longevity of cratonic lithosphere, and this 1000-fold increase is indeed expected for highly depleted cratonic lithosphere [Jordan, 1979; Phipps Morgan, 1997]. Therefore, the assumed rigidity of lithosphere is probably justified for our modeling of small-scale convection. The reference viscosity for asthenosphere, $\mu_{1}$, is set at either $10^{18}$ or $10^{19} \mathrm{~Pa}$ s. The viscosity ratio between asthenosphere and the mantle transition zone $\left(\mu_{1} / \mu_{2}\right)$ is either 1 or 100 , to identify the effect of viscosity layering on small-scale convection. Two values of activation energy (200 and $400 \mathrm{~kJ} / \mathrm{mol})$ are tested.

The computational domain is discretized with $51 \times 71$ variable-size $2-D$ quadrilateral elements (Figure 6-2c). 2-D numerical solutions to the above coupled differential equations are obtained using the finite element method, the implementation of which is similar to that of ConMan [King et al., 1990], except for an optional 3-D mean-field approximation (Chapter 5). The system is integrated for $200 \mathrm{~m} . \mathrm{y}$. The Pangea supercontinent was formed during the mid-Carboniferous $(-320 \mathrm{Ma})$, and its integrity was maintained until the late Jurassic ( $170 \mathrm{Ma})$ [Scotese, 1984], although incipient rifting events were recorded since the mid-Triassic [Veevers, 1989]. Thus, the adopted computational period is sufficient to monitor the dynamics of small-scale convection that might have been present beneath the supercontinent. Because our interest is limited to dynamics with a time scale of 100 m.y. or so, the rigid bottom boundary may be valid to describe the boundary between the upper and lower mantle. Both viscosity contrast and endothermic phase transition may temporarily hamper material flux through the base of the upper mantle [Honda et al., 1993; Tackley et al., 1993; Solheim and Peltier, 1994; Tackley, 1995]. Though the characteristics of the convective system are often described in terms of 
the Rayleigh number and the Nusselt number, their use in a transient cooling problem with a nonuniform lithospheric lid is limited. Temporal variation in surface heat flux is very small because of rigid lithosphere. It is also not obvious how to define a proper system height to calculate the interior Rayleigh number [Nataf and Richter, 1982; Christensen, 1984] with our model geometry. The evolution of the system is thus monitored in terms of maximum and minimum vertical velocity components, total kinetic energy, and the average internal temperature of a convecting region. The maximum vertical velocity and the temperature of the convecting domain are most relevant to mantle melting when rifting allows asthenospheric convection to circulate through shallower depths.

\subsection{Results}

For each model geometry, there are eight different combinations of mantle rheology parameters (i.e., two asthenospheric viscosities, two viscosity contrasts, and two choices of activation energy), and all combinations are tested. The thinnest lithosphere is initially $100-\mathrm{km}$ thick in both types of geometry, so small-scale convection with the velocity scale exceeding $10 \mathrm{~cm} / \mathrm{yr}$ is expected beneath the thin spot, based on boundary layer theory (Figure 6-1a), though the presence of a conducting, cratonic side wall can considerably reduce the strength of convection (e.g., Chapter 5). Because the system is only cooled from above, asthenospheric temperature must gradually decrease, and a nearly linear decrease with a gradient of $\sim 80^{\circ} \mathrm{C} / 100 \mathrm{~m} . \mathrm{y}$. is observed in all cases. In general, higher reference viscosity and higher activation energy result in more sluggish convection, as expected (e.g., Figure 6-1). The temporal evolution of sublithospheric convection is, however, found to be highly sensitive to the particular choice of rheology parameters, even though our parameter range is rather limited. In the following, we will present some representative examples of model evolution and discuss them in detail.

\subsubsection{2-D Solutions}

The evolution of model A with $\mu_{1}=10^{18} \mathrm{~Pa} \mathrm{~s}, \mu_{1} / \mu_{2}=100$, and $E=200 \mathrm{~kJ} / \mathrm{mol}$ is shown in Figure 6-3. The maximum and minimum vertical velocities as well as the average temperature of the convecting interior are measured for asthenospheric mantle in the 
vicinity of cratonic lithosphere $(\mathrm{z}>400 \mathrm{~km}$ and $325<\mathrm{x}<975 \mathrm{~km})$. The kinetic energy is calculated for the entire model domain. The largest peak in the minimum vertical velocity (Figure 6-3b) as well as the highest kinetic energy (Figure 6-3c) observed at $\sim 5$ m.y. corresponds to the formation of the first cold downwelling from the base of thin lithosphere. This onset of instability excites small-scale convection, and the maximum upwelling velocity fluctuates between $10-20 \mathrm{~cm} / \mathrm{yr}$ during the first $40 \mathrm{~m} . \mathrm{y}$. Due to a $100-$ fold increase in viscosity at the depth of $400 \mathrm{~km}$, this convection is almost confined to the asthenosphere (Figure 6-3e and f). There is persistent downwelling along the cratonic side wall, but during the first 40 m.y., there is no indication that small-scale convection is modulated by cratonic lithosphere. Upwellings are mainly controlled by downwellings from the base of thin lithosphere, and they migrate through time. This is because the lateral temperature gradient within the cratonic lithosphere is smaller than the vertical temperature gradient in the thin lithosphere. The generation of negative buoyancy due to side wall cooling is not large enough to compete with random downwellings beneath the thin lithosphere.

After the first 40 m.y. or so, however, the emergence of another convective pattern is observed. Whereas the minimum vertical velocity is reduced by about $50 \%$, the maximum vertical velocity is still as high as $10 \mathrm{~cm} / \mathrm{yr}$ (Figure $6-3 \mathrm{a}$ and b). This corresponds to strong upwelling from the base of the cratonic lithosphere to shallow asthenosphere, and this seems to suppress the formation of random downwelling from the thin lithosphere (Figure 6-3g and h). Though downwelling along the cratonic side wall is still present, which tends to deflect this upwelling (Figure 6-3h), the location of upwelling is persistent through time. This stable, larger-scale convection originates from differential cooling imposed by the variation in lithospheric thickness. Whereas the vigorous smallscale convection efficiently cools the asthenosphere beneath the thin lithosphere, the mantle beneath the cratonic lithosphere is relatively uncooled. This leads to the development of a large-scale lateral temperature gradient in the sublithospheric mantle, by which relatively hot, deep mantle flows to shallower depths. This large-scale flow is 
conceptually similar to a mechanism advocated by King and Anderson $[1995 ; 1998]$ for the generation of continental flood basalts.

Differential surface cooling, however, does not always result in such large-scale convection. Figure 6-4 shows the evolution of model $\mathrm{A}$, with no viscosity contrast at the base of asthenosphere, but with otherwise identical mantle rheology. Downwellings initiated at the base of lithosphere extend to the bottom, and the strength of convection is relatively uniform beneath the lithosphere, due to the uniform reference viscosity (Figure 6-4e-h). The convecting domain is thus well mixed, preventing the formation of a large-scale lateral temperature gradient. The pattern of convection is mostly determined by downwellings from the base of the thin lithosphere and along the cratonic side wall. This edge-driven convection [e.g., King and Anderson, 1998] at the cratonic side wall is persistently observed over the period of 100 m.y. (e.g., Figure $6-4 f$ and h), except when sporadic, stronger downwellings from the thin lithosphere (Figure 6-4b) modulate the pattern of convection (Figure 6-4g). Note that a low viscosity for the entire upper mantle is unrealistic, and the purpose of this example is simply to illustrate the effect of a viscosity contrast on the pattern of sublithospheric convection. Similar model evolution with reduced convective vigor is observed by using the reference viscosity of $10^{19} \mathrm{~Pa} \mathrm{~s}$. An increase in activation energy does not modify the well-mixed nature of convection.

Another example of model A evolution with $\mu_{1}=10^{19} \mathrm{Pas}, \mu_{1} / \mu_{2}=100$, and $E=200 \mathrm{~kJ} / \mathrm{mol}$ is shown in Figure 6-5. The maximum vertical velocity is on the order of 1$2 \mathrm{~cm} / \mathrm{yr}$ (Figure 6-5a), which is about an order of magnitude smaller than the velocity scale observed in the model with $\mu_{1}=10^{18} \mathrm{~Pa}$ (Figure 6-3a). A reduction in velocity is expected to be proportional to the square root of an increase in viscosity (equation 6.6), and the discrepancy from this prediction suggests that the velocity scale based on the boundary layer theory is somewhat overestimated, which results in underestimating the effect of temperature-dependent viscosity. Snapshots of a temperature field show a gradual increase in the thickness of the top thermal boundary layer (Figure 6-5e-h). This thickening acts to slow down the generation of negative buoyancy and to reduce the depth extent of the low viscosity layer, both of which in turn result in further thickening of the boundary layer. The 
effect of differential surface cooling is thus only weakly reflected in sublithospheric convection, and a large-scale lateral thermal gradient does not form.

Results with model $\mathrm{B}$ are in general similar to those with model $\mathrm{A}$, except that stronger effects of side walls are observed. Figure 6-6 shows the evolution of model B with $\mu_{1}=10^{18} \mathrm{~Pa} \mathrm{~s}, \mu_{1} / \mu_{2}=100$, and $E=200 \mathrm{~kJ} / \mathrm{mol}$. The maximum vertical velocity is about $8 \mathrm{~cm} / \mathrm{yr}$ for the first $100 \mathrm{~m} . \mathrm{y}$. (Figure 6-6a), which is slightly reduced compared to model A with the same rheological parameters (Figure 6-3a). Because of the closely spaced cratonic side walls, however, the pattern of small-scale convection is fixed. Return flow to downwellings along side walls and from the base of the thin spot takes place as focused upwellings into the thin spot as a return flow (Figure 6-6f-h). The effect of differential surface cooling is also observed; a broad cold downwelling from the center of the thin spot brings uncooled mantle beneath the cratonic lithosphere into the thin spot (Figure 6-6g and h). Unlike in model A, this larger-scale flow coincides with the pattern of preexisting small-scale convection.

During the very early evolution of this model, the highest maximum vertical velocity (>15 cm/yr) is observed (Figure 6-6a), and this is worth some explanation. Snapshots of convection during this high-energy period are shown in Figure 6-7. It is clearly seen that vigorous small-scale convection results from the formation of the initial downwellings along the side walls, and that focused upwelling in the model center is simply due to this model geometry, because this type of initial upwelling is not observed in model A (Figure 6-3a). The mechanism of this rapid convection is essentially the same as that of the convection models of Mutter et al. [1988] and Boutilier and Keen [1999]. Although we prepared the initial temperature field by solving heat conduction to create a smooth thermal structure within the lithospheric lid, having a uniformly hot mantle next to cold cratonic side walls results in a rapid development of an unstable vertical thermal boundary layer within the asthenosphere. This initial small-scale convection, therefore, depends solely on how we prepare the initial temperature field, and its origin is somewhat artificial. Indeed, this rapid convection quickly reduces its strength within the first 2 m.y. 
(Figure 6-6a). We would like to emphasize that small-scale convection developed later (>10 m.y.) originates mainly in cooling from above, which is of our primary interest.

The strong side wall effect on the strength of convection can be seen in another model run with a 10 -fold increase (compared to the previous model) in asthenospheric viscosity (Figure 6-8). Small-scale convection severely retarded by side walls cannot prevent the growth of the thermal boundary layer, and the thin spot is closed within the first 100 m.y.

\subsubsection{3-D Mean-Field Solutions}

The analysis of the convective instability of an isoviscous fluid in the presence of conducting side wall suggests that the preferred planform of convection is always 3-D, in which convection cells are aligned perpendicular to a side wall (Chapter 5). The velocity reduction due to side walls is also shown to be mitigated in $3-\mathrm{D}$ convection. To explore this potential importance of 3-D convection in our transient cooling problem, we computed 3-D mean-field solutions using the method developed in Chapter 5. Though the 3-D meanfield approximation can handle only single-mode convection in the out-of-plane coordinate, it suffices for our purpose to observe whether 3-D convection is excited in this transient problem. Model parameters are identical to that used for 2-D solutions. The initial out-of-plane temperature field has random perturbations with an amplitude of $0.1 \mathrm{~K}$. The out-of-plane wavenumber is set as $\pi$ for the cases with $\mu_{1} / \mu_{2}=1$, and as $2 \pi$ for the cases with $\mu_{1} / \mu_{2}=100$, given the expected spatial scale of convection. In all cases, out-of-plane convection is eventually excited, and the strength of convection tends to be increased compared to 2-D cases as expected. Though it is probably reasonable to use the 3-D meanfield approximation to investigate the pattern of convection confined in asthenosphere such as observed in the cases with a 100-fold viscosity contrast, it is less certain how to interpret the structure of larger-scale flow such as induced by differential cooling, because the outof-plane wavenumber is prescribed to the scale of small-scale convection. Thus, we focus on small-scale convection in the following examples.

Figure 6-9 shows the evolution of model A with $\mu_{1}=10^{19} \mathrm{~Pa} \mathrm{~s}, \mu_{1} / \mu_{2}=100$, and $E=200 \mathrm{~kJ} / \mathrm{mol}$. The mode of out-of-plane convection is excited at $\sim 20 \mathrm{~m}$.y. (Figure $6-9 \mathrm{~d}$ ), 
which coincides with the formation of the first downwelling (Figure 6-9b). The contribution of the out-of-plane kinetic energy to the total kinetic energy gradually increases in the next $30 \mathrm{~m} . \mathrm{y}$. until its proportion reaches $\sim 0.5$. Due to this excitation of a $3-$ D convective planform, the evolution of sublithospheric convection becomes very different from that observed in the 2-D calculation (Figure 6-5). Whereas the maximum vertical velocity is only $1-2 \mathrm{~cm} / \mathrm{yr}$ in the $2-\mathrm{D}$ case, it is about $5 \mathrm{~cm} / \mathrm{yr}$ in the fully developed $3-\mathrm{D}$ convection state (Figure 6-9a). This enhanced convection brings relatively uncooled mantle from the mantle transition zone into asthenosphere (Figure 6-9g), slowing down the cooling of asthenosphere (Figure 6-9e). The mean-field kinetic energy is comparable with the out of-plane kinetic energy, so the 3-D velocity field has a broad, sheet-like upwelling (Figure 6-9h), which is similar to the pattern of 3-D mean-field steady-state convection in a fluid bounded by conducting side walls studied in Chapter 5 .

A similar enhancement of convection is also observed for model B. An example of model evolution with $\mu_{1}=10^{18} \mathrm{~Pa} \mathrm{~s}, \mu_{1} / \mu_{2}=100$, and $E=200 \mathrm{~kJ} / \mathrm{mol}$ is shown in Figure 6-10. As in the 2-D case, downwellings along side walls tend to focus upwelling into the thin spot. The excitation of out-of-plane convection follows this pattern of convection modulated by side walls, as expected from the fact that the out-of-plane temperature field must share the same type of symmetry with the mean-field temperature field (Chapter 5). We note that the rapid convection observed at the beginning $(<2$ m.y.; Figure $6-10 \mathrm{a}$ and b), which is already discussed for the 2-D case in terms of the artificiality of an initial temperature condition, does not excite the mode of out-of-plane convection (Figure 6-10d). The preference of a 3-D convective planform in a fluid bounded by side walls, as suggested in Chapter 5, is only for stationary state convection, and this example indicates that it does not apply to the early stage of transient convection.

A summary of all model runs is presented in terms of the maximum vertical velocity and the viscosity of the convecting domain (Figure 6-11). The 20-m.y.-average during $200 \mathrm{~m}$.y. is calculated for both parameters. Because the system is simply cooled from above, the interior temperature gradually decreases through time, resulting in the observed trend with a negative slope. The functional dependence of the vertical velocity on 
the interior viscosity is generally consistent with the prediction from the boundary layer theory, though several factors of difference can be seen in the absolute values of the vertical velocity. The closest match is observed for the 3-D mean-field solution of model A, with $\mu_{1}=10^{18} \mathrm{~Pa} \mathrm{~s}, \mu_{1} / \mu_{2}=100$, and $E=200 \mathrm{~kJ} / \mathrm{mol}$, which shows only a factor of $\sim 2$ difference (Figure 6-11a). This is expected because the simple energetics argument becomes more applicable with low viscosity and low activation energy as well as a high viscosity contrast. The effect of side walls is minimal in the 3-D mean-field calculation with the model A geometry. The major source of this small discrepancy probably comes from the episodic nature of convection, which results in a more complicated flow pattern than assumed in the boundary layer theory. This tendency of overpredicting the velocity scale by equation 6.6 results in a rather optimistic estimate of the influence of temperaturedependent viscosity (Figure 6-1b). Increasing the activation energy from $200 \mathrm{~kJ} / \mathrm{mol}$ to $400 \mathrm{~kJ} / \mathrm{mol}$ can considerably reduce the strength of convection, even for asthenospheric viscosity of $10^{18} \mathrm{~Pa} \mathrm{~s}$ (Figure 6-11a). For the majority of the model runs, the maximum vertical velocity lies in a range of $1-10 \mathrm{~cm} / \mathrm{yr}$. Given that a typical rifting rate is on the order of $1 \mathrm{~cm} / \mathrm{yr}$, our result suggests that mantle upwelling could be 10 times more rapid than surface divergence, and that the intensity of this active mantle upwelling is sensitive to both mantle rheology and the structure of continental lithosphere.

\subsection{Discussion and Conclusion}

As a preliminary attempt to explore the possibility of sublithospheric convection beneath a supercontinent, several important complications pertinent to the Earth's mantle have been left for future work, such as non-Newtonian rheology and internal heat generation. Because the dynamic origin of small-scale convection is the instability of a top thermal boundary layer, and because the strength of convection is sensitive to the portion of the boundary layer that can be detached, the role of stress-dependent viscosity in destabilizing the boundary layer is probably important for finite-amplitude convection [e.g., Solomatov, 1995; Larsen and Yuen, 1997a; Larsen and Yuen, 1997b]. Internal heat generation acts to buffer the interior temperature, so more steady evolution of sublithospheric convection is 
expected. Heat flux expected from radioactive heat generation within a 500 -km-thick mantle column ranges from $\sim 10 \mathrm{~mW} / \mathrm{m}^{2}$ to $\sim 20 \mathrm{~mW} / \mathrm{m}^{2}$, depending on the estimate of the concentrations of radioactive elements in the upper mantle [e.g., Hart and Zindler, 1986; Anderson, 1989], so it is not sufficient to completely prevent the secular cooling of asthenosphere. Heat flux into the base of cratonic lithosphere, however, could be much smaller than $20 \mathrm{~mW} / \mathrm{m}^{2}$, because of internal heat generation within continental crust and cratonic lithosphere [Jordan, 1988; Jaupart et al., 1998; Rudnick et al., 1998], so internal heating may be able to sustain the mantle temperature beneath cratonic lithosphere.

To apply the idea of strong sublithospheric convection to rifting magmatism, it will be necessary to consider how rifting affects this preexisting convection. Because smallscale convection is driven by negative buoyancy, the strength of upwellings probably remains similar as long as the distribution of negative buoyancy is not severely destroyed by rapid rifting. As rifting proceeds and evolves into seafloor spreading, mantle flow will be eventually dominated by plate-driven flow, and it may be important to investigate the time scale of this transition as well as the temporal variation in the planform of convection. Mantle melting may add further complication to the dynamics of small-scale convection [e.g., Tackley and Stevenson, 1993].

The purpose of this paper is not to challenge the importance of plumes in mantle dynamics. Though they have not been seismically imaged in a convincing manner, a number of numerical studies of whole-mantle convection show that plumes are the most preferred style of upwelling. Our intention is to point out that "normal" asthenosphere can exhibit the rich dynamics of small-scale convection. A currently prevailing tendency in studies of large igneous provinces is that anything that cannot be explained by the passive upwelling of normal asthenosphere is regarded as the influence of a mantle plume. We have shown that, using an acceptable range of rheological parameters for the Earth's upper mantle, vigorous upwelling in asthenosphere is a likely state of sublithospheric mantle. A variation in the thickness of continental lithosphere can modulate the pattern of convection in some cases, and as a result, upwelling tends to be focused toward a thinner spot, which is also a potential locus for rifting. Given the limited parameter space of mantle rheology 
we explored, it is somewhat surprising to observe a great variety of dynamical behavior among different model runs, in terms of the strength of convection, the pattern of convection, and the style of temporal evolution. Because the upper mantle is not expected to be isothermal in the first place, due to various kinds of previous tectonic history [e.g., Anderson et al., 1992], the strength of small-scale convection and the temperature of convecting region may also be influenced by preexisting thermal anomalies. When we are to investigate the origin of some "anomalous" magmatism, therefore, it may be worth first questioning what could happen without mantle plumes. 
(a)

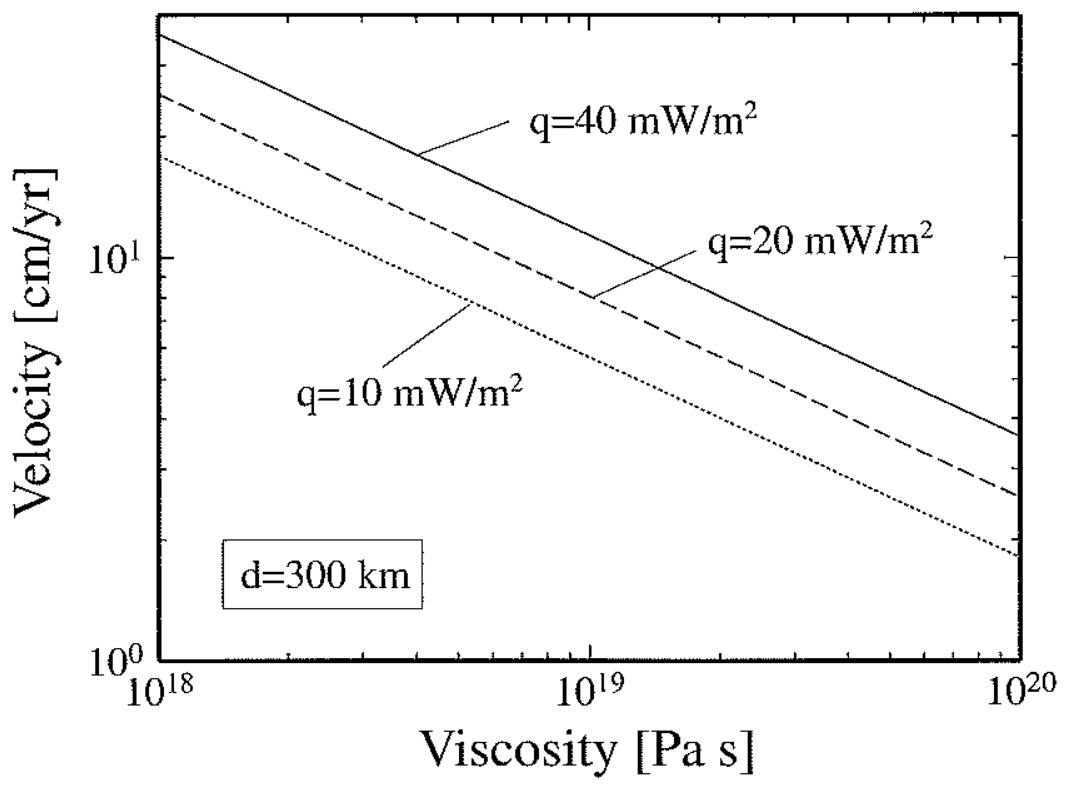

(b)

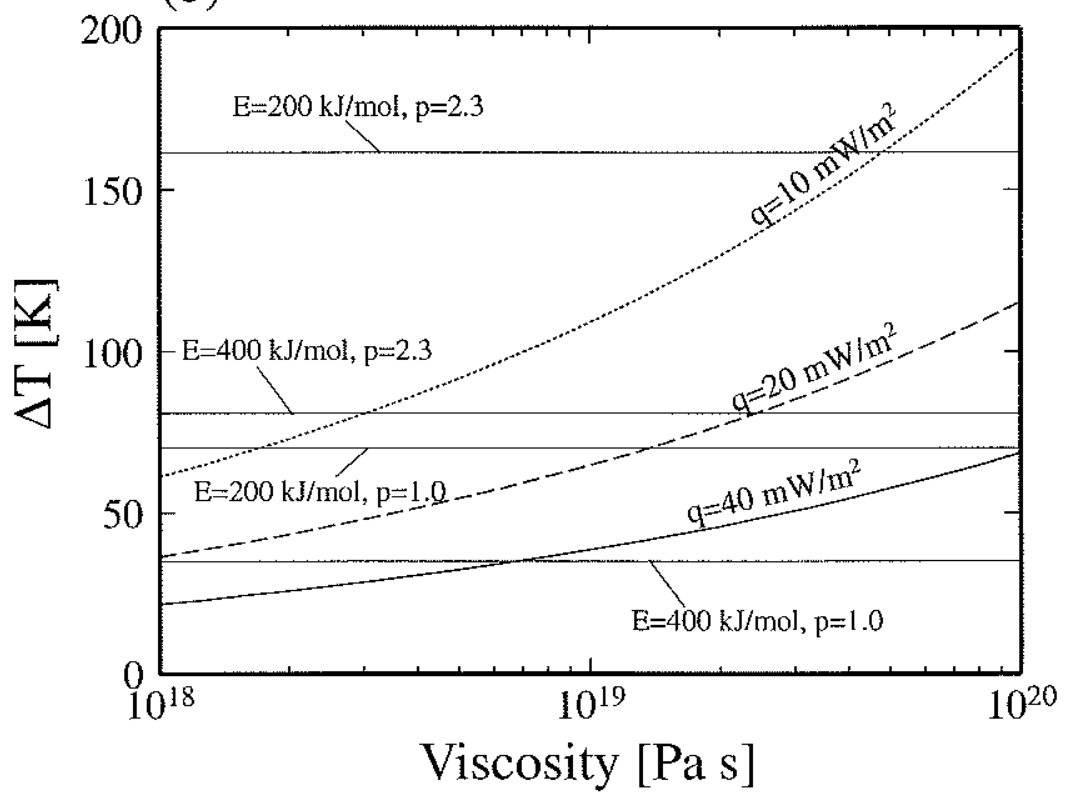

Figure 6-1. (a) Velocity scale of isoviscous convection subject to a given surface heat flux (equation 6.6) is plotted as a function of viscosity. The depth scale of convection is set as $300 \mathrm{~km}$. (b) Temperature drop across cold thermal boundary layer (equation 6.4) is compared with the maximum temperature drop imposed by temperature-dependent viscosity (straight lines, equation 6.8). The reference temperature $T_{o}$ is set as $1573 \mathrm{~K}$. Cases for two values of activation energy ( 200 and $400 \mathrm{~kJ} / \mathrm{mol}$ ) and two values of logarithmic viscosity contrast ( 1.0 and 2.3 , corresponding to the viscosity contrasts of 3 and 10 , respectively) are illustrated. 
(a)

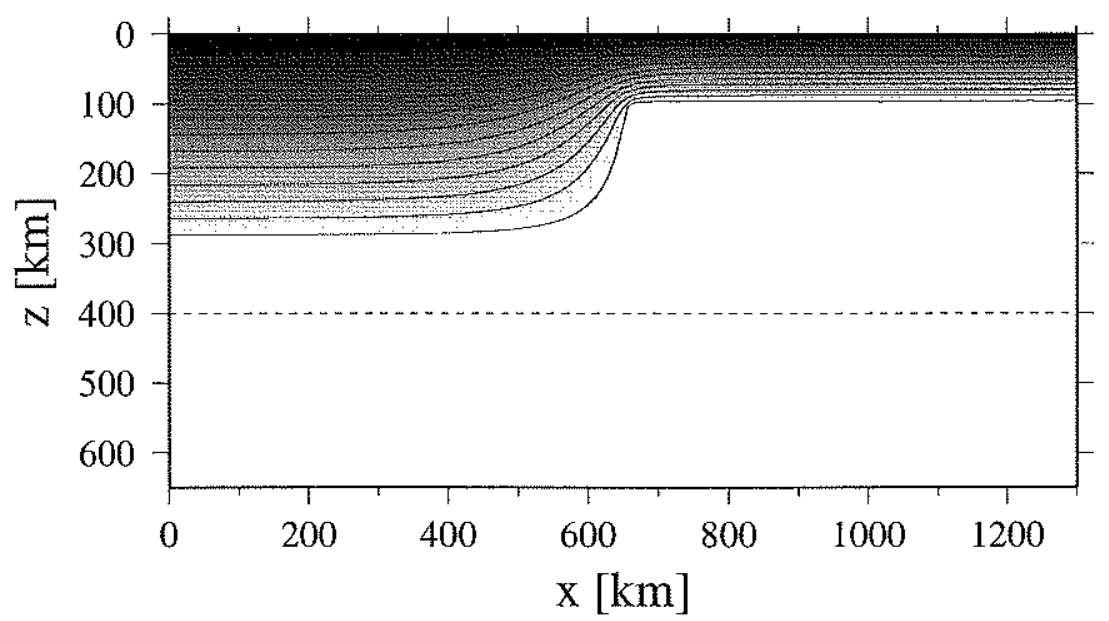

(b)

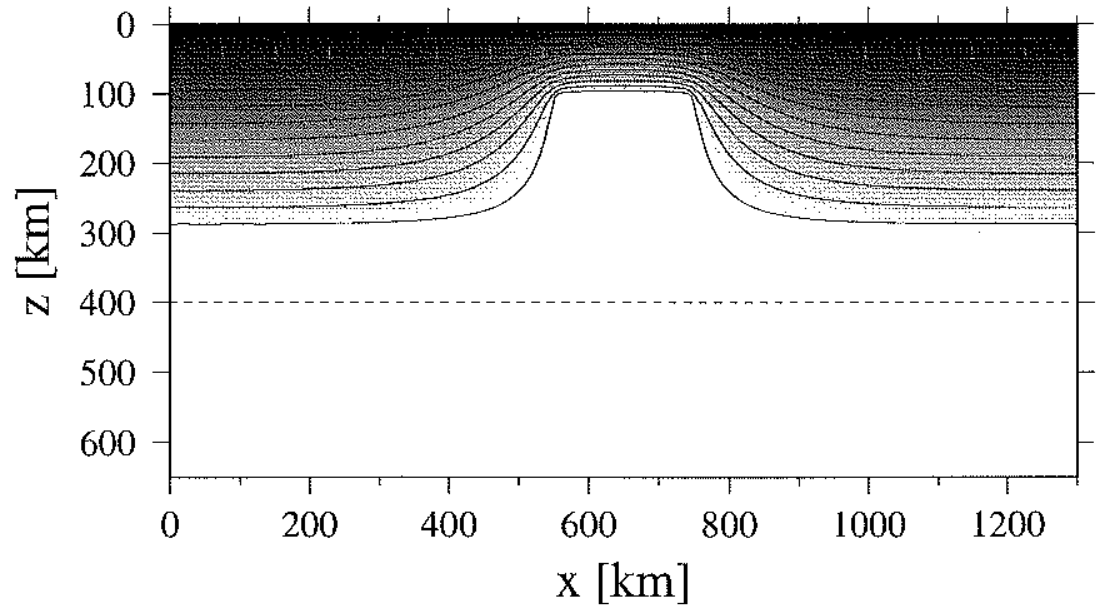

(c)

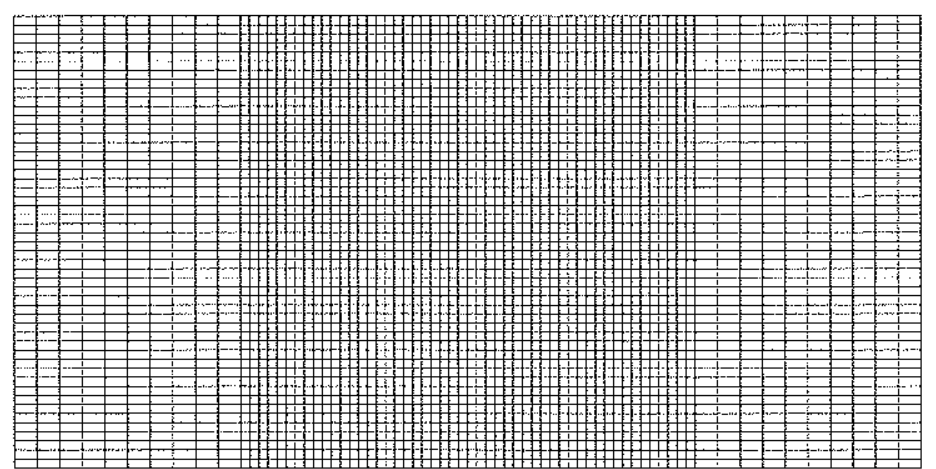

Figure 6-2. Initial temperature field for finite element modeling. (a) Model A, in which $100-\mathrm{km}$-thick lithosphere is juxtaposed to 300-km-thick lithosphere. (b) Model B, in which $200-\mathrm{km}$-wide and $100-\mathrm{km}$-thick lithosphere is emplaced in $300-\mathrm{km}$-thick lithosphere. Contours are at $100 \mathrm{~K}$ interval. Finite element mesh is shown in (c). 


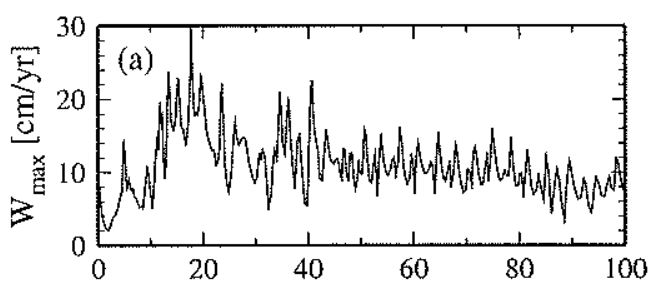

\section{(e)}
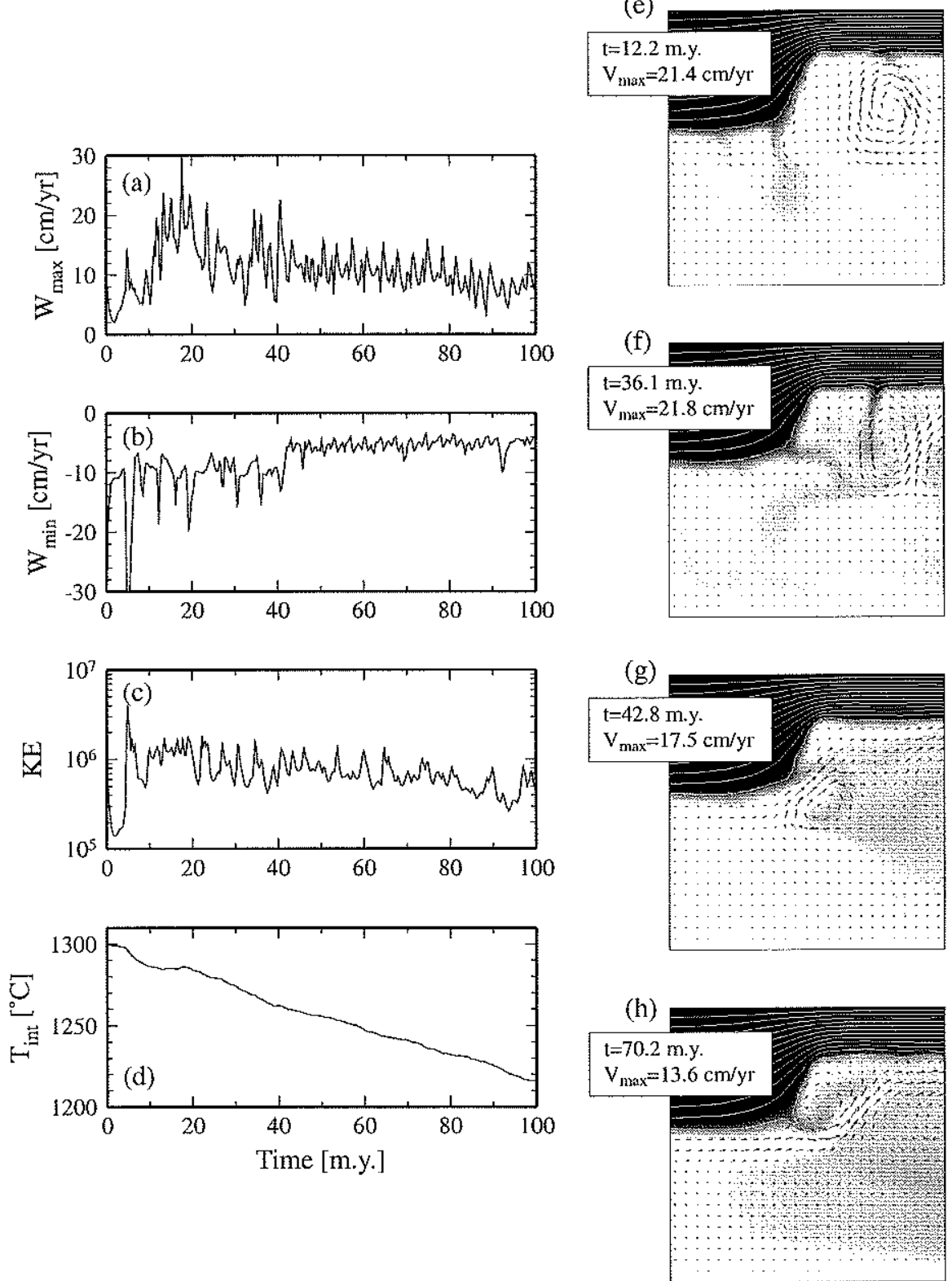

Figure 6-3. Evolution of model A, with $\mu_{1}=10^{18} \mathrm{~Pa} \mathrm{~s}, \mu_{1} / \mu_{2}=100$, and $E=200 \mathrm{~kJ} / \mathrm{mol}$. The time series of (a) maximum vertical velocity, (b) minimum vertical velocity, (c) kinetic energy, and (d) average temperature of the convecting region are shown for the first 100 m.y. While kinetic energy is calculated for the entire computational domain, other parameters are measured for asthenosphere in the vicinity of thick cratonic lithosphere $(z>400 \mathrm{~km}$ and $325<x<975 \mathrm{~km})$. Snapshots of partial temperature field $(325<x<975 \mathrm{~km})$ are also shown in $(\mathrm{e})-(\mathrm{h})$, with corresponding velocity field. Gray scales are set to vary from $T_{\max }-120$ (black) to $T_{\max }-20$ (white) at each instant, to enhance subtle temperature variations in convecting mantle. $100^{\circ} \mathrm{C}$ contours are also shown. Velocity arrows are also normalized by the maximum velocity at each instant, and the scale is given in inset. 

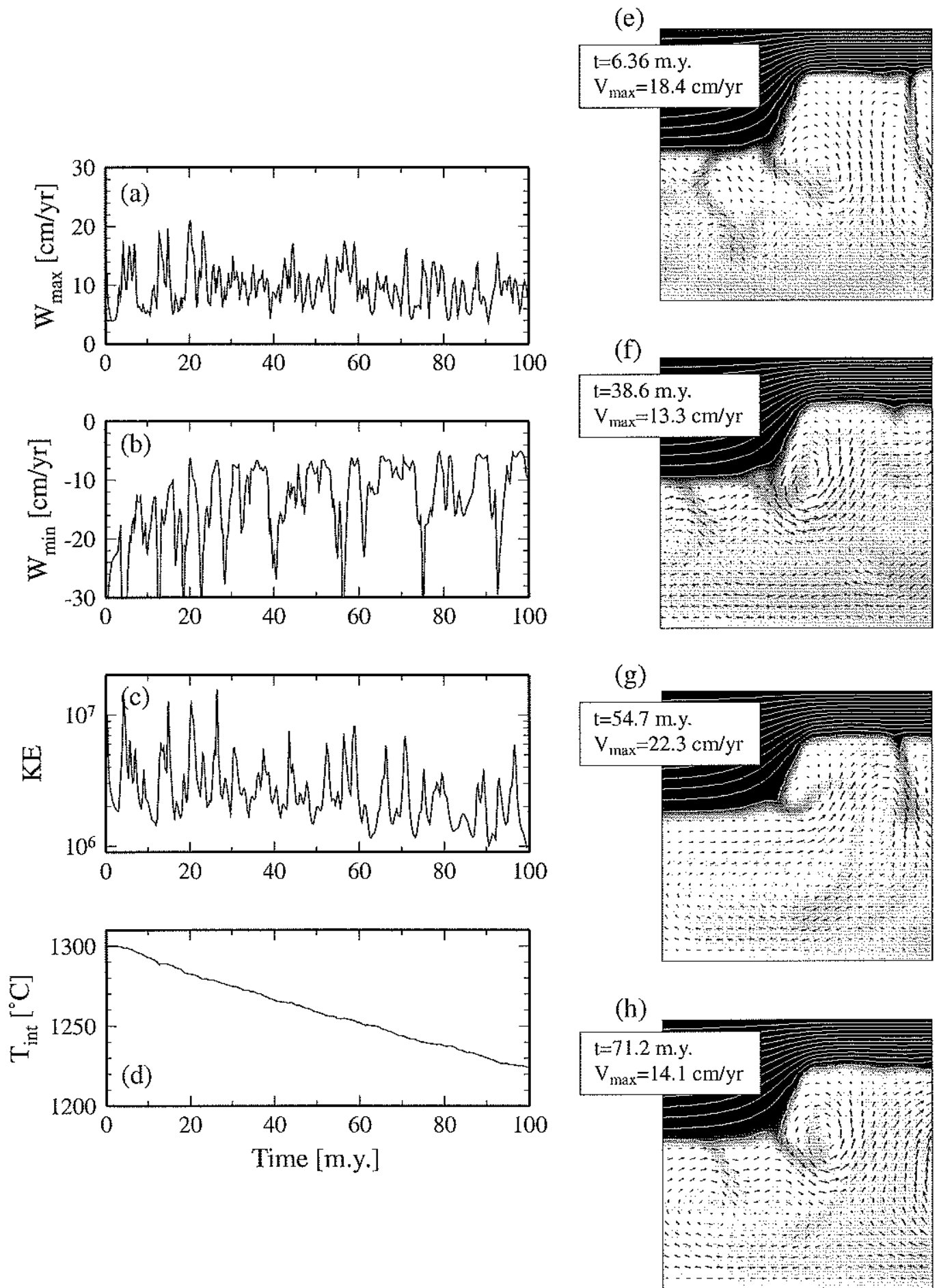

Figure 6-4. Same as Figure 6-3, but with $\mu_{1}=10^{18} \mathrm{~Pa} \mathrm{~s}, \mu_{1} / \mu_{2}=1$, and $E=200 \mathrm{~kJ} / \mathrm{mol}$. Temperature variation in the convecting region is more subtle in this example, and gray scales are set to vary from $T_{\max }-80$ (black) to $T_{\max }$ (white) at each instant. 

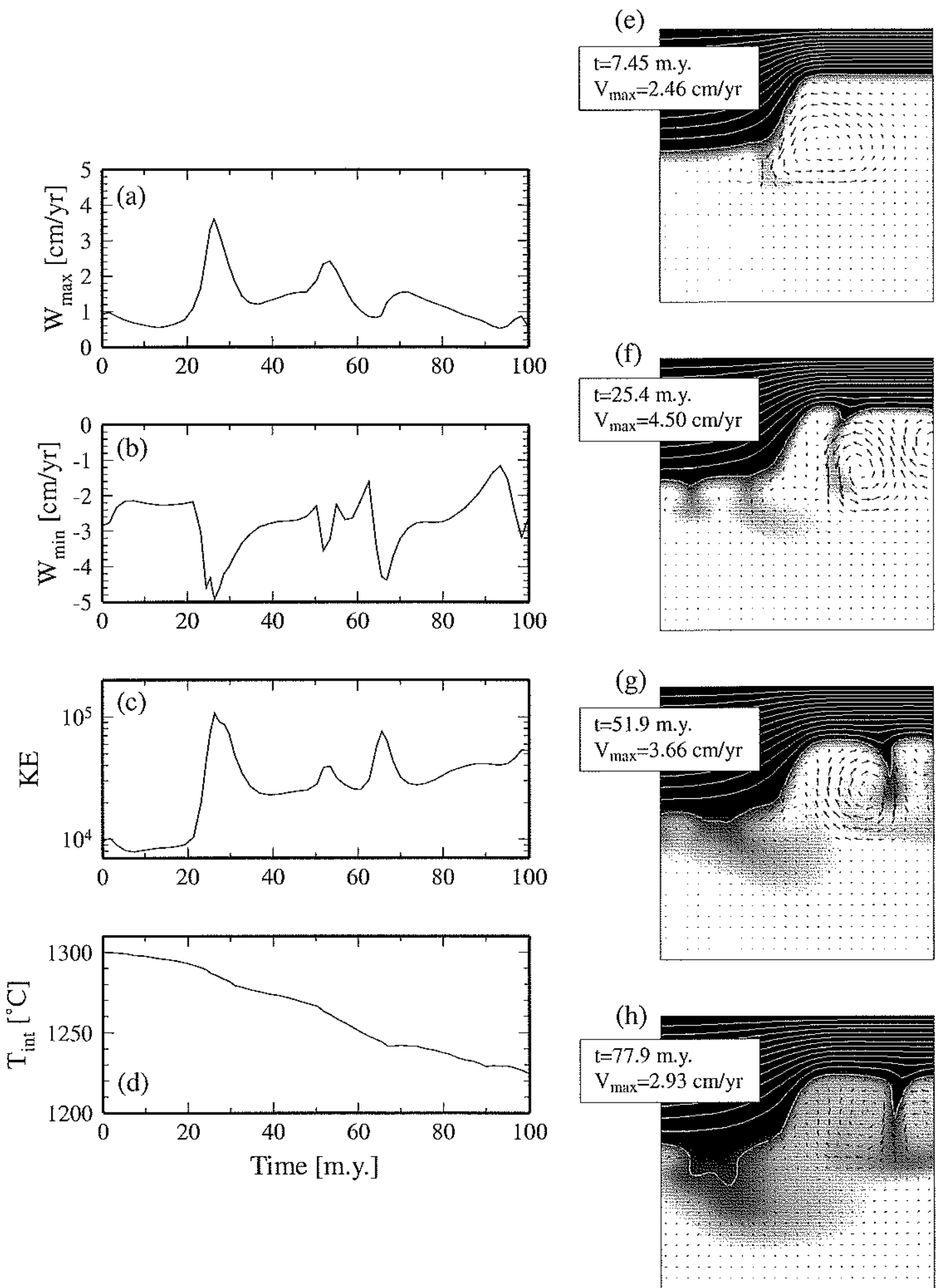

Figure 6-5. Same as Figure 6-3, but with $\mu_{1}=10^{19} \mathrm{~Pa} \mathrm{~s}, \mu_{1} / \mu_{2}=100$, and $E=200 \mathrm{~kJ} / \mathrm{mol}$. 
(e)
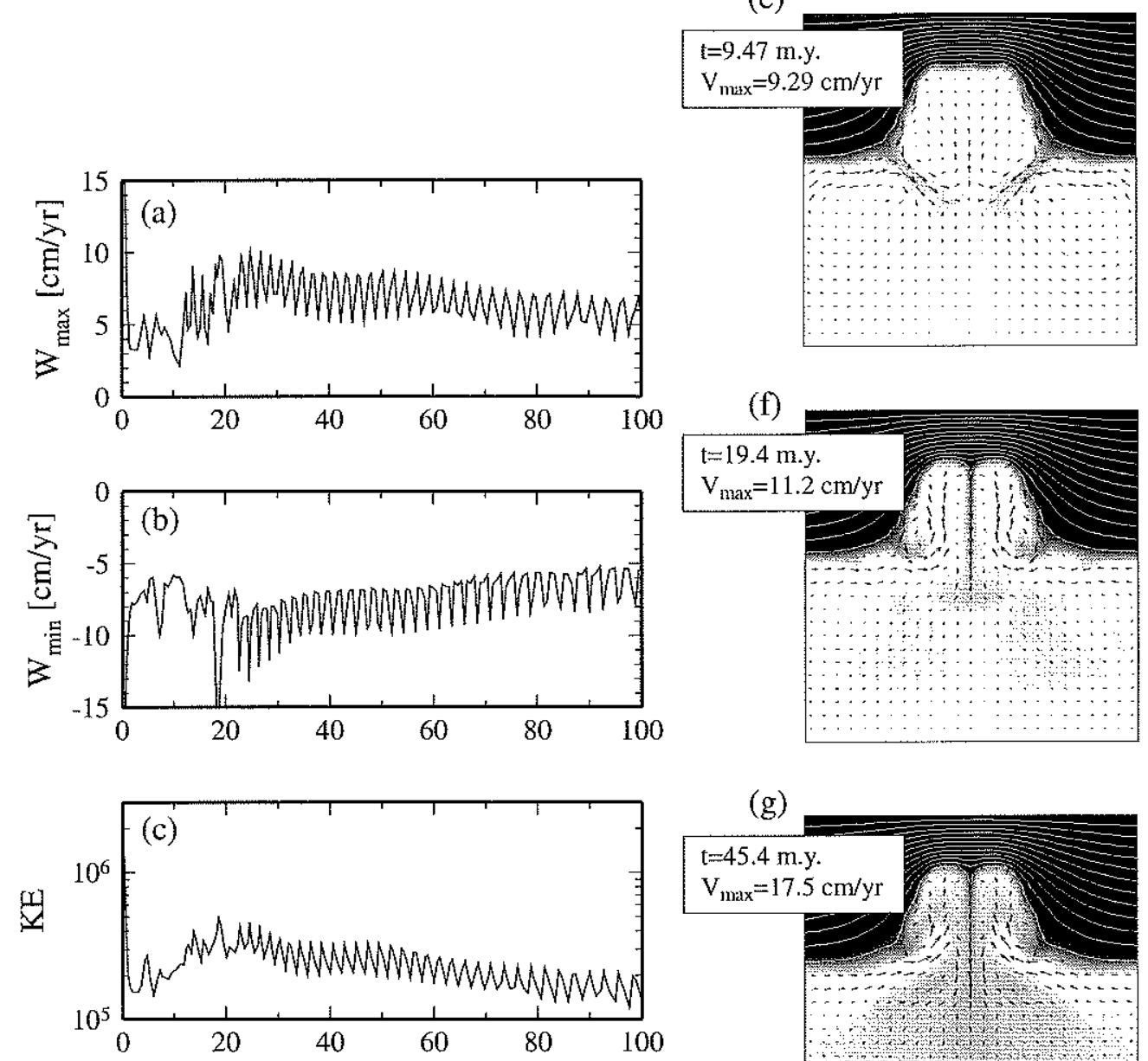

(g)
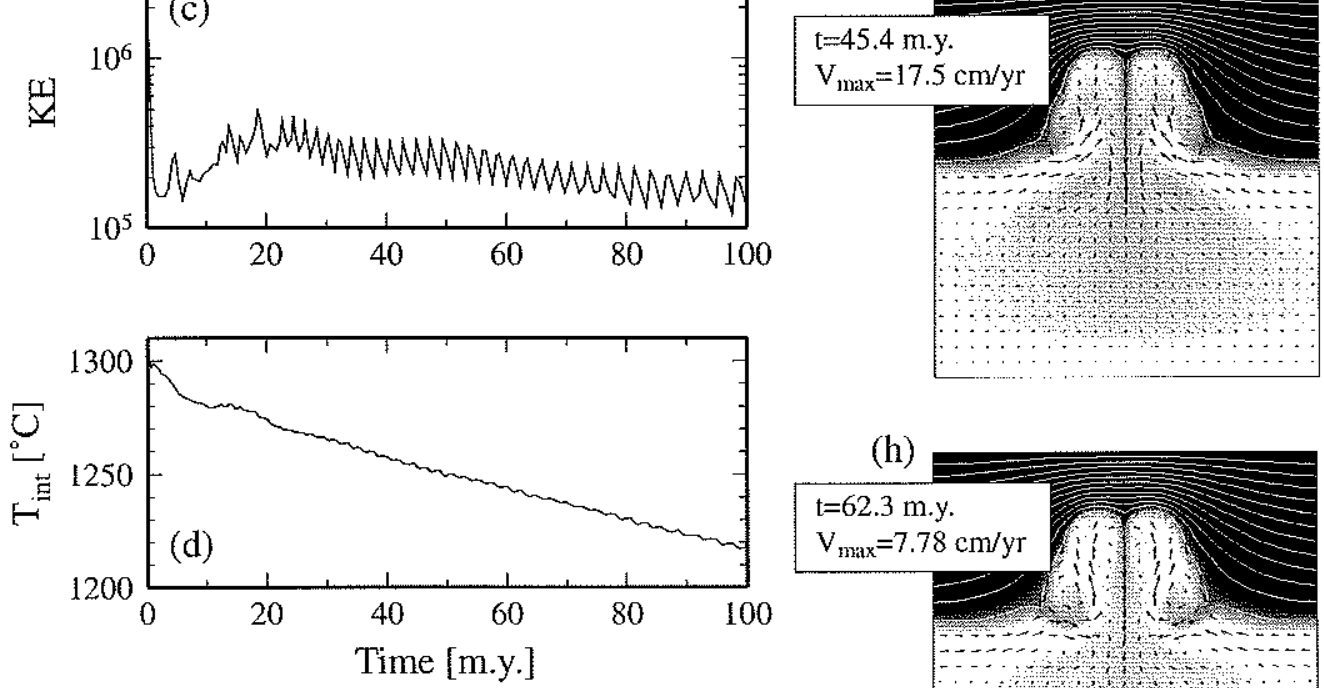

\section{(h)}

$t=62.3$ m.y.

$\mathrm{V}_{\max }=7.78 \mathrm{~cm} / \mathrm{yr}$

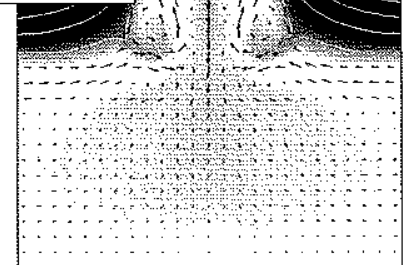

Figure 6-6. Evolution of model $\mathrm{B}$, with $\mu_{1}=10^{18} \mathrm{~Pa} \mathrm{~s}, \mu_{1} / \mu_{2}=100$, and $E=200 \mathrm{~kJ} / \mathrm{mol}$. Time series and snapshots are shown in the same manner of Figure 6-3. 
(a)

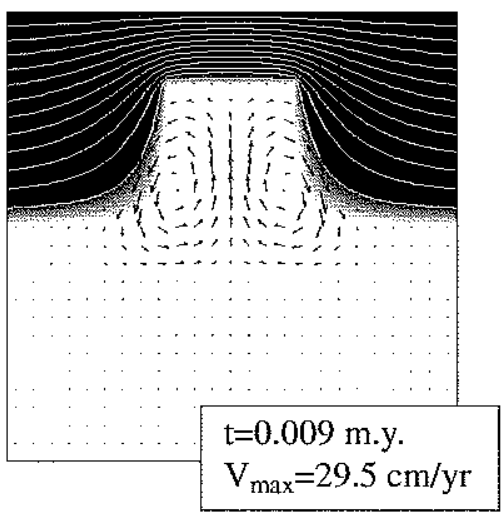

(c)

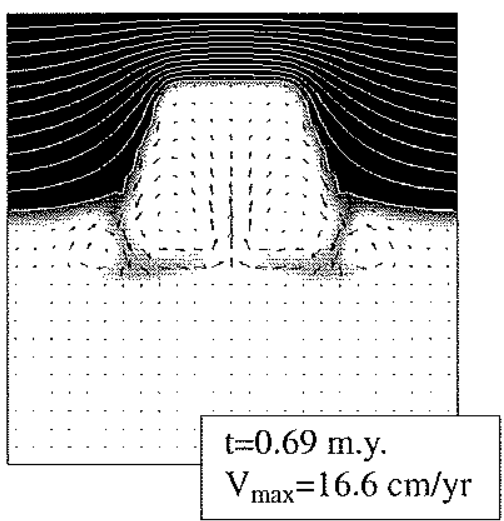

(b)

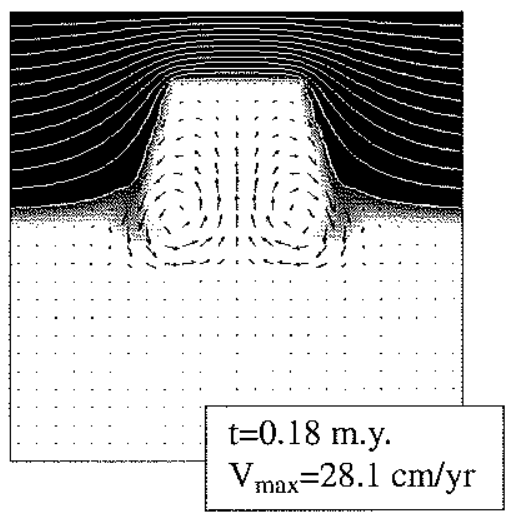

(d)

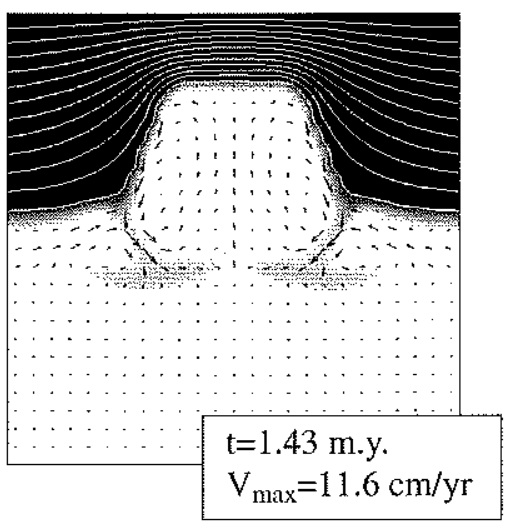

Figure 6-7. Snapshots for the very early evolution of model B presented in Figure 6-6. 


\section{(e)}
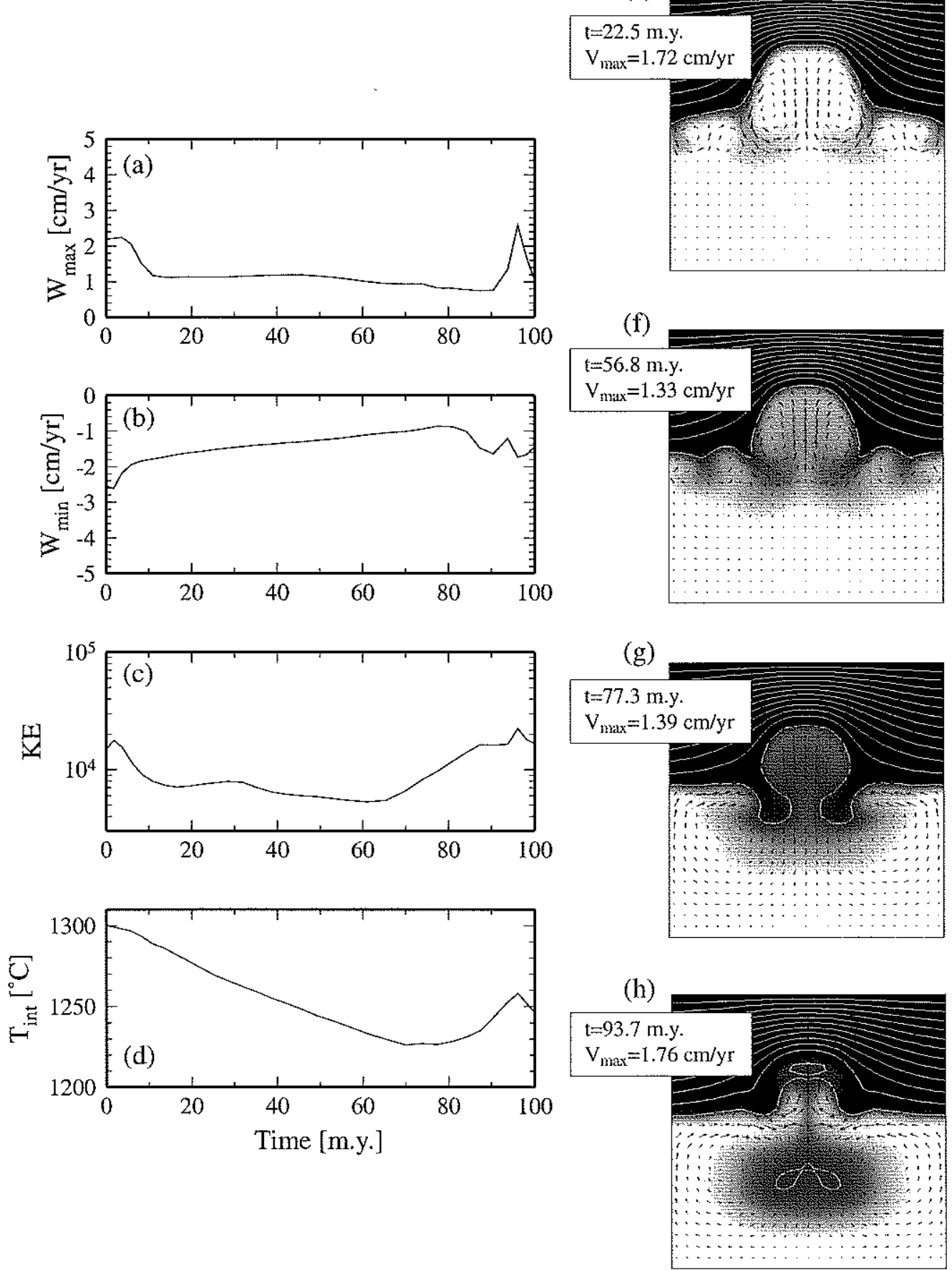

Figure 6-8. Same as Figure $6-6$, but with $\mu_{1}=10^{19} \mathrm{~Pa} \mathrm{~s}, \mu_{1} / \mu_{2}=100$, and $E=200 \mathrm{~kJ} / \mathrm{mol}$. 

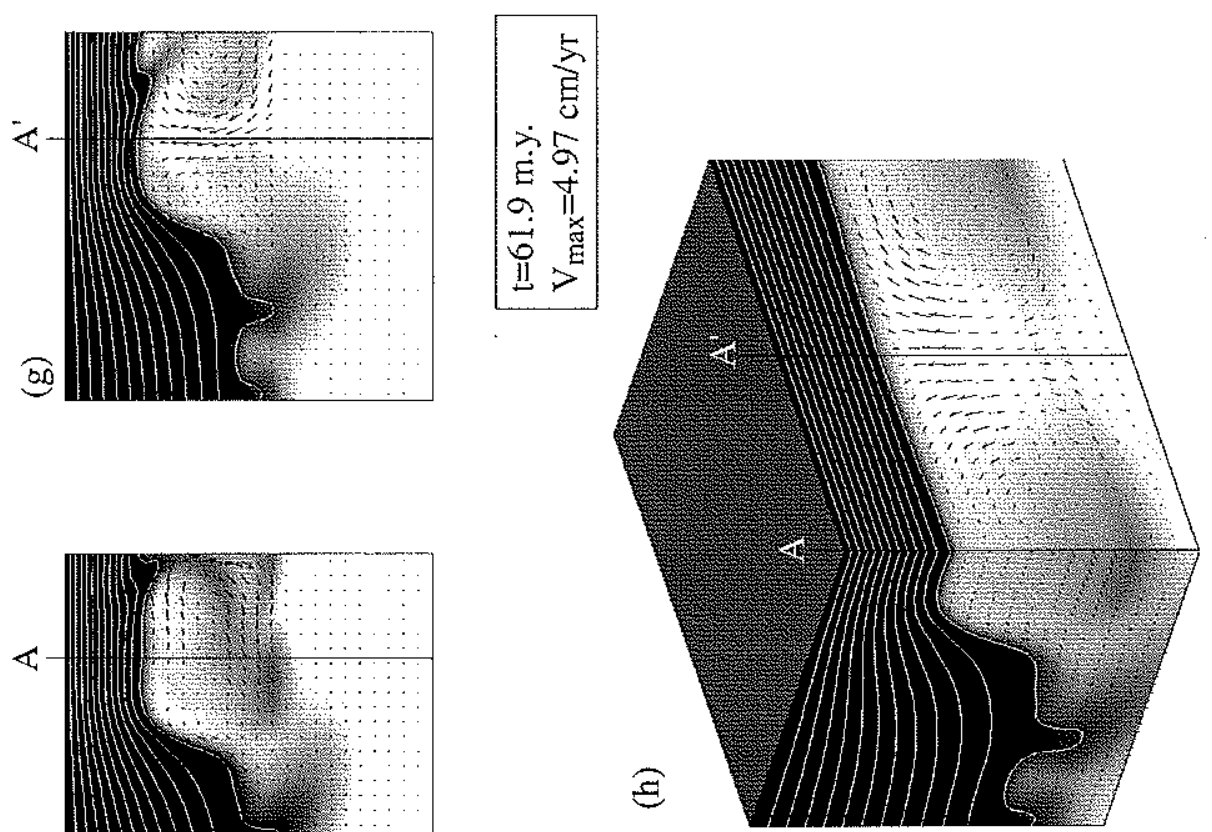

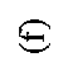
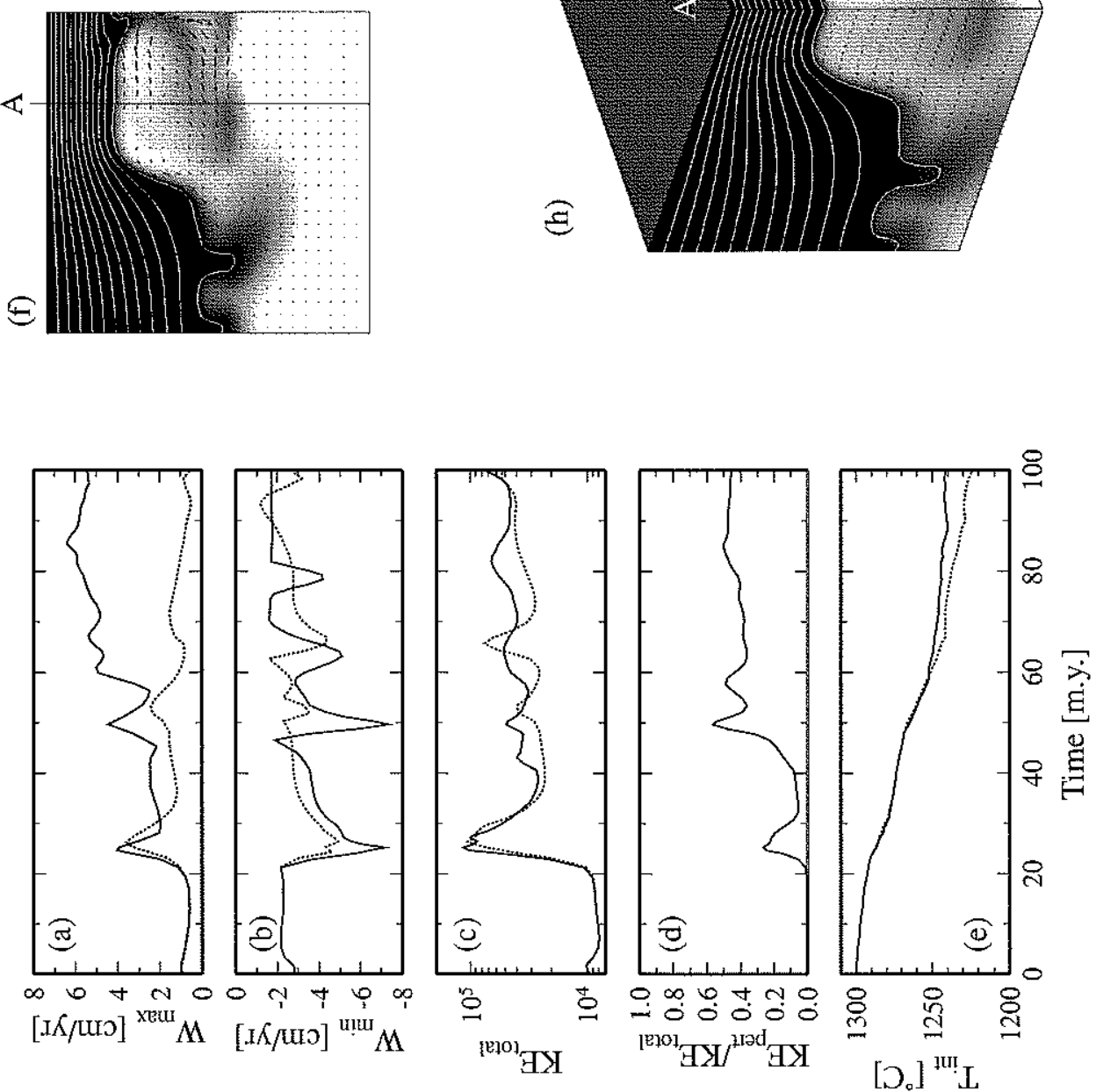

Figure 6-9. 3-D mean-field solution for model $\mathrm{A}$, with $\mu_{1}=10^{19} \mathrm{~Pa} \mathrm{~s}, \mu_{1} / \mu_{2}=100$, and $E=200 \mathrm{~kJ} / \mathrm{mol}$. (a) Maximum vertical velocity, (b) minimum vertical velocity, (c) total kinetic energy, (d) the fraction of out-of-plane kinetic energy in total kinetic energy, and (e) average temperature of the convecting region. While total kinetic energy is calculated for the entire computational domain, other parameters (shown in a, b, d, and e) are measured for asthenosphere in the vicinity of thick cratonic lithosphere $(z>400 \mathrm{~km}$ and $325<x<975 \mathrm{~km})$. Dotted lines denote measurements from the 2-D solution as in Figure 6-5. Two slices of a snapshot at $61.9 \mathrm{~m} . \mathrm{y}$. are also shown with a $3-\mathrm{D}$ perspective plot. Gray scale varies from $1180^{\circ} \mathrm{C}$ (black) to $1280^{\circ} \mathrm{C}$ (white). 

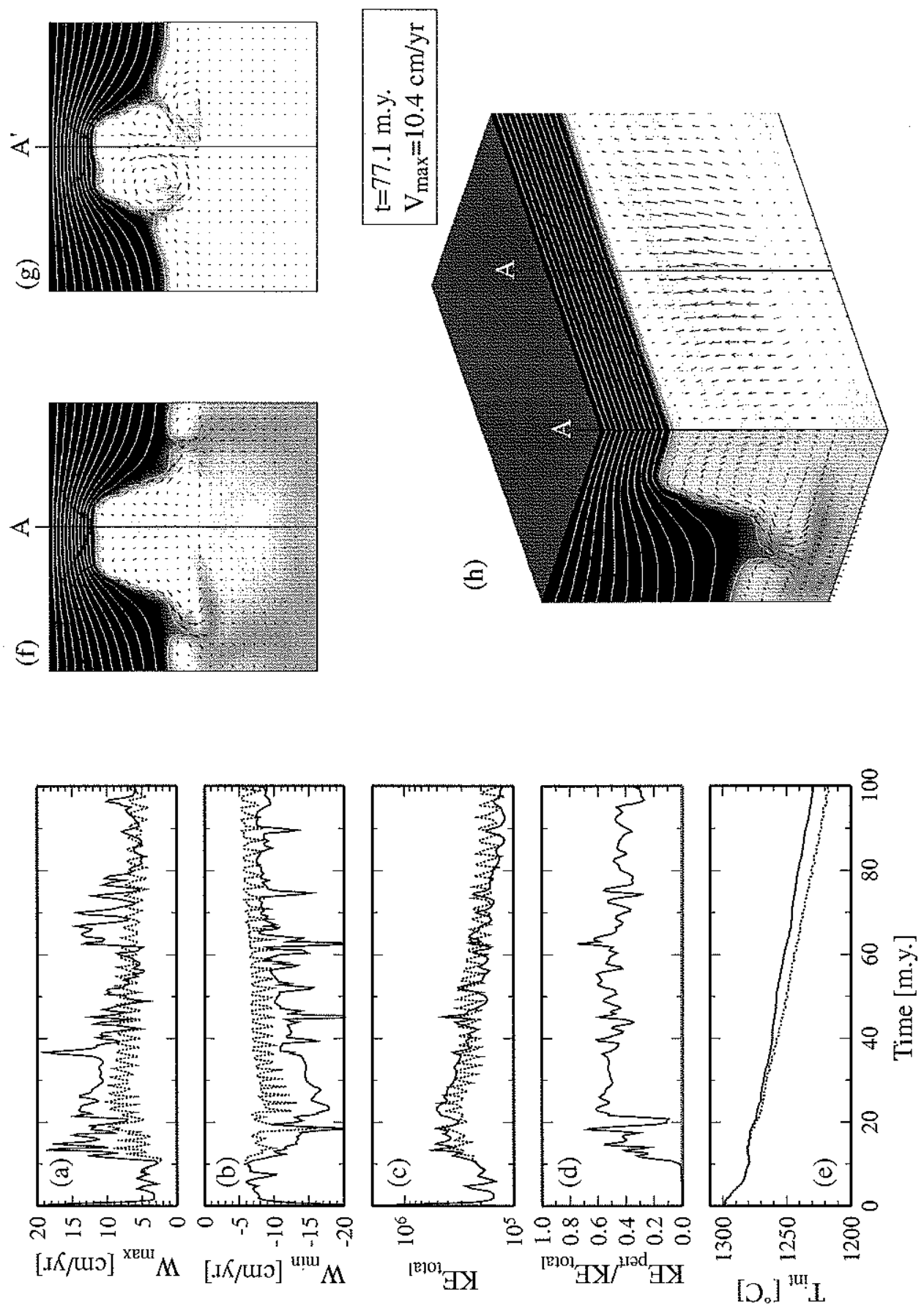

Figure 6-10. Same as Figure 6-9, but for model B with $\mu_{1}=10^{18} \mathrm{~Pa} \mathrm{~s}, \mu_{1} / \mu_{2}=100$, and $E=200 \mathrm{~kJ} / \mathrm{mol}$. A snapshot is shown at $77.1 \mathrm{~m} . \mathrm{y}$. 


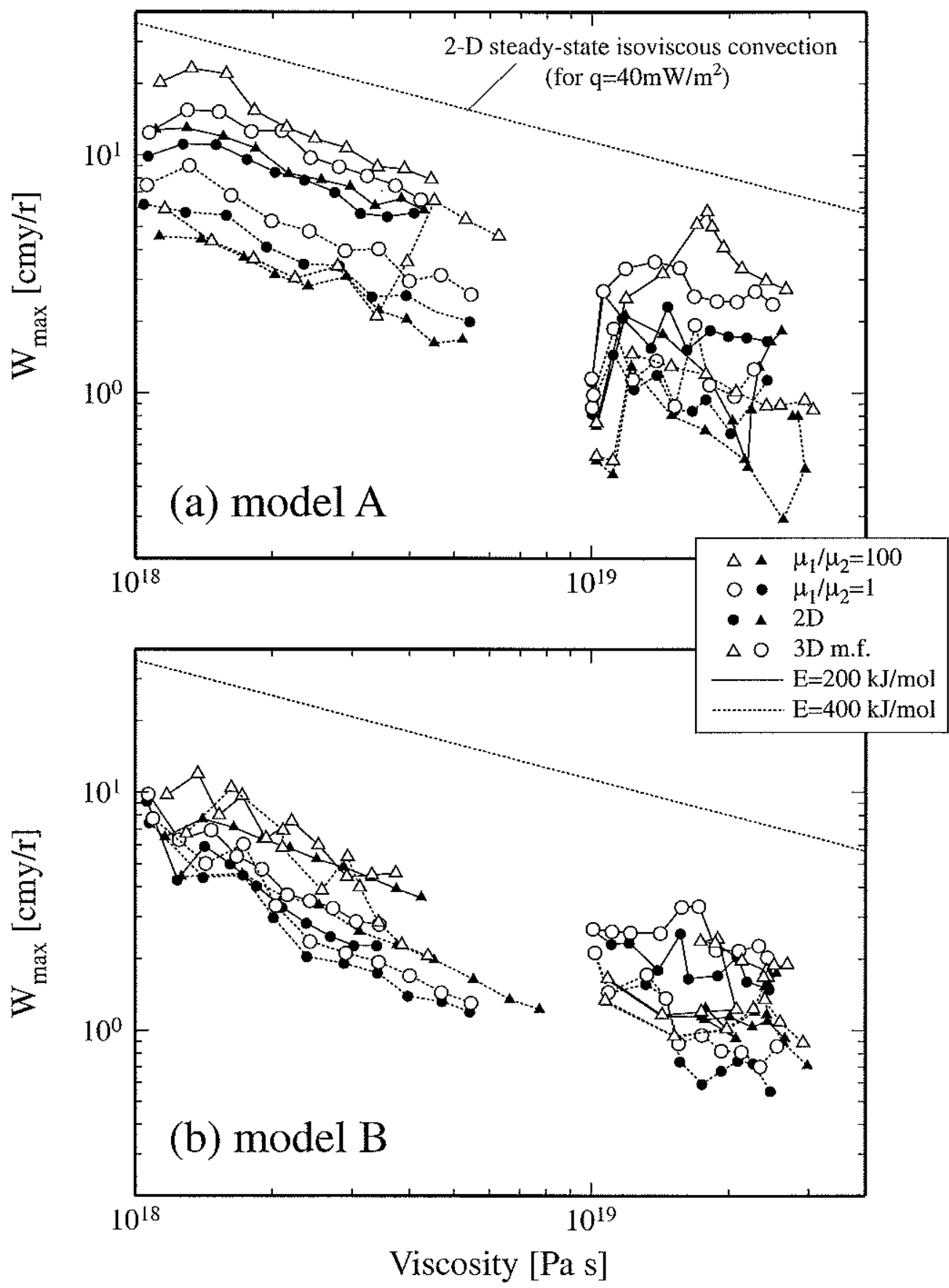

Figure 6-11. Summary of model evolution in terms of maximum vertical velocity and the viscosity of convecting domain calculated from its average temperature. Average values for a time interval of 20 m.y. are calculated for each transient solution with the duration of 200 m.y. Dotted line denotes the velocity scale of 2-D steady-state isoviscous convection corresponding to surface heat flux of $40 \mathrm{~mW} / \mathrm{m}^{2}$ estimated from the boundary layer theory (Figure 6-1a). 


\section{References}

Ahern, J.L., and D.L. Turcotte, Magma migration beneath an ocean ridge, Earth Planet Sci. Lett., 45, 115-122, 1979.

Ahrens, T.J., and G. Schubert, Gabbro-eclogite reaction rate and its geophysical significance, Rev. Geophys., 13, 383-400, 1975.

Aki, K., and P.G. Richards, Quantitative Seismology, W. H. Freeman \& Co., New York, 1980.

Allègre, C.J., M. Treuil, J.-F. Minster, B. Minster, and F. Albarède, Systematic use of trace element in igneous process, I. Fractional crystallization processes in volcanic suites, Contrib. Mineral. Petrol., 60, 57-75, 1977.

Allègre, C.J., and D.L. Turcotte, Implicatoins of a 2-component marble-cake mantle, Nature, 323, 123-127, 1986.

Anderson, D.L., Theory of the Earth, 366 pp., Blackwell Scientific Publications, Boston, 1989.

Anderson, D.L., Superplumes or supercontinents?, Geology, 22, 39-42, 1994.

Anderson, D.L., The EDGES of the Mantle, in The Core-Mantle Boundary Region, pp. 255-271, Americal Geophysical Union, Washington DC, 1998a.

Anderson, D.L., The scales of mantle convection, Tectonophysics, 284, 1-17, 1998 b.

Anderson, D.L., T. Tanimoto, and Y.-S. Zhang, Plate tectonics and hotspots; the third dimension, Science, 256, 1645-1651, 1992.

Asimow, P.D., A model that reconciles major- and trace-element data from abyssal peridotites, Earth Planet Sci. Lett., 169, 303-319, 1999.

Asimow, P.D., M.M. Hirschmann, and E.M. Stolper, An analysis of variations in isentropic melt productivity, Phil. Trans. R. Soc. Lond. A, 355, 255-281, 1997.

Baker, M.B., and E.M. Stolper, Determining the composition of high-pressure mantle melts using diamond aggregates, Geochim. Cosmochim. Acta, 58, 2811-2827, 1994.

Barnouin-Jha, K., E.M. Parmentier, and D.W. Sparks, Buoyant mantle upwelling and crustal production at oceanic spreading centers: On-axis segmentation and off-axis melting, J. Geophys. Res., 102,11979-11989, 1997.

Barton, A.J., and R.S. White, Crustal structure of Edoras Bank continental margin and mantle thermal anomalies beneath the North Atlantic, J. Geophys. Res., 102, 3109$3129,1997 \mathrm{a}$.

Barton, A.J., and R.S. White, Volcanism on the Rockall continental margin, J. Geol. Soc. Lond., 154, 531-536, 1997b.

Barton, P.J., The relationship between seismic velocity and density in continental crust - A useful constraint?, Geophys. J. R. astr. Soc., 87, 195-208, 1986.

Bender, J.F., F.N. Hodges, and A.E. Bence, Petrogenesis of basalts from the project FAMOUS area: experimental study from 0 to $15 \mathrm{kbars,} \mathrm{Earth} \mathrm{Planet} \mathrm{Sci.} \mathrm{Lett.,} 41$, 277-302, 1978.

Bender, J.F., C.H. Langmuir, and G.N. Hanson, Petrogenesis of basalt glasses from the Tamayo region, East Pacific Rise, J. Petrol., 25, 213-254, 1984.

Benn, K., A. Nicolas, and Y. Reuber, Mantle-crust transition zone and origin of wehrlitic magmas: Evidence from the Oman ophiolite, Tectonophysics, 151, 75-85, 1988.

Bernstein, B., High-pressure fractionation in rift-related basaltic magmatism: Faeroe plateau basalts, Geology, 22, 815-818, 1994.

Bernstein, S., P.B. Kelemen, and C.K. Brooks, Highly depleted spinel harzburgite xenoliths in Tertiary dykes from East Greenland: Restites from high degree melting, Earth Planet Sci. Lett., 154, 221-235, 1998.

Berryman, J.G., Mixture theories for rock properties, in A Handbook of Physical Constants: Rock Physics and Phase Relations, pp. 205-228, AGU, 1995. 
Bickel, S.H., Velocity-depth ambiguity of reflection traveltimes, Geophysics, 55, 1990.

Birch, F., The velocity of compressional waves in rocks to 10 kilobars, part 2, J. Geophys. Res., 66, 2199-2224, 1961.

Bishop, T.N., K.P. Bube, R.T. Cutler, R.T. Langan, P.L. Love, J.R. Resnick, R.T. Shuey, D.A. Spindler, and H.W. Wyld, Tomographic determination of velocity and depth in laterally varying media, Geophysics, 50, 903-923, 1985.

Blakely, R.J., Potential Theory in Gravity and Magnetic Applications, 441 pp., Cambridge University Press, New York, 1995.

Booker, J.R., Thermal convection with strongly temperature-dependent viscosity, J. Fluid Mech., 76, 741-754, 1976.

Bottinga, Y., and D.F. Weill, Densities of liquid silicate systems calculated from partial molar volumes of oxide components, Am. J. Sci., 269, 169-182, 1970.

Boudier, F., and A. Nicolas, Nature of the Moho Transition Zone in the Oman ophiolite, $J$. Petrol., 36, 777-796, 1995.

Boudier, F., A. Nicolas, and B. Ildefonse, Magma chambers in the Oman ophiolite: Fed from the top or the bottom?, Earth Planet Sci. Lett., 144, 239-250, 1996.

Boutilier, R.R., and C.E. Keen, Small-scale convection and divergent plate boundaries, $J$. Geophys. Res., 104, 7389-7403, 1999.

Boyd, F.R., Compositional distinction between oceanic and cratonic lithosphere, Earth Planet Sci. Lett., 96, 15-26, 1989.

Boyd, F.R., N.P. Pokhilenko, D.G. Pearson, S.A. Mertzman, N.V. Sobolev, and L.W. Finger, Composition of the Siberian cratonic mantle: Evidence from Udachnaya peridotite xenoliths, Contrib. Mineral. Petrol., 128, 228-246, 1997.

Brandsdóttir, B., W. Menke, P. Einarsson, R.S. White, and R.K. Staples, Färoe-Iceland Ridge Experiment 2. Crustal structure of the Krafla central volcano, J. Geophys. Res., 102, 7867-7886, 1997.

Brooks, A.N., and T.J.R. Hughes, Streamline upwind/Petrov-Galerkin formulations for convection dominated flows with particular emphasis on the incompressible Navier-Stokes equations, Comp. Meth. Appl. Mech. Eng., 32, 199-259, 1982.

Bube, K.P., R.T. Langan, and J.R. Resnick, Theoretical and numerical issues in the determination of reflector depths in seismic reflection tomography, J. Geophys. Res., 100, 12449-12458, 1995.

Buck, W.R., and E.M. Parmentier, Convection beneath young oceanic lithosphere: Implications for thermal structure and gravity, J. Geophys. Res., 91, 1961-1974, 1986.

Busse, F.H., On the stability of two-dimensional convection in a layer heated from below, J. Math. Phys., 46, 140-150, 1967.

Busse, F.H., A model of time-periodic flow, Geophys. J. R. astr. Soc., 52, 1-12, 1978.

Campbell, I.H., and R.W. Griffiths, Implications of mantle plume structure for the evolution of flood basalts, Earth Planet Sci. Lett., 99, 79-93, 1990.

Cannat, M., How thick is the magmatic crust at slow spreading oceanic ridges?, $J$. Geophys. Res., 101, 2847-2857, 1996.

Carlson, R.L., and C.N. Herrick, Densities and porosities in the oceanic crust and their variations with depth and age, J. Geophys. Res., 95, 9153-9170, 1990.

Carlson, R.L., and G.S. Raskin, Density of the ocean crust, Nature, 311, 555-558, 1984.

Cerveny, V., I. Molotkov, and I. Psencik, Ray Method in Seismology, University of Karlova, Prague, Czechoslovakia, 1977.

Chandrasekhar, S., Hydrodynamic and hydromagnetic stability, 654 pp., Dover, New York, 1981.

Charlson, G.S., and R.L. Sani, Thermoconvective instability in a bounded cylindrical fluid layer, Int. J. Mass Heat Transfer, 13, 1479-1496, 1970.

Charlson, G.S., and R.L. Sani, On thermoconvective instability in a bounded cylindrical fluid layer, Int. J. Mass Heat Transfer, 14, 2157-2160, 1971. 
Chen, Y.-Y., Boundary conditions and linear analysis of finite-cell Rayleigh-Bénard convection, J. Fluid Mech., 241, 549-585, 1992.

Chen, Y.J., A second melt lens at fast spreading mid-ocean ridges? (abstract), EOS, F837, 1998.

Chian, D., C. Keen, I. Reid, and K.E. Louden, Evolution of nonvolcanic rifted margins: New results from the conjugate margins of the Labrador Sea, Geology, 23, 589$592,1995$.

Christensen, N.I., Compressional wave velocities in possible mantle rocks to pressures of 30 kilobars, J. Geophys. Res., 79, 407-412, 1974.

Christensen, N.I., Compressional wave velocities in rocks at high temperatures and pressures, critical thermal gradients, and crustal low-velocity zones, J. Geophys. Res., 84, 6849-6857, 1979.

Christensen, N.I., and W.D. Mooney, Seismic velocity structure and composition of the continental crust: A global view, J. Geophys. Res., 100, 9761-9788, 1995.

Christensen, N.I., and M.H. Salisbury, Structure and constitution of the lower oceanic crust, Rev. Geophys., 13, 57-86, 1975.

Christensen, N.I., and G.H. Shaw, Elasticity of mafic rocks from the Mid-Atlantic Ridge, Geophys. J. R. astr. Soc., 20, 271-284, 1970.

Christensen, N.I., and J.D. Smewing, Geology and seismic structure of the northern section of the Oman ophiolite, J. Geophys. Res., 86, 2545-2555, 1981.

Christensen, N.I., and R.H. Wilkens, Seismic properties, density, and composition of the Icelandic crust near Raydarfjördur, J. Geophys. Res., 87, 6389-6395, 1982.

Christensen, U., A numerical model of coupled subcontinental and oceanic convection, Tectonophysics, 95, 1-23, 1983.

Christensen, U., Convection with pressure- and temperature-dependent non-Newtonian rheology, Geophys. J. R. astr. Soc., 77, 343-384, 1984.

Christensen, U.R., Time-dependent convection in elongated Rayleigh-Benard cells, Geophys. Res. Lett., 14, 220-223, 1987.

Clarke, D.B., Tertiary basalts of Baffin Bay: possible primary magmas from the mantle, Contrib. Mineral. Petrol., 25, 203-224, 1970.

Clarke, D.B., and M.J. O'Hara, Nickel, and the existence of high-MgO liquids in nature, Earth Planet Sci. Lett., 44, 153-158, 1979.

Clever, R.M., and F.H. Busse, Transition to time-dependent convection, J. Fluid Mech., 65, 625-645, 1974.

Coffin, M.F., and O. Eldholm, Large igneous provinces: crustal structure, dimensions, and external consequences, Rev. Geophys., 32, 1-36, 1994.

Cordery, M.J., and J. Phipps Morgan, Convection and melting at mid-ocean ridges, $J$. Geophys. Res., 98, 19477-19503, 1993.

Daines, M.J., and F.M. Richter, An experimental method for directly determining the interconnectivity of melt in a partially molten system, Geophys. Res. Lett., 15, $1459-1462,1988$.

Davaille, A., and C. Jaupart, Transient high-Rayleigh-number thermal convection with large viscosity variations, $J$. Fluid Mech., 253, 141-166, 1993.

Davaille, A., and C. Jaupart, Onset of thermal convection in fluids with temperaturedependent viscosity: Application to the oceanic mantle, J. Geophys. Res., 99, 19853-19866, 1994.

Davis, S.H., Convection in a box: linear theory, J. Fluid Mech., 30, 465-478, 1967.

Deal, M.M., and G. Nolet, Nullspace shuttles, Geophys. J. Int., 124, 372-380, 1996.

Demant, A., Mineral chemistry of volcanic sequences from Hole 917A, Southeast Greenland margin, Proc. Ocean Drill. Prog. Sci. Res., 152, 403-416, 1998.

DePaolo, D.J., Trace element and isotopic effects of combined wallrock assimilation and fractional crystallization, Earth Planet Sci. Lett., 53, 189-202, 1981.

Dick, H.J.B., R.L. Fisher, and W.B. Bryan, Mineralogic variability of the uppermost mantle along mid-ocean ridges, Earth Planet Sci. Lett., 69, 88-106, 1984. 
Dick, H.J.B., P.S. Meyer, S. Bloomer, S. Kirby, D. Stakes, and C. Mawer, Lithostratigraphic evolution of an in-situ section of oceanic layer 3, Proc. Ocean Drill. Program, Sci. Res., 118, 439-538, 1991.

Dick, H.J.B., P.T. Robinson, and P.S. Meyer, The plutonic foundation of a slow-spreading ridge, in Synthesis of Results from Scientific Drilling in the Indian Ocean, pp. 1-39, American Geophysical Union, 1992.

Dijkstra, E.W., A note on two problems in connection with graphs, Numer. Math., 1, 269$271,1959$.

Doin, M.-P., L. Freitout, and U. Christensen, Mantle convection and stability of depleted and undepleted continental lithosphere, J. Geophys. Res., 102, 2771-2787, 1997.

Duncan, R.A., H.C. Larsen, J.F. Allan, and the Shipboard Scientific Party, Proc. Ocean Drill. Prog. Init. Rep., Ocean Drilling Program, College Station, TX, 1996.

Elder, J., The Bowels of the Earth, Oxford University Press, 1976.

Eldholm, O., J. Thiede, and E. Taylor, The Norwegian continental margin: tectonics, volcanic and paleoenvironmental framework, Proc. Ocean Drill. Program, Sci. Results, 104, 5-26, 1989.

Falloon, T.J., D.H. Green, C.J. Hatton, and K.L. Harris, Anhydrous partial melting of a fertile and depleted peridotite from 2 to $30 \mathrm{~kb}$ and application to basalt petrogenesis, J. Petrol., 29, 1257-1282, 1988.

Fischer, R., and J.M. Lees, Shortest path ray tracing with sparse graphs, Geophysics, 58, 987-996, 1993.

Fleitout, L., and D.A. Yuen, Secondary convection and the growth of the oceanic lithosphere, Phys. Earth Planet. Int., 36, 181-212, 1984.

Forte, A.M., and J.X. Mitrovica, New inferences of mantle viscosity from joint inversion of long-wavelength mantle convection and post-glacial rebound data, Geophys. Res. Lett., 23, 1147-1150, 1996.

Foster, T.D., Stability of a homogeneous fluid cooled uniformly from above, Phys. Fluids, $8,1249-1257,1965$.

Fowler, S.R., R.S. White, G.D. Spence, and G.K. Westbrook, The Hatton Bank continental margin - II. Deep structure from two-ship expanding spread seismic profiles, Geophys. J., 96, 295 309, 1989.

Fram, M.S., and C.E. Lesher, Geochemical constraints on mantle melting during creation of the North Atlantic basin, Nature, 363, 712-715, 1993.

Fram, M.S., and C.E. Lesher, Generation and polybaric differentiation of East Greenland Early Tertiary flood basalts, J. Petrol., 38, 231-275, 1997.

Fram, M.S., C.E. Lesher, and A.M. Volpe, Mantle melting systematics: transition from continental to oceanic volcanism on the Southeast Greenland margin, Proc. Ocean Drill. Prog. Sci. Res., 152, 373-386, 1998.

Frey, F.A., C.-Y. Suen, and H.W. Stockman, The Ronda high temperature peridotite: Geochemistry and petrogenesis, Geochim. Cosmochim. Acta, 49, 2469-2491, 1985.

Frick, H., and R.M. Clever, The influence of side walls on finite-amplitude convection in a layer heated from below, J. Fluid Mech., 114, 467-480, 1982.

Fujii, T., and C.M. Scarfe, Composition of liquids coexisting with spinel lherzolite at 10 kbar and the genesis of MORBs, Contrib. Mineral. Petrol., 90, 18-28, 1985.

Gaherty, J.B., and T.H. Jordan, Lehmann discontinuity as the base of an anisotropic layer beneath continents, Science, 268, 1468-1471, 1995.

Gallo, G., and S. Pallottino, Shortest path methods: A unifying approach, Mathematical Programming Study, 26, 38-64, 1986.

Golitsyn, G.S., Simple theoretical and experimental study of convection with some geophysical applications and analogies, J. Fluid Mech., 95, 567-608, 1979.

Grand, S.P., Mantle shear structure beneath the Americas and surrounding oceans, $J$. Geophys. Res., 99, 11591-11621, 1994.

Granser, H., Nonlinear inversion of gravity data using the Schmidt-Lichtenstein approach, Geophysics, 52, 88-93, 1987. 
Gregory, R.T., and H.P. Taylor, Jr., An oxygen isotope profile in a section of Cretaceous oceanic crust, Samail ophiolite, Oman: Evidence for $\mathrm{d}^{18} \mathrm{O}$ buffering of the oceans by deep $(>5 \mathrm{~km})$ seawater-hydrothermal circulation at mid-ocean ridges, $J$. Geophys. Res., 86, 2737-2755, 1981.

Grove, T.L., R.J. Kinzler, and W.B. Bryan, Fractionation of mid-ocean ridge basalt (MORB), in Mantle Flow and Melt Generation at Mid-Ocean Ridges, pp. 281-310, American Geophysical Union, 1992.

Guspi, F., General 2D gravity inversion with density contrast varying with depth, Geoexploration, 26, 253-265, 1990.

Hager, B.H., and R.W. Clayton, Constraints on the structure of mantle convection using seismic observations, flow models, and the geoid, in Mantle Convection and Global Dynamics, edited by W.R. Peltier, pp. 657-763, Gordon and Breach Science Publishers, 1989.

Hager, B.H., R.W. Clayton, M.A. Richards, R.P. Comer, and A.M. Dziewonski, Lower mantle heterogeneity, dynamic topography and the geoid, Nature, 313, 541-545, 1985.

Hamilton, E.L., Sound velocity-density relations in sea-floor sediments and rocks, $J$. Acoust. Soc. Am., 63, 366-377, 1978.

Hansen, U., and A. Ebel, Time-dependent thermal convection - a possible explanation for a multi-scale flow in the earth's mantle, Geophys. J., 94, 181-191, 1988.

Hanson, G.N., and C.H. Langmuir, Modelling of major elements in mantle-melt systems using trace element approaches, Geochim. Cosmochim. Acta, 42, 725-741, 1978.

Hart, S.R., and K.E. Davis, Nickel partitioning between olivine and silicate melt, Earth Planet Sci. Lett., 40, 203-219, 1978.

Hart, S.R., and A. Zindler, In search of a bulk-earth composition, Chem. Geol., 57, 247$267,1986$.

Hashin, Z., and S. Shtrikman, A variational approach to the elastic behavior of multiphase materials, J. Mech. Phys. Solids, 11, 127-140, 1963.

Hauri, E.H., Major-element variability in the Hawaiian mantle plume, Nature, 382, 415$419,1996$.

Hemond, C.H., N.T. Arndt, U. Lichtenstein, A.W. Hofmann, N. Oskersson, and S. Steinthorsson, The heterogeneous Iceland plume: Nd-Sr-O isotopes and trace element constraints, J. Geophys. Res., 98, 15833-15850, 1993.

Henstock, T.J., A.W. Woods, and R.S. White, The accretion of oceanic crust by episodic sill intrusion, J. Geophys. Res., 98, 4143-4161, 1993.

Herring, J.R., Investigation of problems in thermal convection, J. Atmos. Sci., 20, 325-338, 1963.

Hestenes, M.R., Pseudoinverses and conjugate gradients, Comm. ACM, 18, 40-43, 1975.

Hill, R.I., Starting plumes and continental breakup, Earth Planet Sci. Lett., 104, 398-416, 1991.

Hill, R.I., I.H. Campbell, G.F. Davies, and R.W. Griffiths, Mantle plumes and continental tectonics, Science, 256, 1992.

Hinz, K., A hypothesis on terrestrial catastrophes wedges of very thick oceanward dipping layers beneath passive continental margins, Geol. Jb., E22, 3-28, 1981.

Hirose, K., and I. Kushiro, Partial melting of dry peridotites at high pressures: Determination of compositions of melts segregated from peridotite using aggregates of diamond, Earth Planet Sci. Lett., 114, 477-489, 1993.

Hirth, G., and D.L. Kohlstedt, Water in the oceanic mantle: Implications for rheology, melt extraction, and the evolution of the lithosphere, Earth Planet Sci. Lett., 144, 93108, 1996.

Holbrook, W.S., and P.B. Kelemen, Large igneous province on the US Atlantic margin and implications for magmatism during continental breakup, Nature, 364, 433-436, 1993. 
Holbrook, W.S., H.C. Larsen, J. Korenaga, T. Dahl-Jensen, I.D. Reid, P.B. Kelemen, J.R. Hopper, G.M. Kent, D. Lizarralde, S. Bernstein, and R.S. Detrick, Mantle thermal structure and melting processes during continental breakup in the North Atlantic, submitted to EPSL, 1999.

Holbrook, W.S., G.M. Purdy, R.E. Sheridan, L.G. III, M. Talwani, J. Ewing, and D. Hutchinson, Seismic structure of the U. S. Mid-Atlantic continental margin, $J$. Geophys. Res., 99, 17871-17891, 1994a.

Holbrook, W.S., E.C. Reiter, G.M. Purdy, D. Sawyer, P.L. Stoffa, J.A. Austin, Jr., J. Oh, and J. Makris, Deep structure of the U. S. Atlantic continental margin, offshore South Carolina, from coincident ocean bottom and multichannel seismic data, $J$. Geophys. Res., 99, 9155-9178, 1994b.

Honda, S., D.A. Yuen, S. Balachandar, and D. Reuteler, Three-dimensional instabilities of mantle convection with multiple phase transitions, Science, 259, 1308-1311, 1993.

Hopper, J.R., J.C. Mutter, R.L. Larson, C.Z. Mutter, and Northwest Australia Study Gruop, Magmatism and rift margin evolution: Evidence from northwest Australia, Geology, 20, 853-857, 1992.

Horsefield, S.J., R.B. Whitmarsh, R.S. White, and J.-C. Sibuet, Crustal structure of the Goban Spur rifted continental margin, NR Atlantic, Geophys. J. Int., 119, 1-19, 1994.

Howard, L.N., Convection at high Rayleigh number, in Proceedings of the Eleventh International Congress of Applied Mechanics, edited by H. Gortler, pp. 1109-1115, Springer-Verlag, New York, 1966.

Huang, P.Y., and S.C. Solomon, Centroid depths of mid-ocean ridge earthquakes: Dependence on spreading rate, J. Geophys. Res., 93, 13445-13477, 1988.

Hughes, T.J.R., The Finite Element Method: Linear Static and Dynamic Finite Element Analysis, 803 pp., Prentice-Hall, New Jersey, 1987.

Irving, A.J., A review of experimental studies of crystal/liquid trace element partitioning, Geochim. Cosmochim. Acta, 42, 743-770, 1978.

Ito, G., J. Lin, and C.W. Gable, Dynamics of mantle flow and melting at a ridge-centered hotspot: Iceland and the Mid-Atlantic Ridge, Earth Planet Sci. Lett., 144, 53-74, 1996.

Iturrino, G.J., N.I. Christensen, S. Kirby, and M.H. Salisbury, Seismic velocities and elastic properties of oceanic gabbroic rocks from Hole 735B, in Proceedings of the Ocean Drilling Program, Scientific Results, edited by R.P. Von Herzen, P.T. Robinson, and e. al., pp. 227-244, Ocean Drilling Program, College Station, 1991.

Iturrino, G.J., D.J. Miller, and N.I. Christensen, Velocity behavior of lower crustal and upper mantle rocks from a fast-spreading ridge at Hess Deep, in Proceedings of the Ocean Drilling Program, Scientific Results, edited by C. Mével, K.M. Gillis, A.J. F., and P.S. Meyer, pp. 417-440, Ocean Drilling Program, College Station, 1996.

Jackson, I., R.L. Rudnick, S.Y. O'Reilly, and C. Bezant, Measured and calculated elastic wave velocities for xenoliths from the lower crust and upper mantle, Tectonophysics, 173, 207-210, 1990.

Jakobsson, S.P., J. Jónsson, and F. Shido, Petrology of the western Reykjanes Peninsula, Iceland, J. Petrol., 19, 669-705, 1978.

Jannane, M., W. Beydoun, E. Crase, D. Cao, Z. Koren, E. Landa, M. Mendes, A. Pica, M. Noble, G. Roeth, S. Singh, R. Snieder, A. Tarantola, D. Trezeguet, and M. Xie, Wavelengths of earth structures that can be resolved from seismic reflection data, Geophysics, 54, 906-910, 1989.

Jaques, A.L., and D.H. Green, Anhydrous melting of peridotite at $0-15 \mathrm{~kb}$ pressure and the genesis of tholeiitic basalts, Contrib. Mineral. Petrol., 73, 287-310, 1980.

Jaupart, C., J.C. Mareschal, L. Guillou-Frottier, and A. Davaille, Heat flow and thickness of the lithosphere in the Canadian Shield, J. Geophys. Res., 103, 15269-15286, 1998. 
Jaupart, C., and B. Parsons, Convective instabilities in a variable viscosity fluid cooled from above, Phys. Earth Planet. Int., 39, 14-32, 1985.

Johnes, J.H., Experimental trace element partitioning, in A Handbook of Physical Constants: Rock Physics and Phase Relations, pp. 73-104, AGU, 1995.

Johnson, K.T.M., H.J.B. Dick, and N. Shimizu, Melting in the oceanic upper mantle: An ion microprobe study of diopsides in abyssal peridotites, $J$. Geophys. Res., 95, 2661-2678, 1990.

Jordan, T.H., Mineralogies, densities and seismic velocities of garnet lherolites and their geophysical implications, in The Mantle Sample: Inclusions in Kimberlites and Other Volcanics, pp. 1-14, AGU, 1979.

Jordan, T.H., Structure and formation of the continental tectosphere, in J. Petrol. Spec. Vol., pp. 11-37, 1988.

Karato, S., and P. Wu, Rheology of the upper mantle: A synthesis, Science, 260, 771-778, 1993.

Karner, G.D., and A.B. Watts, On isostacy at Atlantic-type continental margins, $J$. Geophys. Res., 87, 2923-2948, 1982.

Keen, C.E., and R.R. Boutilier, Lithosphere-asthenosphere interactions below rifts, in Rifted Ocean-Continent Boundaries, pp. 17-30, Kluwer, 1995.

Kelemen, P.B., and E. Aharonov, Periodic formation of magma fractures and generation of layered gabbros in the lower crust beneath oceanic spreading centers, in Faulting and Magmatism at Mid-Ocean Ridges, pp. 267-289, American Geophysical Union, 1998.

Kelemen, P.B., H.J.B. Dick, and J.E. Quick, Formation of harzburgite by pervasive melt/rock reaction in the upper mantle, Nature, 358, 635-641, 1992.

Kelemen, P.B., S.R. Hart, and S. Bernstein, Silica enrichment in the continental upper mantle via melt/rock reaction, Earth Planet Sci. Lett., 164, 387-406, 1998.

Kelemen, P.B., G. Hirth, N. Shimizu, M. Spiegelman, and H.J.B. Dick, A review of melt migration processes in the adiabatically upwelling mantle beneath oceanic spreading ridges, Phil. Trans. R. Soc. London A, 355, 283-318, 1997a.

Kelemen, P.B., and W.S. Holbrook, Origin of thick, high-velocity igneous crust along the U.S. East Coast Margin, J. Geophys. Res., 100, 10077-10094, 1995.

Kelemen, P.B., K. Koga, and N. Shimizu, Geochemistry of gabbro sills in the crust/mantle transition zone of the Oman ophiolite: Implications for the origin of the oceanic lower crust, Earth Planet Sci. Lett., 146, 475-488, 1997b.

Kelemen, P.B., N. Shimizu, and V.J.M. Salters, Extraction of mid-ocean-ridge basalt from the upwelling mantle by focused flow of melt in dunite channels, Nature, 375, 747 $753,1995$.

Kern, H., and J.M. Tubia, Pressure and temperature dependence of P- and S-wave velocities, seismic anisotropy and density of sheared rocks from the Sierra Alpujata massif (Ronda peridotites, Southern Spain), Earth Planet Sci. Lett., 119, 191-205, 1993.

King, S.D., and D.L. Anderson, An alternative mechanism of flood basalt formation, Earth Planet Sci. Lett., 136, 269-279, 1995.

King, S.D., and D.L. Anderson, Edge-driven convection, Earth Planet Sci. Lett., 160, 289296, 1998.

King, S.D., A. Raefsky, and B.H. Hager, ConMan: vectorizing a finite element code for incompressible two-dimensional convection in the earth's mantle, Phys. Earth Planet. Int., 59, 195-207, 1990.

Kinzler, R.J., Melting of mantle peridotite at pressures approaching the spinel to garnet transition: Application to mid-ocean ridge basalt petrogenesis, J. Geophys. Res., 102, 852-874, 1997.

Kinzler, R.J., and T.L. Grove, Primary magmas of mid-ocean ridge basalts 2. Applications, J. Geophys. Res., 97, 6907-6926, 1992. 
Kinzler, R.J., and T.L. Grove, Corrections and further discussion of the primary magmas of mid-ocean ridge basalts, 1 and 2, J. Geophys. Res., 98, 22339-22347, 1993.

Kinzler, R.J., T.L. Grove, and S.I. Recca, An experimental study on the effect of temperature and melt composition on the partitioning of nickel between olivine and silicate melt, Geochim. Cosmochim. Acta, 54, 1255-1265, 1990.

Klein, E.M., and C.H. Langmuir, Global correlations of ocean ridge basalt chemistry with axial depth and crustal thickness, J. Geophys. Res., 92, 8089-8115, 1987.

Klein, E.M., C.H. Langmuir, and H. Staudigel, Geochemistry of basalts from the Southeast Indian Ridge, $115^{\circ} \mathrm{E}-138^{\circ} \mathrm{E}, J$. Geophys. Res., 96, 2089-2107, 1991.

Kogiso, T., K. Hirose, and E. Takahashi, Melting experiments on homogeneous mixtures of peridotite and basalt: application to the genesis of ocean island basalts, Earth Planet Sci. Lett., 162, 45-61, 1998.

Korenaga, J., and P.B. Kelemen, Melt migration through the oceanic lower crust: A constraint from melt percolation modeling with finite solid diffusion, Earth Planet Sci. Lett., 156, 1-11, 1998.

Kuster, G.T., and M.N. Toksöz, Velocity and attenuation of seismic waves in two-phase media: Part I. Theoretical formulations, Geophysics, 39, 587-606, 1974.

Langmuir, C.H., Geochemical consequences of in situ crystallization, Nature, 340, 199 $205,1989$.

Langmuir, C.H., and G.N. Hanson, An evaluation of major element heterogeneity in the mantle sources of basalts, Phil. Trans. R. Soc. Lond. A, 297, 383-407, 1980.

Langmuir, C.H., E.M. Klein, and T. Plank, Petrological systematics of mid-ocean ridge basalts: Constraints on melt generation beneath ocean ridges, in Mantle Flow and Melt Generation at Mid-Ocean Ridges, pp. 183-280, American Geophysical Union, 1992.

Larsen, H.C., and R.A. Duncan, Introduction: Leg 163 background and objectives, Proc. Ocean Drill. Prog. Init. Rep., 163, 5-13, 1996.

Larsen, H.C., and S. Jakobsdóttir, Distribution, crustal properties and significance of seawards-dipping sub-basement reflectors off E Greenland, in Early Tertiary Volcanism and the Opening of the NE Atlantic, edited by A.C. Morton, and L.M. Parson, pp. 95-114, Geological Society of London, 1988.

Larsen, H.C., A.D. Saunders, P.D. Clift, and the Shipboard Scientific Party, Proc. Ocean Drill. Prog. Init. Rep., Ocean Drilling Program, College Station, TX, 1994.

Larsen, L.M., J.G. Fitton, and M.S. Fram, Volcanic rocks of the Southeast Greenland margin in comparison with other parts of the North Atlantic Tertiary igneous province, Proc. Ocean Drill. Prog. Sci. Res., 152, 315-330, 1998.

Larsen, T.B., and D.A. Yuen, Fast plumeheads: Temperature-dependent versus nonNewtonian rheology, Geophys. Res. Lett., 24, 1995-1998, 1997a.

Larsen, T.B., and D.A. Yuen, Ultrafast upwelling bursting through the upper mantle, Earth Planet Sci. Lett., 146, 393-399, 1997b.

LASE Study Group, Deep structure od the US East Coast passive margin from large aperture seismic experiments (LASE), Mar. Petrol. Geol., 3, 234-242, 1986.

Lawver, L.A., and R.D. Müller, Iceland hotspot track, Geology, 22, 311-314, 1994.

Lenardic, A., and L.-N. Moresi, Some thoughts on the stability of cratonic lithosphere: Effects of buoyancy and viscosity, J. Geophys. Res., 104, 12747-12758, 1999.

Lister, C.R.B., On the penetration of water into hot rock, Geophys. J. R. astr. Soc., 39, 465$509,1974$.

Lister, C.R.B., The basic physics of water penetration into hot rocks, in Hydrothermal Processes at Seafloor Spreading Centers, pp. 141-168, Plenum, New York, 1983.

Lizarralde, D., and W.S. Holbrook, U.S. mid-Atlantic margin structure and early thermal evolution, J. Geophys. Res., 102, 22855-22875, 1997.

Lundstrom, C.C., J. Gill, Q. Williams, and M.R. Perfit, Mantle melting and basalt extraction by equilibrium porous flow, Science, 270, 1958-1961, 1995. 
Malkus, W.V.R., The heat transport and spectrum of thermal turbulence, Proc. $R$. Soc. Lond. A, 225, 196-212, 1954.

Manning, C.E., and C.J. MacLeod, Fracture-controlled metamorphism of Hess Deep gabbros, Site 894: Constraints on the roots of mid-ocean ridge hydrothermal systems at fast-spreading centers, Proc. Ocean Drill. Program, Sci. Res., 147, 189$212,1996$.

Matarese, J.R., Nonlinear traveltime tomography, Ph.D. thesis, Massachusetts Institute of Technology, 1993.

Mavko, G., T. Mukerji, and J. Dvorkin, The Rock Physics Handbook, 329 pp., Cambridge University Press, New York, 1998.

McCollom, T.M., and E.L. Shock, Fluid-rock interactions in the lower oceanic crust: Thermodynamic models of hydrothermal alteration, J. Geophys. Res., 103, 547$575,1998$.

McDonough, W., and F. Frey, Rare earth elements of upper mantle rocks, in Geochemistry and Mineralogy of Rare Earth Elements, edited by B.R. Lipin, and G.R. McKay, pp. 99.-145, Mineralogical Society of America, Washington DC, 1989.

McDonough, W.F., and S.-S. Sun, The composition of the Earth, Chem. Geol., 120, 223$253,1995$.

McKenzie, D., Some remarks on the development of sedimentary basins, Earth Planet Sci. Lett., 40, 25-32, 1978.

McKenzie, D., The generation and compaction of partially molten rock, J. Petrol., 25, 713$765,1984$.

McKenzie, D., and M.J. Bickle, The volume and composition of melt generated by extension of the lithosphere, J. Petrol., 29, 625-679, 1988.

McKenzie, D.P., J.M. Roberts, and N.O. Weiss, Convection in the earth's mantle: towards a numerical simulation, J. Fluid Mech., 62, 465-538, 1974.

Menke, W., and D. Sparks, Crustal accretion model for Iceland predicts 'cold' crust, Geophys. Res. Lett., 22, 1673-1676, 1995.

Meyer, P.S., H. Sigurdsson, and J.-G. Schilling, Petrological and geochemical variations along Iceland's neovolcanic zones, J. Geophys. Res., 90, 10043-10072, 1985.

Minster, J.F., J.B. Minster, M. Treuil, and C.J. Allègre, Systematic use of trace elements in igneous process, II. Inverse problem of the fractional crystallization process in volcanic suites, Contrib. Mineral. Petrol., 61, 49-77, 1977.

Mitrovica, J.X., and G.T. Jarvis, A numerical study of thermal convection between rigid horizontal boundaries, Geophys. Astrophys. Fluid Dynamics, 38, 193-224, 1987.

Mjelde, R., P. Digranes, H. Shimamura, H. Shiobara, S. Kodaira, H. Brekke, T. Egebjerg, N. Sørenes, and S. Thorbjørnsen, Crustal structure of the northern part of the Vøring Basin, mid-Norway margin, from wide-angle seismic and gravity data, Tectonophysics, 293, 175-205, 1998.

Montagner, J.-P., and T. Tanimoto, Global upper mantle tomography of seismic velocities and anisotropies, J. Geophys. Res., 96, 20337-20351, 1991.

Morgan, J.V., and P.J. Barton, A geophysical study of the Hatton Bank volcanic margin: a summary of the results from a combined seismic, gravity and magnetic expriment, Tectonophysics, 173, 517-526, 1990.

Morgan, J.V., P.J. Barton, and R.S. White, The Hatton Bank continental margin - III. Structure from wide-angle OBS and multichannel seismic refraction profiles, Geophys. J., 96, 367-384, 1989.

Morrison, M.A., R.N. Thompson, and A.P. Dickin, Geochemical evidence for complex magmatic plumbing during development of a continental volcanic center, Geology, $13,581-584,1985$.

Moser, T.J., Shortest path calculation of seismic rays, Geophysics, 56, 59-67, 1991.

Moser, T.J., G. Nolet, and R. Snieder, Ray bending revisited, Bull. Seis. Soc. Am., 82, 259$288,1992$. 
Mutter, C.Z., and J.C. Mutter, Variations in thickness of layer 3 dominate oceanic crustal structure, Earth Planet Sci. Lett., 117, 295-317, 1993.

Mutter, J.C., W.R. Buck, and C.M. Zehnder, Convective partial melting; 1, A model for the formation of thick basaltic sequences during the initiation of spreading, $J$. Geophys. Res., 93, 1031-1048, 1988.

Mutter, J.C., M. Talwani, and P.L. Stoffa, Origin of seaward-dipping reflectors in oceanic crust off the Norwegian margin by "subaerial sea-floor spreading", Geology, 10, 353-357, 1982.

Mutter, J.C., M. Talwani, and P.L. Stoffa, Evidence for a thick oceanic crust adjacent to the Norwegian Margin, J. Geophys. Res., 89, 483-502, 1984.

Mutter, J.C., and C.M. Zehnder, Deep crustal structure and magmatic processes: the inception of seafloor spreading in the Norwegian-Greenland Sea, in Early Tertiary Volcanism and the Opening of the NE Atlantic, edited by A.C. Morton, and L.M. Parson, pp. 35-48, Blackwell, Boston, 1988.

Nabelek, P.I., Nickel partitioning between olivine and liquid in natural basalts: Henry's law behavior, Earth Planet Sci. Lett., 48, 293-302, 1980.

Nakamura, Y., P.L. Donoho, P.H. Roper, and P.M. McPherson, Large-offset seismic surveying using ocean-bottom seismograms and air guns: Instrumentation and field technique, Geophysics, 52, 1601-1611, 1987.

Nakanishi, I., and K. Yamaguchi, A numerical experiment on nonlinear image reconstruction from first-arrival times for two-dimensional island arc structure, $J$. Phys. Earth, 34, 195-201, 1986.

Nataf, H.C., C. Froidevaux, J.L. Levrat, and M. Rabinowicz, Laboratory convection experiments: Effect of lateral cooling and generation of instabilities in the horizontal boundary layers, J. Geophys. Res., 86, 6143-6154, 1981.

Nataf, H.C., and F.M. Richter, Convection experiments in fluids with highly temperaturedependent viscosity and the thermal evolution of the planets, Phys. Earth Planet. Int., 29, 320-329, 1982.

Nicholson, H., M. Condomines, A.E. Fallick, J.G. Fitton, K. Grönvold, and G. Rogers, Geochemical and isotopic evidence for crustal assimilation beneath Krafla, Iceland, J. Petrol., 32, 1005-1020, 1991.

Nicolas, A., and A. Prinzhofer, Cumulative or residual origin for the transition zone in ophiolites: structural evidence, J. Petrol., 24, 188-206, 1983.

Nicolas, A., I. Reuber, and K. Benn, A new magma chamber model based on structural studies in the Oman ophiolite, Tectonophysics, 151, 87-105, 1988.

O'Hara, M.J., Geochemical evolution during fractional crystallisation of a periodically refilled magma chamber, Nature, 266, 503-507, 1977.

O'Hara, M.J., and R.E. Matthews, Geochemical evolution in an advancing periodically replenished, periodically tapped, continuously fractionated magma chamber, $J$. Geol. Soc. London, 138, 237-277, 1981.

Ogawa, M., G. Schubert, and A. Zebib, Numerical simulations of three-dimensional thermal convection in a fluid with strongly temperature-dependent viscosity, $J$. Fluid Mech., 233, 299-328, 1991.

Oldenburg, D.W., The inversion and interpretation of gravity anomalies, Geophysics, 39 , 526-536, 1974.

Olson, P., A comparison of heat transfer laws for mantle convection at very high Rayleigh numbers, Phys. Earth Planet. Int., 48, 153-160, 1987.

Paige, C.C., and M.A. Saunders, LSQR: An algorithm for sparse linear equations and sparse least squares, ACM Trans. Math. Software, 8, 43-71, 1982.

Pallister, J.S., and C.A. Hopson, Samail Ophiolite plutonic suite: Field relations, phase variation, cryptic variation and layering, and a model of a spreading ridge magma chamber, J. Geophys. Res., 86, 2593-2644, 1981. 
Papazachos, C., and G. Nolet, $P$ and $S$ deep velocity structure of the Hellenic area obtained by robust nonlinear inversion of travel times, J. Geophys. Res., 102, 8349-8367, 1997.

Parker, R.L., The Rapid calculation of potential anomalies, Geophys. J. R. astr. Soc., 31, 447-455, 1972 .

Parker, R.L., and S.P. Huestis, The Inversion of magnetic anomalies in the presence of topography, J. Geophys. Res., 79, 1587-1593, 1974.

Parsons, B., and D. McKenzie, Mantle convection and the thermal structure of the plates, J. Geophys. Res., 83, 4485-4496, 1978.

Parsons, B., and J.G. Sclater, An analysis of the variation of ocean floor bathymetry and heat flor with age, J. Geophys. Res., 82, 803-827, 1977.

Pasay, Q.R., Upper mantle viscosity derived from the difference in rebound of the Provo and Bonneville shorelines: Lake Bonneville basin, Utah, J. Geophys. Res., 86, 11701-11708, 1981.

Pedersen, A.K., Reaction between picrite magma and continental crust: early Tertiary silicic basalts and magnesian andesites from Disko, West Greenland, Grønlands Geologiske Unders $\emptyset$ gelse Bulletin, 152, 1-126, 1985.

Phipps Morgan, J., The generation of a compositional lithosphere by mid-ocean ridge melting and its effect on subsequent off-axis hotspot upwelling and melting, Earth Planet Sci. Lett., 146, 213-232, 1997.

Phipps Morgan, J., and Y.J. Chen, The genesis of oceanic crust: Magma injection, hydrothermal circulation, and crustal flow, J. Geophys. Res., 98, 6283-6297, 1993.

Plank, T., and C.H. Langmuir, Effects of the melting regime on the composition of the oceanic crust, J. Geophys. Res., 97, 19749-19770, 1992.

Plank, T., M. Spiegelman, C.H. Langmuir, and D.W. Forsyth, The meaning of "mean F": Clarifying the mean extent of melting at ocean ridges, J. Geophys. Res., 100, 15045-15052, 1995.

Planke, S., Geophysical response of flood basalts from analysis of wire line logs: Ocean Drilling Program Site 642, Vøring volcanic margin, J. Geophys. Res., 99, 9279. 9296, 1994.

Planke, S., and H. Cambray, Seismic properties of flood basalts from Hole 917A downhole data, Southeast Greenland volcanic margin, in Proceedings of the Ocean Drilling Program, Scientific Results, pp. 453-462, Ocean Drilling Program, College Station, TX, 1998.

Presnall, D.C., S.A. Dixon, T.H. O'Donnell, N.L. Brenner, R.L. Schrock, and D.W. Dycus, Liquidus phase relations on the join Diopside-Forsterite-Anorthite from 1 atm to 20 kbar: Their bearing on the generation and crystallization of basaltic magma, Contrib. Mineral. Petrol., 66, 203-220, 1978.

Prothero, W.A., W.J. Taylor, and J.A. Eickemeyer, A fast, two-point, three-dimensional raytracing algorithm using a simple step search method, Bull. Seis. Soc. Am., 78, $1190-1198,1988$.

Quareni, F., and D.A. Yuen, Mean-field methods in mantle convection, in Methematical Geophysics, edited by N.J. Vlaar, G. Nolet, M.J.R. Wortel, and S.A.P.L. Cloetingh, pp. 227-264, D. Reidel Publishing Co., 1988.

Quareni, F., D.A. Yuen, G. Sewell, and U.R. Christensen, High Rayleigh number convection with strongly variable viscosity: A comparison between mean field and two-dimensional solutions, J. Geophys. Res., 90, 12633-12644, 1985.

Quick, J.E., and R.P. Denlinger, Ductile deformation and the origin of layered gabbro in ophiolites, J. Geophys. Res., 98, 14015-14027, 1993.

Rabinowicz, M., G. Ceuleneer, and A. Nicolas, Melt segregation and flow in mantle diapirs below spreading centers: Evidence from the Oman ophiolite, J. Geophys. Res., 92, 3475-3486, 1987. 
Rabinowicz, M., B. Lago, and C. Froidevaux, Thermal transfer between the continental asthenosphere and the oceanic subducting lithosphere: Its effect on subcontinental convection, J. Geophys. Res., 85, 1839-1853, 1980.

Raitt, R.W., The crustal rocks, in The Sea, edited by M.N. Hill, pp. 85-102, WileyInterscience, New York, 1963.

Reid, I., and H.R. Jackson, Crustal structure of northern Baffin Bay: Seismic refraction results and tectonic implications, J. Geophys. Res., 102, 523-542, 1997.

Ribe, N.M., U.R. Christensen, and J. Theißing, The dynamics of plume-ridge interaction, 1: Ridge-centered plumes, Earth Planet Sci. Lett., 134, 155-168, 1995.

Richards, M.A., R.A. Duncan, and V.E. Courtillot, Flood basalts and hot-spot tracks: Plume heads and tails, Science, 246, 103-107, 1989.

Richter, F.M., Dynamical models for sea floor spreading, Rev. Geophys. Space Phys., l1, 223-287, 1973.

Ringwood, A.E., Compositions and petrology of the Earth's mantle, McGraw-Hill, New York, 1975.

Ringwood, A.E., and D.H. Green, Experimental investigations bearing on the nature of the Mohorovicic discontinuity, Nature, 201, 566-567, 1964.

Ringwood, A.E., and D.H. Green, An experimental investigation of the gabbro-eclogite transformation and some geophysical implications, Tectonophysics, 3, 383-427, 1966.

Ritzwoller, M.H., and E.M. Lavely, Three-dimensional seismic models of the earth's mantle, Rev. Geophys., 33, 1-66, 1995.

Roberts, D.G., J. Backman, A.C. Morton, J.W. Murray, and J.B. Keene, Evolution of volcanic rifted margins: Synthesis of Leg 81 results on the west margin of Rockall Plateau, Init. Rep. Deep Sea Drill. Proj., 81, 883-911, 1984.

Roeder, P.L., and R.F. Emslie, Olivine-liquid equilibrium, Contrib. Mineral. Petrol., 29, 275-289, 1970.

Ross, W.S., The velocity-depth ambiguity in seismic traveltime data, Geophysics, 59, 830$843,1994$.

Rudnick, R.L., W.F. McDonough, and R.J. O'Connell, Thermal structure, thickness and composition of continental lithosphere, Chem. Geol., 145, 395-411, 1998.

Sato, H., Nickel content of basaltic magmas: identification of primary magmas and a measure of the degree of olivine fractionation, Lithos, 10, 113-120, 1977.

Scales, J.A., and R. Snieder, To Bayes or not to Bayes?, Geophysics, 62, 1045-1046, 1997.

Scarrow, J.H., and K.G. Cox, Basalts generated by decompressive adiabatic melting of a mantle plume: a case study from the Isle of Skye, NW Scotland, J. Petrol., 36, 322, 1995.

Schilling, J.-G., Upper mantle heterogeneities and dynamics, Nature, 314, 62-67, 1985.

Schilling, J.-G., P.S. Meyer, and R.H. Kingsley, Evolution of the Iceland hotspot, Nature, 296, 313-320, 1982.

Schilling, J.-G., M. Zajac, R. Evans, T. Johnson, W. White, J.D. Devine, and R. Kingsley, Petrologic and geochemical variations along the Mid-Atlantic Ridge from $29^{\circ} \mathrm{N}$ to $73^{\circ} \mathrm{N}$, Am. J. Sci., 283, 510-586, 1983.

Scotese, C.R., Paleomagnetism and the assembly of Pangaea, American Geophysical Union, 1984.

Scott, D.R., Small-scale convection and mantle melting beneath mid-ocean ridges, in Mantle Flow and Melt Generation at Mid-Ocean Ridges, pp. 327-352, American Geophysical Union, 1992.

Shapiro, S.S., Structure and dynamics of the continental tectosphere, PhD thesis, Massachusetts Institute of Technology, Cambridge, 1995.

Shaw, P.R., and J.A. Orcutt, Waveform inversion of seismic refraction data and applications to young Pacific crust, Geophys. J. R. astr. Soc., 82, 375-414, 1985.

Shor, G.G., Jr., H.W. Menard, and R.S. Raitt, Structure of the Pacific Basin, in The Sea, edited by A.E. Maxwell, pp. 3-27, Wiley-Interscience, New York, 1970. 
Simons, M., and B.H. Hager, Localization of the gravity field and the signature of glacial rebound, Nature, 390, 500-504, 1997.

Sinton, J.M., and R.S. Detrick, Mid-ocean ridge magma chambers, J. Geophys. Res., 97, 197-216, 1992.

Slater, L., Melt generation beneath Iceland, Ph.D. thesis, University of Cambridge, Cambridge, England, 1996.

Sleep, N.H., Hydrothermal circulation, anhydrite precipitation, and thermal structure at ridge axes, J. Geophys. Res., 96, 2375-2387, 1991.

Smith, W.H.F., and D.T. Sandwell, Marine gravity field from declassified Geosat and ERS-1 altimetry (abstract), Eos Trans. AGU, 76, F156, 1995.

Sobolev, A.V., and N. Shimizu, Ultra-depleted primary melt included in an olivine from the Mid-Atlantic Ridge, Nature, 363, 151-154, 1993.

Sobolev, S.V., and A.Y. Babeyko, Modeling of mineralogical composition, density and elastic wave velocities in anhydrous magmatic rocks, Surveys in Geophysics, 15, 515-544, 1994.

Solheim, L.P., and W.R. Peltier, Avalanche effects in phase transition modulated thermal convection, J. Geophys. Res., 99, 6997-7018, 1994.

Solomatov, V.S., Scaling of temperature- and stress-dependent viscosity convection, Phys. Fluids, 7, 266-274, 1995.

Sparks, D.W., E.M. Parmentier, and J. Phipps Morgan, Three-dimensional mantle convection beneath a segmented spreading center - implications for along-axis variations in crustal thickness and gravity, J. Geophys. Res., 98, 8153-8171, 1993.

Sparks, R.S.J., P. Meyer, and H. Sigurdsson, Density variation amongst mid-ocean ridge basalts: Implications for magma mixing and the scarcity of primitive lavas, Earth Planet Sci. Lett., 46, 419-430, 1980.

Spiegelman, M., Geochemical consequences of melt transport in 2-D: the sensitivity of trace elements to mantle dynamics, Earth Planet Sci. Lett., 118, 115-132, 1996.

Spudich, P., and J. Orcutt, A new look at the seismic velocity structure of the oceanic crust, Rev. Geophys. Space Phys., 18, 627-645, 1980.

Srivastava, S.P., and C.R. Tapscott, Plate kinematics of the North Atlantic, in The Western North Atlantic Region, edited by P.R. Vogt, and B.E. Tucholke, pp. 379-404, Geological Society of America, Boulder, CO, 1986.

Staples, R.K., R.S. White, B. Brandsdóttir, W. Menke, P.K.H. Maguire, and J.H. McBride, Färoe-Iceland Ridge Experiment 1. Crustal structure of northeastern Iceland, $J$. Geophys. Res., 102, 7849-7866, 1997.

Stoffa, P.L., J.T. Fokkema, R.M. de Luna Freire, and W.P. Kessinger, Split-step Fourier migration, Geophysics, 55, 410-421, 1990.

Stolper, E., and D. Walker, Melt density and the average composition of basalt, Contrib. Mineral. Petrol., 74, 7-12, 1980.

Stork, C., Singular value decomposition of the velocity-reflector depth tradeoff, Part 1: Introduction using a two-parameter model, Geophysics, 57, 927-932, 1992a.

Stork, C., Singular value decomposition of the velocity-reflector depth tradeoff, Part 2: High-resolution analysis of a generic model, Geophysics, 57, 933-943, 1992b.

Stork, C., and R.W. Clayton, Linear aspects of tomographic velocity analysis, Geophysics, 483-495, 1991.

Su, W., and W.R. Buck, Buoyancy effects on mantle flow under mid-ocean ridges, $J$. Geophys. Res., 98, 12191-12205, 1993.

$\mathrm{Su}$, W., R.L. Woodward, and A.M. Dziewonski, Degree 12 model of shear velocity heterogeneity in the mantle, J. Geophys. Res., 99, 6945-6980, 1994.

Tackley, P.J., On the penetration of an endothermic phase transition by upwellings and downwellings, J. Geophys. Res., 100, 15477-15488, 1995.

Tackley, P.J., and D. Stevenson, A mechanism for spontaneous self-perpetuating volcanism on the terrestorial planets, in Flow and Creep in the Solar System: 
Observations, Modeling and Theory, edited by D.B. Stone, and S.K. Runcorn, pp. 307-321, Kluwer Academic, 1993.

Tackley, P.J., D.J. Stevenson, G.A. Glatzmaier, and G. Schubert, Effects of an endothermic phase transition at $670 \mathrm{~km}$ depth in a spherical model of convection in the Earth's mantle, Nature, 361, 699-704, 1993.

Takahashi, E., Partitioning of $\mathrm{Ni}^{2+}, \mathrm{Co}^{2+}, \mathrm{Fe}^{2+}, \mathrm{Mn}^{2+}$ and $\mathrm{Mg}^{2+}$ between olivine and silicate melts: compositional dependence of partition coefficient, Geochim. Cosmochim. Acta, 42, 1829-1844, 1978.

Takahashi, E., and I. Kushiro, Melting of a dry peridotite at high pressures and basalt magma genesis, Am. Mineral., 68, 859-879, 1983.

Takahashi, E., K. Nakajima, and T.L. Wright, Origin of the Columbia River basalts: melting model of a heterogeneous plume head, Earth Planet Sci. Lett., 162, 63-80, 1998.

Tarantola, A., Inverse Problem Theory: Methods for Data Fitting and Model Parameter Estimation, 613 pp., Elsevier, New York, 1987.

Tarantola, A., and B. Valette, Inverse problems = quest for information, J. Geophys., 50, $159-170,1982$.

Tegner, C., C.E. Lesher, L.M. Larsen, and W.S. Watt, Evidence from the rare-earthelement record of mantle melting for cooling of the Tertiary Iceland plume, Nature, $395,591-594,1998$.

Telford, W.M., L.P. Geldart, and R.E. Sheriff, Appllied Geophysics, Cambridge University Press, New York, 1990.

The MELT Seismic Team, Imaging the deep seismic structure beneath a mid-ocean ridge: The MELT Experiment, Science, 280, 1215-1218, 1998.

Thompson, R.N., M.A. Morrison, A.P. Dickin, L.L. Gibson, and R.S. Harmon, Two contrasting styles of interaction between basic magma and continental crust in the British Tertiary Volcanic Province, J. Geophys. Res., 91, 5985-5997, 1986.

Thy, P., C.E. Lesher, and M.S. Fram, Low pressure experimental constraints on the evolution of basaltic lavas from Site 917, Southeast Greenland continental margin, Proc. Ocean Drill. Prog. Sci. Res., 152, 359-372, 1998.

Toomey, D.R., and G.R. Foulger, Tomographic inversion of local earthquake data from the Hengill-Grensdalur central volcano complex, Iceland, J. Geophys. Res., 94, 17497 $17510,1989$.

Toomey, D.R., S.C. Solomon, and G.M. Purdy, Microearthquakes beneath the median valley of the Mid-Atlantic Ridge near $23^{\circ} \mathrm{N}$ : Hypocenters and focal mechanisms, $J$. Geophys. Res., 90, 5443-5458, 1985.

Toomey, D.R., S.C. Solomon, and G.M. Purdy, Tomographic imaging of the shallow crustal structure of the East Pacific Rise at $9^{\circ} 30^{\prime}$ N, J. Geophys. Res., 99, 2413524157, 1994.

Tréhu, A.M., A. Ballard, L.M. Dorman, J.F. Gettrust, K.D. Klitgord, and A. Schreiner, Structure of the lower crust beneath the Carolina Trough, U.S. Atlantic continental margin, J. Geophys. Res., 94, 10585-10600, 1989.

Turcotte, D.L., and J. Phipps Morgan, The physics of magma migration and mantle flow beneath a mid-ocean ridge, in Mantle Flow and Melt Generation at Mid-Ocean Ridges, pp. 155-182, American Geophysical Union, 1992.

Turcotte, D.L., and G. Schubert, Geodynamics: Applications of continuum physics to geological problems, John Wiley \& Sons, New York, 1982.

Ulmer, $\mathrm{P}$., The dependence of the $\mathrm{Fe}^{2+}{ }^{2} \mathrm{Mg}$ cation-partitioning between olivine and basaltic liquid on pressure, temperature and composition: An experimental study to 30 kbars, Contrib. Mineral. Petrol., 101, 261-273, 1989.

Um, J., and C. Thurber, A fast algorithm for two-point seismic ray tracing, Bull. Seis. Soc. Am., 77, 972-986, 1987. 
Van Avendonk, H.J.A., An investigation of the crustal structure of the Clipperton transform fault area using 3D seismic tomography, Ph.D. thesis, University of California, San Diego, 1998.

Van Avendonk, H.J.A., A.J. Harding, and J.A. Orcutt, A two-dimensional tomographic study of the Clipperton transform fault, J. Geophys. Res., 103, 17885-17899, 1998.

Van Avendonk, H.J.A., A.J. Harding, and J.A. Orcutt, Error estimates for the shortest path method and ray bending for traveltime tomography applications, submitted to Geophysics, 1999.

Veevers, J.J., Middle-late Triassic $(230+5 \mathrm{Ma})$ singularity in the stratigraphic and magmatic history of the Pangean heat anomaly, Geology, 17, 784-787, 1989.

Velte, W., Stabilitätsverhalten und verzweigung stationärer lösungen der NavierStokesschen Gleichungen, Arch. Rat. Mech. Anal., 16, 97-125, 1964.

Vogt, P.R., Bermuda and Appalachian-Labrador rises: Common non-hotspot processes?, Geology, 19, 41-44, 1991.

Vogt, P.R., W.-Y. Jung, and J. Brozena, Arctic margin gravity highs remain puzzling, EOS Trans. $A G U, 79,601-606,1998$.

Walter, M.J., Melting of garnet peridotite and the origin of komatiite and depleted lithosphere, J. Petrol., 39, 29-60, 1998.

Watt, J.P., G.F. Davies, and R.J. O'Connell, The elastic properties of composite minerals, Rev. Geophys., 14, 541-563, 1976.

Watts, A.B., Gravity anomalies, crustal structure and flexure of the lithosphere at the Baltimore Canyon trough, Earth Planet Sci. Lett., 89, 221-239, 1988.

Watts, A.B., and C. Marr, Gravity anomalies and the thermal and mechanical structure of rifted continental margins, in Rifted Ocean-Continent Boundaries, edited by E. Banda, M. Torné, and M. Talwani, pp. 64-94, Kluwer Academic, Norwell, MA, 1995.

Watts, A.B., and S. Zhong, Observations of flexture and the rheology of oceanic lithosphere, submitted to Geophys. J. Int., 1999.

Weaver, J.S., and C.H. Langmuir, Calculation of phase equilibrium in mineral-melt systems, Comp. Geosci., 16, 1-19, 1990.

Weertman, J., The creep strength of the Earth's mantle, Rev. Geophys. Space Phys., 8, 146$168,1970$.

Weins, D.A., and S. Stein, Implications of oceanic intraplate seismicity for plate stresses, driving forces and rheology, Tectonophysics, 116, 143-162, 1985.

White, D.B., The planforms and onset of convection with a temperature-dependent viscosity, J. Fluid Mech., 191, 247-286, 1988.

White, D.J., and R.M. Clowes, Shallow crustal structure beneath the Juan de Fuca Ridge from 2-D seismic refraction tomography, Geophys. J. Int., 100, 349-367, 1990.

White, R., and D. McKenzie, Magmatism at rift Zones: The generation of volcanic continental margins and flood basalts, J. Geophys. Res., 94, 7685-7729, 1989.

White, R.S., and D. McKenzie, Mantle plumes and flood basalts, J. Geophys. Res., 100, 17543-17585, 1995.

White, R.S., D. McKenzie, and R.K. O'Nions, Oceanic crustal thickness from seismic measurements and rare earth element inversions, J. Geophys. Res., 97, 19683$19715,1992$.

White, R.S., G.D. Spence, S.R. Fowler, D.P. McKenzie, G.K. Westbrook, and A.N. Bowen, Magmatism at rifted continental margins, Nature, 330, 439-444, 1987.

Wilkens, R.H., G.J. Fryer, and J. Karsten, Evolution of porosity and seismic structure of upper oceanic crust: Importance of aspect ratios, J. Geophys. Res., 96, 17981$17995,1991$.

Wilson, J.T., Did the Atlantic close and then re-open?, Nature, 211, 676-681, 1966.

Wolfe, C.J., G.M. Purdy, D.R. Toomey, and S.C. Solomon, Microearthquake characteristics and crustal velocity structure at $29^{\circ} \mathrm{N}$ on the Mid-Atlantic Ridge; the 
architecture of a slow spreading segment, J. Geophys. Res., 100, 24449-24472, 1995.

Wood, D.A., J.-L. Joron, M. Treuil, M. Norry, and J. Tarney, Elemental and Sr isotope variations in basic lavas from Iceland and the surrounding ocean floor, Contrib. Mineral. Petrol., 70, 319-339, 1979.

Yilmaz, Ö., Seismic Data Processing, 526 pp., Society of Exploration Geophysicists, Tulsa, 1987.

Yuen, D.A., and L. Fleitout, Stability of the oceanic lithosphere with variable viscosity: an initial-value approach, Phys. Earth Planet. Int., 34, 173-185, 1984.

Zehnder, C.M., J.C. Mutter, and P. Buhl, Deep seismic and geochemical constraints on the nature of rift-induced magmatism during breakup of the North Atlantic, Tectonophysics, 173, 545-565, 1990.

Zelt, C.A., and R.B. Smith, Seismic traveltime inversion for $2-D$ crustal velocity structure, Geophys. J. Int., 108, 16-34, 1992.

Zhang, J., U.S. ten Brink, and M.N. Toksöz, Nonlinear refraction and reflection travel time tomography, J. Geophys. Res., 103, 29743-29757, 1998.

Zhang, J., and M.N. Toksöz, Nonlinear refraction traveltime tomography, Geophysics, 63, 1726-1737, 1998.

Zhang, K., and F.-H. Busse, Convection in spherical fluid shells with an outer crust of variable thickness, Phys. Earth Planet. Int., 104, 283-294, 1997.

Zhang, Y.-S., and T. Tanimoto, High-resolution global upper mantle structure and plate tectonics, J. Geophys. Res., 98, 9793-9823, 1993.

Zindler, A., S.R. Hart, F.A. Frey, and S.P. Jakobsson, Nd and Sr isotope ratios and REE abundances in Reykjanes Peninsula basalts: Evidence for mantle heterogeneity beneath Iceland, Earth Planet Sci. Lett., 45, 249-262, 1979. 


\section{Acknowledgements}

By looking back the last five years and half in the MIT/WHOI Joint Program, I was simply amazed by how fortunate I have been about advisers and how many people have influenced me. I had three great advisors: Steve Holbrook, whose scientific leadership and clear strategic thinking were always great support for my otherwise unsteady research path; Peter Kelemen, who single-handedly revolutionized the way I think about science through his guidance on igneous petrology; and Tom Jordan, whose scientific vision and theoretical elegance deeply influenced my research style.

Bob Detrick, Brad Hager, Jack Whitehead, and Maria Zuber kindly agreed to be on my thesis committee and provided a number of constructive comments and helpful suggestions. Uri ten Brink and Don Anderson also read a part of my thesis and provided several important suggestions.

My geophysics mentors also include Dan Lizarralde, Rafi Katzman, Graham Kent, John Collins, Ralph Stephen, Harm Van Avendonk, Greg Hirth, and Shijie Zhong. I couldn't imagine how dark my days at WHOI would have been if Dan weren't there. If Rafi didn't pushed me so hard to work with Tom, I couldn't have such an exciting time for the last two years of my $\mathrm{PhD}$ program. It was very fortunate for me that Shijie moved from Caltech to MIT just around the time when I started to work on mantle convection.

As my geochemistry mentors, I'd like to thank Ken Koga, Nobu Shimizu, Henry Dick, Peter Meyer, Stan Hart, and Tim Grove. It was sheer luck that I shared a house with Ken when I started to work with Peter. After dinner, almost every day, we talked about various topics in earth sciences for hours, and he was very patient to teach me the very basics of petrology and geochemistry.

I was also very lucky with having so many friends. A relaxing atmosphere at Woods Hole was colored by Javier Escartin, Garrett Ito, Gary Jarslow, Emilie Hooft, Laura Magde, Stefan Hussenoeder, Kelsey Jordahl, Rob Greaves, Debbie Hassler, Alberto Saal, Bill Shaw, Sheri White, Allegra Hosford, Jennifer Georgen, and Mike Braun. At MIT, I had great times with Frederik Simons, Mark Behn, Hrafnkell Kárason, Li Zhao, Jeff McGuire, Rebecca Saltzer, Eliza Richardson, Phil Tracadas, Oded Aharonson, Clint Conrad, Jim Van Orman, Kirsten Nicolaysen, Steve Parman, Marion Freybourger, Sebastian Chevrot, John Castle, Laurent Montesi, Liangjun Chen, and Hugh Cox.

My work at the WHOI ocean bottom laboratory was always cheerfully supported by software and hardware super-experts like Jim Dolan, Dave Dubois, Beecher Wooding, Ken Peal, and Peter Lemmond. Linda Meinke and Amanda Bourque were very helpful to facilitate my life at MIT.I would like to thank the Joint Program education office for maintaining such an inspiring $\mathrm{PhD}$ program, especially Abbie Charlotte Alvin, Jake Peirson, Julia Westwater, Marcey Simon, and Ronni Schwartz. 